\title{
Macroheterocyclic Compounds - a Key Building Block in New Functional Materials and Molecular Devices
}

\author{
Oskar I. Koifman, ${ }^{\mathrm{a}, \mathrm{b} @ 1}$ Tatyana A. Ageeva, ${ }^{\mathrm{a}}$ Irina P. Beletskaya, $^{\mathrm{c}, \mathrm{d} @ 2}$ Alexei D. Averin, $^{\mathrm{c}, \mathrm{d}}$ \\ Alexei A. Yakushev, ${ }^{\mathrm{c}}$ Larisa G. Tomilova, ${ }^{\mathrm{c}, \mathrm{e} @ 3}$ Tatiana V. Dubinina, $^{\mathrm{c}, \mathrm{e}}$ Aslan Yu. Tsivadze, ${ }^{\mathrm{d}, \mathrm{f}}$ \\ Yulia G. Gorbunova, ${ }^{\text {d,f }}$ Alexander G. Martynov, ${ }^{\text {d@4 }}{ }^{\text {Dmitri V. Konarev, }}{ }^{\text {g@5 }}$ Salavat S. Khasanov, $^{\text {h }}$ \\ Rimma N. Lyubovskaya, ${ }^{\text {g }}$ Tatyana N. Lomova, ${ }^{\text {b@6 }}$ Viktor V. Korolev, ${ }^{\text {b }}$ Eduard I. Zenkevich, ${ }^{\text {i@7 }}$ \\ Thomas Blaudeck, ${ }_{r}{ }^{\text {,k }}$ Christian von Borczyskowski, ${ }^{1}$ Dietrich R.T. Zahn, ${ }^{k, 1}$ Andrey F. Mironov, ${ }^{\text {m } @ 8}$

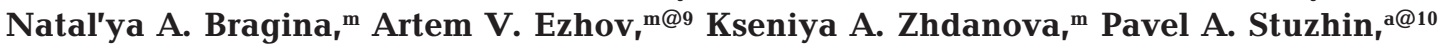 \\ Georgy L. Pakhomov, ${ }^{n}$ Nataliya V. Rusakova, ${ }^{\circ 11}$ Nikolay N. Semenishyn, ${ }^{\circ}$ Sergey S. Smola ${ }^{\circ}$ \\ Vladimir I. Parfenyuk, ${ }^{\text {b } 12}$ Arthur S. Vashurin, ${ }_{r}{ }^{@ 13}$ Sergei V. Makarov, ${ }^{\text {a }}$ Ilia A. Dereven'kov, ${ }^{\text {a }}{ }^{14}$ \\ Nugzar Zh. Mamardashvili, ${ }^{\text {b15 }}$ Tigran S. Kurtikyan, ${ }^{p}$ Garik G. Martirosyan, ${ }^{\text {p@16 }}$ \\ Vladimir A. Burmistrov ${ }^{a}{ }^{\circledR 17}$ Viktor V. Aleksandriiskii, ${ }^{a}$ Igor V. Novikov ${ }^{a}{ }^{a}$ Dmitriy A. Pritmov ${ }^{\mathrm{m}}$ \\ Mikhail A. Grin ${ }^{\mathrm{m} @ 18}$ Nikita V. Suvorov $^{\mathrm{m}}{ }^{\mathrm{An}}$ Antoliy A. Tsygankov, ${ }^{\mathrm{q}}$ Alexey Yu. Fedorov, ${ }^{\mathrm{r} @ 19}$ \\ Natalia S. Kuzmina, ${ }^{r}$ Alexander V. Nyuchev ${ }_{r}{ }^{r}$ Vasilii F. Otvagin, ${ }^{\mathrm{r}}$ Andrey V. Kustov ${ }_{r}^{\mathrm{a}, \mathrm{b}}$ \\ Dmitry V. Belykh, ${ }^{\mathrm{s}}$ Dmitry B. Berezin, ${ }^{\mathrm{a} @ 20}$ Anna B. Solovieva, ${ }^{\mathrm{\varrho} 21}{ }^{\mathrm{r}}$ Peter S. Timashev $_{\mathrm{r}}^{\mathrm{t}, \mathrm{u}}$

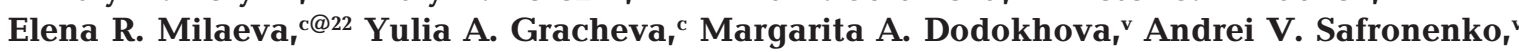
Dmitry B. Shpakovsky, ${ }_{r}$ Sergey A. Syrbu, ${ }^{\text {b }}$ Yury A. Gubarev, ${ }^{\text {b23 }}{ }^{2}$ Alexey N. Kiselev, ${ }^{\text {b }}$ Mikhail O. Koifman $_{r}{ }^{a}$ Natalia Sh. Lebedeva, ${ }^{b}$ and Elena S. Yurina ${ }^{b}$

\section{Dedicated to the 90th Anniversary of Ivanovo State University of Chemistry and Technology}

\begin{abstract}
${ }^{a}$ Research Institute of Macroheterocyclic Compounds, Ivanovo State University of Chemistry and Technology, 153000 Ivanovo, Russia

${ }^{\mathrm{b}}$ G.A. Krestov Institute of Solution Chemistry of the Russian Academy of Sciences, 153045 Ivanovo, Russia 'Department of Chemistry, Lomonosov Moscow State University, 119991 Moscow, Russia

${ }^{\mathrm{d}}$ A.N. Frumkin Institute of Physical Chemistry and Electrochemistry of the Russian Academy of Sciences, 119071 Moscow, Russia
\end{abstract}

${ }^{\mathrm{e}}$ Institute of Physiologically Active Compounds of the Russian Academy of Sciences, 142432 Chernogolovka, Moscow Region, Russia

${ }_{\mathrm{f}}^{\mathrm{f}}$ N.S. Kurnakov Institute of General and Inorganic Chemistry of the Russian Academy of Sciences, 119991 Moscow, Russia ${ }^{\mathrm{g}}$ Institute of Problems of Chemical Physics of the Russian Academy of Sciences, 142432 Chernogolovka, Moscow region, Russia ${ }^{\mathrm{h}}$ Institute of Solid State Physics of the Russian Academy of Sciences, 142432 Chernogolovka, Moscow region, Russia iBelarussian National Technical University, 220013 Minsk, Belarus

${ }^{\mathrm{j}}$ Fraunhofer Institute for Electronic Nano Systems (ENAS), Technologie-Campus 3, 09126 Chemnitz, Germany

${ }^{\mathrm{k}}$ Chemnitz University of Technology, Research Center for Materials, Architectures and Integration of Nanomembranes (MAIN), 09107 Chemnitz, Germany

${ }^{1}$ Chemnitz University of Technology, Institute of Physics, 09107 Chemnitz, Germany

${ }^{\mathrm{m}}$ MIREA-Russian Technological University, 119454 Moscow, Russia

${ }^{\mathrm{n}}$ Institute for Physics of Microstructures of the Russian Academy of Sciences, GSP-105, 603950 Nizhny Novgorod, Russia ${ }^{\circ}$ A.V. Bogatsky Physico-Chemical Institute of the National Academy of Sciences of Ukraine, 65080 Odessa, Ukraine ${ }^{\mathrm{p} S}$ Scientific Technological Center of Organic and Pharmaceutical Chemistry NAS RA, Molecule Structure Research Centre, 0014 Yerevan, Armenia

${ }^{\mathrm{q}}$ Institute of Basic Biological Problems of Russian Academy of Sciences, 142290 Pushchino, Moscow region, Russia ${ }^{\mathrm{r}}$ N.I. Lobachevsky State University of Nizhny Novgorod, 603950 Nizhny Novgorod, Russia

${ }^{\mathrm{s}}$ Institute of Chemistry of the Federal Research Center "Komi Scientific Center of the Ural Branch of the Russian Academy of Sciences", 167000, Syktyvkar, Russia

${ }^{t}$ N.N. Semenov Federal Research Center for Chemical Physics of the Russian Academy of Sciences, 119991 Moscow, Russia "Institute for Regenerative Medicine, I.M. Sechenov University, 119991 Moscow, Russia

${ }^{\mathrm{v}}$ Rostov State Medical University, 344022 Rostov-on-the-Don, Russia

E-mails: @11koifman@isuct.ru; @2 beletska@org.chem.msu.ru; @3tom@med.chem.msu.ru;

@4Martynov.Alexandre@gmail.com; ${ }^{55}$ konarev3@yandex.ru; $@{ }^{1}$ tnl@isc-ras.ru; @7 zenkev@tut.by; ${ }^{@ 8}$ mironov@mitht.ru;

@99artem.ejov@gmail.com; @10 stuzhin@isuct.ru; @11 natavrusakova@gmail.com; @12vip@isc-ras.ru; ${ }^{13}$ asvashurin@mail.ru; 
Macroheterocyclic Compounds - a Key Building Block in New Functional Materials and Molecular Devices

@14derevenkov@gmail.com; @15.ngm@isc-ras.ru; @16 ggmartirosyan@gmail.com; @17burmistrov@isuct.ru;

@18michael_grin@mail.ru; @19afedorovnn@yandex.ru; ${ }^{920}$ berezin@isuct.ru; ${ }^{21}$ ann.solovieva@gmail.com;

@22milaeva@med.chem.msu.ru; @23yury.gu@gmail.com

The review discusses the latest advances in the directed synthesis and application of macroheterocyclic compounds in science, engineering and technology, viz. as catalysts for various processes in photo- and electrocatalysis, optical chemosensors for metal cations, selective receptors of organic compounds, inductors and selectors, in nonlinear optics, organic electronics, as magnets, photosensitizers for PDT of a number of oncological diseases and for antimicrobial PDT, etc.

Keywords: Tetrapyrrolic compounds, porphyrins, phthalocyanines, metal complexes, chlorins, cobalamins, synthesis, application, catalysis, photosensitizers, photovoltaics, optical properties, magnetic properties, sensors, interaction with proteins.

\section{Макрогетероциклические соединения - ключевое звено в созАании новых функциональных материалов и молекулярных устройств}

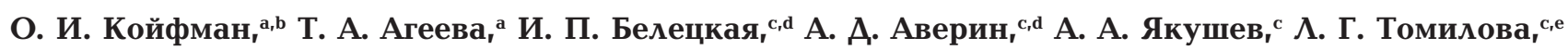
Т. В. Аубинина, ${ }^{\text {,e }}$ А. Ю. ЦиваАзе, ${ }^{d, f}$ Ю. Г. Горбунова, ${ }^{d, f}$ А. Г. Мартынов, ${ }_{r}^{d}$. В. Конарев, ${ }^{g}$

С. С. Хасанов, ${ }^{\text {h }}$ Р. Н. Аюбовская,,${ }^{9}$ Т. Н. Аомова, ${ }^{b}$ В. В. Королев, ${ }^{\text {b }}$ Э. И. Зенькевич, ${ }^{i}$ Т. Блаудек, ${ }^{j, k}$ К. фон Борцисковски, ${ }^{1}$ A. Р. Т. Цан, ${ }_{r}{ }^{k, l}$ А. Ф. Миронов, ${ }^{m}$ Н. А. Брагина, ${ }^{\mathrm{m}}$ А. В. Ежов, ${ }^{\mathrm{m}}$ К. А. ЖАанова, ${ }^{\mathrm{m}}$

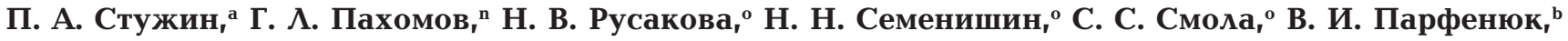

А. С. Вашурин, ${ }^{a}$ С. В. Макаров, ${ }^{a}$ И. А. Аеревеньков, ${ }^{a}$ Н. Ж. Мамардашвили, ${ }^{b}$ Т. С. Куртикян, ${ }^{p}$ Г. Г. Мартиросян, ${ }^{p}$ В. А. Бурмистров, ${ }^{a}$ В. В. Александрийский, ${ }^{a}$ И. В. Новиков, ${ }^{a}$ А. А. Притьмов, ${ }^{\mathrm{m}}$ М. А. Грин, ${ }^{\text {m }}$ Н. В. Суворов, ${ }^{\text {m }}$ А. А. Цыганков, ${ }^{q}$ А. Ю. ФёАоров, ${ }^{\mathrm{r}}$ Н. С. Кузьмина, ${ }^{\mathrm{r}}$ А. В. Нючев, ${ }^{\mathrm{r}}$ В. Ф. Отвагин, ${ }^{r}$ А. В. Кустов, ${ }^{a, b}$ A. В. Белых, ${ }_{r}^{\text {s }}$ А. Б. Березин, ${ }^{a}$ А. Б. Соловьёва, ${ }^{t}$ П. С. Тимашев, ${ }_{r}^{\text {t,u }}$ Е. Р. Милаева, ${ }^{\mathrm{c}}$ Ю. А. Грачёва, ${ }^{\mathrm{c}}$ М. А. Аодохова, ${ }^{\mathrm{v}}$ А. В Сафроненко, ${ }^{\mathrm{v}}$ А. Б. Шпаковский, ${ }^{\mathrm{c}}$ С. А. Сырбу,

\section{0-летию Ивановского госуgарственного химико-технологического университета посвящается}

\footnotetext{
`Институт макрогетероциклических соединений, Ивановский государственный химико-технологический университет, 153000 Иваново, Россия

${ }^{\mathrm{b}}$ Институт химии растворов им. Г.А. Крестова РАН, 153045 Иваново, Россия

${ }^{\mathrm{c}}$ Химический факультет, Московский государственный университет имени М.В. Ломоносова, 119991 Москва, Россия

${ }^{\mathrm{d}}$ Институт физической химии и электрохимии им. А.Н. Фрумкина РАН, 119071 Москва, Россия

' Институт физиологически активных соединений РАН, 142432 Черноголовка, Московская обл., Россия

${ }^{\mathrm{f}}$ Институт общей и неорганической химии им. Н.С. Курнакова РАН, 119991 Москва, Россия

' Институт проблем химической физики РАН, 142432 Черноголовка, Московская обл., Россия

h Институт физики твердого тела РАН, 142432 Черноголовка, Московская обл., Россия

іБелорусский национальный технический университет, 220013 Минск, Беларусь

јИнститут электронных наносистем им. Фраунгофера (ENAS), 09126 Хемнии, Германия

${ }^{\mathrm{k}}$ Технологический университет Хемница, Исследовательский иентр материалов, архитектуры и интеграции наномембран (MAIN), 09107 Хемнии, Германия

${ }^{1}$ Хемницкий технологический университет, Институт физики, 09107 Хемнии, Германия

${ }^{\mathrm{m}}$ МИРЭА-Российский технологический университет, 119454, Москва, Россия

${ }^{\mathrm{n}}$ Институт физики микроструктур РАН, 603950 Нижний Новгород, Россия

${ }^{\circ}$ Физико-химический институт им. А.В. Богатского НАНУ, 65080 Одесса, Украина

р Научно-технологический центр органической и фармацевтической химии НАН РА, Центр исследования структуры молекул, 0014 Ереван, Армения

qИнститут фундаментальных проблем биологии РАН, 142290 Пущчин, Московская область, Россия ${ }^{\mathrm{r}}$ Нижегородский государственный университет им. Н.И. Лобачевского, 603950 Нижний Новгород, Россия 'Институт химии Коми научного иентра Уральского отделения РАН, 167000, Сыктывкар, Россия
} 
'Федеральный исследовательский центр химической физики им. Н.Н. Семенова РАН, 119991 Москва, Россия

"Институт регенеративной медицины Университета им. И.М. Сеченова, 119991 Москва, Россия

vРостовский государственный медииинский университет, 344022 Ростов-на-Дону, Россия

В обзоре рассмотрены последние достижения в области направленного синтеза и применения макрогетероичиклиеских соединений в науке, технике и технологии, а именно: в качестве катализаторов различньх процессов, в фото- и электрокатализе, оптических хемосенсоров для катионов металлов, селективных рецепторов органических соединений, индукторов и селекторов, в нелинейной оптике, органической электронике, в качестве магнитов, фотосенсибилизаторов для фотодинамической терапии (ФДТ) ряда онкозаболеваний и для антимикробной ФДТ и т.д.

Ключевые слова: Тетрапиррольные соединения, порфирины, фталоцианины, металлокомплексы, хлорины, кобаламины, синтез, применение, катализ, фотосенсибилизаторы, фотовольтаика, оптические свойства, магнитные свойства, сенсорные свойства, взаимодействие с белками.

\section{Content}

Introduction.

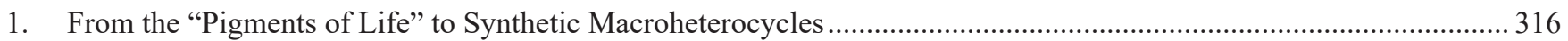

2. Synthesis of Porphyrin-Azamacrocycle Conjugates via Pd-Catalyzed Amination Reactions .........................................320

3. New Advances in the Application of Phthalocyanines and Their Analogues .......................................................... 325

4. Design of Single Molecule Magnets Based on Binuclear Lanthanide Complexes with Tetrapyrrolic Ligands............. 332

5. Influence of Axial Coordination and Functional Cations on Optical and Magnetic Properties

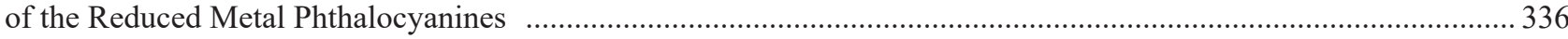

6. Synthesis of Paramagnetic Metalloporphyrins as Promising Materials with Magnetocaloric Activity ........................ 343

7. Functional Porphyrins as Specific Probes of Surface Morphology, Optical Properties, and Exciton Relaxation Processes for Semiconductor Quantum Dots .................................................................. 353

8. Tetrapyrroles and Related Compounds for Photovoltaics....................................................................... 360

9. 1,2,5-Chalcogenadiazole Analogues of Phthalocyanines - Perspective Acceptors for Organic Electronics................. 364

10. Luminescence of Lanthanides in Complexes with Polytopic Tetrapyrrole Macrocycles ...........................................369

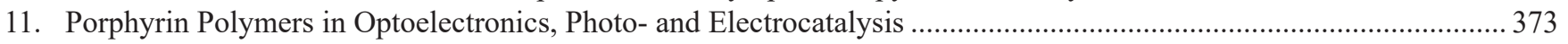

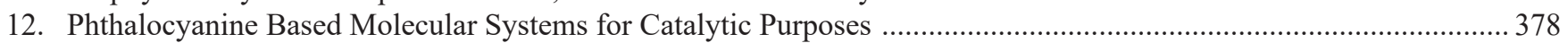

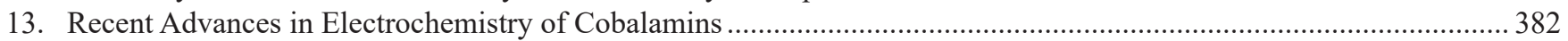

14. Macrocyclic Receptors for Recognition and Selective Binding of Substrates of Different Nature.............................385

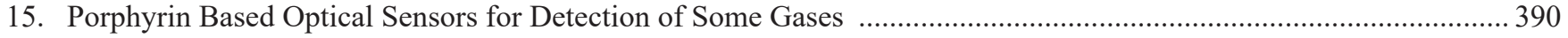

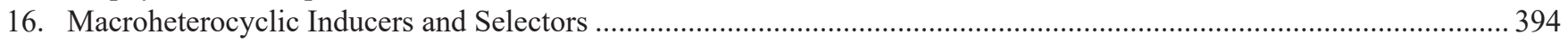

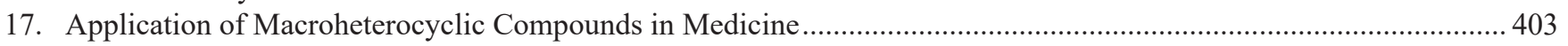

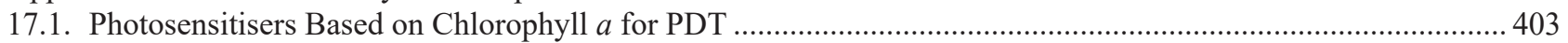

17.2. Fundamental and Applied Aspects of Bacteriochlorophyll Derivatives Chemistry ......................................... 410

17.3. The Porphyrinoid-Based Conjugates as a New Paradigm in Targeted Photodynamic Therapy of Cancer ........... 423

17.4. Photodynamic Inactivation of Pathogenic Microorganisms with the Chlorin $e_{6}$-Type Sensitizers: from in vitro to in vivo Studies.

17.5. Polymeric Porphyrin-Containing Systems for Antimicrobial Photodynamic Therapy ..................................... 435

17.6. Attenuation of in vivo Toxicity of the Organotin Compound by the Porphyrin Modified with Peripheral 2,6-Di-tert-butylphenol Antioxidant Groups........................................................................ 438

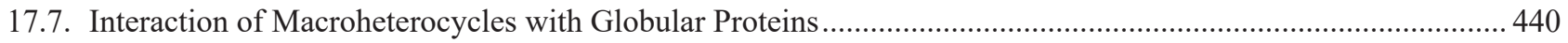

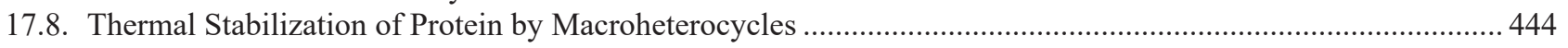

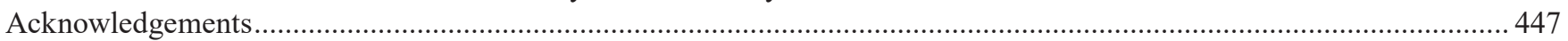

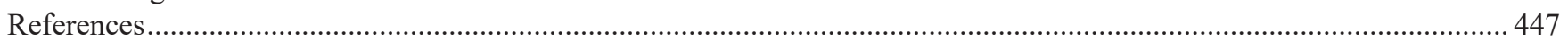




\section{Introduction}

Macroheterocyclic compounds (MHCs) have a wide range of unique properties: high stability, high catalytic activity in chemical, photochemical and electrochemical processes, biological activity.

The potential capabilities of MHCs are fully realized in the composition of hybrid and composite materials, giving them a certain functionality. As a result, there are ample opportunities for creating new materials based on MHCs: catalysts for a number of important processes, materials for medical and agricultural purposes, materials for separating structurally similar compounds, optical limiters, photosensitizers, solar energy converters, optosensor and electrically conductive materials, etc.

Many aspects of the synthesis and application of MHCs were described in the monograph Functional Materials Based on Tetrapyrrole Macroheterocyclic Compounds, Moscow: URSS, 2019. 848 p.

It can be assumed that further studies of MHCs will expand the areas of possible application of these compounds.

This issue of the journal publishes a review of domestic and foreign scientists working in the field of synthesis and application of macroheterocyclic compounds. A team of authors from 22 universities and research institutes have presented their vision in 17 sections of the review.

Obviously, this review is not able to cover all aspects of the problem under consideration; however, one would like to hope that it will serve the further development of studies in the field of application of this promising class of compounds.

\section{From the "Pigments of Life" to Synthetic Macroheterocycles}

In the course of evolution, nature has created a number of tetrapyrrole macroheterocyclic compounds, without which life on Earth is impossible. Their most famous representatives are chlorophylls, bacteriochlorophylls, and hemes of plant, bacterial, or animal origin, called "life pigments". ${ }^{[1-3]}$ Green pigments chlorophylls and bacteriochlorophylls, red-brown hemes, red cobalamins, vitamin $\mathrm{B}_{12}$, participating in the most important processes of life, such as plant and bacterial photosynthesis, respiration, enzymatic catalysis, sulfite and nitroreduction, methanogenesis, etc., are the source of life support on our planet. At the initial stage of the development of life, the main source of organic carbon was marine phytoplankton, bacteria and blue-green algae, but in modern conditions such a source is the higher living organisms that live or grow in the Earth's biosphere. During the evolution of life, there was a change in organisms that represent the bulk of biogenic carbon on Earth, and the relative amount and structural diversity of individual tetrapyrrolic pigments in the biosphere changed. ${ }^{[4,5]}$ A huge amount of tetrapyrrole pigments, represented mainly by magnesium, iron or cobalt complexes of porphyrins and chlorins, included in certain quantities and in a strictly defined position in the native polymer matrix, ${ }^{[6-11]}$ is present and functioning in biosphere layers today. As shown in Figures 1 and 2 by the example of bacteriochlorophyll (BChl) and heme in human oxygenase, the self-organization of tetrapyrroles with the surrounding biopolymer macromolecules leads to the formation of strictly defined complexes necessary to perform the functions assigned to them.

(A)

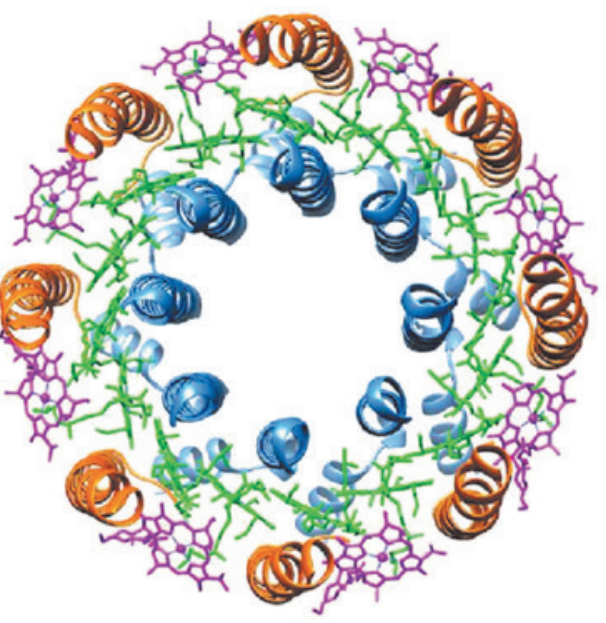

(B)

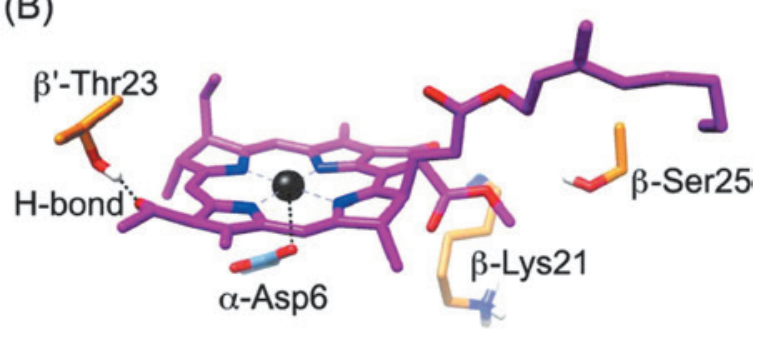

Figure 1. Overall structure (top view) (A) and the structure in the proximity of B800 BChl $a$ in molischianum-LH2 (B). Protein Data Bank entry 1LGH. B800 and $\mathrm{B} 850 \mathrm{BChl}$ a are colored magenta and green, respectively. The $\alpha$ - and $\beta$-polypeptides are colored sky blue and orange, respectively. ${ }^{[12]}$

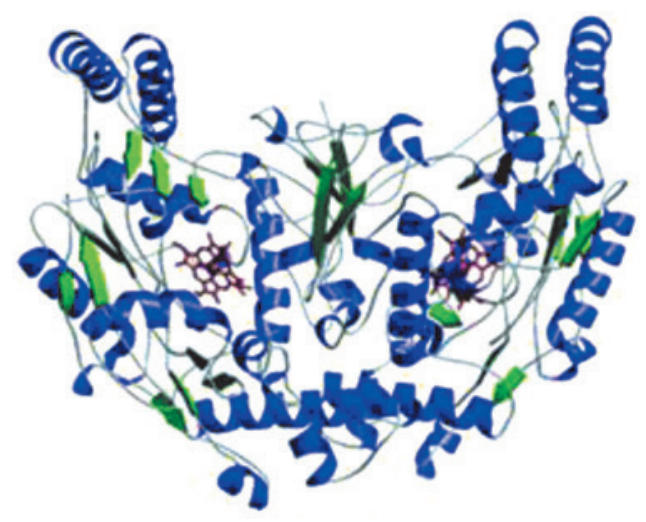

Figure 2. Crystal structure of the human oxygenase component. ${ }^{[9]}$.

A special place is given to the green plant pigments, chlorophylls, which play a key role in photosynthesis, 
the most important life-support process on Earth. ${ }^{[13-15]}$ At present, over 50 chlorophylls have been isolated and characterized from higher plants, algae, and photosynthetic bacteria, which differ in their chemical structure, color, and distribution in living organisms. ${ }^{[16,17]}$ This amount of photosynthetic pigments on Earth is determined by the variety of natural conditions in which the process of photosynthesis takes place, primarily the amount of light energy that enters the light-absorbing complexes.

Photosynthesis is the only process in the Earth's biosphere associated with the conversion of the energy of sunlight into the energy of chemical bonds, enclosed in organic substances. This complex physical, chemical and biological process of redox transformations of water and carbon dioxide to hydrate, amino acids, lipids and other organic compounds is induced by the chlorophyll $a$ photocatalytic system and proceeds in the photosynthetic apparatus of the plant.

Light-harvesting chlorophyll-protein complexes in natural conditions are formed by self-assembly due to noncovalent interactions (electrostatic, hydrogen, coordination, $\pi$ - $\pi$-stacking), which are due to the presence of active functional groups on the periphery of the tetrapyrrole macroheterocyclic compound and magnesium ion in the coordination center. Therefore, photosynthesis can occur only in the presence of pigment-protein complexes, which have a strictly defined organization, which ensures the capture of photons of different wavelengths and the transfer of excitation energy to the reaction centers. The efficiency of the light-harvesting complex is determined by the fraction of absorbed light energy that reaches to reaction center of the photosystem.

Another natural pigment, heme, is a prosthetic group of iron-containing proteins (hemoglobin, cytochrome myoglobin, peroxidase, catalase, etc.) that provides the main functions of these proteins: binding (myoglobin and hemoglobin) and oxygen transport (hemoglobin), the work of the electron transport chain (cytochromes), oxygen reduction to water (cytochrome oxidase), microsomal oxidation (cytochrome P450), peroxide decomposition (catalase and peroxidase). ${ }^{[7,9,10,19-21]}$

Cobalamins, vitamin $\mathrm{B}_{12}$, are involved in the body's isomerization and methylation reactions, play an important role in the synthesis of a number of compounds essential for life: amino acids, nucleic acids, choline, promote the formation of heme in erythrocytes, participate in cellular metabolism and affect DNA synthesis. ${ }^{[1,22-25]}$

Attempts to create artificial systems similar to the natural photosynthetic apparatus, blood substitutes, oxygen carriers, catalytic systems similar to natural enzymes, worried scientists in the past and in the last century remain a priority task. Therefore, chlorophyll, which has been known for more than 200 years, still takes the first place in the number of studies, and research in the field of natural tetrapyrroles was awarded the Nobel Prizes in 1915, 1930, 1961, 1962, 1964, 1965, 1988..$^{[14,26]}$

Comprehensive studies of natural porphyrin polymer complexes in various biological processes, especially in photosynthesis, have opened the way to the creation of artificial photocatalytic complexes, the effectiveness of which is not limited by the physiological characteristics of living organisms. The design of systems similar to natural ones is associated with the creation of materials that can effectively collect light energy, transmit and separate charges and carry them to the necessary centers. Using biomimetic principles, to create artificial models of natural porphyrin polymers, specific key interactions of tetrapyrrole pigments with natural with natural or synthetic oligomeric or polymer molecules are put at the forefront. ${ }^{[27-29]}$ First of all, the isolation of tetrapyrrole pigments from natural sources, the establishment of the structure and properties of individual molecules allowed us to identify the general characteristics and patterns of functioning of natural tetrapyrroles.

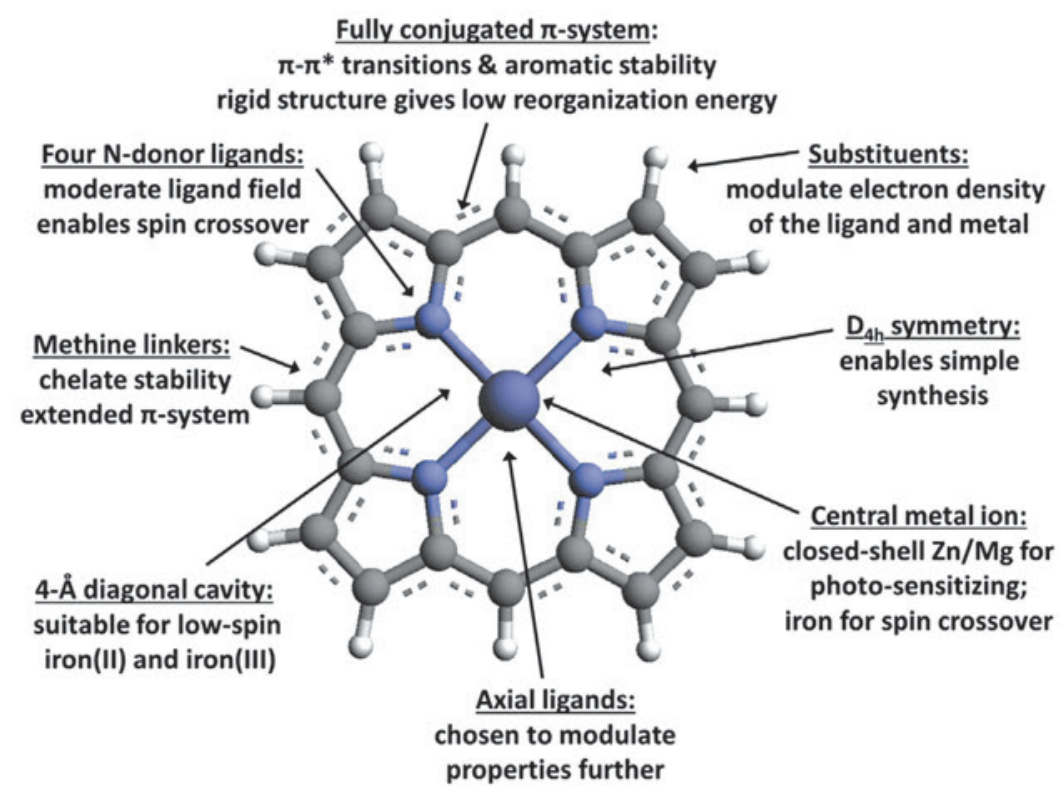

Figure 3. The combination of properties, that porphyrin ligand offers, makes it uniquely suited for life's most important processes, photosynthesis and $\mathrm{O}_{2}$-management. ${ }^{[7]}$ 
The "pigments of life" are based on the tetrapyrrole macroheterocycle, the versatility and uniqueness of its properties define it as an universal molecule of vital processes. Several fundamental properties of the porphyrin ligand are summarized in Figure 3 and reflect its polyfunctionality, which can be manifested in various ways and used in various applications. The tetrapyrrole macroheterocycle has a highly conjugated $\pi$-system, a powerful chromophore, high $\mathrm{D}_{4 \mathrm{~h}}$ symmetry, and a characteristic coordination center with a cavity of $4 \AA$ in diameter, which makes it easy to incorporate metal ions into it.

Porphine without peripheral substituents is the ancestor of the class of porphyrins and their analogues (Figure 4, structure $\boldsymbol{a}$ ). Hydrogenation of one pyrrole ring of the macroheterocycle leads to the formation of dihydroporphine or chlorin (Figure 4, b). Chlorin, a close analogue of porphine, is a common structural unit in the class of photosynthetic pigments of plant origin. ${ }^{[12,29,30]}$ The hydrogenation of two double bonds in the opposite pyrrole rings of the macroheterocycle opens the way to the production of tetrahydroporphyrins or bacteriochlorins (Figure $4, c$ ), the basis of the most ancient photosynthetic pigments - bacteriochlorophylls. ${ }^{[31-33]}$ Hydrogenation of two neighboring pyrrole rings in the macroheterocycle gives a structural bacteriochlorin isomer, isobacteriochlorin (Figure $4, \boldsymbol{d}$ ), which is also a structural unit of some "life pigments". ${ }^{[33]}$ No less important structural analogue of porphine is phorbine (Figure $4, \boldsymbol{e}$ ), a chlorin derivative, which has a cyclopentene ring (exocycle) at the third pyrrole fragment.

Porphyrins with a phorbine structure of macrocycle are often found in oils and shale, and cyclopentenone porphyrins, phorbine derivatives, in which the $\mathrm{V}$ ring at one of the carbon atoms is oxidized to a ketone group, form a large group of chlorophylls.

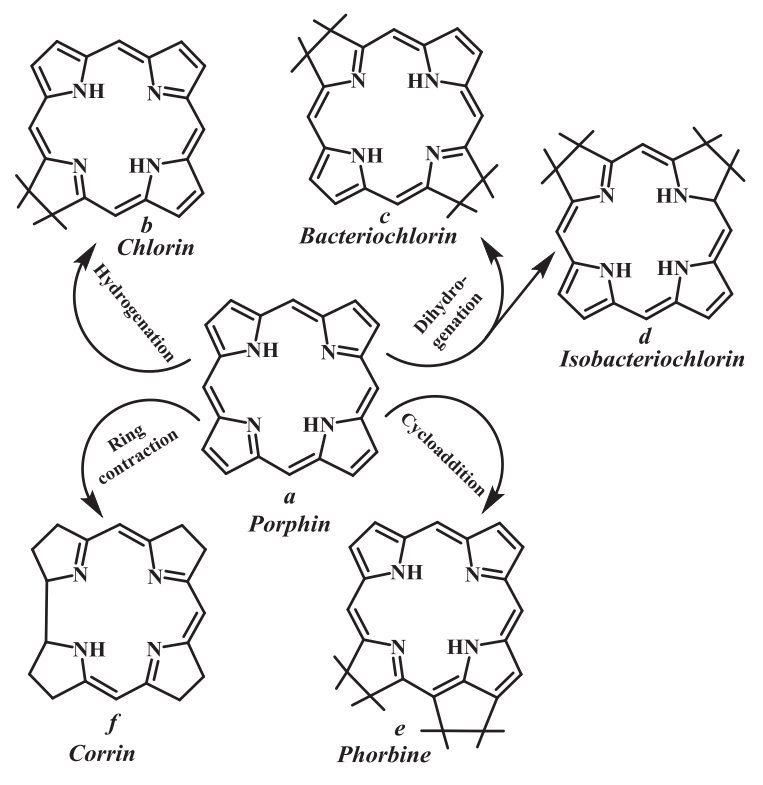

Figure 4. Structural variations of the tetrapyrrole macroheterocycle in the "pigments of life".

The alteration of tetrapyrrolic macroheterocycles by ring contraction or ring expansion has led to the emer- gence of a large group of porphyrin analogues called porphyrinoids. $^{[35]}$ The porphyrinoid closest to the structural analogue of porphine is corrin (Figure $4, \boldsymbol{f}$ ), the ancestor of the corrinoid group, which is the basis of cobalamins, vitamin $\mathrm{B}_{12} \cdot{ }^{[36-38]}$

Thus, the main groups of porphyrins that arise during the modification of the macroheterocycle and preserve its basic properties are represented in the "pigments of life". Each of these tetrapyrroles is a key link in its biological systems and performs a strictly defined function for it.

All natural porphyrins are asymmetrically substituted macroheterocycles with various substituents on the periphery of the molecule: alkyl, vinyl, formyl, propionyl substituents, which provide the fixation of the macroheterocycle in one or another way in the polymer matrix. The synthetic way of obtaining such compounds is very laborious and the syntheses of chlorophyll and hemine carried out by R. Woodward and H. Fischer are of purely fundamental importance. Therefore, it is more expedient to isolate bioporphyrins of the chlorophyll and heme groups from natural raw materials and, if necessary, modify them. ${ }^{[39,40]}$

The unique properties of chlorophyll $a$ and bacteriochlorophyll $a$ have shown great possibilities of creating photosensitizers based on them for antimicrobial and anticancer photodynamic therapy. Chemical modification of side substituents of the initial natural macroheterocycle can significantly increase the stability of its derivatives, since the pigments themselves are highly liable and also increase the tropism for malignant neoplasms, improve the spectral and other physicochemical properties of the compound. ${ }^{[41-46]}$

The blood pigment, heme, can be easily modified also by chemical transformations of peripheral substituents of the macroheterocycle. ${ }^{[47,48]}$ On the basis of hematoporphyrin, a drug for photodynamic therapy and fluorescence diagnostics "Photohem" has been obtained and is successfully used. ${ }^{[47]}$ Porphyrins derived from heme are more stable than pigments in green plants and algae. On the other hand, heme derivatives without a macromolecular environment become unstable to the effects of the environment, light, and temperature.

The study of synthetic tetrapyrrole macroheterocyclic compounds and the search for technological approaches to the their synthesis have opened a large group of synthetic porphyrins and analogues that are more stable than natural ones.

The synthetic chemistry of porphyrins began with the preparation of symmetric tetraarylporphyrins. The methodology proposed by Rothmund 85 years ago and subsequently developed by Adler-Longo-Lindsey underlies most of the methods that form the porphyrin macroheterocycle. ${ }^{[49-51]}$ The main synthon of tetrapyrroles is either unsubstituted pyrrole, which reacts with condensation with compounds capable of forming methylene bridges of the porphyrin ring, or having $\alpha$-methylene groups and cyclotetramerizing into a macroheterocycle. The necessary functional substituents on the porphyrin periphery can be formed by including them in the initial pyrrole or aldehyde. The condensation of pyrroles with aldehydes produced a large amount of porphyrins, which have various substituents both in the $\beta$-positions of the pyrrole rings and in the meso-positions of the macrocycle. This method 
allows one to obtain symmetrically substituted porphyrins, unsymmetrical derivatives, with their subsequent separation by chromatographic methods. The simplicity of the technique and rather high yields of the reaction made this method one of the most used in the preparation of synthetic porphyrins for various purposes.

Another direction in the synthesis of new porphyrins is the modification of the periphery of tetrapyrrole macroheterocycle. ${ }^{[52]}$ Any position in the porphine macrocycle (Figure 5) can be used for modification, which makes it possible to obtain a large number of new compounds of the porphyrin and analogues with various properties.

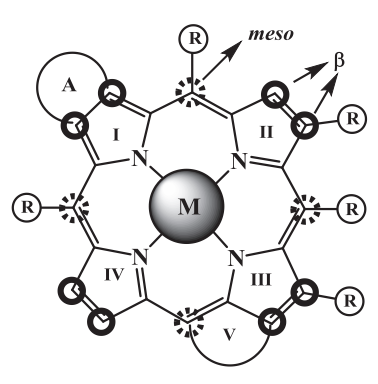

$$
\begin{aligned}
& \text { - meso-position } \\
& \text { of the macroheterocycle } \\
& \text { - } \beta \text {-position } \\
& \text { of the macroheterocycle }
\end{aligned}
$$

Figure 5. Possible directions of porphine functionalization.

Functionalization of the porphine periphery includes the introduction of various substituents at the $\beta$-positions ( $\beta$-functionalization) $)^{[53]}$ and at the meso-positions (mesosubstitution) of the macroheterocycle, ${ }^{[54,55]}$ as well as annulation at the $\beta, \beta$-positions of the pyrrole rings, which allow to obtain benzo-, naphthalo-, anthra-, phenanthro- and other derivatives of porphyrins with extended $\pi$-conjugation. ${ }^{[6,57]}$ In addition, similar natural chlorophylls, peripheral modification of the macroheterocycle is possible due to the formation of an exocycle (V-ring) or its transformation in both natural and synthetic porphyrins. ${ }^{[58,59]}$ The functionalization of macroheterocycles significantly affects its physicochemical properties: stability, reactivity, solubility, etc., while maintaining the basic properties inherent in the class of porphyrins. The main methods of functionalization are classical reactions of introduction of peripheral substituents by electrophilic or nucleophilic substitution, as well as nucleophilic addition reactions. ${ }^{[50]}$

In recent years, cross-coupling reactions are widely used for the functionalization of porphyrins. ${ }^{[50,60]}$ The palladium-catalyzed cross-coupling reaction of halogen substituted porphyrins with $\mathrm{N}, \mathrm{O}, \mathrm{P}$, and $\mathrm{S}$ nucleophiles is one of the promising methods for introducing heterosubstituents at the meso- and $\beta$-positions of the porphyrin macrocycle. The derivatives of porphyrins inaccessible via other methods can be obtained with high yields through the use of this method. The sequential Suzuki and Sonogashira crosscoupling strategy makes it possible to obtain porphyrin dimers, trimers, and oligomers. Only the use of the strategy of cross-coupling reactions catalyzed by palladium or other metals opened the way to the synthesis of new linear and cyclic polyporphyrins with a diverse structure and size of the macromolecule. ${ }^{[61,62] .}$

Even greater prospects for the creation of highly stable tetrapyrrole macroheterocyclic compounds are opening up in the synthesis of tetrapyrroles with a hetero atom in the meso position of the macroheterocycle, most often nitrogen. ${ }^{[63]}$ Porphyrazines, usually obtained by template assembly, have no analogues in nature, but serve as the basis for many, including hybrid functional materials. The most famous representatives of this group of tetrapyrroles, phthalocyanines, are stable synthetic pigments. Phthalocyanines have been widely used for more than half a century, both as pigments and as heterogeneous catalysts for various redox processes. The synthetic chemistry of these compounds is as diverse as the chemistry of porphyrins, and the possibilities of creating new porphyrazines are also endless. ${ }^{[64,65]}$

Thus, the advances in synthetic organic chemistry opens up unlimited possibilities for the preparation of various tetrapyrrole macroheterocyclic compounds. The use of new methodologies that facilitate the methods of their binding with oligomeric and polymeric compounds makes it possible to obtain new hybrid materials, the functional properties of which are determined by synthetic analogues of natural pigments.

Natural and synthetic polymers of linear and network structure are used today for binding the porphyrins. Macrocyclic compounds are incorporated into the matrix as a part of polymer chain, a crosslinking fragment or end groups, imparting specific properties to the resulting polymer. ${ }^{[62]}$ Any methodology of polymer chemistry can be successfully implemented for the design of porphyrin-polymer materials. An important point in this process is the directed synthesis of porphyrins of the required structure with the targeted formation of reactive groups in the macrocycle.

The anchoring of a porphyrin on a polymer support offers a number of advantages that are absent in the case of free porphyrins. Among them are cooperative interactions in polymer chains, separation of active centers, specific binding of various substrates to active centers, a rise in stability of a tetrapyrrole pigment, and a reduction in its toxicity with respect to biological media.

A special property of tetrapyrrole macroheterocyclic compounds is the ability to form coordination compounds with metal ions. All natural porphyrins perform their biological functions only as part of metal complexes; therefore, interest in the creation of new macroheterocyclic materials inevitably turns to synthetic metalloporphyrins.

Metalloporphyrins representing a large group of chelates are most stable of all existing coordination compounds. The incorporation of a metal ion into the coordination center of a tetrapyrrole macroheterocycle results in its significant stabilization and provides the metal complex with a number of important properties making it possible to exhibit its functionality. The structures of such complexes can be finely tuned by varying both the porphyrin ligand structures and the nature of the metal ion in the coordination center of tetrapyrrole macroheterocycles (Figure 5). Therefore, the formed structure of the coordination center of the macroheterocycle is a key factor that determines the stability of the formed complex, reactivity, and physicochemical properties. ${ }^{[66-69]}$

The most important property of metalloporphyrins is their ability to coordinate axially different molecules. This process provides a chemical bond between metal complexes and the environment, including $\mathrm{H}_{2} \mathrm{O}, \mathrm{O}_{2}$, solvent molecules, 


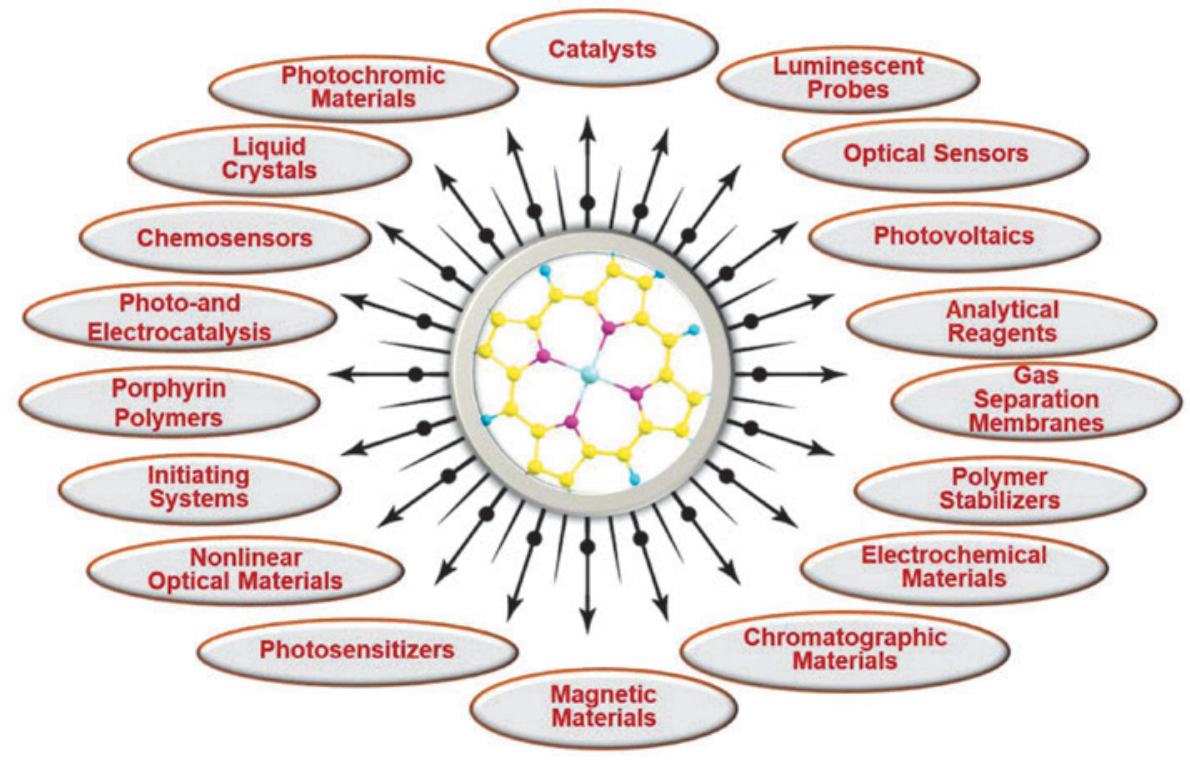

Figure 6. Applications of tetrapyrrole macroheterocyclic compounds.

and various biologically active compounds. The high activity of the metalloporphyrins in various chemical and biochemical processes primarily is achieved due to their ability to axially coordinate additional ligands.

The ability of metalloporphyrins to form intermolecular bonds based on axial coordination of ligands with central metals is inherent in many square-planar porphyrin complexes. Therefore tetrapyrrole macroheterocyclic metallocomplexes are remarkable building blocks of supramolecular systems, and the rapid development of this chemistry led to assemblies possessing various architectures and properties. The synthetic and physical chemistry of metalloporphyrins and supramolecular systems and multimerization ensembles based on them is being intensively developed today to create artificial photosynthetic systems ${ }^{[70,71]}$ and new generation functional materials. ${ }^{[72]}$ Non-covalent interactions leading to the formation of dimeric, oligomeric, and polymer structures of metalloporphyrins are one of the most effective tools for studying the relationship between the structural and functional properties of both natural photosynthetic systems and synthetic assemblies that form the basis of new functional materials and devices. ${ }^{[73]}$ The reversibility of the non-covalent interaction of these compounds is reflected in the design of the most stable and mobile supramolecular formations that quickly respond to any external changes. The development of synthetic chemistry of macroheterocycles and the emergence of new synthetic analogues of natural pigments open up new promising directions in supramolecular design based on smart materials. $^{[74-80]}$

Thus, due to a number of diverse sites for interaction with the environment, porphyrins and their metal complexes are perspective starting blocks that preserve the diversity of the properties of tetrapyrroles for new functional materials with various purposes from high-efficiency energy converters to therapeutic and diagnostic medicines (Figure 6). ${ }^{[81]}$

The intensive development of the chemistry of tetrapyrrole macroheterocyclic compounds and the emergence of new areas of application of the properties of both "life pigments" and their synthetic analogues allow us to hope that the testament of the Nobel Prize laureate F. J. Curie is being implemented:

«I believe in the future of atomic energy and I am sure in the importance of this discovery. However, I am convinced that the real change in the energetics will come only when we'll be able to synthesize on the mass scale the molecules analogous to chlorophyll and even higher quality species....»

\section{Synthesis of Porphyrin-Azamacrocycle Conjugates via Pd-Catalyzed Amination Reactions}

Up to date numerous researches on the catalytic modifications of porphyrins have been undertaken. In 2012 they were enough abundant so as to be reviewed in a Handbook of Porphyrin Science. ${ }^{[50]}$ From 1994 onward various approaches were shown to be applicable for the construction of carbon-carbon and carbon-heteroatom bonds like Suzuki coupling, ${ }^{[82-84]}$ Sonogashira reaction, ${ }^{[85-87]}$ as well as Heck, ${ }^{\left[{ }^{[8]}\right.}$ Stille, ${ }^{[89]}$ and so-called "click" reactions. ${ }^{[90]}$ The $\operatorname{Pd}(0)$-catalzyed amination reactions according to Buchwald-Hartwig protocol began to be employed in the porphyrins modifications since 2001. ${ }^{[91-97]}$ This type of porphyrins transformations is of great importance for us as we focused on the synthesis of polymacrocyclic conjugates of porphyrins with $\mathrm{N}$ - and $\mathrm{O}$-containing macrocycles in order to obtain promising molecules for fluorescent detection of metal cations. Initial studies included the search for the best conditions of the Pd-catalyzed amination of 5-(4-bromophenyl) and 5,15-di(4-bromophenyl)porphyrins with monoamines, ${ }^{[98,99]}$ and after this main efforts were put forward in the reactions with diamines and especially $\mathrm{N}$-containing macrocycles. It is to be noted that the synthesis of pure porphyrin dyads and triads was also addressed 
by us by means of the catalytic amination, ${ }^{[100-103]}$ however, it is out of scope of this mini-review which is focused solely on the porphyrin-macrocycles conjugates. Apart from our approach other catalytic transformations (e.g. Heck ${ }^{[104]}$ and Suzuki ${ }^{[105]}$ reactions) were reported for the construction of polymacrocycles of such type. All these facts strongly support efficiency and versatility of the catalytic methodologies in the porphyrin transformations.

\section{Pd(0)-Catalyzed Amination of meso-(Bromophenyl) Substituted Porphyrins with Aza- and Diazacrown Ethers}

Our investigations started from the preparation of $\mathrm{Zn}$ (II) 5-(4-bromophenyl) and 5,15-di(4-bromophenyl) substituted octaalkyl porphyrinates (1 and $\mathbf{5}$, respectively) and the first reaction with macrocyclic compounds employed 1-aza-15-crown-5 ether (Scheme 1). ${ }^{[106,107]}$ The reactions with the $\mathrm{Zn}$ (II) complexes of porphyrins were chosen to prevent possible undesirable coordination of the cations by the tetrapyrrolic moiety, though further it was demonstrated that free porphyrins could be equally introduced in the palladium-catalyzed amination reactions, however, with some restrictions. It is worth noting that the macrocycles under investigation are in fact secondary cyclic amines whose arylation is not a simple task. Standard and most versatile catalytic system $\mathrm{Pd}(\mathrm{dba})_{2} / \mathrm{BINAP}(\mathrm{dba}=$ dibenzylideneacetone, BINAP $=2,2^{\prime}$-bis(diphenylphosphino)-1,1-binaphthalene) was found to be insufficiently active and all reactions were carried out using a more electron-donor phosphine DavePhos (2-dicyclohexylphosphino-2'-dimethylaminobiphenyl). It helped to obtain the bismacrocycle 2 in an excellent $90 \%$ yield. The reactions with 1,10-diaza-18-crown-6 and $N, N$ ' $N$ ''-triBoccyclen provided corresponding conjugates 3 and $\mathbf{4}$ in lower yields (Scheme 1).

The introduction of $\mathrm{Zn}$ (II) 5,15-di(4-bromophenyl) porphyrinate $\mathbf{5}$ in the reactions with the aza- and diazac- rown ethers was equally successful (Scheme 2), it was possible to obtain trismacrocyclic diaminated products 6 and 8 in almost the same yields (64 and $63 \%$ respectively), in each case a substantial amount of the monoaminated derivatives 7 and 9 were obtained due to a partial catalytic reduction of the $\mathrm{C}-\mathrm{Br}$ bond. This process became more important in the reaction with $N, N^{\prime} N^{\prime \prime}$-triBoccylen and only the monoaminated bismacrocyclic conjugate $\mathbf{1 0}$ could be isolated. Compound $\mathbf{8}$ was reacted with the porphyrinate $\mathbf{1}$ to produce an interesting pentamacrocycle $\mathbf{1 1}$ containing three porphyrins and two diazacrown ether moieties. ${ }^{[101]}$

It was also possible to synthesize the conjugate of another composition, by reacting bismacrocycle $\mathbf{3}$ with $\mathrm{Zn}$ (II) porphyrinate $\mathbf{1}$ under similar conditions, this reaction provided the trismacrocyclic system 13 in $56 \%$ yield which contained two porphyrins and one diazacrown moiety. The possibility to introduce another metal in the conjugate was shown by the reaction of $\mathbf{3}$ with $\mathrm{Ni}$ (II) porphyrinate $\mathbf{1 2}$, and in this case the yield of the trismacrocyclic derivative $\mathbf{1 4}$ was equally good $(50 \%)$.

The increase in the number of active bromine atoms in 5,10,15,20-tetrakis(4-bromophenyl)-tetrabenzoporphyrin 15 resulted in a more important role of the concurrent hydrodebromination process (Scheme 3). The reaction of this compound with excess of 1-aza-18-crown- 6 resulted in an inseparable mixture of tetra- and triamination products 16 and 17..$^{[108]}$

Another combination of the porphyrin and azacrown moieties was achieved by introducing a propane-1,3-diamine spacer (Scheme 4). Preliminary prepared diamino derivatives of azacrown ethers $\mathbf{1 8}$ and $\mathbf{2 1}$ were reacted with $\mathrm{Zn}$ (II) porphyrinates 5 and $\mathbf{1 9}$ or with the free porphyrin 22 to give corresponding bis- and trismacrocyclic derivatives 20, 23 and 24. ${ }^{[109,110]}$ Their yields were quite humble (15-20\%), moreover, all attempts to introduce a free base analogue of the $\mathrm{Zn}$ (II) complex 19 failed.
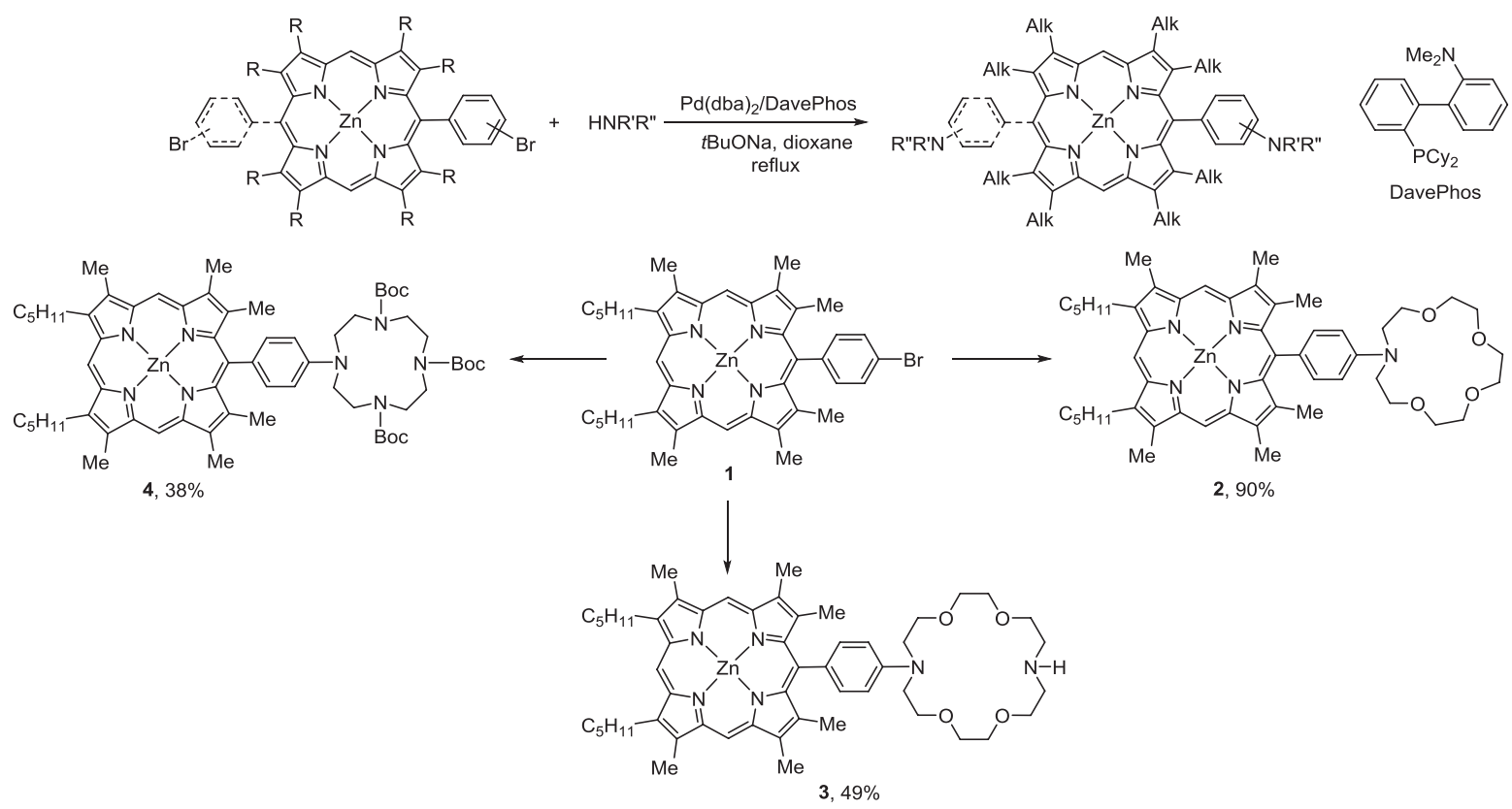

Scheme 1. 
Macroheterocyclic Compounds - a Key Building Block in New Functional Materials and Molecular Devices
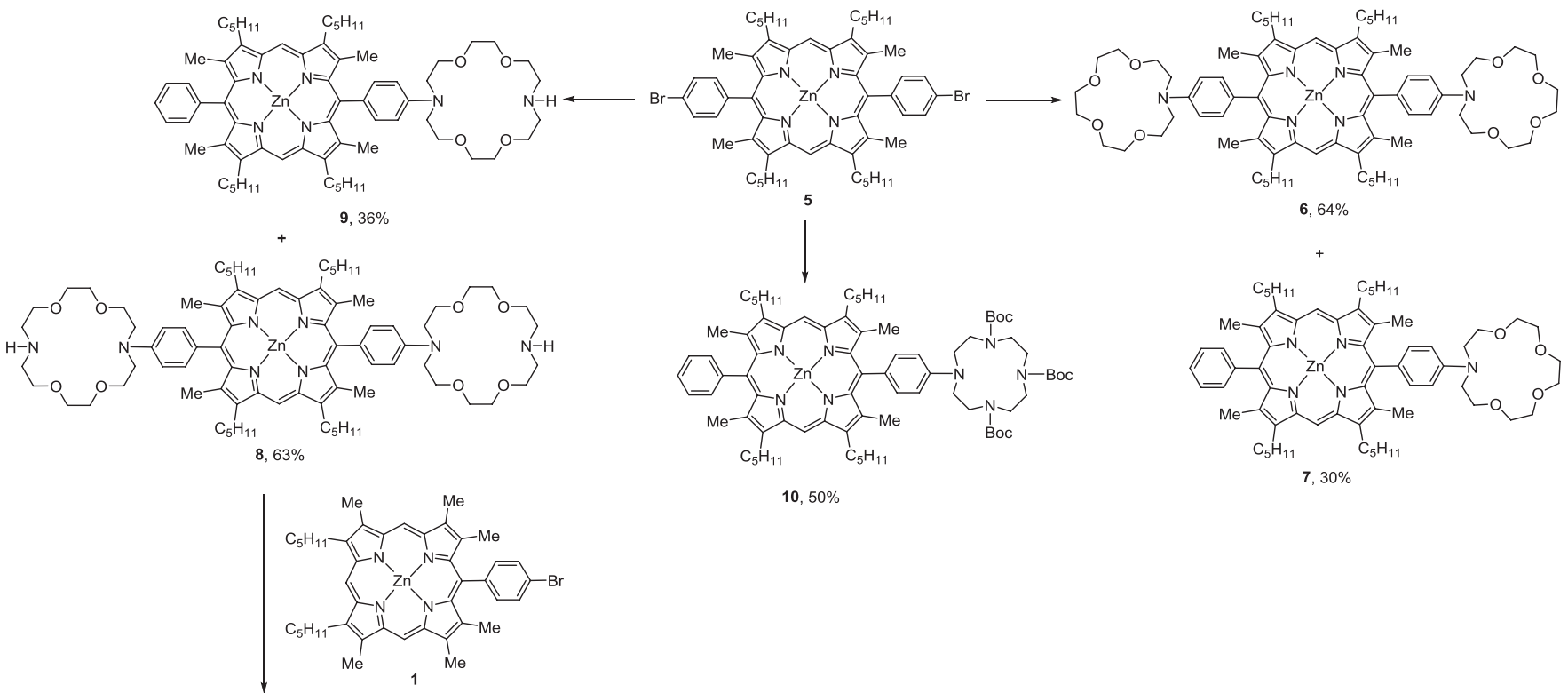

$10,50 \%$
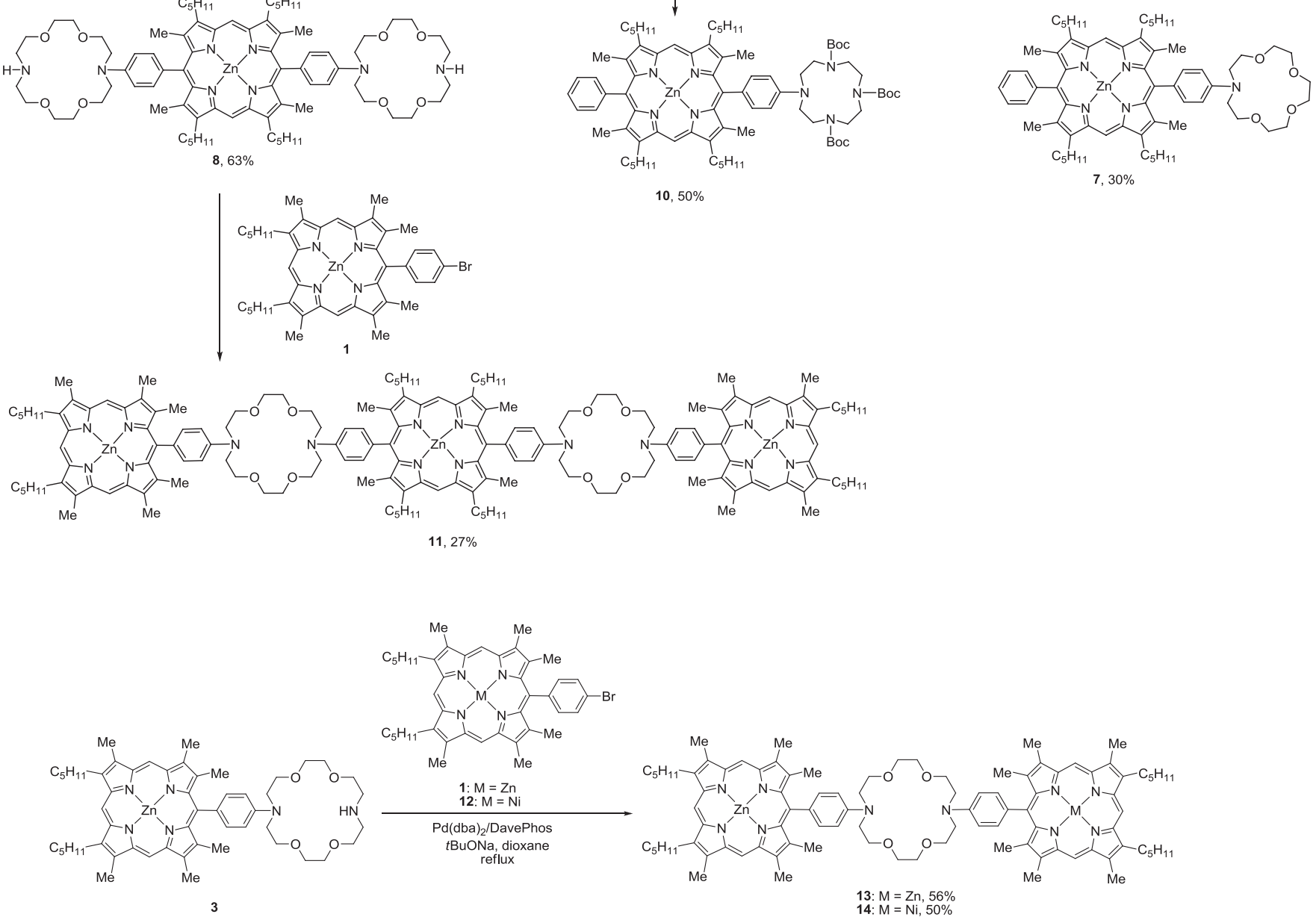

Scheme 2.
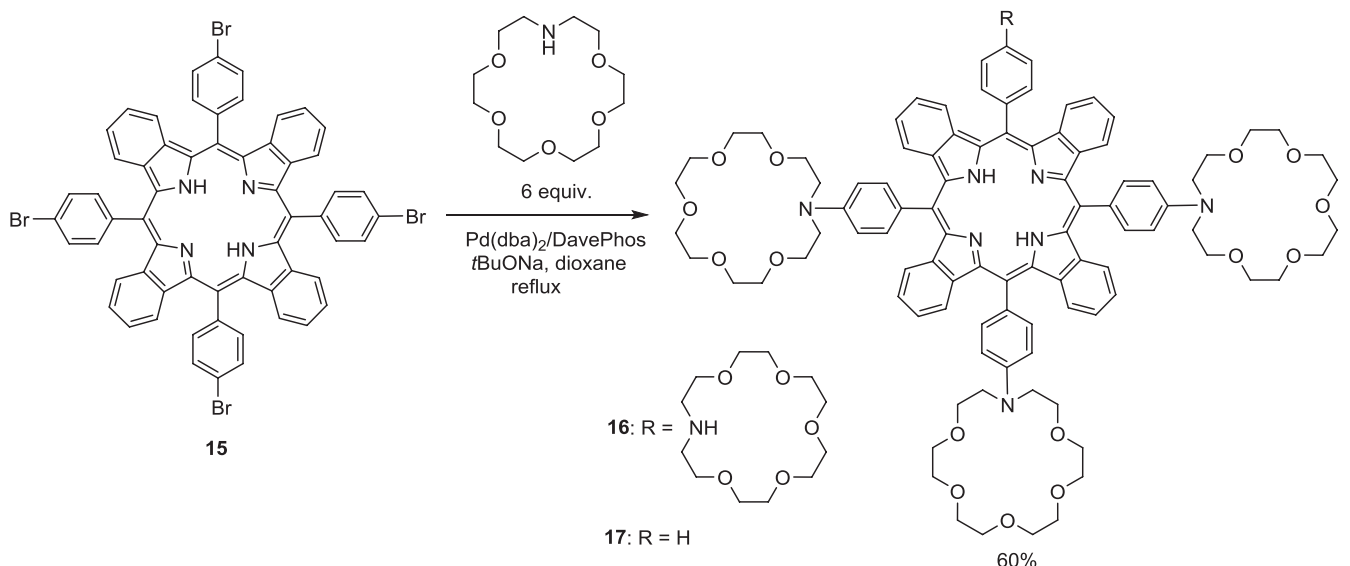

Scheme 3. 


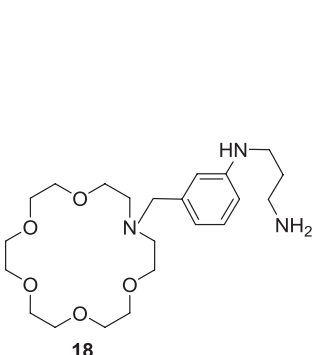

18

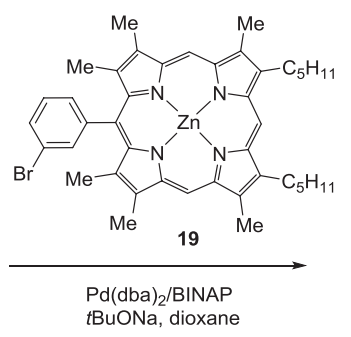

tBuONa, dioxan

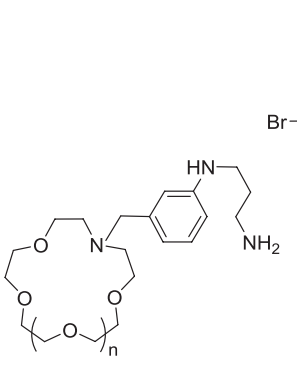

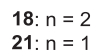

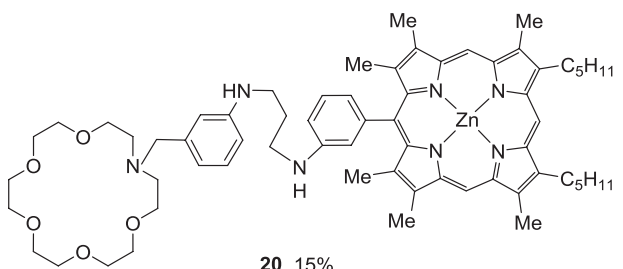

20, $15 \%$

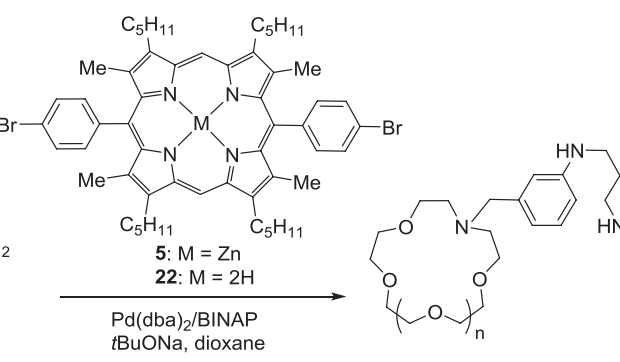

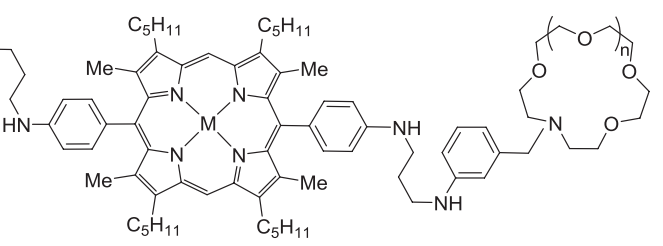

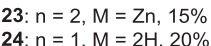

\section{Scheme 4.}
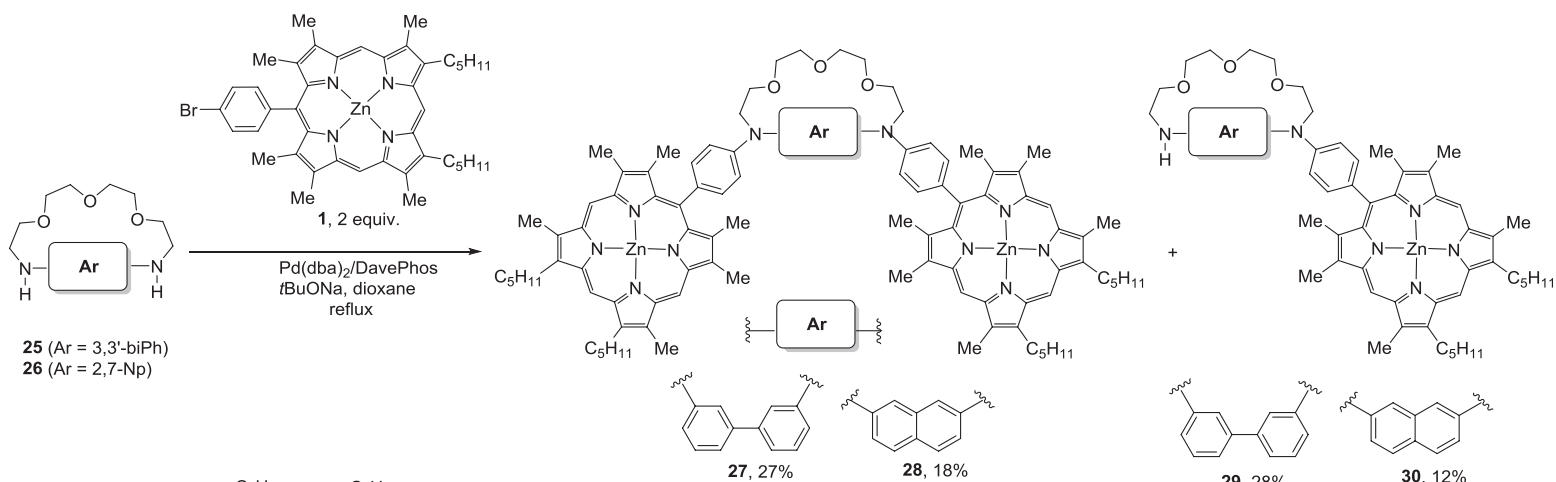

$26(\mathrm{Ar}=2,7-\mathrm{Np})$

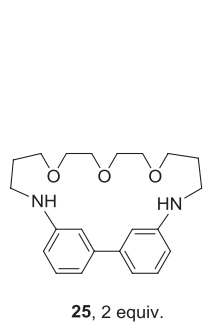

25, 2 equiv.

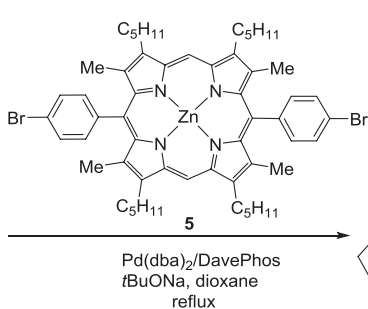

reflux

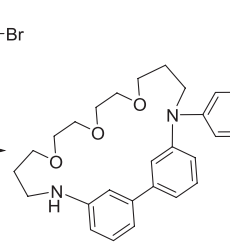

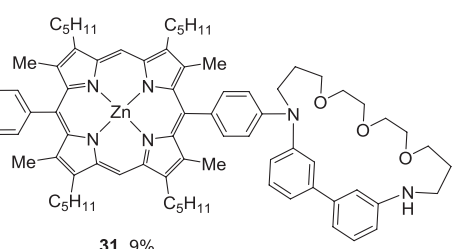

$31,9 \%$

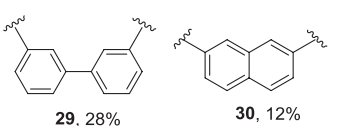

$29,28 \%$

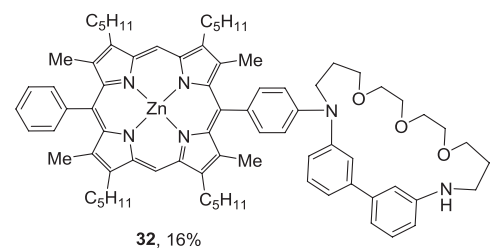

Scheme 5.

Catalytic Arylation of Diazapolyoxamacrocycles, Macrobicycles and Diaminocalix[4]arenes

Another approach to porphyrin-macrocycles conjugates utilized the reactions of $\mathrm{Zn}(\mathrm{II})$ porphyrinate $\mathbf{1}$ with the diazatrioxamacrocycles comprising 3,3'-disubstituted biphenyl and 2,7-disubstituted naphthalene moieties (25 and 26 respectively). The arylation of these compounds with 2 equiv. of 1 provided corresponding trismacrocycles 27 and $\mathbf{2 8}$, however, monoarylated products were obtained as well in comparable yields (Scheme 5). ${ }^{[11]}$

Similar reaction of 2 equivs. of $\mathbf{2 5}$ with $\mathrm{Zn}$ (II) 5,15-di(4bromophenyl)porphyrinate $\mathbf{5}$ was less selective giving a number of products among which were isolated the desirable trismacrocycle 31 (in only $9 \%$ yield) and bismacrocycle $32(16 \%)$ formed due to the reduction of one $\mathrm{C}-\mathrm{Br}$ bond in the porphyrin..$^{[112]}$

An interesting modification of the cryptands 33, 34 and 37 previously obtained by us from diazacrown ethers and trioxadiamines ${ }^{[113]}$ was achieved by the reactions of these compounds with 2 equiv. of isomeric Zn(II) 5-(bromophenyl)porphyrinates 1 and 19 (Scheme 6) ${ }^{[112]}$ Tetramacrocyclic compounds $\mathbf{3 5}$ and $\mathbf{3 6}$ were isolated in similar yields 20-23\%, the compound 38 was obtained in lower yield (11\%) while the bismacrocyclic derivative 39 - the product of monoarylation - was isolated in $16 \%$ yield, probably due 

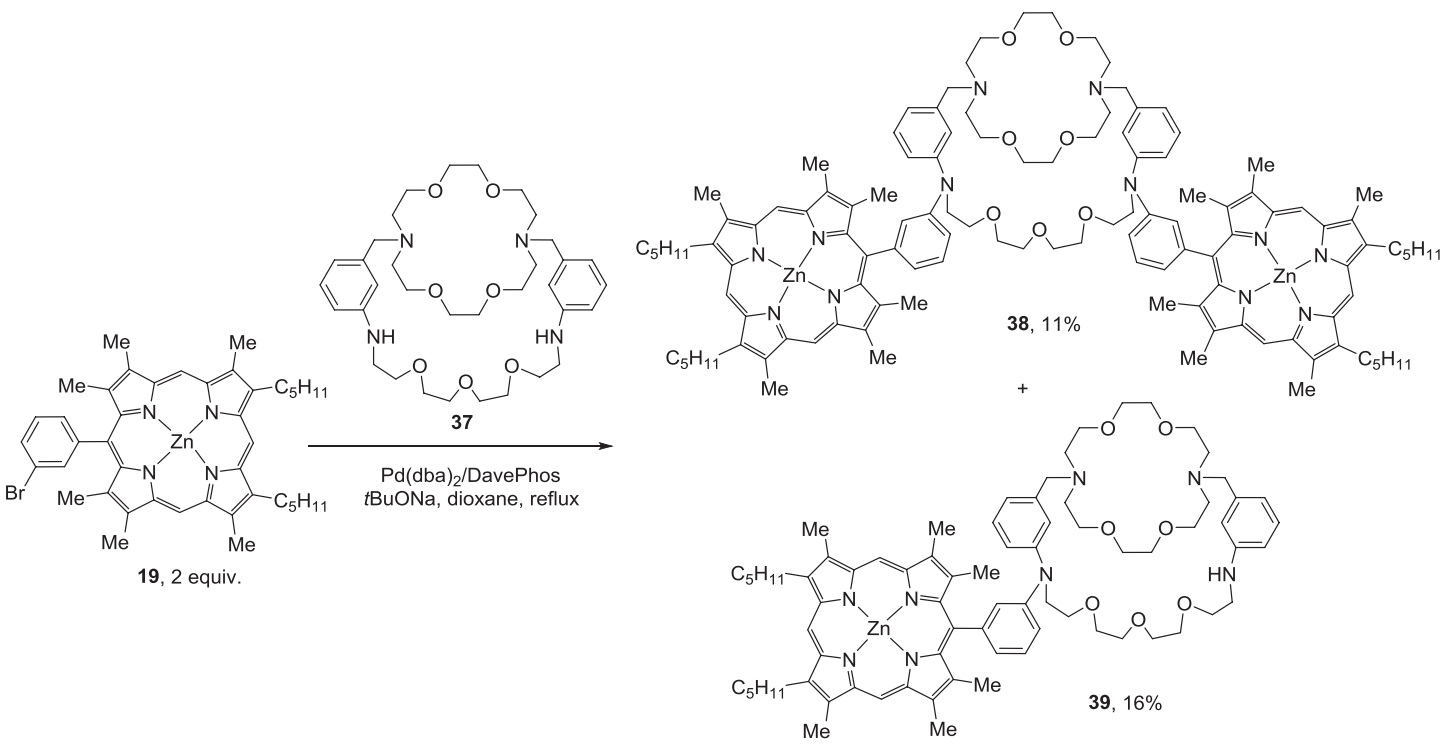

Scheme 6.

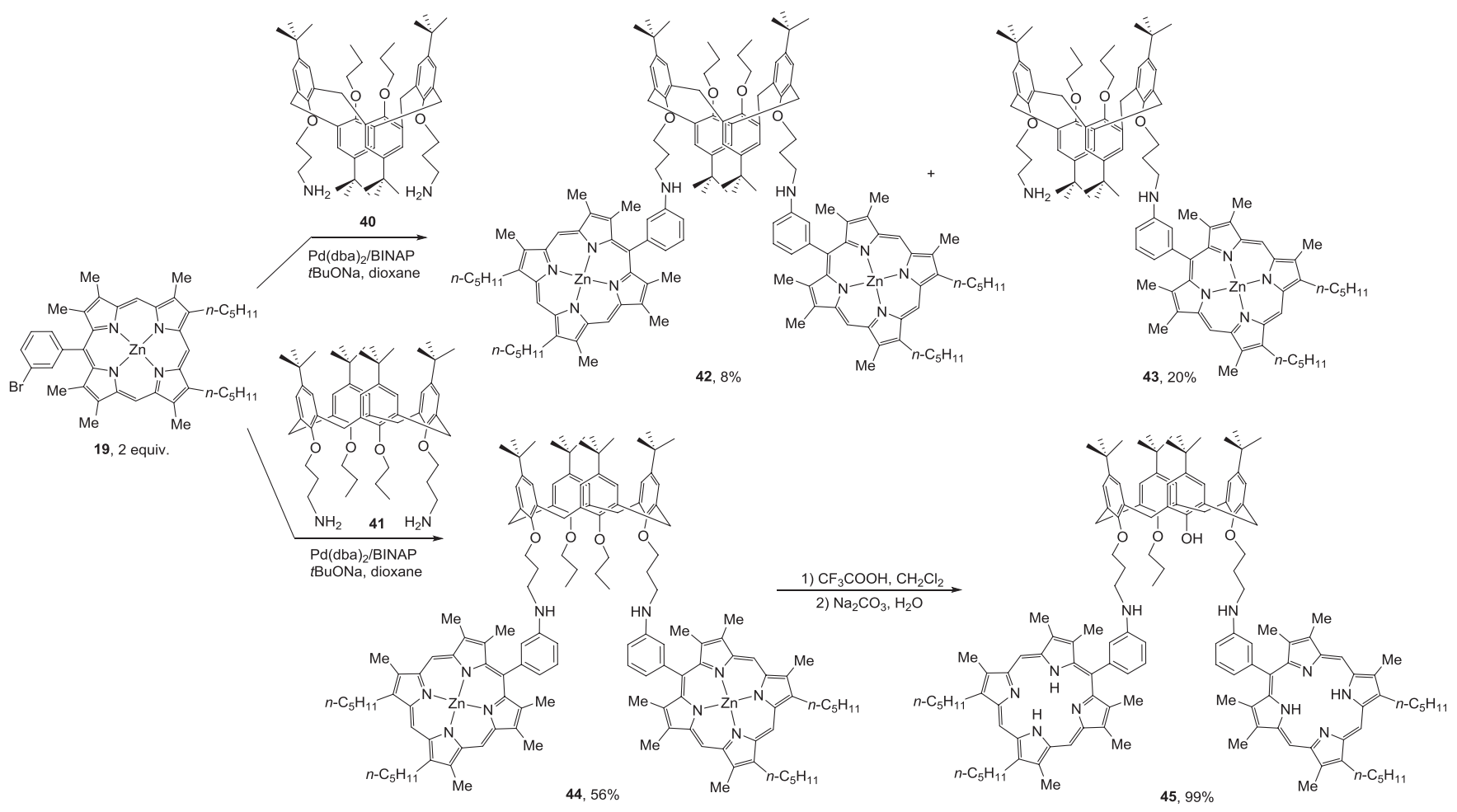

Scheme 7.

to lower reactivity of the bromine in position 3 in the starting porphyrinate 19 compared to a more reactive bromine in position 4 in compound $\mathbf{1}$.

The reaction of the $\mathrm{Zn}(\mathrm{II})$ porphyrinate 19 with diaminocalix[4]arenes $\mathbf{4 0}$ and $\mathbf{4 1}$ was helpful for the synthesis of corresponding porphyrin-calix[4] arene conjugates (Scheme 7). ${ }^{[14]}$ 1,3-Alternate 40 was obviously less reactive and its bis(porphyrin) derivative $\mathbf{4 2}$ was isolated only in $8 \%$ yield though the monoarylated product 43 was obtained in a higher yield. Much better result was observed in the reaction with the full cone isomer $\mathbf{4 1}$ as the desired dia- rylated product 44 was synthesized in $56 \%$ yield. The latter was subjected to a treatment with $\mathrm{CF}_{3} \mathrm{COOH}$ to get the free base, it was quite successful though the acidic hydrolysis of one propoxy group took place affording conjugate 45 .

Investigations of the UV-Vis and fluorescence spectra of many of the porphyrin-macrocycle conjugates thus obtained demonstrated that the majority of them could serve as chemosensors or molecular probes for $\mathrm{Cu}$ (II) cations due to full quenching of the emission and notable changes in the UV-Vis spectra of these compounds associated with the peculiar oxidation of the porphyrin moiety by this metal 
into non-planar dication. In some cases $\mathrm{Cu}(\mathrm{II})$ selectively quenched the emission (e.g. of compounds 28 and 36), in other cases both $\mathrm{Cu}(\mathrm{II})$ and $\mathrm{Al}$ (III) similarly affected the fluorescence (conjugates 42 and 44), rarely $\mathrm{Cr}$ (III) could also significantly quench the emission (compound 27). On the other hand, the conjugate $\mathbf{4 5}$ with free-base porphyrins was found to be a possible molecular probe for $\mathrm{Cu}(\mathrm{II}), \mathrm{Zn}(\mathrm{II})$ and $\mathrm{Cd}(\mathrm{II})$, as each of these metals led to a unique change in the spectrum of fluorescence.

To sum up, the $\operatorname{Pd}(0)$-catalyzed amination reaction proved to be a powerful tool for the construction of the porphyrin-macrocycle conjugates of versatile architecture, the yields of these polymacrocyclic compounds differed substantially depending on the nature of starting $\mathrm{N}$-macrocycles, and many of them were found to be useful for the development of fluorescent detectors of metal cations.

\section{New Advances in the Application of Phthalocyanines and Their Analogues}

The history of the discovery of phthalocyanines dates back to the 90s of the XIX century, when Pinner discovered an intense blue coloration of the reaction mixture in the preparation of imidoesters from aromatic dinitriles. However, he didn't think anything of it. In 1907 Braun has separated blue compound in a low yield after heating of $o$-cyanobenzamide in ethanol. ${ }^{[115]}$ Until the 30 th of the XX century, there was no data on the structure of the compounds obtained.
Linstead is widely credited to be the pioneer of phthalocyanine chemistry, who first studied in detail the methods of their synthesis and some properties. ${ }^{[116]}$ His findings gave rise to global investigation of these compounds ${ }^{[117,118]}$, that led to discovering of its unique spectral, electrochemical, magnetic and semiconductor properties caused by multi-contour aromatic system and ability to form complexes with most of the elements of the periodic system. Thanks to the presence of these properties phthalocyanines and their analogs are currently finding an increasing number of application areas: from medicine ${ }^{[119]}$ and catalysis to alternative energy sources (materials for photovoltaics), ${ }^{[120]}$ digital technologies, for instance, in devices for the formation of images in a near IR region.

In recent years, the development of synthetic approaches to multiple-decker complexes inspires the interest of researchers. ${ }^{[121-123]} \mathrm{J}$. Jiang et al. described examples of discrete multiple-decker complexes, containing from four to six phthalocyanine macrocycles, the decks-macroheterocycles of which are not linked by the covalent bonds ${ }^{[121,122,124]}$ (Figure 7). These compounds are prospective single molecule magnets.

Quadruple-decker complexes, linked by rigid or flexible spacers ${ }^{[125,126]}$ (in addition to coordination bonds with lanthanide ions) or metal ions (e.g. sodium), ${ }^{[127]}$ draw an outstanding interest of researchers in investigation of magnetic properties.

For the first time our research group demonstrated the possibility of forming of multiple-decker complexes based on clamshell-type ligands containing 6-10 phthalo-
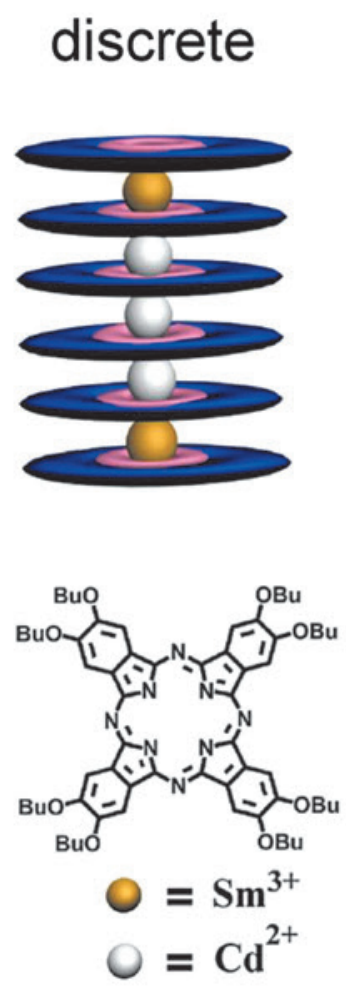
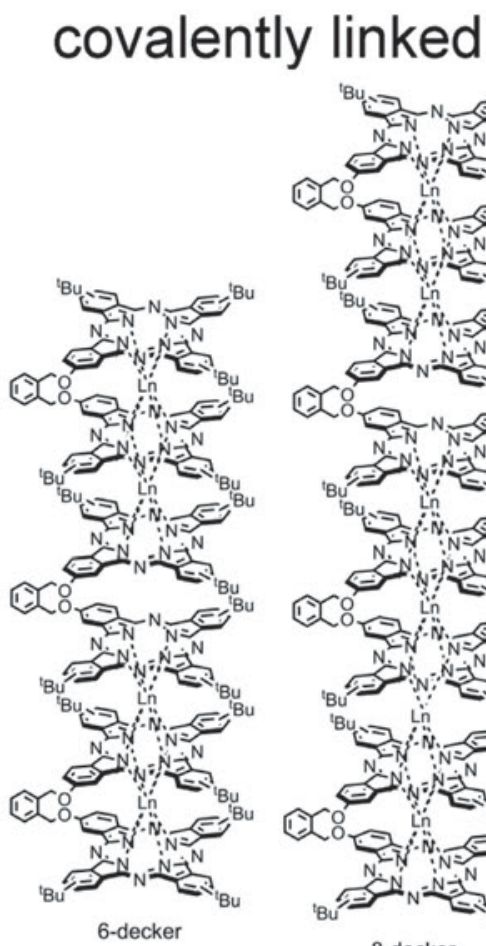

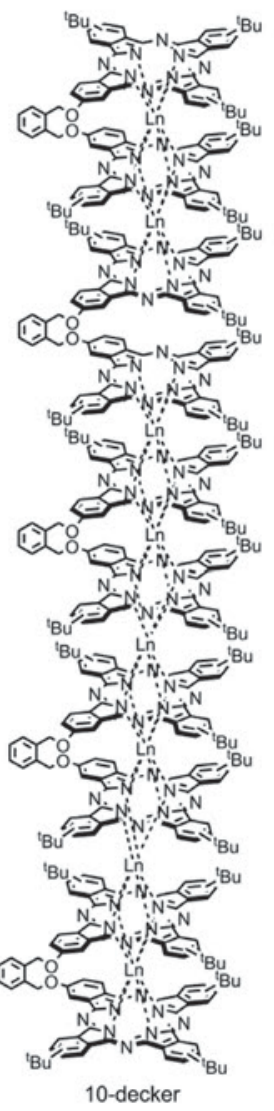

Figure 7. Structure of discrete multiple-decker complexes ${ }^{[121]}$ and complexes containing covalently-linked macrocycles. ${ }^{[128]}$ 
cyanine macrocycles ${ }^{[128]}$ (Figure 7). These compounds are prone to formation of ordered nanoscale architectures.

A relatively new is the synthesis of $J_{-}{ }^{[129]}$ or $H$-type ${ }^{[130]}$ dimers, characterized by an unusual type of assembly using weak intermolecular interactions: hydrogen bonds, coordination of the central ion and/or $\pi$ - $\pi$-stacking. These dimers are also prone to manifestation of magnetic properties.

Another important area associated with the preparation of substituted phthalocyanines is the synthesis of conjugates. L.G. Tomilova et al. obtained novel covalently linked dyad of $\mathrm{A}_{3} \mathrm{~B}$-type phthalocyanine and methyl pheophorbide. ${ }^{[131,132]}$ The resulting conjugates demonstrate high value of singlet oxygen quantum yields and are promising compounds for theranostics - modern trend in medicine that combines therapy and preliminary treatment of oncological diseases. Optical properties of dyads indicate that the practical use of this new class is highly promising.

This Section will focus on the most relevant application areas of coordination compounds of phthalocyanines of various structures, developed in our scientific group and its comparison with literature data.

\section{Phthalocyanines with Absorption in a Near IR Region}

Compounds with absorption in the near IR region attract the attention of researchers for several reasons. At wavelengths in the range of $0.75-3 \mu \mathrm{m}$, light penetrates deep into the human skin; this area is called the "therapeutic window". This, together with the ability to generate singlet oxygen, makes it possible to consider these compounds as promising photosensitizers for photodynamic therapy of oncological diseases. ${ }^{[133]}$ The presence of absorption in the near IR region is an important factor in the design of optical limiters. For instance, the generation of the first harmonic of Nd YAG laser occurs at $1064 \mathrm{~nm}$, and for $\mathrm{Nd}$ $\mathrm{YVO}_{4}-$ at $914 \mathrm{~nm}$. Thus, the best nonlinear response can be achieved if there is an absorption maximum in these regions.

$50 \%$ of solar irradiance spectrum fall at the near IR region, therefore, it is especially important to use in the composition of photocells compounds that have intense absorption in the near IR region. This approach allows to increase an efficacy of solar cells. ${ }^{[134,135]}$

Phthalocyanines and their analogs with absorption in the near IR region can be divided into two main types: planar and sandwich architecture. Planar phthalocyanines have absorption in the near IR region due to: 1) presence of substituents at $\alpha$-positions of phthalocyanine macrocycle; 2) extension of peripheral $\pi$-system, or 3 ) introduction of $P$, $\mathrm{V}$ or Mn as central ions.

As it was shown by the calculations carried out by $\mathrm{N}$. Kobayashi et al. ${ }^{[136]}$ the most promising substituents for introduction into $\alpha$-position of phthalocyanine macrocycle are the groups containing $\mathrm{S}$ or Se. An increase in the total $\pi$-system of the complex is achieved due to the effective interaction between the phthalocyanine $\pi$-orbitals and $3 p$ orbitals of $\mathrm{S}$ or $\mathrm{Se}$ atoms. This hypothesis is confirmed by the fact that phosphorus phthalocyanine containing $\mathrm{PhSe}$ groups at the $\alpha$-positions of the phthalocyanine macrocycle is characterized by absorption at $1033 \mathrm{~nm}$.
In the case of extension of peripheral $\pi$-system linear benzoannelation of phthalocyanine results in two analogues, which are stable at aerobic conditions: 2,3-naphthalocyanines and antracocyanines. Due to the extension of $\pi$-system going from phthalocyanine macrocycle to 2,3-naphthalocyanine strong destabilization of HOMO and insignificant destabilization of LUMO orbital is observed. Due to this reason the energy gap between HOMO and LUMO decreases and the absorption spectrum exhibits a bathochromic shift of the absorption maximum to the near IR region by almost $100 \mathrm{~nm}$. This phenomenon is called the "100 nm" rule. ${ }^{[137]}$ In accordance with it the absorption maximum of 2,3-naphthalocyanines lies in the region of 700-800 $\mathrm{nm}$ and for antracocyanines -800 $900 \mathrm{~nm} \cdot{ }^{[138]}$

In 2010 an interesting example of isomer of 2,3-naphthalocyanine - azulenocyanine with absorption shifted beyond $1000 \mathrm{~nm}$ was described by A. Muranaka et al. (Figure 8) ${ }^{[139]}$ Donor-acceptor complexes with fullerenes, ${ }^{[140]}$ which are of interest for photovoltaics, were successfully obtained on the basis of these compounds.

The preparation of bi- and polynuclear (na) phthalocyanines sharing rigid aromatic spacers is one of the most promising field. ${ }^{[141]}$ We have shown that an increase in the length of such a bridge from benzene to naphthalene leads to a weakening of the interaction between phthalocyanine macrocycles; the greatest bathochromic shift of the $Q$ band can be achieved in the case of polynuclear complexes with a common benzene bridge. We were lucky to obtain novel binuclear naphthalocyanine complexes with absorption maximum bathochromically shifted beyond $900 \mathrm{~nm} \cdot{ }^{[142]} \mathrm{S}$. Makarov et al. prepared trinuclear phthalocyanine with absorption maximum at $944 \mathrm{~nm} \cdot{ }^{[143]}$ As in the case of binuclear complexes, the extension of $\pi$-system of aromatic bridge going from benzene to naphthalene leads to hypsochromic shift of absorption band of trinuclear magnesium complex obtained by us. ${ }^{[144]}$

The design of materials for near IR region can be realized using sandwich-type phthalocyanine. Due to $\pi$-radical nature of these complexes the absorption bands corresponding to transitions of an unpaired electron (red valence $(R V)$ and inter valence $(I V))$ are observed in the near IR region spectra of these compounds. By the analogy with planar complexes, because of the destabilization of HOMO going from diphthalocyanine to dinaphthalocyanine the bathochromic shift of main absorption maximum - Q band is observed ("100 nm" rule). ${ }^{[145,146]}$ By varying the nature of the central ion, it is also possible to persistently obtain compounds with a tunable absorption range. For instance, with an increase in the ionic radius of REEs, bathochromic shift of the $I V$ band is observed (from 1262 to $1618 \mathrm{~nm}$ for the $\mathrm{Lu}$ and $\mathrm{Eu}$ complexes, respectively) ${ }^{[126]}$ (Figure 9).

In conjunction with the foregoing, the preparation and study of the properties of sandwich complexes based on bridging phthalocyanine ligands with a spacer structure opens up new prospects for their practical use as materials for organic electronics, in particular, as components of solar cells and single-molecule magnets. 

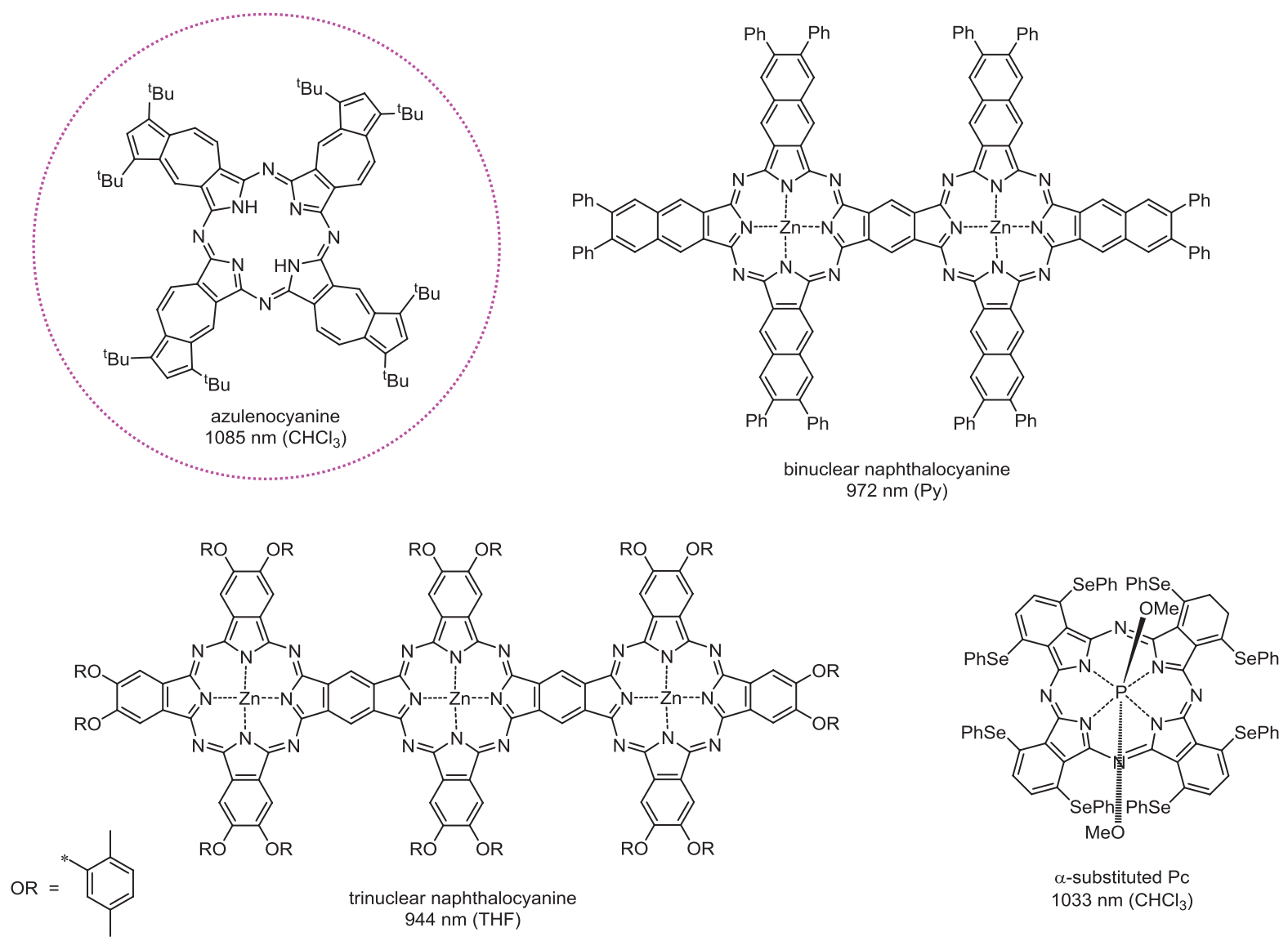

Figure 8. The structures of phthalocyanines - record holders in a shift of absorption maximum to the near IR region.

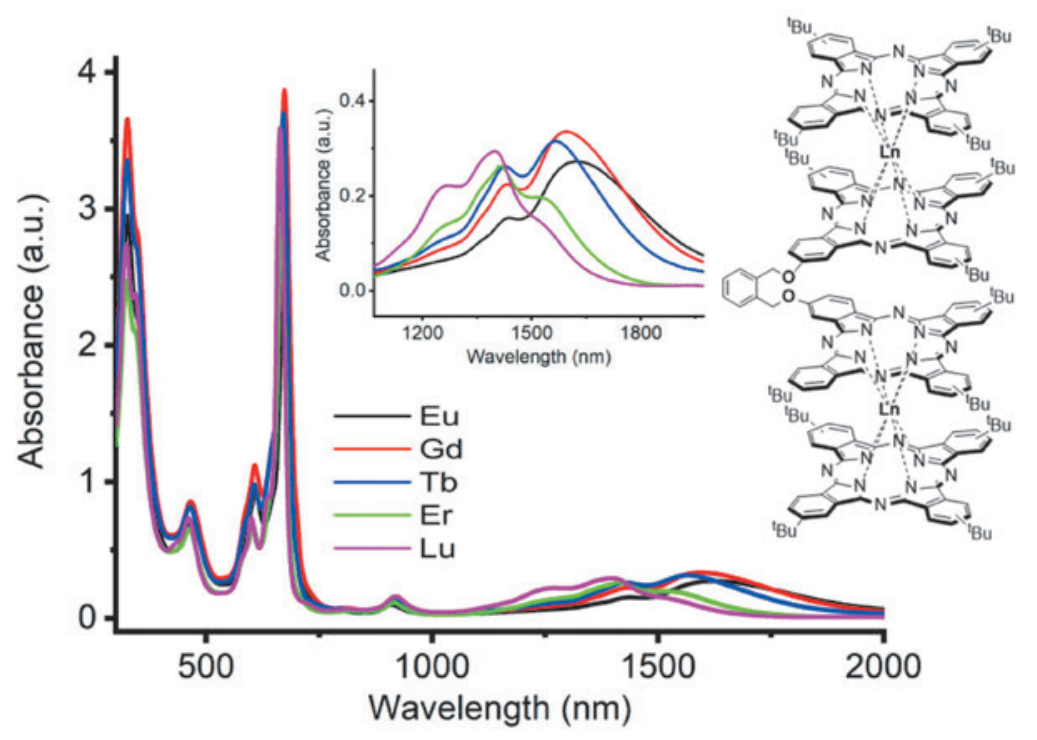

Figure 9. UV-Vis spectra of quadruple-decker complexes in the near IR region in $\mathrm{CCl}_{4}$.

Development of Effective Sensors

for Detection of Antibiotics in Surface Water

Pollution of surface waters by pharmaceuticals, including antibiotics, is a serious global challenge. ${ }^{[147,148]}$ Significant antibiotic contamination of surface waters occurs from the discharge of waste and wastewater from pharmaceutical companies, hospitals, livestock farms and household waste. This leads to the appearance of local pollution focus, where the concentration of antibiotics exceeds the minimum inhibitory concentration and creates conditions for the emergence of resistant bacteria. ${ }^{[147,149]}$ As a result, this leads to the spread of resistant bacteria in the environment as a result of horizontal gene transfer 
and pathogenic antibiotic resistant bacteria. In the European Union, more than 25,000 people per a year die from infections caused by resistant pathogenic bacteria. ${ }^{[150,151]}$ The Russian Federation is also at risk, as there are no or very limited programs to monitor surface waters for the availability of pharmaceuticals.

The analytical methods for the determination of antibiotics currently used can be divided into three main groups: instrumental methods, microbiological tests, and biosensors. Expensive laboratory methods such as liquid chromatography-mass spectrometry (LC-MS) are commonly used to detect pharmaceuticals in surface waters. ${ }^{[152-154]}$ A significant disadvantage of this method is the time-consuming procedure of sample preparation, the need for trained staff, and a specialized laboratory. For economic and practical reasons, the possibilities of using this method for wide and systematic monitoring are limited.

Microbiological tests based on the inhibition of bacterial growth by various antibacterial agents were originally developed for milk quality control. This method is relatively inexpensive and easy to implement. However, it is time-consuming, does not allow for on-line analysis, is not specific, and has several obstructive factors.

The detection of antibiotics by electrochemical sensors is carried out using various electrode materials and techniques for electrochemical experiments. ${ }^{[155]}$ In electrochemical biosensors, the electrode is modified with receptor molecules that can reversibly and with high selectivity bind to the analyte. ${ }^{[156]}$

By a group a of K. De Wael new strategy for the photoelectrochemical determination of amoxicillin was developed due to the photocatalytic oxidation of the phenolic fragment in its structure in the presence of a photosensitizer and subsequent amperometric detection of the reaction product. ${ }^{[157]}$ This approach allows detection of up to $20 \mathrm{nM}$ of amoxicillin in $1 \mu \mathrm{L}$ of a sample. Fluoro-substituted zinc phthalocyanine $\left({ }^{\mathrm{F} 64} \mathrm{PcZn}\right)$ was tested as a catalyst. ${ }^{[157]}$.

Hydroquinone (HQ), which can be reversibly oxidized to benzoquinone under the action of singlet oxygen, can serve as a convenient object for testing sensor activity.

With the participation of our scientific group, an easy-to-use express method for testing the photocatalytic activity of photosensitizers generating singlet oxygen has been developed. ${ }^{[158]}$ The method is based on the process of photooxidation of hydroquinone on a graphite electrode in an organic solvent (Figure 10).

This study is the first example of a one-pot combination of electrochemical and photosensitized reactions in an organic solvent environment. The photocatalytic reaction was initiated using LED lasers with operating wavelengths of 532 and $659 \mathrm{~nm}$. Phthalocyanine and subphthalocyanine dyes of various structures were selected as photosensitizers; pheophorbide $a$ and Rose Bengal dye were used as reference samples.

Figure 11 shows typical amperometric measurements obtained by the method we have developed. First, it is required to stabilize the background current for 1-2 minutes and only after that the laser is turned on. Switching on the laser leads to the appearance of a photocurrent signal of $130 \mathrm{nA}$ if the solution contains both a photosensitizer ( $\left.{ }^{\mathrm{OH}, t-\mathrm{Bu}} \mathrm{PcAlCl}\right)$ and hydroquinone (HQ) (Figure 11, red curve). In the presence of only $\mathrm{HQ}$, the photocurrent is not recorded, and in the presence of only the photosensitizer, the minimum photocurrent is recorded (Figure 11b). The presence of this small photocurrent can be explained by the electrochemical reduction of the $\mathrm{O}_{2}^{-}$superoxide anion or hydrogen peroxide, which can form in side reactions with the participation of ${ }^{1} \mathrm{O}_{2}$, or due to the electrochemical reduction of ${ }^{1} \mathrm{O}_{2}$ formed near the electrode surface. Due to the fact that the lifetime of singlet oxygen in methanol is rather short $\left(7 \mu \mathrm{s}^{[159]}\right)$, the expected diffusion distance should not exceed $200 \mathrm{~nm}$.

It is important to note that turning on and off the light source allows instantaneous and accurate measurement of the photocurrent, taking into account the background signal, without additional blank experiments. Moreover, the fact that the photocurrent value is stable over time means that the system reached equilibrium almost instantly, since this method does not require the accumulation of the reaction product. This is an important advantage of the developed method in comparison with spectroscopic methods coupled with the use of optical or paramagnetic traps. ${ }^{[160]}$

Linear correlation between the value of photocurrent, which is need for reduction of benzoquinone to hydroquinone, concentration of hydroquinone and singlet oxygen
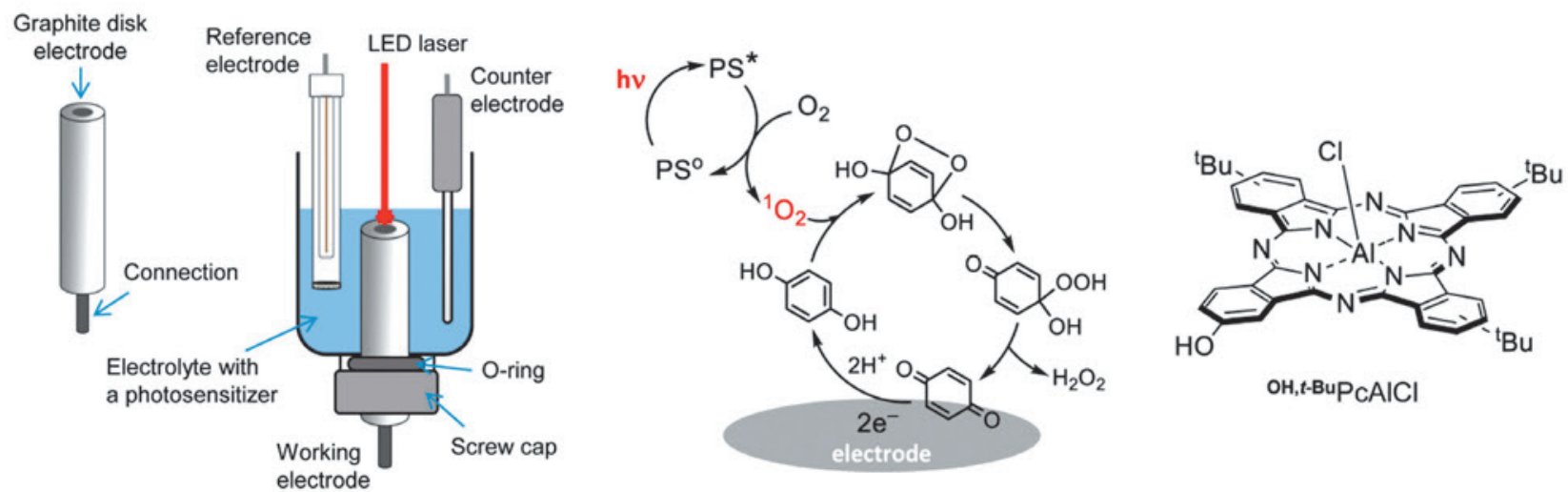

Figure 10. Schematic illustration of three-electrode cell equipped with graphite dick electrode and scheme of reversible process of oxidation of hydroquinone into benzoquinone under the singlet oxygen, generating by photosensitizer $\left({ }^{\mathrm{OH}, t-\mathrm{Bu}} \mathrm{PcAlCl}\right) \cdot{ }^{[158]}$ 

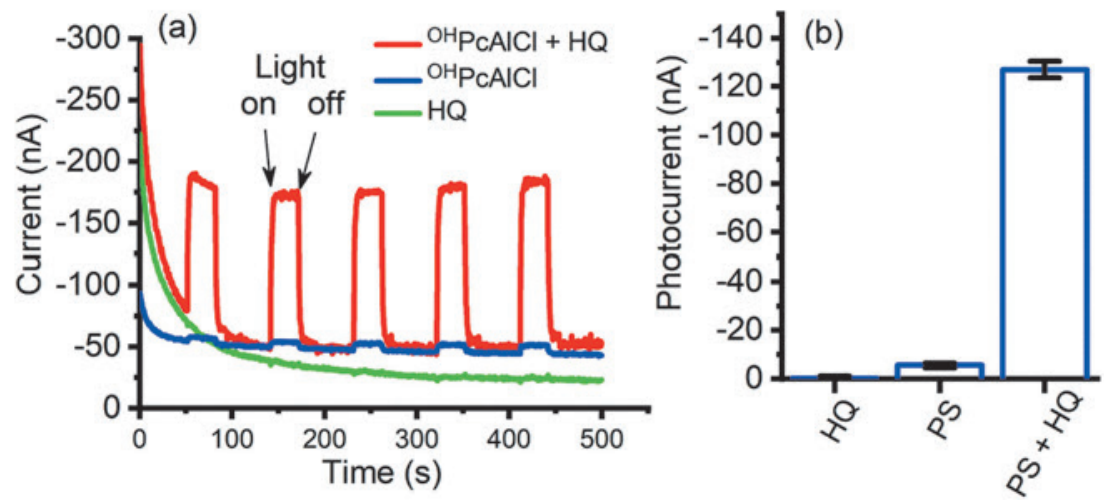

Figure 11. Amperometric behavior of ${ }^{\mathrm{OH}, t-\mathrm{Bu}} \mathrm{PcAlCl}$ in the presence and the absence of $\mathrm{HQ}$, and $\mathrm{HQ}$ in the absence of the photosensitizer PS (a). The potential applied $-0.1 \mathrm{~V}$; red laser $659 \mathrm{~nm} ; 30 \mathrm{~mW}$. Average photocurrents obtained for $\mathrm{HQ},{ }^{\mathrm{OH}, \mathrm{t}-\mathrm{Bu}} \mathrm{PcAlCl}$ and ${ }^{\mathrm{OH}}, \mathrm{t}-\mathrm{Bu} \mathrm{PcAlCl}+$ HQ (background corrected) ${ }^{[158]}$.

quantum yield $\left(\Phi_{\Delta}\right)$ was determined. $\Phi_{\Delta}$ value for target phthalocyanines and their analogs can be calculated as:

$\Phi_{\Delta^{\prime} \mathrm{PS}}=\Phi_{\Delta^{\prime} \mathrm{REF}} \times \alpha_{\mathrm{REF}} / \alpha_{\mathrm{PS}} \times$ Photocurrent $_{\mathrm{PS}} /$ Photocurrent $_{\mathrm{REF}}$,

where $\alpha=\left(1-10^{-\mathrm{Abs}}\right.$ at the working wavelength of laser $)$.

The following correlations were found between the values of $\Phi_{\Delta}$ and the structure of photosensitizers. In comparison with the corresponding monomeric complex, dimeric aluminum phthalocyanine showed a worse response. Comparing to aluminum complexes, photosensitizers containing zinc central ion are more effective. Thus tert-butylsubstituted zinc phthalocyanine demonstrated the highest singlet oxygen quantum yield (working wavelength of laser $\lambda_{\text {laser }}=659 \mathrm{~nm}$ ).

At the working wavelength of laser $\lambda_{\text {laser }}=532 \mathrm{~nm}$ the fluoro-substituted subphthalocyanine of boron showed the highest singlet oxygen quantum yield. Due to the cone geometry of subphthalocyanine molecule, ${ }^{[166]}$ it demonstrated better solubility and absence of aggregation comparing to phthalocyanine complexes. For this reason singlet oxygen quantum yields of subphthalocyanines are higher, than those for phthalocyanines.

Thus, the developed method for determining the photocatalytic activity of phthalocyanines and their analogs is well suited for determining high values of the yield

Table 1. Optical spectral data, values of photocurrent and singlet oxygen quantum yields for phthalocyanine photosensitizers. ${ }^{[158]}$
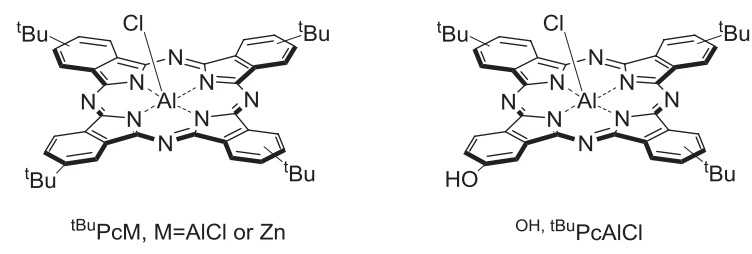

$\mathrm{OH}, \mathrm{tBu} P \mathrm{AICl}$

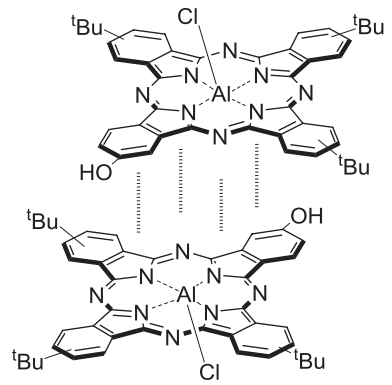

$\left({ }^{\mathrm{OH}, \mathrm{tBu}} \mathrm{PCAICl}\right)_{2}$

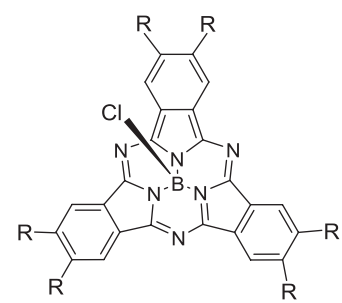

RSubPcBCl, R = tBu, F, PhO

\begin{tabular}{|c|c|c|c|c|}
\hline Compound & $\begin{array}{c}\lambda_{\max }(\mathrm{nm}) \\
\text { in } \mathrm{CH}_{3} \mathrm{OH}\end{array}$ & $\begin{array}{c}\text { Absorbance at the working } \\
\text { wavelength of laser } \lambda_{\text {laser }}\end{array}$ & Photocurrent $(\mu \mathrm{A})$ & $\Phi_{\Delta}$ \\
\hline t-BupcAlCl & 680 & $0.231 \pm 0.008$ & $-0.236 \pm 0.022$ & $0.15 \pm 0.01$ \\
\hline oH,t-BupcAlCl ${ }^{[129]}$ & 684 & $0.241 \pm 0.002$ & $-0.156 \pm 0.004$ & $0.095 \pm 0.002$ \\
\hline$\left({ }^{\mathrm{OH}, t-\mathrm{Bu}} \mathrm{PcAlCl}\right)_{2}{ }^{[129]}$ & 685 & $0.256 \pm 0.002$ & $-0.039 \pm 0.003$ & $0.023 \pm 0.002$ \\
\hline Pheophorbide $a$ (reference sample) & 666 & $0.248 \pm 0.003$ & $-1.040 \pm 0.050$ & $0.62^{[162]}$ \\
\hline${ }^{\mathrm{F}} \mathrm{SubPcBCl}{ }^{[158]}$ & 550 & 0.236 & $-0.683 \pm 0.032$ & $0.76 \pm 0.04$ \\
\hline${ }_{t \text {-Bu SubPcBCl }}$ & 565 & $0.236 \pm 0.004$ & $-0.407 \pm 0.051$ & $0.45 \pm 0.05 ; 0.47^{[163]}$ \\
\hline${ }^{\mathrm{PhO}} \mathrm{SubPcBCl}{ }^{[164]}$ & 569 & $0.245 \pm 0.003$ & $-0.613 \pm 0.059$ & $0.67 \pm 0.07$ \\
\hline
\end{tabular}


of singlet oxygen generation $\Phi_{\Delta}>0.5$ and for low yields $\Phi_{\Delta}<0.05$. It is characterized by rapidity and good reproducibility: the value of the relative standard deviation for five independent measurements was only $7.7 \%$.

In contrast to the commonly used trap method, ${ }^{[167]}$ this approach does not require a long experiment time, solution stirring, or complex measuring equipment. The developed method can be used to study the activity of photosensitizers deposited onto the surface of printed electrodes.

Based on the experiments performed, it can be concluded that the investigated aluminum phthalocyanine complexes and subphthalocyanine complexes are effective photosensitizers. The results obtained made it possible to start the development of effective biosensors using phthalocyanines for the detection of antibiotics in surface waters.

\section{Phthalocyanines - Prospective Catalysts in the Synthesis of Novel Biologically Active Compounds}

Our group studied the catalytic activity of new phthalocyanine complexes containing various metals in their structure, electron-donor and acceptor peripheral substituents to determine the most active catalysts.

Since in our group previously we practically did not study the catalytic properties of the newly synthesized complexes, understandably, we started with the simplest processes, using free ligands and their complexes with $\mathrm{Fe}, \mathrm{Co}, \mathrm{Cu}, \mathrm{Al}, \mathrm{In}, \mathrm{Ga},{ }^{[168-173]}$ including those supported on surfaces or grafted to polymer substrates. The following carriers were chosen: melamine sponges, Nafen nanofibers (NAFEN) and modified hypercrosslinked polystyrene PS-3. According to the low-temperature nitrogen adsorption method (in the BET approximation), their specific surface areas are 800,155 , and $1100 \mathrm{~m}^{2} / \mathrm{g}$, respectively.

In addition, we covalently immobilized 2-hydroxy9(10),16(17),23(24)-tri-tert-butyl phthalocyanine of cobalt(II) to a styrene-divinylbenzene copolymer PS-100, activated by introducing chloromethyl groups. At the same time, high catalytic activity was found in the oxidation of phenol to benzoquinone. ${ }^{[169]}$

We succeeded in the preparation of heterogeneous catalysts for model oxidation of phenol using immobilized substituted phthalocyanine complexes of $\mathrm{Co}$ and $\mathrm{Cu}$ on polymer supports with highly developed surfaces. ${ }^{[169]}$

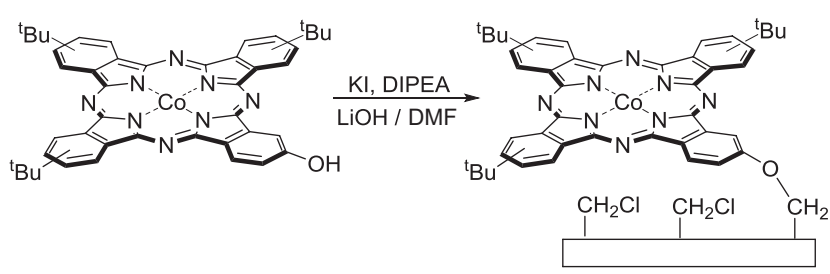

PS-100

Scheme 8. Synthesis of Co(II) phthalocyanine complex covalently linked to PS-100.

A general approach to the preparation of palladium complexes of phthalocyanines containing electron donor $\left(\mathrm{OPh}, \mathrm{OMe}, \mathrm{OEt}, \mathrm{OBu},{ }^{t} \mathrm{Bu}\right)$ and electron acceptor $\left(\mathrm{NO}_{2}\right.$, $\mathrm{CN}, \mathrm{F}, \mathrm{Cl})$ peripheral substituents has been developed. Their high catalytic activity in the palladium-catalyzed Sonogashira reaction between phenylacetylene and various aryl halides was shown. It was demonstrated that the yield of the target diphenylacetylenes reached $90 \%$, which significantly exceeds the efficiency of the catalytic systems developed to date. ${ }^{[174]}$

Using a palladium catalyst, several new 5-alkynyl-substituted uracil and uridine derivatives have been synthesized in high yields at room temperature.

All compounds were tested for antimycobacterial activity against Mycobacterium bovis and Mycobacterium tuberculosis (H37Ra) at concentrations of $1-100 \mu \mathrm{g} / \mathrm{mL}$ using the MABA test. The results obtained showed that the most of the studied derivatives of uracil have high antimycobacterial activity $($ MIC50 $=1.1-19.2 \mu \mathrm{g} / \mathrm{ml})$ versus therapeutic agents such as rifampicine, isoniazid, and D-cycloserine. ${ }^{[175]}$

We were the first to use palladium phthalocyanine as a homogeneous catalyst in the Sonogashira reaction at room temperature, promoted by $\mathrm{Pd} / \mathrm{Cu} \cdot{ }^{[176]}$ Based on the studies carried out, the leading compounds were selected - candidates for extended preclinical studies: 2-phenyl-1-benzoylindole3-carboxylic and 2-phenyl-1-benzoylindole-3-acetic acids.

\section{Anion-Selective Electrodes Based on Phthalocyanine Complexes}

Due to the diversity of coordination chemistry, metal phthalocyanines and their analogues are widely used as active components of potentiometric sensors for various

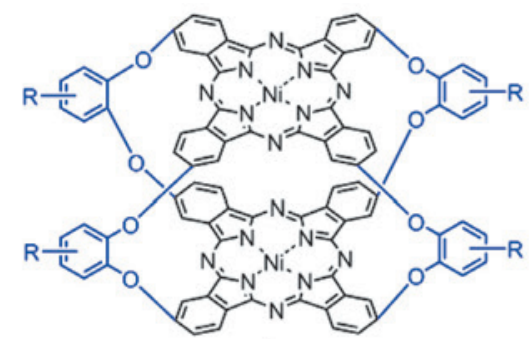

A

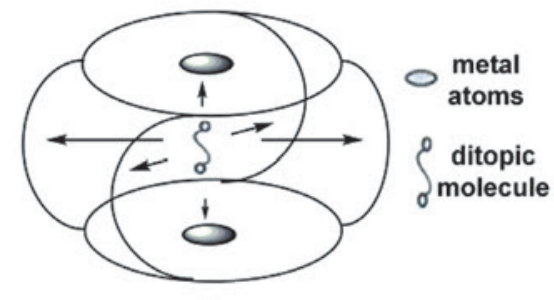

B

Figure 12. Structure of binuclear Ni(II) tetra(4-tert-butylphenylene-1,2-dioxy)bisphthalocyanine (A) and schematic presentation of interactions between ionophore and two coordination centers of ditopic molecule (B) ${ }^{[179]}$ 
anions. The selectivity of such sensors is based on the specific interaction of the central metal with the detected anion. For example, due to the ability of the metal to coordinate additional ligands in the axial position of the initial compound, or as a result of the exchange reaction between the extra ligand and the target ion. ${ }^{[177,178]}$.

The binuclear Ni(II) tetra(4-tert-butylphenylene1,2-dioxy)-bisphthalocyanine is able to selectively coordinate the anions of dicarboxylic acids (Figure 12). ${ }^{[179]}$ Ion selective electrodes (ISE) with a plasticized membrane based on this compound demonstrate a response in solutions of maleic and terephthalic acids. The resulting electrode was used by us to determine the terephthalate dianion in drinking water "Holy Spring", stored in polyethylene terephthalate packaging.

Metal phthalocyanines have found use in solid-state ion-selective electrodes. ${ }^{[180]}$ For the first time, subphthalocyanines were chosen for ion-selective electrodes. ${ }^{[181,182]}$

Thus, based on the studies carried out, it can be concluded that phthalocyanines have been successfully used to create ISEs of various structures for a number of organic and inorganic anions with high selectivity to the ion being determined. In some cases, ISEs with outstanding characteristics were obtained. By varying the central metal in the ionophore complex and its surrounding, a researcher gets many opportunities for creating new, highly selective, and highly sensitive sensors for anions.

\section{Other Application Areas of Phthalocyanines}

\section{Nonlinear Optics}

Optical limiters are materials that reduce the intensity of powerful laser radiation, protecting the sensitive optics of the device or the observer's eyes. The presence of pronounced nonlinear optical properties allows one to consider phthalocyanines and hybrid materials based on them as promising optical limiters. The nonlinear optical properties of phthalocyanines and their analogues can be characterized using the $\mathrm{Z}$ scan technique. ${ }^{[183]}$

T. Nyokong's group has carried out large-scale studies to improve nonlinear optical characteristics (ratio of cross sections of the ground and excited states $\sigma_{1} / \sigma_{0}$, nonlinear absorption coefficient) by obtaining hybrid materials based on phthalocyanines of various structures and nanoparicles, ${ }^{[184,185]}$ quantum dots ${ }^{[186]}$ and alloys. ${ }^{[187]}$

Of particular interest is the identification of correlations between the nonlinear optical response and the nature of the substituents or the central ion in the phthalocyanine macrocycle. In the works of our group, a study of the nonlinear optical properties of complexes of planar and sandwich structures of elements of the beginning of the series $(\mathrm{Nd}, \mathrm{Pr})$ was carried out by the method of Z scanning. ${ }^{[188]}$

It was found that $\mathrm{Nd}$ (III) monophthalocyanines are characterized by larger values of $\sigma_{1}$ and $\sigma_{1} / \sigma_{0}$ than for the corresponding $\operatorname{Pr}(\mathrm{III})$ complexes. This is probably due to differences in the electronic structure of these lanthanide ions. An increase in the value of $\sigma_{1} / \sigma_{0}$ is observed upon passing from octa- to hexadeca-chlorosubstituted phthalocyanine complexes. This can be explained by an increase in the electron density deficit on the phthalocyanine macrocycle with an increase in the number of chlorine atoms. Monophthalocyanine complexes showed higher values of nonlinear optical response than double-deck complex. This is probably due to a decrease in symmetry upon passing from the bisphthalocyanine complex to monophthalocyanine complexes.

We have also obtained hybrid nanoparticles based on hexadecachloro-substituted lanthanide phthalocyanines and $\mathrm{Au}$ nanoparticles with diameters of 20 and $30 \mathrm{~nm}$. It was found that the nonlinear optical response is more pronounced for hybrids compared to the individual erbium complex and rises with an increase of the Au nanoparticles size, reaching $\beta=18 \cdot 10^{-12} \mathrm{~cm} / \mathrm{W}$ for a hybrid with nanoparticles with a diameter of $30 \mathrm{~nm} \cdot{ }^{[189]}$

The comparison of properties was carried out for hexadecachloro-substituted complexes. ${ }^{[190]}$ The influence of the central lanthanide ion nature on their nonlinear optical properties is illustrated in Figure 13. It is shown that terbium phthalocyanine demonstrates the best nonlinear optical response.

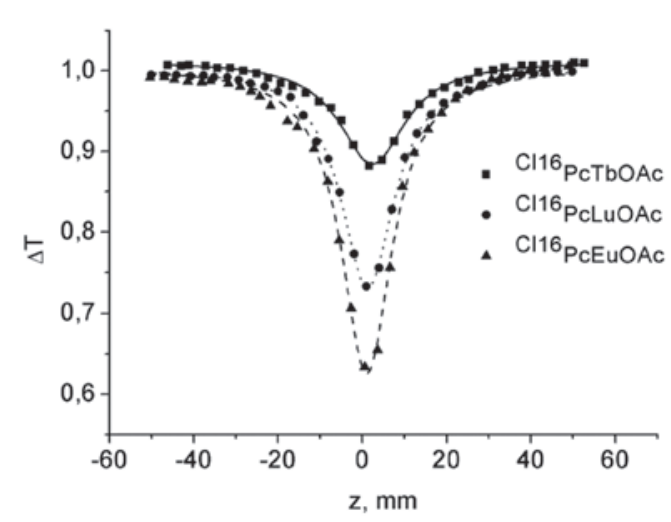

Figure 13. Comparison of transmittance dependencies for samples $\left(\mathrm{Cl}_{16} \mathrm{PcEuOAc}\right.$ - triangles, dashed line - approximation;

$\mathrm{Cl}_{16} \mathrm{PcTbOAc}$ - squares, solid line - approximation, $\mathrm{Cl}_{16} \mathrm{PcLuOAc}$ - dots, dotted line - approximation) (open aperture Z-scan).

Thus, due to their pronounced nonlinear optical properties, the synthesized complexes can be used to create optical limiters of high-power laser radiation on their basis.

\section{Materials with Resistive Switching Effect}

In modern microelectronics, the development of fast, compact and inexpensive electronic memory elements is one of the priority tasks. ${ }^{[191]}$ The use of resistive switching effect in materials and structures of various types can lead to efficient and easy-to-use memory elements with basic performance parameters at least comparable to other competing devices. ${ }^{[192,193]}$ In particular, we are talking about devices based on organic materials.

Based on tris(hexadecachlorophthalocyaninato)dilutetium and CdSe nanoplates (NP CdSe) with mass concentrations of $55 \%$ and $10 \%$, respectively in a polymer matrix, we obtained a composite material exhibiting the effect of resistive switching (Figure 14) ${ }^{[194]}$ The possibility of stabilizing intermediate states in the process of the resistive switching 

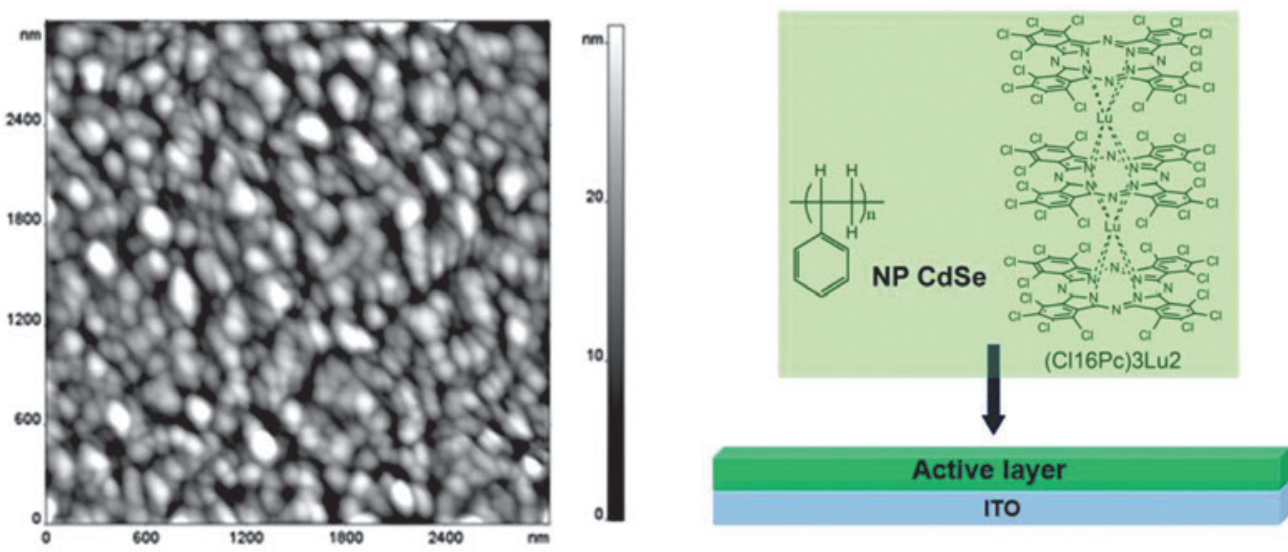

Figure 14. Structure of composite material. ${ }^{[194]}$

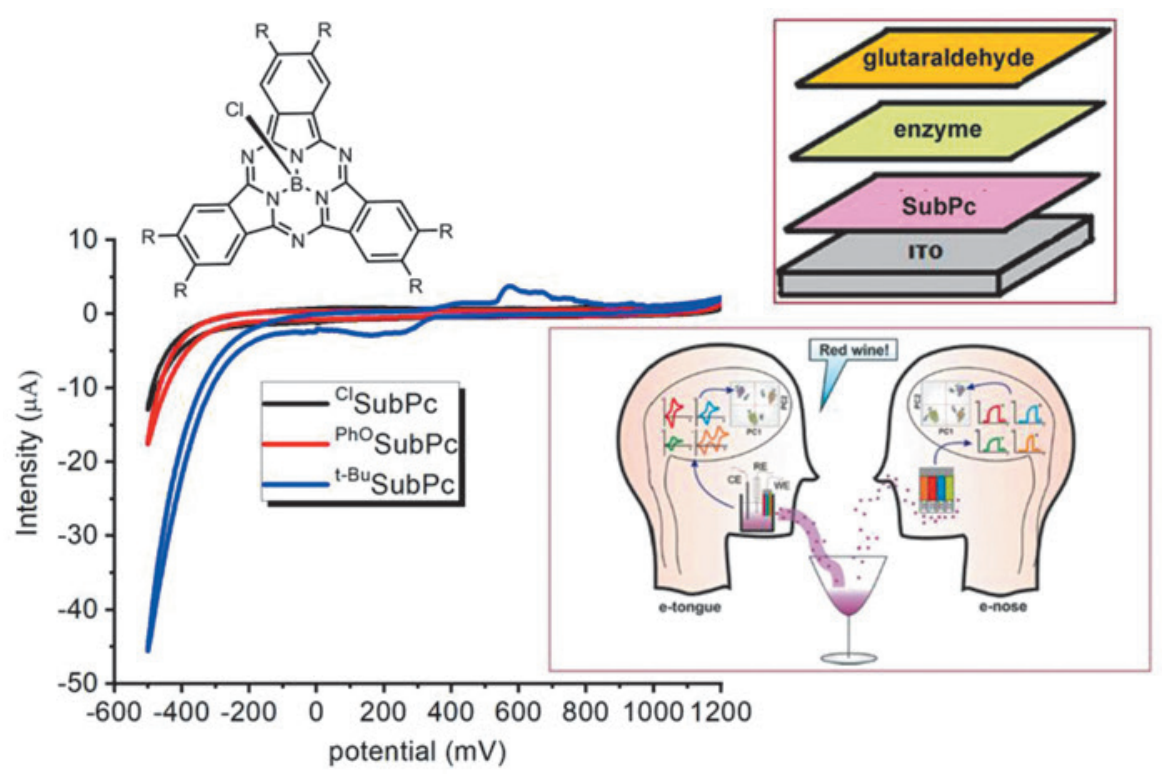

Figure 15. Cyclic voltammetry of subphthalocyanines and sensor device diagram. ${ }^{[195]}$

effect is shown, which makes it possible to increase the number of states of a memory cell.

\section{Multisensor Systems}

The detection of phenols is an important task for food chemistry. In collaboration with University of Valladolid ${ }^{[195]}$ thin-film sensor based on enzymes (tyrosinase and laccases) were developed for detection of dibasic alcohols (pyrocatechine and hydroquinone). Novel subphthalocyanine complexes were introduced into the sensor as additive and played the role of electron mediators between enzymes and electrode.

The sensors based on enzymes, without the addition of subphthalocyanine, demonstrated detection limits, which was close in value to $10^{-6} \mathrm{M}$. The presence of subphthalocyanine resulted in the detection limit of alcohols till $10^{-7}$ M. The sensor based on phenoxy-substituted subphthalocyanine showed the best response. This can be explained by $\pi-\pi$ stacking interactions between active sites of enzymes and peripheral substituents of subphthalocyanine. The investigation of three samples of red wine, provided by "Instituto
Tecnológico Agrario de Castilla y León (ITACyL) (Valladolid, Spain), was conducted. ${ }^{[195]}$ The wines were prepared from the same sort of grape (Tempranillo), but total polyphenol indexes were different for all three wines (Figure 15).

\section{Design of Single Molecule Magnets Based on Binuclear Lanthanide Complexes with Tetrapyrrolic Ligands}

Development of new approaches to information storage is an urgent task of modern science, which is solved by cooperation of different fields of physics, chemistry and materials science. One way to solve this problem is to miniaturize elements of memory storage devices, preferably up to the molecular level using multistable molecules which can undergo transition between states under external physical influence. ${ }^{[196]}$ From this point of view, high-spin $d$ - and $f$-metal complexes are of particular interest, as some of them behave like single molecule magnets (SMM). ${ }^{[197]}$ 

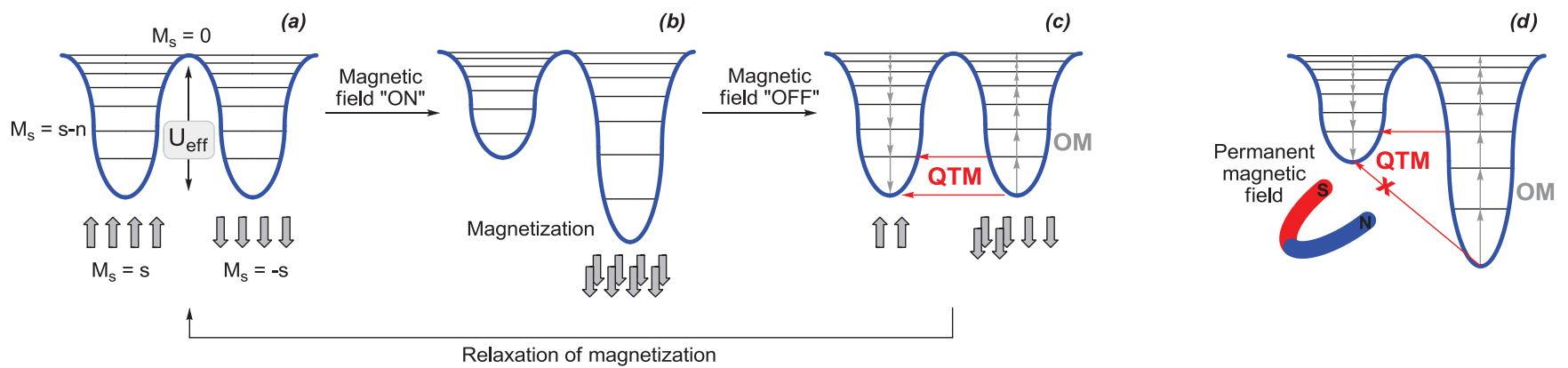

Figure 16. $(a-c)$ - Schematic diagram demonstrating the magnetization $(b)$ and magnetic relaxation $(c)$ processes in a single molecule magnet, OM stands for thermally activated Orbach mechanism of relaxation, QTM - for quantum tunneling of magnetization. (d) The principle of suppression of QTM in de field.

The phenomenon of SMM behaviour in high spin complexes is explained by the appearance of energy barrier $\mathrm{U}_{\text {eff }}$ between spin states $\mathrm{M}_{\mathrm{s}}=\mathrm{S}$ and $\mathrm{M}_{\mathrm{s}}=-\mathrm{S}$, which is provided by large negative values of zero field splitting $\mathrm{D}$ in the ligand field (Figure 16a). Once the SMM is placed into the magnetic field, the degeneration between spin states with different signs is removed and the magnetisation occurs due to the population of the more favourable spin state (Figure 16b). Turning-off the field results in magnetization relaxation (Figure 16c) and its rate depends on its predominant mechanism:

-thermally-activated Orbach mechanism (OM) provides slow relaxation, which ensures that the magnetization remains below the so-called blocking temperature for a relatively long time needed for information storage and processing.

-barrier-free quantum tunnelling of magnetization (QTM) is fast and this process should be efficiently supressed. This can be done by application of external direct current (dc) magnetic field, affording therefore the so called field-induced SMM (Figure 16d). Another approach is elaboration of multinuclear systems, where QTM is suppressed by exchange bias in ferromagnetically coupled bi- or polynuclear systems: assuming that the probability of QTM in mononuclear SMMs is $P_{\mathrm{QTM}}$, its probability in the coupled binuclear system is $\mathrm{P}_{\mathrm{QTM}}{ }^{2}<\mathrm{P}_{\mathrm{QTM}}$.

The first SMM was reported in 1993 on the example of a 12-core mixed-valent carboxylate manganese cluster $\left[\mathrm{Mn}_{12} \mathrm{O}_{12}(\mathrm{OAc})_{16}\left(\mathrm{H}_{2} \mathrm{O}\right)_{4}\right]^{\left[{ }^{[198]}\right.}$ At temperatures below $4 \mathrm{~K}$ it could be magnetized in an external magnetic field followed by a slow relaxation of magnetization after removal of the field, which was characterized by the bar-

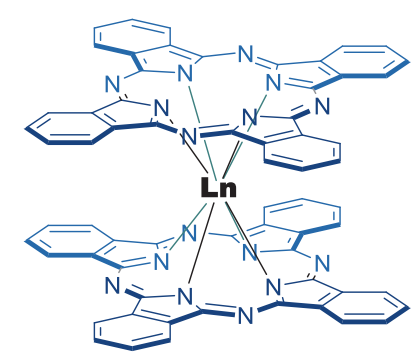

Figure 17. Double-decker bisphthalocyaninates $\operatorname{Ln}(\mathrm{Pc})_{2}, \mathrm{Ln}=\mathrm{Tb}$, Dy as parent building blocks for lanthanide-based SMMs. rier $\mathrm{U}_{\mathrm{eff}}=43 \mathrm{~cm}^{-1}$, and the blocking temperature, at which magnetization remains for $100 \mathrm{~s} \mathrm{~TB}_{100}=3.1 \mathrm{~K}$.

The important breakthrough in the field of SMM occurred in 2003 when N. Ishikawa reported discovered slow magnetization relaxation on the example of $\mathrm{Tb}(\mathrm{III})$ and Dy(III) bisphthalocyaninates $\left[\mathrm{Ln}\left(\mathrm{Pc}^{2-}\right)_{2}\right]^{-}\left(\mathrm{NBu}_{4}\right)^{+}$(Figure 17). ${ }^{[199,200]}$ These complexes were first examples of both $f$-metal based SMMs and monoionic SMMs. Slow relaxation of $\mathrm{Tb}(\mathrm{III})$ complex was observed up to $14 \mathrm{~K}$, which significantly exceeded the values ever obtained previously for any $d$-metal based SMM. It was found that nearly ideal $D_{4 d}$ coordination surrounding of lanthanide ions provides high magnetic anisotropy resulting in high $\mathrm{U}_{\text {eff }}$ and $\mathrm{T}_{\mathrm{B} 100}$ values $\left(\mathrm{U}_{\text {eff }}=230\right.$ and $28 \mathrm{~cm}^{-1}, T_{B 100}=15.6 \mathrm{~K}$ и $2.4 \mathrm{~K}$ for Tb and Dy complexes respectively).

Numerous studies of SMM properties of $\operatorname{Ln}(\mathrm{III})$ complexes with tetrapyrrolic ligands allowed to summarize the influence of structural and electronic factors on the rates and mechanisms of magnetic relaxation. ${ }^{[201]}$ Moreover, peculiar coordination chemistry of lanthanide ions in tetrapyrrolic surrounding allowed to elaborate multinuclear systems with ferromagnetically coupled metal centres. This small review is aimed to give examples of coupled systems constructed via coordination, covalent or supramolecular assembling of $\operatorname{Ln}(\mathrm{III})$ sandwich complexes with tetrapyrrolic ligands.

Binuclear coordination compounds include, first of all, vast family of triple-decker complexes composed of various combinations of paramagnetic nuclei and tetrapyrrolic ligands (Figure 18). The investigation of heteronuclear triple-decker complexes revealed the effect of $f-f$ interaction between the proximal paramagnetic lanthanides separated by $c a .3 .5 \AA$, which was reported firstly in 2002 on the example of heteroleptic trisphthalocyaninates $\left[(\mathrm{BuO})_{8} \mathrm{Pc}\right] \mathrm{M}^{*}(\mathrm{Pc})$ $\mathrm{M}(\mathrm{Pc}){ }^{\left[{ }^{[202]}\right.}$ Later it was demonstrated that the appearance of $f-f$ interaction results in growth of the relaxation time in $\left[(\mathrm{BuO})_{8} \mathrm{Pc}\right] \mathrm{Tb}(\mathrm{Pc}) \mathrm{Tb}(\mathrm{Pc})$ complex in comparison with heteronuclear complexes $\left[(\mathrm{BuO})_{8} \mathrm{Pc}\right] \mathrm{Tb}(\mathrm{Pc}) \mathrm{Y}(\mathrm{Pc})$ and $\left[(\mathrm{BuO})_{8} \mathrm{Pc}\right] \mathrm{Y}(\mathrm{Pc}) \mathrm{Tb}(\mathrm{Pc})$ containing only one paramagnetic ion. ${ }^{[203]}$ The temperatures of slow relaxation of magnetization were found to be $7 \mathrm{~K}$ and $18 \mathrm{~K}$ for heteronuclear complexes respectively and $27 \mathrm{~K}$ for the homonuclear complex.

Importantly, the elongation by $\sim 0.25 \AA$ of bonds between metal ions and nitrogen atoms of inner Pc ligands 


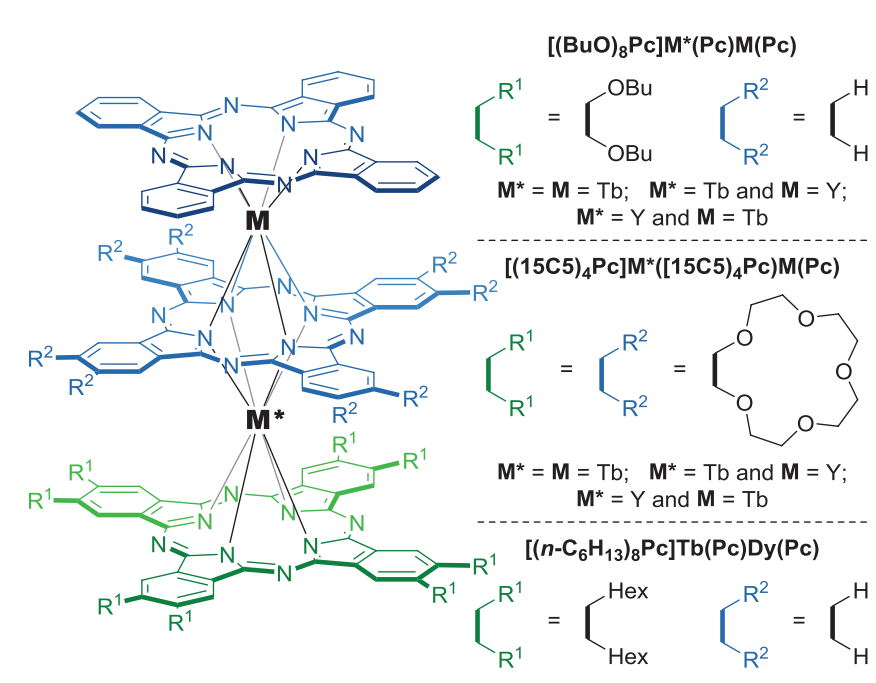

Figure 18. Heteroleptic homo- and heteronuclear triple-decker complexes possessing $\mathrm{SMM}$ properties $-\left[(\mathrm{BuO})_{8} \mathrm{Pc}\right] \mathrm{M}(\mathrm{Pc})$ $\mathrm{M}(\mathrm{Pc}),{ }^{[203]}\left[(15 \mathrm{C} 5)_{4} \mathrm{Pc}\right] \mathrm{M}\left[(15 \mathrm{C} 5)_{4} \mathrm{Pc}\right] \mathrm{M}(\mathrm{Pc})^{[204]}$ and $\left[\left(n-\mathrm{C}_{6} \mathrm{H}_{13}\right)_{8} \mathrm{Pc}\right]$ $\mathrm{Tb}(\mathrm{Pc}) \operatorname{Dy}(\mathrm{Pc}) \cdot{ }^{[205]}$

in triple-decker complexes resulted in decrease of symmetry of the coordination polyhedron from idealized $D_{4 d}$ to $C_{4 d}$ which activated the QTM mechanism, therefore the heteronuclear complexes containing only one $\mathrm{Tb}^{3+}$ ion revealed field-induced SMM behaviour. To the contrast, the binuclear complex exhibited slow relaxation of magnetization even in zero field.

In the case of heteroleptic crown-substituted tripledeckers $\left[(15 \mathrm{C} 5)_{4} \mathrm{Pc}\right] \mathrm{M} *\left[(15 \mathrm{C} 5)_{4} \mathrm{Pc}\right] \mathrm{M}(\mathrm{Pc})$ homonuclear di$\mathrm{Tb}(\mathrm{III})$ complex also revealed better SMM properties with higher $\mathrm{U}_{\text {eff }}\left(159 \mathrm{~cm}^{-1}\right)$ in comparison with $\mathrm{Tb}(\mathrm{III}) / \mathrm{Y}(\mathrm{III})$ counterparts $\left(117 \mathrm{~cm}^{-1}\right.$ for $\left[\mathrm{Y}^{*}, \mathrm{~Tb}\right]$ and $90 \mathrm{~cm}^{-1}$ for $\left.\left[\mathrm{Tb}^{*}, \mathrm{Y}\right]\right) \cdot{ }^{[204]}$ It was also shown that the value of $U_{\text {eff }}$ correlated with the value of axial component of the magnetic susceptibility tensor deduced from crystallographic and NMR studies. These results emphasize the role of coordination surrounding in SMM behaviour of triple-decker complexes. ${ }^{[206]}$

The complex $\left[\left(n-\mathrm{C}_{6} \mathrm{H}_{13}\right)_{8} \mathrm{Pc}\right] \mathrm{Tb}[(\mathrm{Pc}) \mathrm{Dy}(\mathrm{Pc})$ refer to the sole example of triple-deckers containing two different paramagnetic ions whose SMM properties have been

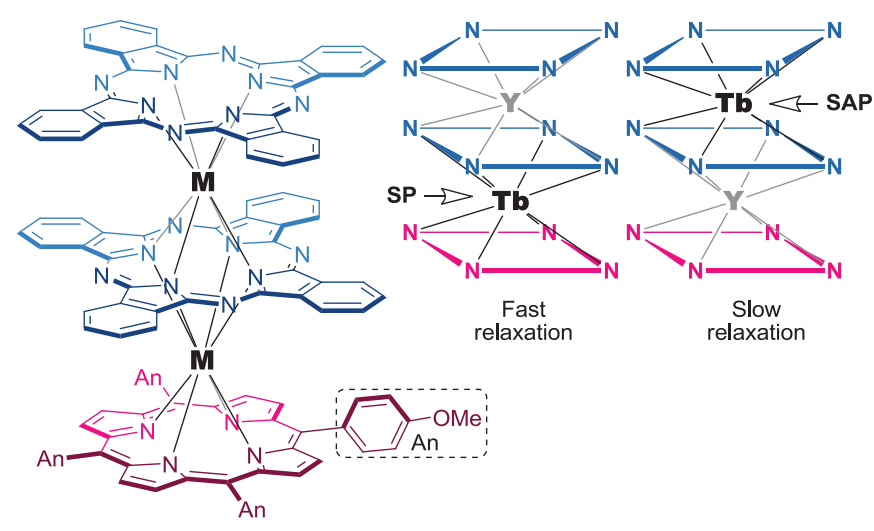

Figure 19. Heteroleptic triple-decker complex containing Por and Pc ligands providing square-prismatic (SP) and squareantiprismatic (SAP) coordination surrounding of REE ions. ${ }^{[27]}$ investigated.$^{[205]}$ A weak, yet notable, ferromagnetic interaction between the $\mathrm{Tb}^{3+}$ and $\mathrm{Dy}^{3+}$ ions was detected affecting the magnetic behaviour of the complex. Importantly, magnetic properties of the complex are essentially preserved when isolated molecular units were deposited on the surface of highly ordered pyrolytic graphite.

The combination of Por and Pc ligands can be used to tune the coordination polyhedron of the lanthanide ion in sandwich complexes. For example, there were synthesized heteroleptic complexes [meso-(MeOPh) $\left.{ }_{4} \mathrm{Por}\right] \mathrm{M}^{*}(\mathrm{Pc}) \mathrm{M}(\mathrm{Pc})$, $\mathrm{M}, \mathrm{M}^{*}=\mathrm{Tb}$ and $\mathrm{Y}$ (Figure 19). ${ }^{[207]}$ Depending on the position of $\mathrm{Tb}$ ion it can be in square antiprismatic (SAP, M $=\mathrm{Tb}$, $\mathrm{M}^{*}=\mathrm{Y}$ ) or prismatic ( $\left.\mathrm{SP}, \mathrm{M}=\mathrm{Y}, \mathrm{M}^{*}=\mathrm{Tb}\right)$ surrounding. In the former case there was observed slow magnetization relaxation, while in the latter case the relaxation was faster because of QTM. This mechanism was suppressed once two $\mathrm{Tb}$ ions were introduced into the molecule due to the dipolar $f-f$ interaction between lanthanide ions separated by $\sim 3.6 \mathrm{~A} .{ }^{[208]}$

One more type of binuclear SMMs refer to complexes where one bridging $\mathrm{Pc}^{2-}$ ligand is coordinated to two paramagnetic nuclei surrounded by tetramethylheptanedionate (thd) counterions - $\left[\mathrm{PcLn}_{2} \text { (thd) }\right)_{4}$ (Figure 20). These complexes were firstly isolated in $1983^{[209]}$ and the exchange coupling between $\mathrm{Ln}^{3+}$ ions was detected in 1986, ${ }^{[210]}$ however their SMM behaviour attracted attention only in 2017, when Dy(III) and $\operatorname{Er}($ III) complexes forming crystalline solvates with benzene molecules were studied. It was shown that the solvate $\left[\mathrm{PcEr}_{2}(\text { thd })_{4}\right] \times 2 \mathrm{C}_{6} \mathrm{H}_{6}$ exhibited fast magnetic relaxation with barrier of $2.6 \mathrm{~cm}^{-1}$ under $600 \mathrm{Oe}$ dc field, however soaking of these crystals in dichloromethane afforded the exchange of solvate molecules giving the solvate $\left.\left[\mathrm{PcEr}_{2} \text { (thd }\right)_{4}\right] \times 2 \mathrm{CH}_{2} \mathrm{Cl}_{2}$ with $\mathrm{SMM}$ behaviour and barrier of $34.3 \mathrm{~cm}^{-1}$, showing therefore the rare example of subtle control of relaxation by lattice solvents. ${ }^{[211]}$ On the example of Dy(III) complex $\left[\mathrm{PcDy}_{2}(\text { thd })_{4}\right] \times 2 \mathrm{C}_{6} \mathrm{H}_{6}$ it was shown that the solvate benzene molecules in the crystal lattice can be reversibly released and recovered which also affected the dynamics of magnetic relaxation. ${ }^{[212]}$

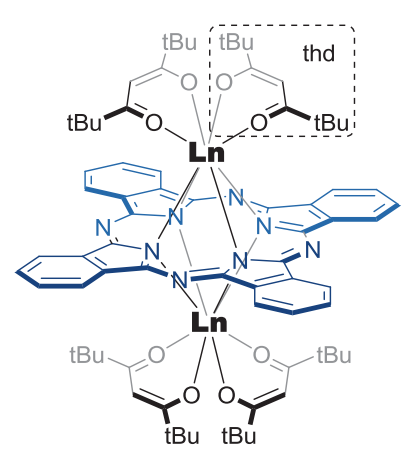

Figure 20. Binuclear monophthalocyaninates $\left[\operatorname{PcLn}_{2}(\text { thd })_{4}\right]$, $\mathrm{Ln}=\mathrm{Er}^{[211]}$ and Dy. ${ }^{[212]}$

Coordination binding of double-decker building blocks with $\mathrm{Cd}^{2+}$ ions allowed to construct extended binuclear sandwiches containing from four to five and six decks. In this sequence the Ln...Ln distance increased from 6.6 to 9.9 and $13 \AA$ (Figure 21), which renders these molecules as 


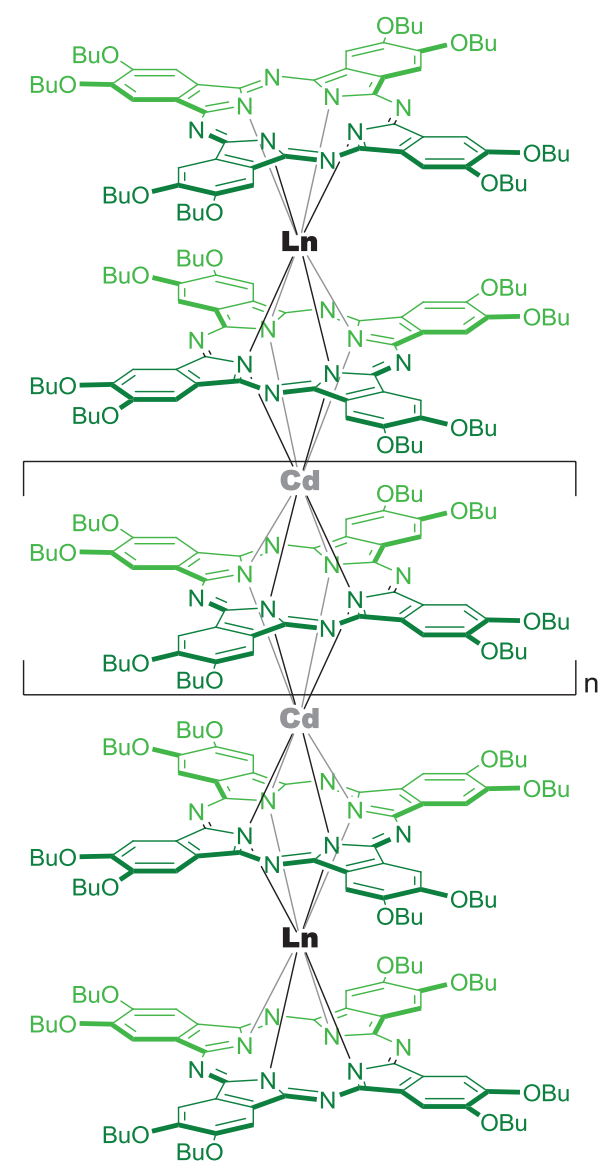

Figure 21. Quadruple- $(n=0)$, quintuple- $(n=1)$ and sextupledecker $(n=2)$ sandwich phthalocyaninates, $L n=T b, D y$.

excellent objects to study long-range $f$ - $f$ interactions in controlled arrangement of spin centres. ${ }^{[213,214]}$ It was shown that even at the longest distance in sextuple-decker molecule notable interaction between $\mathrm{Dy}^{3+}$ ions is responsible for partial suppression of QTM. ${ }^{[215]}$

Conjugated binuclear Pc ligand was used to form the binuclear complex with two fused double-decker blocks, affording the coupling between unpaired $\pi$ and $f$-electrons together with $f$ - $f$ interactions between ions in $D_{4 d}$ surrounding (Figure 22a). ${ }^{[216]}$ Altogether, it resulted in efficient QTM suppression, and the fused binuclear complex showed enhanced SMM properties $\left(\mathrm{U}_{\text {eff }}=699 \mathrm{~cm}^{-1}\right)$ in comparison with the binuclear triple-decker counterpart $\mathrm{Tb}_{2}\left[(\mathrm{BuO})_{8} \mathrm{Pc}\right]_{3}\left(\mathrm{U}_{\text {eff }}=230 \mathrm{~cm}^{-1}\right)$. NMR studies evidenced that the axial magnetic anisotropy in the fused complex is by $\sim 60 \%$ larger than that of the classical triple-decker complex, which also correlates with the higher $U_{\text {eff }}$ value of the former sandwich.

Connection of two double-decker units with an $o$-xylyl bridge $^{[217]}$ afforded clamshell-type binuclear quadrupledecker complex (Figure 22b). Magnetic measurements revealed ferromagnetic $\mathrm{Tb} \cdots \mathrm{Tb}$ interaction, which was responsible for the enhancement of SMM properties in comparison with the monomeric complex. ${ }^{[218]}$ To diminish the flexibility of the bridging unit and to obtain the welldefined arrangement of the interacting units, $\mathrm{Cd}^{2+}$ ion was incorporated into the clamshell cavity (Figure 22c). However, it impaired strongly the SMM properties of the binuclear system, for example $\mathrm{U}_{\text {eff }}$ reduced from 547 to $117 \mathrm{~cm}^{-1}$. This happened because of the symmetry lowering - binding of cadmium resulted in elongation of $\mathrm{Tb}-\mathrm{N}$ bonds formed with the internal Pc ligands.

To construct the dimer of double-deckers with welldefined architecture the supramolecular approach was used. Thus, the heteroleptic $\mathrm{Tb}(\mathrm{III})$ complex $\left[(15 \mathrm{C} 5)_{4} \mathrm{Pc}\right]$ $\mathrm{Tb}(\mathrm{Pc})$ with unsubstituted and crown-substituted ligands was treated with potassium acetate resulting in cationinduced assembling (Figure 23a). Such binding retained the lanthanide polyhedron unaffected since the $U_{\text {eff }}$ in monomer $\left(360 \mathrm{~cm}^{-1}\right)$ and dimer $\left(350 \mathrm{~cm}^{-1}\right)$ remained essentially the same. ${ }^{[219]}$ However, efficient suppression of QTM upon dimerization resulted in drop of relaxation rate from 0.001 to $1 \mathrm{~s}$ at $2 \mathrm{~K}$. The distance between interacting $\mathrm{Tb}^{3+}$ ions $(6.15-7.31 \AA$ ) was estimated using NMR data and this estimation was refined later by single-crystal $\mathrm{X}$-ray diffraction analysis of the dimer, formed by the triple-

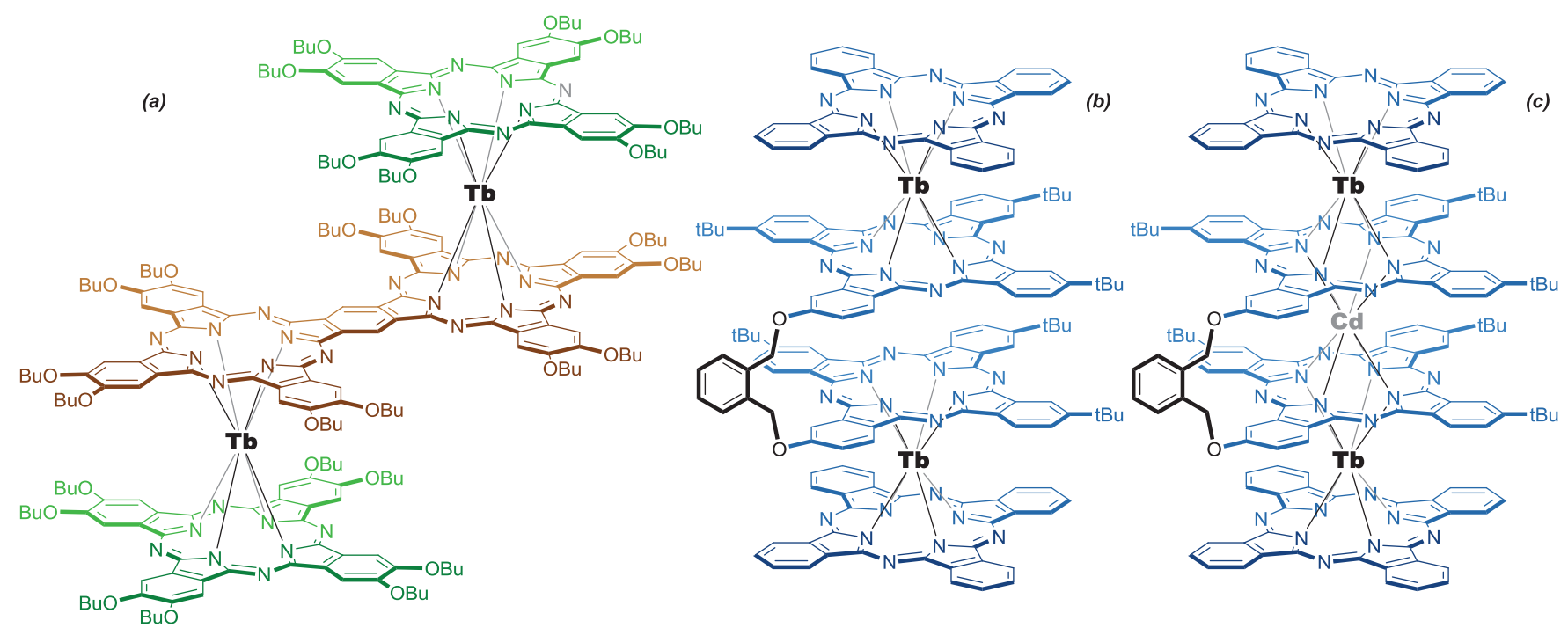

Figure 22. (a) - Dimeric complex formed from two fused Tb(III) bisphthalocyaninate units. ${ }^{[216]}$ (b) - Flexible clamshell-type dimer formed by two $\mathrm{Tb}(\mathrm{III})$ bisphthalocyaninate units joined via $o$-xylyl bridge; (c) rigid derivative of the clamshell dimer, where two units are coordinated to $\mathrm{Cd}^{2+}$ ion. ${ }^{[218]}$ 

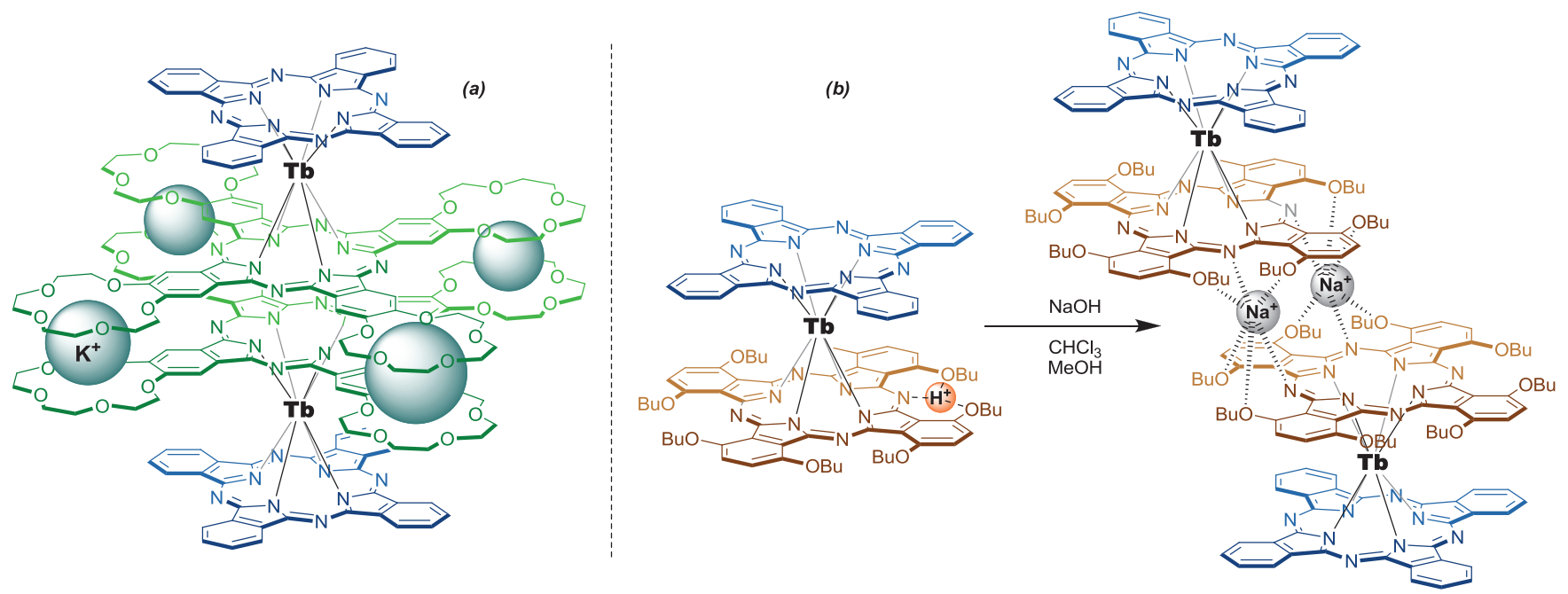

Figure 23. (a) - Supramolecular dimer, formed by $\left[(15 \mathrm{C} 5)_{4} \mathrm{Pc}\right] \mathrm{Tb}(\mathrm{Pc})$ in the presence of potassium cations. ${ }^{[219]}(b)$ - Preparation of the supramolecular quadruple-decker complex via deprotonation and cation-induced assembling of the complex $\left[(\alpha-\mathrm{BuO})_{8} \mathrm{PcH}\right] \mathrm{Tb}(\mathrm{Pc}){ }^{[127]}$

decker complex $\left[(15 \mathrm{C} 5)_{4} \mathrm{Pc}\right] \mathrm{Y}(\mathrm{Pc}) \mathrm{Y}(\mathrm{Pc})$ in the presence of $\mathrm{KBPh}_{4}$. In this case the distance between proximal REE ions was equal to $6.249 \AA \AA^{[220]}$ The later example also provides unique approach to the assemblies containing four paramagnetic nuclei and their SMM properties are yet to be studied.

One more example of the supramolecular dimer revealing SMM properties was prepared by $\mathrm{Na}^{+}$-induced dimerization of the protonated heteronuclear double-decker complex bearing nonperipherally-substituted Pc ligand $\left[(\alpha-\mathrm{BuO})_{8} \mathrm{PcH}\right] \mathrm{Tb}(\mathrm{Pc})$ (Figure 23b). ${ }^{[127]}$ The proton in this complex forms trifurcated hydrogen bond with meso-N atom and two oxygen atoms of $\alpha$-BuO-groups, which results in severe distortion of the molecular geometry including the deviation of the skew angle from $45^{\circ}$ to $28^{\circ}$ which leads to weak magnetic performance with $\mathrm{U}_{\text {eff }}=120 \mathrm{~cm}^{-1}$ and blocking temperature $2 \mathrm{~K}$. However, deprotonation of the complex with $\mathrm{NaOH}$ results in the formation of the quadruple-decker system with strongly improved SMM properties $-\mathrm{U}_{\text {eff }}=367 \mathrm{~cm}^{-1}$ and blocking temperature $25 \mathrm{~K}$. This spectacular improvement was associated with the fine optimization of the $\mathrm{Tb}^{3+}$ coordination polyhedron (skew angle $34^{\circ}$ ), intensification of the coordination field strength and significant enhancement of molecular magnetic anisotropy.

To conclude, it should be noted that elaboration of SMM materials is among the most emerging research fields in chemistry of Ln-based complexes with tetrapyrrolic ligands. The summarized results emphasize the need in proper design of molecular blocks including the control of coordination polyhedron geometry and coordination field strength. The influence of these features on SMM properties can be studied using paramagnetic NMR spectroscopy owing to well-defined correlations between structural features and spectral behavior. ${ }^{[221-224]}$ Finally, mighty means of coordination, organic and particularly supramolecular chemistry can be used as tools for the further fine tuning of magnetic properties of sandwich complexes promising new achievements in near future.

\section{Influence of Axial Coordination and Functional Cations on Optical and Magnetic Properties of the Reduced Metal Phthalocyanines}

Metal phthalocyanines find applications as dyes, pigments, electronic materials and catalyst. ${ }^{[225,226]}$ Properties of metal phthalocyanines can be modified by changing the periphery of the macrocycle or by axial coordination of different nitrogen and oxygen-containing ligands to the central metal atoms of phthalocyanines. Additional electron appears on the macrocycle under reduction or oxidation of metallophthalocyanines and that can essentially modify their optical, magnetic and conducting properties. Oxidation of metal phthalocyanines with the formation of $\pi$-stacks from the macrocycles provides one-dimensional conductivity of metallic type which in some cases is stable down to liquid helium temperatures. ${ }^{[227]}$ Oxidation of iron(III) metal phthalocyanines with axial cyano-ligands yields paramagnetic metal atoms integrated into conducting system and such compounds show giant magnetoresistance when magnetic field can affect their conductivity. ${ }^{[228]}$ Reduction can be centered on the central metal atoms of metal phthalocyanines ${ }^{[22-231]}$ or on the macrocycles providing the formation of the $\mathrm{Pc}^{\bullet 3-}$ radical trianions. ${ }^{[232,233]}$ In the latter case rather effective magnetic coupling of spins can be observed at close macrocycles packing in the layers or dimmers. ${ }^{[230,233-235]}$ Working with metal phthalocyanines we found that in some cases coordination of the ligands to metallophthalocyanines is accompanied by an electron transfer from this ligands or metal atoms to the macrocycles. In this case unpaired electron appears on the macrocycle and they can manifest rather strong magnetic coupling of spins. ${ }^{[236,238]}$ Electron transfer between two different anionic ligands coordinated to one metal center is also possible. ${ }^{[239]}$ In this section we also consider reduction of metal phthalocyanines accompanied by the coordination of $N$-methylimidazole. That allows to obtain different types of metal phthalocyanine compounds: formally neutral compounds containing radical 
Table 2. Composition of the compounds discussed in this section of the review.

\begin{tabular}{|c|c|c|}
\hline No. & Compound & Reference \\
\hline I & $\left\{\mathrm{CpFe}^{\mathrm{II}}(\mathrm{CO})_{2}\left[\mathrm{Sn}^{\mathrm{II}}\left(\mathrm{Pc}^{\bullet-3-}\right)\right]\right\}^{0}$ & [236] \\
\hline II & $\left\{\mathrm{Cp} * \mathrm{Fe}^{\mathrm{II}}(\mathrm{CO})_{2}\left[\mathrm{Sn}^{\mathrm{II}}\left(\mathrm{Nc}^{\bullet 3-}\right)\right]\right\}^{0} \times 2 \mathrm{C}_{6} \mathrm{H}_{4} \mathrm{Cl}_{2}$ & [236] \\
\hline III & $\left\{\mathrm{CpFe}^{\mathrm{II}}(\mathrm{CO})_{2}\left[\mathrm{Sn}^{\mathrm{II}}\left(\mathrm{TPP}^{\bullet}{ }^{-}\right)\right]\right\}^{0}$ & [236] \\
\hline IV & $\left\{\mathrm{CpFe}(\mathrm{CO})_{2}\left[\operatorname{In}^{\mathrm{I}}\left(\mathrm{Pc}^{2-}\right)\right]\right\}^{0}$ & [236] \\
\hline $\mathbf{V}$ & $\left\{\mathrm{Ph}_{5} \mathrm{CpRu}(\mathrm{CO})_{2}\left[\mathrm{Sn}^{\mathrm{II}}\left(\mathrm{Pc}^{\bullet 3-}\right)\right]^{0}\right.$ & [237] \\
\hline VI & $\left\{\right.$ cryptand $\left.\left(\mathrm{K}^{+}\right)\right\}\left\{(\text {cis-indigo- } O, O)^{2-} \mathrm{Ti}^{\mathrm{IV}}\left(\mathrm{Pc}^{2-}\right)\right\}\left(\mathrm{Cl}^{-}\right) \times \mathrm{C}_{6} \mathrm{H}_{4} \mathrm{Cl}_{2}$ & [238] \\
\hline VII & $\left\{\right.$ cryptand $\left.\left(\mathrm{K}^{+}\right)\right\}\left\{(\text {cis-thioindigo- } O, O)^{2-} \mathrm{In}^{\mathrm{III}}\left(\mathrm{Pc}^{2-}\right)\right\}^{-} \times \mathrm{C}_{6} \mathrm{H}_{4} \mathrm{Cl}_{2}$ & [238] \\
\hline VIII & $\left(\mathrm{Bu}_{4} \mathrm{~N}^{+}\right)_{2}\left\{\left[\mathrm{Sn}^{\mathrm{II}}\left(\mathrm{Pc}^{\bullet-3-}\right)\right](\text { cis-indigo- } N, N)^{2-} \mathrm{Cp} * \mathrm{Ir}^{\mathrm{III}}\right\}_{2}^{-} \times 0.5\left(\mathrm{H}_{2}\right.$ Indigo $) \times 2.5 \mathrm{C}_{6} \mathrm{H}_{4} \mathrm{C}_{12}$ & [239] \\
\hline IX & $\left\{\operatorname{cryptand}\left(\mathrm{K}^{+}\right)\right\}_{2}\left\{\mathrm{Sn}^{\mathrm{IV}}\left(\mathrm{NC}^{-}\right)_{2}\left(\mathrm{Pc}^{4}\right)\right\}^{2-} \times \mathrm{C}_{6} \mathrm{H}_{4} \mathrm{Cl}_{2}$ & [240] \\
\hline $\mathbf{X}$ & $\left\{\operatorname{cryptand}\left(\mathrm{K}^{+}\right)\right\}_{2}\left\{\mathrm{Sn}^{\mathrm{IV}}\left(\mathrm{CN}^{-}\right)_{2}\left(\mathrm{Nc}^{4-}\right)\right\}^{2-} \times 2 \mathrm{C}_{6} \mathrm{H}_{4} \mathrm{Cl}_{2}$ & [240] \\
\hline $\mathbf{X I}$ & {$\left[\mathrm{Al}^{\mathrm{III}}(N-\mathrm{MeIm})_{2}\left(\mathrm{Pc}^{\bullet-}-\right)\right]^{0} \times N-\mathrm{MeIm}$} & [241] \\
\hline XII & {$\left[\mathrm{V}^{\mathrm{II}}(N-\mathrm{MeIm})_{2}\left(\mathrm{Pc}^{2-}\right)\right]^{0} \times N-\mathrm{MeIm}$} & [242] \\
\hline XIII & $\left\{\operatorname{cryptand}\left(\mathrm{Na}^{+}\right)\right\}\left\{\mathrm{Mg}^{\mathrm{II}}(N-\mathrm{MeIm})\left(\mathrm{Pc}^{\bullet-3-}\right)\right\}^{\bullet-} \times 2.25 \mathrm{C}_{6} \mathrm{H}_{4} \mathrm{Cl}_{2} \times 0.25 \mathrm{C}_{6} \mathrm{H}_{14}$ & this work \\
\hline XIV & $\left\{\mathrm{Cp}_{2}^{*} \mathrm{Cr}^{+}\right)\left\{\mathrm{Fe}^{\mathrm{l}}\left(\mathrm{Pc}^{2-}\right)\right\}^{-} \times 4 \mathrm{C}_{6} \mathrm{H}_{4} \mathrm{Cl}_{2}$ & [231] \\
\hline XV & $\left(\mathrm{CV}^{+}\right)\left\{\mathrm{Sn}^{\mathrm{IV}} \mathrm{Cl}_{2}\left(\mathrm{Pc}^{\bullet-3-}\right)\right\}^{\bullet-} \times 0.4 \mathrm{C}_{6} \mathrm{H}_{4} \mathrm{Cl}_{2}$ & [243] \\
\hline
\end{tabular}

Cryptand[2.2.2]: abbreviation is cryptand.

trianion $\mathrm{Pc}^{\bullet 3-}$ macrocycles, compounds with transition metals in low oxidation state $\left(\mathrm{V}^{\mathrm{II}}\right.$ and $\left.\mathrm{Cr}^{\mathrm{II}}\right)$ as well as to reduce compounds which cannot be reduced in normal conditions. ${ }^{[241,242]}$ Optical and magnetic properties of metallophthalocyanine compounds can also be affected by the introduction of the cations having strong absorption in visible or NIR ranges (crystal violet cations) or paramagnetic cations like decamethylchromocenium. All these topics will be the subject of present section. The compounds discussed are listed in Table 2. Their composition was determined from $\mathrm{X}$-ray diffraction on single crystals, we have studied their optical and magnetic properties as well. Due to the presence of air-sensitive components ( $\mathrm{Pc}^{\bullet 3-}, \mathrm{V}^{\mathrm{II}}$, dianion dye ligands) these compound are rather air-sensitive, and work with them was carried out in the Glovebox MBraun 150B-G with controlled atmosphere and the content of $\mathrm{H}_{2} \mathrm{O}$ and $\mathrm{O}_{2}$ less than $1 \mathrm{ppm}$ in the solvents which were carefully purified from oxygen and water.

\section{Coordination Complexes of Tin and Indium Macrocycles with Transition Metals}

Tin or indium macrocycles can form coordination complexes with different transition metals. Charge of the macrocycle does not affect noticeably on the coordination of transition metals to tin(II) phthalocyanine. ${ }^{[237]}$ However, compounds with the $\mathrm{Pc}^{\bullet-}$ radical anions are preferable to manifest strong magnetic coupling of spins.

Coordination of metal-containing ligands to tin(II) phthalocyanine can provide compounds with different properties. Interaction of $\left\{\mathrm{Sn}^{\mathrm{II}}\left(\mathrm{Pc}^{2-}\right)\right\}$ with (pentamethylcyclopentadienyl)iridium(III) diiodide dimer, $\left(\mathrm{Cp}^{*} \mathrm{Ir}^{\mathrm{III}} \mathrm{I}_{2}\right)_{2}$, yields the $\left\{\mathrm{Cp}^{*} \mathrm{Ir}^{\mathrm{III}} \mathrm{I}_{2}\left[\mathrm{Sn}^{\mathrm{II}}\left(\mathrm{Pc}^{2-}\right)\right]\right\} \cdot 2 \mathrm{C}_{6} \mathrm{H}_{4} \mathrm{Cl}_{2}$ complex in which the $\mathrm{Pc}^{2-}$ macrocycles preserve dianion state. ${ }^{[20]}$ Formally neutral $\left\{\mathrm{Cp}\left({ }^{*}\right) \mathrm{Fe}^{\mathrm{II}}(\mathrm{CO})_{2} \mathrm{Sn}^{\mathrm{II}}\left(\mathrm{Pc}^{\bullet 3-}\right)\right\}^{0}$ complexes
(I) can be obtained at the interaction of $\left\{\mathrm{Sn}^{\mathrm{II}}\left(\mathrm{Pc}^{2-}\right)\right\}$ with an excess of the $\left\{\mathrm{CpFe}^{\mathrm{I}}(\mathrm{CO})_{2}\right\}_{2}$ or $\left\{\mathrm{Cp}^{*} \mathrm{Fe}^{\mathrm{I}}(\mathrm{CO})_{2}\right\}_{2}$ dimers. In these complexes unusual intrinsic charge transfer from $\mathrm{Fe}^{\mathrm{I}}$ to the $\mathrm{Pc}^{2-}$ macrocycle is observed accompanied by the formation of $\mathrm{Fe}^{\mathrm{II}}$ and paramagnetic $\mathrm{Pc}^{\bullet 3-}$ radical trianions. ${ }^{[237]}$ Tin(II) naphthalocyanine (Nc, Figure 24a) or tetraphenylporphyrin (TPP) also form such complexes with intrinsic charge transfer which also provides the formation of radical trianion naphthalocyanine and porphyrin macrocycles. ${ }^{[237]}$ Neutral $\left\{\mathrm{Cp}^{(*)} \mathrm{Fe}^{\mathrm{II}}(\mathrm{CO})_{2} \mathrm{Sn}^{\mathrm{II}}\left(\mathrm{Mac}^{\bullet 3-}\right)\right\}^{0}$ complexes $(\mathrm{Mac}=\mathrm{Pc}, \mathrm{Nc})$ do not contain cations and as a result they are closely packed in the $\pi$-stacking dimers with effective interaction between the radical trianion macrocycles. ${ }^{[236]}$ Additional $\pi-\pi$ interactions between the macrocycles from the neighboring dimers can appear due to essential shift of the macrocycles in these dimers providing the formation of one-dimensional chains as shown in Figure 24b for II. Magnetic behavior of the dimers and chains from radical trianion macrocycles can be described well by the Heisenberg models for dimers and chains with rather large intradimer interactions $J / k_{\mathrm{B}}$ of $-54 \div-85 \mathrm{~K}$. The largest coupling is observed for the naphthalocyanine containing compound $\left\{\mathrm{CpFe}^{\mathrm{II}}(\mathrm{CO})_{2} \mathrm{Sn}^{\mathrm{II}}\left(\mathrm{Nc}^{\bullet 3-}\right)\right\}^{0}$ (II) with intra- and interdimer interactions $\left(J / k_{\mathrm{B}}\right)$ of -140 and $-40 \mathrm{~K}$, respectively. ${ }^{[236]}$ Due to the absence of any $\pi-\pi$ interactions between the radical trianion porphyrin macrocycles in $\left\{\mathrm{CpFe}^{\mathrm{II}}(\mathrm{CO})_{2} \mathrm{Sn}^{\mathrm{II}}\left(\mathrm{TPP}^{\bullet-3-}\right)\right\}^{0}$ (III) only weak magnetic coupling of spins with Weiss temperature of $-5 \mathrm{~K}$ is observed. Similar compound with indium phthalocyanine, $\left\{\mathrm{CpFe}^{\mathrm{II}}(\mathrm{CO})_{2} \operatorname{In}^{\mathrm{I}}\left(\mathrm{Pc}^{2-}\right)\right\}^{0} \quad$ (IV), was also obtained. ${ }^{[236]}$ In contrast to I-III it contains diamagnetic and EPR silent $\mathrm{Pc}^{2-}$ macrocycle. Most probably charge transfer is also possible in this compound since state of $\mathrm{CpFe}^{\mathrm{II}}(\mathrm{CO})_{2}$ in $\mathbf{I V}$ is rather similar to those in $\mathbf{I}-\mathbf{I I I}$. In this case charge transfer from iron occurred to the indium(III) center which transfers to diamagnetic and EPR silent $\operatorname{In}^{\mathrm{I}}$. 

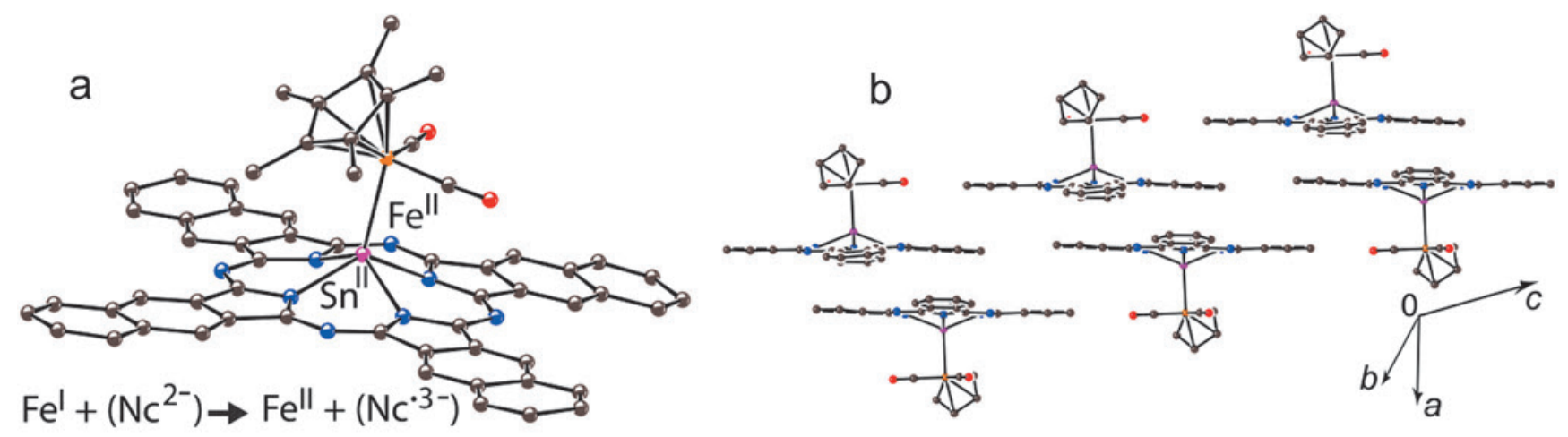

Figure 24. (a) View on the molecular structure of neutral coordination unit $\left\{\left[\mathrm{Cp}^{*} \mathrm{Fe}^{\mathrm{II}}(\mathrm{CO})_{2}\right] \mathrm{Sn}^{\mathrm{II}}\left(\mathrm{Nc}^{\bullet 3-}\right)\right\}^{0}$ in II, the scheme of charge transfer is shown below; (b) the packing of these units in II to form one-dimensional chain structure. ${ }^{[236]}$

Formally neutral coordination compounds can be obtained when the $\left\{\mathrm{Sn}^{\mathrm{II}}\left(\mathrm{Pc}^{\bullet 3^{-}}\right)\right\}^{\bullet-}$ radical anion substitutes chloride anions in the transition metal fragment. For example, $\left\{\mathrm{Sn}^{\mathrm{II}}\left(\mathrm{Pc}^{\bullet 3^{-}}\right)\right\}^{\bullet-}$ substitutes chloride at the $\mathrm{Ru}^{\mathrm{II}}$ atom in $\mathrm{Ph}_{5} \mathrm{CpRu}(\mathrm{CO})_{2} \mathrm{Cl}$ and that is accompanied by the formation of $\mathrm{Sn}^{\mathrm{II}}-\mathrm{Ru}^{\mathrm{II}}$ bond to form formally neutral compound $\left\{\mathrm{Ph}_{5} \mathrm{CpRu}(\mathrm{CO})_{2}\left[\mathrm{Sn}^{\mathrm{II}}\left(\mathrm{Pc}^{\bullet-}\right)\right]^{0}\right.$ which however contains paramagnetic $\mathrm{Pc}^{\bullet-}$ radical trianions. The arrangement of the $\mathrm{Pc}^{\bullet 3-}$ macrocycles in the $\pi$-stacking dimers yields effective coupling of spins with exchange interaction of $J / k_{\mathrm{B}}=-183 \mathrm{~K}^{\left[{ }^{[237]}\right.}$

\section{Coordination of Indigo Type Dianion Ligands to Metallophthalocyanines}

Generally the indigo type ligands have trans-conformation of the molecule which cannot transfer to cis-conformation due to the presence of double central $\mathrm{C}=\mathrm{C}$ bond. However, under reduction especially up to the dianion state such rotation becomes possible since the central $\mathrm{C}=\mathrm{C}$ bond transfers to the single C-C bond as shown in Figure 25a. Molecule can transfer to cis-conformation providing coordination of both oxygen or potentially nitrogen atoms to the metal centers. Therefore, metallophthalocyanines can coordinate the dye dianions. Since generally dyes have strong absorption in the visible range such coordination can modify properties of metallophthalocyanines. That is possible not only for the dyes of indigo type but for other vat type dyes having carbonyl groups which under reduc- tion can transfer to strongly coordinating $-\mathrm{O}^{-}$groups. As a result, dianions of indigo or thioindigo can substitute chloride anions in $\mathrm{Ti}^{\mathrm{IV}} \mathrm{Cl}_{2}\left(\mathrm{Pc}^{2-}\right)$ or $\mathrm{In}^{\mathrm{III}} \mathrm{Cl}\left(\mathrm{Pc}^{2-}\right)$ phthalocyanines forming neutral $\left\{\left(\right.\right.$ cis-indigo $\left.\left.{ }^{2-}\right) \mathrm{Ti}^{\mathrm{IV}}\left(\mathrm{Pc}^{2-}\right)\right\}$ or anionic $\left\{\left(\text { cis-thioindigo }{ }^{2-}\right) \mathrm{In}^{\mathrm{III}}\left(\mathrm{Pc}^{2-}\right)\right\}^{-}$species (Figure 25b) with two chromophore ligands at one metal centers. ${ }^{[238]}$ Donor properties of the cis-indigo and cis-thioindigo dianions are not strong enough to transfer electron density to the phthalocyanine macrocycles, and these macrocycles preserve diamagnetic $\mathrm{Pc}^{2-}$ state. As a result, complexes are diamagnetic in all studied temperature range (1.9-300 K). At the same time at the using of the dianions of other dyes an electron transfer between two chromophore ligands becomes possible affecting optical and magnetic properties of the coordination units. A phthalocyanine ligand shows more intense absorption bands in visible range than the dye dianions and the bands of cis-indigo or cis-thioindigo dianions are manifested in the spectra of VI and VII only as shoulders of more intense absorption bands of phthalocyanine. ${ }^{[238]}$

\section{Charge Transfer between Two Chromophores Coordinated to One Metal Center}

Compounds with two functional ligands coordinated to one metal center are of a special interest. Indigo dye ${ }^{[239]}$ and tin(II) phthalocyanine ${ }^{[244]}$ form coordination complexes with pentamethylcyclopentadienyl iridium(III), $\mathrm{Cp}^{*} \operatorname{Ir}^{\mathrm{III}}$. As a result, both ligands can be fixed at one metal center

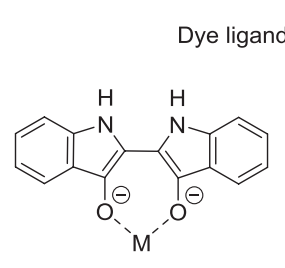

Cis-indigo dianion

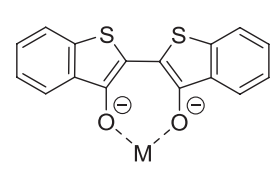

Cis-thioindigo dianion

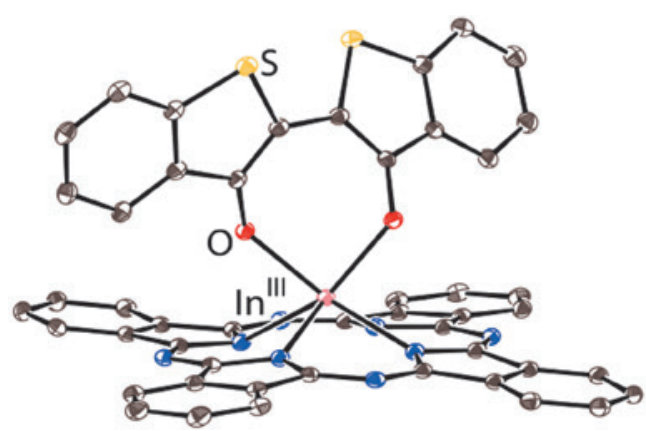

Figure 25. (a) Dianion indigo-type ligands in cis-conformation used for coordination with metal phthalocyanines; (b) the molecular structure of the $\left\{(\text { cis-thioindigo- } O, O)^{2-} \operatorname{In}^{111}\left(\mathrm{Pc}^{2-}\right)\right\}^{-}$anion in $7 .{ }^{[238]}$ 
forming coordination anionic $\left(\mathrm{Bu}_{4} \mathrm{~N}^{+}\right)\left(\mathrm{Cp}^{*} \operatorname{Ir}^{\mathrm{III}}\left(\right.\right.$ cis-indigo $\left.{ }^{2-}\right)$ $\left\{\mathrm{Sn}^{\mathrm{II}}\left(\mathrm{Pc}^{\bullet 3-}\right)\right\}^{\bullet-}$ (VIII) assembly, containing doubly deprotonated cis-indigo ${ }^{2-}$ dianions and tin(II) phthalocyanine radical anions. ${ }^{[239]}$ In accordance with the presence of $\left\{\operatorname{Sn}^{\mathrm{II}}\left(\mathrm{Pc}^{\bullet 3-}\right)\right\}^{\bullet-}$, this complex has effective magnetic moment of $1.71 \mu_{\mathrm{B}}$ and shows a broad EPR signal characteristic to $\left\{\operatorname{Sn}^{\mathrm{II}}\left(\mathrm{Pc}^{\bullet 3^{-}}\right)\right\}^{\bullet-}$ coordinated to the transition metals. Narrow signals which can be attributed to the reduced indigo are also present but their intensity is only about $6 \%$ from that of the broad signal at low temperatures. Temperature increase above $160 \mathrm{~K}$ provides growth of the intensity of the narrow signals whereas relative intensity of the broad signal decreases. This indicates temperature induced transfer of spin and electron density from $\left\{\mathrm{Sn}^{\mathrm{II}}\left(\mathrm{Pc}^{\bullet 3-}\right)\right\}^{\bullet-}$ to $c$ is-indigo ${ }^{2-}$ and, correspondingly, the formation of diamagnetic $\left\{\mathrm{Sn}^{\mathrm{II}}\left(\mathrm{Pc}^{2-}\right)\right\}^{0}$ and paramagnetic cis-indigo ${ }^{\bullet-}$ radical trianions at the temperature increase. This charge transfer does not affect the magnetic moment of the complex which preserves value close to $1.7 \mu_{\mathrm{B}}$ but delocalization of spin and electron density between two ligands obviously is changed under heating. ${ }^{[239]}$ Potentially such electron transfer changes properties of the coordination complex since diamagnetic and paramagnetic species of both indigo and phthalocyanine show different optical and magnetic properties. Therefore, optical properties can be tuned by the temperature change. Potentially dye ligands with different redox properties can be used in the design of such complexes providing different types of electron transfer between the chromophore ligands.

\section{Coordination Induced Charge Transfer from Metal to the Macrocycle}

Coordinationinduced chargetransferwas found fortin(II) phthalocyanine $(\mathrm{Pc})$ and naphthalocyanine $(\mathrm{Nc})$ when two cyano-,cyanato-orimidazolato-anionscoordinate to thetin(II) atoms in the $\left\{\text { cryptand }\left(\mathrm{M}^{+}\right)\right\}_{2}\left\{\mathrm{Sn}^{\mathrm{IV}} \mathrm{L}_{2}\left(\text { Macrocycle }^{4-}\right)\right\}^{2-}$ $\left(\mathrm{M}=\mathrm{Na}, \mathrm{K}, \mathrm{L}=\mathrm{CN}^{-}, \mathrm{CNO}^{-}, \mathrm{Im}^{-}\right.$, Macrocycle is Pc or Nc) salts (Figure 26). ${ }^{[240]}$ Two electron from $\mathrm{Sn}(\mathrm{II})$ at such transition are transferred to the dianion $\mathrm{Pc}^{2-}$ macrocycle forming tin(IV) and the tetraanion $\mathrm{Pc}^{4-}$ macrocycle. That is supported by calculations, essential distortion of the Pc macrocycles with the appearance of short and long C-N bonds, blue shift of the Q-band and the appearance of new bands in the NIR range. Initially tin(II) atoms have too large size in $\mathrm{Sn}^{\mathrm{II}}\left(\mathrm{Pc}^{2-}\right)$ displacing out of the Pc plane by $1.275 \AA^{[245]}$ and positioning only from one side of the macrocycle. Correspondingly, the $\mathrm{Sn}(\mathrm{II})$ atoms cannot coordinate simultaneously two ligands which approach from both sides of the macrocycle. At the same time it is known that $\operatorname{tin}(\mathrm{IV})$ atoms in $\left\{\mathrm{Sn}^{\mathrm{IV}} \mathrm{Cl}_{2}\left(\mathrm{Pc}^{2-}\right)\right\}^{[246]}$ are positioned exactly in the center of the Pc macrocycle and coordinate two $\mathrm{Cl}$ ligands in the axial position. Therefore, tin(II) atoms for coordination of two ligands should transfer two electrons to the macrocycle forming the tin(IV) atoms.

Besides this, it was found that orientation of the $\mathrm{CN}^{-}$ ligands at the tin(IV) atoms is defined essentially by cationic surrounding. Close approach of the cations reorients the $\mathrm{CN}^{-}$ group by nitrogen atom towards tin(IV) atom. That elongates the Sn-N bond $(2.339(1) \AA)$ in comparison with the Sn-C bond (2.266(3) $\AA$ ), but electrostatic interaction of $\mathrm{NC}^{-}$with the cationic surrounding is enhanced due to larger negative charge on $\mathrm{NC}^{-}$in such orientation. Generally the $\mathrm{CN}^{-}$group is coordinated to metals by carbon atom. ${ }^{[240]}$

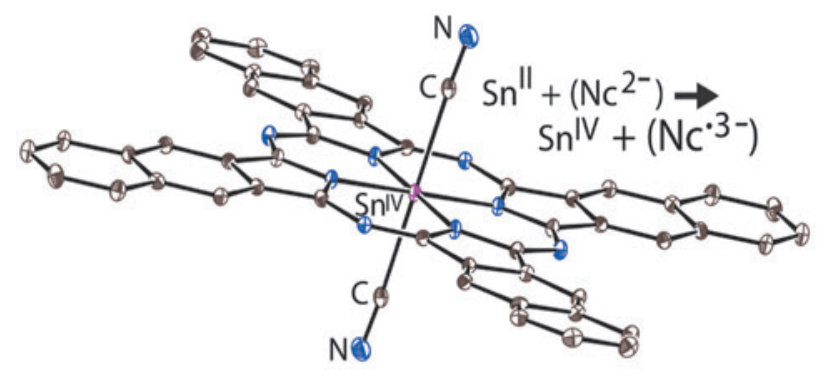

Figure 26. Molecular structure of the $\left\{\mathrm{Sn}^{\mathrm{IV}}\left(\mathrm{CN}^{-}\right)_{2}\left(\mathrm{Nc}^{4-}\right)\right\}^{2-}$ dianion in $\mathbf{X}^{[240]}$

\section{The Role of N-Methylimidazole in the Reduction Reactions}

Many ligands as pyridine, imidazole, $N$-methylimidazole $(N$-MeIm) and other nitrogen and oxygen containing molecules can form complexes with metal phthalocyanines axially coordinated to the metal atoms. For example, series of dipyridine complexes of manganese(II), iron(II) and cobalt(II) phthalocyanines is obtained and an essential effect of such coordination on their magnetic properties is shown. ${ }^{[247,248]}$ Here we will show how coordination of $\mathrm{N}$-MeIm during the reduction allows to obtain different types of compounds. The $\left[\mathrm{Al}^{\mathrm{III}} \mathrm{Cl}\left(\mathrm{Pc}^{2-}\right)\right]^{0}$ phthalocyanine can be selectively reduced in $o$-dichlorobenzene by sodium fluorenone ketyl in the presence of tetrabutylammonium cations to form the $\left(\mathrm{Bu}_{4} \mathrm{~N}^{+}\right)_{2}\left[\mathrm{Al}^{\mathrm{III}}\left(\mathrm{HFl}-\mathrm{O}^{-}\right)\left(\mathrm{Pc}^{\bullet-}\right)\right]^{\bullet-}\left(\mathrm{Br}^{-}\right) \cdot 1.5 \mathrm{C}_{6} \mathrm{H}_{4} \mathrm{Cl}_{2}$ salts $\left(\mathrm{HFl}-\mathrm{O}^{-}=\right.$Fluoren-9-olato ${ }^{-}$anion is formed from the fluorenone ketyl reductant). ${ }^{[249]}$ Addition of several drops of $\mathrm{N}$-MeIm into the reaction mixture allows to carry out reduction by sodium fluorenone ketyl and in this case crystals of formally neutral compound $\left[\mathrm{Al}^{\mathrm{III}}(N-\mathrm{MeIm})_{2}\left(\mathrm{Pc}^{\bullet 3-}\right)\right]^{0} \cdot N$-MeIm (XI) were obtained. Solved crystal structure shows that two $N$-MeIm ligands are coordinated to aluminium(III) in the axial positions (Figure 27a) and an average length of the Al-N(MeIm) bonds is 2.055(2) $\AA .{ }^{[241]}$ Negative charge of the paramagnetic radical trianion $\mathrm{Pc}^{\bullet 3-}$ macrocycle is compensated in $\mathbf{X I}$ by the $\mathrm{Al}^{\mathrm{III}}$ trications. Radical trianion nature of the macrocycle in XI is supported by characteristic changes in its geometry, appearance of new bands in the NIR range and observation of one unpared $S=1 / 2$ spin per a formula unit. Similar compound was obtained with gallium(III) phthalocyanine. In spite of metal phthalocyanines having generally poor solubility in unpolar solvents the compounds of such type are well soluble in them. ${ }^{[241]}$

The $N$-MeIm ligand can stabilize phthalocyanines with metals in the low oxidation states. For example, chromium(III)chloride or vanadyl phthalocyanines can be reduced by sodium fluorenine ketyl in the presence of several drops of $\mathrm{N}$-MeIm and stoichiometric amount of chromium(II) chloride or samarium(II) iodide. $\mathrm{Cr}^{\mathrm{II}} \mathrm{Cl}_{2}$ or $\mathrm{Sm}^{\mathrm{II}} \mathrm{I}_{2}$ are used to accept chloride or oxygen atoms from the reduced metal phthalocyanines. Interesting that 

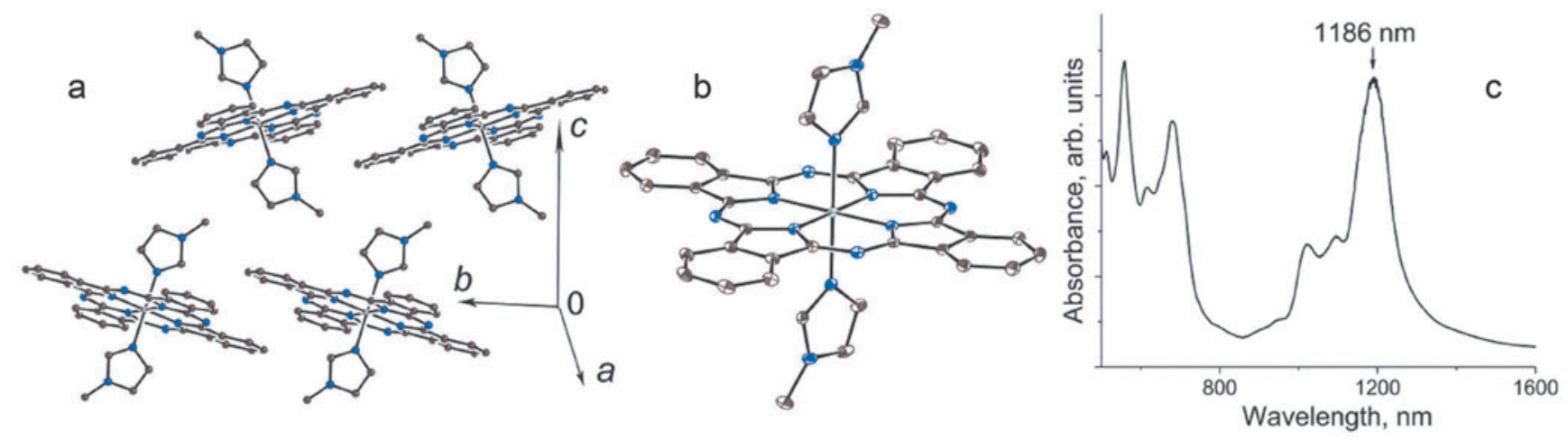

Figure 27. (a) View on the packing of the $\left[\mathrm{Al}^{\mathrm{III}}(\mathrm{MeIm})_{2}\left(\mathrm{Pc}^{\bullet-}\right)\right]^{0}$ units in $\mathbf{X I} \mathbf{I}^{[241]}(\mathrm{b})$ the molecular structure of the $\left[\mathrm{V}^{\mathrm{II}}\left(\mathrm{MeIm}_{2}\left(\mathrm{Pc}^{2-}\right)\right]^{0} \mathrm{units}\right.$ in XII ; ${ }^{[242]}$ (c) UV-Vis-NIR spectrum of XII in $\mathrm{KBr}$ pellet prepared under anaerobic conditions. ${ }^{[242]}$

chromium(II) chloride can also act as a reductant but in this case slower reduction is observed. Two compounds were obtained as single crystals $\left[\mathrm{V}^{\mathrm{II}}(\mathrm{MeIm})_{2}\left(\mathrm{Pc}^{2-}\right)\right]^{0} \cdot N$-MeIm (XII) and $\left[\mathrm{Cr}^{\mathrm{II}}(\mathrm{MeIm})_{2}\left(\mathrm{Pc}^{2-}\right)\right]^{0} \cdot 2 \mathrm{C}_{6} \mathrm{H}_{4} \mathrm{Cl}_{2}$. Chromium(II) phthalocyanine solvated by pyridine is already known, ${ }^{[250]}$ but solvated vanadium(II) phthalocyanine is prepared for the first time. Compounds containing vanadium(II) or chromium(II) in low oxidation state coordinate two $\mathrm{N}$-MeIm ligands (Figure $27 \mathrm{~b})$ with the length of the $\mathrm{M} \cdots \mathrm{N}(N$-MeIm) bonds of 2.15 and $2.09 \AA$, respectively. In contrast to $\mathbf{X I}$ in this case no electron transfer to the macrocycles is observed preserving their diamagnetic $\mathrm{Pc}^{2-}$ state. According to the values of magnetic moment chromium(II) have the $S=1$ spin state whereas vanadium(II) is in the high $S=3 / 2$ spin state. As a result, both compounds show broad EPR signals. Complex XII shows strong absorption in the NIR range with maximum at $1186 \mathrm{~nm}$. This absorption is very intense and is comparable with those of the bands in the UV-visible range (Figure 27c). Similar absorption band but of weaker intensity with maximum at $1178 \mathrm{~nm}$ is also observed for solvated chromium(II) phthalocyanine. Absorption in the NIR range is explained by unusually small HOMO-LUMO gaps for the $N$-MeIm solvated $\mathrm{Cr}^{\mathrm{II}} \mathrm{Pc}$ and $\mathrm{V}^{\mathrm{II}} \mathrm{Pc}$ phthalocyanines. Essential diradicaloid character of the Pc macrocycles was found for $\left[\mathrm{Cr}^{\mathrm{II}}(\mathrm{MeIm})_{2}\left(\mathrm{Pc}^{2-}\right)\right]^{0} \cdot 2 \mathrm{C}_{6} \mathrm{H}_{4} \mathrm{Cl}_{2}$, in which $\alpha$ - and $\beta$-orbitals are distributed in different regions of the macrocycle. ${ }^{[242]}$ That is rare phenomena for metal phthalocyanines and it was found only for some extended corroles. ${ }^{[251]}$

Usually phthalocyanines of different metals can be reduced to the radical anion state. ${ }^{[232,233]}$ However, phthalocyanines with zinc(II), manganese(II) and magnesium(II) cannot be obtained in form of solid radical anion salt since they coordinate chloride or other type ligands and cannot be reduced in this state. Magnesium(II) phthalocyanine contains coordinated water which under reduction transforms to the $\mathrm{OH}^{-}$group coordinated to one of two $\mathrm{Mg}^{\mathrm{II}}\left(\mathrm{Pc}^{2-}\right)$ units. ${ }^{[252,253]}$ Addition of $\mathrm{N}$-MeIm during the synthesis of the salt allows to substitute these ligands by $N$-MeIm. For example, interaction of $\mathrm{Mg}^{\mathrm{II}}\left(\mathrm{Pc}^{2-}\right)$ with sodium fluorenone ketyl in the presence of cryptand[2.2.2] and several drops of $N$-MeIm provides dissolution of this phthalocyanine to form deep blue solution from which slow mixing with $n$-hexane yields the crystals of $\left\{\operatorname{cryptand}\left(\mathrm{Na}^{+}\right)\right\}$ $\left\{\mathrm{Mg}^{\mathrm{II}}(N-\mathrm{MeIm})\left(\mathrm{Pc}^{\bullet 3-}\right)\right\}^{\bullet-} \cdot 2.25 \mathrm{C}_{6} \mathrm{H}_{4} \mathrm{Cl}_{2} \cdot 0.25 \mathrm{C}_{6} \mathrm{H}_{14}$

(XIII).
In this compound only one $N$-MeIm group is coordinated to magnesium(II) in spite of an excess of this ligand in the synthesis. At that there are two orientation of $N$-MeIm in XIII with short N...Mg bonds of 2.056(4) $\AA$ (major orientation) and essentially longer bond of 2.209(4) $\AA$ (minor orientation). This difference can be explained by short contacts with neighboring strongly disordered solvent molecules occupied position near the coordinated $N$-MeIm ligand (these solvent molecules are not shown in Figure 28a). The formation the $\mathrm{Pc}^{\bullet 3-}$ radical trianion is supported by the alternation in the length of the $\mathrm{N}_{\text {meso }}-\mathrm{C}$ bonds, since four shorter and four longer bonds appear in the macrocycles with difference between them of $0.019 \AA$. These bonds are arranged by such a way that shorter and longer bonds belong to two oppositely located isoindole units. Spectrum of pristine $\mathrm{Mg}^{\mathrm{II}}\left(\mathrm{Pc}^{2-}\right)$ contains Soret band at $347 \mathrm{~nm}$ and split Q-band at 630,691 and $818 \mathrm{~nm}$ (Figure 28b). The formation of $\mathrm{Pc}^{\bullet 3-}$ shifts the components of Q-band in blue side (appear at 584 and $662 \mathrm{~nm}$ ) and new band is manifested in the NIR range at $973 \mathrm{~nm}$ (Figure 28b). Magnetic properties of XIII were studied by SQUID and EPR techniques. Effective magnetic moment of the system of $1.71 \mu_{\mathrm{B}}$ at $300 \mathrm{~K}$ (Figure 29a) indicates the contribution from one $S=1 / 2$ spin per a formula unit which weakly antiferromagnetically coupled with the neighboring spins since Weiss temperature is only $-5 \mathrm{~K}$ (Figure 29b). The reason of that is a large distance between the $\mathrm{Pc}^{\bullet-}$ macrocycles (Figure 28a). Salt manifests intense narrow Lorentzian EPR signal with $g=2.0029$ and the linewidth $(\Delta H)$ of $0.378 \mathrm{mT}$ (Figure 29c). The signal slightly shifts to larger g-factors and narrows with temperature decrease. Below $50 \mathrm{~K}$ it splits into two narrow lines with $g_{1}=2.0024(\Delta H=0.258 \mathrm{mT})$ and $g_{2}=2.0035(\Delta H=0.234 \mathrm{mT})$ (Figure 29d). Thus, synthesis with $N$-MeIm allows to reduce $\mathrm{Mg}^{\mathrm{II}}\left(\mathrm{Pc}^{2-}\right)$ obtaining crystalline radical anion salt with radical trianion $\mathrm{Pc}^{\bullet-3-}$ macrocycles. Previously only one compound with the reduced manganese(II) phthalocyanine was known $-\left[\left(\mathrm{K}^{+} \mathrm{DME}_{2}\right)_{2}\right]\left[\mathrm{Mg}^{\mathrm{II}}\left(\mathrm{OH}^{-}\right)\left(\mathrm{Pc}^{\bullet 3-}\right)\right] \cdot{ }^{[252]}$

\section{Functional Cations in the Synthesis of Phthalocyanine Radical Anion Salts}

Cations of different size and shape can be used in the synthesis of metal phthalocyanine radical anion salts. Variation of cations allows to affect packing of the macrocy- 

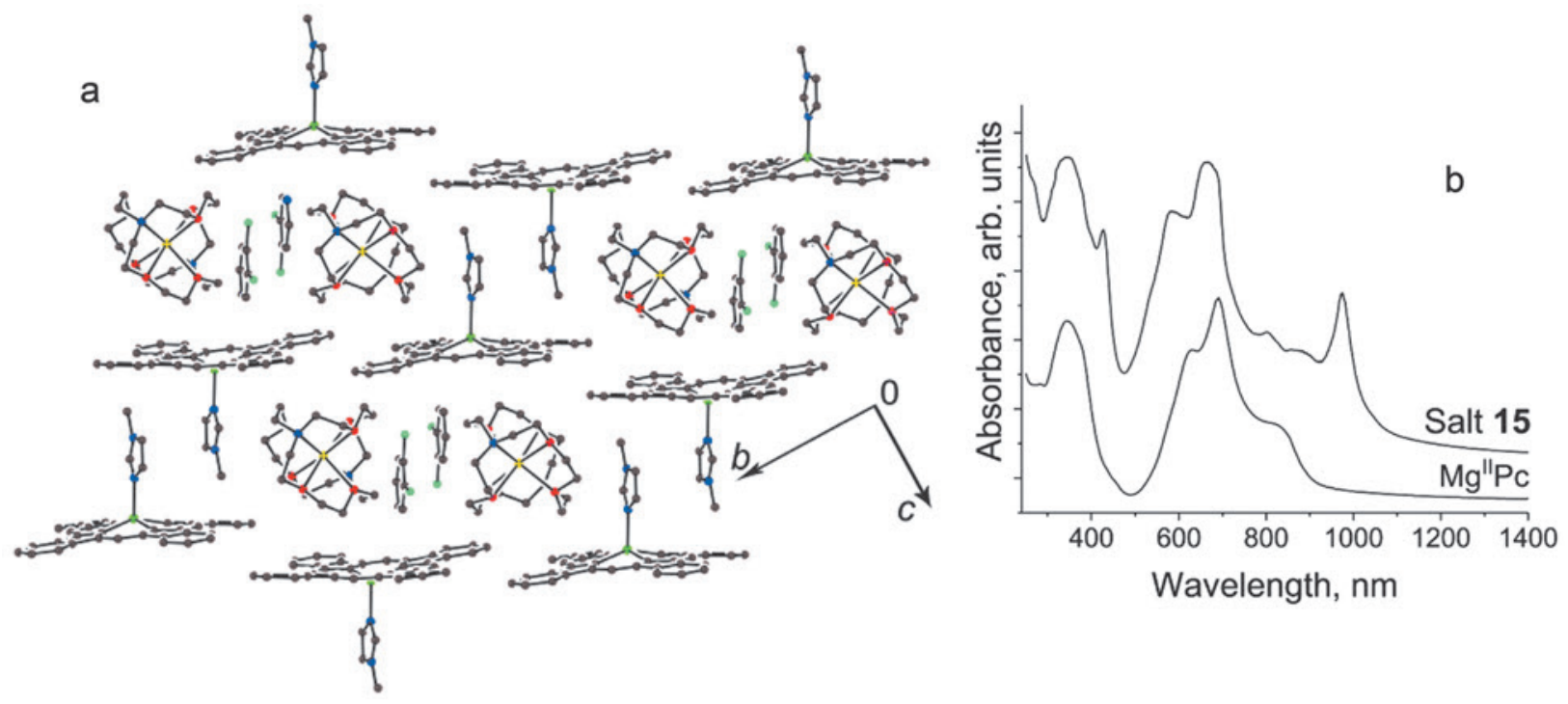

Figure 28. View on the packing of the $\left\{\mathrm{Mg}^{\mathrm{II}}(\mathrm{MeIm})\left(\mathrm{Pc}^{\bullet-}\right)\right\}^{\bullet-}$ radical anions in XIII together with the $\left\{\mathrm{Cryptand}[2.2 .2]\left(\mathrm{Na}^{+}\right)\right\}$cations and solvent $o-\mathrm{C}_{6} \mathrm{H}_{4} \mathrm{Cl}_{2}$ molecules along the $a$ axis.

a

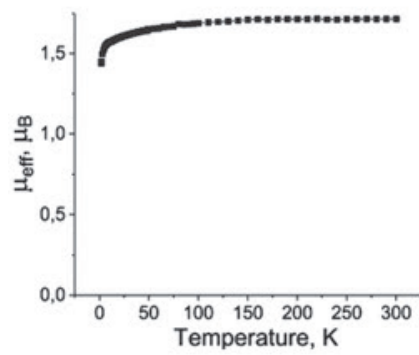

b

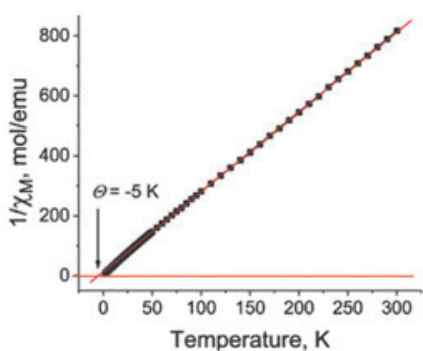

C

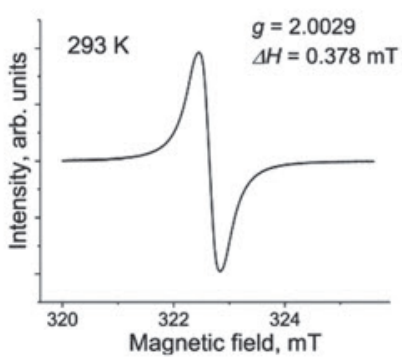

d

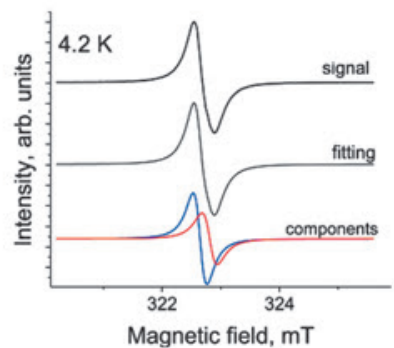

Figure 29. Magnetic data for polycrystalline salt XV: temperature dependence of effective magnetic moment (a) and reciprocal molar magnetic susceptibility (b); EPR signal from polycrystalline $\mathbf{X V}$ at room temperature (293 K, c) and $4.2 \mathrm{~K}$ (d). For (d) the fitting of the signal by two Lorentzian lines (red and blue curves) is shown below.

cles in a crystal and correspondingly their optical and magnetic properties. Such possibilities are shown for a large series of salts based on vanadyl and titanyl phthalocyanine anions. ${ }^{[235]}$ Cations with different functional properties can introduce new functionality into the radical anion salts. For example, paramagnetic decamethylchromocene cations introduce magnetic component allowing the preparation of molecular magnets. ${ }^{[231]}$ Different cations with strong absorption in the visible range can also affect optical properties of the radical anion salts. ${ }^{[243]}$

First molecular magnet with metal phthalocyanine was obtained by the reduction of iron(II) phthalocyanine by decamethylchromocene $\left(\mathrm{Cp}_{2}{ }_{2} \mathrm{Cr}\right)$ forming $\left(\mathrm{Cp}^{*}{ }_{2} \mathrm{Cr}^{+}\right)$ $\left\{\mathrm{Fe}^{\mathrm{I}}\left(\mathrm{Pc}^{2-}\right)\right\}^{-} \cdot 4 \mathrm{C}_{6} \mathrm{H}_{4} \mathrm{Cl}_{2}$ (XIV). ${ }^{[231]}$ The $\left\{\mathrm{Fe}^{\mathrm{I}}\left(\mathrm{Pc}^{2-}\right)\right\}^{-}$and $\mathrm{Cp}_{2}{ }_{2} \mathrm{Cr}^{+}$ ions alternated in XIV in the $\pi$-stacks (Figure $30 \mathrm{a}$ ). Alternation of species with different spin state $\left(\mathrm{Fe}^{\mathrm{I}}\right.$ and $\mathrm{Cr}^{\mathrm{III}}$ have the $S=1 / 2$ and $3 / 2$ spin states, respectively) provides antiferromagnetic ordering of spins in the stacks below $4.7 \mathrm{~K}$. However, magnetic moment of $\mathrm{Cr}^{\mathrm{III}}$ is larger than that of $\mathrm{Fe}^{\mathrm{I}}$ and as a result uncompensated moment from the $\mathrm{Cr}^{\mathrm{III}}$ spins is manifested in the ordered state below $4.7 \mathrm{~K} .{ }^{[231]}$ Behavior of system with such ordering is indistinguishable from that of the system with ferromagnetic ordering of $S=1$ spins within the stacks $(S=3 / 2-S=1 / 2)$. Compound XIV shows a difference between zero field and field cooling curves and magnetic hysteresis below $4.7 \mathrm{~K}$ (Figure $30 \mathrm{~b}$ ) characteristic for molecular magnets. Later we obtained charge transfer complexes of $\mathrm{Cp}_{2}{ }_{2} \mathrm{Cr}^{+}$with paramagnetic metal-free phthalocyanine radical anions and corrole radical dianions with similar alternation of the ions in the $\pi$-stacks. ${ }^{[254,255]}$ However, essentially weaker magnetic coupling of spins was found in these complexes (the strongest interactions were found for the corrole based system with Weiss tempeature of $-31 \mathrm{~K}) .{ }^{[254]}$ The reason of that is the position of the $\mathrm{Cp}^{*}$ ligand of $\mathrm{Cp}_{2}^{*} \mathrm{Cr}^{+}$exactly over the $\mathrm{Fe}^{\mathrm{I}}$ atom of $\left\{\mathrm{Fe}^{\mathrm{I}}\left(\mathrm{Pc}^{2-}\right)\right\}$ in XIV as shown in Figure 30c. As a result, linear chains of alternated paramagnetic metal atoms are formed. That is also a result of good overlapping of $\pi$-orbitals of $\mathrm{Cp}^{*}{ }_{2} \mathrm{Cr}^{+}$ and $\left\{\mathrm{Fe}^{\mathrm{I}}\left(\mathrm{Pc}^{2-}\right)\right\}^{-}$in XI at their close approach to each other (the interplanar distance is equal to $3.46 \AA$ ). ${ }^{[231]}$ Contrary, the $\mathrm{Cp}^{*}$ ligand is positioned nearly over the empty central fragment of the metal-free macrocycle providing worse $\pi-\pi$ overlapping and weaker magnetic coupling between the radical anions and the $\mathrm{Cr}^{\mathrm{III}}$ centers. ${ }^{[254,255]}$ 

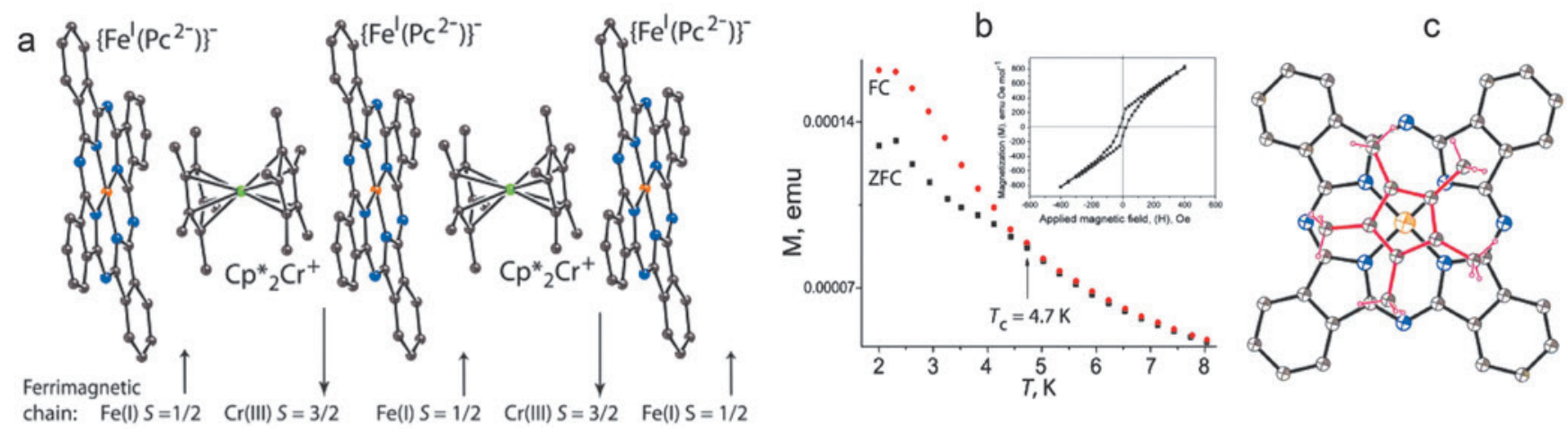

Figure 30. (a) View on the chains of alternating $\mathrm{Cp}_{2}^{*} \mathrm{Cr}^{+}$and $\left\{\mathrm{Fe}^{\mathrm{I}}\left(\mathrm{Pc}^{2-}\right)\right\}^{-}$ions in $\mathbf{X I V}{ }^{[231]}$ and scheme of ferrimagnetic ordering is shown below; (b) temperature dependence of magnetization of XIV in zero field cooling (ZFC) and field ( $1 \mathrm{kOe})$ cooling conditions and inset shows the hysteresis curve; ${ }^{[231]}(\mathrm{c})$ view on the overlapping between the $\mathrm{Cp} *$ ligand (bonds are shown by red color) and the $\left\{\mathrm{Fe}^{\mathrm{I}}\left(\mathrm{Pc}^{2-}\right)\right\}^{-}$ anion in XIV.
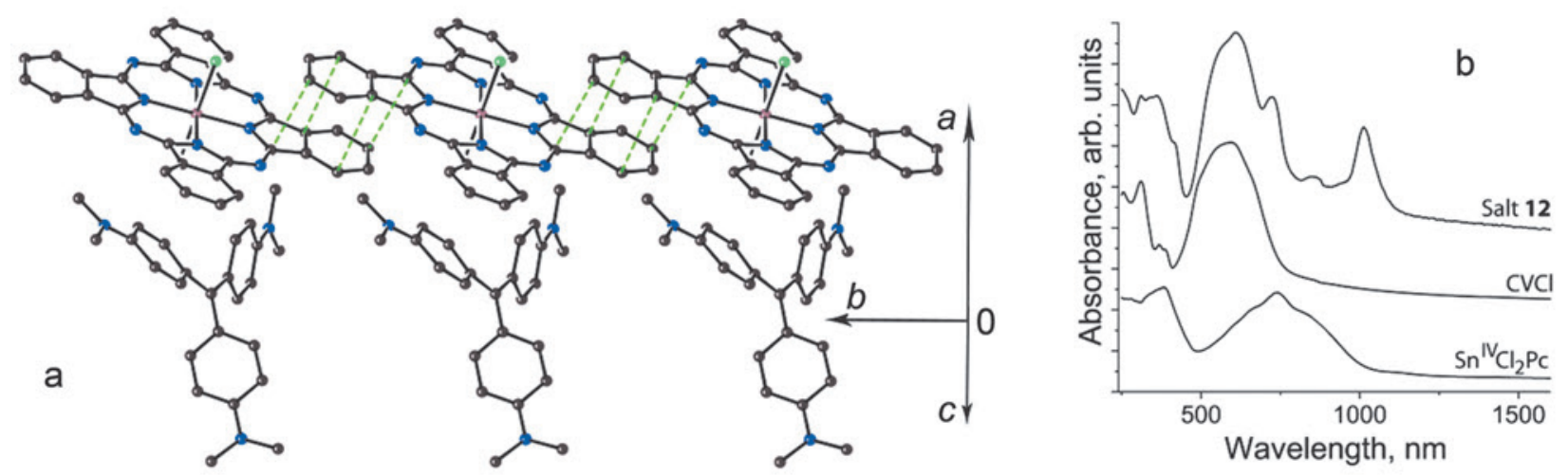

Figure 31. (a) View on the stacking chains from the $\left\{\mathrm{Sn}^{\mathrm{IV}} \mathrm{Cl}_{2}\left(\mathrm{Pc}^{\bullet}{ }^{3-}\right)\right\}^{\bullet-}$ radical anions in $\mathbf{X V}$; the UV-Vis-NIR spectra of $\mathbf{X V}$ and reference compounds. ${ }^{[243]}$

Another possibility to affect optical properties of metal phthalocyanine compounds is the introduction of chromophore cations. Such cations as crystal violet $\left(\mathrm{CV}^{+}\right)$have intense absorption band at $595 \mathrm{~nm}$ (Figure 31b). Compound with these cations was obtained by simple dissolution of two equivalents $\left(\mathrm{CV}^{+}\right)\left(\mathrm{Cl}^{-}\right)$and $\left\{\mathrm{Sn}^{\mathrm{II}}\left(\mathrm{Pc}^{2-}\right)\right\}^{0}$ in $o$-dichlorobenzene. ${ }^{[243]}$ Phthalocyanine is completely dissolved forming deep blue solution characteristic of the $\mathrm{Pc}^{\bullet 3-}$ radical trianions. Slow mixing of the obtained solution with $n$-hexane produces crystals of the radical anion $\left(\mathrm{CV}^{+}\right)\left\{\mathrm{Sn}^{\mathrm{IV}} \mathrm{Cl}_{2}\left(\mathrm{Pc}^{\bullet-3-}\right)\right\}^{\bullet-\cdot} \cdot 0.4 \mathrm{C}_{6} \mathrm{H}_{4} \mathrm{Cl}_{2}$ salt (XV). Probably, the formation of this salt without any reductants is accompanied by coordination induced charge transfer from $\mathrm{Sn}^{\text {II }}$ to the $\mathrm{Pc}^{2-}$ macrocycles to form $\mathrm{Sn}^{\mathrm{IV}}$ and the $\mathrm{Pc}^{4-}$ macrocycles as described previously for coordination of cyano-, cyanato-, and imidazolato-ligands to $\left\{\mathrm{Sn}^{\mathrm{II}}\left(\mathrm{Pc}^{2-}\right)\right\}^{0}$. In this case two chloride anions are coordinated to $\mathrm{Sn}^{\mathrm{II}}$. The $\mathrm{Pc}^{4-}$ macrocycle is a very strong reductant whereas the $\mathrm{CV}^{+}$cations are redox active with first reduction to $\mathrm{CV}^{\bullet}$ at $-0.77 \mathrm{~V}$ vs SCE. ${ }^{[256]}$ Therefore, $\mathrm{Pc}^{4-}$ can reduce one $\mathrm{CV}^{+}$cation forming $\left\{\mathrm{Sn}^{\mathrm{IV}} \mathrm{Cl}_{2}\left(\mathrm{Pc}^{\bullet 3-}\right)\right\}^{\bullet-}$ which is observed in the solid state. Salt XV contains $\pi$-stacks from the $\left\{\mathrm{Sn}^{\mathrm{IV}} \mathrm{Cl}_{2}\left(\mathrm{Pc}^{\bullet-}\right)\right\}^{\bullet-}$ radical anions with effective $\pi-\pi$ interactions between the phenylene substituents of $\mathrm{Pc}^{\bullet-3-}$. As a result rather effective magnetic coupling of spins is observed in the stacks of $\mathbf{X V}$. Both Pc $\mathrm{Pc}^{\bullet-}$ and $\mathrm{CV}^{+}$ions absorb strongly in the visible and NIR ranges up to about $1200 \mathrm{~nm}$ (Figure 28b). That absorption band of $\mathrm{CV}^{+}(610 \mathrm{~nm})$ is even higher in intensity in comparison with the Q-band of $\left\{\mathrm{Sn}^{\mathrm{IV}} \mathrm{Cl}_{2}\left(\mathrm{Pc}^{\bullet 3-}\right)\right\}^{\bullet-}(723 \mathrm{~nm})$ (Figure 31b). Band in the NIR range at $1013 \mathrm{~nm}$ in the spectrum of $\mathbf{X V}$ (Figure 30b) was attributed to the $\mathrm{Pc}^{\bullet 3-}$ radical trianions. Generally these bands are manifested at 920-1040 nm in the spectra of the radical anion salts of metal phthalocyanines, exact position is defined by the type of central metal atom. ${ }^{[232,233]}\left(\mathrm{CV}^{+}\right)\left(\mathrm{Cl}^{-}\right)$can also form salts due to the coordination of chloride anions to different metal phthalocyanines but in this case no charge transfer from metal to $\mathrm{Pc}^{2-}$ is observed. For example, $\left.\mathrm{Fe}^{\mathrm{III}} \mathrm{Cl}\left(\mathrm{Pc}^{2-}\right)\right\}^{0}$ interacts with $\left(\mathrm{CV}^{+}\right)\left(\mathrm{Cl}^{-}\right)$forming isostructural to $\mathrm{XV}$ salt $\left(\mathrm{CV}^{+}\right)$ $\left\{\mathrm{Fe}^{\mathrm{III}} \mathrm{Cl}_{2}\left(\mathrm{Pc}^{2-}\right)\right\} \cdot 0.5 \mathrm{C}_{6} \mathrm{H}_{4} \mathrm{Cl}_{2}$, the structure and properties of which were also studied. ${ }^{[243]}$

Thus, the data show how the coordination of different ligands and the using of functional cations can affect optical and magnetic properties of the reduced metal phthalocyanines. Coordination of transition metal fragments as ligands can directly introduce paramagnetic component or provides charge transfer from the transition metal to the macrocycle with the formation of $\mathrm{Pc}^{\bullet 3-}$ radical trianions. Formally neutral compounds were obtained allowing close packing of paramagnetic macrocycles in the crystals and effective magnetic coupling of spins. Organic dyes with carbonyl-containing groups can also coordinate in the dianion state to the metal 
phthalocyanines. Such complexes are already received with indigo and thioindigo dianions but the using of other vat dyes is possible. So far, no charge transfer has been detected from the dye dianions to the macrocycles. However, the using of dye dianions with different redox properties can allow to observe charge transfer between dyes and phthalocyanines and it is expected that in some cases it can be reversible. Charge transfer affects optical and magnetic properties of metallophthalocyanines making these systems interesting for switchers and transducers. Example of the compound in which electron is transferred from the tin(II) phthalocyanine radical anion to the dye dianion at the temperature increase is even obtained. Another interesting possibility of charge transfer exists when chloride, cyano-, cyanato- or imidazolato-anions are coordinated to tin(II) phthalocyanine providing two electron transfer from tin(II) to the macrocycle forming $\mathrm{Sn}^{\mathrm{IV}}$ and tetraanion $\mathrm{Pc}^{4-}$ macrocycles. That is possible when tin(II) atoms of phthalocyanine have two large size to be in the phthalocyanine plane. As a result, transition from $\mathrm{Sn}$ (II) to $\mathrm{Sn}(\mathrm{IV})$ is observed at coordination of two ligands since the $\mathrm{Sn}$ (IV) atoms due to smaller size are arranged exactly in the Pc plane allowing the coordination of two ligands. Complex formation reaction with $\mathrm{N}$-MeIm under reduction is also interesting and can yield new types of compounds. Phthalocyanines of trivalent metals under reduction in the presence of $\mathrm{N}$-MeIm yield formally neutral compounds which, however, contain the $\mathrm{Pc}^{\bullet 3-}$ radical trianions. In the presence of $\mathrm{N}$-MeIm the phthalocyaninates of vanadium(II) and chromium(II) can be obtained as the $N$-MeIm solvates. In these solvates the metals preserve in the high-spin state and these compounds show unusually strong NIR absorption due to small HOMO-LUMO energy gap in these phthalocyanines. Complex formation with $N$-MeIm also allows to obtain some metal(II) phthalocyanine radical anion salts in solid state which cannot be obtained at normal reduction conditions, like magnesium(II) phthalocyanine. The size and shape of counter cations in the radical anion salts define distances and overlapping between $\mathrm{Pc}^{\bullet 3-}$ and as a result their optical and magnetic properties. Some cations can have additional functionality which can modify essential properties of metal phthalocyanine salts. For example, paramagnetic metallocene cations alternating with metal-containing and metal-free phthalocyanine anions or radical anions can yield molecular magnets or compounds with essential magnetic coupling of spins. Different chromophore cations like crystal violet can absorb strongly in visible range and extend absorption of the salts to the NIR range. Potentially such salts are interesting as materials with photo- and thermo-induced charge transfer between the chromophore components.

\section{Synthesis of Paramagnetic Metalloporphyrins as Promising Materials with Magnetocaloric Activity}

Tetrapyrrole complexes with the transition $\mathrm{M}^{2+}$ cations having vacant axial coordination sites can demonstrate ferromagnetic behavior due to organizing in stacking structures with a large positive exchange interaction. ${ }^{[257-259]}$ These are, for example, MnPc ( $\mathrm{Pc}$ is phthalocyanine dianion) with the ground state $d$-electronic configuration $\left(d_{\mathrm{xy}}\right)^{2}\left(d_{\mathrm{yz}} d_{\mathrm{zx}}\right)^{2}\left(d_{z}^{2}\right)^{1}$ which displays the ferromagnetic behavior by ordering of $\mathrm{Mn}^{2+}$ with $S=3 / 2$ state ${ }^{[257,260]}$ and FePc in a metastable $\alpha$-polymorph shown magnetic order below $10 \mathrm{~K}$ due to the shorter $\mathrm{Fe}-\mathrm{Fe}$ distance compared with the not ferromagnetic stable b-polymorph. ${ }^{[257,261]} 2 \mathrm{D}$ polyphthalocyanines coordinated with $\mathrm{M}^{2+}$ such as manganese(II) and rhenium(II) being also ferromagnetic are of interest for spintronic applications together with ferromagnets above. ${ }^{[262]}$ Despite the lack of column self-assembly capabilities, paramagnetic MPcXs ( $\mathrm{X}$ is anionic axial ligand) with the sterically hindered axial positions can exhibit spontaneous magnetization at temperatures above $0 \mathrm{~K}$ without supramolecular organization. Magnetic anisotropy appears in this case in the other way than that in single molecule magnets (SMMs) based on $d$-metal clusters caused by the magnetic interactions among high-spin $3 d$ metal ions in a molecule. ${ }^{[257]}$ Many LnPcXs (Ln is lanthanide ion) and their porphyrin analogs (LnPXs) were studied as zero-dimensional SMMs when the second tetrapyrrole macrocycle plays the role of the axial ligand. ${ }^{[201,263,264]}$ The effective energy barrier against magnetic relaxation is determined in these complexes by the ligand field around a lanthanide ion. ${ }^{[265]}$ This parameter for double-decker complexes, $\mathrm{LnPc}_{2}$, is quantitatively very similar to the zero-field energy gap between the two lowest sublevels of the ground multiplets. For example, the effective barrier of $230 \mathrm{~cm}^{-1}$ that is to date larger than the one for any $3 d$-based SMM was described for $\left[\mathrm{Pc}_{2} \mathrm{~Tb}\right]^{-} \mathrm{TBA}^{+}$(TBA is tetrabutylammonium). ${ }^{[264,265]}$ The ligand-field effect controlled by an electronic structure of the aromatic macrocycles was by the example of electro neutral double-decker $[(\mathrm{Pc}) \mathrm{Tb}(\mathrm{Pc})]^{0}$ shown is improving when another paramagnetic center, unpaired $\pi$-electron is available in the structure. The effective energy barrier against magnetic relaxation achieves $400 \mathrm{~cm}^{-1}$ for the mentioned SMM. ${ }^{[264]}$ The mixed triple-decker tetrapyrrole complexes of Dy-Dy, $\mathrm{Y}-\mathrm{Dy}, \mathrm{Dy}-\mathrm{Y}^{[266]}$ and Dy-Dy ${ }^{[267]}$ demonstrating also SMM behavior are characterized by intramolecular $f-f$ and antiferromagnetic dipole-dipole interaction dominating in their magnetic properties. The introduction of the paramagnetic metal cation in a porphyrin molecule cavity allows deriving chemical compounds having a changeable $\mathrm{p}$ electron system and, consequently, spin carrier state. The participation of a metal atom in electron density delocalization not only determines MPcXs/MPXs magnetic behavior but is also reflected most evidently in a hyper-type electron absorption spectrum (UV-Vis) and increases stability of a coordination center. ${ }^{[268]}$ The paramagnetic state of the tetrapyrrole complexes changes dramatically their NMR spectra. To insight into the historical evolution of the mentioned SMMs, their $f$-electronic structure, main mechanisms of the achieving relaxation of the magnetization, and structure-function relationships, the reader should be referred to the exhaustive review. ${ }^{[264]}$

Magnetocaloric effect (MCE), i.e. change of the temperature of a magnet with a change in an external magnetic field, is most pronounced at the temperatures near the magnetic phase transition. It was above mentioned that it is the low-temperature region for the tetrapyrrole-based paramagnets. Deriving the magnets with high MCE arises from the attempt to use the magnetic materials for magnetic 
refrigeration and as cryogenic magnetic coolants. ${ }^{[269,270]}$ Moreover, the discovery of the SMMs quantum nature led to potential applications in quantum computing. ${ }^{[271]}$ The results in the field of molecular magnetism obtained since the beginning of the development of this new discipline in the field of magnetochemistry are presented in an exhaustive review article in $2013 .{ }^{[272]}$ The structure, synthesis, composition, magnetic properties, and suitability in various fields of magnetic $3 d, 3 d-4 f$, and $4 f$-refrigerants were studied. It was shown that gadolinium(III) and less commonly other lanthanides can be used in the case of monomeric compounds as the inclusion in $3 d$ metal clusters and as homometallic compounds. Both paths led to an increase in the MCE in comparison with the use of only $3 d$ metals. The review covers manganese clusters with added lanthanides, $3 d-4 f$ phosphonate compounds instead of metals, copper(II)/nickel(II) - gadolinium clusters, chromium(III) - gadolinium fluorides, giant metal frames, compact multi-metal frames, $4 d-4 f$ scaffolds, and cubic cobalt(II) lanthanides. The metal cations in them are in a more or less complex ligand environment. The homometallic gadolinium compounds were considered in different clusters in which the magnetic component of the change in entropy $-\Delta S_{\mathrm{M}}$ that is a measure of the MCE are reached about $27 \mathrm{~J} / \mathrm{kg}$ at $5 \mathrm{~K}$ and a change in the field from zero to 1 $\mathrm{T}$. In the case of porphyrin-based gadolinium compounds, only two complexes with $\mathrm{H}_{2} \mathrm{TPP}^{[273]}$ were mentioned. Study of MCE manifested by paramagnets at the room temperature allows receiving the data about magnetic ordering, exchange and magnetocrystalline interactions, the transformation of magnetic structures under magnetic fields, and improving of the molecule spin state by variation in the chemical structure. Here, we focus on the field of MPXs/MPcXs magnetocaloric behavior at the temperature close to room depending on the paramagnet's molecular structure. To reveal trends of changes in the magnetic activity of porphyrin complexes, variation of both a macrocycle composition in $3 d$ complexes and an axial ligand in lanthanide complexes was used. MCE of the tetrapyrrole complexes was determined in adiabatic conditions by the direct microcalorimetry method ${ }^{[274,275]}$ at the temperatures from 278 to $343 \mathrm{~K}$ in magnetic fields from 0 to $1.0 \mathrm{~T}$. MCE related to the completely thermostatic calorimetric cell with solid paramagnets and water above it was observed in the first works. ${ }^{[273,276-279]}$ A paramagnet heat capacity was observed in the same experiment as for MCE values and calculated according to equation (1). The heat capacity of solid paramagnets was evaluated as a difference (2). In the subsequent works, ${ }^{[275,280-286]}$ a new method for MCE determination was based on using the equation (3). The paramagnet true MCE values were obtained by this method. The heat capacity of paramagnets was independently observed by the direct DSC method in the cited works.

$$
\begin{aligned}
& C_{\mathrm{p}}=Q / \Delta T-W \\
& C_{\mathrm{p}(\mathrm{M})}=\left(C_{\mathrm{p}}-C_{\mathrm{p} \text { water }} \omega_{\text {water }}\right) / \omega_{(\mathrm{M})}
\end{aligned}
$$

Here $Q$ is the quantity of heat being introduced into the calorimetric cell by use of a calibrated heater, DT is temperature changing of the calorimetric cell due to introduced joule heat with account for heat exchange in initial and final periods; $W-$ the calorimeter constant. $C_{\mathrm{p}(\mathrm{M})}, C_{\mathrm{p}}, C_{\mathrm{p} \text { water }}$ and $\omega_{(\mathrm{M})}, \omega_{\text {water }}$ are the specific heat capacities of the solid paramagnet, thermostatic cell, water and the mass portions of the solid paramagnet, water, respectively.

$$
Q_{\mathrm{MCE}}=Q_{\mathrm{J}}\left(\Delta T / \Delta T_{\mathrm{J}}\right)=m_{(\mathrm{M})} C_{\mathrm{p}(\mathrm{M})} \Delta T_{\mathrm{MCE}}
$$

Here $Q_{\mathrm{J}}$ is Joule heat, injected in the calorimetric experiment, $\Delta T_{\mathrm{J}}$ and $\Delta T$ are the temperature change of calorimetric cell as the result of injecting of Joule heat and because of a magnetic field change, respectively, $\Delta T_{\mathrm{MCE}}$ - the temperature change that is true $\mathrm{MCE}, m_{(\mathrm{M})}$ is mass of a paramagnetic material.

\section{Structural Modifications and Magnetocaloric Behavior of Manganese(III) porphyrins}

There are no data about the study of manganese(III) tetrapyrrole complexes MCE at the temperatures near the magnetic phase transition. According to the work, ${ }^{[257]}$ manganese(II) phthalocyanine acting due to the ordering of $\mathrm{Mn}^{2+}$ with $S=3 / 2$ state in the $\beta$-polymorph display ferromagnetic behavior below Currie temperature. The molecular magnets based on manganese porphyrin/phthalocyanine building blocks are described.[287-290] The paramagnetic nature of the $\mathrm{Mn}^{3+}$ porphyrins confirmed not only by their spectral properties but also by the effective magnetic moment $\left(\mu_{\text {eff }}\right)$ magnitude $4.8-5.0 \mu_{\mathrm{B}}$ allows to expect magnetocaloric activity in these complexes. Room-temperature MCE of manganese(III) porphyrins/porphyrazines was studied by the microcalorimetric method above, in which the direct temperature change measurement in the adiabatic process was selected from two possible methods of MCE determining - the one and calculation of the magnetic entropy change in an isothermal process with respect to the variation of the magnetic field. ${ }^{[291]}$

\section{Octaethylporphyrins}

$(2,3,7,8,12,13,17,18$-Octaethylporphinato)manganese(III) chloride, $(\mathrm{Cl}) \mathrm{MnOEP}$ displays the noticeable positive MCE value which increases with the growth of the magnetic field induction at all temperatures and decreases with the temperature growth at all magnetic fields (Figures 32, 33 created using the data from the article cited). ${ }^{[276]}$

Dependence of specific heat capacity of the studied paramagnets on the value of magnetic field induction has a maximum at $0.2-0.3 \mathrm{~T}$ at all temperatures as in the case of high disperse particles with ferrimagnetic and ferromagnetic structures. ${ }^{[269-271]}$ The tendency to heat capacity value decrease in the fields higher $0.4 \mathrm{~T}$ (Figure 32b) was attributed to the regulation of the complex magnetic structure. The temperature growth leads to the increase of the specific heat capacity in all magnetic fields.

Change of magnetic molar entropy $\Delta S_{\mathrm{M}}(H, T)$ was calculated using equation (4):

$$
\Delta S_{M}=-\frac{\Delta C_{M} \cdot \Delta T}{T}
$$

Here $\Delta C_{\mathrm{M}}$ is a change in the magnetic part of the specific heat with a change in the field (equal $\left.C_{\mathrm{p}}(\mathrm{H})-C_{\mathrm{p}}(0)\right), \Delta T$ is MCE, $T$ is the temperature. Isotherms of the $\Delta S_{\mathrm{M}}^{\mathrm{p}}(H, T)$ 


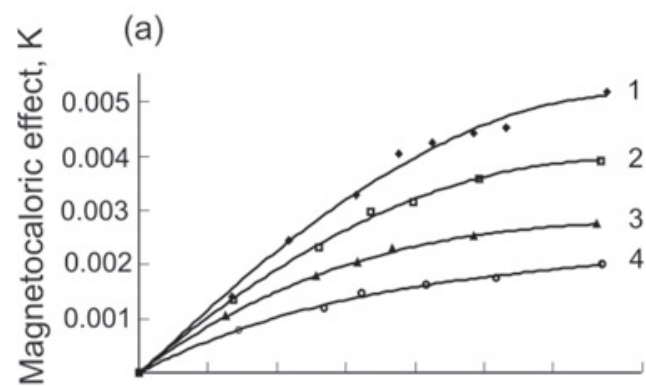

(b)

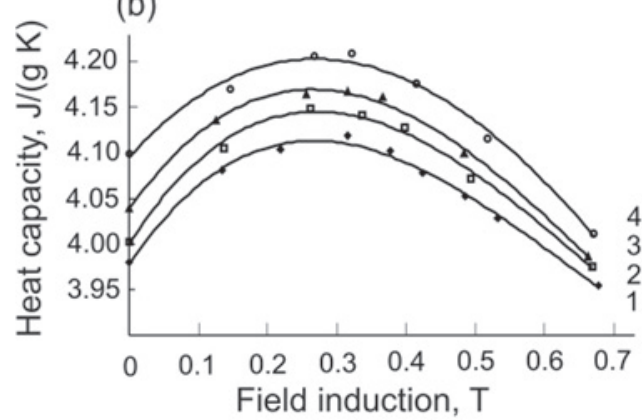

Figure 32. Dependence of the manganese complex MCE (a) and the specific heat capacity (b) at the temperatures of 298 (1), 313 (2), 328 (3), and 343 (4) K (a) on the magnitude of the magnetic field induction.

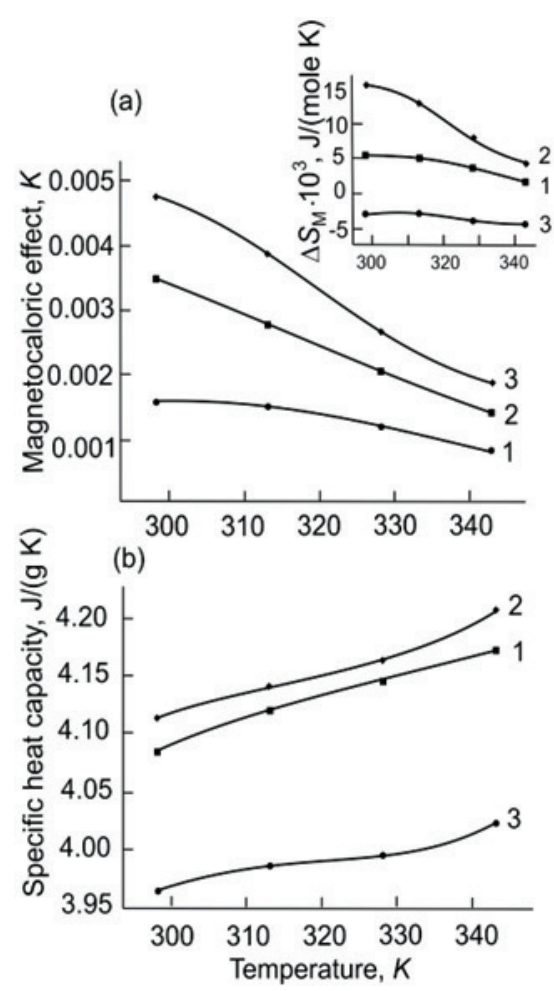

Figure 33. MCE, the changes of the magnetic part of the molar entropy (insert) (a) and specific heat capacity (b) of (Cl)MnOEP as a function of temperature in various applied magnetic fields. $B, \mathrm{~T}: 0.15$ (1), 0.32 (2), $0.6(3)$.

dependence on magnetic field induction have extreme character with maxima at induction of $0.3-0.4 \mathrm{~T}$, the height of which decreases with the temperature increase. $\Delta S_{\mathrm{M}}$
$(H, T)$ values decrease with the temperature growth and become negative when induction is equal to $0.65 \mathrm{~T}$ (Figure 33a, insert).

The absence of anomalies in the descending curves of the MCE temperature dependence with similar ascending curves in coordinates $C_{\mathrm{p}}-T$ (Figure 33) gives grounds to characterize the contribution of the lattice component to the MCE value as the main one.

An idea to control of MPXs/MPcXs magnetocaloric behavior by the macrocycle chemical structure modifications is based on the spin density redistribution data obtained for the paramagnetic manganese(III) complexes of substituted phthalocyanine, (AcO) $\mathrm{MnPc}\left(3,5-\mathrm{di}^{t} \mathrm{BuPhO}\right)_{8}{ }^{\left[{ }^{[292]}\right.}$ Figure 34 represented here by the results of the cited work shows that spin density of the spin carrier, $\mathrm{Mn}^{3+}$ is delocalized with the participation of both four coordinated $\mathrm{N}$ atoms and axial ligand.

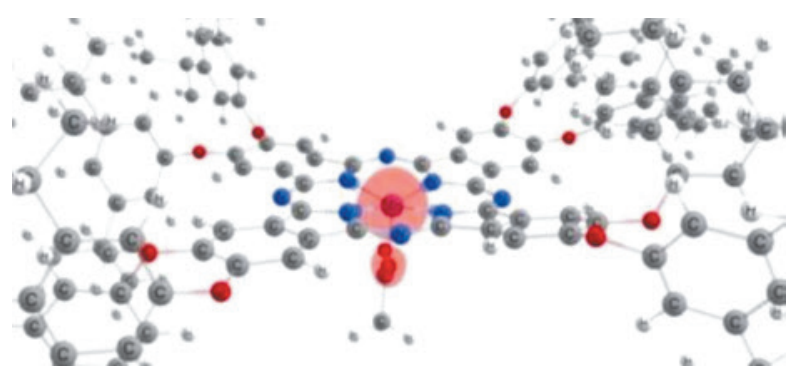

Figure 34. Spin density distribution quintet states of $(\mathrm{AcO}) \mathrm{MnPc}\left(3,5-\mathrm{di}-{ }^{ } \mathrm{BuPhO}\right)_{8}$ calculated by UB3LYP*+D3BJ/6-31G method (Cutoff at 0.01 a.u.). ${ }^{t} \mathrm{Bu}$ substituents are not shown.

Based on this, the next stage of the research included the transition to the manganese(III) complexes of meso-tetraphenylporphyrins with the electron-deficient macrocycles compared to $(\mathrm{Cl}) \mathrm{MnOEP}$. Modification of meso-phenyl groups opens wide prospective to the variation of macrocycle electronic state. Axial ligand changing gives additional opportunities.

\section{Tetraphenylporphyrins}

Magnetocaloric properties of high spin (5,10,15,20-tetraphenylporphyrinato)manganese(III) chloride, bromide, and acetate were determined at $298 \mathrm{~K}$ in a magnetic field of $0-1.0 \mathrm{~T}$ by the microcalorimetric method. ${ }^{[278]}$ For $(\mathrm{Cl}) \mathrm{MnOEP}$, the $\mathrm{MCE} / \mathrm{C}_{\mathrm{p}}$ values were obtained at the magnetic inductions of 0.8 and $1.0 \mathrm{~T}$ (Table 3). It was established that when the magnetic field was applied the temperature of the systems increases, leading to positive MCE values: the higher the magnetic field induction, the higher the values. The highest MCE value is exhibited by $(\mathrm{Cl}) \mathrm{MnOEP}$. For all inductions taken, the MCE values for (Cl)MnTPP and (Br)MnTPP are lower than those for $(\mathrm{Cl}) \mathrm{MnOEP}$ and differ inappreciably. Comparing these values, we can conclude that the MCE is affected by macrocycle substitution nature, and the effect from axial ligand is somewhat less. The MCE change during transition from the octaethylporphin complex to the similar tetraphenylpor- 

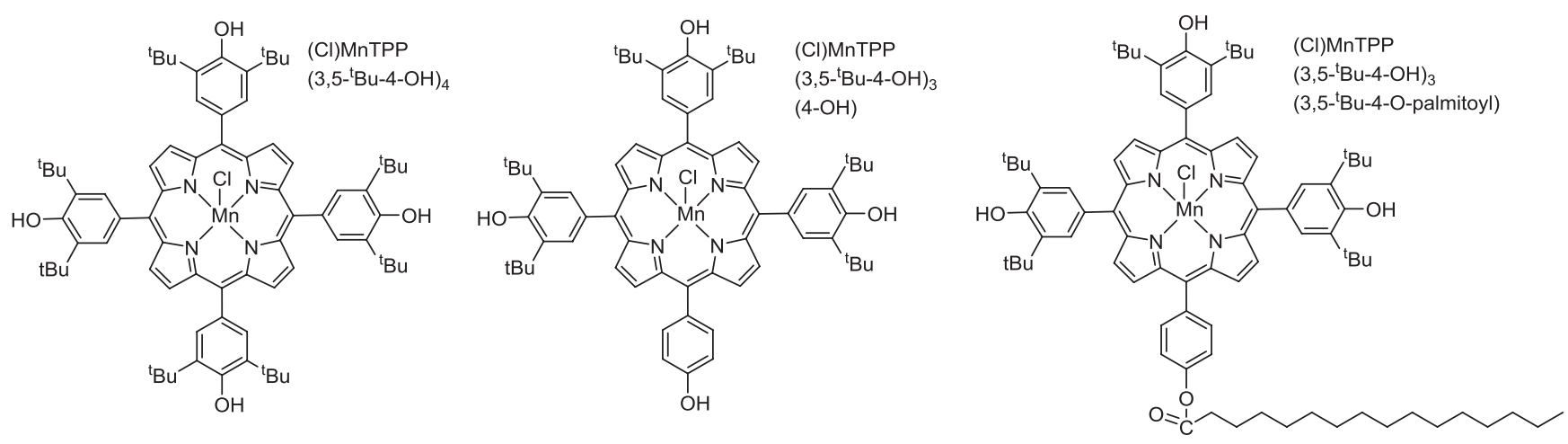

Figure 35. Formulas of manganese(III) porphyrins with phenol moiety in the molecular structure.

phin one is explained by destabilization/occupation reduction of $a_{1 u} / a_{2 u}$ HOMO involved in charge transfer to the $d$ orbitals of $\pi$ symmetry $\left(d_{\mathrm{xz}}, d_{\mathrm{yz}}\right.$, and $\left.d_{\mathrm{z}^{2}}\right)$. The lowest MCE values are obtained for (AcO)MnTPP. The same regularities are observed in comparing the dynamics of the change of enthalpy $\left(\Delta H_{\text {enth }}\right)$ values upon the magnetization of manganese complexes. ${ }^{[278]}$

Table 3. The magnetocaloric effect, $\Delta T_{\mathrm{MCE}}$, the specific heat capacity, $C_{\mathrm{p}}$, and the change in the magnetic part of entropy, $\Delta S_{\mathrm{M}}$ of manganese(III) porphyrin complexes at $298 \mathrm{~K}$ in the magnetic field of $1.0 \mathrm{~T}$.

\begin{tabular}{lccc}
\hline \multicolumn{1}{c}{ Complex } & $\Delta T_{\mathrm{MCE}}, \mathrm{K}$ & $C_{\mathrm{p}}, \mathrm{J} /(\mathrm{g} \cdot \mathrm{K})$ & $\Delta S_{\mathrm{M}} 10^{-3}, \mathrm{~J} /(\mathrm{mol} \cdot \mathrm{K})$ \\
\hline$(\mathrm{Cl}) \mathrm{MnTPP}$ & 0.069 & 0.66 & -1.7 \\
$(\mathrm{AcO}) \mathrm{MnTPP}$ & 0.065 & - & - \\
$(\mathrm{Br}) \mathrm{MnTPP}$ & 0.069 & 0.70 & -0.3 \\
$(\mathrm{Cl}) \mathrm{MnOEP}$ & 0.082 & 0.74 & -1.4 \\
\hline
\end{tabular}

The heat capacity of the (X)MnTPP solid particles depended strongly on the magnetic field induction was also determined. The field dependences of the heat capacity of manganese complexes have a maximum in the average fields whose value depends on the electron nature of the macrocycle substituents as it is shown on the $\mathrm{H}_{2}$ TPP and $\mathrm{H}_{2} \mathrm{OEP}$ examples. The influence of the coordinated axial ligands is less pronounced. The heat capacity in the area above $0.6 \mathrm{~T}$ is less than that in the zero fields. The magnetic field dependences of the change in magnetic entropy $\Delta S_{\mathrm{M}}$ have their maximum in fields of $0.2-0.4 \mathrm{~T}$. The amplitude of the maximum is also associated with modifications in the structure of the macrocycle and coordination center.

MCE and heat capacity during the magnetization of (5,10,15,20-tetrakis(3,5-di-tert-butyl-4-hydroxyphenyl) porphinato)-, (5-(4-hydroxyphenyl)-10,15,20-tris(3,5-ditert-butyl-4-hydroxyphenyl)porphinato)-, and (5-(4-palmitoyloxyphenyl)-10,15,20-tris(3,5-di-tert-butyl-4-hydroxyphenyl)porphinato)manganese(III) chloride were determined over the temperature range of 278-320 K and in a magnetic induction of $0-1 \mathrm{~T}^{[286]}$

Dependences of MCE on temperature had weak maxima at all magnetic inductions (Figure 36) which were connected with the intermolecular hydrogen bonding of the hydroxyl groups (see below). MCE was increased at all temperatures under the palmitoyl substituent incorporation into one of the phenol groups. The heat capacity of the studied complexes increased slightly with the temperature growth (Figure 36, insert). Dependences of heat capacity on temperature show that the magnetic component of heat capacity due to the presence of the manganese atom acting as a paramagnetic center in complexes did not appear.

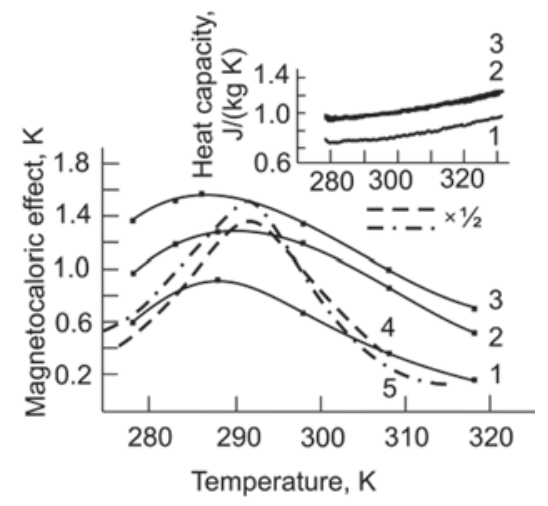

Figure 36. Temperature dependences of MCE and heat capacity (insert) at the magnetic induction of $1 \mathrm{~T}$ in (Cl)MnTPP $\left(3,5-{ }^{t} \mathrm{Bu}-4-\mathrm{OH}\right)_{4}(1),(\mathrm{Cl}) \mathrm{MnTPP}\left(3,5-{ }^{t} \mathrm{Bu}-4-\mathrm{OH}\right)_{3}(4-$ $\mathrm{OH})(2),(\mathrm{Cl}) \mathrm{MnTPP}\left(3,5-{ }^{t} \mathrm{Bu}-4-\mathrm{OH}\right)_{3}\left(3,5-{ }^{t} \mathrm{Bu}-4-\mathrm{O}-\right.$ palmitoyl $)(3)$, polycrystalline gadolinium according to experimental data from the work ${ }^{[281]}$ (4) and the work ${ }^{[293]}(5)$.

The relation between the complexes structure and their magnetocaloric properties was analyzed. Manganese(III) porphyrins containing 3,5-di-tert-butyl-4-hydroxyphenyl, 4-hydroxyphenyl, and 3,5-di-tert-butyl-4-palmitoyloxy groups in meso-positions reveal the paramagnetic properties and are characterized by "giant magnetocaloric effect" in the fields of $0-1 \mathrm{~T}$. The magnitudes of MCE are twenty or more times as large as compared with all complexes above as well as the octa- $\beta$-(4-tert-butylphenyl)substituted porphyrazine manganese(III) complex discussed in the next section. The changes of magnetocaloric properties resulted from the replacement of functional substituent in one of phenol groups as well as with passing to unsubstituted analog were caused by electronic substitution effects and, to an even greater degree, by the conditions of the formation of inter- 
molecular hydrogen bonds in the paramagnetic materials. Due to the presence of hydroxyl and palmitoyloxy groups in benzene rings of macrocycle the intermolecular hydrogen bonding occurs and it makes the main contribution into MCE change with less considerable electron influence of the substituents. This conclusion was established by an analysis of UV-vis quantitative characteristics (the relative band intensities) of the complexes in the solvents with various hydrogen bonding ability. ${ }^{[286]}$ Here we should note that the contribution from the ferromagnetic coupling between the spin carrier and the phenolic oxygen atoms which has a radical-like character ${ }^{[24]}$ is also possible.

Thus, it was first shown that the magnetocaloric effect in a porphyrin complex of a transition $d$-metal can reach a value comparable to that in the expensive polycrystalline gadolinium, the magnetic phase transition in which are cased at the temperature of $292 \mathrm{~K}$ (Figure 36).

\section{Substituted Manganese(III) Porphyrazines}

MCE, heat capacity, and magnetization enthalpy/ entropy of high-spin (acetato)(2,3,7,8,12,13,17,18-octa-paratert-butylphenyltetraazaporphinato)manganese(III) were determined microcalorimetrically at $298 \mathrm{~K}$ in magnetic fields of $0.1-1.0 \mathrm{~T},{ }^{[279]}$ that is, under the conditions comparable with those for $(\mathrm{Cl}) \mathrm{MnOEP}$. The polycrystalline complex was found to have paramagnetic properties displaying positive MCE values. These values grew as magnetic field induction increased (Figure 37, line 5). The presence of aza groups in the structure of the complex decreases the MCE value compared with the porphyrin complexes (Figure 37, lines 1-4). More effective charge transfer from macroring orbitals to the manganese cation in the complex with tetraazaporphyrin explains a decrease in MCE. This conclusion is based on the data ${ }^{[295,296]}$ for mutual arrangement of the frontier MOs and $d$-orbitals of (porphyrinato)iron(III) and $\quad(2,3,7,8,12,13,17,18$-octaphenyltetraazaporphynato) iron(III) chlorides $\left((\mathrm{Cl}) \mathrm{Fe}^{\mathrm{III}} \mathrm{P}\right.$ and $(\mathrm{Cl}) \mathrm{Fe}^{\mathrm{III}} \mathrm{OPTAP}$, respectively) in the intermediate spin state $S=3 / 2$ which can be considered analogs to the manganese(III) complexes.

The specific heat capacity of the complex strongly depends on the magnetic field value; field dependences had a maximum close to $0.3 \mathrm{~T}$. Changes in the $\Delta S_{\mathrm{M}}(H, T)$ were

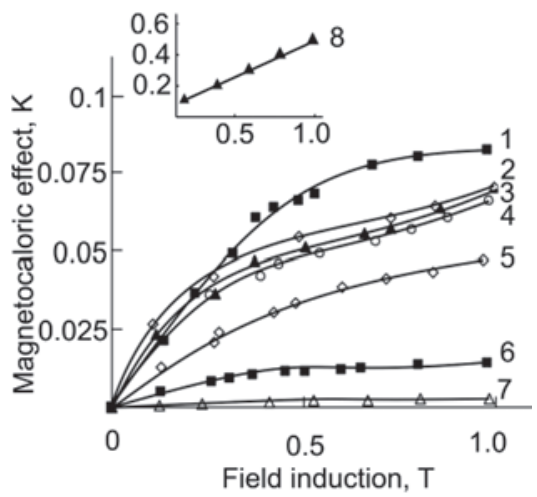

Figure 37. $\mathrm{MCE}$ dependences of $(\mathrm{Cl}) \mathrm{MnOEP}(1),(\mathrm{Cl}) \mathrm{MnTPP}$ (2), (Br)MnTPP (3), (AcO)MnTPP (4), (AcO)MnTAP $\left(p-{ }^{t} \mathrm{BuPh}\right)_{8}$ (5), $\mathrm{MnCl}_{2}(6), \mathrm{Ni}(7),{ }^{[297,298]}$ and $(\mathrm{AcO}) \mathrm{MnTAP}\left(3-\mathrm{CF}_{3} \mathrm{Ph}\right)_{8}(\mathrm{MCE}$ obtained by equation (3)) (8) on magnetic induction at $298 \mathrm{~K}$. also extreme and had maxima in magnetic fields $0.3-0.4 \mathrm{~T}$. The height of the maximum is also related to macroring structural modifications.

Comparative analysis of the MnPXs and MnTAPX magnetocaloric properties shown that macroring structural modification controls magnetocaloric behavior more effectively compared with a change of acido ligands in the five-coordinate manganese(III) complexes. Incorporation of manganese(III) spin carrier into equatorial macrocyclic and axial ligand fields leads to the significant increase in MCE compared with the simple salt $\mathrm{MnCl}_{2}$ or metallic magnets (Figure 37).

The magnetocaloric effect related to solid paramagnets only, heat capacity, and magnetization thermodynamic parameters of the high-spin (octakis-trifluoromethylphenyltetraazaporphinato)manganese(III) acetate, (AcO) $\operatorname{MnTAP}\left(3-\mathrm{CF}_{3} \mathrm{Ph}\right)_{8}$ (Figure 38) were obtained at the temperature from 275 to $320 \mathrm{~K}$ in magnetic fields of $0.1-1.0 \mathrm{~T}^{[280]}$

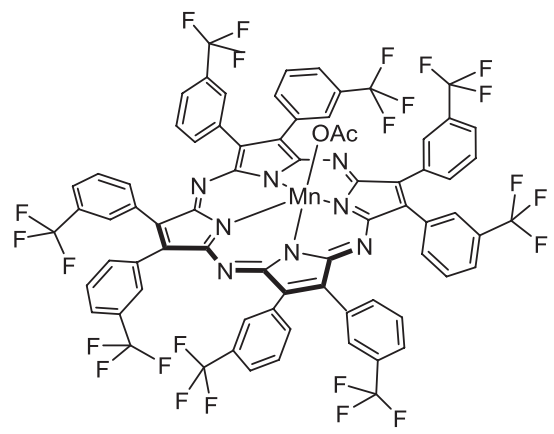

Figure 38. Chemical structure of (octakis-trifluoromethylphenyltet raazaporphinato)manganese(III) acetate.

As paramagnet, that complex exhibit "a large magnetocaloric effect" (up to $0.85 \mathrm{~K}$ when the magnetic induction is changed from 0 to $1 \mathrm{~T}$ ) at the temperature close to the room, which could be employed for cooling in home and industrial refrigerators and hyperthermia in diagnostics and therapy. The effect of the magnetic field on the temperature dependence of the specific heat capacity for $(\mathrm{AcO}) \mathrm{MnTAP}\left(3-\mathrm{CF}_{3} \mathrm{Ph}\right)_{8}$ has been shown to be practically invisible. The specific heat capacity of the complex sharply increases with temperature (Figure 39), which may be connected with thermal changes in the crystal lattice of the complex and achieves a value of $1.01 \mathrm{~J} /(\mathrm{g} \cdot \mathrm{K})$ at $318 \mathrm{~K}$. Such form of MCE- $T$ and $C_{\mathrm{p}}-T$ curves (Figure 39) is related to the change in the spin subsystem during a magnetization along with the thermal changes in its lattice subsystem.

\section{A Spin Carrier Changing Effect}

\section{Cobalt(II) Porphyrins}

The article ${ }^{[284]}$ represents the first unique data about new cobalt(II) porphyrin paramagnets with both vacant axial positions and a carbon nanoform composited with metal porphyrin via donor-acceptor bonding. Magnetocaloric effect values/magnetization thermodynamic parameters and specific heat capacity have been directly determined for $(5,10,15,20$-(tetra-4-tert-butylphenyl)- 


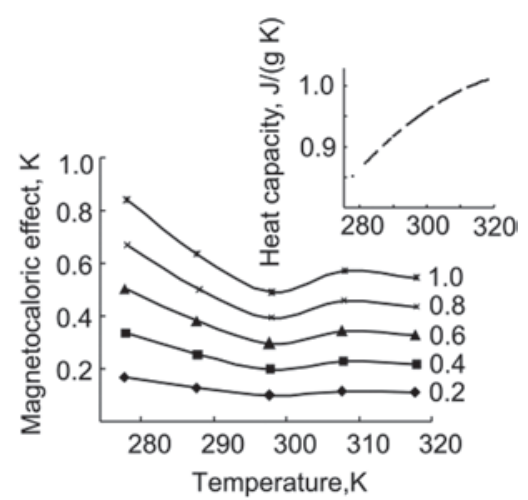

Figure 39. Temperature dependences of $\Delta T_{\mathrm{MCE}}$ in $(\mathrm{AcO})$ $\operatorname{MnTAP}\left(3-\mathrm{CF}_{3} \mathrm{Ph}\right)_{8}$ at magnetic induction and of its specific heat capacity (insert) in zero magnetic fields.

21H,23H-porphinato)cobalt(II) $\left(\mathrm{CoTPP}\left(p-{ }^{t} \mathrm{BuPh}\right)_{4}\right)$ and its donor-acceptor triad with 1'-N-methyl-2'-(pyridin-4-yl) pyrrolidino[3',4':1,2][60]fullerene, $\quad\left((\mathrm{PyF})_{2} \operatorname{CoTPP}(p-\right.$ $\left.\left.{ }^{t} \mathrm{BuPh}\right)_{4}\right)$. The samples display the positive magnetocaloric effect (Figure 40 ) that has been determined at $278 \mathrm{~K}$ and 1.0 $\mathrm{T}$ to be equal to 0.028 , and $0.0075 \mathrm{~K}$ for $\operatorname{CoTPP}\left(p{ }^{t} \mathrm{BuPh}\right)_{4}$ and $(\mathrm{PyF})_{2} \mathrm{CoTPP}\left(p-{ }^{t} \mathrm{BuPh}\right)_{4}$, respectively. The magnetocaloric effect values observed are comparable with those in metal-based magnetic materials ${ }^{[299,300]}$ but unlike those, the studied triad demonstrates a photoinduced electron transfer. ${ }^{[284,301]}$

To analyze the contributions of aromatic electron systems to the magnetization thermodynamic parameters, direct determinations of $\mathrm{MCE} / \mathrm{magnetization}$ thermodynamic parameters and heat capacity of $\mathrm{C}_{60}$ and $1^{\prime}-N$-methyl-2'(pyridin-4-yl)pyrrolidino[3',4':1,2][60]fullerene (PyF) were performed using the microcalorimetric method and DSC, respectively. ${ }^{[275]}$ Both materials display positive MCE that has been determined to be 0.004 and $0.016 \mathrm{~K}$ in $\mathrm{C}_{60}$ and $\mathrm{PyF}$, respectively, at $278 \mathrm{~K}$ and $1.0 \mathrm{~T}$. The increase in PyF MCE is observed although the molar heat capacity values of this paramagnets are two times higher than that of $\mathrm{C}_{60}$ (Figure 41a). Using the temperature dependences of $Q_{\mathrm{MCE}}$, $\Delta H$, (Figure 4lb) $\Delta S$ of magnetization and specific heat capacity (Figure 41a) along with calculated full energies, bond lengths, and the charges on atoms according to Mulliken in fullerenes (PM3 and the Hartree-Fock in the basis of 3-21G, using Gaussian03), ${ }^{[284]}$ they have explained that fact by the presence of aromaticity and antiaromaticity areas state in fullerene moiety.

The (PyF) $)_{2}$ CoTPP $\left(p-{ }^{t} \mathrm{BuPh}\right)_{4} \mathrm{MCE}$ decrease compared with the precursors $\left(\mathrm{PyF}\right.$ and $\left.\operatorname{CoTPP}\left(p-{ }^{t} \mathrm{BuPh}\right)_{4}\right)$ is caused by the triad higher specific heat capacity. Taken into account this fact the authors ${ }^{[284]}$ have suggested the need to move to pentacoordinate cobalt(II) porphyrin complexes, in which there is only one axial coordination site for the substituted fullerene bonding.

\section{Iron(III) Porphyrins}

For iron(III) porphyrins, MCE during the magnetization of chloride ligated pentacoordinate iron(III) 5,10,15,20-tetraphenylporphin, (Cl)FeTPP and dimethylfor- (a)

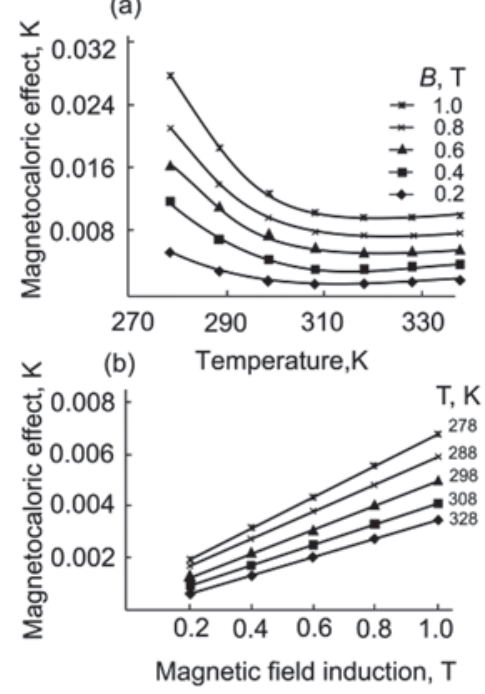

Figure 40. MCE magnitude in $\left(\operatorname{CoTPP}\left(p-{ }^{t} \mathrm{BuPh}\right)_{4}\right)$ as a function of temperature (a) and in $(\mathrm{PyF})_{2} \operatorname{CoTPP}\left(p-{ }^{t} \mathrm{BuPh}\right)_{4}$ as a function of magnetic field induction (b).

(a)
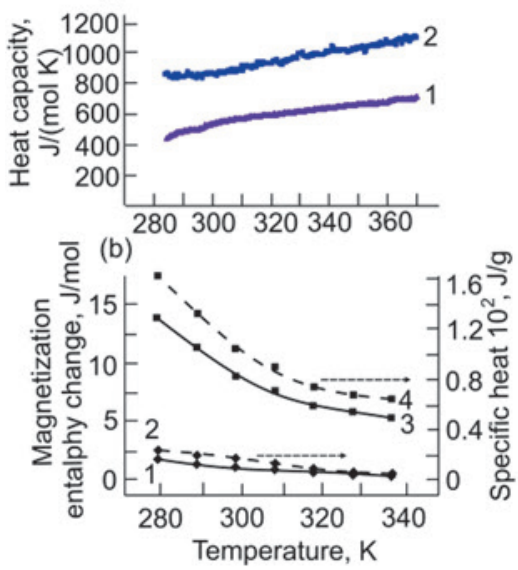

Figure 41. The temperature dependences of $\mathrm{C}_{60} / \mathrm{PyC}_{60}$ heat capacity $(1 / 2)$ in zero magnetic fields (a) and enthalpy $(1,3) /$ specific heart change $(2,4)$ due to magnetization of $\left.\mathrm{C}_{60}(1,2)\right)$ and $\operatorname{PyF}(3,4))$ at the magnetic induction of $1.0 \mathrm{~T}(\mathrm{~b})$.

mamide ligated six-coordinated iron(III) mesoporphyrin IX, $\left[(\mathrm{DMF})_{2} \mathrm{FeMP}^{+} \mathrm{Cl}^{-}\right.$over the temperature range of $278-$ $338 \mathrm{~K}$ and in magnetic fields from 0 to $1 \mathrm{~T}$ were determined by the direct microcalorimetric method. ${ }^{[302]}$ Specific heat capacity in the solid of $(\mathrm{Cl}) \mathrm{FeTPP} /\left[(\mathrm{DMF})_{2} \mathrm{FeMP}\right]^{+} \mathrm{Cl}^{-}$ has been directly obtained depending on a temperature in zero magnetic fields using DSC. Thermodynamic parameters of iron(III) complexes magnetization namely a heat, an enthalpy/entropy change was determined. Specific spin-admixed $\mathrm{S}=5 / 2,3 / 2$ state of $\left[(\mathrm{DMF})_{2} \mathrm{FeMP}\right]^{+} \mathrm{Cl}^{-}$ and pure high-spin state of (Cl)FeTPP were established by temperature-depended ${ }^{1} \mathrm{H}$ NMR spectrometry. The difference in the spin states leads to specific magnetocaloric behavior in this case.

$\left[(\mathrm{DMF})_{2} \mathrm{FeMP}\right]^{+} \mathrm{Cl}^{-}$and $(\mathrm{Cl}) \mathrm{FeTPP}$ display positive MCE of 0.028 and $0.020 \mathrm{~K}$ at the magnetic induction of $1.0 \mathrm{~T}$ and the temperature of 283 and $278 \mathrm{~K}$, respec- 
tively. The MCE values in both paramagnets are the same at $300 \mathrm{~K}$. The ones at higher temperatures get higher in $(\mathrm{Cl}) \mathrm{FeTPP}$ compared $\left[(\mathrm{DMF})_{2} \mathrm{FeMP}\right]^{+} \mathrm{Cl}^{-}$. The sharp rise of the $\left[(\mathrm{DMF})_{2} \mathrm{FeMP}\right]^{+} \mathrm{Cl}^{-} C_{\mathrm{p}}$ values from 1.0 to $1.75 \mathrm{~J} /(\mathrm{g} \cdot \mathrm{K})$ at the low taken temperatures (from 275 to $310 \mathrm{~K}$ ) and its deceleration in the high-temperature area was established and interpreted because of the iron(III) porphyrin spin state changing. The $S=5 / 2$ contribution increases in the spinadmixed $S=5 / 2,3 / 2$ state in $\left[(\mathrm{DMF})_{2} \mathrm{FeMP}^{+} \mathrm{Cl}^{-}\right.$with the temperature growth. MCE values are accordingly associated with both the iron(III) porphyrin spin state and redistribution of spin density between the spin carrier and the macrocycle $\pi$-electron system. The latter is controlled by macrocycle meso/peripheral substitution.

\section{Summary of 3d-Complexes Magnetocaloric Activity}

The advantages of $3 d$ metal porphyrins/porphyrazines over other paramagnets consist firstly in the fact that we can control their magnetocaloric properties through molecule structural modification that is unlimited in the chemistry of tetrapyrroles. Figure 42 designed using the cited above works gives a comparative picture of magnetocaloric activity paramagnets studied.

Manganese(III) complexes characterized by the more pronounced effect of a macrocycle structure on the MCE value compared with an axial ligand display the highest MCE among the complexes of $3 d$ metals taken. Paramagnetic iron(III) and cobalt(II) porphyrins demonstrate the lowest MCE acting without supramolecular organization due to an axial ligand or a bulk substituent in a molecular structure (Figure 42). It is interesting that a supramolecular structure built via hydrogen bonding in solid manganese(III) complexes with phenolic groups in Figure 42 leads to "giant MCE" of $0.98-1.57 \mathrm{~K}$ at the magnetic induction changed from zero to $1 \mathrm{~T}$. Positive contribution in MCE is probably connected also with ferromagnetic coupling between

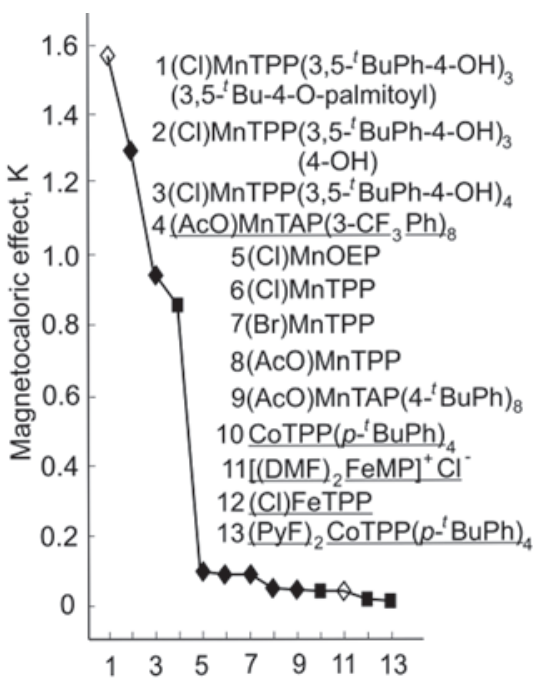

Figure 42. The MCE sensitivity graph of polycrystalline MPXs/ $(\mathrm{AcO}) \mathrm{MnTAP}\left(3-\mathrm{CF}_{3} \mathrm{Ph}\right)_{8}$ (underlined) and of the cell with $\mathrm{MnPXs} /(\mathrm{AcO}) \mathrm{MnTAP}\left(4-{ }^{\mathrm{B}} \mathrm{BuPh}\right)_{8}$ plus water to modifications in the axial or equatorial ligand. Temperature, $\mathrm{K} \boldsymbol{\square}-278, \diamond-283$, -298 . the spin carrier and the radical-like antioxidant $\mathrm{PhOH} /$ PhOR groups in the molecular structure.

MCE decrease in $\operatorname{CoTPP}\left(p-{ }^{t} \mathrm{BuPh}\right)_{4}$ in comparison with manganese(III) porphyrin complexes is explained by the electron configuration of the spin carrier in the lowspin cobalt(II) complex and high-spin state of manganese(III) porphyrins. On the other hand, the prospects for using cobalt(II) porphyrins in creating photoactive fullerenebased hybrids are based on their high coordination ability along the axial direction. The constant of the donor-acceptor $(\mathrm{PyF})_{2} \mathrm{Co}\left(p-{ }^{t} \mathrm{BuPh}\right)_{4}$ triad formation is $(1.34 \pm 0.12) \cdot 10^{10}$ $\left(\mathrm{L}^{2} / \mathrm{mol}^{2}\right),{ }^{[284]}$ which is one of the highest values in "axial" metal porphyrin chemistry. ${ }^{[268]}$

While contribution in MCE from a high spin paramagnetic carrier is always positive, the $\pi$ dative bonding metal macrocyclic ligand effects depending not only from the macrocycle's electron structure but from the electron configuration of a central ion. For example, MCE in manganese(III) tetraazaporphyrins is higher for the $3-\mathrm{CF}_{3} \mathrm{Ph}$ substituted complex than for the $4-{ }^{t} \mathrm{BuPh}$ derivative in spite of striving for manganese to achieve a stable electronic configuration $d^{5}$. It is probable that the main role belongs here to the redistribution of $\mathrm{Mn}$ atom spin density with axial acetate ion (see Figure 34). It should be borne in mind that the negative contribution in a MCE value from growing interaction with nuclear spins depends on a coordination center geometry that can be changed by aza substitution in a macrocycle.

\section{Design of the REE Porphyrin Structures to Improve Magnetocaloric Activity}

\section{Axially Coordinated Lanthanide(III) Tetraphenylporphyrins}

MCEs in (5,10,15,20-tetraphenylporphyrinato)acetatogadolinium(III), (AcO)GdTPP and (5,10,15,20-tetraphenylporphyrinato)chlorogadolinium(III), (Cl)GdTPP determined in a range of temperatures from 278 to $318 \mathrm{~K}$ and magnetic fields from 0 to $1 \mathrm{~T}^{[273]}$ are positive (Figure 43a), i.e. temperature of the complexes increases at applying a magnetic field in the adiabatic conditions. The substitution of chloride ligand by acetate in (X)GdTPP leads to a significant increase in MCE and its temperature dependence. MCE in $(\mathrm{Cl})$ GdTPP does not actually depend on temperature. The heat capacity of the complexes slightly increases with an increase in temperature and more noticeably in the case of (AcO)GdTPP (Figure 43b). A magnetic component of heat capacity is revealed only in (AcO)GdTPP at temperatures above $298 \mathrm{~K}$, which is connected perhaps with a temperature change in the crystal lattice of the complex and influence of the magnetic properties of gadolinium ion on this change. Despite the higher (AcO)GdTPP $C_{\mathrm{p}}$ values than that for (Cl)GdTPP, higher MCE is observed (Figure 43) showing on the contribution in MCE from the gadolinium(III) complexes electronic subsystem. Transition from (Cl)GdTPP to the acetate analog in which $\mathrm{AcO}^{-}$is coordinated bidentately ${ }^{[303]}$ is accompanied by the additional displacement of the central ion from the macrocyclic plane. Related to this, lowering the conditions for the Gd↔N $\pi$-interaction leads to the enhancement of paramagnetic properties of the central ion, which gives rise to changes in the MCE values. 


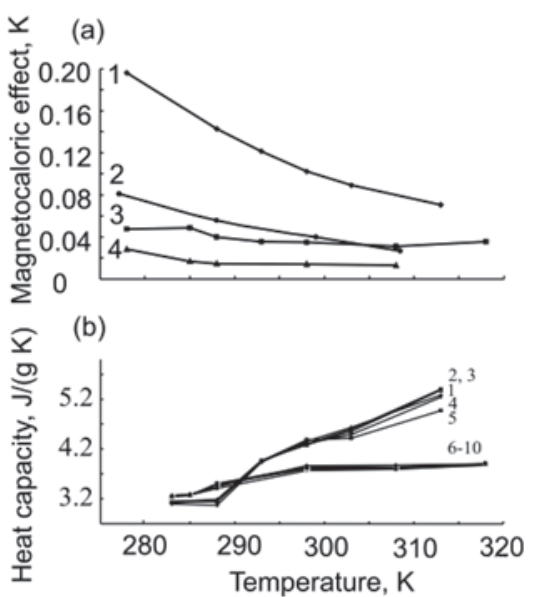

Figure 43. Temperature dependences of magnetocaloric effect in (AcO)GdTPP (1), (Cl)EuTPP (2), (Cl)GdTPP (3), and (Cl) TmTPP (4) at the magnetic field of $1.0 \mathrm{~T}$ (a) and of specific heat capacity for $(\mathrm{AcO}) \mathrm{GdTPP}(1-5)$ and $(\mathrm{Cl}) \mathrm{GdTPP}(6-10)$ in the magnetic fields of $0(1,10), 0.25(2,9), 0.50(3,8), 0.75(4$, $7)$, and $1.0(5,6) \mathrm{T}(\mathrm{b})$.

In contrast to gadolinium(III) complexes, $\pi$ dative interaction starts to prevail over the geometrical in the case of lanthanide complexes with unsymmetrically filled $f$ shells. In the case of chlorides, MCE values fall along with the Eu > $\mathrm{Gd}>\mathrm{Tm}$ series at temperatures from 275 to $300 \mathrm{~K}$ (Figure 43) despite more planar lanthanide ion disposition in the porphyrin plane due to a lanthanide compression effect in the same series. The maximum MCE value for $(\mathrm{Cl})$ EuTPP complex is in good agreement with the properties of its $f$ shell with the $f^{6}$ electronic configuration, particularly with its tendency to reach a stable $f^{7}$ configuration by means of the effective $\mathrm{N} \rightarrow \mathrm{Eu} \pi$ dative interaction. Variation of the axial ligand is a more important factor for controlling magnetocaloric properties than changes in the nature of the lanthanide. To finally be convinced of the decisive role not only of the presence, but also of the direction of the dative Ln-N $\pi$ bond in the redistribution of the spin density of the central atom, one should turn to the data on MCE/the thermodynamic parameters of magnetization and the specific heat capacity of the solid samples obtained for ytterbium(III) complexes (Cl)YbTPP and (Cl)YbTPP $\left(p-{ }^{t} \mathrm{Bu}\right){ }_{4} \cdot{ }^{\left[{ }^{[04]}\right.}$ The gradual increase in the (Cl)YbTPP $C_{\mathrm{p}}-T$ dependence is consistent with the corresponding MCE decrease. Not high curve maximum obtained for $(\mathrm{Cl}) \mathrm{YbTPP}\left(p-{ }^{t} \mathrm{Bu}\right)_{4}$ was interpreted because of structure improvement in the solid with the temperature growth. ${ }^{[305]}$ The $C \mathrm{p}$ values of both paramagnets differ little. The highest MCE value at $278 \mathrm{~K}$ and at the magnetic induction of $1.0 \mathrm{~T}$ is 0.028 and $0.0046 \mathrm{~K}$ for $(\mathrm{Cl}) \mathrm{YbTPP}$ and $(\mathrm{Cl}) \operatorname{YbTPP}\left(p-{ }^{t} \mathrm{Bu}\right)_{4}$, respectively, which is one-two orders of magnitude lower compared with europium(III), gadolinium(III), and thulium(III) tetraphenylporphine complexes. Wherein, Eu and $\mathrm{Yb}$ in the $(\mathrm{Cl}) \mathrm{LnTPP}$ structure are similar to each other in the desire to complement their $f$-shell to stable $4 f^{7}$ and $4 f^{14}$, respectively, which can be achieved through dative $\mathrm{p}$ bonding $\mathrm{Ln}-\mathrm{N}$. The contribution to the $(\mathrm{Cl})$ YbTPP spin state from the dative $\mathrm{Yb}-\mathrm{N} \pi$ bonds is negative, unlike in the case of $(\mathrm{Cl})$ EuTPP in which the spin improvement is achieved.
We must emphasize that MCE in the real experiment ${ }^{[273]}$ covers generally porphyrin paramagnets with the water in a cell. The MCE values assigned only to lanthanide(III) porphyrin complex are $0.127-1.45 \mathrm{~K}$ at the temperature close to the room and at the magnetic induction of $1 \mathrm{~T} \cdot{ }^{[297,298]}$ Characteristically, the view of temperature/field dependences of MCE obtained for paramagnet + water and for paramagnet only is similar to each other. Mentioned MCE values are higher than these for polycrystalline $\mathrm{Gd}$ in the ferromagnetic state (at $90 \mathrm{~K}, 1 \mathrm{~T}$ ), the magnetocaloric effect of which is accepted as regular. Polycrystalline gadolinium and gadolinium ferrite $\left(\mathrm{GdFeO}_{3} \mathrm{FeO}\right)$ display $\mathrm{MCE}$ values of $2.56(293 \mathrm{~K}$, field change $0-1.0 \mathrm{~T})$, and of $0.42 \mathrm{~K}(298 \mathrm{~K}$, $0-0.65 \mathrm{~T})$, respectively. ${ }^{[306,307]}$

Extension of the $\pi$ networks along the axis perpendicular to the macrocycle plane due to chelating acetylacetone anion by gadolinium(III) porphyrin is described in the work. ${ }^{[281]}$ Magnetocaloric properties of $(5,10,15,20$-tetraphenyl-21H,23H-porphinato)gadolinium(III) acetylacetonate, (Acac)(AcacH) GdTPP (Figure 44) as a function of both temperature and magnetic induction are studied. (Acac)GdTPP exhibits large MCE of $0.37 \mathrm{~K}$ at $278 \mathrm{~K}$, when the magnetic induction is changed from zero to $1 \mathrm{~T}$, which could be employed for magnetic cooling.

MCE in the acetylacetonate complex is two - four and approximately one and a half times less than that in the acetate and chloride complex, respectively. This ratio in the MCE values does not reflect the one in specific heat capacity. The (Acac)GdTPP $C_{\mathrm{p}}$ values increase sharply with temperature and have a value of $0.9 \mathrm{~J} /(\mathrm{g} \cdot \mathrm{K})$ at $278 \mathrm{~K}$ and of $1.7 \mathrm{~J} /(\mathrm{g} \cdot \mathrm{K})$ at $330 \mathrm{~K}$. Effect of the magnetic field on the temperature dependence of $C_{\mathrm{p}}$ practically does not appear. $C_{\mathrm{p}}$ value at the temperature of $288 \mathrm{~K}$ in zero magnetic fields is equal to $1.19,1.04$, and $1.04 \mathrm{~J} /(\mathrm{g} \cdot \mathrm{K})$ for the acetate, acetylacetonate, and chloride axial complex of gadolinium porphyrin. The ratio in MCE does not reflect the one in the location of the $\pi \rightarrow \pi^{*}$ transition band at 553, 554, and $555 \mathrm{~nm}$ in the UV-Vis spectrum of (Acac)GdTPP, (AcO)GdTPP, and (Cl)GdTPP, respectively. This parameter is quantitatively very similar to an effective energy barrier against magnetic relaxation. ${ }^{[264,265]}$

The absence of correlation between MCE and $C_{\mathrm{p}} / \lambda_{\max }$ indicates zero contribution of the effects in the lattice subsystem on magnetic relaxation of (Acac)GdTPP. It means that MCE in (Acac)GdTPP is connected with the changes in the electronic subsystem. Formation of the six-member aromatic ring by axial ligand in (Acac)GdTPP (Figure 44)

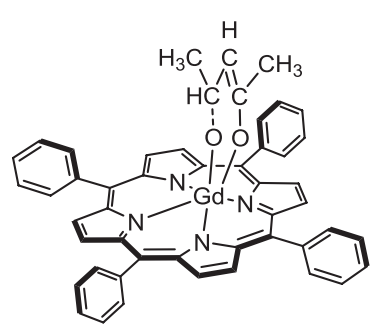

Figure 44. Chemical structure of $(5,10,15,20$-tetraphenyl$21 H, 23 H$-porphinato)gadolinium(III) acetylacetonate, (Acac) GdTPP. Molecular ligands are not shown. 
leads to $\pi$ dative bonding (both $\mathrm{O} \rightarrow \mathrm{Gd}$ and $\mathrm{O} \leftarrow \mathrm{Gd}$ ) in the complex and to the lowering MCE.

\section{Lanthanide(III) Phthalocyanine Acetates}

Lanthanide(III) ions situated in a coordination site of phthalocyanine macrocycle as molecular paramagnets with a large magnetocaloric effect at the temperatures close to the room are presented for the first time on the examples of gadolinium and thulium. ${ }^{[282]}$ Magnetocaloric properties of (AcO) GdPc and (AcO)TmPc are studied at 278-338 K in the magnetic field of $0-1.0 \mathrm{~T}$. The $\mathrm{Gd}^{3+} / \mathrm{Tm}^{3+}$ ions retain their paramagnetic properties being in a phthalocyanine ligand field. Maximum MCE values are observed at the magnetic induction of $1.0 \mathrm{~T}$ (Figure 45). The higher values of mass and specific heat capacity together with the lower paramagnetic moment in the trivalent state ${ }^{[308]}$ do not lead to the significant lowering of maximum MCE value in (AcO)TmPc compared with (AcO)GdPc. MCE at $278 \mathrm{~K}$ and at the magnetic induction of $1.0 \mathrm{~T}$ is equal to 0.35 and $0.47 \mathrm{~K}$, respectively.

Less radius of Tm compared with Gd caused higher planarity and stability of the coordination center and correspondingly the spin carrier interactions with nuclear spins does not change the mentioned MCE ratio. The positive con-

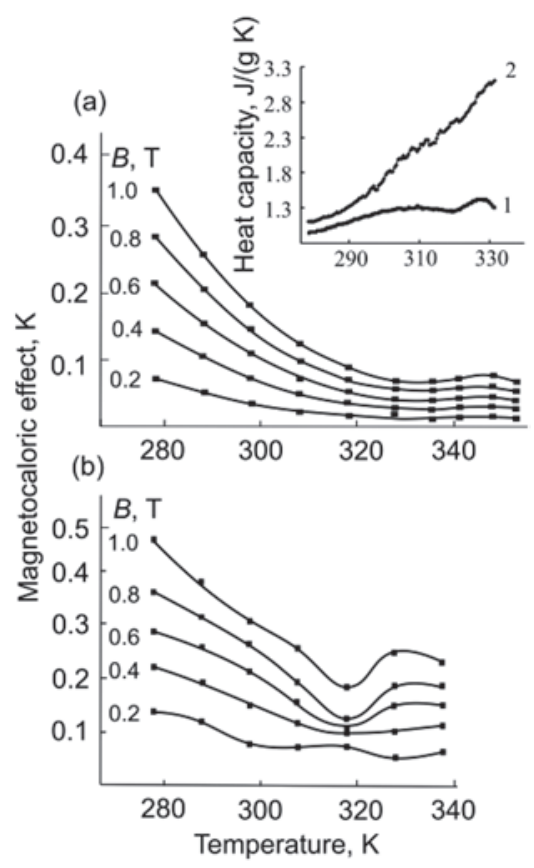

Figure 45. Temperature dependences of $\mathrm{MCE}$ in (AcO)TmPc (a) and in $(\mathrm{AcO}) \mathrm{GdPc}(\mathrm{b})$ at the field induction shown in Figure and the specific heat capacity in zero fields (insert) in (AcO) GdPc (1) and (OAc) TmPc (2). tribution in the spin state of thulium-containing paramagnet is $\pi$-dative interaction $\mathrm{Tm} \rightarrow \mathrm{N}$, which starts to notable factor for spin density delocalization in the case of lanthanide complexes with asymmetrically filled $f$-shells.Doubledecker gadolinium(III) phthalocyanine

Gadolinium is useful in the room temperature magnetic refrigeration due to its large magnetocaloric effect occurring at temperatures close to the room. This element was choused as the central atom in the double-decker phthalocyanine complex, $\mathrm{GdPc}_{2}{ }^{\bullet}$, in which one of two aromatic macrocycle had an unpaired electron as an additional spin carrier. ${ }^{[283]}$ Thus, an attempt was made to implement the strategy used in the work $^{[309]}$ to improve the spin state of the complex (Figure 46). Although only a spin $(S=7 / 2)$ contribution (due to the absence of spin-orbit coupling) SMM behavior of the gadolinium(III) complex unlike the terbium(III) and dysprosium(III) analogs was established for bis(oct aethyltetraazaporphyrinato) complexes by DC magnetic susceptibility experiments. ${ }^{[263]}$ That promises to simplify the understanding of ferromagnetic coupling nature in $\mathrm{GdPc}_{2}$ and in such magnetics.

Magnetocaloric properties (a magnetocaloric effect, specific heat capacity, heat released due to MCE, enthalpy/ entropy change) of $\mathrm{GdPc}_{2}{ }^{\bullet}$ at $278-338 \mathrm{~K}$ in the magnetic fields of $0-1.0 \mathrm{~T}$ were studied for the first time in the family of these paramagnets. ${ }^{[283]}$ The study of magnetocaloric properties and specific heat capacity were performed in two independent experiments by microcalorimetry and DSC, respectively. Positive MCE values increased with the magnetic field induction and decreased with temperature growth in the studied ranges of temperature/magnetic induction exhibit maximums at $288 \mathrm{~K}$ (Figure 47 created using the reported data $\left.{ }^{[283]}\right)$. The character of a changing of $\mathrm{MCE}$ is largely determined by the temperature dependence of the specific heat capacity. In accordance with equation (1), the MCE magnitude decreases sharply at the temperature corresponding to the one of maximum on the curve $C_{\mathrm{p}}-T$ (Figure 47). That can indicate the lattice subsystem contribution in MCE. Really, analysis of the temperature dependences of the magnetization thermodynamic parameters, $\Delta H_{\mathrm{M}} / \Delta S_{\mathrm{M}}$ together with the decrease of MCE values in the series of (AcO)GdTPP, (AcO)GdPc, $\mathrm{GdPc}_{2}{ }^{\bullet}$ indicates that the intramolecular oxidation-activated spin coupling does not prevail over the intermolecular coupling of two radical spins. ${ }^{[283]}$ However, the absence of correlation $\mathrm{MCE}-C_{\mathrm{p}}$ (the latter is equal to $1.19,1.05$ and $1.19 \mathrm{~J} \cdot \mathrm{g}^{-1} \cdot \mathrm{K}^{-1}$ in zero magnetic fields at the temperature of $288 \mathrm{~K}$ in the series mentioned) and view of $\mathrm{MCE} / C_{p}$ temperature dependences at the temperatures higher than the one at $C_{p}$ maximum means the presence of an electronic subsystem contribution in $\mathrm{MCE}$ in the gadolinium(III) complexes under consideration.

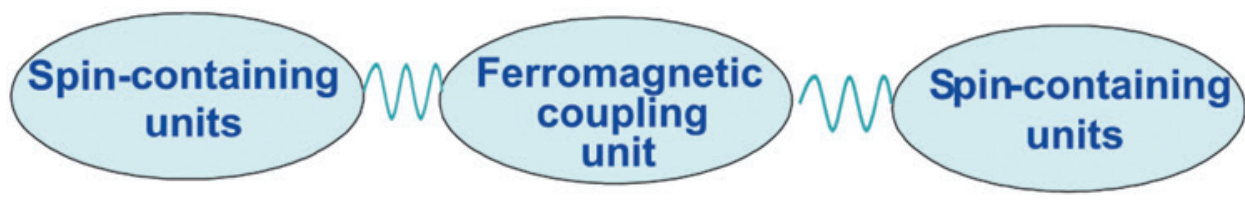

Figure 46. The strategy of spin state improving in a tetrapyrrole complex. 
Table 4. A magnetocaloric effect, $\Delta T_{\mathrm{MCE}}$ of lanthanide(III) porphyrin/phthaloyanine complexes at the magnetic induction of $1.0 \mathrm{~T}$.

\begin{tabular}{|c|c|c|c|c|c|c|c|c|}
\hline \multirow{2}{*}{ Complex } & \multicolumn{8}{|c|}{ Temperature, $\mathrm{K}$} \\
\hline & 278 & 283 & 285 & 288 & 290 & 293 & 296 & 298 \\
\hline (AcO)GdTPP & 1.45 & 1.01 & 0.893 & 0.801 & 0.663 & & 0.592 & \\
\hline (Cl)GdTPP & 0.449 & 0.347 & - & 0.326 & - & 0.281 & - & 0.306 \\
\hline (Acac)GdTPP & 0.38 & - & - & 0.30 & 0.23 & $0.28^{\mathrm{a}}$ & $0.205^{\mathrm{b}}$ & \\
\hline (Cl)EuTPP & 0.23 & 0.490 & - & 0.326 & - & 0.250 & - & \\
\hline (Cl)TmTPP & 0.281 & 0.209 & - & 0.173 & - & 0.127 & - & 0.127 \\
\hline$(\mathrm{AcO}) \mathrm{GdPc}$ & 0.455 & & - & 0.385 & - & - & - & 0.304 \\
\hline$(\mathrm{AcO}) \mathrm{TmPc}$ & 0.355 & & - & 0.270 & - & - & - & 0.190 \\
\hline $\mathrm{GdPc}_{2} \cdot$ & 0.050 & 0.070 & - & 0.085 & $0.038^{\mathrm{a}}$ & $0.035^{\mathrm{b}}$ & - & 0.040 \\
\hline
\end{tabular}

aat $308 \mathrm{~K},{ }^{b}$ at $318 \mathrm{~K}$.

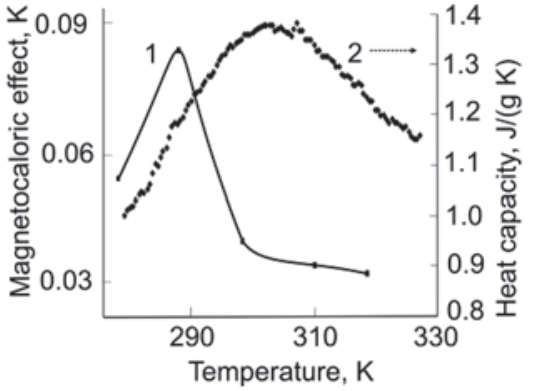

Figure 47. Temperature dependence of $\mathrm{MCE}$ in $\mathrm{GdPc}_{2}{ }^{\bullet}$ at applied magnetic induction $1.0 \mathrm{~T}(1)$ and of its specific heat capacity in zero magnetic fields (2).

\section{Summary on Lanthanide(III) Complexes Magnetocaloric Effect and Heat Capacity}

The lanthanide(III) complexes exhibit a large magnetocaloric effect at temperatures close to room, e.g. up to 1.45, 0.455 , and $0.38 \mathrm{~K}$ in (AcO)GdTPP, (AcO)GdPc, and (Acac) GdTPP, respectively, when the magnetic induction is changed from zero to $1 \mathrm{~T}$ (Table 4). That is only slightly lower than one in $3 d-4 f$ clusters/homo-metallic gadolinium compounds at higher fields $(2.4-3 \mathrm{~K}$ at $\Delta B$ of $0-7 \mathrm{~T}) .^{[272]}$

Contributions in a MCE value of (porphyrinato)/ (phthalocyaninato) lanthanide(III) complexes include a positive and negative part. Ligand-field splitting, spin density delocalization with the participation of macrocycle $\mathrm{p}$ electron system, namely $\pi$ dative interaction M-N depending on Ln electron configuration of lanthanide complexes with asymmetrically filled $f$-shells $(\mathrm{N} \rightarrow \mathrm{Eu}$ $\left(f^{6}\right)$ and $\mathrm{N} \leftarrow \mathrm{Tm}\left(f^{12}\right) \pi$ bonding in the case of $(\mathrm{Cl})$ EuTPP and $(\mathrm{Cl}) \mathrm{TmPc}$, respectively) are responsible for appearing and improving of MPXs/MPcXs spin state. The negative contribution is obtained from increasing of mass and specific heat capacity, the spin carrier interactions with nuclear spins (the increasing of macrocycle bonding), and depending on electron configuration, from the $\pi$ dative interaction metal - macrocyclic ligand $\left(\mathrm{N} \rightarrow \operatorname{Tm}\left(f^{12}\right)\right.$ $\pi$ bonding in $(\mathrm{Cl}) \mathrm{TmPc})$.
Finally, an important role in the formation of a porphyrin/phthalocyanine-based paramagnet spin state belongs to antiferromagnetic coupling if the one is possible. MCE of $0.47 \mathrm{~K}$ in $(\mathrm{AcO}) \mathrm{GdPc}$ at $278 \mathrm{~K}$ when the magnetic field is changed from zero to $1 \mathrm{~T}$ decreases almost an order of magnitude by the transition to the sandwich analog, $\mathrm{GdPc}_{2}{ }^{\bullet}$ in which one of two macrocycles has been oxidized. The intramolecular oxidation-activated spin coupling as you could see does not prevail over the intermolecular coupling of two radical spins of the two neighboring molecules. ${ }^{\text {[283] }}$

Porphyrin/phthalocyanine complexes with paramagnetic ions are of particular interest as both SMMs at 0-55 K and paramagnets at the temperatures above the Curie temperature. Studies on introducing magnetic properties into REE porphyrin catalysts to simplify methods deriving solid catalysts and increasing their catalytic activity and recyclability were performed. ${ }^{[310]}$ Since porphyrins/ phthalocyanines and their complexes can selectively accumulate in a tumor of a human body, the tetrapyrrole complexes under consideration promise to expand opportunities of hyperthermia in cancer diagnostics and therapy. Magnetic properties of rare earth porphyrin/phthalocyanine complexes are already used in photosensitizing of active singlet oxygen. ${ }^{[311,312]}$ These ions can be used to accumulate paramagnetic ions that are capable of emitting a signal under the influence of an external magnetic induction in magnetic resonance tomography, ${ }^{[310]}$ e.g., a conjugate of 2-(1'-hexyloxyethyl)-2-divinylpyropheophorbide $a$ with aminophenyl-diethylene-triamine-pentaacetic acid (DTPA) coordinated by gadolinium(III). Although magnetic characteristics of molecular magnetics are on the whole more modest than those of nonorganic magnets, the highest MCE values of the paramagnetic porphyrin/phthalocyanine $3 d /$ REE complexes, as was shown above are comparable with some nanomagnets. It could be employed for cooling in home and industrial refrigerators and other devices. As the environmentally friendly paramagnets, the complexes of this class can replace the toxic compounds used in a vapor-compression cycle. The gadolinium porphyrins/ phthalocyanines contain only a small percentage of expensive gadolinium in comparison with the polycrystalline gadolinium. MPXs/MPcXs-based paramagnets are soluble 
in organic media what allows the formation of nanostructures higher order from their molecules. As solution-processable or easily sublimated, they can be conjugated with inorganic coats, quantum dots, and Ag nanoparticles giving promising nonlinear optical and shift-reagent properties ${ }^{[313]}$ or with ferromagnetic nickel substrate, ultrathin cobalt, and nickel films and a perovskite manganite. ${ }^{[314]}$ However, it is firstly important in a view of the advantages of paramagnets of this class over other ones, we can control their magnetocaloric properties through modifications in a molecule structure. Scientific interest to MPXs/MPcXs-based paramagnets consists in obtaining information about contributions in their magnetocaloric behavior namely in their spin state formation. For this, the temperature depended study of magnetization thermodynamic parameters must be accompanied by all means by specific heat capacity measurements in the temperature/magnetic induction field. Keep in mind that unlike the quantitative determination of the spin multiplicity of such paramagnets at room temperature ${ }^{[315]}$ the studies above resulting in exact magnetocaloric parameters give an only qualitative presentation of spin density redistribution in MPXs/MPcXs-based paramagnets.

\section{Functional Porphyrins as Specific Probes of Surface Morphology, Optical Properties, and Exciton Relaxation Processes for Semiconductor Quantum Dots}

At the moment, it is well-documented that besides their pivotal role on Earth (pigments of life ${ }^{[2,316]}$ ) tetrapyrrolic derivatives are considered and used as powerful tools for various applications, e.g., molecular electronics, artificial light harvesting, and biomedicine..$^{[317-322]}$ They also serve as building blocks for supramolecular nanoassembly. ${ }^{[323-325]}$ In addition, because of the versatile structural, chemical and photophysical properties of tetrapyrrolic derivatives, the application of artificial porphyrin systems is attracting significant attention (being used as molecular probes) for the detection of various chemical species as well as for molecular recognition and sensing. ${ }^{[326-328]}$ Thus, taking into account the recent advances in synthetic protocols, considerable extension in the applications of porphyrin-based or porphyrin-related compounds is expected.

On the other hand, semiconductor nanocrystals (or quantum dots, QDs) hybridized with functional organic dye molecules represent a prominent class of composite nanomaterials. ${ }^{[329-332]}$ Over the past decade the development of these new nanoscale building blocks has intensified as they offer significant advantages in a lot of applications, ${ }^{[333-339]}$ especially for purposes of fluorescent imaging and probing, mainly of cells and tissues. ${ }^{[340]}$ Tunable photophysics, selective solubility, enhanced energy transfer, charge separation, and effective sensitization have all been realized recently with applications in areas ranging from single biomolecule tracking to solid state device fabrication. In the coming years, progress in this area is likely to continue with a number of significant applications realized. Functional organic dye ligands allow for incorporation of QDs into areas where their unique photophysics can be exploited.
Energy and charge transfer between the ligands and QDs also result in enhanced physical properties that can be tuned by the choice of ligand architecture and by varying the specific electronic interactions with chemical entities attached to QD surfaces and interfaces. ${ }^{[341-343]}$ It should be noted in this respect, that the main barrier on the path to develop functional QD-based nanodevices is our limited understanding of QD surfaces, their interaction with surface ligated molecules, and their impact on charge or energy transfer between QDs and attached molecules. As a matter of fact, due to the high surface-to-volume ratio for QDs of various types the physical and chemical properties at the interface play a significant role in determining the overall QD properties. $^{[344]}$ Correspondingly, the QD physical and chemical properties might be tuned and probed specifically via their interaction with functional organic molecules attached to their surface. Experimental and theoretical results show that functionalized organic molecules (being used as specific probes) possess a broad scenario for modifying and studying QD surface morphology, energy states, optical properties, and pathways of exciton relaxation. ${ }^{[344-356]}$

It follows from above considerations that the qualitative and quantitative analysis of surface and interface processes in QD-organic ligand nanoassemblies (which may be tuned by tailoring the surface state energy and by varying the specific electronic interactions with dye molecules attached to QDs) is a key challenge for those working on hybrid nanomaterials and their applications. Calculations predict ${ }^{[357,358]}$ that ligand molecules may cause (depending on their concrete structure and number per QD) surface reconstruction of QD, modify even on a single ligand base the electronic states or the electron-phonon coupling, and the hot carrier relaxation. On the other hand, modern spectral-kinetic methods (including steady-state, time- and spatially resolved spectroscopy in a wide spectral range, detection of single nanoobjects, light scattering measurements, TEM, etc.) present a good background for the study of spectral-kinetic properties of QD-dye nanoassemblies as well as the verification of some theoretical conclusions. ${ }^{[333,342,343,347,348,352,355]}$ Unfortunately, none of the experimental methods allows sufficiently resolved visualization of the structure of the colloidal QD surface and some details of attachment of the dye molecule to it, in order to understand the corresponding structure-spectral-energetic correlations. Thus, a microscopic understanding of the interaction of dye molecule(s) with the QD surface is far from being at hand. Therefore, it is a challenge for experimentalists to reach on a single ligand (namely dye) level a sufficient sophistication of QD surface characterization in order to finally achieve tailoring of the electronic properties on demand.

We attempted to compare calculations and experiments on the level of a single (or at least a few) dye molecules on a QD surface. From the methodological point of view, it means that a functionalized dye molecule attached to a QD surface may be considered as a specific probe for testing the QD surface morphology, optical properties, and energy states in QD-dye nanoassemblies. Our approach includes the so-called self-assembly approach for the formation of QD-dye nanoassemblies. It is based on the attachment of one (or at most few) organic dye molecule since the dye acts as a kind of spectator for efficient and direct optical 

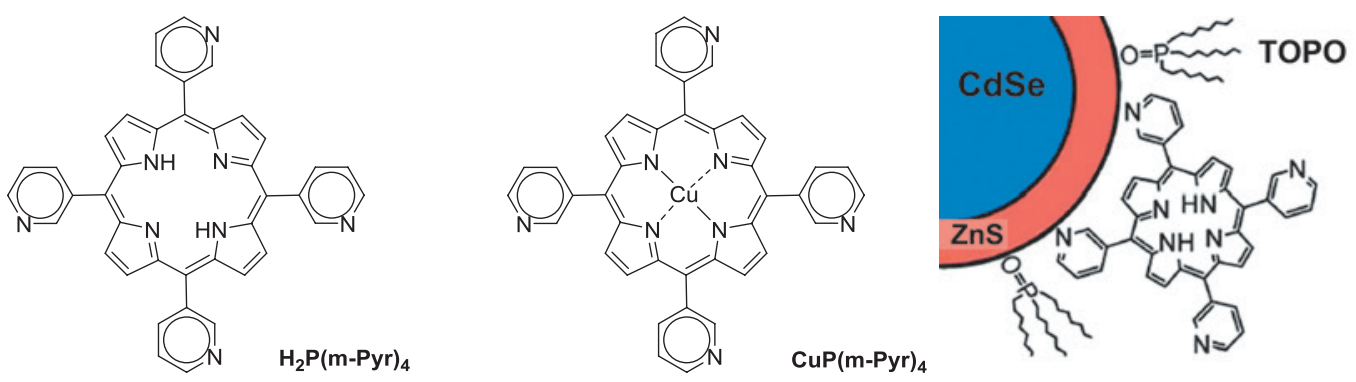

Figure 48. Structures of 5,10,15,20-meso-meta-pyridyl porphyrin, $\mathrm{H}_{2} \mathrm{P}(m \text {-Pyr })_{4}$ and its $\mathrm{Cu}$-complex $\mathrm{CuP}(m \text {-Pyr })_{4}$ as well as schematic presentation of a QD-porphyrin nanoassembly based on CdSe/ZnS QD and $\mathrm{H}_{2} \mathrm{P}(m \text {-Pyr })_{4}$. The capping ligands tri- $n$-octyl phosphine oxide (TOPO) are also shown. The formation of CdSe/ZnS-porphyrin nanoassemblies is realized via two-fold coordination of nitrogens of the porphyrin meso-pyridyl rings with $\mathrm{Zn}$ atoms of the QD ZnS shell ("key-lock" principle).

access. Such an approach was used by us recently for TOPOand amine-capped CdSe/ZnS QDs being attached via coordination interactions with functionalized organic molecules (tetra-pyridylporphyrins and perylene bisimides) in toluene and other non-polar solvents. ${ }^{[333,352-354,359,360]}$

This mini-review highlights our recent results in the use of functionalized tetrapyrrolic macrocycles for surface decoration of QDs of various nature thus playing the role of specific molecular probes in the experimental and theoretical analysis of some structural properties of QD themselves as well as formation principles, interface/surface phenomena, energy states, and competitive relaxation pathways in QD-porphyrin nanoassemblies. We chose porphyrins as molecular probes since their attachment to the QD surface was uniquely identified for a whole bunch of porphyrins and QDs of various sizes. ${ }^{[320,323,333,339,342,351-354]}$ Moreover, porphyrins allow due to their intrinsic nature for a wide range of functionalization (including the number and positions of functional side groups as well as their charge) and modification of the central ion. ${ }^{[320,323,333]}$ Basic results of this study may be useful in the directed formation of new nanoscale organic-inorganic building blocks and offer significant advantages in a wide areas of applications.

Colloidal TOPO (n-trioctylphosphine oxide) caped $\mathrm{CdSe} / \mathrm{ZnS}$ QDs (diameter variation $\mathrm{d}_{\mathrm{CdSe}}=2.1 \div 5.2 \mathrm{~nm}$, with 2 capping $\mathrm{ZnS}$ monolayers) were obtained from Evident Technologies, Inc, Troy, NY, USA. Tetraphenyl porphyrins with different number of meso-phenyl rings, $\mathrm{H}_{2} \mathrm{P}(\mathrm{Pyr})_{n}$ $(\mathrm{n}=1 \div 4)$ were used for the controllable formation of QDporphyrin nanoassemblies via a coordination "key-lock" principle during titration experiments. The synthesis of pyridyl substituted porphyrins, spectral details, and the titration procedure were described by us earlier. ${ }^{[323,342,361,362]}$ The structure of 5,10,15,20-meso-meta-pyridyl substituted porphyrin, $\mathrm{H}_{2} \mathrm{P}(m-\mathrm{Pyr})_{4}$, as well as a schematic representation (not on scale) of a QD-porphyrin nanoassembly and mutual arrangement of a $\mathrm{H}_{2} \mathrm{P}$ molecule with respect to the QD surface are shown in Figure 48.

Toluene, methylcyclohexane (Aldrich HPLC grade) were used without further purification. Steady-state and time-resolved fluorescence measurements were carried out in quartz optical cuvettes (Hellma QS 27 111, path length $1 \mathrm{~cm})$. The optical density of the solutions was OD $\leq 0.15$ in order to avoid reabsorption effects. Steady-state UV-Vis absorption spectra were recorded with a Shimadzu 3001
UV/Vis or a Cary-500 M Varian spectrometer. Emission spectra were measured with a Shimadzu RF-5001PC. Time-resolved PL measurements for QDs and porphyrin molecules in solutions (time correlated single photon counting mode, TCSPC) were performed using a home-made experimental setup described by us earlier ${ }^{[342,352,354]}$ where instrumentation details as well as the analysis of PL decay curves are presented.

As noted above ${ }^{[320,323,331,333,337,341-347,352,353,358,360]}$ and discussed in recent publications, ${ }^{[363-365]}$ the detailed evaluation of the structural and energetic properties of QD-dye nanoassemblies as well a thorough description of relaxation processes in these complexes presents itself as a rather complicated task which may be solved using both a variety of modern experimental techniques and corresponding theoretical approaches. Moreover, the kind and concentration of organic dyes in nanoassemblies may control the energies of surface states and their influence on the optical properties of QDs which is important for the analysis of photoluminescence (PL) quenching mechanisms for QDs. It means, that in certain cases functionalized organic molecules (like porphyrins in our study) being attached to a QD surface may be considered also as specific markers for elucidation of some structural and/or spectral and energetic QD properties which may not be obtained by direct measurements. Keeping this idea in mind, some important results obtained from studying the interaction of porphyrin-based ligands (as probes) with QDs of two types are reviewed below.

\section{Spatial Displacement of the Porphyrin Macrocycle on the QD Surface}

During titration experiments our approach was based on the replacement of one or at most a few capping TOPO molecules on a CdSe/ZnS QD surface by exactly one porphyrin molecule since the porphyrin molecule is used as a kind of spectator for efficient and direct optical access. Typically, the increase of the molar ratio $x=\left[C_{\mathrm{Porph}}\right] /\left[C_{\mathrm{OD}}\right]$ leads to QD PL quenching manifesting itself in a relative PL intensity decrease and decay shortening. Noteworthy, in all cases the QD absorption spectra of the QD-porphyrin nanoassemblies are essentially a linear combination of the corresponding absorption spectra of QDs and porphyrin ligands, while the QD PL is considerably quenched upon titration by meso-pyridyl substituted porphyrins. Common spectral- 

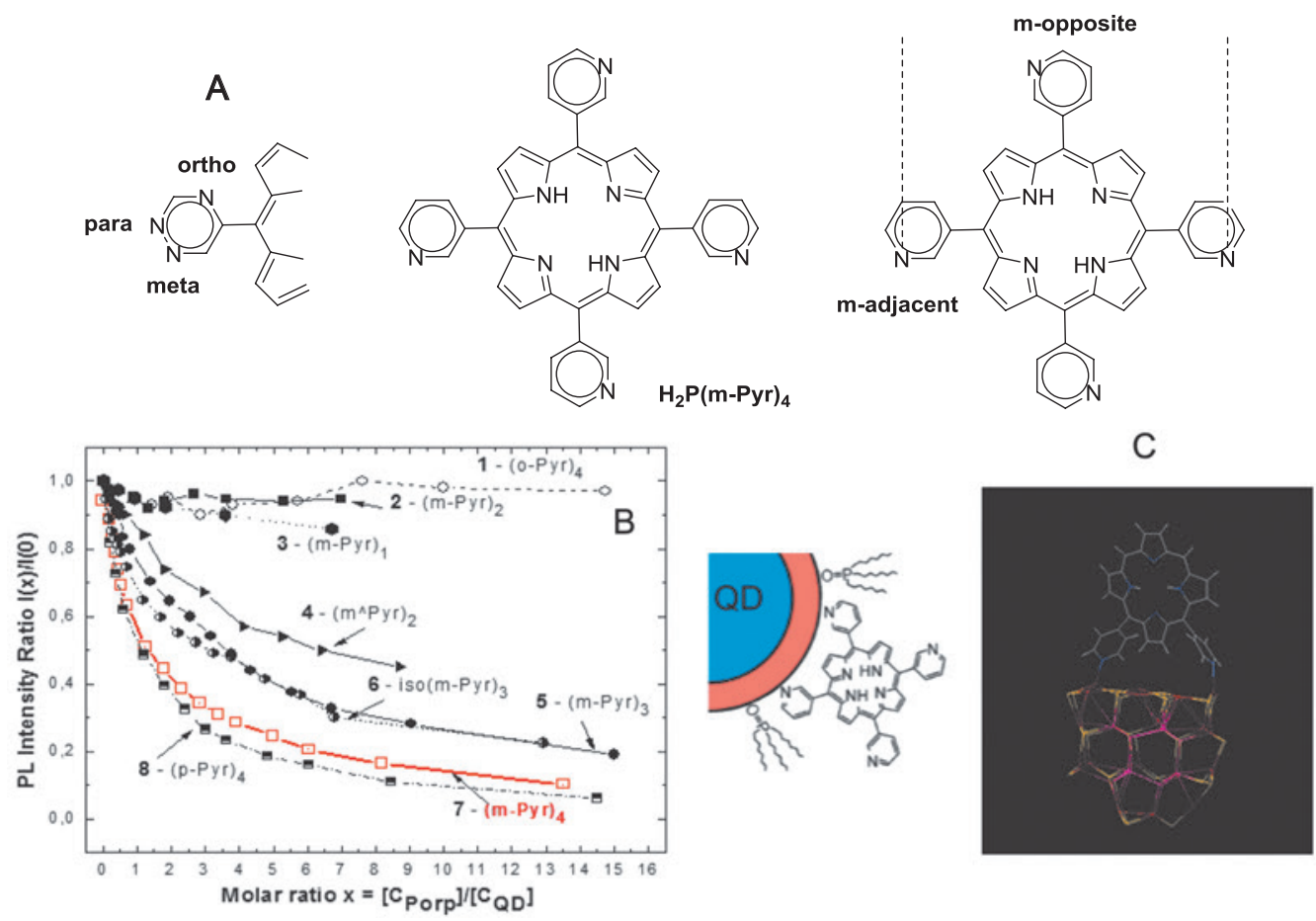

Figure 49. Chemical structures, abbreviation and positions of nitrogen for meso-pyridyl substituted porphyrins as probes. B: Relative photoluminescence intensity dependence $I(x) / I(0)$ for CdSe $/ \mathrm{ZnS}$ QD $\left(\mathrm{d}_{\mathrm{CdSe}}=2.5 \mathrm{~nm}, 2 \mathrm{ZnS}\right.$ monolayers, $\left.C_{\mathrm{QD}}=4 \cdot 10^{-7} \mathrm{M}\right)$ on the molar ratio $\left(x=\left[C_{\mathrm{Porph}}\right] /\left[C_{\mathrm{QD}}\right]\right)$ for various porphyrin structures. C: Relative displacement of the porphyrin macrocycle with respect to the QD surface based on quantum chemical calculations ${ }^{[348]}$ (optimization: $\mathrm{Cd}_{33} \mathrm{Se}_{33}+\mathrm{H}_{2} \mathrm{P}\left(m^{\wedge} \mathrm{Pyr}\right)_{2}$ HyperChem 7.0, DFT).

kinetic features of these effects were described and analyzed by us in detail earlier. ${ }^{[323,342,351,353]}$ Here, we would like to evaluate the spatial displacement of the porphyrin macrocycle with respect to the QD surface in order to use this information for the further quantitative studies of the energy relaxation pathways in nanoassemblies. The comparative analysis is based on the results of titration experiments for $\mathrm{CdSe} / \mathrm{ZnS}$ QD with fixed parameters $\left(\mathrm{d}_{\mathrm{CdSe}}=2.5 \mathrm{~nm}\right.$, $\mathrm{ZnS}$ monolayers $\mathrm{n}=2, C_{\mathrm{OD}}=4 \cdot 10^{-7} \mathrm{M}$ ) but using as probes, meso-pyridyl substituted porphyrins $\mathrm{H}_{2} \mathrm{P}(\mathrm{mPyr})_{n}$ with varying properties such as: ${ }^{[351]}$ (i) the number of pyridyl-rings from 1 to 4 including the two variants for the displacement ( $m$-Pyr $)_{2}$ - and $\left(m^{\wedge} \mathrm{Pyr}\right)_{2}-$, where pyridyl rings are opposite or adjacent to each other, respectively; (ii) for the four-fold pyridyl substituted $\mathrm{H}_{2} \mathrm{P}$ the type of $\mathrm{N}$-substitution within the pyridyl ring from meta- $(m)$ to ortho- $(o)$ and para- $(p)$ $\mathrm{N}$ positions (Figure 49A).

As can be seen from Figure 49B $\mathrm{H}_{2} \mathrm{P}(o-\mathrm{Pyr})_{4}$ and $\mathrm{H}_{2} \mathrm{P}(m-\mathrm{Pyr})_{2}$ porphyrins almost do not quench the QD PL (curves 1,2), whereas the quenching is strongest for $\mathrm{H}_{2} \mathrm{P}(p \text {-Pyr })_{4}$ and $\mathrm{H}_{2} \mathrm{P}(m-\mathrm{Pyr})_{4}$ ones. The absence of QD $\mathrm{PL}$ quenching in the case of $\mathrm{H}_{2} \mathrm{P}(o-\mathrm{Pyr})_{4}$ and $\mathrm{H}_{2} \mathrm{P}(m-\mathrm{Pyr})_{2}$ porphyrins indicates that despite the flexibility of pyridyl rings with respect to the $\mathrm{H}_{2} \mathrm{P}$ macrocycle, a parallel orientation of the macrocycle with respect to the QD surface is obviously not favored. On the other hand, an orientation of the porphyrin macrocycle nearly perpendicular to the QD surface would be easily possible for meta and para $\mathrm{N}$ positions in pyridyl substituted porphyrins (Figure 49A). Theoretical calculations of the optimized geometry for $\mathrm{CdSe} / \mathrm{ZnS} \mathrm{QD}-\mathrm{H}_{2} \mathrm{P}\left(m^{\wedge} \mathrm{Pyr}\right)$, nanoassemblies (having adjacent meta-pyridyl rings) also predict presumably per- pendicular displacement of the porphyrin macrocycle with respect to the QD surface (Figure 49C) ${ }^{[348]}$ Correspondingly, for further analysis we use experimental and theoretical results obtained for nanoassemblies based on $\mathrm{CdSe} / \mathrm{ZnS}$ QDs and 5,10,15,20-meso-meta-pyridyl porphyrin, $\mathrm{H}_{2} \mathrm{P}(m \text {-Pyr })_{4}$, as far as for this molecular ligand the QD PL quenching is the strongest (curve 7 in Figure 49B) and the displacement on the QD surface is well-established.

\section{Quantitative Testing Non-Radiative Energy Transfer $Q D \rightarrow$ Porphyrin and Photoinduced Charge Transfer}

It follows from the experimental results (Figure 49B) that the formation of QD-porphyrin nanoassemblies is accompanied by QD PL quenching. It reflects the increase of nonradiative relaxation channels in the excited states of QD while the fluorescence of the attached porphyrin is not quenched. From the basic considerations, ${ }^{[319,320,324,333,336,346,348]}$ these features may be connected with non-radiative energy transfer of Foerster type ${ }^{[366]}$ (FRET) QD $\rightarrow$ porphyrin or with photoinduced charge transfer (CT) processes. Correspondingly, we used various porphyrin macrocycles as probes for the interaction with CdSe/ZnS QDs in order to quantitatively check the role of the above processes in the non-radiative deactivation of QD excited states in nanoassemblies. Usually in the FRET case, the direct verification of the energy transfer process as the reason of PL quenching is the comparison of the experimental values of FRET efficiencies via the donor (QD) PL quenching and the sensitization of the acceptor (porphyrin) fluorescence. Such a quantitative comparison was carried out by us for QD- $\mathrm{H}_{2} \mathrm{P}(m \text {-Pyr })_{4}$ nanoassemblies. ${ }^{[351,353]}$ Figure 50A shows the comparison of $\mathrm{CdSe} / \mathrm{ZnS}$ QD PL quenching data 

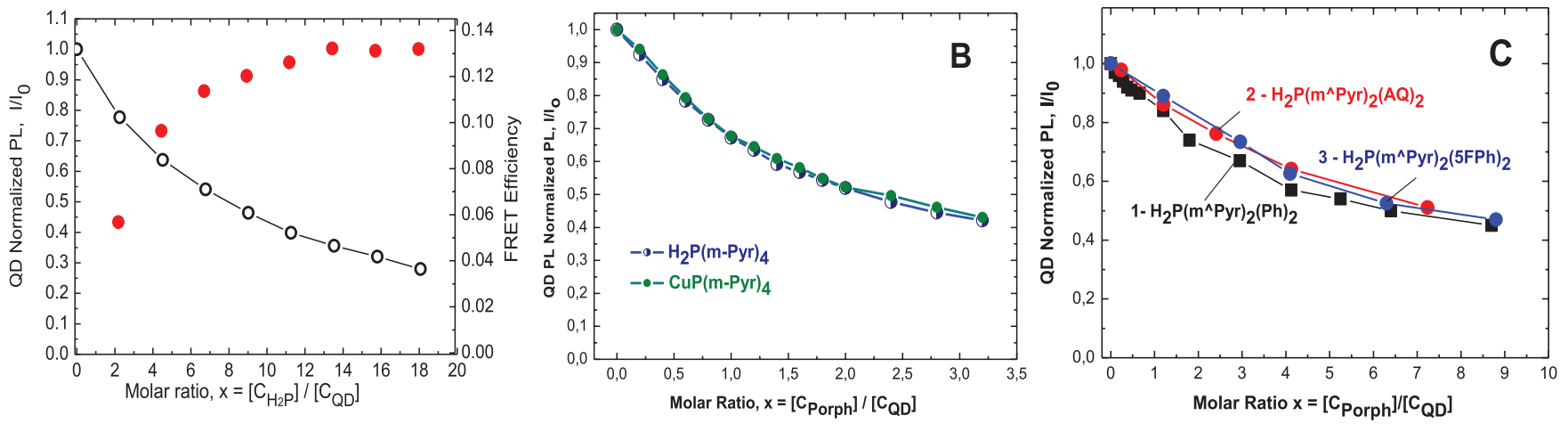

Figure 50 Photoluminescence quenching of TOPO-capped CdSe/ZnS QD ( $\mathrm{d}_{\mathrm{CdSe}}=3.0 \mathrm{~nm}, 2 \mathrm{ZnS}$ monolayers) by various porphyrin molecules in QD-Porphyrin nanoassemblies with the same displacement on QD surface as function of the molar ratio $x=\left[C_{\mathrm{Porp}}\right] /\left[C_{\mathrm{QD}}\right]$ in toluene at $295 \mathrm{~K}$. A: Comparison of QD PL quenching $I / I_{0}$ (2, left scale) and FRET efficiency $\Phi_{\text {FRET }}$ values (1, red points, right scale) estimated via the porphyrin fluorescence sensitization for QD- $\mathrm{H}_{2} \mathrm{P}(m-\mathrm{Pyr})_{4}$ nanoassemblies. B: Comparison of QD PL quenching $I / I_{0}$ by two porphyrins, $\mathrm{H}_{2}(m \text {-Pyr })_{4}$ and $\mathrm{CuP}(m-\mathrm{Pyr})_{4}$ possessing different spectral overlaps $\left[\mathrm{J}(\mathrm{v})=3.65 \cdot 10^{-14} \mathrm{~cm}^{6} \cdot \mathrm{M}^{-1}\right.$ and $\mathrm{J}(v)=7.1 \cdot 10^{-14} \mathrm{~cm}^{6} \cdot \mathrm{M}^{-1}$, correspondingly $\left.{ }^{374}\right]$ with the QD PL band. C: Comparison of QD PL quenching $I / I_{0}$ by porphyrins without electron accepting groups, $\mathrm{H}_{2} \mathrm{P}\left(m^{\wedge} \mathrm{Pyr}\right)_{2}(\mathrm{Ph})_{2}$, and those having side strong electron acceptors: ${ }^{[367]}$ anthraquinone, $\mathrm{H}_{2} \mathrm{P}\left(m^{\wedge} \mathrm{Pyr}\right)_{2}(\mathrm{AQ})_{2}$, and pentafluorinated phenyls, $\mathrm{H}_{2} \mathrm{P}\left(m^{\wedge} \mathrm{Pyr}\right)_{2}(5 \mathrm{FPh})_{2}$.

and FRET efficiency values $\mathrm{F}_{\mathrm{FRET}}$ found via the porphyrin $\mathrm{H}_{2} \mathrm{P}(m-\mathrm{Pyr})_{4}$ sensitization at every titration step. It follows from these results that for every molar ratio $x, \mathrm{~F}_{\mathrm{FRET}}$ values (found from the acceptor sensitization data, red points 1, right scale) do not exceed $\sim 6 \div 14 \%$ and are significantly smaller than those estimated from the QD PL quenching efficiency $\left[\mathrm{F}_{\mathrm{QUENCH}}=1-I / I_{0} \sim 50,80 \%\right.$, curve 2 , left scale $]$. Our calculations, carried out in the frame of the Foerster FRET model for $\mathrm{QD}-\mathrm{H}_{2} \mathrm{P}(m \text {-Pyr })_{4}$ nanoassemblies with a well-defined geometry (Figures 48 and 49C) showed, ${ }^{[342,348,351,353]}$ that if FRET is the only reason of QD PL quenching in nanoassemblies, already upon attachment of one single acceptor molecule, the maximal achievable FRET efficiency may reached up to $60-70 \%$ for various $\mathrm{CdSe} / \mathrm{ZnS}$ QDs $\left(\mathrm{d}_{\mathrm{CdSe}}=3 \mathrm{~nm}, 2 \mathrm{ZnS}\right.$ monolayers) and FRET should be realized within a time scale $>10 \div 25 \mathrm{~ns}$. In contrast, for QD- $\mathrm{H}_{2} \mathrm{P}(m-\mathrm{Pyr})_{4}$ nanoassemblies the QD PL mean decay times $<\mathrm{t}>\approx 10 \div 13$ ns containing presumably shorter components. ${ }^{[342,348,351,353]}$

Moreover, Figure 50B shows that the QD PL quenching effect is the same for QD-porphyrin nanoassemblies of the same geometry but having porphyrins (energy acceptors $), \mathrm{H}_{2} \mathrm{P}(m-\mathrm{Pyr})_{4}$ and $\mathrm{CuP}(m-\mathrm{Pyr})_{4}$, with different absorption spectra and thus possessing essentially different spectral overlaps with the QD PL band. If FRET would be the dominant quenching process in the given nanoassemblies, the corresponding quenching curves would have different slopes according to the FRET theory. ${ }^{[366]}$ Thus, the analysis of these results leads to the basic conclusion that in toluene at ambient temperature the energy transfer QD $\rightarrow$
Porphyrin cannot be the main reason of QD PL quenching in nanoassemblies.

Finally, the data presented in Figure 50C show also that the observed QD PL quenching in QD-porphyrin nanoassemblies does not depend on electron-accepting properties of the porphyrin ligand (due to the existence of additional pentafluor-substituted meso-phenyl rings and anthraquinone substituents). In addition, the fluorescence parameters (quantum efficiency and decay) of porphyrin ligands in joint nanoassemblies practically do not change with respect to those measured for individual ligands. ${ }^{[367]}$ These results are in contrast with those obtained by us for multiporphyrin complexes (triads) containing porphyrin ligands on electron-accepting groups where the photoinduced electron transfer manifested itself in a fluorescence strong quenching for both electron and acceptor moieties and was verified by femtosecond pump-probe experiments. ${ }^{[323]}$ Correspondingly, one may conclude that photoinduced electron transfer QD $\rightarrow$ Porphyrin with participation of molecular orbitals of the porphyrin macrocycle is probable low and cannot be considered as a reason for QD PL quenching in QD-porphyrin nanoassemblies.

\section{Electron Tunneling in Conditions of Quantum Confinement and Creation of New Surface Trap States}

The experimental findings and theoretical estimations presented above show that besides FRET some other competitive non-FRET processes may be responsible

Table 5. Mean $<K_{\mathrm{sv}}(x)>$ values and PL quenching rate constants $k_{q}$ for CdSe/ZnS QDs

\begin{tabular}{lcccc}
\hline \multicolumn{1}{c}{$\mathrm{d}_{\mathrm{QD}}, \mathrm{nm}$} & 4.3 & 5.2 & 6.3 & 7.4 \\
\hline$<K_{\mathrm{SV}}(x)>$ & $0.65 \pm 0.10$ & $0.115 \pm 0.015$ & $0.055 \pm 0.007$ & $0.020 \pm 0.003$ \\
$k_{\mathrm{q}}, \mathrm{ns}^{-1}$ & $0.041 \pm 0.016$ & $0.0057 \pm 0.0023$ & $0.0027 \pm 0.0016$ & $0.0015 \pm 0.0009$ \\
\hline
\end{tabular}

Note: In all cases QD PL quenching in QD-Porphyrin nanoassemblies is caused by to at least two reasons (small FRET and dominant non-FRET). FRET efficiency does not exceed $\sim 10-14 \%$ over the total titration range, and becomes sequentially lower with increasing QD size. $\left\langle K_{\text {SV }}(x)>\right.$

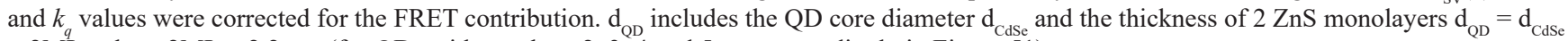
$+2 \mathrm{ML}$, where $2 \mathrm{ML}=2.2 \mathrm{~nm}$ (for QDs with numbers 2, 3, 4 and 5, correspondingly in Figure 51). 


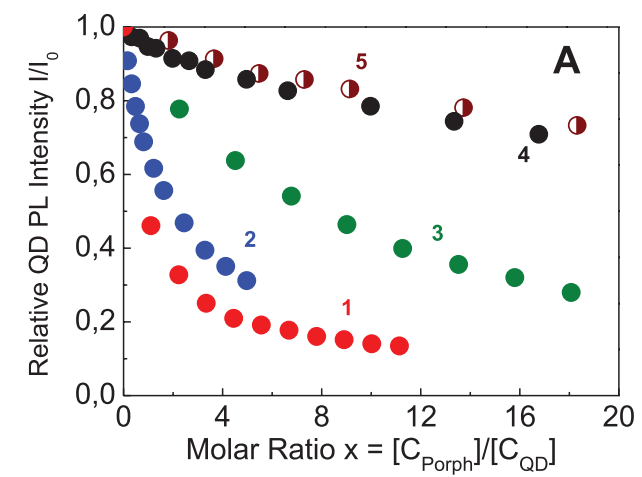

Figure 51. QD PL quenching $I(x) / I_{0}$ for TOPO capped $\mathrm{CdSe} / \mathrm{ZnS}$ QDs of various sizes upon titration by $\mathrm{H}_{2} \mathrm{P}(m-\mathrm{Pyr})_{4}$ as function of the molar ratio $x=\left[C_{\mathrm{Porph}}\right] /\left[C_{\mathrm{QD}}\right]$ in toluene at $295 \mathrm{~K}$ : $1-\mathrm{d}_{\mathrm{CdSe}}=3.5 \mathrm{~nm} / 0 \mathrm{ML}$ (monolayers) $\mathrm{ZnS} ; 2-\mathrm{d}_{\mathrm{CdSe}}=2.1 \mathrm{~nm} / 2 \mathrm{ML}$ $\mathrm{ZnS} ; 3-\mathrm{d}_{\mathrm{CdSe}}=3.0 \mathrm{~nm} / 2 \mathrm{ML} \mathrm{ZnS} ; 4-\mathrm{d}_{\mathrm{CdSe}}=4.1 \mathrm{~nm} / 2 \mathrm{ML} \mathrm{ZnS}$; $5-\mathrm{d}_{\text {CdSe }}=5.2 \mathrm{~nm} / 2 \mathrm{ML} \mathrm{ZnS}$.

for the observed QD PL quenching upon attachment of functionalized porphyrin molecules to the QD surface. In this respect it should be noted that due to quantum confinement $^{[332,333,338,341,344,368]}$ the exciton in a QD is highly sensitive to local charges or distortions of the charge distribution. The excitonic wave function probes these local charges effectively because of its spatial extension even beyond the QD core and -if present- ZnS capping layers. Following these ideas, we proposed that this non-FRET mechanism for QD PL quenching in nanoassemblies may be connected with the extension of the wave function of the exciton beyond the QD core.

In our study, the experimental background was the comparative analysis of QD PL quenching by one type of porphyrin $\mathrm{H}_{2} \mathrm{P}(m \text {-Pyr })_{4}$ molecule (as a best quencher among other porphyrins, see Figure 49B) being attached by the same manner to QDs of different sizes. ${ }^{[352,353]}$ Clearly, porphyrin molecules in this case might act as specific probes of the quantum confinement effect. Typical QD PL quenching curves are shown in Figure 51 together with Table 5 containing experimental PL quenching parameters.

It follows from Figure 51 that at the same molar ratios $x$ the QD PL quenching is more effective for smaller QDs. The appropriate analysis of the QD PL quenching as a function of the number of porphyrin molecules per QD for various QD sizes was carried out using the modified Stern-Volmer formalism. ${ }^{[352,353]}$ As a result, the Stern-Volmer parameters $K(x)$ determined experimentally for every QD were presented as $K(x)=k_{q}(x) \cdot \tau_{0}$, where $k_{q}$ corresponds to the total quenching rate induced by the number of attached quencher molecules and $\tau_{0}$ is the intrinsic PL lifetime of the QD in absence of porphyrin molecules. Data collected in Table 5 show that for CdSe/ZnS QDs having $2 \mathrm{ZnS}$ monolayers, $k_{q}$ values decay considerably upon increase of the QD core diameter. In addition, for CdSe QDs without a $\mathrm{ZnS}$ shell a much stronger QD PL quenching is found compared to that obtained for the respective core-shell analogue (curve 1, Figure 51).

As far as the electronic structure and properties of attached $\mathrm{H}_{2} \mathrm{P}(m \text {-Pyr) })_{4}$ porphyrin (probe) are the same in these experiments the observed QD PL quenching in QD- porphyrin nanoassemblies with various QDs has to be related with interface phenomena. From a physico-chemical background it means that upon interaction of the attached $\mathrm{H}_{2} \mathrm{P}(m \text {-Pyr })_{4}$ molecule with the QD surface, the electron wave function may be locally modified forming a surface local state capable to trap the electron of the photogenerated electron-hole pair. As in any related quantum mechanical problems the wave function of the delocalized electron may extend beyond the imposed barrier or, in other words, the corresponding particle may tunnel through the barrier. With respect to QDs, quantum confinement of the excitonic wave function in its most simple version confines the wave function of the exciton (electron-hole pair) to a spherical potential box. ${ }^{[369]}$ Thus, a general scheme of the QDporphyrin interaction and QD PL quenching model under the conditions of quantum confinement with the electron wave function at the outer interface of the QD may be presented schematically as shown in Figure 52A. It implies that the electron of the photogenerated electron-hole pair becomes partly localized in a volume element in the vicinity of the attachment site. ${ }^{[352,353]}$ Consequently, upon porphyrin attachment on the QD surface, specific trap states are formed giving rise to a trapping of the delocalized electron.

Taking into account this model the observed size dependence of the PL quenching (as expressed by the quenching rates $k_{q}$ ) was directly compared with the probability density $\Psi^{2}(r)$ calculated from the quantumconfined $s$-type electronic wave function $\Psi$ for a $1 s$ electron using the Schrödinger equation (simplified particle-in-a-box model in the effective-mass approximation for a spherical core/shell QD). ${ }^{[352,353]}$ The results of such a comparison are presented in Figure 52B. A remarkable coincidence is seen between experimental values $k_{q}$ (symbols) and theoretical calculations $\Psi^{2}(r)$ (lines). On the basis of these results we conclude that one point-like charge density perturbation caused by the organic linker group of the porphyrin at the QD interface forces the electron of the delocalized exciton of the QD to become localized. In fact, the dependence of QD PL quenching induced by even only one single porphyrin molecule on the QD surface clearly resembles the tunneling of an electron (through the $\mathrm{ZnS}$ barrier) to the outer interface of the QD. Such tunneling is followed by the (self-) localization of the electron. This corresponds to the creation or modification of trap states in the semiconductor band gap. Such traps might be subject to non-radiative channels $e . g$. via enhanced electron-phonon coupling.

Summarizing, one may conclude that non-FRET quenching in QD-Porphyrin nanoassemblies is related to exchange and/or depletion of TOPO ligands by the respective porphyrin molecules followed up by creating more and even new quenching states according to the amplitude of the tunnelling ("leaking") excitonic wave function.

\section{Low Temperature Transformation of QD Capping TOPO Ligand Shell Caused by Attached Porphyrins}

It is well-known that the elucidation of exciton relaxation pathways for QDs alone and QD-dye nanoassemblies upon temperature lowering is a necessary step for the understanding the photophysics and photochemistry of quantumconfined nanoassemblies. Working in this direction we found 

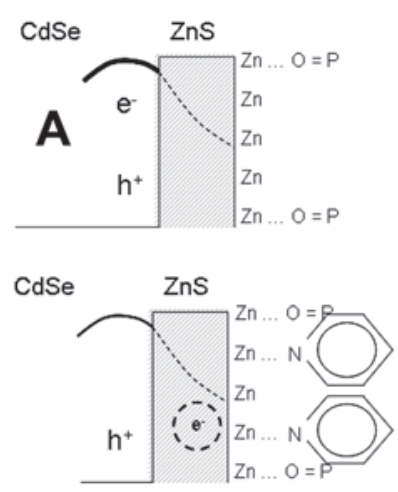

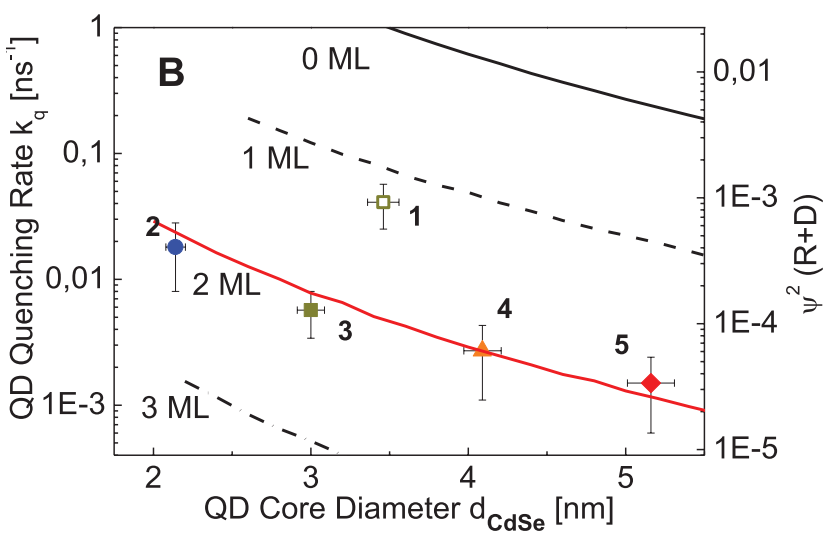

Figure 52. Basic scheme of the QD PL quenching model and comparison of experimental results and theoretical calculations for QD$\mathrm{H}_{2} \mathrm{P}(m \text {-Pyr })_{4}$ nanoassemblies. (A): Scheme of the QD-porphyrin interaction and PL quenching model: Upon excitation, an electronhole pair (exciton) is created in the CdSe core of the QD. In the case of finite barriers, electron e- and hole $\mathrm{h}+$ are subject to tunnelling from the core through the $\mathrm{ZnS}$ shell into the environment (QD surface). Because of the presence of a pyridyl-coordinated porphyrin molecule, the electron becomes partly localized in a volume element in the vicinity of the attachment site. On the QD surface, specific trap states are formed giving rise to the trapping of the delocalized electron. It means that charged QD remains dark. (B): Comparison of the experimentally determined quenching rate constants $k_{q}$ (symbols with error bars, left axis) and calculated probability density functions $\Psi^{2}(r=R+D)$ (lines, right axis) of a $1 s$ electron at the outer interface $(r=R+D)$ between the $\mathrm{ZnS}$ shell and the environment as a function of the QD radius $\mathrm{R}\left(\mathrm{QD}\right.$ core diameter $\left.\mathrm{d}_{\mathrm{CdSe}}=2 R\right)$ and $\mathrm{ZnS}$ shell thickness $D$. The QD PL quenching rate for various CdSe/ZnS QDs is presented as $k_{q}(r)=C \Psi^{2}(r)$. The constant $\mathrm{C}$ was adjusted with respect to $\Psi^{2}(R+D)$ to fit the experimental value at $\mathrm{d}_{\text {CdSe }}=4.1 \mathrm{~nm}$, $\mathrm{n}_{\mathrm{ZnS}}=2$. All theoretical lines correspond to calculations of $\Psi^{2}(R)$ with the same proportionality constant C. More details on the simulation constraints are found elsewhere. ${ }^{[370]}$

that upon temperature lowering (293 K $\rightarrow 77 \mathrm{~K}$ ) for QDs and QD-porphyrin nanoassemblies dissolved in typical liquid solvents (in contrast to rigid matrixes such as polymers, resins, or glasses), some specific spectral and energetic effects may take place caused by the so-called "phase transition" of the capping ligand (TOPO or amine) layer. ${ }^{[333,342,354,371,372]}$ Here, we show how porphyrin molecules as probes seem to be useful for the detection of the ligand layer low temperature transformation as well as for the microscopic understanding of the nanoassembly formation (including ligand dynamics) and related mechanisms of QD PL quenching in QD-porphyrin nanoassemblies.

As can be seen from Figure 53A that upon temperature lowering, the maximum of the band-edge absorption band as well as the maximum of PL spectra for TOPO-capped $\mathrm{CdSe} / \mathrm{ZnS}$ QDs are blue shifted accompanied by a PL line width (FWHM) narrowing and intensity rise. ${ }^{[354,371,372]}$ It is also noticeable that the temperature dependences of the respective peak energies for absorption and PL data are non-monotonous upon temperature lowering. Interestingly, this non-monotonous behavior (which is called "kink" for simplicity) is observed for these QDs between 200 and $240 \mathrm{~K}$, far from the glass formation temperatures of the methylcyclohexane/toluene (6:1) mixture (151.6 K). It means that $\mathrm{CdSe} / \mathrm{ZnS}$ QDs undergo a so-called low temperature "phase transition" in the sense, that the "freezing" TOPO ligand shell exerts strain on the $\mathrm{ZnS}$ shell thus creating trap states with reduced PL quantum yields.

Moreover, this phase transition becomes more pronounced for QD-porphyrin nanoassemblies (Figure 53B). In fact, the attachment of $\mathrm{H}_{2} \mathrm{P}(m-\mathrm{Pyr})_{4}$ and molecules to QD surface enhances QD PL decrease at the phase transition temperature (curves 2 and 3, Figure 53B), and the effect is strongest for $\mathrm{CuP}(m-\mathrm{Pyr})_{4}$ (curve 3$)$. On the other hand, tetraphenylporphyrin molecules without meso-pyridyl anchoring rings do not enlarge the QD PL decrease at the "kink" (curve 4) because of self-assembly absence in the latter case.

Thus, the presented results clearly demonstrate that the capping ligand and attached dye molecules noticeably influence the optical properties at ligand controlled phase transitions. The scheme presented in Figure 53C shows qualitatively how the lowest band edge states respond to depletion of the ligand shell either due to geometric constraints or ligand replacement following the porphyrin attachment. The basic conclusion is that the porphyrin molecule removes ligands from (specific) surface sites thus acting as a new "ligand" creating a modified set of new surface states. This explains specifically why non-FRET processes of QD PL quenching upon QD-porphyrin nanoassembly formation are often not exclusively related to energy (FRET) or charge transfer. In this respect, the observed QD PL changes (for QDs alone and QD-porphyrin nanoassemblies) upon temperature lowering (especially for liquid solutions) should be analyzed not only from the common approach (including thermally activated carrier trapping, ${ }^{[374]}$ and/or the electron-phonon coupling decrease ${ }^{[368,375,376]}$ ) but take into account also the influence of surface states formed by ligand attachment and depending on QD surface transformations upon temperature changes.

We use porphyrin molecules as single molecular surface probes and showed experimentally for the first time, that only one porphyrin molecule is capable to change the surface related distribution of near-band-edge or surface state energies. In addition, it follows from quantum-chemical calculations $^{[350,372,373]}$ and the results discussed above that the QD itself may not be presented as homogeneous ball, but as a QD with various facets having their own properties and traps. The gen- 

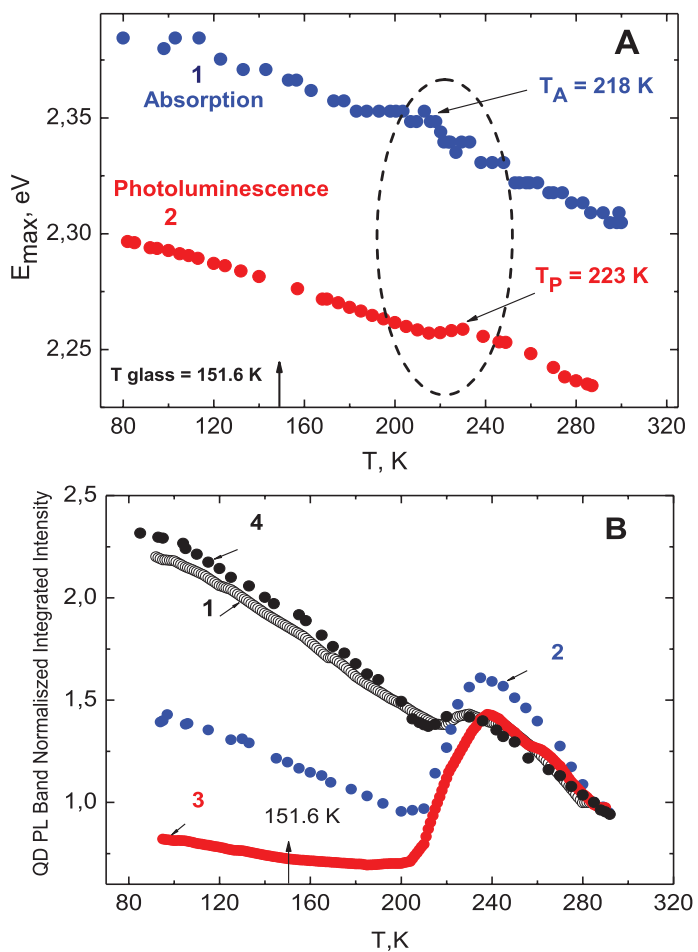

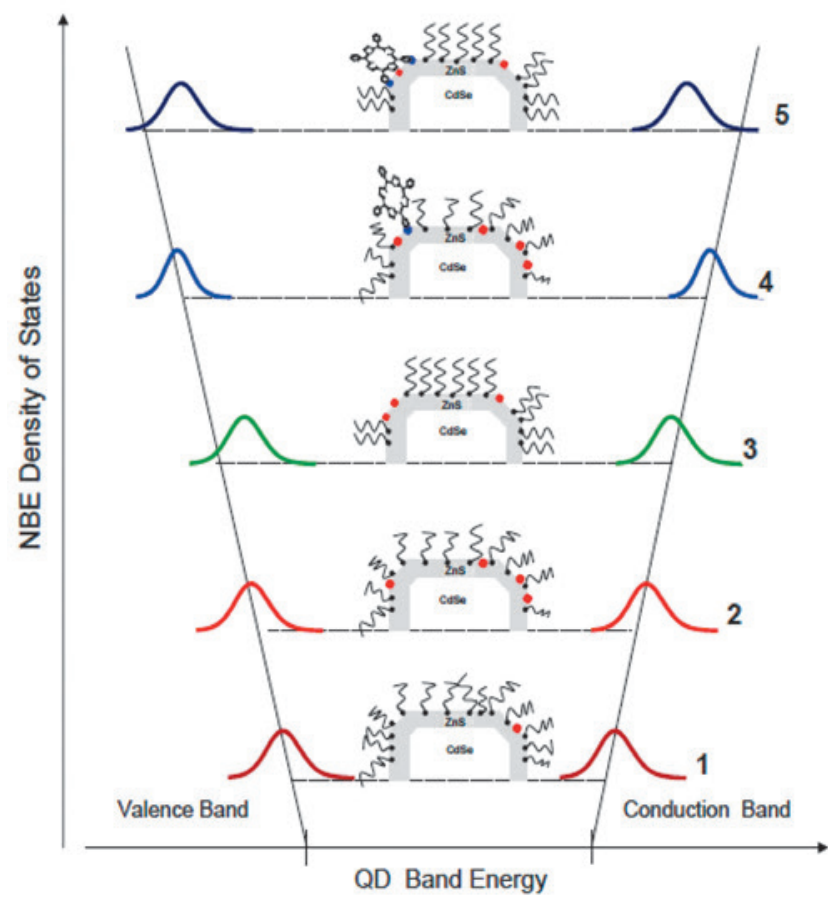

QD Band Energy

Figure 53. Temperature dependence of absorption and PL properties for TOPO capped CdSe $/ \mathrm{ZnS}$ QDs $\left(\mathrm{d}_{\text {Cdse }}=3.0 \mathrm{~nm}, 2 \mathrm{ZnS}\right.$ monolayers) and QD-porphyrin nanoassemblies in methylcyclohexane/toluene (6:1) mixture (A, B) and schematic qualitative presentation for the density of near band energy (NBE) states as a function of QD surface organization $(\mathbf{C})$, based on considerations described in ${ }^{[372,373]}$ ). The solvent glass transition temperature $\left(\mathrm{T}_{\text {glass }}=151.6 \mathrm{~K}\right)$ is indicated by solid arrow. $(\mathbf{A})$ : Temperature dependence of the first absorption peak (1) and PL maximum $\left(2, \lambda_{\text {exc }}=450 \mathrm{~nm}\right)$ for QDs. Dashed ellipse indicates temperature range at which the optical properties change (showing "kink" for absorption at $\mathrm{T}_{\mathrm{A}}=218 \mathrm{~K}$ and for PL at $\mathrm{T}_{\mathrm{P}}=223 \mathrm{~K}$ ). $(\mathbf{B})$ : Temperature dependence of QD PL band normalized integrated intensities $\left(\lambda_{\text {exc }}=450 \mathrm{~nm}\right)$ for nanoassemblies based on TOPO-capped CdSe/ZnS QDs $\left(\mathrm{d}_{\text {Cdse }}=3.0 \mathrm{~nm}, 2 \mathrm{ZnS}\right.$ monolayers) and various porphyrin molecules at a molar ratio $x=\left[C_{\mathrm{H}_{2} \mathrm{p}}\right] /\left[C_{\mathrm{QD}}\right]=1$. All curves are normalized to the integrated band intensity of QD PL at $285 \mathrm{~K}$. Experimental temperature dependences are shown for the same type of TOPO-capped CdSe/ZnS QD under various conditions and attached porphyrins: (1) QDs; (2) QD $+-\mathrm{H}_{2} \mathrm{P}(m-\mathrm{Pyr})_{4}$; (3) QD $+\mathrm{CuP}(m \text {-Pyr) })_{4}$ 4) QD+tetraphenylporphyrin (having no pyridyl rings). (C): (1) complete coverage of QD surface by capping ligand molecules; (2) partial coverage of QD surface by capping ligand molecules caused by steric constraints; (3) "phase transition" of capping layer (e.g. crystallization and ordering) followed by the formation of additional surface states; (4) competing attachment of a porphyrin molecule on QD surface before "phase transition" leading to the removal of few capping ligand molecules due to steric interactions; (5) competing two-point attachment of a porphyrin molecule on QD surface after "phase transition" in the vicinity of surface defects. The phase transition is accompanied by creation of surface traps (red points) due to strain formation and increased ligand coverage.

eral features of our considerations are shown in Scheme 9 with respect to the electronic properties of the QD.

Thus, using a "bottom-up" self-assembly approach for the directed formation of nanoassemblies based on colloidal TOPO-capped semiconductor CdSe/ZnS QDs of various sizes and porphyrin macrocycles in solutions we demonstrated that functionalized pyridyl substituted porphyrin molecules can be considered as extremely sensitive probes for studying the complex interface physics and exciton relaxation processes in QDs. Based on a quantitative experimental analysis of PL properties for QDs and QD-Porphyrin nanoassemblies in combination with corresponding theoretical considerations we tested some surface-mediated processes with participation of porphyrin molecules which determine QD properties and several of the most interesting and potentially useful photophysical phenomena on their surfaces. Some principal moments are the following: i) Presumably perpendicular displacement of the porphyrin macrocycle with respect to the QD surface was proven which allowed further quantitative studies of the energy relaxation pathways in QD- porphyrin nanoassemblies. ii) It was quantitatively shown that FRET QD $\rightarrow$ porphyrin plays a minor role in the overall QD PL quenching, and basic non-FRET processes can be understood in terms of electron tunneling beyond the CdSe core under conditions of quantum confinement; the competition between FRET and non-FRET quenching processes drastically depends on the solvent and ligand properties. iii) The observed non-FRET quenching of QD PL induced by the attachment of one porphyrin molecule can be explained by the presence of a limited number of empty or easily accessible sites on the QD surface followed by ligand competitive exchange; this non-FRET quenching is related to the depletion of capping TOPO ligands by the respective porphyrin molecules followed-up by creating more and even new quenching states according to the amplitude of the tunnelling ("leaking") excitonic wave function followed by the effective population of deep intraband states. iv) Temperature variation $(77 \div 295 \mathrm{~K})$ and related changes in QD absorption and emission reveal drastic changes of the ligand shell structure in a narrow temperature range 

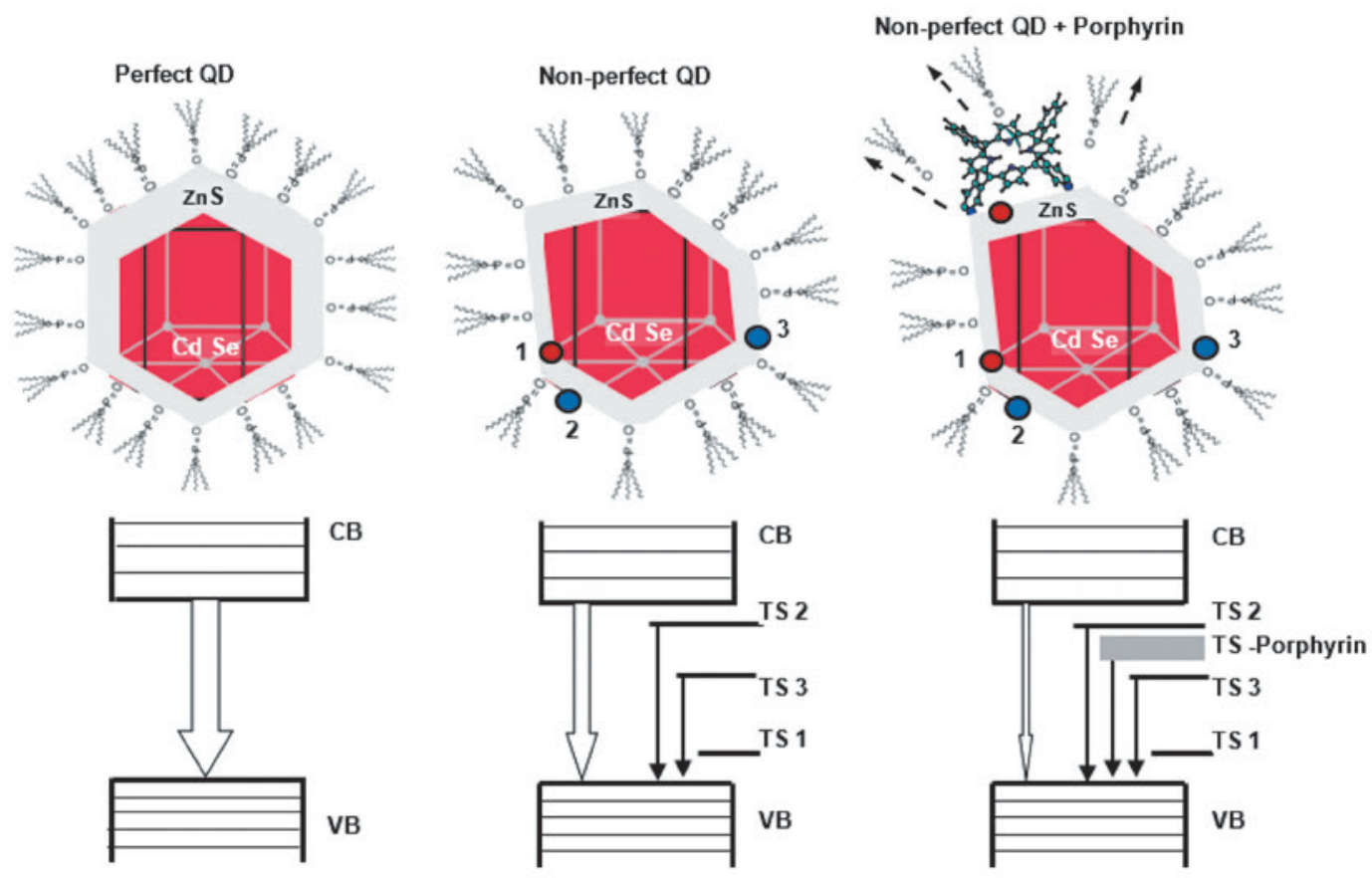

Scheme 9. Schematic presentation of perfect TOPO-capped CdSe/ZnS QD, non-perfect QDs and a "QD-porphyrin" nanoassembly as well as arbitrary excitonic and trap states (TS) and main relaxation pathways. For the nanoassembly an induced ligand detachment is shown by broken arrows. 1 is a hole trap, 2 and 3 are electron traps; "TS-Porphyrin" are trap states caused by the porphyrin attachment.

for the TOPO ligand shell (so-called low-temperature "phase transition" of capping layer); upon attachment of only one or a few porphyrin molecules to the QD surface the corresponding "phase transition" changes considerably the QD PL intensities, energies, and dynamics.

Basically, our findings show the high sensitivity of the optical properties of CdSe/ZnS QDs to their surface chemistry which may be selectively probed and tuned by surface-attached functionalized porphyrin molecules. These findings indicate also that hybrid nanoassemblies formed by semiconductor QDs with stable and tunable photophysically active molecules or dielectric materials may be used as building blocks for optoelectronic sensing solutions, e.g. in structural health monitoring ${ }^{[37,379]}$ and load detection ${ }^{[380]}$ of lightweight structures or as tagging labels for molecular motors ${ }^{[381]}$ paving the way for network-based biocomputation (NBC) or as diagnostic motility assays for environmental biodetection. ${ }^{[382]}$

Finally, it should be mentioned that very recently, we have also realized the presented approach for the studying structural and energetic properties of QD-dye nanoassemblies based on Ag-In-S/ZnS core/shell QDs (AIS/ $\mathrm{ZnS})$ stabilized by glutathione (GSH) as native ligand and attached with 5,10,15,20-(tetra- $N$-methyl-4-pyridyl) porphyrin tetraiodide molecules via Coulomb attraction between negatively charged terminal groups of GSH and positively charged sites of $\mathrm{H}_{2} \mathrm{P}\left(\mathrm{PyMe}^{+}\right)_{4}$ molecules in water. ${ }^{[383-385]}$ We chose porphyrins as molecular probes since their attachment to the QD surface was uniquely identified for a whole bunch of porphyrins and QDs of various sizes. As a result, using functionalized porphyrins as probes in spectral-kinetic measurements as well as the constructed size-proportional quantum chemical atomistic 3D model for $\mathrm{AIS} / \mathrm{ZnS} / \mathrm{GSH}$ QD surface we succeeded to present a realistic scenario of the interactions between porphyrin molecules and GSH-stabilized AIS QDs. Consequently, a very close displacement of the porphyrin molecule to the QD surface leads to a very effective non-radiative deactivation QD excited states. The results presented open the possibility to analyze quantitatively the nature of these quenching processes. The analysis of these results is under the way in our group and will be presented in a forthcoming paper.

\section{Tetrapyrroles and Related Compounds for Photovoltaics}

The fast depletion of fossil fuels reserves, together with the severe environmental impact associated with their combustion, push the development of energy production technologies based on clean and renewable energy sources. ${ }^{[386]}$ Our Sun is an abundant source of free and clean energy and in recent decades the world has been motivated to develop and improve photovoltaic (PV) devices - solar cells - that enable the capture of sunlight and conversion directly to electricity. ${ }^{[387]}$

Although the prospects of crystalline silicon $(\mathrm{c}-\mathrm{Si})$ solar cells are promising, issues, such as their complicated fabrication process, long energy payback time, and use of hazardous chemicals, may open up growth-opportunities for other PV technologies in the future. Third-generation PV technologies, such as dye-sensitized solar cells (DSSC) or perovskite solar cells, have high potential for industrial scale manufacturing, as they can be produced while using established scalable manufacturing methods, such as screen printing, inkjet printing, or slot die coating. ${ }^{[388]}$ 
One of the most important DSSC components is a dye sensitizer, which is an electron pump driven by photos (light) from a DSSC device. This allows the injection of electrons into the semiconductor conduction band and the conversion of visible light photons into electricity. Dyes in DSSC act as absorbers of visible light or sensory photons; thus, the semiconductor that only works in the UV region can work in the visible light region. ${ }^{[389]}$ Since the past decade, great efforts have been focused on the advancement of dye-sensitizers. ${ }^{[390,391]}$

This section focuses on the use of tetrapyrroles as sensitizers in DSSC, the main trends in the design of the molecular structure of such dyes, as well as some practical applications of DSSC in various devices.

\section{DSSC Components}

A schematic representation of a typical Grätzel cell is shown in Figure 54. On the left, an electron transfer scheme is shown taking into account the energy levels in such a system. Under the light, the dye transitions to an excited state and an electron are transferred to the conduction band of the semiconductor (titanium dioxide). The electrode and the counter electrode are placed in a redox electrolyte solution, with the participation of which redox reactions take place, returning the dye to its original state. In practical implementation, the components of the cell are enclosed in a housing made of transparent material, for example, glass or plastic.

Under the influence of light, six main processes can occur in a cell, leading to the generation of an electric current. These processes are represented by equations $(5)-(10)$ :

(5) under the action of light energy, the dye transitions into an excited state, i.e. the transition of the electron to a higher energy level occurs;

(6) an electron is transferred from an excited dye molecule to a semiconductor material, provided that the energy of the corresponding molecular orbital is higher than the energy of the semiconductor conduction band;

(7) due to the reverse transfer of an electron to the dye, charge recombination can occur, without generating an electric current in the circuit;

(8) the dye is reduced to the ground state by the iodide anion (or another ion of the redox pair) dissolved in the electrolyte, which is oxidized to triiodide;

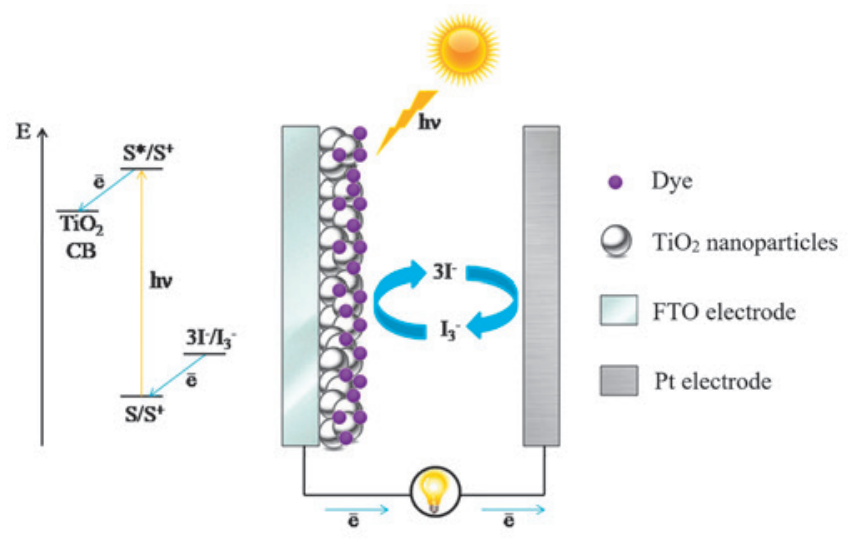

Figure 54. DSSC circuit and energy diagram of electron transfer in DSSC. ${ }^{[392]}$
(9) moving along the electric circuit from the photoanode, the electron reaches the counter electrode, on the surface of which the redox process of reduction of triiodide to iodide takes place;

(10) upon contact of the iodide anion with a semiconductor, its reduction can also occur, which corresponds to another undesirable recombination process. ${ }^{[393]}$

$$
\begin{aligned}
& \mathrm{TiO}_{2}\left|\mathrm{~S}+\mathrm{h} v \rightarrow \mathrm{TiO}_{2}\right| \mathrm{S}^{*} \\
& \mathrm{TiO}_{2}\left|\mathrm{~S}^{*} \rightarrow \mathrm{TiO}_{2}\right| \mathrm{S}^{+}+\mathrm{e}_{\mathrm{cb}} \\
& \mathrm{TiO}_{2}\left|\mathrm{~S}^{+}+\mathrm{e}_{\mathrm{cb}} \rightarrow \mathrm{TiO}_{2}\right| \\
& \mathrm{TiO}_{2}\left|\mathrm{~S}^{+}+3 / 2 \mathrm{I}^{-} \rightarrow \mathrm{TiO}_{2}\right| \mathrm{S}+1 / 2 \mathrm{I}_{3}^{-} \\
& 1 / 2 \mathrm{I}_{3}^{-}+\mathrm{e}_{(\mathrm{Pt})} \rightarrow 3 / 2 \mathrm{I}^{-} \\
& \mathrm{I}_{3}^{-}+2 \mathrm{e}_{\mathrm{cb}} \rightarrow 3 \mathrm{I}^{-}
\end{aligned}
$$

Each solar cell element contributes to the efficiency of photoconversion. ${ }^{[394]}$ As a result, there are many works devoted to the study and modification of various constituent parts of the DSSC. For example, titanium dioxide is most often used as a semiconductor layer, but $\mathrm{ZnO}$ is also widely studied as an alternative, with the use of which it is possible to achieve an efficiency of up to $7.5 \%{ }^{[395]}$ There are also works devoted to the study of other semiconductor materials: $\mathrm{SnO}_{2}, \mathrm{SrTiO}_{3}, \mathrm{Nb}_{2} \mathrm{O}_{5}$ and $\mathrm{WO}_{3} \cdot{ }^{[396]}$ In recent years, more and more attention has been paid to the creation of complex materials and composites that combine the advantages of individual elements. Thus, the $\mathrm{ZnO} / \mathrm{TiO}_{2}$ nanocomposite provided a significant increase in photoconversion as compared to each semiconductor separately. ${ }^{[397]}$ It was also reported about the creation of a binary hybrid photoanode, combining microsized hollow spheres of $\mathrm{ZnO}$ and $\mathrm{SnO}$ nanoparticles, which is a mass ratio of 8:2 provided an almost fourfold increase in efficiency compared to $\mathrm{SnO}$ nanoparticles separately. ${ }^{[398]}$ Similar results were obtained for semiconductor layers obtained from a mixture of $\mathrm{ZnO}$ and $\mathrm{SnO}_{2}$ nanoparticles, which are superior to analogs that include only one of these materials. ${ }^{[399]}$ The same effect was observed for the mixture $\mathrm{ZnO}$ with $\mathrm{Nb}_{2} \mathrm{O}_{5} \cdot{ }^{[400]}$

An equally important cell element is the redox electrolyte. The function of electrolyte is a charge-transfer carrier, and its performance is closely relevant to the viscosity and conductivity of electrolytes which determines the rate and quantity of charge transfer respectively. ${ }^{[401-403]}$ The "gold standard" is still the liquid ionic electrolyte based on redox couple of $\mathrm{I}^{-} / \mathrm{I}_{3}^{-}$due to many advantages in high diffusion rate, low cost, easy to prepare, and is beneficial to the practical application. ${ }^{[404]}$ Despite the high results demonstrated by iodine-containing electrolytes, they have several disadvantages: they absorb light in the blue region of the spectrum, reducing the short-circuit current density $\left(\mathrm{J}_{\mathrm{SC}}\right)$ of the device; $\mathrm{I}_{3}^{-}$has a corrosive effect, in particular on silver or copper elements used in the practical embodiment of solar panels; the redox potential of the $\mathrm{I}^{-} / \mathrm{I}_{3}{ }^{-}$pair limits the maximum open-circuit voltage $\left(\mathrm{V}_{\mathrm{OC}}\right)$ of the device. ${ }^{[405]} \mathrm{As}$ a result, several alternative redox pairs have been proposed, among which the most studied and effective are various pyridinium complexes of cobalt. ${ }^{[406]}$ In addition to the redox 
pair, electrolyte also often contains various additives that increase the performance of the solar cell. ${ }^{[407]}$ An example is the widely used tert-butylpyridine, which, by binding to the titanium dioxide surface, lowers the conduction band edge and also prevents the recombination process (which is described by equation (8) above) by preventing the iodide anion from contacting with the semiconductor surface. Electrolyte modifications are not limited to the components of the solution: the solvent itself can also affect. Recently, ionic liquids are increasingly used as a solvent, which can simultaneously be both a solvent and a carrier of the redox ions of the mediator. The most common ionic liquids are based on alkylimidazoles. ${ }^{[408]}$ In addition to various variations of liquid electrolytes, the use of quasi-solid and solid electrolytes has been described, the main advantage of which is long-term stability. ${ }^{[388]}$ Quasi-solid electrolytes include, first of all, gel electrolytes based on various polymer matrices impregnated with a liquid electrolyte. ${ }^{[409,410]}$ This approach provides excellent stability along with significant efficiencies of up to $10 \% \cdot{ }^{[411]}$ Nevertheless, quasi-solid electrolytes still contain a liquid, which does not completely solve the problem associated with the potential for evaporation during longterm operation. This problem can be solved by creating completely solid polymer electrolytes. For them, the main task is to improve the ability to transfer charge, which is solved by the development of various polymer composites, such as $\left(\mathrm{I}^{-} / \mathrm{I}_{3}{ }^{-}\right)$-incorporated poly(ethylene oxide)/polyaniline. ${ }^{[412]}$

\section{Tetrapyrroles in DSSC}

Of course, the dye can be considered the central element of the DSSC, since it depends on the dye how effective the absorption of light and the injection of an electron into the semiconductor layer will be. Many dyes have been proposed to date, ${ }^{[413-415]}$ including those based on ruthenium complexes, ${ }^{[416]}$ oligothiophenes, ${ }^{[417,418]}$ triarylamines, ${ }^{[419,420]}$ squarains, ${ }^{[421-424]}$ various natural dye, ${ }^{[425-427]}$ etc. It is often difficult for new dyes to be assigned to anyone of these classes. One of the main approaches to sensitizer design is the creation of a donor- $\pi$-acceptor (D- $\pi$-A) structure or different variations with the introduction of additional donors or auxiliary acceptors. For example, the structures with triphenylamine or carbazole as a donor and various thiophene structures as a $\pi$-bridge are common. ${ }^{[428,429]}$

Tetrapyrrole compounds such as porphyrins and phthalocyanines occupy a special place among organic dyes.
Porphyrins are an important part of natural photosynthetic systems, due to which they are widely used in artificial photosynthetic systems. ${ }^{[430]}$ Due to the efficiency of energy transfer and electron transfer in natural light-harvesting systems, porphyrins and their analogs are widely used in photovoltaic devices. ${ }^{[431]}$ These compounds are used as dyes for DSSC both in the free base form and in the form of metal complexes; however, in contrast to ruthenium dyes, more readily available zinc one is most often used. Another feature of the metal complexes of porphyrins and phthalocyanines is that the organic part of the molecule has a significant effect on the photoelectrochemical properties, including light-harvesting properties.

As with other dyes, the most effective porphyrin sensitizers are characterized by the D- $\pi$-A structure, where porphyrin simultaneously plays the role of a $\pi$-bridge, as well as a convenient synthetic platform around which all the necessary elements are assembled. The most widespread are porphyrins with the $\mathrm{AB}_{2} \mathrm{C}$-type structure (Figure 55), where donor and acceptor fragments are located on opposite sides of the porphyrin core, and the two remaining mesopositions serve to introduce hydrophobic alkyl substituents, the role of which is discussed below. The features of the choice of each of the elements are discussed in detail in the section. ${ }^{[432]}$

Anchor groups play an important role in the structure of the sensitizer. They determine the efficiency of binding of a dye with a semiconductor, as well as the efficiency of electron injection. ${ }^{[431,433]}$ For porphyrins and other tetrapyrrole compounds, anchor groups, which are also used in other groups of dyes, are characteristic. There are porphyrins with pyridinium, ${ }^{[434,435]}$ 2-hydroxy- and 2-carboxypyridinium, ${ }^{[436]}$ phosphate and phosphodiester, ${ }^{[437,438]}$ phosphine, ${ }^{[439]}$ hydroxyquinoline, ${ }^{[40]}$ sulfate, ${ }^{[441]}$ and salicylic ${ }^{[442,443]}$ anchor groups. However, the leading positions in terms of frequency of use are occupied by carboxyl and cyanoacrylate groups. From the point of view of synthetic approaches these groups are relatively easy to introduce, and yet, in the vast majority of comparative studies, they provide greater efficiency compared to other anchor groups.

In addition to the nature of anchor groups, the effectiveness of DSSCs can be influenced by their number, position, and the presence of a spacer connecting them with the macrocycle. As for the number of groups, as a rule, the introduction of more than one group is impractical, since due to the peculiarities of the geometry of porphyrins,

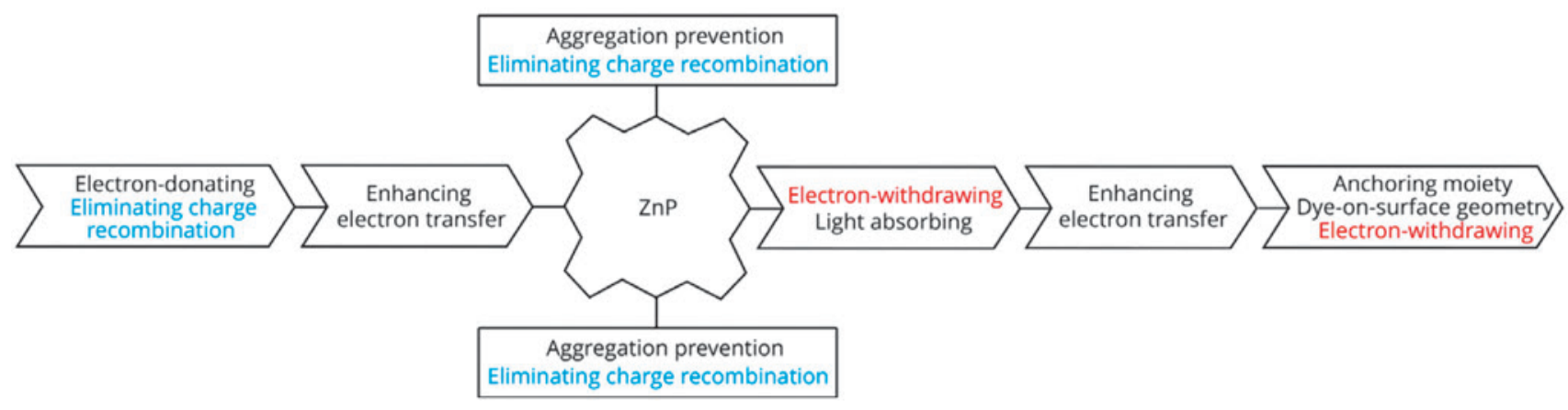

Figure 55. Functions of peripheral meso-substituents in the state-of-art zinc porphyrin design for DSSC. Reprinted from ${ }^{[432]}$ with permission. Copyright 2017 WILEY-VCH Verlag GmbH \& Co. KGaA, Weinheim. 
the remaining groups can act as an acceptor of electron density, but not participate in binding with a semiconductor, thus providing a lower efficiency of electron transfer. ${ }^{[444,445]}$ However, if the anchor groups are located enough close in the macrocycle (for example, in the $\beta$-positions of neighboring pyrrole rings), this, on contrary, can have a favorable effect. ${ }^{[46]}$ In the presence of one anchor group, its position can change the position of the plane of the tetrapyrrole cycle relative to the titanium dioxide surface from perpendicular to almost parallel, which slows down the recombination process, but reduces the overall efficiency of light absorption. When minimizing the recombination processes, the paracarboxyphenyl anchor fragment in the phthalocyanine molecule turned out to be the most effective. ${ }^{[447]}$ The work $^{[448]}$ also concludes that the para-position of the carboxyl group is superior to the meta-position.

The nature and length of the spacer between the macrocycle and the anchor group are also important. In the case of the conjugated alkene system, switching from 2-propenoic acid to 2,4-pentadiene acid resulted in a slight increase in efficiency. ${ }^{[466]}$ A similar increase in the length of the spacer, but in the case of replacing phenyl with biphenyl between the carboxyl group and the macrocycle, on the contrary, had a negative effect. ${ }^{[449]}$

An important factor for maximizing the cell open-circuit voltage is minimizing surface recombination losses. ${ }^{[40]}$ One of the main strategies for overcoming such recombination losses, along with the use of co-adsorbents, is the introduction of a hydrophobic "barrier" directly into the dye structure. Such a hydrophobic insulating layer typically consists of alkyl substituents or less commonly bulky aromatic hydrocarbons. Along with the insulating function, the alkyl chains introduced into the dye structure perform another important function: they increase the uniformity of the dye application and prevent aggregation, to which many tetrapyrrole sensitizers are prone. Dye aggregation leads to significant intermolecular energy transfer, reducing the efficiency of electron transfer to the semiconductor. ${ }^{[451]}$ A number of works are devoted to comparative studies to identify the relationship "structure and position of the hydrophobic substituent - the efficiency of the light-converting cell". ${ }^{452-456]}$

Finally, tetrapyrroles and their metal complexes have a limited absorption region. Although deposition on a photoanode leads to a significant broadening of all characteristic bands and an increase in the intensity of the Q bands as a result of the binding of the compound with titanium dioxide, the maximum quantum yield cannot be achieved at all wavelengths of the incident light. For this reason, co-sensitization is often used, i.e. the use of two or more dyes to ensure maximum light absorption in the entire region of the solar spectrum. ${ }^{[457]}$ For example, zinc metal complexes of porphyrins exhibit weak absorption in the green region. The combination with indolelenium dye made it possible to achieve a synergistic effect: individually, each dye provided an efficiency of 5.6-5.7 \%, and upon co-sensitization $-6.9 \%{ }^{[458]}$

\section{Use of DSSC in Various Devices}

Of course, the development of any technology at some point must be implemented in the practical incarnation. One of the largest completed projects using DSSC technol- ogy is the facade of the Swiss Tech Convention Center in Switzerland. However, the widespread use of DSSC today is limited by a number of factors such as price/performance ratio and number of scaling and long-term stability issues. However, even before a practical solution to these problems, DSSCs can find their place in compact and self-contained portable devices that do not require a significant amount of power to function.

To date, a number of such devices have been proposed. For example, it has been proposed to use the DSSC series with a total surface area of $16 \mathrm{~cm}^{2}$ for the functioning of an artificial neural network used for pattern recognition in images (for example, photographs of printed text). ${ }^{[459]}$ The device was operated under 1000 lux fluorescent lamp, which is quite achievable in any home without the use of any special equipment.

Another interesting device is a portable immunosensor that works on the DSSC principle and is aimed at detecting a tumor marker - carcinoembryonic antigen in urine samples. FTO electrode was modified with anti-CEA by means of a bottom-up approach. This electrode acted as a counter electrode of a dye-sensitized solar cell, and the electrical output of this cell was monitored for the different concentrations of CEA. Under optimized conditions, the device displayed a linear behaviour against CEA concentration, from $5 \mathrm{pg} / \mathrm{mL}$ to $15 \mathrm{ng} / \mathrm{mL}$. ${ }^{[460]}$

Since such stand-alone devices can be used in a variety of environments, it would be convenient to add an energy storage function. One approach for these purposes is to create a hybrid 2-in-1 device. Such power pack incorporates a series-wound dye-sensitized solar cell (DSSC) and a lithium ion battery (LIB) on the same Ti foil that has double-sided $\mathrm{TiO}_{2}$ nanotube (NTs)arrays. The power pack can be charged to about $3 \mathrm{~V}$ in about $8 \mathrm{~min}$, and the discharge capacity is about $38.89 \mu \mathrm{Ah}$ under the discharge density of $100 \mu \mathrm{A} .^{[461]}$

The development of hybrid devices has led to ideas such as textile-based energy harvesting (TBEH). TBEH is realized by integrating fabric triboelectric nanogenerator (TENG) and fiber-shaped dye-sensitized solar cell (FDSSC) to scavenge the energy of human motion and solar energy, respectively (Figure 56). The hybrid power-textile is demonstrated to be soft, flexible, wearable, and promising for applications in wearable electronics or smart textiles. ${ }^{\text {[462] }}$

A number of other examples of devices using DSSC operating principles are presented in the overview. ${ }^{[463]}$
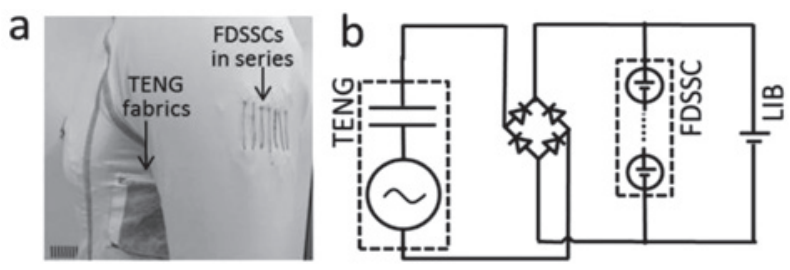

Figure 56. The integrated power-textile. a) A photo of a powertextile with a pair of TENG fabrics underneath the arm and seven FDSSCs in series on the shoulder. b) An equivalent circuit of a self-charging system with hybrid TENG fabrics and FDSSCs as energy-harvesting devices and LIB as an energy storage devices. Reprinted with permission from ${ }^{[462]}$. Copyright 2016 WILEY-VCH Verlag GmbH \& Co. KGaA, Weinheim. 


\section{Use of DSSC in Photoelectrochemical Cells}

Photoelectrochemical cells operating on the DSSC principle can be distinguished into a separate group of devices. This group of devices is primarily used for water splitting and generate hydrogen. One of the first systems for the photocatalytic decomposition of water was a system of $\mathrm{TiO}_{2}$ photoanode and $\mathrm{Pt}$ cathode. ${ }^{[464]}$ When the light is irradiated on $\mathrm{TiO}_{2}$, the absorbed light energy makes it possible to decompose water into hydrogen and oxygen. The main disadvantage, in this case, is that such a photoelectrochemical cell only absorbs UV light, which makes up only $5-7 \%$ of the solar energy that hits the ground. In this case, the principle used in DSSC can help. ${ }^{[465]}$ With this approach, the cell layout could be represented as shown in Figure 57. Here the photoanode is a mesoporous $\mathrm{TiO}_{2}$ electrode, the dye is a monolayer of sensitizer-capped $\mathrm{IrO}_{2} \cdot \mathrm{nH}_{2} \mathrm{O}$ particles. The $\left[\mathrm{Ru}(\mathrm{bpy})_{3}\right]^{2+}$ sensitizer is modified with both phosphonate and malonate ligands in the 4-positions of the 2,2'-bipyridyl ligands in order to adsorb strongly to $\mathrm{TiO}_{2}$ and $\mathrm{IrO}_{2} \cdot \mathrm{nH}_{2} \mathrm{O}$, respectively. The cathode is a $\mathrm{Pt}$ wire electrode. ${ }^{[466]}$

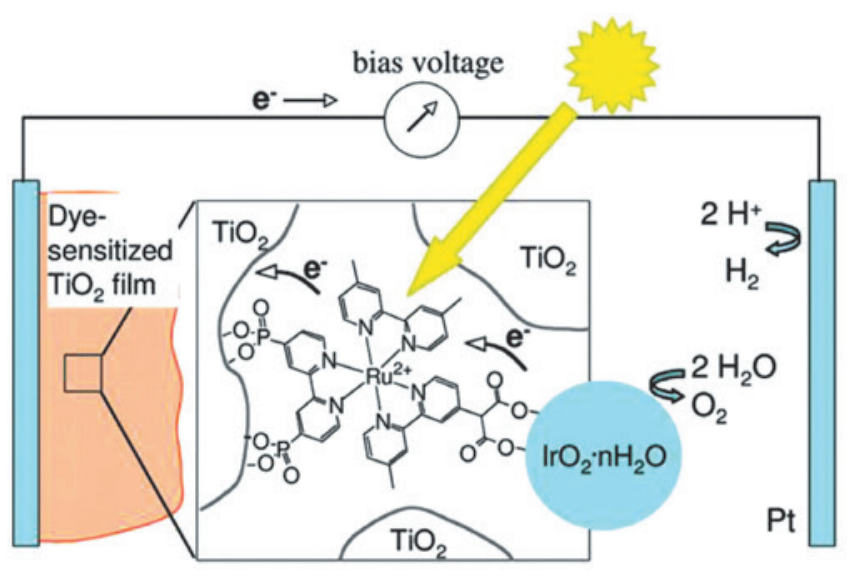

Figure 57. Schematic diagram of a water-splitting dye-sensitized solar cell. The inset illustrates a sensitizer-capped $\mathrm{IrO}_{2} \cdot n \mathrm{H} 2 \mathrm{O}$ catalyst particle in the mesopores of the $\mathrm{TiO}_{2}$ electrode film. Adapted with permission from ${ }^{[466]}$. Copyright 2009 American Chemical Society.

In practice, various tandem schemes are used, for example, those described in work ${ }^{[467]}$. A thin film of nanocrystalline such as $\mathrm{WO}_{3}$ or $\mathrm{Fe}_{2} \mathrm{O}_{3}$ serves as the top electrode absorbing light from the blue region of the solar spectrum. The valence-band holes $\left(\mathrm{h}^{+}\right)$, created by the bandgap excitation of the film oxidize water to oxygen, and the conductionband electrons are fed into the second photosystem consisting of DSSC. The latter is placed under the $\mathrm{WO}_{3}$ film, capturing the green and red region of the solar spectrum that is transmitted through the top electrode. The photovoltage generated by the DSSC enables hydrogen to be generated by the conduction-band electrons.

A variety of photoelectrochemical cell designs using DSSC principles is highlighted in reviews. ${ }^{[468-470]}$ Since tetrapyrrole sensitizers show some of the best results in the functioning of DSSCs, they are also widely used in photoelectrochemical cells..$^{[471-473]}$

Solar energy can be considered one of the most promising alternative energy sources. DSSC can become an important member of the family of photovoltaic devices, but for their practical implementation, it is necessary to continue research, including those aimed at improving the efficiency of such devices. Work in this area concerns all the key elements of DSSC: semiconductor material, redox electrolyte, and of course, a sensitizer. Tetrapyrrole compounds and their metal complexes occupy one of the leading positions among the dyes used. To date, a number of major trends in the molecular design of the most effective dyes can be identified. These include the creation of a donor- $\pi$ acceptor structure, the introduction of alkyl substituents, the use of carboxyl-containing anchor groups, and auxiliary acceptor units for their attachment. Despite the existing limitations that prevent large-scale use of DSSC, a number of compact devices that use the energy provided by such cells have been developed. In addition, DSSC principles have found applications in photoelectrochemical cells, which are used for water splitting.

\section{1,2,5-Chalcogenadiazole Analogues of Phthalocyanines - Perspective Acceptors for Organic Electronics}

Phthalocyanines (Pcs) and their analogues (Scheme 10) are widely studied as perspective functional materials for organic electronics. ${ }^{[225,474,475]}$ Unsubstituted phthalocyanines are commonly used as $p$-type organic semiconductors. Halogenation of phthalocyanines increases their electron affinity and can endow them with $n$-type conducting properties. ${ }^{[476,477]}$ Substitution of annulated benzene rings in phthalocyanines by aromatic heterocycles is another way for modulation of their electronic properties ${ }^{[478]}$ Thus, fusion of $\pi$-electron-deficient heterocycles instead of benzene rings enhances the acceptor properties of the phthalocyanine-type macrocycle. Octaazaanalogues of phthalocyanines containing pyrazine rings instead of benzene rings - tetrapyrazinoporphyrazines (TPyzPAs) ${ }^{[479]}$ - are characterized by much easier reduction and stronger electron affinity and are actively studied as perspective materials for different application fields. ${ }^{[400]}$ The acceptor properties of the macrocycle can be further increased by substitution of the peripheral ethylene fragment in pyrazine rings by a chalcogen atom ( $\mathrm{S}, \mathrm{Se}$, or Te). 1,2,5-Chalcogenadiazoles due to their remarkable electron-acceptor properties ${ }^{[481]}$ have found application in material chemistry, particularly in organic photovoltaics..$^{[482,483]}$ The detailed study of porphyrazines bearing annulated 1,2,5-thiadiazole and 1,2,5-selenadiazole rings have been started by Ercolani, Stuzhin and co-workers more than two decades ago ${ }^{[484,485]}$ and the works published till 2006 were reviewed. ${ }^{[486]}$ In this section we give a brief overview of the synthesis of porphyrazines with fused 1,2,5-chalcogenadiazole rings (TXDPAs) and consider their physico-chemical properties determining application perspectives as functional materials in organic electronics. 

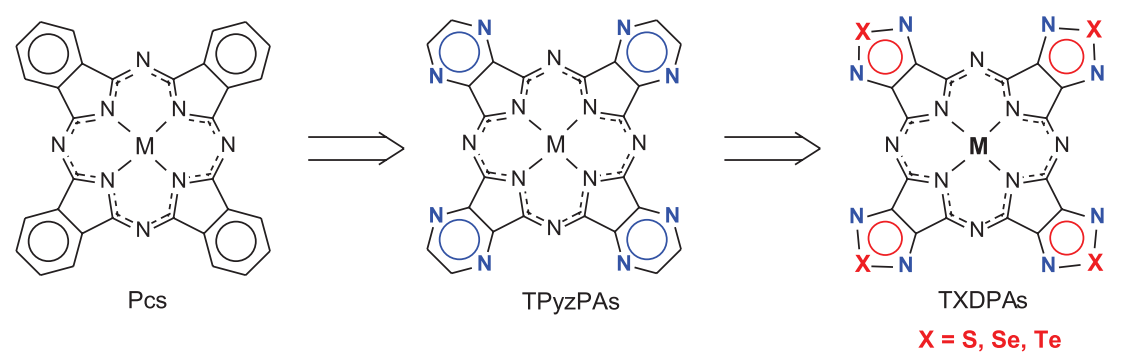

Scheme 10. Phthalocyanines and their heterocyclic analogues.

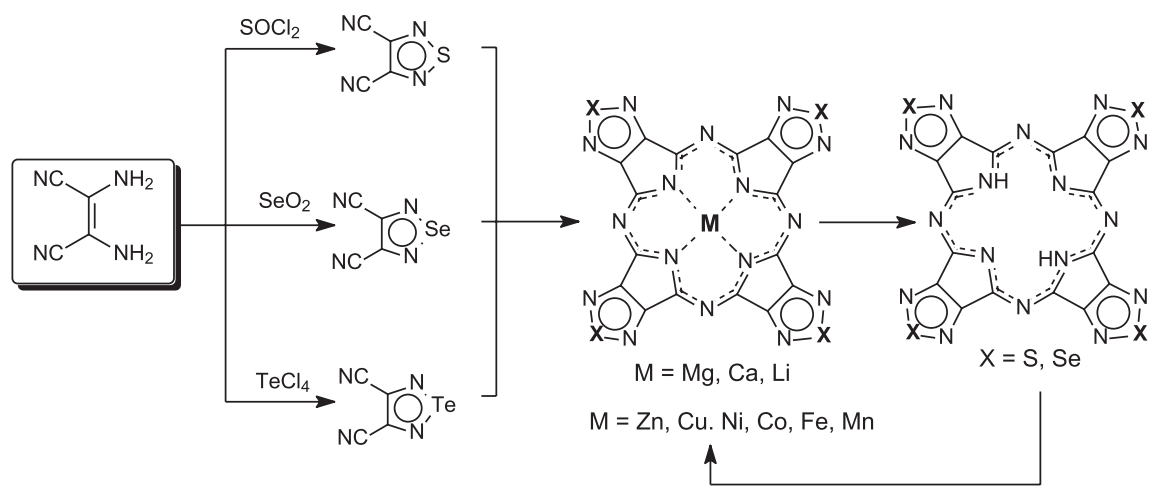

Scheme 11. Synthesis of tetra(1,2,5-chalcogenadiazolo)porphyrazines.

\section{Synthesis Aspects}

The dinitrile precursors for porphyrazines with fused 1,2,5-chalcogenadiazole rings - 1,2,5-chalcogenadiazolo3,4-dicarbonitriles $\left(\mathrm{XN}_{2} \mathrm{C}_{2}\right)(\mathrm{CN})_{2}-$ are easily available by condensation of diaminomaleodinitrile (DAMN) with thionyl chloride, selen(IV) oxide or tellurium tetrachloride, for $\mathrm{X}=\mathrm{S}$, Se, Te, respectively. ${ }^{[484,485,487]}$ These dinitriles are cyclotetramerized in the presence of $\mathrm{Mg}^{\mathrm{II}}$ alcoholates with formation of the corresponding $\mathrm{Mg}^{\mathrm{II}}$ porphyrazines (Scheme 11), isolated usually as aquacomplexes [TXDPAMg$\left.\left(\mathrm{H}_{2} \mathrm{O}\right)\right]\left(\mathrm{X}=\mathrm{S},{ }^{[484]} \mathrm{Se},{ }^{[485]} \mathrm{Te}^{[488]}\right)$.

$\mathrm{Ca}^{\mathrm{II}}$ complex, ${ }^{[489]}$ [TSDPACa], and $\mathrm{Li}^{\mathrm{I}}$ complex ${ }^{[490]}$ existing as lithate salt [TSDPALi] $\mathrm{Li}^{+}$were similarly obtained. Complexes with these metals are unstable in the presence of acids and metal free porphyrazines [TSDPAH $]^{[484]}$ and $\left[\mathrm{TSeDPAH}_{2}\right]^{[485]}$ are prepared by their demetallation, e.g. by treatment of the $\mathrm{Mg}^{\mathrm{II}}$ complexes with $\mathrm{CF}_{3} \mathrm{COOH}$. Complexes with bivalent $d$-metals [TSDPAM] ( ${ }^{3}=\mathrm{Zn}^{\mathrm{II}}$, $\mathrm{Cu}^{\mathrm{II}}, \mathrm{Ni}^{\mathrm{II}}, \mathrm{Co}^{\mathrm{II}}, \mathrm{Fe}^{\mathrm{II}}, \mathrm{Mn}^{\mathrm{II}}{ }^{[491]}$ and $\left.\mathrm{Cd}^{\mathrm{II}}{ }^{[492]}\right)$ and [TSeDPAM] $\left(\mathrm{M}=\mathrm{Zn}^{\mathrm{II}}, \mathrm{Cu}^{\mathrm{II}}, \mathrm{Ni}^{\mathrm{II}}, \mathrm{Co}^{\mathrm{II}}, \mathrm{Mn}^{\mathrm{II}[485,493]}\right)$ can be obtained by complexation of corresponding metal salt with metal free macrocycle in a donor type solvent (pyridine, DMSO), while direct template cycloteramerization of dinitriles is not effective or give only poor yields of these complexes. Recently first complexes with rare earth metals [TSDPAM(acac)] $\left(\mathrm{M}=\mathrm{Y}^{\mathrm{III}}, \mathrm{Lu}^{\mathrm{III}}\right)$ have been obtained by reaction of [TSDPAH $\left.{ }_{2}\right]$ with corresponding acetylacetonates in $o$-dichlorobenzene in the presence of strong base (DBU). ${ }^{[494]}$ At the same time formation of porphyrazine macrocycle by template condensation of 1,2,5-thiadiazole-3,4-dicarbonitrile is possible in the case of metals having lower coordination affinity to $\mathrm{N}$ and $\mathrm{S}$ atoms of heterocyclic ring (Scheme 12). Thus, com-

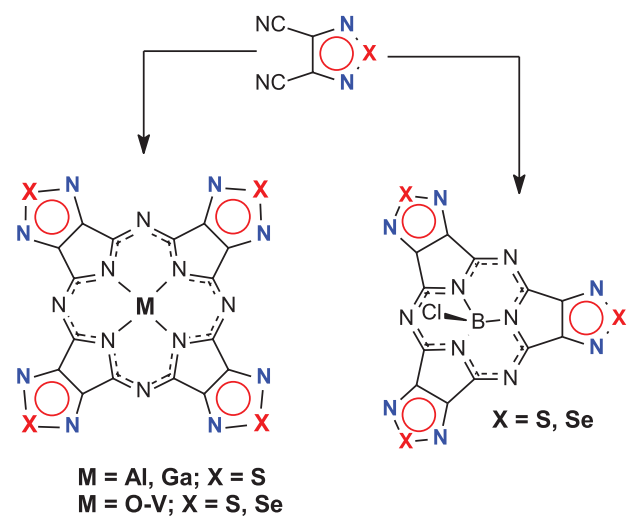

Scheme12. Direct template synthesis of porphyrazines and subporphyrazines.

plexes with $\mathrm{Al}^{\mathrm{III}}, \mathrm{Ga}^{\mathrm{III}}$, [TSDPAAl$\left.(\mathrm{Cl})\right]$ and [TSDPAGa $\left.(\mathrm{Cl})\right]$, were obtained by direct reaction of S-containing dinitrile with corresponding metal chlorides in quinoline. ${ }^{[495]}$ Cyclotetramerization of S- or Se-containing dinitriles in the presence of $\mathrm{VCl}_{3}$ in pyridine or $\mathrm{VOSO}_{4}$ in DMSO was used for preparation of the vanadyl(IV) complexes $[\mathrm{TSDPAV}=\mathrm{O}]^{[496]}$ and $[\mathrm{TSeDPAV}=\mathrm{O}],{ }^{[497]}$ respectively. In the presence of boron(III) halides S- and Se-containing dinitriles are cyclotrimerized with formation of subporphyrazines with fused 1,2,5-chalcogenadiazole rings. ${ }^{[488,499]}$

1,2,5-Chalcogenadiazole-3,4-dicarbonitriles can be also involved in mixed cyclotetramerization with substituted phthalonitriles, ${ }^{[500-502]}$ maleo- or fumarodinitriles ${ }^{[503]}$ and pyrazine-2,3-dicarbonitriles ${ }^{[504]}$ leading to low-symmetry porphyrazines containing from one to three $1,2,5$-chalcogenadiazole rings. 
A

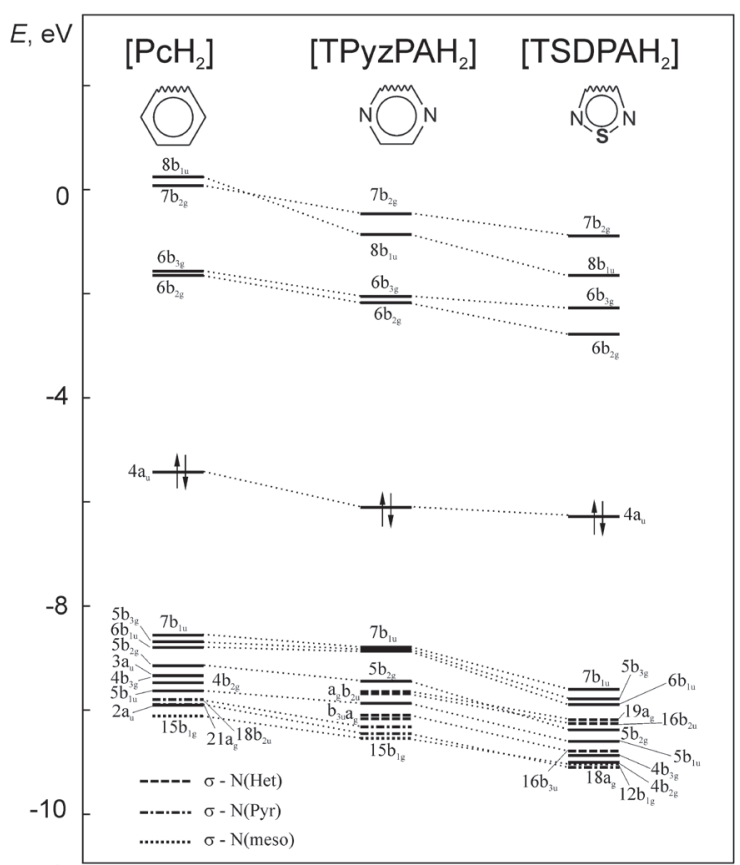

$B$

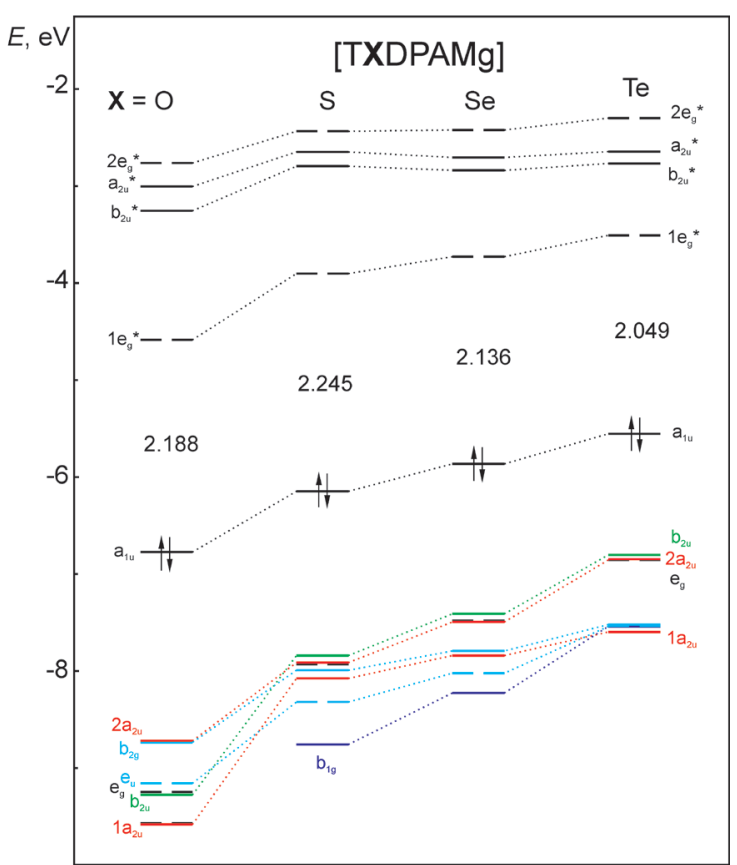

Figure 58. The energy levels calculated for (A) metal free phthalocyanine and its pyrazine and 1,2,5-thiadiazole analogues using AM1 $\operatorname{method~}^{[505]}$ (left); and for (B) $\mathrm{Mg}^{\mathrm{II}}$ complexes of porphyrazines with fused 1,2,5-chalcogenadiazole rings (DFT B3LYP method ${ }^{[511]}$ ).

Table 6. Reduction potentials for selected 1,2,5-thiadiazole fused porphyrazines and their analogues.

\begin{tabular}{|c|c|c|c|c|c|}
\hline \multirow{2}{*}{ Compound } & \multicolumn{3}{|c|}{ Potential $E_{1 / 2}, \mathrm{~V} v s . \mathrm{SCE}$} & \multirow{2}{*}{ Solvent } & \multirow{2}{*}{ Ref. } \\
\hline & $1^{\text {st }}$ reduction & $2^{\text {nd }}$ reduction & $1^{\text {st }}$ oxidation & & \\
\hline$\left[\mathrm{PcH}_{2}\right]$ & -0.66 & -1.06 & +0.64 & DMF & $*$ \\
\hline$\left[\mathrm{TSDPAH}_{2}\right]$ & -0.19 & -0.51 & & DMSO & [508] \\
\hline$[\mathrm{PcMg}]$ & -0.92 & -1.26 & +0.65 & DMF & $*$ \\
\hline [TSDPAMg] & -0.36 & -0.80 & & DMF & [508] \\
\hline$\left[\mathrm{Py}_{8} \mathrm{TPyzPAMg}\right]$ & -0.40 & -0.79 & & Py & [480] \\
\hline [PcZn] & -0.89 & -1.33 & +0.67 & DMF & * \\
\hline [TSDPAZn] & -0.44 & -0.80 & & DMSO & [508] \\
\hline$[\mathrm{PcAlCl}]$ & -0.66 & -0.98 & +0.94 & DMF & [503] \\
\hline [TSDPAAlCl] & -0.07 & -0.54 & & Py & [503] \\
\hline$\left[\mathrm{Cl}_{8}\right.$ TPyzPAAlCl $]$ & -0.17 & -0.90 & & DMSO & [480] \\
\hline
\end{tabular}

*Data from L'Her M., Pondaven A. Electrochemistry of Phthalocyanines. Chart 4. In: The Porphyrin Handbook, Vol. 16 (Kadish K.M., Smith K.M., Guilard R., Eds.), Academic Press, 2003. p. 117-170.

\section{Electronic Properties}

Substitution of benzene rings in phthalocyanine by 1,2,5-thiadiazole fragments leads to considerable stabilization of the frontier $\pi$-molecular orbitals. ${ }^{[505-507]}$ As is illustrated in Figure 58A for the case of metal free macrocycles fusion of 1,2,5-thiadiazole rings have even stronger effect than annulation of pyrazine rings. Similar results are obtained also by calculation performed by DFT method for metal complexes. ${ }^{[508-510]}$ It was also shown that the increase of the atomic number of the chalcogen leads to destabilization of the frontier $\pi$-MO (Figure 58B) and to narrowing of the HOMO-LUMO gap..$^{[511]}$

Such effect of heteroatom substitution in benzene rings of phthalocyanine strongly influences the redox potentials of phthalocyanine type macrocycles (Table 6). Thus, first reduction of the macrocycle in $\left[\mathrm{TSDPAH}_{2}\right]$ and its metal complexes occurs at much less negative potentials than in the case of corresponding phthalocyanines and even easier than for tetrapyrazinoporphyrazines bearing peripheral electron acceptor substituents (pyridyl groups or chlorine atoms). While phthalocyanines can be oxidized at $0.6-0.9 \mathrm{~V}$, 

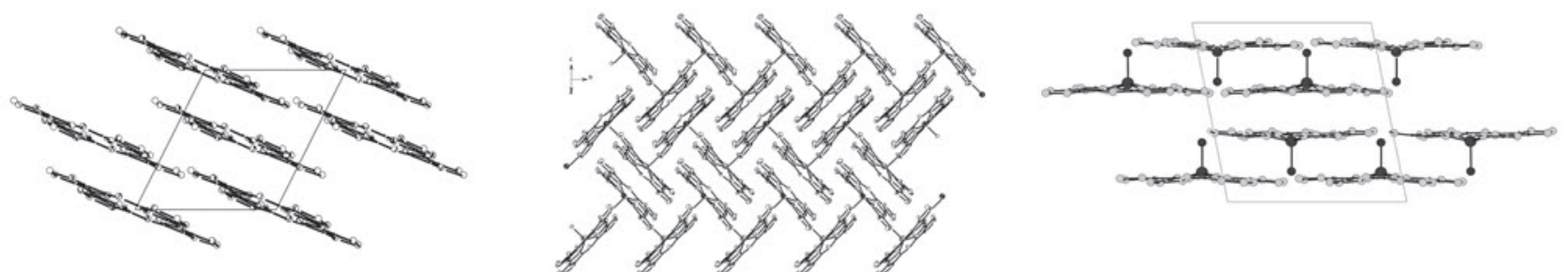

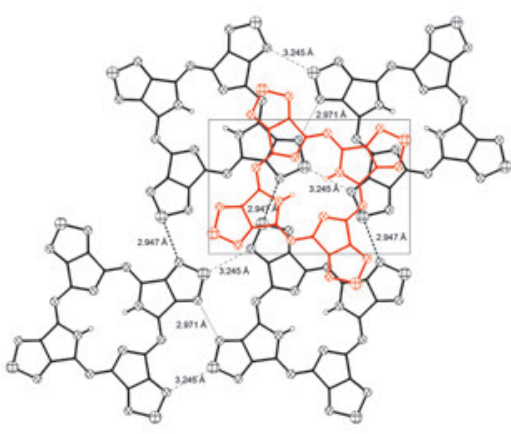

[TSDPAH $\left.{ }_{2}\right]$

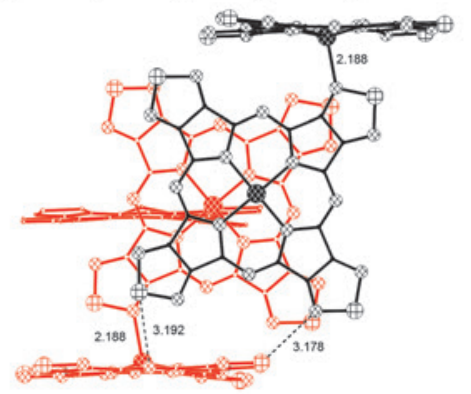

[TSDPAZn]

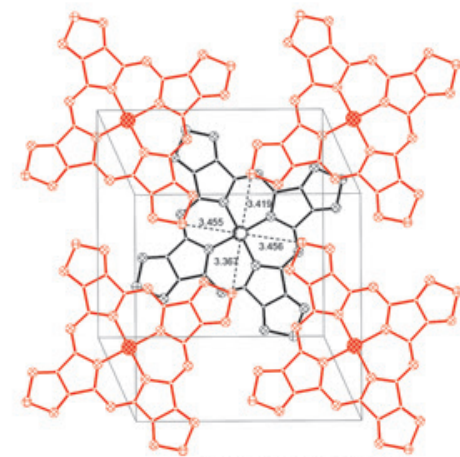

[TSDPAAl $(\mathrm{Cl})$ ]

Figure 59. Molecular packing of $\left[\mathrm{TSDPAH}_{2}\right]{ }^{[512]}[\mathrm{TSDPAZn}]^{[513]}$ and [TSDPAAl $\left.(\mathrm{Cl})\right]^{[495]}$ Redrawn from ${ }^{[486]}$

no oxidation could be observed for TSDPA macrocycle even at higher potentials.

The high electron affinity of the macrocycle is a necessary prerequisite for application of $\left[\mathrm{TSDPAH}_{2}\right]$ and its complexes as acceptor materials with $n$-type conductivity in organic electronics. Another important property is peculiar molecular arrangement observed in solid state and thin films.

\section{Crystal Structure}

[TSDPAH $\left.{ }_{2}\right]$ and its metal complexes can be sublimed in vacuum above $500{ }^{\circ} \mathrm{C}$. It was shown by mass-spectrometry that vacuum sublimation of [TSDPAZn] is accompanied by partial decomposition of the macrocycle with formation of the dinitrile precursor. ${ }^{[509]}$ In this work the structure of [TSDPAZn] in the gas phase was determined by gaseous electronography. Single crystals of $\left[\mathrm{TSDPAH}_{2}\right]$ and its metal complexes suitable for X-ray diffraction analysis were obtained by Awaga and co-workers, ${ }^{[512,513]}$ who have used sublimation at $\sim 10^{-3}$ bar in the continuous flow of $\mathrm{N}_{2}$ gas at $450-550{ }^{\circ} \mathrm{C}$. Molecular packing of $\left[\mathrm{TSDPAH}_{2}\right]$ and its complexes is primarily determined by intermolecular interactions of $\mathrm{N}$ and $\mathrm{S}$ atoms in the 1,2,5-thiadiazole rings. In the case of $\left[\mathrm{TSDPAH}_{2}\right]$ and its $\mathrm{Cu}^{\mathrm{II}}$ and $\mathrm{Ni}^{\mathrm{II}}$ complexes this leads to a unique layered structure with short intermolecular N...S contacts (2.95-3.25 $\AA$ ) and interlayer distances (3.2-3.3 $\AA$ ) evidencing about $\pi \pi$-interaction.

In the case of $\mathrm{Fe}^{\mathrm{II}}, \mathrm{Co}^{\mathrm{II}}$, and $\mathrm{Zn}^{\mathrm{II}}$ complexes additional interaction between nitrogen atoms in the 1,2,5-thiadiazole rings and metal atom in the neighbouring molecule with formation of the $\mathrm{N}_{\mathrm{h}} \rightarrow \mathrm{M}$ bonds (N-Zn bond $-2.19 \AA$ ) leads to coordination polymers with interchain $\pi \pi$ interaction (3.3-3.4 $\AA$ ). Complexes of tervalent metals [TSDPAM(Cl)] $\left(\mathrm{M}=\mathrm{Al}^{\mathrm{III}}\right.$. $\left.\mathrm{Ga}^{\mathrm{III}}\right)$ are arranged in $2 \mathrm{D}$ layers due to $\mathrm{Cl} \cdots \mathrm{S}$ interactions $(\sim 3.4 \AA)$ and inter-plane separation 3.3-3.4 $\AA$ ). ${ }^{[487]}$ The crystals of $\mu$-oxo dimer [TSDPAAl] ${ }_{2} \mathrm{O}$ also characterized by layered molecular packing. ${ }^{[514]}$ In the case of vanadyl(IV) complex [TSDPAV=O] formation of two different polymorphs is predominantly determined by intermolecular interactions between oxygen and mesonitrogens (2.93 $\AA$ in the $\alpha$-form). ${ }^{[496]}$

Vacuum deposition technology was also used for fabrication of the thin films of 1,2,5-thiadiazole-fused porphyrazines.

\section{Thin Films}

Several noteworthy studies on the properties of thin films of $\left[\mathrm{TSDPAH}_{2}\right]$ and its complexes obtained by the standard vacuum sublimation technique were published by Awaga et al. Thin films of [TSDPAH ${ }_{2}$ ] obtained by vacuum vapor deposition on various substrates are highly ordered and form unique $\pi$-stacked $2 \mathrm{D}$ arrays lying parallel to the substrate surface with an interlayer separation of $3.3 \AA . .^{[515]}$ These films are easily reduced at $-0.12 \mathrm{~V}$ exhibiting electrochromism and $n$-type behavior. Interestingly, that thin films of $\left[\mathrm{TSDPAH}_{2}\right]$ can influence crystallinity and molecular orientation of the films deposited over them. Such "templating effect" of $\left[\mathrm{TSDPAH}_{2}\right]$ was observed ${ }^{[516]}$ on the structure and optical properties of the $p$-type semiconductor $[\mathrm{PcCu}]$, which usually forms edge-on-type thin films. However, when deposited on the $\left[\mathrm{TSDPAH}_{2}\right]$ thin film the molecules of $[\mathrm{PcCu}]$ replicating the structure of the bottom layer have face-on orientation leading to appearance of new low-energy electronic transitions.

Unlike $\left[\mathrm{TSDPAH}_{2}\right]$, thin films of metal complexes [TSDPAM] often lack crystalline ordering. Formation of amorphous sublimed films was observed by Awaga et al. for complexes with vanadyl(IV) ${ }^{[496]}$ and cobalt(II), ${ }^{[517]}$ and by Pakhomov et al. for complexes with iron(II), ${ }^{[518]}$ zinc(II). ${ }^{[519]}$ While thin films of [TSDPACo] on polyimide or glass substrates ${ }^{[517]}$ are amorphous, transition to crystalline state can be triggered by introducing appropriate seed layer before the film deposition, e.g. perylene-3,4,9,10tetracarboxylic dianhydride layer. [TSDPAV $=\mathrm{O}$ ] forms well-ordered film corresponding to $4 \times 4$ superstructure when 


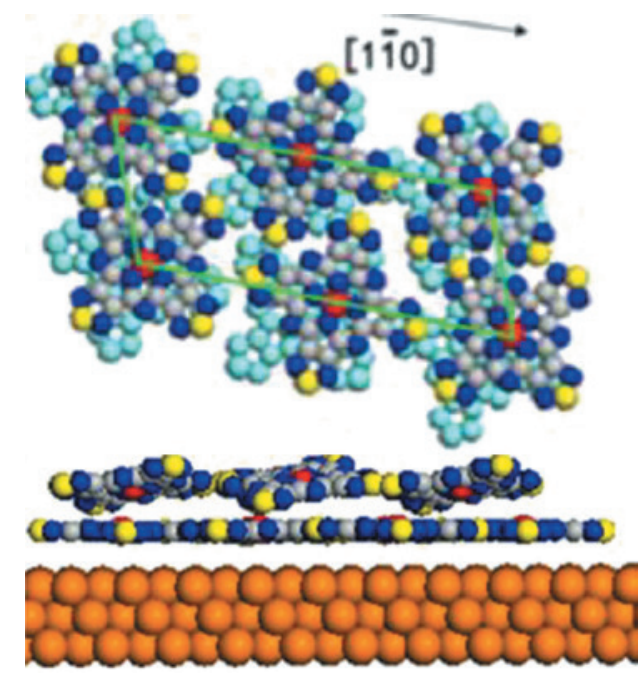

Figure 60. Model of the top and side views of the two layers of [TSDPACo] on $\mathrm{Au}$ [111] surface. Reproduced with permission from ACS Omega 2020, 5, 6676-6683 (Ref. ${ }^{[521]}$ ). Further permissions related to the material excerpted should be directed to the ACS.

deposited on $\mathrm{Au}(111)$ surface. ${ }^{[520]}$ Deposition on the $\mathrm{Au}(111)$ surface have also strong effect on the structural, electronic, and magnetic properties of thin films formed by [TSDPACo]. Molecules in the first layer have an ordered molecular lattice azimuthal rotation by $60^{\circ}$ allowing stronger intermolecular interaction between the $\mathrm{S}$ and meso- $\mathrm{N}$ atom leading to compact flat-lying configuration (Figure 60). Molecules in the second layer are tilted to avoid steric repulsion. ${ }^{[521]}$

\section{Prototypes of Electronic Devices}

Awaga and co-authors have also studied electrical and magnetic properties of the vacuum deposited thin films of $\left[\mathrm{TSDPAH}_{2}\right]$ and its complexes and revealed $n$-type semiconductivity. To determine the sign of majority charge carriers, they carried out the measurements in a field effect transistor (FET) and a Schottky-type photocell geometries. It was shown ${ }^{[522]}$ that $\left[\mathrm{TSDPAH}_{2}\right]$ act as an $n$-semiconductor in the Schottky-type photocells ITO/ $\left[\mathrm{TSDPAH}_{2}\right] / \mathrm{Au}$ and ITO/[TSDPAH $\left.{ }_{2}\right] / \mathrm{Al}$ (Figure 61, left). The thin-film field effect transistor on the basis of crystalline $\left[\mathrm{TSDPAH}_{2}\right]$ (Figure 61, right) operated by the gates of an ionic liquid and $\mathrm{SiO}_{2}$ exhibit the field effect mobility of $7.2 \cdot 10^{-4} \mathrm{~cm}^{2} \cdot \mathrm{V}^{-1} \cdot \mathrm{s}^{-1}$ and an on/off ratio of $10^{4}$. The mobility becomes impressively higher $\left(2.8 \cdot 10^{-2} \mathrm{~cm}^{2} \cdot \mathrm{V}^{-1} \cdot \mathrm{s}^{-1}\right)$ when
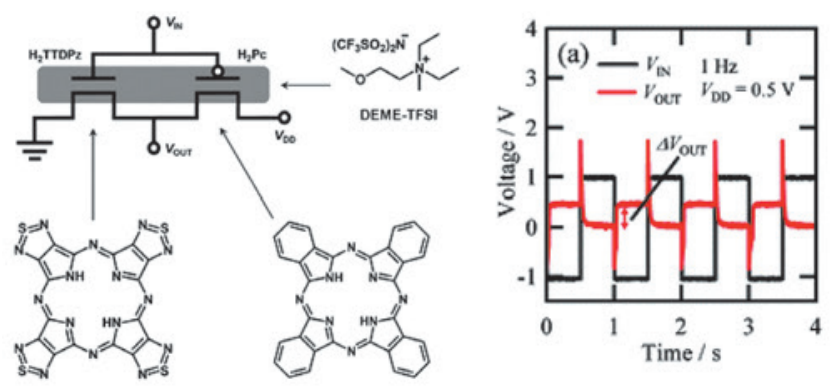

Figure 62. Complementary OTFTs inverter on the basis of $\left[\mathrm{TSDPAH}_{2}\right]$ and $\left[\mathrm{PcH}_{2}\right]$ and its dynamic response characteristics measured under $1.0 \mathrm{~V}$ peak-to-peak square input signal at $1 \mathrm{~Hz}$ frequency with $V_{\mathrm{DD}}=0.5 \mathrm{~V}$. Adapted from Ref. ${ }^{[523]}$

the amorphous vanadyl complex [TSDPAV=O] is used as $n$-type semiconducting layer. ${ }^{[496]}$

Using $\left[\mathrm{TSDPAH}_{2}\right]$ and $\left[\mathrm{PcH}_{2}\right]$ as $n$-type and $p$-type semiconductors, respectively, a complementary organic inverter with the ionic-liquid gate dielectrics was fabricated (Figure 62) and its high performance with a very low operation voltage below $1.0 \mathrm{~V}$ and a dynamic response up to $20 \mathrm{~Hz}$ was observed. ${ }^{[523]}$ The complementary inverters composed from [TSDPAV $=\mathrm{O}]$ co-deposited with $[\mathrm{PcV}=\mathrm{O}]$ worked in the first and third quadrants with a large signal gain over $10 .^{[517]}$

Ambipolar electric-double layer transistor was obtained when $[\mathrm{TSDPAV}=\mathrm{O}]$ was co-deposited with $[\mathrm{PcV}=\mathrm{O}]$; an improved mobility $\left(2.3 \cdot 10^{-3}\right.$ and $1.0 \cdot 10^{-4} \mathrm{~cm}^{2} \cdot \mathrm{V}^{-1} \cdot \mathrm{s}^{-1}$ for $p$ - and $n$-type), threshold voltage and a high on/off ratio were observed. ${ }^{[524]}$ By analyzing X-ray diffraction patterns of the co-deposited films (supported by theoretical estimations of crystal energies), AFM images, optical spectra, cyclic voltammetry data and FET performance, the authors arrive to the conclusion that the charge transport in the mixed phases is governed by the two-dimensional percolations of the $n$ - and $p$-type semiconductor domains. Although an acceptor-like behavior of thiadiazole fused porphyrazine with respect to unsubstituted phthalocyanine should be anticipated in this pair, the charge transfer of D/A type is strictly denied.$^{[521]}$ Note that in contrast with the $p / n$ junctions composed of conventional inorganic semiconductiors, the organic-based heterojunction cells rely on the D/A model of the photoinduced charge separation at the interface. ${ }^{[525]}$

Thin-film photovoltaic cells with fully molecular (hetero-)junctions have been considered by Pakhomov and co-
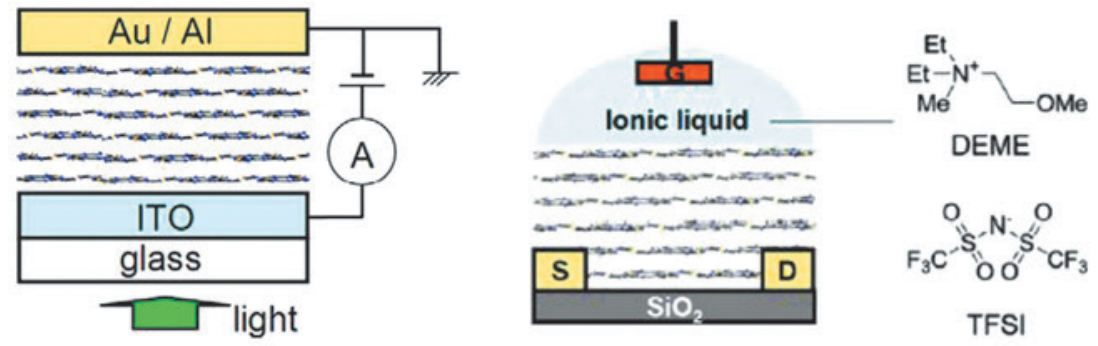

Figure 61. Schemes of photocells (left) and field effect transistor (right) on the basis of [TSDPAH ${ }_{2}$. Adapted from Ref. ${ }^{[522]}$ 

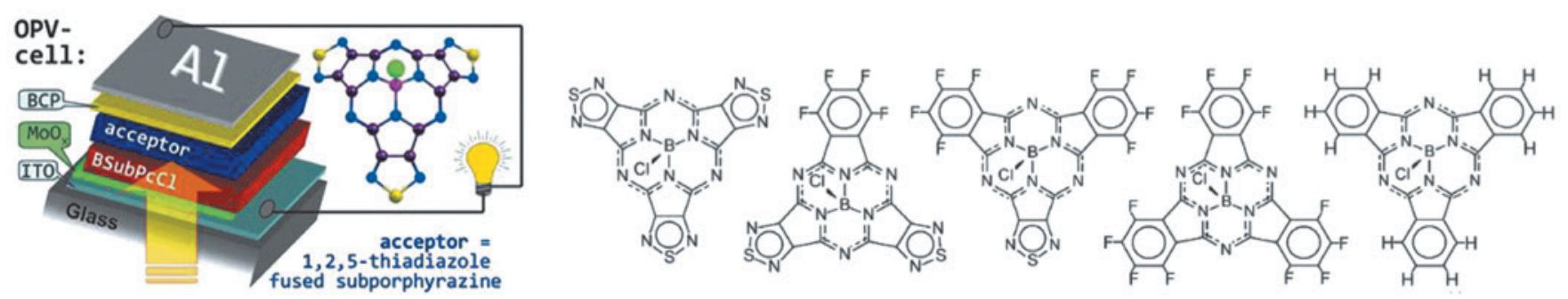

Figure 63. Organic photovoltaic cell consisting from subphthalocyanines as donor and subporphyrazines with fused 1,2,5-thiadiazole rings as acceptor.

workers. The archetypal devices with a planar heterojunction formed by subphthalocyanines [SubPcBCl] as a donor and $\mathrm{Fe}^{\mathrm{II}}$ complex of 1,2,5-thiadiazole fused porphyrazine [TSDPAFe] as an acceptor were fabricated and studied. ${ }^{[525]}$ It was shown that these $\mathrm{D} / \mathrm{A}$ pair generate photovoltage of more than $0.4 \mathrm{~V}$ measured in the unbuffered, unoptimized cells.

$\mathrm{Zn}^{\text {II }}$ complex [TSDPAZn] was used for fabrication of planar and sandvich-type photovoltaic devices and their output characteristics were measured under various conditions (atmosphere, illumination temperature). It was shown $^{[525]}$ that strong photovoltaic effect appears when $p$-[PcZn] $/ n$-[TSDPAZn] is assembled within the multilayer heterojunction organic-based cell. Although the power conversion efficiency of unoptimized cell is low, it is by a factor of two greater than for $p$-[SubPcBCl] $/ n$-[TSDPAFe] pair.

Later, this theme was continued by studying photovoltaic parameters of planar molecular heterojunctions where the acceptor component of D/A pair were heterocyclic analogues of perfluorinated subphthalocyanines - subporphyrazines (sPAs) containing fused 1,2,5-thiadiazole rings instead one, two or all three tetrafluorobenzene rings. ${ }^{[526]}$ Photovoltaic activity of these sPAs as acceptors was examined in the schematically equivalent cells incorporating a sister non-substituted subphthalocyanines as a donor component (Figure 63). Although the cell photovoltage in this series varied within $0.7-0.9 \mathrm{~V}$, the output parameters were still inferior to those of the devices based on traditional fullerene acceptor. However, in the regime of a photodiode, the sPAsbased devices showed the signal as high as $>10^{5} .{ }^{[26]}$ More effort should be spent on investigating solid-state properties of subporphyrazine-type compounds and on searching new approaches to their device-oriented applications.

The obtained data indicate that porphyrazines and subporphyrazines with fused 1,2,5-thiadiazole rings can be considered as perspective acceptor materials for organic electronics, especially for design of photovoltaic devices.

\section{Luminescence of Lanthanides in Complexes with Polytopic Tetrapyrrole Macrocycles}

Intensive studies of tetrapyrrolic macroheterocycles and their complexes with lanthanide ions $(\operatorname{Ln}(\mathrm{III}))$ are obliged to their high demand in photochemistry, catalysis, luminescence spectroscopy, solar energy conversion technologies, etc. ${ }^{[527-531]}$ The variety of structures synthesized over quite short period of time is striking: starting from simple alkyl- and arylporphyrins to derivatives that contain fragments capable of molecular recognition, regulation of polarity, redox potentials, solubility and other properties that are key for the use of polytopic nitrogen-containing macrocycles in practice. ${ }^{[533,652]}$

Among all lanthanides, only $\mathrm{Yb}(\mathrm{III}), \mathrm{Nd}(\mathrm{III})$ and $\operatorname{Er}(\mathrm{III})$ are capable for $4 f$-emission in complexes with macrocyclic tetrapyrroles. The reason of such a limitation is the low values of the donor triplet levels of tetrapyrroles in phosphorescence region (at about $800 \mathrm{~nm}$ ) and only named lanthanide ions have low resonance levels matching for the excitation energy transfer (Figure 64).

In order to gain effective $4 f$-luminescence design of multichromophoric systems is very promising because of the presence of several sources of excitation energy for the $\operatorname{Ln}(\mathrm{III})$ ion. Most often, such compounds are polytopic, because they are capable of additional coordination of the second metal ion with the formation of polynuclear complexes.

\section{Lanthanide Porphyrinates}

All lanthanide porphyrinates are labile systems, which is caused by:

- the inability of lanthanides to be in $d^{2} s p^{3}$-hybridization (it is impossible to form a strong covalent bond with the ligand because of the shielding of $d$-orbitals);

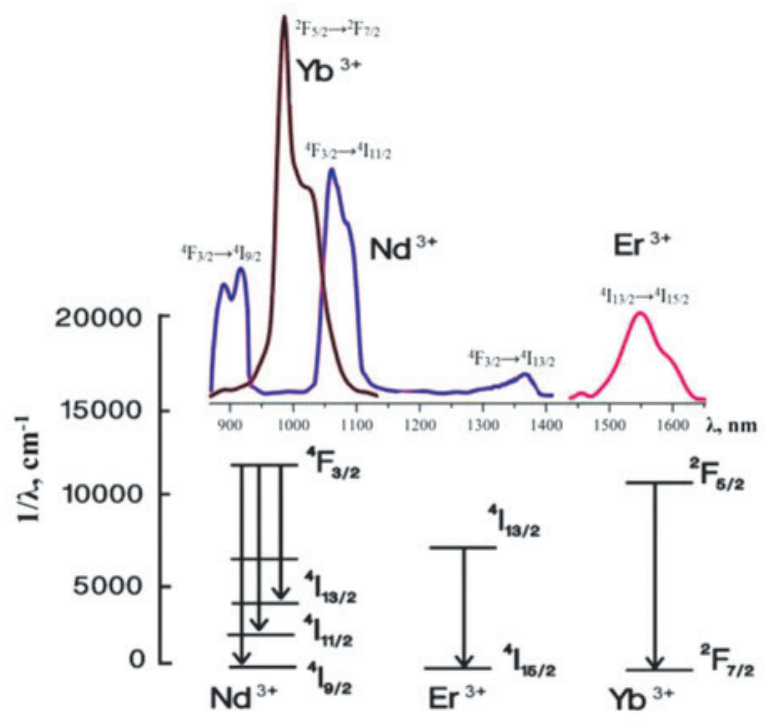

Figure 64. Profiles of $4 f$-luminescence spectra of $\mathrm{Yb}(\mathrm{III}), \mathrm{Nd}(\mathrm{III})$ and $\operatorname{Er}(\mathrm{III})$ ions, and the corresponding electronic transitions. 


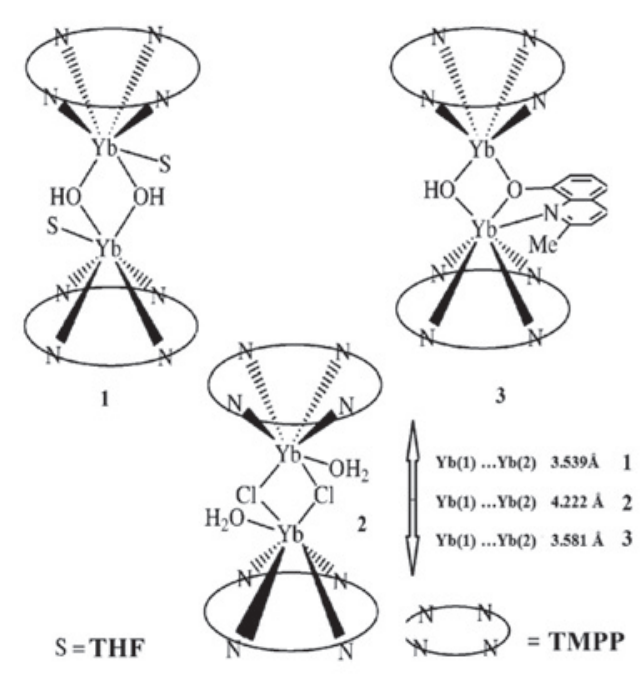

a

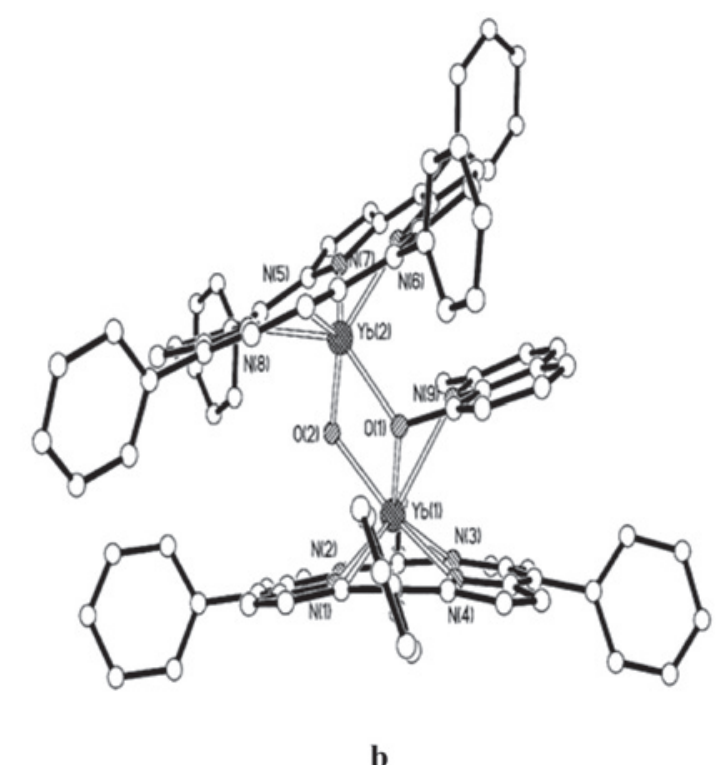

b

Figure 65. Diytterbium complexes of tetra( $p$-methoxyphenyl)porphyrin (TMPP) (a) and the structure of the complex $[\mathrm{Yb}(\mathrm{TMFP})(\mu-\mathrm{OH})]_{2}(\mathrm{THF}) \cdot 2 \mathrm{H}_{2} \mathrm{O}(\mathrm{b})$.

- shielding of $f$-electrons by external electronic layers, therefore $\pi$-bonds are formed;

- strong displacing of the lanthanide ion from the plane of nitrogen atoms of the macrocycle because of high values of covalent radii;

- facilitation of dissociation of $\mathrm{Ln}-\mathrm{N}$ bonds, because of coordination with extra-ligand. ${ }^{[534]}$

The creation of polynuclear complexes of porphyrins is possible only in the presence of additional complexing fragments in the structure of the ligand or if several tetrapyrrole macrocycle rings linked by spacer groups. Most of these works are devoted to porphyrin complexes with $d$-metals, in which the macrocycles are linked by means of various fragments, allowing varying the distances between porphyrinates, but the nature of the metal itself remains unchanged.

The possibility of dimerization of metalloporphyrins can be also caused by the bridging extra ligands: hydroxo groups, carboxylates, ethers. ${ }^{[335]}$ However, in these systems, bridging ligands quite easily enter into substitution reactions. Porphyrins can also act as a bridging ligand, ${ }^{[536]}$ in this case, there is an equilibrium between the mono- and bimolecular forms, as well as changes in the absorption spectra of the resulting compounds, which was shown by the example of homopolynuclear $f$-f-complexes of porphyrins, in which the lanthanide ion is coordinated by four nitrogen atoms of macrocycle and several extra ligands. As expected, the most labile compounds are those, in which extra ligands are water, methanol, ethanol, dimethylformamide, as well as halogen and hydroxide anions..$^{[537,538]}$ Complexes of this type were isolated only for the lanthanides of the end of the series (Er, $\mathrm{Yb}, \mathrm{Lu}$ ), since the ions of beginning of the series are characterized by large ionic radii, and they cannot be effectively bound with porphyrin. Such systems are quite labile: extra ligands can be substituted with acetate, 8-hydroxyquinoline, diethoxyethane, tetrahydrofuran, 7-azabenzotriazole and other molecules. ${ }^{[539,540]}$ In addition, they are susceptible to dimerization with the implementation of the bridging function of extra ligands (Figure 65a). Structures, one of which is shown in Figure 65b, were obtained by twophase synthesis through the interaction of mono-complexes in dichloromethane at room temperature. Structural analysis showed that the dimer has C2 symmetry and two six-coordinate $\mathrm{Yb}(\mathrm{III})$ ions, the distance between which is $3.581 \AA$, connected by two asymmetric bridging oxygen atoms of hydroxo- and 8-hydroxooxyquinoline extra-ligands.

$4 f$-Luminescent signal was detected in all systems shown in Figure 64 with $\lambda_{\max }=978-982 \mathrm{~nm}$ in the IR region, which is characteristic for the ${ }^{2} \mathrm{~F}_{5 / 2} \rightarrow{ }^{2} \mathrm{~F}_{7 / 2}$ transition of the $\mathrm{Yb}$ (III) ion. The shortest lifetime was recorded for complexes where the bridging functions were performed by water molecules $-1.02 \mu \mathrm{s}$ (complex 1), which is almost one and a half times less than for the mononuclear analogue. In contrast to compound $\mathbf{1}$, an increase of $\tau_{4 \mathrm{f}}$ is observed for compounds $\mathbf{2}$ and $\mathbf{3}$, which is $3.17 \mu \mathrm{s}$ and $2.27 \mu \mathrm{s}$, respectively, and is much higher than the lifetime of fluorescence, which is not completely quenched in these systems, 4.3-4.8 ns. A decrease in the intensity of IR luminescence occurs, according to the authors, with an increase in the number of O-H oscillators in the molecules of the complexes. Much more stable systems were obtained when $\beta$-diketones were used as extra ligands ${ }^{[541-544]}$ and such tridentate ligands as hydridotris (pyrazol-1-yl)borate and cyclopentadienyltris(dialkoxyphosphite)cobaltate(I). ${ }^{[545-547]}$

Compounds of $\mathrm{Gd}(\mathrm{III})$ ions with porphyrins containing diethylenetriaminepentaacetate (dtpa) fragments can serve as an example of polynuclear $f-f$ complexes (Figure 66). The high characteristics of these compounds as contrast agents for the diagnosis of neoplasms were noted. [548,549]

In most cases, the preparation of homopolynuclear complexes based on functionalized porphyrins proceeds in one stage, while, depending on the synthesis conditions, complexation occurs either at the peripheral substituents (Figure 66, left) or at all ligand fragments, including the porphyrin center (Figure 66, right). In contrast to homonuclear 

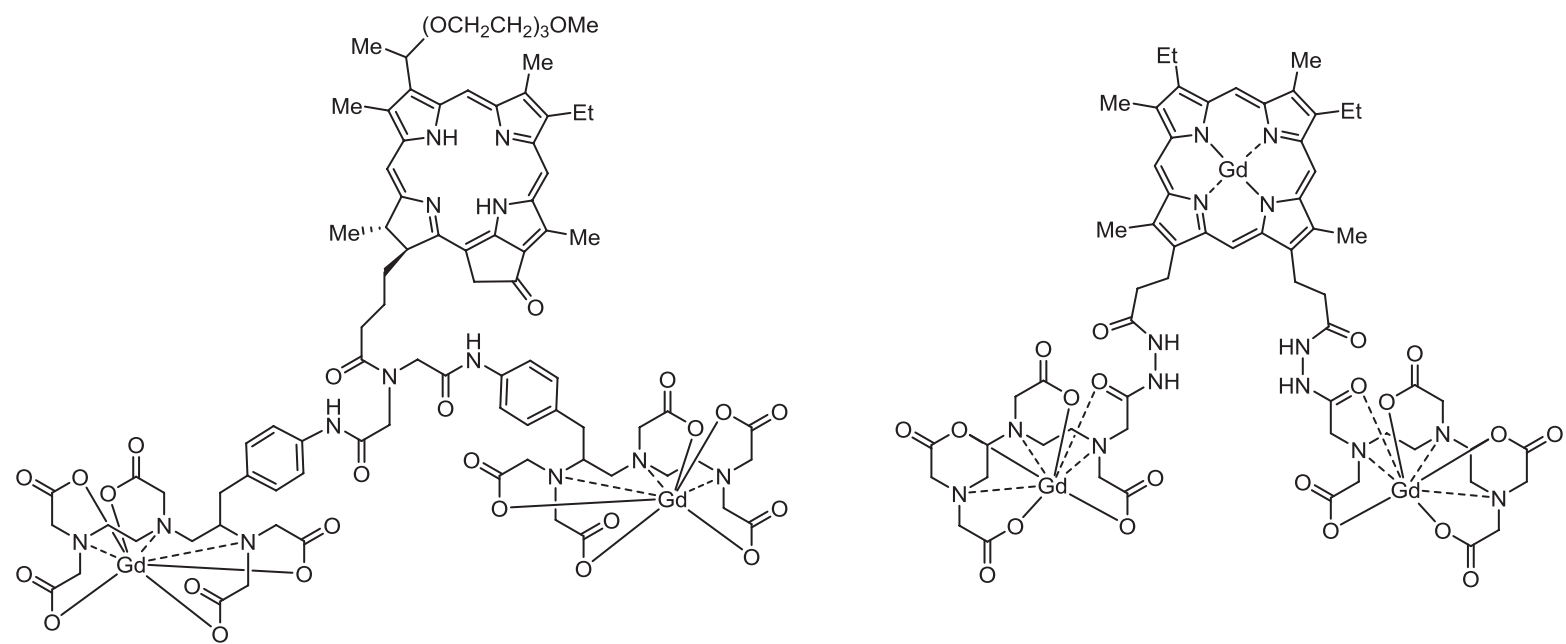

Figure 66. Structure of Gd(III) complexes with synthetic porphyrins modified with dtpa fragments.

complexes, the formation of heteronuclear ones is complicated by the fact that stage-by-stage complexation of metals with polytopic ligands is necessary, which excludes the production of a mixture of products.

$d$-f-Heteropolynuclear complexes with erbium(III) ions were synthesized based on a zinc-containing porphyrin block modified with fragments of 1,10-phenanthroline or 2,2'-dipyridyl. ${ }^{[50]}$ Aromatic substituents were chosen because of two reasons. The first of them is the possibility of the formation of stable $d$-metal complexes. The second reason is the study of the influence of the nature of aromatic spacers, which manifests itself in the mutual arrangement of porphyrinate units: more flexible in the case of nonplanar 2,2'-dipyridyl, in comparison to the rigid binding with condensed 1,10-phenanthroline linker (Figure 67). ${ }^{[50]}$ The obtained compound exhibits sensitized $4 f$-luminescence of the $\operatorname{Er}(\mathrm{III})$ ion in the IR region at $1530 \mathrm{~nm}$ (transition ${ }^{4} \mathrm{I}_{13 / 2} \rightarrow{ }^{4} \mathrm{I}_{15 / 2}$ ), which manifests itself as a result of resonant energy transfer from $\mathrm{Pt}(\mathrm{II})$ porphyrinate to emitting levels of the Er(III) ion. This mechanism of energy transfer is confirmed by the overlap of the luminescence spectra

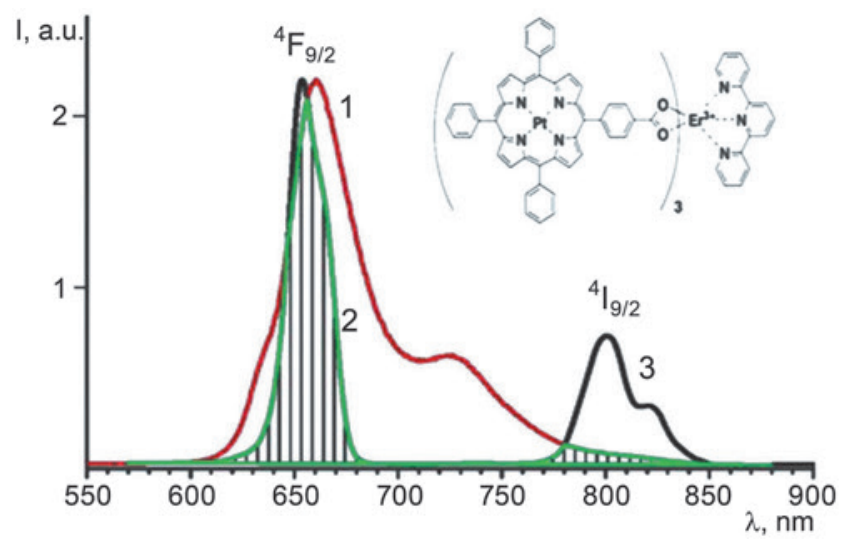

Figure 67. Overlapping (shaded area) of the luminescence spectra of Pt-porphyrinate (1) and the absorption spectra of the Er(III) ion: transitions ${ }^{4} \mathrm{I}_{15 /} 2 \rightarrow{ }^{4} \mathrm{~F}_{9 / 2}(2)$ and ${ }^{4} \mathrm{I}_{15 / 2} \rightarrow{ }^{4} \mathrm{I}_{9 / 2}$ (3). Insert: structure of the Er-Pt complex. of the d-metal porphyrinate and the $f-f$ absorption spectra of erbium ions: transitions from the ground level ${ }^{4} \mathrm{I}_{15 / 2}$ in this case to the levels ${ }^{4} \mathrm{~F}_{9 / 2}$ and ${ }^{4} \mathrm{I}_{9 / 2}$, the maxima whose absorptions are $652 \mathrm{~nm}$ and $801 \mathrm{~nm}$, respectively.

$4 f$-Luminescence of $\mathrm{Nd}$ (III) and $\mathrm{Yb}$ (III) ions was observed $^{[551]}$ in a heteronuclear complex based on $\beta$-monoaminoteraphenylporphyrinate $\mathrm{Pd}(\mathrm{II})$ modified with dodecane tetraacetic acid (dota) derivative (Figure 68). In this work, the preparation of $d$-metal porphyrinate also precedes the stage of introduction of the lanthanide ion into the peripheral binding site.

It is known that palladium complexes of porphyrins have long-lived triplet levels, which can be very effectively quenched in the presence of oxygen in polar media. It was shown that the modification with dota fragment promotes the suppression of oxygen quenching and increases the phosphorescence of palladium complexes by almost an order of magnitude. All luminescence spectra of $\mathrm{Nd}$ (III) and $\mathrm{Yb}(\mathrm{III})$ ions were obtained in aerated and degassed solutions in methanol (deuterated and non-deuterated). $4 f$-Luminescent signal in a deuterated solvent was found to be 4 and 2.5 times increased for $\mathrm{Yb}$ (III) and $\mathrm{Nd}$ (III), respectively, which confirms the sensitivity of the excited ion levels to quenching by $\mathrm{OH}$ vibrations. The excitation spectra of samples in degassed solutions are similar to the absorption spectra of porphyrinates of individual complexes.

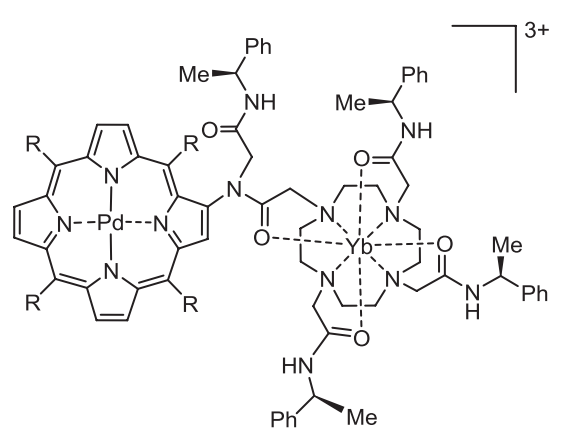

Figure 68. Structure of Yb-Pd-heteronuclear complex with dotafunctionalized porphyrin. 
This indicates, according to the authors, a competitive rate of energy transfer from the triplet levels of the porphyrin part of the complex to the levels of lanthanide ions. Energy transfer in neodymium-containing systems is much more efficient than in $\mathrm{Yb}(\mathrm{III})-\mathrm{Pd}(\mathrm{II})$ complexes. This is a consequence of the larger overlap integrals between the absorption spectra of $\mathrm{Nd}(\mathrm{III})$ ions: absorption maxima at 740 , 794 , and $865 \mathrm{~nm}$ (transitions from the ground level ${ }^{4} \mathrm{I}_{9 / 2}$ to ${ }^{4} \mathrm{~F}_{7 / 2}+{ }^{4} \mathrm{~S}_{3 / 2} \cdot{ }^{4} \mathrm{~F}_{5 / 2}+{ }^{4} \mathrm{H}_{9 / 2},{ }^{4} \mathrm{~F}_{3 / 2}$, respectively) and emission bands of porphyrinates.

A large number of heteronuclear complexes have been obtained based on bridging ligands..$^{[545-547]}$ The creation of $d$-d-heteronuclear complexes by this method is impossible, apparently due to the lower coordination numbers of $d$-metals compared to lanthanides. Unlike previous studies, $f$ - $d$-compounds are synthesized on the basis of lanthanide porphyrinates as the main "block" systems (Figure 69).

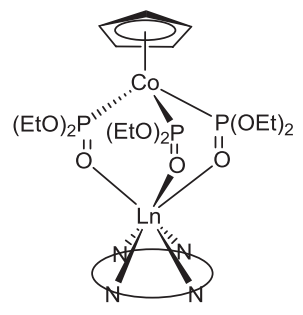

$\operatorname{Ln}(I I I)=N d, E r, Y b, G d$

Figure 69. Structure of lanthanide-containing heteronuclear cobalt compounds.

By the addition of (cyclopentadienyl)tris(diethylphosphinite)cobaltate, Ln-Co complexes were synthesized, in which the effective shielding of the lanthanide ion by a cobalt-containing fragment made it possible to obtain a rather intensive $4 f$-luminescence signal of $\mathrm{Yb}(\mathrm{III}), \mathrm{Nd}(\mathrm{III})$, and $\mathrm{Er}(\mathrm{III})$ ions (Figure 70). The most important and interesting aspect of these compounds is their use as components of electroluminescent devices emitting in the IR region. ${ }^{[552]}$ The need to obtain homogeneous thin films for luminescent layers has attracted considerable attention to the creation of hybrid materials based on lanthanide porphyrinates. ${ }^{[53]}$

Complexes of lanthanides with porphyrins are among the most promising for creating materials for emission

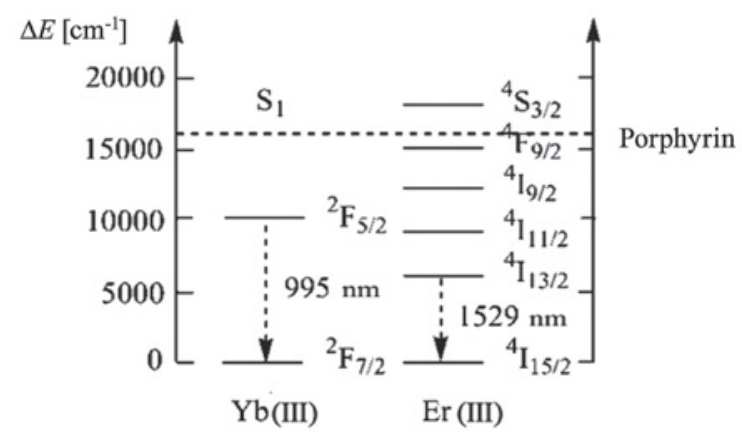

Figure 70. Scheme of energy transfer in lanthanide-containing heteronuclear cobalt compounds. layers. ${ }^{[554,555]}$ Improvement of the efficiency of electroluminescent devices was achieved by the optimal selection of extra ligands in the complexes and matrix materials. In the works of Kango and his co-workers, it was proposed to replace acetylacetonate with a complex of a transition metal - cobalt (Figure 70). A device with a similar structure showed characteristic luminescence even at a voltage of $4 \mathrm{~V}$, and its radiation power was $\sim 0.2-0.6 \mu \mathrm{W} \cdot \mathrm{cm}^{-2}$ at $10-15 \mathrm{~V}$. The replacement of polystyrene by poly(vinylcarbazole) did not affect the characteristics, which indicates that the transport of charge carriers is mainly determined by the properties of $\mathrm{Yb}$ (III) complexes. An attempt to replace the cobalt complex with a pyrazolyl borate ligand made it possible to increase the power of the device by almost an order of magnitude.

\section{Ln(III) Complexes with Phthalocyanines}

Low solubility of phthalocyanine compounds in organic solvents and water complicates their practical use. In order to improve the solubility of phthalocyanines, numerous substituted derivatives have been synthesized. Thus, the binding of bulky substituents, such as naphthyl or crown ethers, into the benzene rings of the phthalocyanine molecule increases the solubility of phthalocyanines in organic solvents. ${ }^{[556-558]}$ In addition, the binding of the crown ether fragment as a substituent of the benzene ring of the isoindole fragment leads to the formation of heteronuclear supramolecular compounds with alkali and alkaline earth metal salts. Thus, obtained supramolecular crown-substituted phthalocyanines are characterized by unique electrophysical and optical properties due to the formation of channels of ionic (crown ether fragments) and semiconducting (phthalocyanine macrocycle) conductivity. On this basis, supramolecular functional materials for ionic and molecular electronics are being developed.

It was shown ${ }^{[556]}$ that for semi-sandwich complexes of Er(III) with tert-butyl and pentyloxy-substituted phthalocyanines under indirect excitation, $4 f$-luminescence of the samples in the solid state and DMSO- $d_{6}$ was detected, whereas in bisphthalocyaninates, the intrinsic emission of $\operatorname{Er}($ III) was absent. However, it is likely that in this work not individual compounds were investigated, as indicated by the absorption spectra.

Binuclear lanthanide complexes were obtained based on hexa-tert-butyl-substituted phthalocyanines. Macrocycles share one benzene ring as a linker. ${ }^{[57]}$ The synthesized complexes with Dy(III), Yb(III), Lu(III) ions are characterized by intense absorption in the near IR region, while the nature of the lanthanide has almost no effect on the electronic spectrum and the positions of the Q-bands. Heteronuclear complexes based on porphyrin dimers were obtained in contrast to the previous lanthanide phthalocyaninates.

The features of $4 f$-luminescence in the IR region were discussed in a many works devoted to various types of lanthanide complexes with tetra(15-crown-5)phthalocyanine: unsubstituted phthalocyaninates, monophthalocyaninates with different axial ligands, di- and triphthalocyaninates. ${ }^{[559-561]}$ In all studied complexes, the coordination of the lanthanide ion $\mathrm{Ln}(\mathrm{III})$ is coordinated with four nitrogen atoms of the tetrapyrrole macroring, and in the case of monophthalocyaninates 
also with donor atoms of the acetate ion, 1,10-phenanthroline, or 1,8-diazabicyclo[5.4.0] undecene (DBU). It has been shown that the $4 f$-luminescence of monophthalocyaninate complexes is affected by the presence of a second ligand with respect to the tetrapyrrole ring: 4 -luminescence in the presence of Phen (DBU) is almost two times higher. $\mathrm{Yb}(\mathrm{III})$ complexes of all types exhibit near-infrared 4fluminescence at $980 \mathrm{~nm}$. The mechanism of this process was proposed. This luminescence can occur probably due to Dexter energy transfer or it can be caused by the photoinduced electron transfer with the reduction of $\mathrm{Yb}$ (III) to $\mathrm{Yb}$ (II) in the electronically excited state of the complex. For all Er(III) complexes intensive $4 f$-luminescence at $1540 \mathrm{~nm}$ was detected with parameters close to known inorganic phosphors which is very promising for the creation of new emitters of the infrared spectrum for telecommunication technologies, as well as in biology and medicine.

\section{4f-Luminescence of Ln(III) in Complexes with Corroles}

Intensive study of corroles started only in 1999 after invention of their high-yield synthesis ${ }^{[562]}$ and the first study on lanthanide-corroles was published only in 2013. ${ }^{[563]}$ The smaller core cavity of corroles relative to porphyrins and phthalocyanines prohibits the formation of stable complexes with lanthanides, which have large ionic radii. Common synthetic procedures for the lanthanide-porphyrins are not suitable for lanthanide-corroles. Nevertheless, few non-luminescent complexes of core-coordinated lanthanidecorroles were synthesized. ${ }^{[564]}$

Sensitization of $4 f$-luminescence (emission of $\mathrm{Nd}, \mathrm{Er}$ and $\mathrm{Yb}$ ions) by corroles was found only in complexes with ditopic corroles (Figure 71). Since lanthanide ion is not bound directly to corrole chromophore, so the latter keeps its molecular emission unaffected by heavy atom effect and formally these compounds are dual-range emitters (VIS/IR).

Ratio of $4 f$-luminescent effectiveness of $\mathrm{Nd}$ and $\mathrm{Yb}$ ions in isostructral compounds is a key and absolutely unpredictable feature of lanthanide-corroles. Thus, luminescence of $\mathrm{Yb}$ (III) is always more effective than $\mathrm{Nd}$ (III) in any isostructural compounds (e.g. $\beta$-diketonates, porphyrinates, complexes with heterocyclic compounds, etc.). It can be easily explained by numerous possible transitions in $4 f$-shell of $\mathrm{Nd}(\mathrm{III})$ ion (in contrast to $\mathrm{Yb}(\mathrm{III})$ with its only one transition), which leads to radiationless energy losses. But corrole complexes with lanthanides reveal opposite trend. For example $\mathrm{Nd}$ (III) complex with edta-derivative has $\varphi_{4 \mathrm{f}}=2.8 \cdot 10^{-3}$ while isostructural $\mathrm{Yb}$-edta-corrole has $\varphi_{4 \mathrm{f}}=5 \cdot 10^{-4}$. Authors ${ }^{[565]}$ explained this phenomenon by changing of the contributions ratio of donation energy from $\mathrm{S} 1$ and $\mathrm{T} 1$ levels in the corrole.

It can be concluded from the analysis of the mentioned works on Ln-tetrapyrroles that the functionalization of macrocyclic ligands with chelating fragments is one of the most promising directions for achieving high luminescence characteristics. In this case, such classes of compounds as porphyrins, phthalocyanines and corroles can be considered as convenient molecular platforms for the creation of heteronuclear IR-luminescent complexes. At the same time two serious shortcomings in the above studies should be noted. First, only a limited number of tetrapyrroles have been studied, and their structure does not initially imply obtaining high luminescent characteristics. This applies, first of all, to natural porphyrins containing a large number of $\mathrm{C}-\mathrm{H}$ bonds, the vibrations of which reduce the luminescence of $\mathrm{Ln}$ (III) ions. Secondly, spectral-luminescent studies are almost not analyzed in terms of the Ln-tetrapyrrolic structure.

\section{Porphyrin Polymers in Optoelectronics, Photo- and Electrocatalysis}

Porphyrins have many properties that allow them to be useful in various practical applications, especially in a wide range of optoelectronic and catalytic possibilities. In addition, these properties are easily modulated by incorporating various metals within the ring or by substitution effects at various places around the ring. The universality of porphyrins as optoelectronic or catalytic blocks makes them attractive elements for inclusion in functional materials. ${ }^{[566,567]}$ Polymers that include porphyrins have been created with a wide variety of structures and are used in a wide range of practical applications. The optical properties of polymers attract a lot of attention because of their potential for optoelectronic applications, such as polymer-based light emitting diodes, organic electrochemical and solar cells. It is possible to optimize the performance of optoelectronic and photonic devices by modifying polymers by introducing certain end groups, copolymerization, grafting, cross-linking, mixing
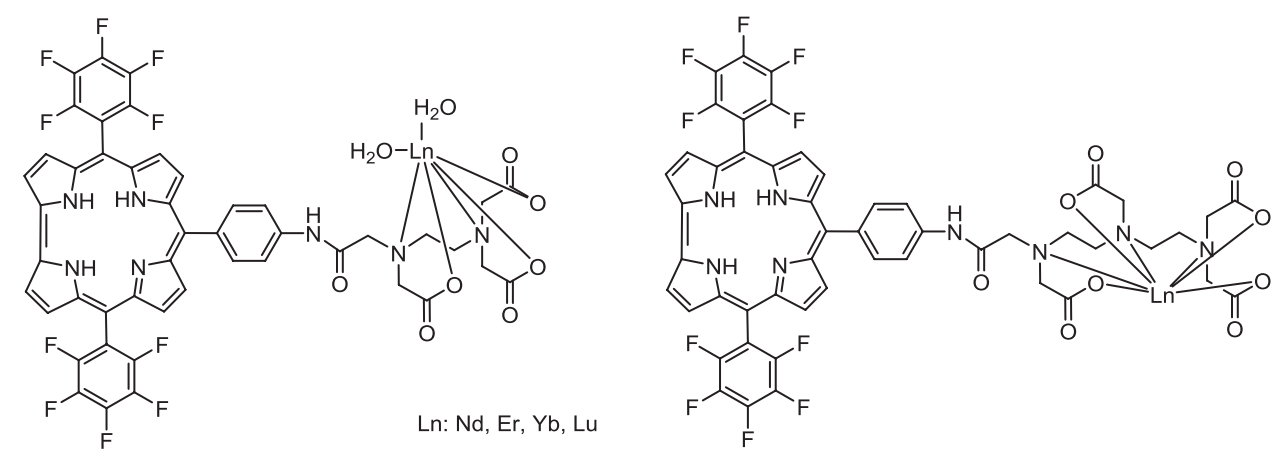

Figure 71. Structure of the lanthanide-corrole compounds based on corrole-edta (left) and corrole-dtpa (right). 
and hybridization with other inorganic polymers. All these methods affect the optical and photocatalytic properties of polymers.

In $1977^{[568,570]}$ it was found that conductive polymer polyacetylene after doping can significantly change its conductivity. Since this discovery, polymers have been widely studied as new materials for electronic and optoelectronic applications. To increase the conductivity of polymers, as well as to give them new optical and electrical properties, four doping processes were used, including chemical, photochemical, electrochemical, and interfacial doping. ${ }^{[569,570]}$ After this, polymers can become suitable materials for optoelectronic and photonic applications.

In the process of research, great progress has been made in understanding the fundamental physical and chemical properties that determine the operation of optoelectronic and photonic devices in order to optimize their effectiveness. However, the correlations between the physical and chemical properties of conductive polymers are not fully understood. One of the most important characteristics of polymers is their optical properties. The optical characteristics of the polymer, such as optical absorption, luminescence spectra, infrared dichroism, and Raman polarization are the most important tools used to study the electronic properties of polymers. ${ }^{[571-574]}$

Organic optoelectronics requires materials combining bright luminescence and efficient ambipolar charge transport. Thiophene-phenylene co-oligomers (TPCOs) are promising highly emissive materials with decent chargecarrier mobility; however, they typically show poor electron injection in devices, which is usually assigned to high energies of their lowest unoccupied molecular orbitals (LUMOs). A widely used approach to lower the frontier orbitals energy levels of a conjugated molecule is its fluorination. In this study, were synthesized three new fluorinated derivatives of one of the most popular TPCOs, 2,2'-(1,4-phenylene) bis[5-phenylthiophene] (PTPTP) and studied them by cyclic voltammetry, absorption, photoluminescence, and Raman spectroscopies. ${ }^{[575]}$ The obtained data reveal a positive effect of fluorination on the properties of PTPTP: LUMO levels are finely tuned, and photoluminescence quantum yield and absorbance are increased. The intensity of the latter subband is higher than that of the former since the central (phenylene) ring is stronger involved in $\pi$-conjugation (Figure 72), and hence its vibration has a stronger impact on it. For PFTPFTPF, only the high-frequency component

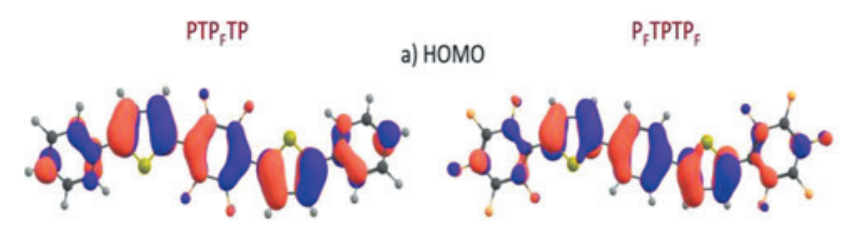

b) LUMO

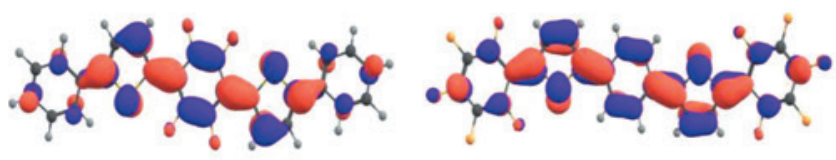

Figure 72. HOMO (a) and LUMO (b) patters for $\mathrm{PTP}_{\mathrm{F}} \mathrm{TP}$ (left) and $\mathrm{P}_{\mathrm{F}}$ TPTP $_{\mathrm{F}}$ (right). ${ }^{[575]}$

is observed, since all the phenyle(ene) rings are fluorinated.

One of the varieties of conductive polymer materials (organic semiconductors) for optoelectronic devices are porphyrin-based polymers. ${ }^{[576-584]}$ Porphyrin polymers are found in a wide range of diverse applications of optoelectronic devices, especially in photovoltaic cells or for use as photo- or electrocatalysts. There are many reports on the development of effective devices using the advantages of the well-known and well-developed optoelectronic and catalytic properties of porphyrin macrocycles. ${ }^{[55-587]}$ These works present the properties of porphyrin polymers, including photochemical characteristics in the excited state and mobility/diffusivity of free charge carriers and excited states (excitons). The effects of increasing/decreasing the molecular organization of porphyrin polymers can dramatically change the diffusion of excitons and free charge carriers. ${ }^{[585,586]}$ However, these two effects quite often mutually exclude each other. There are a number of examples in which increased exciton diffusion led to a decrease in charge mobility and a decrease in the total conversion of solar energy. ${ }^{[587]}$

Figure 73 shows a typical chemical structure of porphyrin, showing four meso- and eight $\beta$-positions (left), a map of the electrostatic potential (in the center), and boundary orbitals of the porphyrin core (right). ${ }^{[567]}$

It is well known that the porphyrin platform has a strong electron-donating ability and an extensive $\pi$-conjugated system. Charge distribution and boundary molecular orbitals (FMO) are graphically depicted in Figure 73. The electrostatic potential map (ESP) clearly showed a completely
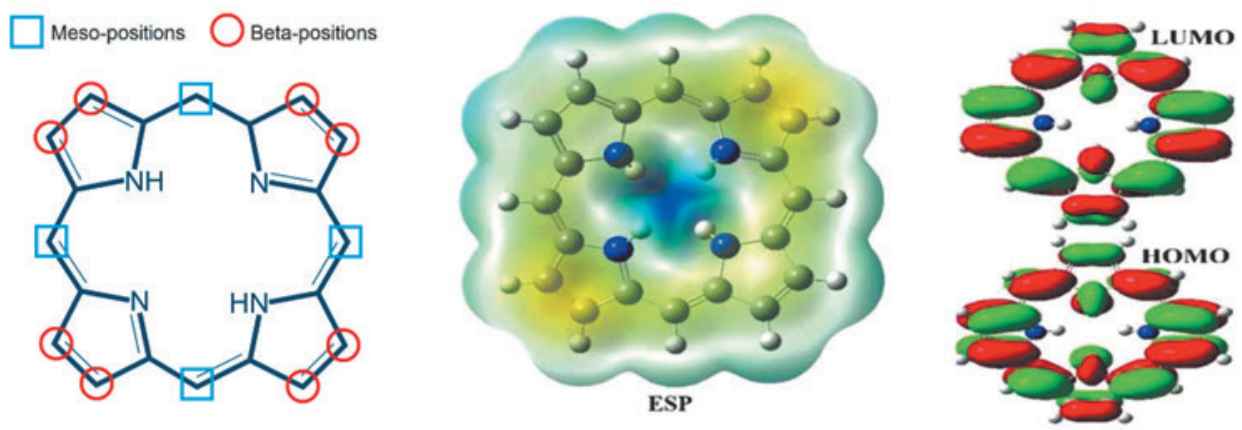

Figure 73. Typical chemical structure of porphyrin. 
green surface, indicating the electron-rich nature of the porphyrin core. The highest occupied (HOMO) and lowest unoccupied (LUMO) molecular orbitals of the distribution of the porphyrin nucleus are almost the same, therefore, electron-dependent groups are needed to transfer the charge from electron-rich porphyrin to electron-deficient chemical compounds. For the formation of organic solar cells (OSCs), the number and nature of electron-determining groups must be carefully selected for their further use as electron donors or acceptors.

As indicated in the $\operatorname{work}^{[566]}$ solution-processable organic planar/bulk heterojunction solar cell devices have several key requirements for efficient conversion of solar photons into electrical power. The active layer requires both $p$-type donor materials and $n$-type acceptors to dissociate excited states into free charge carriers that can be collected at the opposite electrode contacts. Due to the limitations of exciton diffusion in most organic materials ${ }^{[587]}$ the two materials must be intimately blended to ensure that the majority of the excited states reach the $p-n$ interface. The light-absorbing layers must therefore allow for efficient exciton diffusion to occur so that the energy of the absorbed photons can be converted before they recombine radiatively or non-radiatively. In addition, once holes and electrons are generated at that heterojunction interface, the lightabsorbing materials must also efficiently conduct free charge carriers away before they recombine or are trapped within the active layer.

Strategies to increase either hole/electron mobility or exciton diffusion length (LD) include the use of peripheral alkyl substituents or increased conjugation in larger oligomer/polymer systems. The effects of increased/decreased molecular organization can also dramatically alter the exciton/free-charge-carrier diffusion. ${ }^{[585,586]}$ However, the two effects are often not mutually exclusive, with several examples in which increased exciton diffusion led to lower charge mobility and lower overall solar conversion efficiencies. ${ }^{[587]}$

Several types of porphyrin polymer systems synthesized either chemically or electrochemically using a variety of peripheral substituents have been evaluated in photovoltaic configurations. Often these polymeric materials incorporate more readily accessible meso-substituted tetraarylporphyrins whose nonplanar structure (due to the dihedral angle between aryl groups, e.g. phenyl, thienyl, furanyl) limits good charge conduction along the backbone of the polymer. Nevertheless, many groups have investigated mesosubstituted tetraarylporphyrin materials incorporated into a polymer backbone in the main chain or as a side chain often synthesized through electropolymerization. For example, a nanostructured polymeric film of tetra( $p$-aminophenyl) porphyrin (TAPP] was electrochemically deposited onto a fluorine-doped tin oxide electrode to give $\mathrm{V}_{\mathrm{oc}} \sim 0.3 \mathrm{~V}$ and $\mathrm{J}_{\mathrm{sc}}$ $\sim 0.2 \mathrm{~mA} / \mathrm{cm}^{2}{ }^{2588]}$ This device used the nanostructured film to direct the crucial interpenetrating network between p-type material and the $n$-type acceptor $(6,6)$-phenyl$\mathrm{C}_{61}$-butyricacid methyl ester (PCBM), intended to increase the likelihood of exciton dissociation within the photoactive layer (Figure 74).

Using a poly- $\mathrm{H}_{2} \mathrm{~T}\left(p-\mathrm{NH}_{2} \mathrm{Ph}\right) \mathrm{P}$ (poly-TAPP) films deposited on an ITO electrode by the method of superoxide anion-radical deposition from solutions in dimethylsulfoxide (DMSO), ${ }^{[589]}$ we made a laboratory prototype of a photovoltaic device (OSCs) (Figure 75). ${ }^{[590]}$ An LED-A60 11 W LED lamp (spectral temperature $4000 \mathrm{~K}$, luminous flux $990 \mathrm{~lm}$ ) manufactured by ASD was used as a source of radiation in the optical range, located at a distance of $10 \mathrm{~cm}$ from the device. The operation of the photovoltaic device is based on the excitation of excitons by radiation, the redistribution of which leads to a change in the potential of the working electrode in solution $\left(0.5 \mathrm{M} \mathrm{KCl}+0.001 \mathrm{M} \mathrm{K}_{3}\left(\mathrm{Fe}(\mathrm{CN})_{6}\right)\right.$, measured relative to the $\mathrm{Ag} / \mathrm{Ag}^{+}$reference electrode.

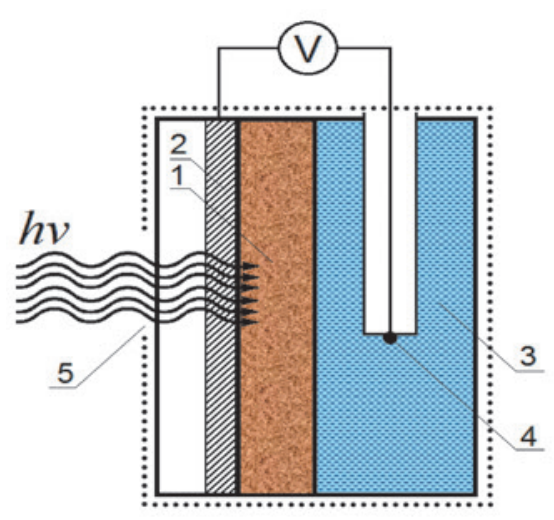

Figure 75. Prototype photovoltaic device. The numbers indicate: $1-$ poly- $\mathrm{H}_{2} \mathrm{~T}\left(p-\mathrm{NH}_{2} \mathrm{Ph}\right) \mathrm{P}$ film, 2 - transparent ITO electrode, 3 - electrolyte $(0.5 \mathrm{M} \mathrm{KCl}+$ $0.001 \mathrm{M} \mathrm{K}_{3}\left(\mathrm{Fe}(\mathrm{CN})_{6}\right), 4-\mathrm{Ag} / \mathrm{Ag}+$ reference electrode, $5-$ a window for visible light enters the device.

As our studies have shown, the stationary state of a photovoltaic device is achieved in no more than 30 minutes, after which reproducible operation of the device is possible.

The stationary potential of the working electrode in dark conditions and under illumination differs by $1.3 \mathrm{mV}$
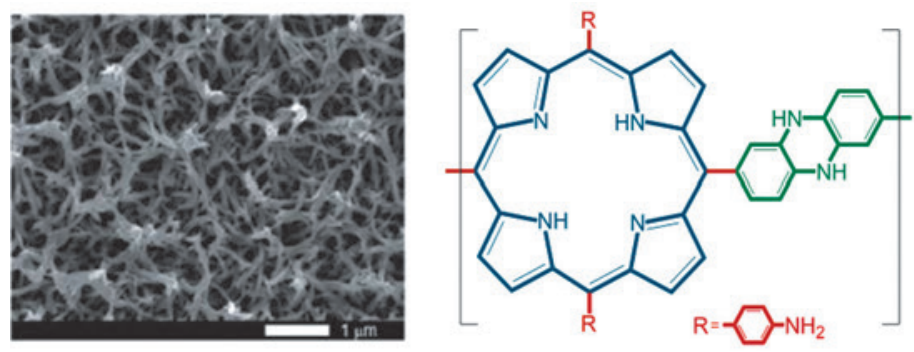

Poly-TAPP:PCBM - OSC

$V_{o c}=0.30 \mathrm{~V}$

$J_{s c}=0.18 \mathrm{~mA} / \mathrm{cm}^{2}$

$\mathrm{ff}=0.29$

$\eta=0.02 \%$

Figure 74. Electropolymerized poly-TAPP nanofibrous films and photovoltaic properties of the films incorporated with PCBM. ${ }^{[588]}$ 

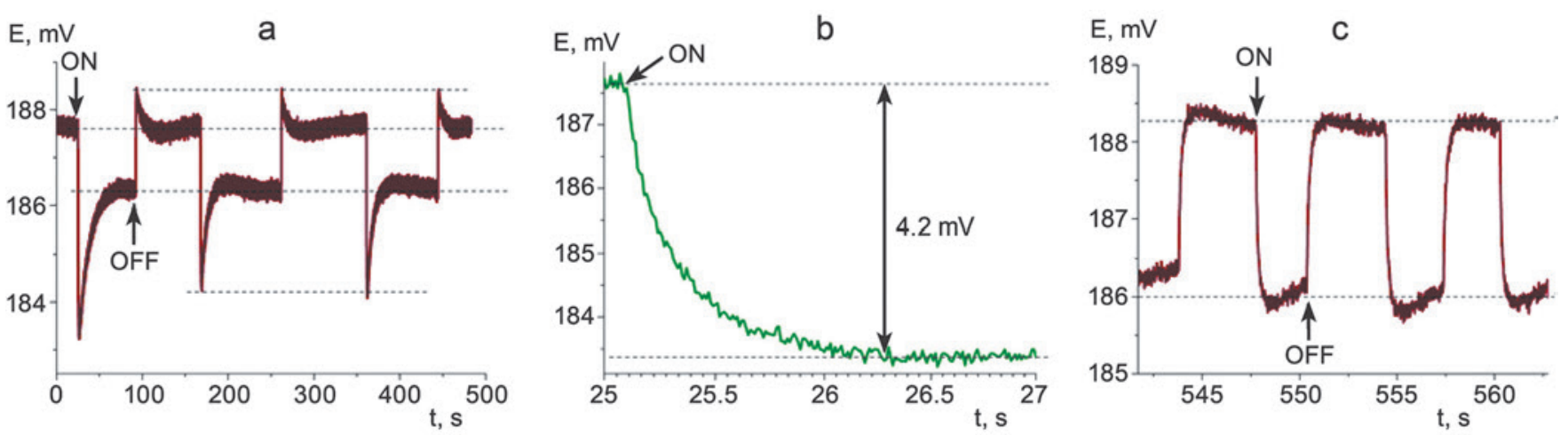

Figure 76. Photovoltaic responses of the OSCs of the device: a) switching with a long exposure of the device in dark and lit conditions and reaching the potential at a stationary value, b) changing the potential after changing the illumination to an extreme value, c) changing the potential during short-term $\mathrm{OFF} / \mathrm{ON}$ switching.

(Figure 76a). The duration of the ON/OFF and OFF/ON transients is about 50 seconds, due to the diffusion of charge carriers and excitons in the film and in solution. During the transition process, the dependence of the potential on time has an extremum, both when the light is turned on and when it is turned off. For ON/OFF and OFF/ON processes, the time to reach the extreme value of the potential is about 1 second (Figure 76b), which is comparable with the duration of the transient processes in the radiation source. The amplitude of the potential change of the OFF/ON transition after a long stay of the device in dark conditions exceeds $4 \mathrm{mV}$. During short-term switching OFF/ON .... OFF/ON (Figure 76c), the potential-time dependence profile has a U-shaped shape, the potential change upon transition from the OFF state to the $\mathrm{ON}$ state is about $2.3 \mathrm{mV}$.

We believe that the main contribution to ON/OFF switching is made by the polymer film deposited on the working electrode. In this case, the switching can be due to the photogeneration of free charge carriers and their redistribution in the film and solution. In the absence of illumination, the stationary potential is formed due to the cathodic process of reduction of ferricyanide ions and the polymer film. When illuminated, the polymer is excited and the electron is transferred from the donor fragment to the acceptor one. As a result, positively and negatively charged centers arise due to the appearance of cation and anion radicals. Further, ferricyanide ions can be reduced by receiving electrons from negatively charged centers, turning into ferrocyanide ions. These ions diffuse into the solution volume and do not affect the electrode potential. The radical cations formed on the electrode surface are easily oxidized and provide a potential shift in the positive direction. There are few radical cations and their "lifetime" is relatively small. Therefore, the potential shift is of little importance. Ferrocyanide ions, due to their large size and low mobility, do not go far from the electrode. When you turn off the light, i.e. reverse switching, the reverse process of electron transfer occurs with the system returning to its original state.

Although porphyrin polymer systems exhibit reduced carrier transport properties, their excellent diffusion ability and well-known catalytic properties continued to stimulate further research into the use of porphyrin polymer systems as photocatalysts.
Research continues on the use of coordination polymer systems based on organometallic (MOFs) and covalent organic scaffolds (COF) as photocatalysts. ${ }^{\left[{ }^{[91,592]}\right.}$ The use of porous porphyrin polymeric materials for catalytic applications is very attractive due to their large surface area, strong light absorption and the possibility of simultaneous formation and storage of the product. A number of recent studies have shown the possibility of using these materials as catalysts for various reactions, including photoelectrolysis of water, which, however, require the presence of water-resistant porphyrin MOF structures. Water-resistant TCPP-based MOF porphyrin (tetrakis(4-carboxyphenyl) porphyrin) (Figure 77) showed evidence of photocatalytic hydrogen evolution using a sacrificial electron donor (ethylenediaminetetraacetic acid) and an acceptor (methyl viologen) to transfer electrons to a colloidal catalyst based platinum for hydrogen evolution. ${ }^{[593]}$ The quantum yields of the system were low, less than $0.01 \%$, probably due to the inability of donor and acceptor materials to diffuse into the MOF structure of porphyrin.

The limiting factor for this system may be the moderate rate of charge transfer through the MOF structure. To improve the performance of these materials, recently developed systems must also exhibit high charge mobility in order to keep up with the rate of catalysis.

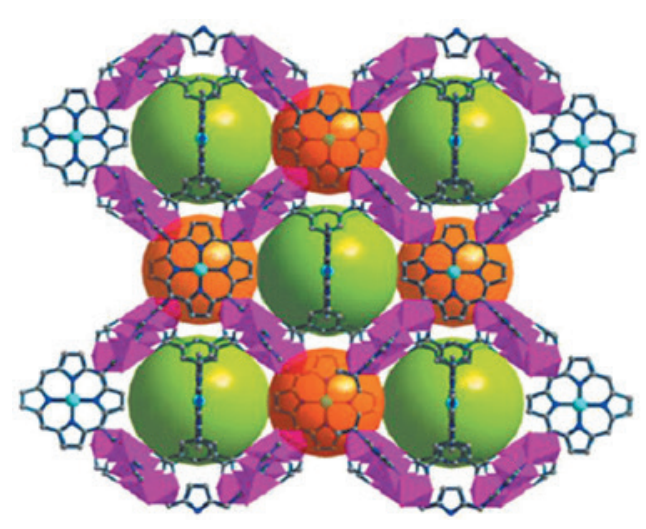

Figure 77. MOF structure of trans-biCPP (gray) coordinated with a variety of metal complexes (purple). Orange and green spheres are used to emphasize cavities available for catalysis. ${ }^{[593]}$ 
As mentioned above, the number and nature of electrondetermining groups must be carefully selected when using porphyrins as precursors for the process of electropolymerization in the electrochemical production of polyporphyrin materials.

A number of our works ${ }^{[594-601]}$ considered the effect of the structure of porphyrin precursors on the processes of film formation and electrocatalytic characteristics of the materials obtained in the reactions of oxygen electroreduction in an alkaline aqueous medium. Reactions of the electrochemical reduction of oxygen in an alkaline medium: four-electron reduction — reaction (11), a series of two-electron reductions - reactions (12) and (13).

$$
\begin{aligned}
& \mathrm{O}_{2}+2 \mathrm{H}_{2} \mathrm{O}+4 \overline{\mathrm{e}} \rightarrow 4 \mathrm{OH}^{-} \\
& \mathrm{O}_{2}+\mathrm{H}_{2} \mathrm{O}+2 \overline{\mathrm{e}} \rightarrow \mathrm{HO}_{2}^{-}+\mathrm{OH}^{-} \\
& \mathrm{HO}_{2}^{-}+\mathrm{H}_{2} \mathrm{O}+2 \overline{\mathrm{e}} \rightarrow 3 \mathrm{OH}^{-}
\end{aligned}
$$

According to experimental results obtained by electrochemical reduction of dissolved oxygen in $0.1 \mathrm{M} \mathrm{KOH}$ on various surfaces for films deposited from DMSO (Figure 77), the beginning of the electrochemical process, current peak potentials, and half-peak potentials are shifted in the positive direction in the presence of polyporphyrin films glassy carbon electrode surface. In addition, in the presence of films of both poly-aminophenylporphyrins and the presence of poly-hydroxyphenylporphyrins, increases in oxygen electroreduction currents are observed at low overvoltages (from -0.35 to $-0.60 \mathrm{~V}$ relative to $\mathrm{Hg} / \mathrm{Hg}_{2} \mathrm{Cl}_{2}, \mathrm{Cl}^{-}(1 \mathrm{M} \mathrm{LiCl})$ ) At the same time, in the potential range from -0.6 to $-0.8 \mathrm{~V}$, lower current densities are observed on polyporphyrin films than on the initial SU. Analyzing the data obtained, it was taken into account that in alkaline electrolytes oxygen can undergo direct four-electron reduction according to reaction (11) or a series of two-electron reduction processes according to reactions (12) and (13). ${ }^{[603]}$ Two-electron electroreduction often occurs on carbon electrodes. Further, the hydroperoxyl ion can be reduced to water at more negative potentials. ${ }^{[603,604]}$

The mechanism of catalytic oxygen reduction, as a rule, involves the formation of a mediator complex between oxygen and the catalyst. The configuration and energy

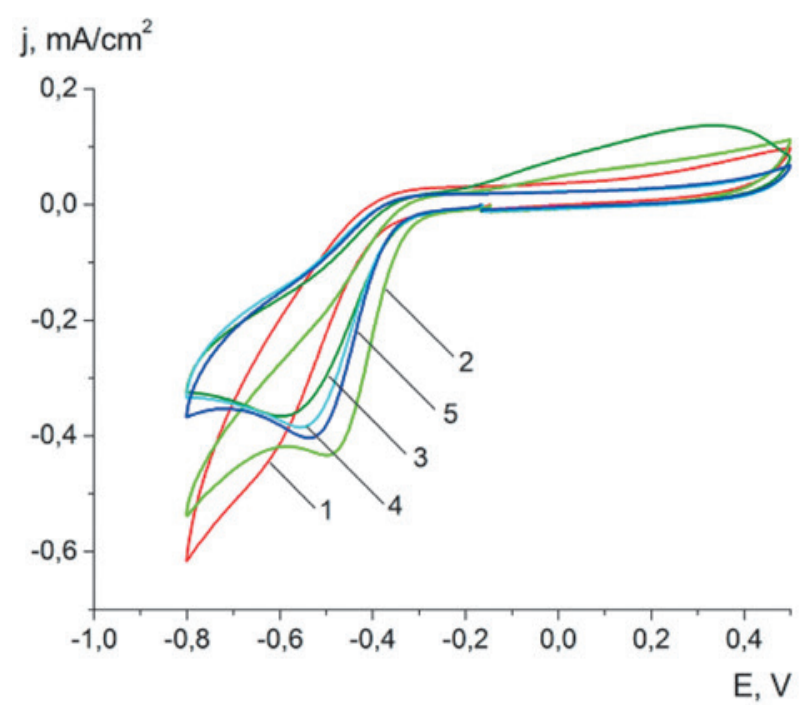

Figure 78. CVA of oxygen electroreduction process in $0.1 \mathrm{M}$ $\mathrm{KOH}$ on various surfaces: 1 - glassy carbon (GC), 2 - GC coated

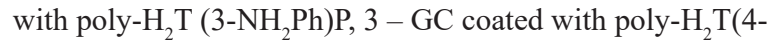
$\left.\mathrm{NH}_{2} \mathrm{Ph}\right) \mathrm{P}, 4-\mathrm{GC}$ coated with poly-H $\mathrm{H}_{2} \mathrm{~T}(3-\mathrm{OHPh}) \mathrm{P}, 5-\mathrm{GC}$ coated with poly- $\mathrm{H}_{2} \mathrm{~T}(4-\mathrm{OHPh}) \mathrm{P}$. The potential sweep rate is $20 \mathrm{mV} / \mathrm{s}$, the potentials are measured relative to $\mathrm{Hg} / \mathrm{Hg}_{2} \mathrm{Cl}_{2}, \mathrm{Cl}^{-}(1 \mathrm{M} \mathrm{LiCl})$.

parameters of this complex depend on the axial ligands and peripheral substituents. ${ }^{[602]}$

The obtained profiles of $J-E$ curves (Figure 78) allow us to conclude that two-electron reactions (12) and (13) are observed on the surface of polyporphyrin films, as well as on GC. The shift in the beginning of the electrochemical process, the potentials of the current peak $E_{\mathrm{p}}$ and the potentials of the half-peak $E_{1 / 2}$, and an increase in the current density in the potential range from -0.35 to $-0.60 \mathrm{~V}$ indicate a catalytic mechanism of the reaction (12). In the potential range from -0.5 to $-0.7 \mathrm{~V}$, the lower value of the current on polyporphyrin films can be explained by the inhibition of reaction (13), which reduces the total current of oxygen electroreduction.

The influence of the structure of porphyrin and the film deposition conditions on the parameters of the process of oxygen electroreduction on polyporphyrin films is pre-

\begin{tabular}{|c|c|c|c|c|}
\hline Electrode surface & $E_{\text {onset }}$ & $E_{\mathrm{p}}, \mathrm{V}$ & $E_{1 / 2}, \mathrm{~V}$ & $I_{\mathrm{p},} \mathrm{mA} / \mathrm{sm}^{2}$ \\
\hline & \multicolumn{4}{|c|}{ Method of superoxide-initiated radical anion precipitation from solutions in DMSO } \\
\hline pure GC & -0.38 & -0.589 & -0.480 & -0.396 \\
\hline poly- $\mathrm{H}_{2} \mathrm{~T}\left(3-\mathrm{NH}_{2} \mathrm{Ph}\right) \mathrm{P}$ & -0.31 & -0.499 & -0.395 & -0.433 \\
\hline poly- $\mathrm{H}_{2} \mathrm{~T}\left(4-\mathrm{NH}_{2} \mathrm{Ph}\right) \mathrm{P}$ & -0.34 & -0.593 & -0.432 & -0.366 \\
\hline poly- $\mathrm{H}_{2} \mathrm{~T}(3-\mathrm{OHPh}) \mathrm{P}$ & -0.34 & -0.553 & -0.433 & -0.385 \\
\hline \multirow[t]{2}{*}{ poly-H $\mathrm{H}_{2} \mathrm{~T}(4-\mathrm{OHPh}) \mathrm{P}$} & -0.34 & -0.537 & -0.428 & -0.403 \\
\hline & \multicolumn{4}{|c|}{ Method for the electrochemical oxidation of a precursor } \\
\hline poly- $\mathrm{H}_{2} \mathrm{~T}\left(4-\mathrm{NH}_{2} \mathrm{Ph}\right) \mathrm{P}$ & -0.33 & -0.56 & -0.46 & -0.68 \\
\hline poly- $\mathrm{H}_{2} \mathrm{~T}(4-\mathrm{OHPh}) \mathrm{P}$ & -0.33 & -0.59 & -0.48 & -0.77 \\
\hline
\end{tabular}

Table 7. The effect of porphyrin structure and deposition conditions on the characteristics of the deposited electrode material in the reaction of electrochemical reduction of oxygen in an alkaline medium. Films are deposited on glassy carbon (GC). 
sented in Table 7. Based on the magnitude of the half-peak potential, we obtained the following series of catalytic activity of polyporphyrin materials. For films of hydroxyand aminophenylporphyrins obtained by the method of superoxide-initiated radical anion deposition from solutions in DMSO: poly- $\mathrm{H}_{2} \mathrm{~T}\left(3-\mathrm{NH}_{2} \mathrm{Ph}\right) \mathrm{P}>$ poly- $\mathrm{H}_{2} \mathrm{~T}(4-$ $\mathrm{OHPh}) \mathrm{P}>$ poly- $\mathrm{H}_{2} \mathrm{~T}\left(4-\mathrm{NH}_{2} \mathrm{Ph}\right) \mathrm{P}>$ poly- $\mathrm{H}_{2} \mathrm{~T}(3-\mathrm{OHPh}) \mathrm{P}$. For films of hydroxy- and aminophenylporphyrins obtained by the electrochemical oxidation of a precursor: poly- $\mathrm{H}_{2} \mathrm{~T}(4-$ $\left.\mathrm{NH}_{2} \mathrm{Ph}\right) \mathrm{P}>$ poly- $\mathrm{H}_{2} \mathrm{~T}(4-\mathrm{OHPh}) \mathrm{P}$.

The characteristics of the electroreduction of oxygen on poly- $\mathrm{H}_{2} \mathrm{~T}(4-\mathrm{OHPh}) \mathrm{P}$ and poly- $\mathrm{H}_{2} \mathrm{~T}\left(4-\mathrm{NH}_{2} \mathrm{Ph}\right) \mathrm{P}$ depend on the precursor structure, both during the deposition of the polymer from DMSO and during the deposition from dichloromethane and ethanol. For materials obtained by direct oxidation of a precursor and for materials obtained by the method of superoxide-initiated precipitation by anion radical from DMSO solutions, a different order of polyporphyrins in the series of catalytic activity was determined, which indicates a significant effect of the deposition conditions on the catalytic properties of the material.

Thus, an analysis of the oxygen electroreduction process on the obtained materials confirms the significant effect on the catalytic properties of both the structure of the porphyrin precursor and the conditions for the formation of the polymer. In addition, the result allows us to consider materials synthesized on the basis of porphyrin ligands as promising metal-free electrocatalysts.

Analysis of the results shows the possibility of using porphyrin-based polymers in a number of practical applications, such as optoelectronics, photo- and electrocatalysis. A brief analysis of scientific reports on the developed effective devices using the advantages of the well-known and welldeveloped optoelectronic and catalytic properties of porphyrin macrocycles is given. A diagram of our laboratory prototype photovoltaic device (OSCs) using a poly- $\mathrm{H}_{2} \mathrm{~T}\left(p-\mathrm{NH}_{2} \mathrm{Ph}\right) \mathrm{P}$ film deposited on an ITO electrode by the method of superoxideinitiated precipitation from solutions in dimethylsulfoxide is presented. As our studies have shown, the stationary state of a photovoltaic device is achieved in no more than $30 \mathrm{~min}$ utes, after which OSCs can operate stably and reproducibly. The influence of the structure of porphyrin precursors and the conditions of film formation on the electrocatalytic characteristics of the obtained material in the reactions of oxygen electroreduction in an alkaline aqueous medium is considered. An analysis of the oxygen electroreduction process on the obtained materials confirms a significant effect on the catalytic properties of both the structure of the porphyrin precursor and the polymer formation conditions. In addition, the results obtained make it possible to consider materials based on porphyrin ligands as promising metal-free electrocatalysts.

\section{Phthalocyanine Based Molecular Systems for Catalytic Purposes}

Catalysis is known as one of the broadest and most promising areas of macroheterocyclic compounds application. Due to the unique physicochemical properties and high chemical stability under various conditions, phthalocyanine macrocycles and materials based on them are of considerable interest as promising solar cells, ${ }^{[605,606]}$ semiconductor materials, ${ }^{[607,608]}$ photosensitizers, ${ }^{[609,610]}$ gas sensors, ${ }^{[611,612]}$ and catalysts. ${ }^{[613,614]}$ It is known ${ }^{[615]}$ that, due to the low solubility in organic and aqueous media, the range of application of these compounds is significantly limited. However, by introducing functional substituents of various nature into the annulated benzene rings of the phthalocyanine macromolecule, the solubility in media, especially in aqueous, is significantly increased. The consideration of phthalocyanine macrocycles as a key link in the creation of new functional materials and molecular devices for oxidation and reduction processes involving inorganic and organic molecules is considered in this section.

The catalysis of redox processes in aqueous solutions of inorganic complex salts by phthalocyanine macrocycles allows reactions to be carried out under mild conditions. For example, in ${ }^{[616]}$, a study was made of the process of water oxidation, where copper(II) tetrasulfophthalocyaninate (CuPcTS) was used as a catalyst. The authors of ${ }^{[616]}$ have found that the oxidation rate reaches its maximum at $\mathrm{pH}=9.5$ and sharply decreases upon transition to a more acidic or more alkaline environment, demonstrating a $\mathrm{pH}$-dependent activity. Kinetic studies showed the rate of water oxidation, where CuPcTS acts as a catalyst, has a second-order dependence on the concentration of the catalyst, showing that the oxidation passes through a bimolecular pathway of radical binding. Extreme dependences of the catalytic activity ${ }^{[617,618]}$ of a number of sulfo- derivatives of copper and cobalt phthalocyanates, which act as homogeneous liquid phase catalysts for the oxidation of sulfur compounds to disulfides and as a part of hybrid materials, were shown. It was found that competing processes ${ }^{[619]}$ dominate the coordination of hydroxide ions with the central cation with respect to substrates in alkaline media and enhance aggregation processes in acidic media. ${ }^{[620,621]}$ Catalytic activity maximum of the phthalocyanine macrocycle was in the $\mathrm{pH}$ range from 9 to 10 for all the systems described.

The classical water-soluble cobalt(II) tetrasulfophthalocyaninate (Co(II)PcTS) can also act as a catalyst in the reduction reactions, for example, of selenite $\left(\mathrm{SeO}_{3}\right)^{2-}$, ${ }^{617]}$ which is one of the most toxic forms of selenium, to slightly toxic $\mathrm{Se}^{0}$ using dithionite ion $\left(\mathrm{S}_{2} \mathrm{O}_{4}\right)^{2-}$ in a neutral aqueous solution. In ${ }^{[617]}$, this process was compared in the presence of phthalocyanine catalyst and without it. It was shown the presence of a catalyst changes the shape of the kinetic curve from an exponential, in the case of a non-catalytic reaction, to a straight line, which indicates a zero order of reaction with respect to $\left(\mathrm{S}_{2} \mathrm{O}_{4}\right)^{2-}$. The mechanism of this process was determined. ${ }^{[617]}$ The results indicate the importance of the further development of phthalocyanine-based catalysts for the deactivation of toxic selenium-based ions.

Nevertheless, the largest number of works is devoted to catalytic reactions involving both small and more complex organic molecules. ${ }^{[622-624]}$ Researchers ${ }^{[625]}$ obtained watersoluble tetracarboxyphthalocyanine and its metal complexes with $\mathrm{Cu}(\mathrm{II}), \mathrm{Fe}(\mathrm{II}), \mathrm{Co}(\mathrm{II})$ for studying the catalytic activity in the cyclohexene epoxidation reaction (Scheme 13). It was shown that metal-complex compounds exhibit higher catalytic activity with a cyclohexene oxide yield in the range 
from $50 \%$ to $58 \%$. Using a free phthalocyanine ligand as a catalyst, cyclohexene oxide was obtained in an yield of about $30 \%$. The key mechanism of the process, which includes the adsorption of molecular oxygen at intermediate with the participation of the phthalocyanine complex, was determined. ${ }^{[621]}$

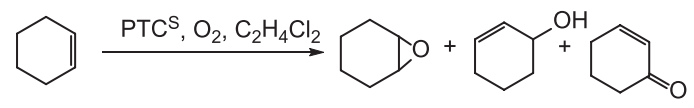

Scheme 13. Scheme of the cyclohexene epoxidation reaction using phthalocyanine derivatives as a catalyst. ${ }^{[625]}$

Aggregation of phthalocyanines in solutions ${ }^{[626]}$ is a significant factor reducing their catalytic activity, for example, during the liquid-phase oxidation of phenol and its derivatives. Meanwhile, pollution with phenolic substances is a rather serious environmental problem due to their high toxicity and high diffusion in the aquatic environment. ${ }^{[627-629]}$ To suppress the aggregation process, the authors of ${ }^{[630]}$ obtained sterically hindered water-soluble complexes of iron phthalocyanine with carboxyphenyloxy- and sulfoquinoline- fragments at the periphery. Both complexes showed high catalytic activity in the oxidation reactions of various phenols in the presence of an oxidizing agent tert-butyl hydroperoxide. Under optimal conditions, the oxidation of 2-chlorophenol takes place over 10 minutes with a conversion of more than $90 \%$. The mechanism of this process includes the formation of a peroxo complex with iron phthalocyaninate (Scheme 14). It should be noted this mechanism in the general case can be extended to the processes of oxidation of other organic substrates in the presence of cobalt phthalocyanines carboxylic acids. ${ }^{[631,632]}$

Meanwhile, complexes of $\mathrm{Co}(\mathrm{II})$ and $\mathrm{Cu}(\mathrm{II})$ with phthalocyanine macrocycles of various nature, having

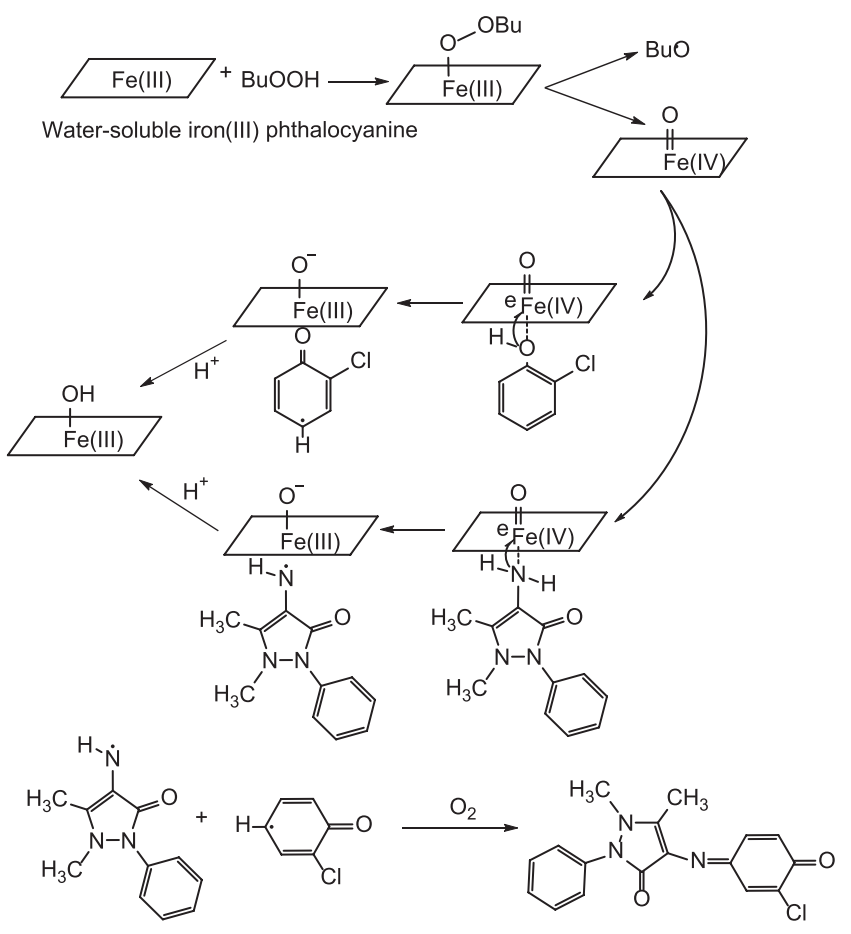

Scheme 14. The mechanism of conversion of chlorophenols into dyes in the presence of a complex of phthalocyanine and $t$-BuOOH. ${ }^{[630]}$

an active center $\mathrm{M}-\mathrm{N}_{4}$, are the most promising catalysts for oxidation processes, for example, 4-nitrophenol, which is also an important environmental pollutant. For this purpose, water-soluble ionic derivatives of 4-(pyridin-4-yl) phenyloxy-substituted complexes with copper and cobalt, which demonstrate H-aggregation, were obtained in ${ }^{[633]}$. The catalytic activity was investigated without the usage of any oxidizing agents, however, the samples were irradiated with light. It was established ${ }^{[633]}$ that compounds with

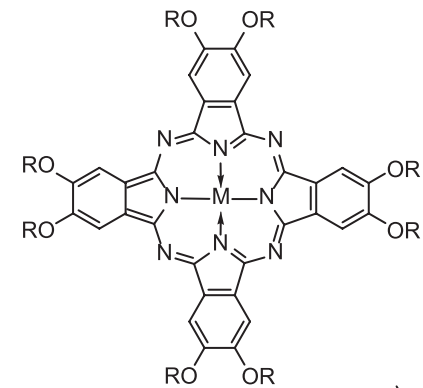

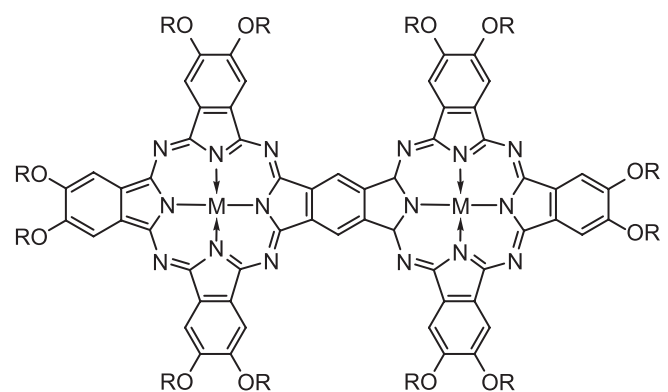<smiles>[R]c1c(C)cccc1C</smiles>
$M=\operatorname{Co}(I I), Z n(I I)$
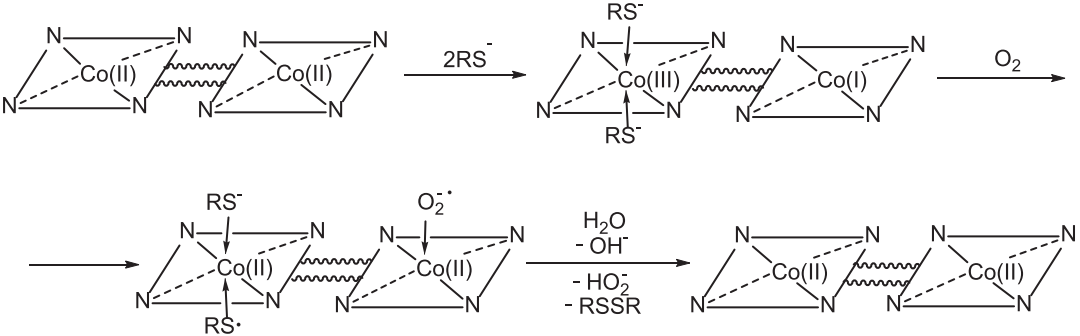

Figure 79. Structure of mono- and binuclear phthalocyanine catalysts and the oxidation mechanism of 2 -mercaptoethanol. ${ }^{[635]}$ 
$\mathrm{Cu}(\mathrm{II})$ and $\mathrm{Co}(\mathrm{II})$, used as photocatalysts in the photooxidation of 4-nitrophenol, showed a conversion of $95 \%$ and $97 \%$, respectively. As reaction products, non-toxic hydroquinone (the main product) and benzoquinone (a by-product) were formd ${ }^{[633]}$ and it was found that the photocatalytic activity for the complex with cobalt gradually decreased from $97 \%$ to $80.3 \%$ with repeated use and to $72 \%$ after the seventh cycle. This was due to the fact that the macrocycle also is decomposed during photooxidation.

Another direction of catalysis is associated with the use of phthalocyanine complexes in the reactions of the formation of chain compounds by oxidation, in particular, of thiols to practically useful disulfides. The process of desulfurization of various oil fractions is industrially important, since sulfur compounds can cause corrosion of equipment, have a choking effect on humans. Cyanosubstituted phthalocyanines are of particular interest in these processes, since they are precursors of polyphthalocyanine structures covalently linked through the periphery. Such compounds open up the possibility of obtaining more complex multinuclear structures in order to increase catalytic activity. So, in the work ${ }^{[634]}$ tetrakis(dicyanophenoxy) phthalocyaninates of copper, cobalt, nickel were obtained and their catalytic activity was studied. It was shown that the liquid-phase oxidation of sodium $N, N$-carbomodithiolate in the presence of such phthalocyanines proceeds more selectively than non-catalytic oxidation. As expected, complexes with cobalt exhibit higher catalytic activity due to a larger partially uncompensated charge on the central cation of the metal of the macroring. Nickel phthalocyanine turned out to be the most catalytically disadvantageous due to the high coordination saturation of the bonds between the metal and the macrocycle, which leads to a low ability to coordinate additional axial ligands. It was noted that copper and zinc complexes showed unexpectedly close activity, which is associated with the steric and electronic action of peripheral substituents that act on the macrocyclic core.

In ${ }^{[635]}$, the authors proved the transition from single to dual core cobalt phthalocyanine complexes (Figure 79), which are connected via a covalently bonded bridge, showed a sharp increase in the catalytic activity of the macrocycle during the oxidation of mercaptans. This fact is explained by the fact that two metal centers work together (one center for the reduction of $\mathrm{O}_{2}$, and the another one for the mercaptan oxidation). The highest oxygen consumption for the oxidation of 2-mercaptoethanol was recorded for the binuclear cobalt complex at $\mathrm{n}=0.25 \mu \mathrm{mol}$ and $\mathrm{n}=0.05 \mu \mathrm{mol}$. The lower TOF values obtained in ${ }^{[635]}$ at a higher catalyst concentration are explained by the slow diffusion of oxygen, whereas at significantly lower TOF concentrations it decreases due to the adsorption of a small amount of catalyst on the walls of the reactor. On the other hand, for phthalocyanines containing a closed zinc shell, the catalytic activity was negligible. As in ${ }^{[634,636,637]}$, the authors adhere to the coordinationadsorption mechanism.

Meanwhile, the authors of ${ }^{[638]}$ used a complex of cobalt phthalocyanine with $\mathrm{N}$-benzylated tetrasulfonamide moieties on the periphery as a catalyst in the oxidation of substituted thiol with aryl moieties, dibenzothiophene, using hydrogen peroxide as an oxidizing agent. This complex showed the best catalytic activity in the desulfination reac- tion of the aryl analogue as compared with unsubstituted cobalt phthalocyanine and with cobalt tetrasulfonamidesubstituted phthalocyanine. Under optimized conditions, the maximum removal of dibenzothiophene was $85 \%$.

Despite the fact that complexes based on a phthalocyanine macrocycle with transition metals, such as iron, nickel, cobalt, demonstrate high catalytic activity, for example, in fuel cells, the substituted ligand complexes with alkaline earth metals, for example, with magnesium, have not been studied as potential catalytic systems although they are environmentally friendly and inexpensive. There are also reports ${ }^{[639]}$ on $\mathrm{MgO}$ can be used as a catalyst in the oxygen reduction reaction. Therefore, the researchers, ${ }^{[640]}$ using quantum chemical calculations (DFT method), analyzed the possibility of using a number of complexes, namely $\operatorname{MgPc}(\mathrm{H})_{16}, \quad \operatorname{MgPc}(\mathrm{OH})_{16}, \quad \operatorname{MgPc}(\mathrm{COOH})_{16}, \quad$ as catalysts in the oxygen reduction reaction. It has been found for functionalized derivatives of $\mathrm{MgPc}$, catalytic activity is enhanced due to a decrease in free energy. Adsorption energy and reversible potential values indicate functionalization with $\mathrm{OH}$ fragments contributes to a good response for catalytic applications. In catalytic systems, it is possible to use not only symmetrically substituted phthalocyanine complexes. The authors of [641] obtained a water-soluble zinc phthalocyanine complex with long-chain saturated fragments at the periphery, where one of the substituents was replaced by an ethoxyhydroxy- fragment, thereby increasing the polarity of the molecule to make it soluble in water. The catalytic activity was tested with hydrogen peroxide in the oxidation reaction of rhodamine $\mathrm{B}$, which is an organic dye that is toxic to humans. As follows from the experimental data, a system of a phthalocyanine catalyst and hydrogen peroxide is capable of destroying $85 \%$ of the dye in 180 minutes.

The introduction of peripheral substituents into the phthalocyanine macrocycle not only gives solubility in the media, but also allows one to obtain various hybrid materials, and the fixation of molecules occurs due to the presence of functional peripheral/non-peripheral substituents. In ${ }^{[642,643]}$, materials for the electrochemical reduction of $\mathrm{CO}_{2}$ were obtained by noncovalent interaction of phthalocyanine complexes with carbon nanotubes. In ${ }^{[642]}$, the unsubstituted complex of phthalocyanine with cobalt shows a lower Faraday efficiency of $88 \%$ compared to the complex of nickel with pyrrolidone peripheral fragments, which reached almost $100 \%$ in a wide range of potentials. The authors of ${ }^{[643]}$ argue that, most likely, the pyrrolidone groups grafted with alkyl chains actually contribute to the reduction of $\mathrm{CO}_{2}$ due to a more uniform distribution of the nickel complex on the surface of the carbon nanotube, thus eliminating aggregation processes that reduce the availability of active centers, as well as for providing local hydrophilic environment to rich the reactants. Carbon nanotubes were also used in ${ }^{[644-646]}$.

The usage of $\mathrm{TiO}_{2}$ as a substrate is quite favorable, since $\mathrm{TiO}_{2}$ is quite inexpensive, non-toxic, stable for a long time. To this end, researchers ${ }^{[647]}$ obtained hybrid materials with carboxy-functionalized $\mathrm{Co}(\mathrm{II})$ or $\mathrm{Zn}$ (II) phthalocyanines, which are attached to the surface of $\mathrm{TiO}_{2}$ via $\mathrm{CO}-\mathrm{O}-\mathrm{TiO}_{2}$ bonds for use in the decomposition of 4-chlorophenol in aqueous media in the presence of visible radiation. The fixation 
of metal phthalocyanines on $\mathrm{TiO}_{2}$ was carried out by impregnation with a solution of complexes in DMSO. Catalysts showed increased decomposition activity of 4-chlorophenol compared to the activity of pure $\mathrm{TiO}_{2}$. The amount of 4-chlorophenol remaining was determined using an internal standard (phenol) using gas chromatography-mass spectrometry, and $99 \%$ decomposition of 4-chlorophenol was observed during the first 30 minutes. In another work ${ }^{[648]}$, quaternized derivatives of zinc phthalocyaninate (at peripheral or nonperipheral positions) with $\mathrm{TiO}_{2}$ were used in model reactions of removal of $\mathrm{Cr}(\mathrm{VI})$ ions from water samples. The use of quaternized functional groups (Figure 80) facilitates electron transfer and also provides a bathochromic shift

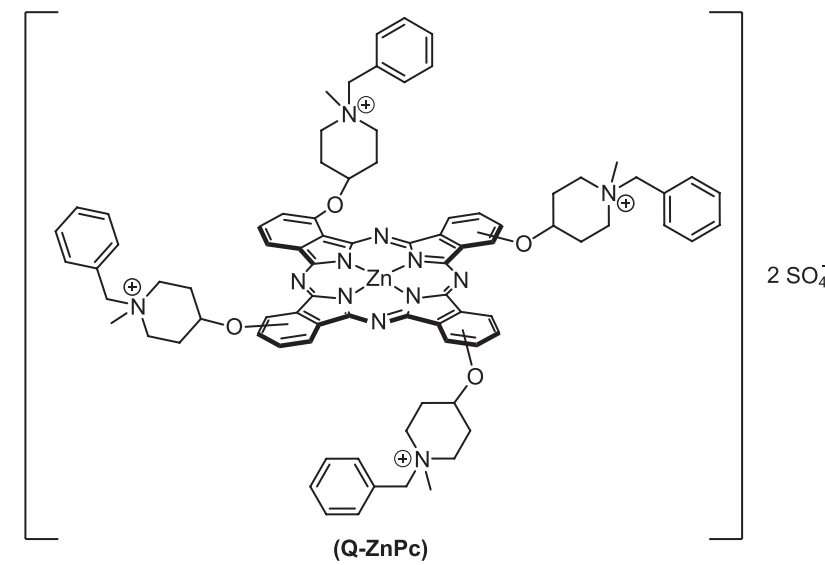

Figure 80. Chemical structure of zinc phthalocyaninate derivatives with quaternized substituents. ${ }^{[648]}$ of absorption maxima. It was shown using a non-peripheral substituted complex in the dark after $150 \mathrm{~min}$, the adsorption of $\mathrm{Cr}(\mathrm{VI})$ reached $62-60 \%$, which is a fairly high rate. Removal of $\mathrm{Cr}(\mathrm{VI})$ up to $97 \%$ was achieved in a relatively short period (30 min) using irradiation.

In ${ }^{[649]}$, porous materials based on copper and cobalt tetrasulfophthalocyanines were obtained by depositing them on $\mathrm{SiO}_{2}$ with an acidified chitosan polymer (Scheme 15) for use in the oxygen reduction reaction. It was shown the obtained material demonstrates highly effective catalytic characteristics in the oxygen reduction reaction in comparison with the $\mathrm{Pt} / \mathrm{C}$ electrode, and also competes with other systems of base metals in a $0.1 \mathrm{M} \mathrm{KOH}$ solution. The increase in catalytic activity in the oxygen reduction reaction was promoted by high porous properties and a high surface area of the obtained material. The mixed use of sulfonated derivatives of phthalocyanine $\mathrm{Cu}$ and $\mathrm{Co}$ helps to balance the density of active centers of electron transport and doping defects.

Using a coprecipitation method, researchers ${ }^{[650]}$ obtained a new catalyst by immobilizing water-soluble cobalt phthalocyanine with cyanuric chloride fragments in annulated benzene rings on $\mathrm{Mg}-\mathrm{Al}$ hydrotalcite in the decomposition of methylene blue in the presence of $\mathrm{H}_{2} \mathrm{O}_{2}$. It was found that using a hybrid material in the presence of $\mathrm{H}_{2} \mathrm{O}_{2}$ significantly reduces the concentration of dyes up to $28.4 \%$ within 7 hours. In another work, ${ }^{[651]}$ the decomposition reaction of methylene blue, cationic quinolinoxy derivatives of zinc phthalocyaninate exhibit high catalytic activity in the form of associates with polyoxometallate, which are a class of metal-oxygen cluster compounds with unique structural and physicochemical properties. ${ }^{[652]}$ It was shown
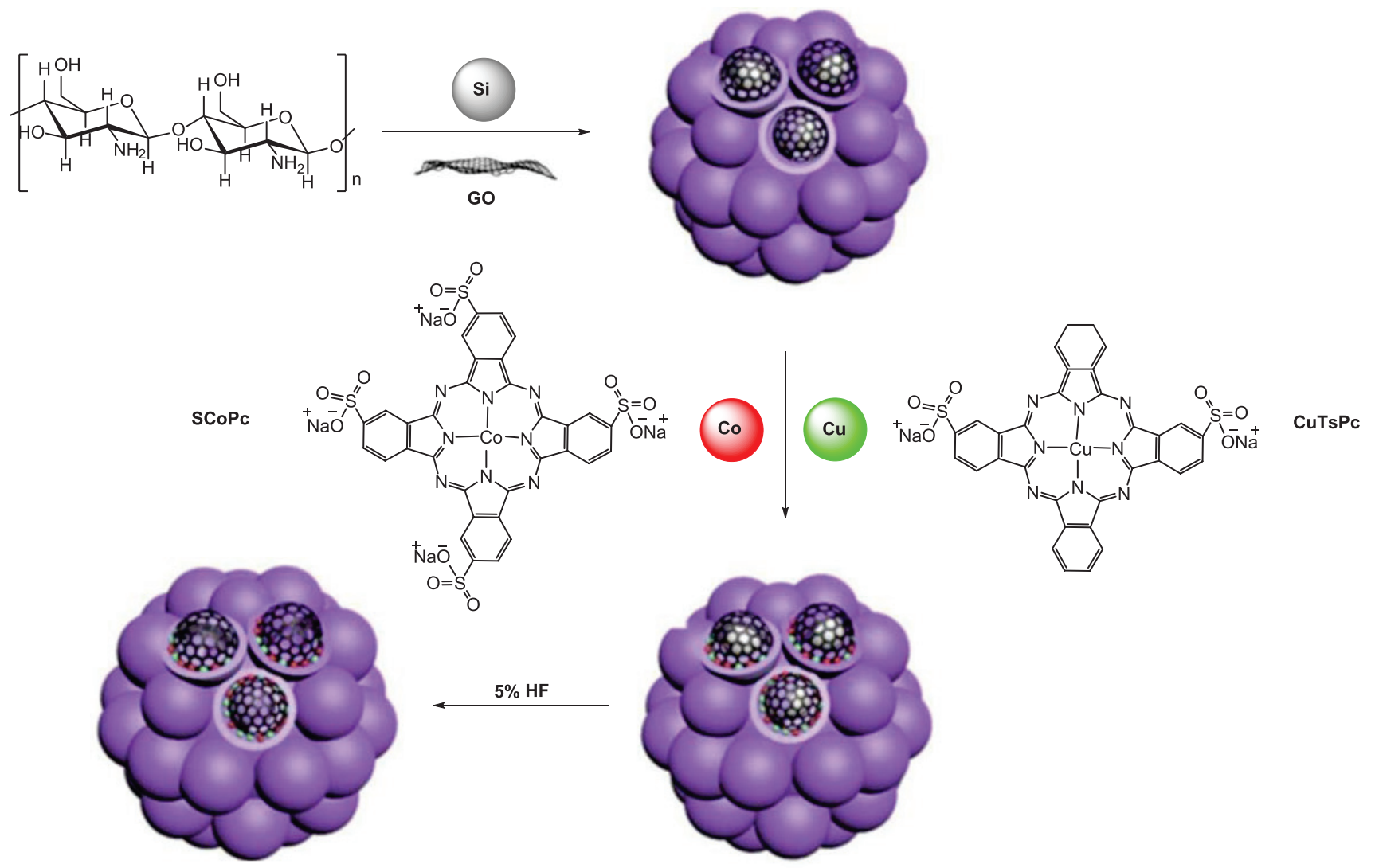

Scheme 15. Scheme of obtaining of porous materials Co-Cu-NSC..$^{[649]}$ 
that upon irradiation, the catalytic activity of the complex and when using the associate increases.

Cellulose is one of the most common, inexpensive, biodegradable and renewable biopolymers on Earth. Therefore, for this purpose, a hybrid material (NCC-PC) ${ }^{[653]}$ based on copper tetrasulfophthalocyanine and crystalline cellulose (NCC) was obtained for the oxidation reaction of alcohols and alkylarenes to the corresponding carbonyl groups in an aqueous solution. It was established that the obtained catalyst has several advantages over other catalysts based on copper tetrasulfophthalocyanine and does not require use of a toxic, dangerous oxidizing agent, high temperatures, the yield of the resulting product being about $99 \%$; does not undergo any deactivation during the catalytic process. In addition, the versatility of this catalyst lies in the fact that it does not require laborious processing conditions, and can be removed using a simple centrifugation process. The catalytic activity for phthalocyanine metal complex immobilized on the surface of a porous polyamide membrane in oxidation reaction of $n$-propylmercaptan and kinetic parameters for such reaction was shown in ${ }^{[654,655]}$.

A rather interesting work is ${ }^{[656]}$, where, based on the asymmetric and symmetric complex of zinc phthalocyanine with fragments of (phenoxy)isophthalic acid and/or tert-butyl groups, a conjugate with covalently bound cobalt ferrite was obtained (Figure 81). Next, the resulting system

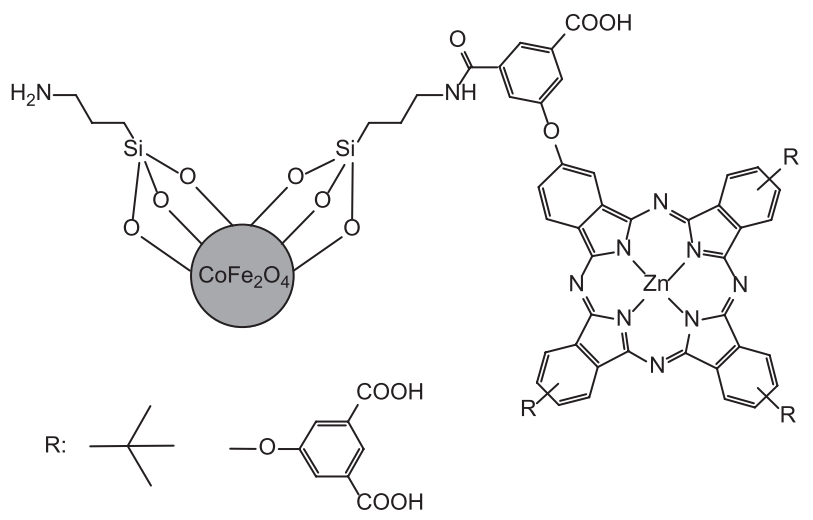

Figure 81. Scheme of hybrid material obtained by conjugation of a cobalt-ferrite phthalocyanine complex. ${ }^{[654]}$ by electrospinning is applied to fibers of polyamide-6. So, for example, the advantage of introducing the conjugate into fibers is that it is protected from decomposition and does not leach out into water, thereby eliminating even more pollution. The resulting system was tested during the destruction of azo dyes. As a result of the study, it was found that the catalytic activity is higher for such conjugates than for their corresponding phthalocyanines fixed on the fibers.

In, ${ }^{[657]}$ the process of applying the phthalocyanine metal complex to polyacrylonitrile fibers by electrospinning to prevent the formation of dimeric and oligomeric phthalocyanine structures was also used. In the presence of hydrogen peroxide, the catalytic activity in the destruction reaction of rhodamine $\mathrm{B}$ and carbamazepine was tested. Phthalocyanine deposited on fibers in the presence of hydrogen peroxide was shown to exhibit the highest catalytic activity. At the same time, the fact that the catalytic oxidizing ability of a nanofiber catalyst has remained virtually unchanged after eight cycles of decomposition of carbamazepine is interesting.

\section{Recent Advances in Electrochemistry of Cobalamins}

Cobalamins (Cbls; Figure 82A) are the most ubiquitous in vivo cobalt complexes. They are involved in numerous reactions in vivo, ${ }^{[1,658]}$ as well as they and their derivatives (e.g., cobinamides, Cbis, and cobyrinates; Figure 82B,C) represent a convenient platform for building new catalysts, ${ }^{[659]}$ sensors and pharmaceuticals. ${ }^{[600]}$ Application of corrinoids mainly depends on redox properties of cobalt ion, which adopts $\mathrm{Co}$ (III), $\mathrm{Co}$ (II) and $\mathrm{Co}(\mathrm{I})$ oxidation states. ${ }^{[11]}$ Conversions between these states are strongly affected by coordination environment of Co-ion, which can be illustrated by redox potentials. ${ }^{[1]}$ $\mathrm{Co}$ (III)-corrinoinds predominantly exist as hexacoordinate complexes, and the reduction of $\mathrm{Co}$ (III)-aqua species to pentacoordinate $\mathrm{Co}$ (II) species is easily accessible (Scheme 16), i.e. the redox potentials of aquacobalamin $\left(\mathrm{H}_{2} \mathrm{OCbl}(\mathrm{III})_{\text {base-on }}\right) / \mathrm{cob}(\mathrm{II})$ alamin $\left(\mathrm{Cbl}(\mathrm{II})_{\text {base-on }}\right)$ and diaquacobinamide $\left(\left(\mathrm{H}_{2} \mathrm{O}\right)_{2} \mathrm{Cbi}(\mathrm{III})\right) / \mathrm{cob}(\mathrm{II})$ inamide $(\mathrm{Cbi}(\mathrm{II}))$ are
(A)

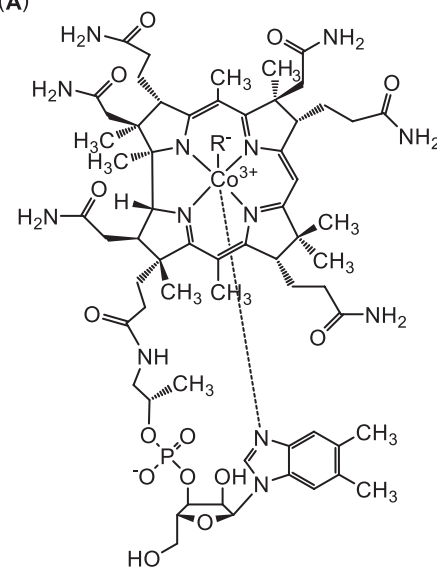

(B)

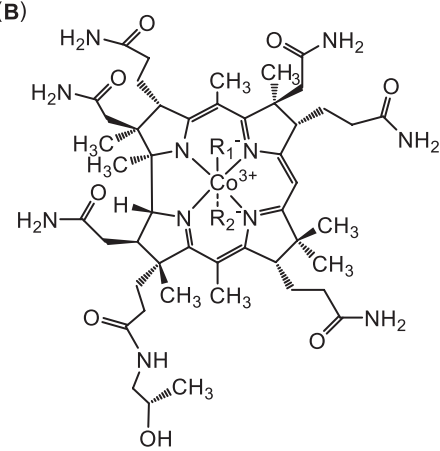

(c)

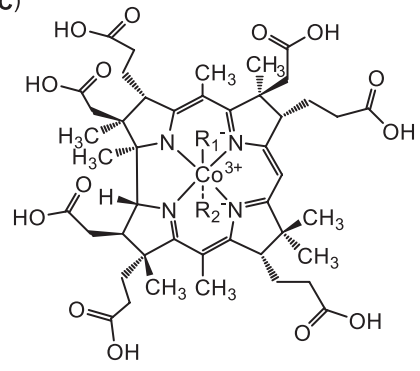

Figure 82. Structures of cobalamins (A), cobinamide (B) and cobyrinic acid (C). 
+0.20 and $+0.51 \mathrm{~V}$ (versus normal hydrogen electrode, NHE, at $22{ }^{\circ} \mathrm{C}$ ), respectively. ${ }^{[1,661]}$ Hereafter, 'base-on' and 'baseoff' refer to $\mathrm{Cbl}$ species with coordinated and dissociated 5,6-dimethylbenzimidazole (DMBI) nucleotide. Binding of ligands (e.g., thiols and thiocyanate) to $\mathrm{Co}(\mathrm{II})$ decreases its redox potential making the complex stronger reductant. ${ }^{[662,663]}$ Further reduction of $\mathrm{Co}(\mathrm{II})$-species to $\mathrm{Co}(\mathrm{I})$ is more challenging, i.e. the redox potentials of $\mathrm{Cbl}(\mathrm{II})_{\text {base-on }} / \mathrm{Cbl}(\mathrm{I})$ and $\mathrm{Cbl}(\mathrm{II})_{\text {base-off }} / \mathrm{Cbl}(\mathrm{I})$ couples are -0.61 and $-0.50 \mathrm{~V}$ (versus NHE; $22^{\circ} \mathrm{C}$ ), respectively. ${ }^{[11,661]}$ Electrochemical reduction of cyanocobalamin $(\mathrm{CNCbl})$ occurs at $-0.76 \mathrm{~V}$ (versus $\mathrm{NHE}$, in DMSO/propanol) and corresponds to two-electron process. ${ }^{[664]}$ In the case of organocobalamins, electrochemical reduction proceeds at more negative redox potentials, i.e. methylcobalamin $(\mathrm{MeCbl})$ is reduced at $-1.20 \mathrm{~V}$ (versus NHE, $20^{\circ} \mathrm{C}$ ) and adenosylcobalamin (AdoCbl) - at $-1.04 \mathrm{~V}$ (in DMF/methanol). ${ }^{[665]}$

The reduction of organocobalamins proceeds via one-electron electron transfer reaction, which produces corrinoid-anion-radical state, that is unstable and decomposes to $\mathrm{Co}(\mathrm{I})$ and alkyl radical (Scheme 16). ${ }^{[11,665]}$ The electrochemical reduction of glutathionylcobalamin (GSCbl) in acidic medium is similar to the reduction of organocobalamins. One-electron reduction produces anion-radical form GSCbl, which is transformed to base-off form in acidic medium. Upon DMBI dissociation, a substantial destabilization of the $\mathrm{Co}$ (III)-S bond occurs that results in formation of $\mathrm{Co}(\mathrm{I})$-state and thiyl radical. ${ }^{[666]}$ Destabilization of the $\mathrm{Co}$ (III)-S bond in Cbi(III) complex with glutathione (GSH) was observed as well. ${ }^{[667]}$

Upon binding to proteins, redox potentials of $\mathrm{Co}$ (III)/ $\mathrm{Co}(\mathrm{II})$ and $\mathrm{Co}(\mathrm{II}) / \mathrm{Co}(\mathrm{I})$ couples are frequently shifted. For example, an increase in the potential of $\mathrm{Co}(\mathrm{II}) / \mathrm{Co}(\mathrm{I})$ couple was observed upon binding of Cbls to ATP:Co(I)rrinoid adenosyltransferases, the enzymes converting $\mathrm{Cbl}(\mathrm{I})$ to AdoCbl. ${ }^{[668]}$ A convenient method of redox-potential determination of protein-bound $\mathrm{Cbl}$ species was suggested. It involves application of potentiometric titration using europium(II) diethylenetriaminepentaacetic acid (DTPA), which possesses strong reducing properties and capable of generating $\mathrm{Co}(\mathrm{I})$-corrinoids. ${ }^{[669]}$

Attempts have been made to predict redox potentials of Cbl-species using DFT, i.e. it was recommended to use PBE functional. ${ }^{[670]}$ Computational determination of redox potential is important for characterization of elusive species, e.g. $\mathrm{Cbl}(\mathrm{I})-\mathrm{HX}$ complexes. ${ }^{[671,672]}$

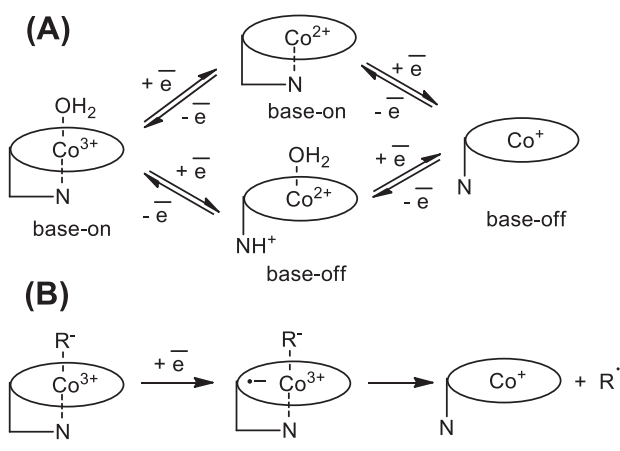

Scheme 16. Pathways of aqua- (A) and organo- (B) Cbls electroreduction.
Although the electrochemistry of corrinoids is studied for a long period, these complexes remain in the focus of several groups extending their scope of application. Next, we discuss recent electrochemical applications of corrinoids.

$\mathrm{CNCbl}$ was employed for fabrication the nanohybrid based on nitrogen-doped graphene, which was further used as electrocatalyst for hydrogen evolution and detection of hydrogen peroxide. ${ }^{[673]}$ Synthesis of this material includes nitrogen doping of graphene oxide by glycine in aqueous medium, $\mathrm{CNCbl}$ incorporation onto surface of nitrogen-doped material in DMF and further fixation of CNCbl-graphene hybrid on glassy carbon electrode. Bare and modified by nitrogen doped graphene glassy carbon electrodes displayed no noticeable response in the entire potential range in aqueous neutral solution in the course of cyclic voltammetry (CV) experiments in contrast to glassy carbon electrode modified by $\mathrm{CNCbl-graphene,} \mathrm{which} \mathrm{indicated} \mathrm{two} \mathrm{sharp} \mathrm{peaks,} \mathrm{i.e.}$ a cathodic peak at $0.28 \mathrm{~V}$ and an oxidation peak at $0.46 \mathrm{~V}$ versus ferrocene/ferrocenium cation $\left(\mathrm{Fc} / \mathrm{Fc}^{+}\right)$. The first peak was attributed to two electron reduction of $\mathrm{CNCbl}$ to $\mathrm{Cbl}(\mathrm{I})$ and the second one - to subsequent $\mathrm{Co}$ (I)-oxidation to $\mathrm{Co}$ (III). CNCbl-graphene/glassy carbon electrode exhibited high stability during repetitive cyclic voltammetry scans as well.

It has been shown that CNCbl-graphene/glassy carbon electrode is capable of efficiently reducing proton to hydrogen. ${ }^{[673]}$ In anaerobic aqueous sulfuric acid solution, efficiency of CNCbl-graphene/glassy carbon electrode is substantially higher than that of glassy carbon electrode modified by nitrogen-doped graphene and lower than that of $\mathrm{Pt} / \mathrm{C}$, whereas bare electrode was almost inactive in this process. Reported Tafel slopes commonly using to characterize hydrogen evolution activity are $60.35,106.3$ and $37.5 \mathrm{mV} / \mathrm{dec}$ for CNCbl-graphene and nitrogen-doped graphene modified glassy carbon electrodes and $\mathrm{Pt} / \mathrm{C}$, respectively. Long-term stability of CNCbl-graphene catalyst during cycling in $0.5 \mathrm{M} \mathrm{H}_{2} \mathrm{SO}_{4}$ was reported. Taking into account relatively low price of $\mathrm{CNCbl}$ in comparison with noble metal catalysts, relatively high efficiency in hydrogen evolution electrocatalysis and superior stability in acidic media, cobalamins and their derivatives must be considered more thoroughly for the construction of new materials for hydrogen evolution.

Heterogeneous hydrogen evolution reaction includes three steps (14)-(16):

$$
\begin{aligned}
& \mathrm{H}_{3} \mathrm{O}^{+}+\overline{\mathrm{e}} \rightarrow \mathrm{H}_{\mathrm{ads}}+\mathrm{H}_{2} \mathrm{O}, \\
& \mathrm{H}_{\mathrm{ads}}+\mathrm{H}_{3} \mathrm{O}^{+}+\overline{\mathrm{e}} \rightarrow \mathrm{H}_{2}+\mathrm{H}_{2} \mathrm{O}, \\
& \mathrm{H}_{\mathrm{ads}}+\mathrm{H}_{\mathrm{ads}} \rightarrow \mathrm{H}_{2},
\end{aligned}
$$

where $\mathrm{H}_{\text {ads }}$ is adsorbed hydrogen, reaction (14) is the Volmer reaction (a discharge step), reaction (15) is the Heyrovsky reaction (a desorption step) and reaction (16) is the Tafel reaction (a recombination step). In the case of CNCbl-graphene electrocatalyst, the rate determining step is assigned to reaction (15). ${ }^{[673]}$ More detailed mechanism of reaction between corrinoid species by proton was proposed during the investigation of photocatalytic hydrogen generation in the presence of Cbi catalyst. ${ }^{[674]}$ The key step of this mechanism is the generation of $\mathrm{Co}(\mathrm{I})$-state of the catalyst, 
which is capable of reacting with proton to give hydridospecies (16). Further interactions involving hydrido-species and protons (reactions 17-21) produce hydrogen and $\mathrm{Co}(\mathrm{II})-$ and $\mathrm{Co}(\mathrm{III})$-corrinoids.

$$
\begin{aligned}
& \mathrm{Cbi}(\mathrm{I})+\mathrm{H}^{+} \rightarrow \mathrm{Cbi}(\mathrm{III})-\mathrm{H}^{-}, \\
& \mathrm{Cbi}(\mathrm{III})-\mathrm{H}^{-}+\mathrm{H}^{+} \rightarrow \mathrm{Cbi}(\mathrm{III})+\mathrm{H}_{2}, \\
& \mathrm{Cbi}(\mathrm{III})-\mathrm{H}^{-}+\overline{\mathrm{e}} \rightarrow \mathrm{Cbi}(\mathrm{II})-\mathrm{H}^{-}, \\
& \mathrm{Cbi}(\mathrm{II})-\mathrm{H}^{-}+\mathrm{H}^{+} \rightarrow \mathrm{Cbi}(\mathrm{II})+\mathrm{H}_{2}, \\
& 2 \mathrm{Cbi}(\mathrm{III})-\mathrm{H}^{-} \rightarrow 2 \mathrm{Cbi}(\mathrm{II})+\mathrm{H}_{2} .
\end{aligned}
$$

Hydrido-corrinoids remain elusive and no solid proof confirming their existence and structure has been obtained. However, there are indirect evidence on their generation in the several redox processes. For example, $\mathrm{Cbl}(\mathrm{I})$ undergoes protonation in acidic medium with $\mathrm{p} K_{\mathrm{a}} c a$. 1 , which likely occurs at Co-ion. ${ }^{[675]}$ Hydrido-corrinoids have been proposed as the key intermediates in the hydrogenation of unsaturated compounds. ${ }^{[676-678]}$ Using spin traps, authors indicated transient generation of $\mathrm{H}^{\circ}$-radical donor, which can be attributed to $\mathrm{Co}(\mathrm{II})-\mathrm{H}^{\cdot}$ species, an electromer of $\mathrm{Co}(\mathrm{III})-\mathrm{H}^{-} .^{[678]}$ Therefore, the primary steps in hydrogen evolution mediated by CNCbl-graphene electrodes involves generation of $\mathrm{Cbl}(\mathrm{I})$-species and their reaction with proton to give Co-hydride species, which, probably, correspond to $\mathrm{H}_{\text {ads }}$ denoted in reactions (14)-(16).

It is interesting to compare hydrogen production assisted by cobalt corrin and tetradehydrocorrin species. ${ }^{[679]}$ Tetradehydrocorrin represents more unsaturated macrocyclic system than corrin including four additional double bonds (Figure 83). In DMF medium, meso-substituted Co(II) tetradehydrocorrin complex exhibits two redox events in cyclic voltammogram, i.e. at -0.5 and $-1.7 \mathrm{~V}$ (versus $\mathrm{Fc} / \mathrm{Fc}^{+}$) $\mathrm{Co}(\mathrm{II}) /$ $\mathrm{Co}(\mathrm{I})$ and $\mathrm{Co}(\mathrm{I}) / \mathrm{Co}(\mathrm{I})$ anion-radical species. $\mathrm{Co}(\mathrm{I})$-species were inactive in hydrogen generation process, probably, due to stabilization of $\mathrm{Co}(\mathrm{I})$ by unsaturated $\pi$-electron system, whereas $\mathrm{Co}(\mathrm{I})$ anion-radical generated $\mathrm{H}_{2}$ after addition $\mathrm{H}_{2} \mathrm{O} / \mathrm{CO}_{2}$ or buffer solution. $\mathrm{Co}(\mathrm{I})$ anion-radical reduces $\mathrm{CO}_{2}$ to $\mathrm{CO}$ as well, but the reaction is characterized by very low yield. ${ }^{[679]}$ The information regarding $\mathrm{Co}(\mathrm{I})$-corrinoid anion-radical (or formally $\mathrm{Co}(0)$ ) species is scarce, i.e. there is only one work reporting preparation of this complex using radiolysis and its reactivity toward $\mathrm{CO}_{2} \cdot{ }^{[680]}$
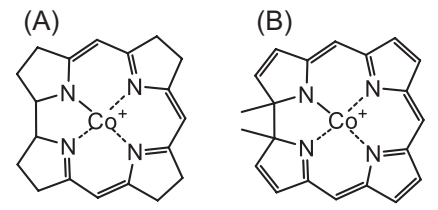

Figure 83. Structures of corrin (A) and tetradehydrocorrin (B).

CNCbl-graphene modified glassy carbon electrode has been successfully employed toward detection of hydrogen peroxide in aqueous medium using $\mathrm{CV}$ and differential pulse voltammetry (DPV). ${ }^{[673]}$ The addition of $\mathrm{H}_{2} \mathrm{O}_{2}$ substantially decreased cathodic peak and increased intensity of anodic peak of CNCbl-graphene hybride. The intensity of the anodic peak is linearly dependent on $\mathrm{H}_{2} \mathrm{O}_{2}$ concentration. Detection limit is $20 \mathrm{nM}$ and several biological species (viz., $\mathrm{K}^{+}, \mathrm{Na}^{+}$, $\mathrm{Ca}^{2+}, \mathrm{Cl}^{-}, \mathrm{NO}_{3}^{-}, \mathrm{SO}_{4}{ }^{2-}, \mathrm{NH}_{4}^{+}, \mathrm{Br}^{-}$, glucose, uric acid, ascorbic acid, dopamine) exhibited no influence on $\mathrm{H}_{2} \mathrm{O}_{2}$ sensing up to 3 -fold excess. Authors proposed coordination of $\mathrm{H}_{2} \mathrm{O}_{2}$ on $\mathrm{Co}$ (III)-center in the course of $\mathrm{H}_{2} \mathrm{O}_{2}$ electrooxidation. However, $\mathrm{H}_{2} \mathrm{O}_{2}-\mathrm{Cbl}$ complexes have not been characterized yet and exact nature of $\mathrm{H}_{2} \mathrm{O}_{2}$ oxidation intermediate requires further study.

$\mathrm{CNCbl}$ was used for the modification of glassy carbon electrode via electropolymerization procedure, i.e. the $\mathrm{CNCbl}$ film was deposited on the electrode surface by cyclic sweeping from -1.2 to $1.0 \mathrm{~V}$ for 20 cycles in DMF containing sodium perchlorate. ${ }^{[681]}$ The modified electrode was successfully employed for peroxynitrite $\left(\mathrm{ONOO}^{-}\right)$ detection. The mechanism of reaction includes the oxidation of $\mathrm{ONOO}^{-}$to $\mathrm{ONO}_{2}^{-}$-radical on the electrode surface and further decomposition of $\mathrm{ONO}_{2}{ }^{-}$to $\mathrm{O}_{2}$ and $\mathrm{NO}_{2}$. $\mathrm{ONOO}^{-}$ detection limit was reported to be $1.0 \cdot 10^{-7} \mathrm{M} \cdot{ }^{[681]}$

Cobalamins (obviously, $\mathrm{CNCbl}$; however, authors did not specify Cbl species) have been employed in membraneless hydrogen peroxide fuel cell. ${ }^{[682]} \mathrm{Cbl}$ and carbon nanotubes were used for modification of surface of glassy carbon electrode. This electrode was used as anode, whereas hemin/carbon nanotube glassy carbon electrode served as cathode. Reactions (22) and (23) represent anode and cathode processes in the cell, respectively. Onset potentials of anode and cathode catalysts are 0.10 and $0.35 \mathrm{~V}$ (versus $\mathrm{Ag} / \mathrm{AgCl}$ ), and the open circuit voltage is $0.25 \mathrm{~V}$. Upon utilizing the fuel cell with $0.1 \mathrm{M} \mathrm{H}_{2} \mathrm{O}_{2}$ in neutral medium, the open circuit voltage and maximum power density are $0.23 \mathrm{~V}$ and $54 \mu \mathrm{W} \mathrm{cm}{ }^{-2}$, respectively.

$$
\begin{aligned}
& \mathrm{H}_{2} \mathrm{O}_{2} \rightarrow \mathrm{O}_{2}+2 \mathrm{H}^{+}+2 \overline{\mathrm{e}}, \\
& \mathrm{H}_{2} \mathrm{O}_{2}+2 \mathrm{H}^{+}+2 \overline{\mathrm{e}} \rightarrow 2 \mathrm{H}_{2} \mathrm{O} .
\end{aligned}
$$

$\mathrm{CNCbl}$ has been employed as an water oxidation catalyst. ${ }^{[683]}$ The controlled potential electrolysis at $1.5 \mathrm{~V}$ (versus $\mathrm{Ag} / \mathrm{AgCl})$ in the presence of $\mathrm{CNCbl}(1 \mathrm{mM})$ under anaerobic conditions at $\mathrm{pH} 7.0$ produces dioxygen, which is detected in the gas phase after several hours. The Faradaic efficiency is $97.50 \%$, and the process is characterized by overpotential of $0.58 \mathrm{~V}$, which is comparable with the other water oxidation catalysts. $\mathrm{CNCbl}$ exhibits high stability in this process within at least 11 hours. To explain the mechanism of the process, authors report that the cyclic and differential pulse voltammograms of $\mathrm{CNCbl}$ solution indicate irreversible oxidation event at ca. $1.2 \mathrm{~V}$ (versus $\mathrm{Ag} / \mathrm{AgCl}$ ) that is attributed to $\mathrm{Co}(\mathrm{III})$-oxidation to $\mathrm{Co}(\mathrm{IV})$. The generated $\mathrm{Cbl}(\mathrm{IV})$ is unstable in aqueous medium and is reduced to $\mathrm{Cbl}$ (III) by water molecule. The proposed reaction mechanism includes cyanide dissociation from $\mathrm{Co}$ (III)-center, further formation of Cbl(IV), proton dissociation and subsequent water attack to give dioxygen (Scheme 17). However, the tight binding cyanide to $\mathrm{Cbl}(\mathrm{III})^{[11]}$ rules out the supposed dissociation of $\mathrm{CN}^{-}$prior to $\mathrm{Co}$ (III)oxidation to $\mathrm{Co}(\mathrm{IV})$. Additionally, $\mathrm{H}_{2} \mathrm{OCbl}$ formed in redox cycle can be easily detected using UV-Vis spectroscopy even in the presence of equal amount of cyanide dissociated from 
the starting complex (reaction between $\mathrm{H}_{2} \mathrm{OCbl}$ and $\mathrm{CN}^{-}$ proceeds relatively slowly in neutral medium). However, the prolonged electrolysis does not induce detectable changes in UV-Vis spectrum of CNCbl. The Scheme $17^{[683]}$ indicates transfer of only one electron, although the generation of one dioxygen molecule from two water molecules requires transfer of four electrons.

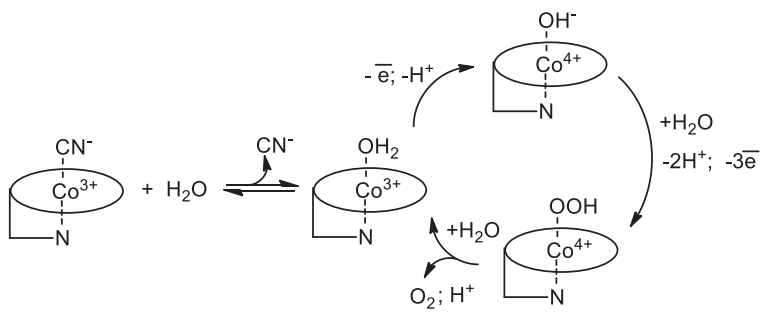

Scheme 17. The mechanism of water electrooxidation in the presence of CNCbl. ${ }^{[683]}$

Corrinoids are thoroughly investigated as the catalysts in the transformation of organic compounds. It is highlighted in depth in the several reviews. ${ }^{[659,684,685]}$ This application is generally based on the generation of $\mathrm{Co}(\mathrm{I})$ species, which possesses strong nucleophilicity or capable of generating Co(III)-hydride. Due to the strong nucleophilicity, Co(I)corrinoids react with alkyl halides to give organo-Co(III), which are reduced in the course of the further electroreduc-

(A)
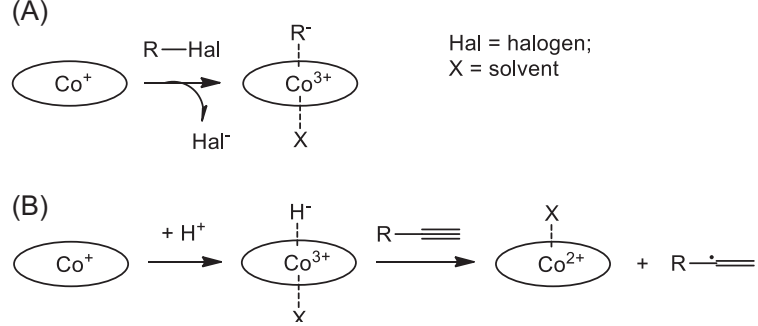

Scheme 18. Primary steps in catalytic cycles of corrinoidcatalyzed reactions involving halogenated (A) and unsaturated (B) organic compounds. tion steps (Scheme 18A). Co(III)-hydride acts as a donor of $\mathrm{H}^{*}$-radical for unsaturated bonds producing organic radical, which undergoes subsequent step to the final products (Scheme 18B).

Therefore, corrinoids, due to their remarkable redox reactivity, represent a convenient platform for electrochemical applications. However, additional studies are required for understanding of mechanistic details of their action, especially regarding methods utilizing electrooxidation of substrates, where generation of high-valent catalyst species is possible.

\section{Macrocyclic Receptors for Recognition and Selective Binding of Substrates of Different Nature}

Design of molecular receptors for a specific type of substrate is an important task of organic and supramolecular chemistry. Porphyrins are extremely useful for this purpose. ${ }^{[76,686-691]}$ Their unique properties are due to the unusual geometric and electronic structure of the porphyrin macrocycle, which contains a developed aromatic conjugated system. Selective chemical modification of porphyrins with fragments of other classes of compounds makes it possible to synthesize molecular systems with a different nature and mutual arrangement of reaction centers. ${ }^{[692-700]}$ This section discusses the results of research in the field of synthesis and study of the receptor ability of porphyrins modified with calix[4]arenes, calix[4]pyrroles, oxacalix[2] arenes, $^{[686]}$ resorcinarenes, as well as bulky carbazolylphenyl substituents of various generations capable of forming inclusion complexes with substrates of various nature. The dependence of the receptor ability of selectively modified porphyrins on the number and position of substituents, as well as on the presence of cations and anions in the reaction system, has been analyzed. The dependence of the complexing ability of the porphyrin base on the conformational mobility of substituents, the possibility of the formation of intramolecular cavities and channels of various shapes in them is shown. The presented material is relevant for researchers

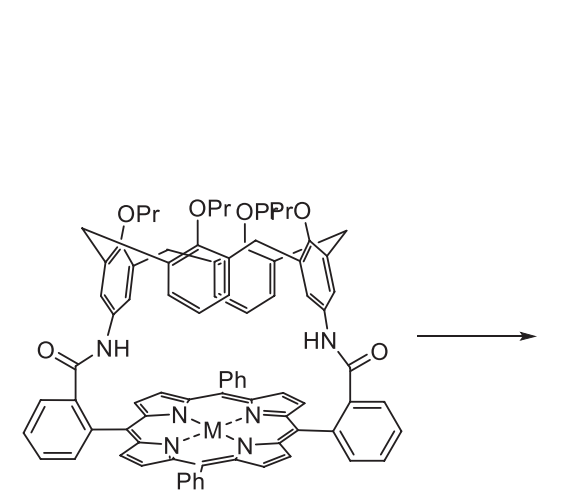

$M=H_{2}(46), Z n(47)$

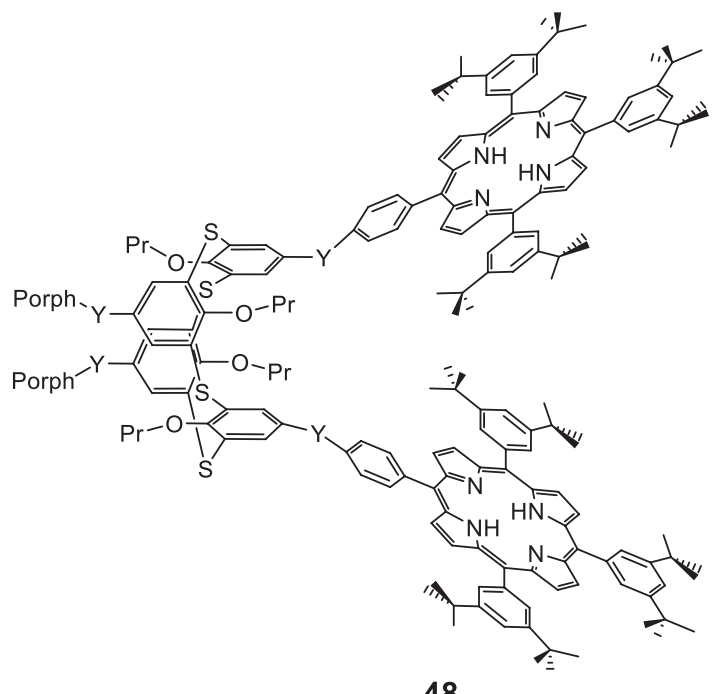

48 
working in the field of creating new "chemo-sensitive" (i.e., selectively interacting with other chemical substances as receptors, sensors, or photo-catalysts) polyfunctional materials.

\section{Molecular Receptors Based on Porphyrin Conjugates with Macrocyclic Compounds of Different Nature}

Interest in the chemistry of porphyrins, initially due to the involvement of these compounds in the processes of photosynthesis, has now acquired a new direction due to the possibility of their use as selective molecular receptors for a certain type of substrate. ${ }^{[701-711]}$

The possibilities of chemical modification of the tetrapyrrole molecule made it possible to synthesize conjugates of porphyrins with other macrocyclic compounds with their own interesting complexing properties with respect to ions and organic molecules of different nature. Among these compounds, covalently bound porphyrin-calix[4]arene macrocyclic receptors are of particular interest.

The first calix[4]arene-porphyrin conjugate 46 with the ability to selectively recognize small organic molecules was described in ${ }^{[688]}$. ${ }^{1} \mathrm{H}$ NMR spectra of porphyrinate 47 in the presence of pyridine (Py) and imidazole (Im) showed two series of signals of aromatic protons, which belong to the protons of free and porphyrinate bound substrate molecules (Figure 84). Moreover, the aromatic protons of bound substrate molecules, due to the shielding effect of the ring current of the porphyrin macrocycle, are displaced into a strong field, compared with the corresponding protons of unbound substrate molecules. It was found by spectrophotometric titration that porphyrinate 47 exhibits the maximum complexing ability with respect to imidazole (Table 8).

«Molecular tweezers» (48) based on thiacalix[4]areneporphyrin conjugate with the ability to selectively recognize fullerene $\mathbf{C}_{70}$ was obtained. ${ }^{[689]}$ The complex-forming ability of receptor $\mathbf{4 8}$ towards fullerenes $\mathbf{C}_{60}$ and $\mathbf{C}_{70}$ was studied by ${ }^{1} \mathrm{H}$ NMR titration. It was found that, in the presence of $\mathbf{C}_{70}$ fullerene, the signals of NH-protons of porphyrin in the ${ }^{1} \mathrm{H}$ NMR spectrum are shifted into a strong field. It was shown
Table 8. Stability constants $K_{\mathrm{a}}$ and binding energy $\Delta G$ by porphyrinate 47 of substrate molecules in chloroform at $298 \mathrm{~K}$.

\begin{tabular}{lcc}
\hline \multicolumn{1}{c}{ Substrarte } & $K_{a}, \mathrm{M}^{-1}$ & $-\Delta G, \mathrm{~kJ} \cdot \mathrm{mol}^{-1}$ \\
\hline Pyridine & $53400 \pm 800$ & 27.0 \\
4-Methylpyridine & $20 \pm 1$ & 7.4 \\
Imidazole & $1920000 \pm 40000$ & 35.8 \\
\hline
\end{tabular}

Table 9. Stability constants $K_{a}$ of complexes 48-C60 and 48-C70 at $25^{\circ} \mathrm{C}$ in toluene.

\begin{tabular}{cc}
\hline Conjugate & $K_{a}, \mathrm{M}^{-1}$ \\
\hline $\mathbf{4 8}_{-} \mathbf{C}_{60}$ & $2.0 \cdot 10^{3}$ \\
$\mathbf{4 8}_{\mathbf{C}} \mathbf{C}_{70}$ & $3.6 \cdot 10^{4}$ \\
\hline
\end{tabular}

(Table 9) that receptor $\mathbf{4 8}$ exhibits high selectivity towards fullerene $\mathbf{C}_{70}$.

The authors ${ }^{[712,713]}$ have developed several synthetic approaches for the synthesis of bis-porphyrins 49a-c, 50ab, 51 with face-to-face orientation of porphyrin fragments in which is provided by the calix[4]arene spacer.

It was found the complexation of calix[4]arenebis(porphyrin) receptors 49c, 50b, 51 with bidentate triethylenediamine (DABCO) proceeds according to the 1:2 interaction type. This assumption is supported by the splitting of the signals of nonequivalent DABCO protons in the ${ }^{1} \mathrm{H}$ NMR spectrum of the complex. That is, due to the discrepancy between the geometric parameters of the porphyrin binding cavity of the receptor and the substrate, single-center binding of the studied bidentate ligand occurs with the formation of an external complex with a stability constant $K_{\mathrm{a}}=4.7 \cdot 10^{5} \mathrm{M}^{-2} \cdot{ }^{[712]}$

However, in the presence of a potassium cation, the complexation of receptor $\mathbf{5 1}$ with DABCO occurs as a 1:1 interaction. This assumption is supported by a clear singlet of equivalent protons of the substrate in the ${ }^{1} \mathrm{H}$ NMR spectrum of the complex. Those, due to conformational

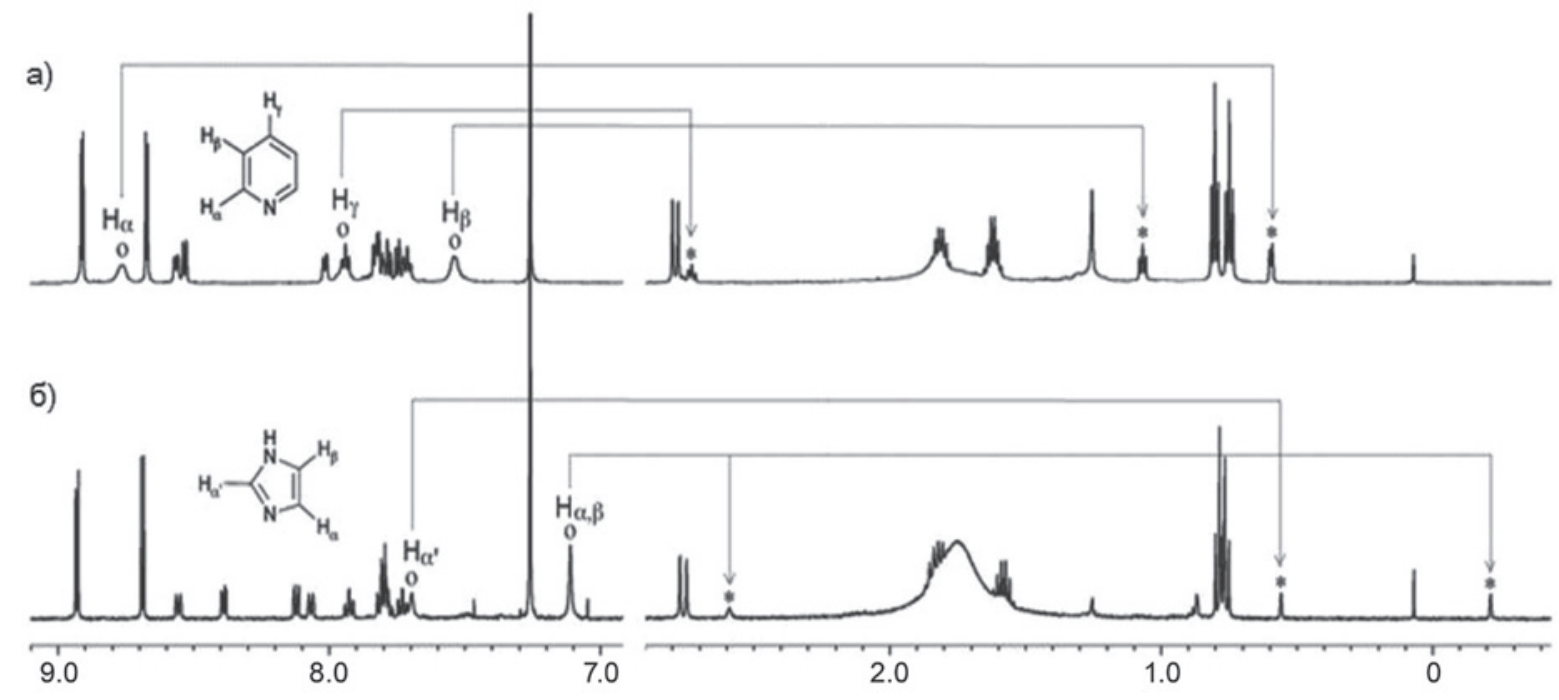

Figure 84. ${ }^{1} \mathrm{H}$ NMR spectra of (a) 47-Py and (b) 47-Im complexes in $\mathrm{CDCl}_{3}$ at $298 \mathrm{~K}$. Signals of free and bound substrate molecules are marked with ${ }^{\circ}$ and $*$ signs. ${ }^{[688]}$ 


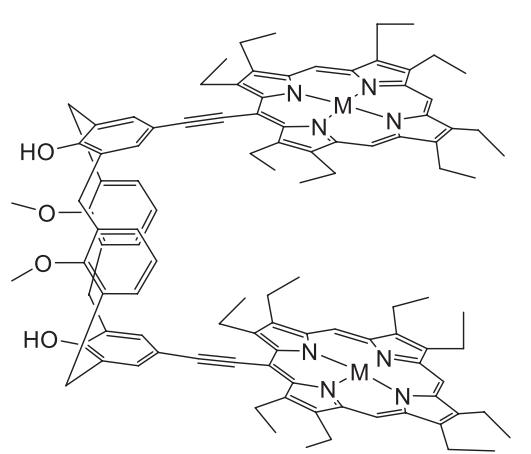

$\mathrm{M}=\mathrm{Ni}(49 \mathrm{a}), 2 \mathrm{H}(49 \mathrm{~b}), \mathrm{Zn}(49 \mathrm{c})$

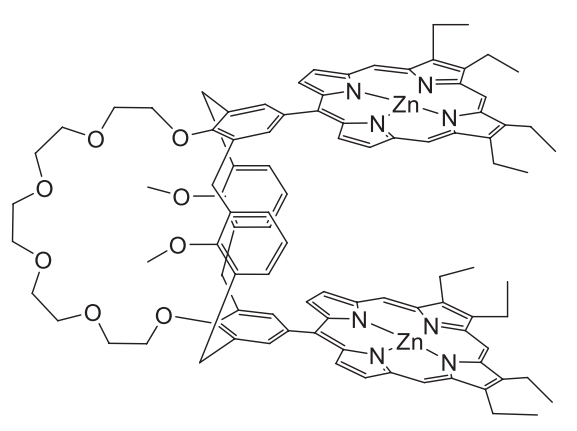

51

changes occurring during the complexation of the crownether fragment of the macrocycle with the potassium cation, the porphyrin fragments of the bis-porphyrin receptor converge and two-center complexation between receptor $\mathbf{5 1}$ and the organic substrate takes place according to the 1:1 type of interaction with the formation of a stable internal complex with the stability constant $\mathrm{K}_{\mathrm{a}}=6.9 \cdot 10^{6} \mathrm{M}^{-1}$.

In ${ }^{[714]}$, the synthesis of the calix[4]pyrrole-bis(porphyrin) conjugate 52 was carried out, in which the role of a spacer holding porphyrin fragments in the face-to-face orientation relative to each other was played by calix[4]pyrrole. It was found by spectrophotometric titration that during the complexation of the calix[4]pyrrole fragment of the receptor 52 with fluorine anions, the Soret band in the absorption spectrum broadens and decreases, and the reaction system is saturated in the region of the molar ratio of reagents 1:1. Those, the complexation of the receptor $\mathbf{5 2}$ with anion occurs with the formation of complexes of the composition 1:1 with a stability constant $K_{a}=15000 \mathrm{M}^{-1}$.

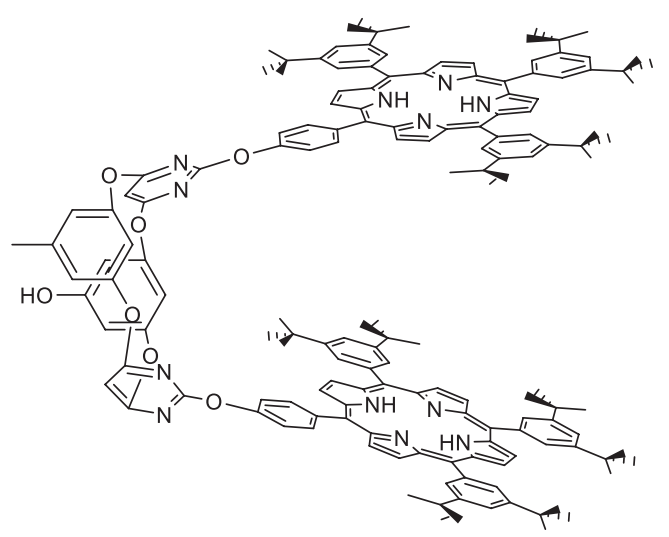

53

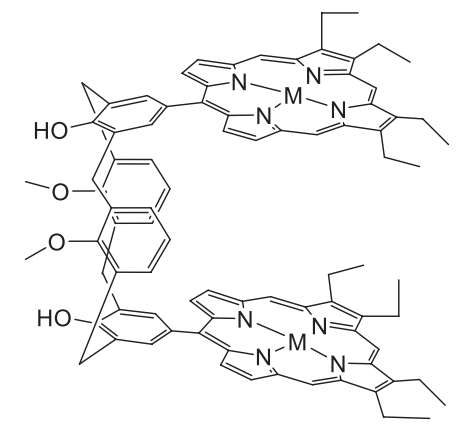

$M=2 H(50 a), Z n(50 b)$

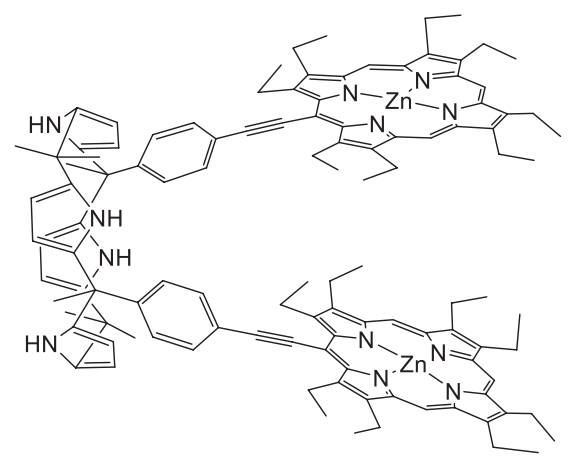

52

The downfield shift of the NH proton signals of the calix[4]pyrrole fragment indicates that complexation leads to a change in the conformation of the spacer from the 1,3-alternate state to the cone state. It has been shown that complexation at the calix[4]pyrrole fragment can be used to control the process of complexation at the porphyrin binding cavity of the receptor 52. In the absence of an anion, the reaction of receptor 52 with DABCO proceeds with the formation of an external complex of 1:2 composition with a stability constant $K_{a}=4.7 \cdot 10^{5} \mathrm{M}^{-2}$. The formation of an external complex with a composition of $1: 2$ is indicated by the splitting of the signals of the substrate protons in the ${ }^{1} \mathrm{H}$ NMR spectrum of the corresponding complex.

Part of the DABCO protons located closer to the macrocycle experiences the maximum screening effect from its $\pi$-electron system and manifests itself in a strong field, while the other part of the protons located at a distance from the macrocycle manifests itself in a weak field. ${ }^{[714]}$

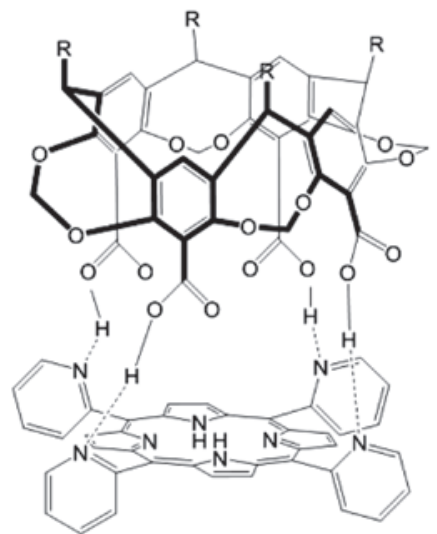

54 
At the same time, it has been shown that in the presence of an anion, complexation proceeds with the formation of an internal complex of 1:1 composition with a stability constant $K_{a}=6.8 \cdot 10^{6} \mathrm{M}^{-1}$. The formation of internal complexes is supported by one signal of the substrate protons located in a strong field. The same screening of substrate protons by porphyrin fragments of the receptor leads to the fact that equivalent protons, under the action of the screening effect from the $\pi$-electron cloud of porphyrin fragments, appear in a strong field. Thus, the conformational changes caused by the complexation of the calix[4]pyrrole fragment with the anion promote the convergence of the porphyrin fragments of the receptor and favor the formation of internal receptor-substrate complexes with two binding points.

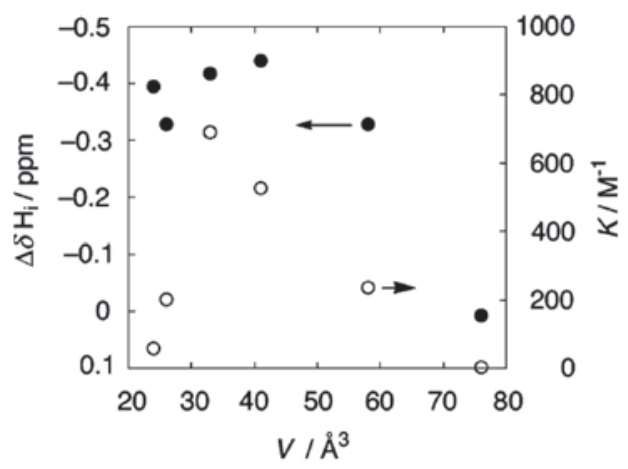

Figure 85. Difference in chemical shifts $\Delta \delta \mathrm{Hi}(\bullet)$ and binding constants $K\left(^{\circ}\right)$ depending on the size of the substrate. $\Delta \delta \mathrm{H}_{\mathrm{i}}=\left(\mathrm{H}_{\mathrm{i}}\right.$ for an encapsulated substrate) - $\left(\mathrm{H}_{\mathrm{i}}\right.$ for an unbound substrate). ${ }^{[715]}$

In ${ }^{[715]}$, the synthesis of oxacalix[2] arene[2]-bis(Znporphyrinate) (53) was carried out. It was shown by ${ }^{1} \mathrm{H}$ NMR titration that receptor $\mathbf{5 3}$ efficiently binds $\mathbf{C}_{70}$ fullerene molecules in solution. ${ }^{[715]}$ At the same time, the authors note that this receptor does not interact with fullerene $\mathbf{C}_{60}$. That is, a good correspondence between the geometric parameters of the porphyrin $\mathbf{5 3}$ binding cavity and the $\mathbf{C}_{\mathbf{7 0}}$ fullerene molecule provides ideal conditions for substrate receptor interactions leading to the formation of stable complexes of the composition of 1:1 with a stability constant $K_{a}=30000 \mathrm{M}^{-1}$.

The authors of ${ }^{[716]}$ have synthesized a stable supramolecular capsule 54 capable of effectively binding small organic molecules of various sizes. It has been shown (Figure 85) that receptor $\mathbf{5 4}$ is able to selectively bind methane, ethane, propane, and ethylene molecules. The stability constant of the complex $\left(\mathrm{M}^{-1}\right.$, at $\left.298 \mathrm{~K}\right)$ changes depending on the nature of the substrate molecule in the following sequence: methane $(56)<$ acetone $(202)<$ ethylene $(690)>$ ethane $(527)>$ propane $(236)>2$-methylpropane (3).

\section{Molecular Receptors Based on Porphyrins Modified at the Periphery of the Macrocycle with Bulky Substituents}

An important direction in the design of tetrapyrrole macrocyclic receptors for a certain type of substrate is the modification of the periphery of the macrocycle with bulky substituents or molecular fragments of different nature. ${ }^{[717-724]}$ Highly branched bulky side substituents are capable of forming additional binding cavities that can be used to recognize and selectively bind certain types of substrates.

In ${ }^{[725]}$, the binding ability of zinc porphyrinates with carbazole branching of the first (55) and second (56) generations was studied. It was found by spectrophotometric titration and ${ }^{1} \mathrm{H}$ NMR that the complexation of zinc porphyrinate (55) with triazole ( $\operatorname{Tr} A)$ proceds with the formation of one type of complexes with a composition of 1:1. The stability constants of the corresponding complexes of receptor $\mathbf{5 5}$ and model zinc tetraphenylporphyrinate (57) with pyridine and triazole are presented in Table 10.

Table 10. Stability constants $K_{a}\left(\mathrm{M}^{-1}\right)$ complexes of $1: 1$ composition between porphyrinates $(\mathbf{5 5}, \mathbf{5 7})$ and organic ligands $(\mathrm{Py}, \operatorname{Tr} \mathrm{A})$ in toluene, $C_{\mathrm{ZnP}} \approx 1.1 \div 1.6 \cdot 10^{-5} \mathrm{M}$.

\begin{tabular}{ccc}
\hline Porphyrin & Py & TrA \\
\hline $\mathbf{5 7}$ & 480 & 5800 \\
$\mathbf{5 5}$ & 110000 & 970000 \\
\hline
\end{tabular}

The high stability of complexes based on receptor 55 can probably be explained by the presence of binding cavities above and below the plane of the coordination center of the tetrapyrrole macrocycle. The authors of ${ }^{[725]}$ studied the complexation of octa-carbazolyl substituted zinc porphyrinates with side branches of the first (55) and second (56) generations with cryptand-2,2,2 (Cr).

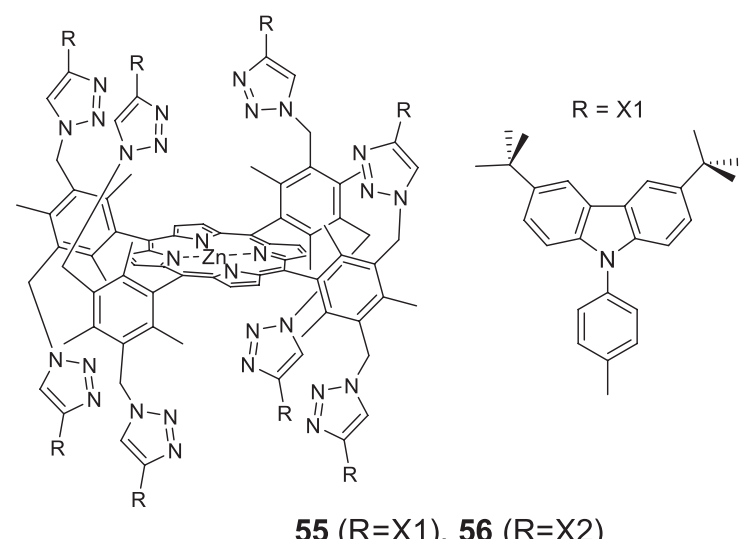

$55(\mathrm{R}=\mathrm{X} 1), 56(\mathrm{R}=\mathrm{X} 2)$
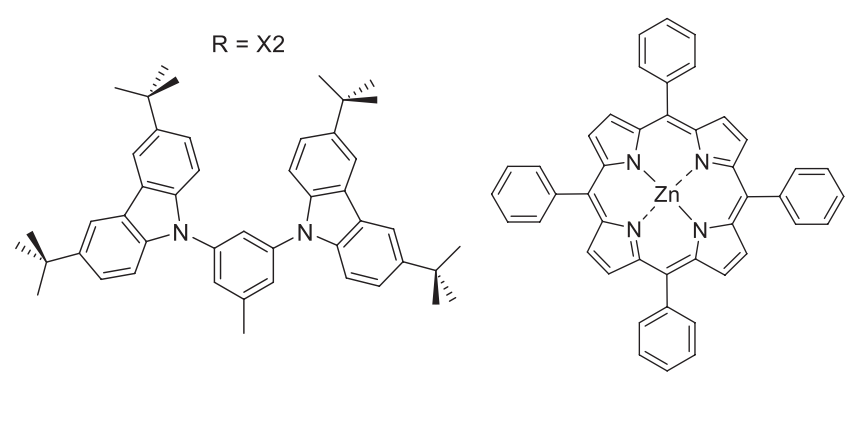

57 
Table 11. Stability constants $(\lg K)$ of $\mathbf{C r}$ complexes with $\mathbf{5 7}$ and $\mathbf{5 5}$ in toluene and a toluene-methanol mixture $(9: 1)$ at $20^{\circ} \mathrm{C}$.

\begin{tabular}{cccc}
\hline $\begin{array}{c}\mathbf{5 7 - C r} \\
\text { (toluene) }\end{array}$ & $\begin{array}{c}\mathbf{5 7 - C r} \\
\text { (toluene- } \\
\text { methanol) }\end{array}$ & $\begin{array}{c}\mathbf{5 7 - C r} \\
\text { (toluene) }\end{array}$ & $\begin{array}{c}\mathbf{5 7 - C r} \\
\text { (toluene- } \\
\text { methanol) }\end{array}$ \\
\hline 2.88 & 2.76 & 5.96 & 5.87 \\
\hline
\end{tabular}

The stability constants of the corresponding complexes are presented in Table 11. The formation of complexes of 1:1 composition with the location of the substrate in the binding cavity of the receptor is confirmed by the cleavage of the signals of the $-\mathrm{OCH}_{2}-$ and $-\mathrm{CH}_{2} \mathrm{~N}$ - substrate protons (Figure 86).

A spectrophotometric study of the interaction between carbazolyl-substituted zinc porphyrinate $\mathbf{5 5}$ and $\mathbf{C r}$ in the presence of alkali metal cations was carried out in a binary solvent toluene-methanol (1:9). It was found that the addition of potassium bromide to the solution of the 55-Cr complex leads to the destruction of the guest-host complex. Those, complexation occurring in the binding cavity of the cryptand affect the complexation of the carbazolyl-substituted porphyrin with the cryptand. That is, the complexation occurring in the binding cavity of the cryptand affects the complexation of the carbazolylsubstituted porphyrin with the cryptand. ${ }^{[725]}$ The obtained experimental data can be explained by the action of two factors. First, as confirmed by the results of calculations, the complexation of cryptand with alkali metal cations is accompanied by a change in the conformation of molecules in general.
Table 12. Stability constants $K_{a} \cdot 10^{4}\left(\mathrm{M}^{-1}\right)$ of the 58G1 and $\mathbf{5 8 G 2}$ complexes with $\mathbf{C}_{60}, \mathbf{C}_{70}$, and $\mathrm{C}_{84}$ in a toluene-acetonitrile mixture $(2: 1), 25^{\circ} \mathrm{C}$.

\begin{tabular}{llll}
\hline & $\mathbf{C}_{60}$ & $\mathbf{C}_{70}$ & $\mathbf{C}_{\mathbf{8 4}}$ \\
\hline $\mathbf{5 8 - G 1}$ & 1.60 & 4.90 & 5.80 \\
\hline $\mathbf{5 8 - G 2}$ & 2.10 & 6.00 & 5.40 \\
\hline
\end{tabular}

Second, in complexes with alkali metal cations, cryptands are in the endo-endo conformation, in which the lone electron pairs of nitrogen atoms are oriented inside the binding cavity of the ligand and, as a consequence, are inaccessible for coordination interaction with the zinc cation of porphyrinate. The combined effect of these factors, on the one hand, leads to the destruction of the porphyrinate-cryptand complex upon addition of a metal cation to the solution, and vice versa, they prevent the interaction of zinc porphyrinate with the cryptate of the corresponding metal cation.

In ${ }^{[726,727]}$, the receptor properties of two-layer dendrimers (58G1 and 58G2) with a carbazole outer and phenylazomethine inner layers were studied. Receptors 58G1 and 58G2 have two types of binding sites: 1) a complexing cavity formed by carbazole fragments of the outer layer of the dendrimer; 2) zinc cation of tetrapyrrole macrocycle.

It was found that 58G1 and 58G2 exhibit selective complexing ability towards fullerenes $\mathbf{C}_{60}, \mathbf{C}_{70}$ and $\mathbf{C}_{84}$. Binding proceeds according to the $1: 1$ interaction with the formation of complexes in which one fullerene molecule is located in a binding cavity formed by carbazole fragments

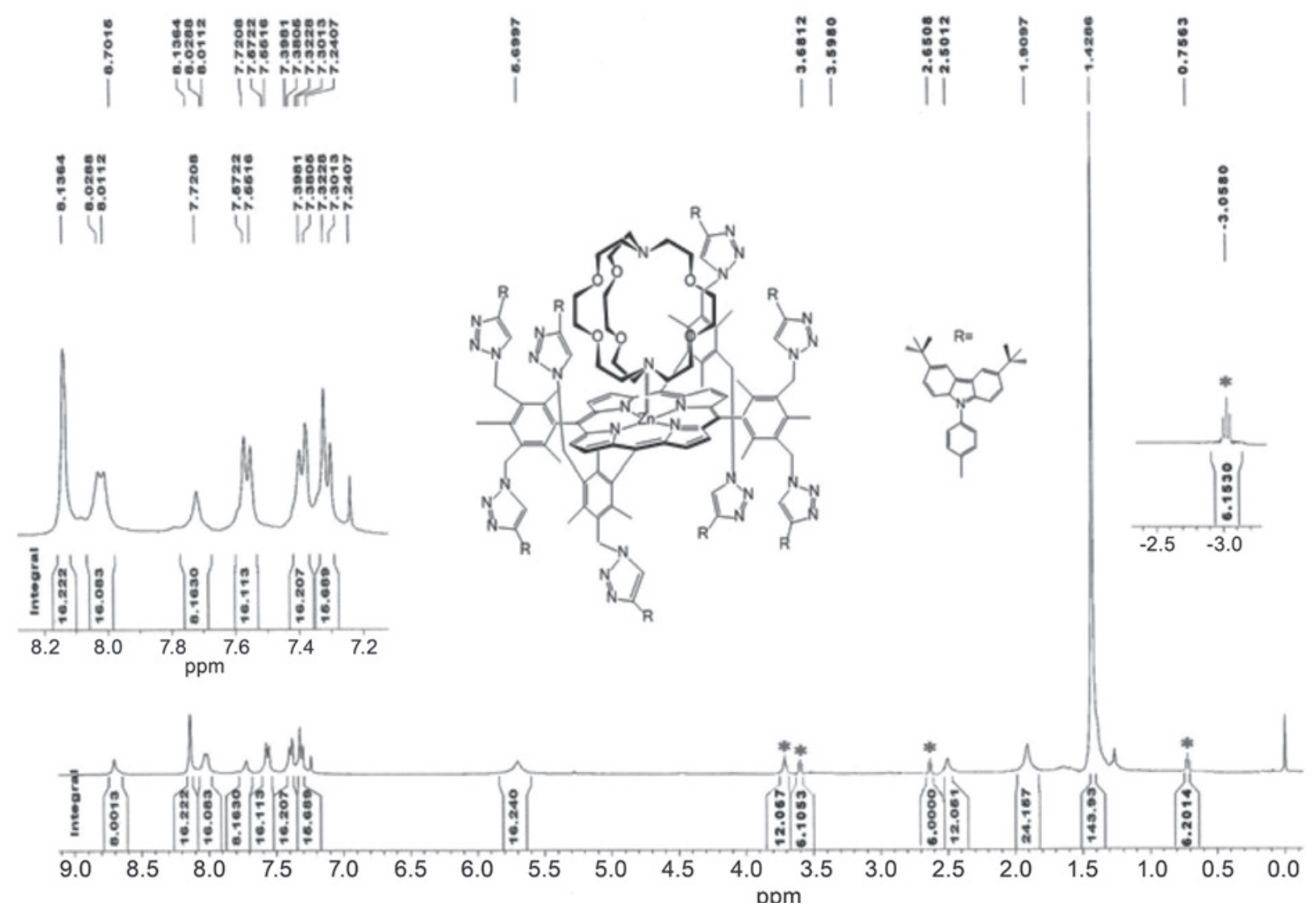

Figure 86. ${ }^{1} \mathrm{H}$ NMR spectrum of $\mathbf{5 5 - C r}$ complex in $\mathrm{CDCl}_{3}(300 \mathrm{MHz}, 298 \mathrm{~K})$. 
$\mathrm{R}=\mathrm{G} 1, \mathrm{G} 2$

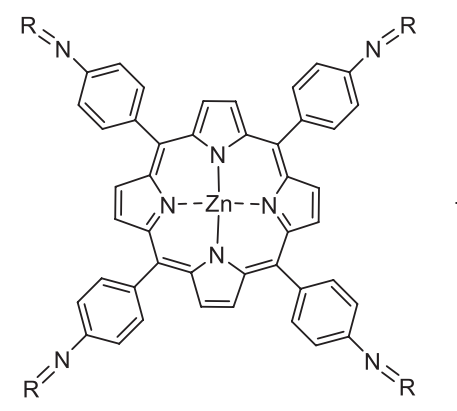

$\mathrm{G} 1(n=2), G 2(n=3)$

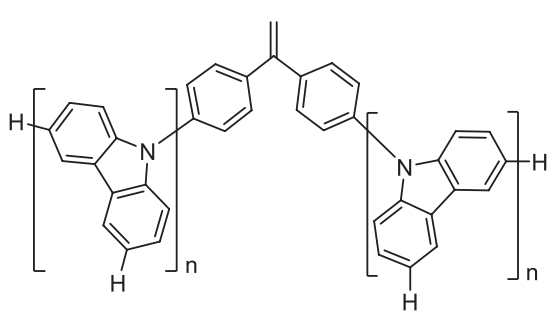

$58 \mathrm{G1}, 58 \mathrm{G} 2$

of the outer layer of the receptor. The stability constants of the corresponding complexes are given in Table 12.

It was shown that in the case of 58G2, the efficiency of $\mathbf{C}_{60}$ binding depends on the presence of phenyl-pyridine (PhPy) in the reaction system (the stability constant of the $\left[\mathbf{5 8 G 2}-\mathbf{C}_{60}\right.$ ]-PhPy complex is 1.5 times higher than

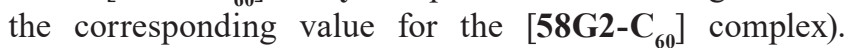
According to ${ }^{[727]}$, complexation of the porphyrinate zinc cation with the phenylpyridine nitrogen atom, causing conformational changes in the dendrimeric environment of the macrocycle, affects the complexing ability of the carbazole outer layer of the $\mathbf{5 8 G 2}$ receptor with respect to $\mathrm{C}_{60}$.

The presented results indicate that porphyrincontaining macrocyclic compounds with a predetermined architecture and functional properties can be used to create ion-controlled molecular devices with a wide spectrum of action. The selectivity and high sensitivity of porphyrins to low-energy external influences makes it possible to control the chemical processes occurring with their participation. These features, together with highly customizable molecular shapes, make these porphyrin-based molecular assemblies ideal receptors for extraction, targeting, and sustained release processes. Given their extremely high extinction coefficients, porphyrins are also excellent molecular detectors for imaging processes affecting the tetrapyrrole macrocycle itself or its surrounding architecture.

\section{Porphyrin Based Optical Sensors for Detection of Some Gases}

The unique chemical and optical properties of porphyrins and their metal complexes have been intensively studied as sensing materials in different fields of application such as analytical, industrial and bio-medical. ${ }^{[326,728-731]}$ This interest based on the ability of porphyrins to form the complexes with most of the metal ions thus providing rich and diverse coordination chemistry. Furthermore, synthetic modification of the macrocycle core and peripheral substituents allows constructing derivatives with specified molecular properties creating different types of porphyrin based sensors with tunable properties. An importance and progress in application of metalloporphyrins as the sensing materials is evident from chapter dedicated to these properties in the Handbook of Porphyrin Science ${ }^{[732]}$ and many of more recent books and reviews. ${ }^{[733-737]}$ Significant interest of many group of scientists over the world to the practical application of porphyrins have led to development of various types of liquid phase and solid state porphyrin based chemical sensors such as chemoresistors, mass transducers, electrochemical (amperometric, potentiometric and voltammetric), photoelectrochemical and optical sensors. ${ }^{[737]}$ Due to the vastness of the field and a huge number of relevant publications it becomes impossible to cover all areas in the one short review. By this reason and experience in optical spectroscopic investigation of the porphyrin and metalloporphyrin reactions with the gaseous molecules the authors are focusing on the recent development in porphyrin based optical sensors. The review presented here will focus mostly on the several specific, biologically important gases such as molecular oxygen, ammonia, nitric oxide and dioxide, hydrogen chloride, sulfur dioxide and hydrogen sulfide.

In optical chemical sensors, the target molecule is recognized by detecting the changes of the optical spectra (as a rule absorbance or fluorescence) when interacting with the target analyte. In general, for development of chemical sensors using optical properties of sensing material the difference in the optical spectra before and after interaction with analytes is used. In terms of optical detection porphyrins and their derivatives represent attractive and widely used class of organic chromophores for sensor elements. Synthetic porphyrins and metalloporphyrins possess high molar extinction coefficients in both the near UV and visible regions and are good fluorescence emitters with relatively high quantum yields. ${ }^{[738]}$ These properties are very suitable for the optical detection and can be tuned by changing metal ion, trans-coordination and/or modification of peripheral substituent groups. The test samples of the optical devices are usually the solid state films deposited on different light-transparent substrates. By this reason, the morphology of the samples (self-organization, uniformity, porosity, thickness and interaction with surfaces) becomes very critical affecting the most important characteristics such as sensitivity, selectivity and reproducibility of the sensors. ${ }^{[732]}$ Various coating techniques, such as dip- and spin-coating, layer-by-layer, Langmuir-Blodgett (LB) and vapor depositions has been explored to obtain reproducible and controlled structures of porphyrin films. ${ }^{[739]}$ In sensing devices, the porphyrins can be also incorporated in light transparent polymer matrices or different composite materials with high permeability for the gases. ${ }^{[735,740-742]}$ 


\section{Hydrogen Chloride and Ammonia}

$\mathrm{HCl}$ and $\mathrm{NH}_{3}$ gases have been widely used as model analytes to test porphyrin-based optical sensors. ${ }^{[737]}$ The early reported porphyrin-based optochemical sensors of these gases are mainly based on the incorporation of porphyrin into glasses, polymers, sol-gel and some solid-state films. ${ }^{[743-746]}$ However, enhancing the long-term stability and shortening the response time for these porphyrin dispersed materials are two major challenges.

Kalimuthu et al. prepared the sensor by coating porphyrin derivatives on glass slide for $\mathrm{HCl}$ sensing. Among the different porphyrin derivatives, meso-tetramesitylporphyrin ( $m$-TMP) coated film showed excellent sensitivity towards $\mathrm{HCl}$. The lowest detection limit of $0.03 \mathrm{ppm} \mathrm{HCl}$ was obtained for $m$-TMP based sensor, which displayed also high stability against humidity. The other porphyrin derivatives, $m$-TPP and meso-tetrakis(4-nitrophenyl)porphyrin coated glass slides were not suitable for $\mathrm{HCl}$ gas sensing due to their irreversible nature towards protonation and deprotonation. ${ }^{[747]}$ Cano et al. fabricated a composite system based on meso-tetra(4-methylpyridinium)porphyrin (TMPyP) as the $\mathrm{HCl}$ gas sensing molecule and columnar optically transparent $\mathrm{TiO}_{2}$ films, as the host material. The inclusion of the TMPyP in the composite entails a remarkable improvement in the sensor, such as fast response, long-term stability and a low surface concentration of the porphyrin. The response of this composite sensor reported to be highly stable and the best detection limit was $0.1 \mathrm{ppm}$ at $80{ }^{\circ} \mathrm{C} .^{[748]}$

Nanofibrous membrane of porphyrinated polyimide (PPI) for the rapid detection of $\mathrm{HCl}$ traces was suggested by $\mathrm{Lv}$ et al. Incorporation of the porphyrin fluorophores into polyimide prevented of porphyrin aggregation and improved the stability of polyimide itself. The nanofibrous membrane of PPI showed very high sensitivity and fast optical response time in presence of analyte, which is promising material for constructing $\mathrm{HCl}$-sensitive gas sensor. ${ }^{[749]}$ Later Lin et al. used PPI to prepare thermally stable honeycomb films for $\mathrm{HCl}$ sensing. The experiments reveal that the honeycomb films show larger fluorescence quenching than that of the corresponding dense films. The films show high thermal stability and high sensitivity to $\mathrm{HCl}$. Recovery experiments by ammonia gas also demonstrate the reusability of the honeycomb films which provide high potential for practical application. ${ }^{[750]} \mathrm{Hu}$ et al. suggested sensitive and fast sensor for gaseous $\mathrm{HCl}$. It is based on the use of the optical probe meso-TPP impregnated in a poly(lactic acid) nanoporous fiber membrane. Porous structure of the sensor facilitates the fast gas absorption and diffusion. The exposure to $\mathrm{HCl}$ causes a color change and initiates quenching of fluorescence. The detection limit of $34 \mathrm{ppb}$, and the response time of $5 \mathrm{~s}$ was reported. The sensor is highly stable after ten cycles and show fully reversible response. ${ }^{[751]}$

Recently Cheng group introduced a facile fluorescent and colorimetric sensor capable of naked-eye detection of gaseous $\mathrm{HCl}$. They used the meso-TPP immobilized in a polystyrene nanoporous fiber membrane and monitored by fluorescence spectrophotometry. Exposure to $\mathrm{HCl}$ gas causes apparent color change of the sensor from pink to yellow-green. The detection limit was found to be $46 \mathrm{ppb}$ and the response time $5 \mathrm{~s}$. The sensor exhibits excellent reversibility and prompt response and recovery. ${ }^{[752]}$ Later, the same group improved the sensor demonstrating the applicability of functionalized hierarchical porous silica nanofiber membrane for the sensitive and rapid tracing $\mathrm{HCl}$ gas at the ppb level by the change of the fluorescence intensity. The sensor used 5,10,15,20-tetra(4-carboxyphenyl)porphyrin immobilized in the hierarchical porous silica nanofiber membrane. The presence of mesopores and macropores promoted the gas adsorption and diffusion exhibiting a highly sensitive and time-efficient response for $\mathrm{HCl}$ gas detection. The sensor showed high sensitivity with a detection limit of $17 \mathrm{ppb}$ and a fast response of $5 \mathrm{~s}$. The device also had a good reversibility, which had a promising to develop a low-cost $\mathrm{HCl}$ gas sensing system. ${ }^{[753]}$

With the aim of $\mathrm{NH}_{3}$-sensing Zuo et al. used spin-coating films based on palladium, nickel, and cobalt tetra (tert-butyl)-5,10,15,20-tetraazaporphyrin $\left(\operatorname{MTAP}(t-B u)_{4}\right)$. The sensors were studied at room temperature following the UV-Vis absorption changes. The $\operatorname{PdTAP}(t-\mathrm{Bu})_{4}$ film exhibited the higher response and recovery times than $\mathrm{CoPc}(t-$ $\mathrm{Bu})_{4}$ and $\mathrm{NiPc}(t-\mathrm{Bu})_{4}$ films, that is explained by the nature of central metal and porous film structure of $\operatorname{PdTAP}(t-\mathrm{Bu})_{4}$. This sensor suggested being a candidate for application as $\mathrm{NH}_{3}$ detector in concentration range of 100-800 ppm. ${ }^{[754]}$ Later Muthukumar et al. synthesized meso-tetrakis(4-nitrophenyl)porphyrin (MTNP), meso-tetrakis(4-pyridyl)porphyrin, 5,15-bis(ferrocenyl)-10,20-bis(4-nitrophenyl)porphyrin and 5,15-bis(4-nitrophenyl)-10,20-bis(3,5-dimethoxyphenyl)porphyrin and incorporated them into Nafion film coated on glass slide to examine the $\mathrm{NH}_{3}$ gas sensing properties by monitor the changes at the Soret band. Among the studied porphyrin derivatives, the Nf-MTNP was most sensitive and detection limit of $0.025 \mathrm{ppm} \mathrm{NH}_{3}$ was achieved. ${ }^{[755]}$

Recently porphyrin/ $\mathrm{TiO}_{2}$ composites has been studied as gas sensors for $\mathrm{NH}_{3}$ through previous protonation of the porphyrin with $\mathrm{HCl}$ followed by subsequent exposure to the basic analyte. Two fluorescent cationic porphyrins, 5-(N-methyl 4-pyridyl)-10,15,20-triphenylporphine chloride (MMPyP) and meso-tetra( $N$-methyl-4-pyridyl)porphine tetrachloride (TMPyP) were anchored to nanostructured thin films of $\mathrm{TiO}_{2}$. However, authors reported significant difference in behavior of two derivatives. While the $\mathrm{MMPyP} / \mathrm{TiO}_{2}$ composite gave reasonable response to $6 \mathrm{ppm}$ of $\mathrm{NH}_{3}$, the TMPyP/ $/ \mathrm{TiO}_{2}$ system showed no response for the same concentration. This result is explained by different attachments of the two porphyrins to the $\mathrm{TiO}_{2}$ matrix. ${ }^{[756]}$ Yoon and co-workers suggested ingenious method in developing $\mathrm{NH}_{3}$ sensors that utilize the formation of transition-metal ammonia complexes to trigger a change in fluorescence. In particular, authors exploring the systems in which reaction of $\mathrm{NH}_{3}$ with a transition-metal complex containing a coordinated fluorophore conjugate resulted in removal of the fluorophore from the coordination sphere with concomitant fluorescence turn-on, in contrast to usually used fluorescence quenching when monitoring an analyte. It was reported that $\mathrm{NH}_{3}$ can displace an axial fluorophore ligand bound to dansylpiperazine(porphyrin-Co(II)) complex, with concomitant six fold fluorescence increase. The detection limit of the sensor calculated to be $40 \mathrm{nM} \cdot{ }^{[757]}$ 
Fiber-optic sensing techniques have attracted a great attention in a chemical and biological sensing and showed success in detecting ammonia with application of porphyrins. ${ }^{[758]}$ Tapered optical fibres with nano-assembled coatings of thicknesses of order tens of nanometres were used for the detection of $\mathrm{NH}_{3}$ gas by Lee and co-workers. The film coating was composed of alternate layers of tetrakis(4sulfophenyl)porphine (TSPP) and poly(allylamine hydrochloride) (PAH). Exposure of a PAH/TSPP nano-assembled optical fiber to $\mathrm{NH}_{3}$ induced significant optical changes in the transmission spectrum and showed a linear sensitivity to the concentration of $\mathrm{NH}_{3}$ in the range of $10-100 \mathrm{ppm}$, with very good response and recovery times. The limit of detection was estimated to be $c a .2$ ppm. ${ }^{[759]}$ The same group reported a long period grating optical fiber sensor coated with a multilayer film of poly(diallyldimethyammonium chloride) (PDDA) and tetrakis(4-sulfophenyl)porphine (TSPP) for $\mathrm{NH}_{3}$ gas detection. The nano-assembled thin film was prepared using a layer-by-layer deposition technique and limit of detection of $0.67 \mathrm{ppm}$ for $\mathrm{NH}_{3}$ gas was achieved. ${ }^{[760]}$ The group have consistently improved the sensors properties and recently reported highly sensitive fiber-optic $\mathrm{NH}_{3}$ sensors fabricated via layer-by-layer deposition of PDDA and TSPP onto the surface of a fiber. The sensitivity of the fiber-optic sensor to $\mathrm{NH}_{3}$ was linear in the range of $0.5-50 \mathrm{ppm}$ showed good response and recovery times and limit of detection of $0.5 \mathrm{ppm} .{ }^{[761]}$ Very recently Sawada and co-workers introduced Co-octaethylporphyrin functionalized graphene sensor which successfully detected sub-ppm of $\mathrm{NH}_{3}$ and demonstrated an excellent selectivity. The detection of low $\mathrm{NH}_{3}$ concentration $(0.04 \mathrm{ppm})$ was shown even under rapid humidity changes that is required for medical breath checkup. ${ }^{[762]}$

\section{Nitrogen Dioxide and Nitrogen Oxide}

The porphyrin films themselves appear to be not good candidates for $\mathrm{NO}_{2}$ gas sensors. The main problem of porphyrin films is their tendency to self-aggregate in time into the clusters which often makes them not uniform and results in unsatisfactory reproducibility from sample to sample or irreversible spectral changes upon interacting with $\mathrm{NO}_{2}{ }^{\left[{ }^{738]}\right.}$ However, insertion of the LB porphyrin films into optically transparent porous host matrix demonstrated an improvement of the sensing properties. Richardson et al. reported that incorporation of 5,10,15,20-tetrakis[3,4-bis(2ethylhexyloxy)phenyl]-21H,23H-porphyrin (EHO) into a calixarene host in the form of a mixed LB film has led to a gas sensing material with a very fast response time. This was explained as a result of the porosity of the calixarene domains originating from the cavities. The study revealed a high sensitivity to $\mathrm{NO}_{2}$ in the very low $130 \mathrm{ppb}-1.2 \mathrm{ppm}$ concentration region. A fast response after gentle heating to around $70-90{ }^{\circ} \mathrm{C}$ has been reported. ${ }^{[763]}$ In more recent study LB mixed films of EHO and p-tert-butylcalix[8] arene demonstrated development of the sensing properties of the porphyrin. This study revealed important thickness dependence that has been related to the surface roughness. ${ }^{[764]}$ Roales et al. prepared composite films based on microstructured $\mathrm{TiO}_{2}$ and free-base porphyrins:
5-(4-carboxyphenyl)10,15,20-triphenyl-21H,23H-porphyrin (MCTPP), 5,10,15,20-tetrakis(4-carboxyphenyl)21H,23H-porphyrin ( $p$-TCPP), and 5,10,15,20-tetrakis(3carboxyphenyl)-21H,23H-porphyrin ( $m$-TCPP) in order to analyze gas-sensing capabilities towards $\mathrm{NO}_{2}$. Authors reported that $m$-TCPP/TiO films are the most stable, showing less aggregation than the other porphyrins. The exposure of films to $\mathrm{NO}_{2}$ has resulted in changes in their UV-Vis spectra and revealing good sensing capabilities in all cases. The improved stability of film made with $m$-TCPP suggests it as the best candidate for the fabrication of $\mathrm{NO}_{2}$ sensor. ${ }^{[765]}$

Optical waveguides are a relatively recent innovation in the field of optical chemical sensors and have been developed for the measurement of trace amounts of a variety of gases at room temperature. ${ }^{[766]}$ In 2012 Woellenstein and co-workers developed the $\mathrm{NO}_{2}$ gas sensing using planar optical waveguide (OWG). The ZnTPP was embedded into a PVC matrix and deposited onto OWG. The sensor showed that $\mathrm{NO}_{2}$ was detectable with a resolution of $1 \mathrm{ppm}$. Exposure of sensor to $5 \mathrm{ppm} \mathrm{NO}_{2}$ induces the color change from purple to yellow. The main disadvantage of implementing ZnTPP is its poor, of several days reversibility. ${ }^{[767]}$ An OWG sensor based on meso-5,10,15,20-tetrakis(4-tertbutylphenyl)porphyrin thin film fabricated by spin coating method was reported by Abudukeremu et al. The sensing behavior of the studied device was tested through mixture of $\mathrm{H}_{2} \mathrm{~S}, \mathrm{SO}_{2}$, and volatile organic compounds (VOC) gases with and without $\mathrm{NO}_{2}$. This sensor exhibited high sensitivity and selectivity to $\mathrm{NO}_{2}$ with fast response time and recovery time and was capable of measuring $\mathrm{NO}_{2}$ down to $10 \mathrm{ppb}$ at ambient temperature. ${ }^{[768]} \mathrm{B}$. Kutilike et al. used 5,10,15,20-(4-hydroxyphenyl)porphyrin as the sensitive material with an OWG. The lowest detection concentration for $\mathrm{NO}_{2}$ was $10 \mathrm{ppb}$, the value at which the sensor demonstrated characteristics of fast response and stability. For $\mathrm{NO}_{2}$ high-concentration $(\geq 100 \mathrm{ppm})$ the sensitive film appeared colorless and could not be recovered. Therefore, in practical application, $\mathrm{NO}_{2}$ gas with low concentration may be detected. The detection can be conducted at ambient temperature and humidity. ${ }^{[769]}$

The optical sensors for nitrogen oxide (NO) gas detection are rare ${ }^{[728]}$ Earlier, pyrrole-based polymers containing porphyrin complexes appeared among one of the candidates for NO gas sensor applications. ${ }^{[770]}$ Recently Miki et al. reported that cobalt tetraphenylporphyrin CoTPP is a promising chemochromic material with excellent sensitivity for detecting NO with an accuracy of $\pm 1 \mathrm{ppb}$. The sensors were fabricated by embedding Co-TPP derivatives into porous nonwoven fabrics. However, the developed sensor response was significantly fluctuated by the change in humidity. ${ }^{[771]}$ In very recent report an optical NO gas sensor, the CoTPP was dispersed in three hydrophobic polymer matrix (polystyrene, ethylcellulose (EC), and polycyclohexyl methacrylate) to improve humidity resistance. The existence of a hydrophobic polymer matrix resulted not only in greater humidity resistivity but also in a more than six times increase in sensitivity due to the suppression of CoTPP aggregation. The limit of detection of NO gas obtained by the CoTPP-EC film was $33 \mathrm{ppb}$, which is lower than that obtained by the CoTPP film (92 ppb). ${ }^{[772]}$ 


\section{Hydrogen Sulfide} and Sulfur Dioxide

Common $\mathrm{H}_{2} \mathrm{~S}$ and $\mathrm{SO}_{2}$ gas sensors are usually based on the metal oxide semiconductors and various hybrid materials. ${ }^{[773]}$ However, in recent years several optical $\mathrm{H}_{2} \mathrm{~S}_{\text {and }} \mathrm{SO}_{2}$ gas sensors have been developed with very promising characteristics. Pellecchia et al. reported binding of hydrogen sulfide $\left(\mathrm{H}_{2} \mathrm{~S}\right)$ to a tetra( $N$-methylpyridyl)porphinatozinc complex (TMPyPZn) and the stabilization of the related zinc hydrosulfido adduct. The coordination of HS- occurs in a reversible manner and modulates fluorescence emission of a zinc porphyrin complex. The results show the potential of TMPyPZn for the implementation of fast and simple $\mathrm{H}_{2} \mathrm{~S}$ sensors via a coordinative-based approach. ${ }^{[774]}$

Metal-organic frameworks (MOF), are relatively new type of hybrid porous coordination polymers with two- or three-dimensional structure and unique properties which gained increasing attention of many research groups for different applications. These materials have ultra-high and tunable porosity, adjustable structure as well as unsaturated metal sites. ${ }^{[775]}$ The integration of porphyrins into MOFs created new characteristics and versatility of applications. ${ }^{[776]} \mathrm{Ma}$ et al. synthesized a $\mathrm{Cu}(\mathrm{II})$ metalated $3 \mathrm{D}$ porous nanoscale metal-organic framework $\left\{\mathrm{CuL}[\mathrm{AlOH}]_{2}\right\}_{\mathrm{n}}$ integrated with meso-tetra(4carboxyl-phenyl) porphyrin as a fluorescence probe for $\mathrm{H}_{2} \mathrm{~S}$ detection. An interaction of $\mathrm{H}_{2} \mathrm{~S}$ molecules with porphyrin ligand based MOF turned on fluorescence. This nanoMOF probe exhibits rapid response, excellent selectivity in in-situ detection of $\mathrm{H}_{2} \mathrm{~S}$. The sensor was also successfully employed as the probe to detect endogenic $\mathrm{H}_{2} \mathrm{~S}$ living cells and represents the most sensitive fluorescence probe for selective $\mathrm{H}_{2} \mathrm{~S}$ detection under physiological $\mathrm{pH} .{ }^{[777]}$

Yimit group very fruitfully used OWG approach to develop various porphyrin based $\mathrm{H}_{2} \mathrm{~S}$ sensors. An optical OWG sensor based on tetrakis(4-nitrophenyl)porphyrin immobilized in a Nafion membrane and deposited onto an glass slide has been reported in 2017 for the detection of $\mathrm{H}_{2} \mathrm{~S}$ and ethanediamine gases. The sensor exhibited excellent reproducibility, reversibility, and selectivity with detection limits for $\mathrm{H}_{2} \mathrm{~S}$ and ethanediamine of 1 and $10 \mathrm{ppb}$, respectively, and has been supposed as promising candidate for use in industrial sensing applications. ${ }^{[778]}$ In very recent paper the same group reported results on a 5,10,15,20-tetrakis(4-methoxyphenyl)porphyrin (TMP) deposited by spin coating on OWG sensor. The device has good response and detects low concentration to $\mathrm{H}_{2} \mathrm{~S}$ and $\mathrm{SO}_{2}$. The TMP film OWG sensor showed high sensitivity and selectivity towards $\mathrm{H}_{2} \mathrm{~S}$ and $\mathrm{SO}_{2}$ gases and exhibits very good response to $1 \mathrm{ppm}$ concentrations.

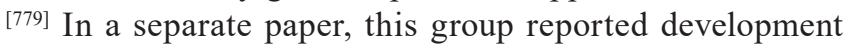
of OWG sensor to detect $\mathrm{SO}_{2}$ and $\mathrm{H}_{2} \mathrm{~S}$ gases based on thin film of 5,10,15,20-(tetra-4-aminophenyl)porphyrin immobilized over the surface of a potassium ion exchange glass. This sensors successfully measure extremely low concentrations of $\mathrm{SO}_{2}$ and $\mathrm{H}_{2} \mathrm{~S}$ (detection limit $=1 \mathrm{ppt}$ ), providing good repeatability at room temperature. The proposed sensor has great potential for future applications in sulfur gases detection. ${ }^{[780]}$

\section{Oxygen}

Among of many kinds of oxygen sensors that have been developed, the optical oxygen sensors based on the fluorescence quenching principle has been of particular interest due to their advantageous features such as fast response, accurate detection, simple operation, high sensitivity. Platinum and palladium porphyrins are often used as oxygensensing probe because display strong room-temperature phosphorescence with high quantum yield and long lifetime. Principles of oxygen sensing and properties devices based on metalloporphyrins dispersed in polymer film were well reviewed by Amao in 2009. ${ }^{[781]}$ Although common Pdand Pt-porphyrins continue attract an attention, ${ }^{[782,783]}$ halogenated porphyrins show better photostability, and oxygen sensors based on them are of interest since they possess high response and recovery time.

A series of fluorinated $\mathrm{Pt}(\mathrm{II})$-porphyrins were synthesized and examined by Arunkumar et al. for oxygen sensitivity in both the solution and in poly(aryletherketone) polymer film. The results demonstrated that by introducing fluorosubstituents at the porphyrin periphery the oxygen sensing properties of the complexes was improved. For example introducing the 3,5-di(trifluoromethylphenyl) group at the meso-position, the oxygen sensitivity is improved twofold. The high sensitivity and the short response/recovery time reveal that these complexes display a higher oxygen sensing ability for the analysis of trace oxygen. ${ }^{[784]}$ Biring et al. have proposed an optical sensor for the dual sensing of oxygen and ammonia gases, which consists of oxygen and ammonia-sensitive fluorescent dyes coated individually on both sides of a glass substrate. An ethyl cellulose matrix doped with platinum(II) meso-tetrakis(pentafluorophenyl) porphyrin serves as the oxygen-sensing material, whereas the $\mathrm{NH}_{3}$-sensing material includes an eosin fluorescent indicator immobilized within a cellulose acetate matrix. The high sensitivity and selectivity of the proposed optical dual sensor suggests the feasibility of the simultaneous sensing of oxygen and ammonia for practical applications. ${ }^{[785]}$

Mao and co-workers developed the oxygen sensors with fluorinated $\mathrm{Pt}$ porphyrins embedded in different matrix. In 2017 they fabricated novel micro-pillar array sensor film for gaseous and dissolved oxygen using platinum(II)5,10,15,20-tetrakis(2,3,4,5,6-pentafluorophenyl)porphyrin (PtTFPP) in polydimethysiloxane matrix. The sensor exhibited unmatched sensitivity and detection limit as low as $0.10 \mu \mathrm{mol} / \mathrm{L}$. Furthermore, the remarkably light intensitychanging characteristic of the sensing film under lower $\mathrm{O}_{2}$ partial pressures facilitates convenient identification of $\mathrm{O}_{2}$ concentrations even with the naked eye. It is very promising candidate in various oxygen sensing fields. ${ }^{[785]}$ Later the group presented a facile approach for the fabrication of honeycomb structured porous films for optical oxygen sensors using a platinum porphyrin-grafted poly(styreneco-4-vinylpyridine) copolymer. The application of these films as oxygen sensors afford exceptional improvement in sensitivity than the solid sensing film owing to the higher accessibility of gas molecules and larger specific surface area. Interestingly, the remarkably light intensity-changing characteristic of the sensing film under lower $\mathrm{O}_{2}$ partial 
pressures facilitates convenient identification of $\mathrm{O}_{2}$ concentrations even with the naked eye. ${ }^{[787]}$ Recently the same group fabricated a high fluorinated platinum porphyringrafted poly(isobutylmethacrylate-co-dodecafluoroheptylmethacrylate) copolymers (PtTFPP-p(IBM-co-DFHMA)) microfibrous thin-films and used them as dissolved oxygen optical sensors. The porous thin-film frameworks afforded an exceptional improvement in sensitivity and exhibited significant higher sensitivity than the solid sensing film owing to the large specific surface area, porous structures and oxygen diffusion enhancement by fluorine elements. This device may be also used as sensor in biological systems. ${ }^{[788]}$ Optical imaging of oxygen in biological systems using porphyrins has been well studied by Vinogradov and coworkers. ${ }^{[789]}$

Although porphyrin-based optical sensors for small gaseous molecules have been intensively studied, there is a grate and endless potential existing for further development of these devices. This potential steams from both, synthetic chemistry of porphyrins and design of the new materials with controlled diffusion of gases into the sensing sites. Improvement of future devices is associated with use of the porphyrins and inorganic or hybrid materials with porous structures, such as honeycomb films, nanoporous fibers, metal-organic frameworks, carbon nanotubes, etc.

\section{Macroheterocyclic Inducers and Selectors}

Macroheterocyclic compounds - porphyrins, phthalocyanines and their analogues, as well as metal complexes based on them - have a number of useful properties that allow them to be used in a number of branches of technology, healthcare, etc. ${ }^{[790,791]}$ At the same time, some specific characteristics of macroheterocycles (MHCs) allow to hope that their potential is far from being exhausted. First of all, this is their high chemical and heat resistance, molecular anisotropy, tendency to axial coordination of various substances, wide possibilities of chemical modification, etc. In addition, MHCs are promising objects of supramolecular chemistry. ${ }^{[74,792,793]}$ All this makes the search for new unconventional areas for the use of MHCs to be actual.
An application of macroheterocycles in liquid-crystalline (LC) systems as dichroic dyes and inducers of helical phases is of particular interest, as well as components of stationary phases and separation membranes with high structural and chiral selectivity. This section is devoted to the analysis of scientific results obtained in the last few years in the field of studying macroheterocycles as inducers of spiral LC phases and selectors in the composition of stationary phases for gas chromatography.

\section{Macroheterocyclic Inducers of Helical Liquid- Crystalline Phases}

The different aspects of chirality in liquid crystals (LC) are of great scientific interest. ${ }^{[795]}$ The most noticeable technical application of helical phases consisting of chiral molecules or induced by chiral dopants in non-chiral LCs is electro-optical devices. ${ }^{[795]}$ However, electro-optics is not the only field of induced LC phases application. Such systems are used in the development of chromatographic stationary phases with high chiral selectivity. ${ }^{[796]}$ Based on them, flexible magnets, ${ }^{[797]}$ photosensitive nanostructures, ${ }^{[798]}$ highly sensitive and selective sensors for gas detection are being developed. ${ }^{[799,800]}$ Two-component LC optically active dopant systems are perspective as chiral media ${ }^{[801]}$ for highly enantioselective reactions. Films based on nematic LC doped with chiral light-driven molecules are used for molecular motors design. ${ }^{[802]}$

The most important field in development of the theory and practice of spiral mesophase induction is molecular design of effective chiral dopants with helical twisting power (HTP) associated with the helix pitch by a simple equation: ${ }^{[790,803]}$

$$
\mathrm{HTP}=(\mathrm{p} \cdot \mathrm{c} \cdot \mathrm{r})^{-1}
$$

where $\mathrm{p}$ - helical pitch; $\mathrm{c}-$ dopant mole fraction; $\mathrm{r}-$ its enantiomeric purity.

In almost all studies, a noticeable effect of the nematic solvent (host effect) on the dopant HTP was noted, which is due to the nature and intensity of the intermolecular interactions solute-solvent. ${ }^{[803,804]}$ It allows to conclude

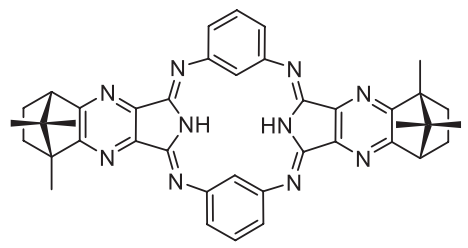

(59)

$(+) \mathrm{HPA}$

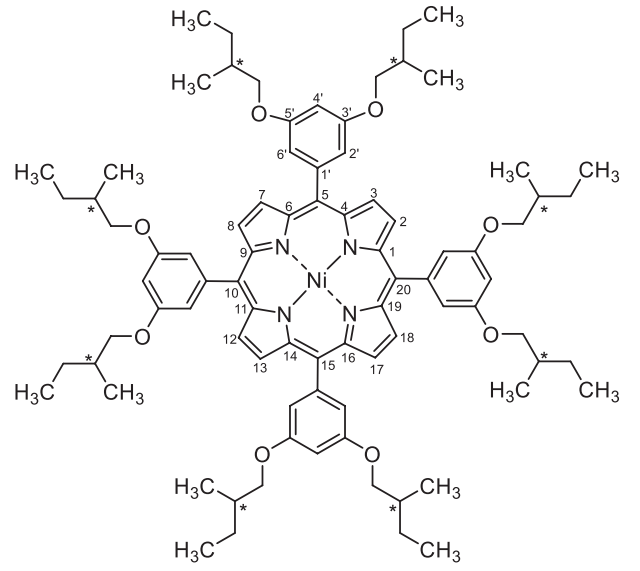

(60)

$\mathrm{Ni}-\mathrm{S}(+) \mathrm{TPP}$ 
that for the effective chiral transfer dopant - LC, optimal orientational correlations of the components due to strong intermolecular interactions must be ensured. The metal complexes ${ }^{[805-808]}$ occupy a special place among chiral dopants. Interest in them is connected with the possibility of strong coordination interactions in the pair complex dopant - LC. Synthetic macroheterocycles and their metal complexes are of great interest because of their natural analogues - heme, chlorophyll, etc., as well as their use as dyes, catalysts, stabilizers, and pharmacological preparations. ${ }^{[809]}$ The presence of a wide variety of chiral porphyrins, phthalocyanines and their analogues ${ }^{[810-812]}$ opens up wide possibilities for using them as chiral dopants for nematic LC. ${ }^{[813]}$

In this regard, in our works, ${ }^{[813,814]}$ we have synthesized the chiral symmetric camphor substituted hemiporphyrazine, (+)HPA (59) and nickel complex of 5,10,15,20tetrakis[3',5'-di-(S)(+)(2"'-methylbutyloxy)phenyl]porphine, Ni-S(+)TPP (60):

Specific optical rotation $(+) \mathrm{HPA}+88.0^{\circ}, \mathrm{Ni}-\mathrm{S}(+) \mathrm{TPP}$ $+103.45^{\circ}$.

The studies of the camphor-substituted hemiporphyrazine $(+)$ HPA and its racemic mixture $( \pm)$ HPA effect on the nematic phase of the six-component mixture of cyanobiphenyls ( $\mathrm{RO}-\mathrm{C}_{6} \mathrm{H}_{4}-\mathrm{C}_{6} \mathrm{H}_{4}-\mathrm{CN}$ ) $\mathrm{CB} 6$ were presented in ${ }^{[813]}$.

Figure 87 shows the Schlieren texture of the initial LC mixture of CB6, characteristic for a nematic phase, the fingerprint texture of the mixture doped with chiral isomer $(+)$ HPA. The latter texture is characteristic of the chiral nematic phase. By the method of polarizing microscopy the clearing temperatures and helix pitch were measured, and the helical twisting power (HTP) was calculated $\left(2.19 \mu^{-1}\right)$. The degree of destabilizing effect of the racemic mixture $( \pm$ ) HPA on the mesophase was shown to be higher than that of the chiral dopant.

The components of dielectric permittivity and its anisotropy $\Delta \varepsilon=\varepsilon-\varepsilon_{\perp}$ are the important characteristics of LC composition using for electro-optical devices. An interesting feature of the dielectric components temperature

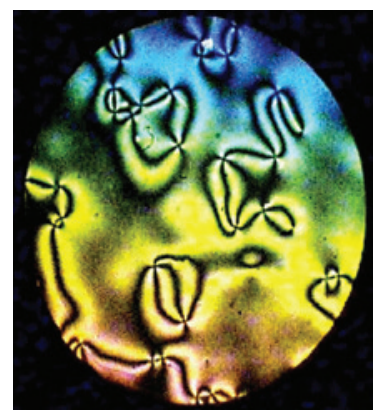

a

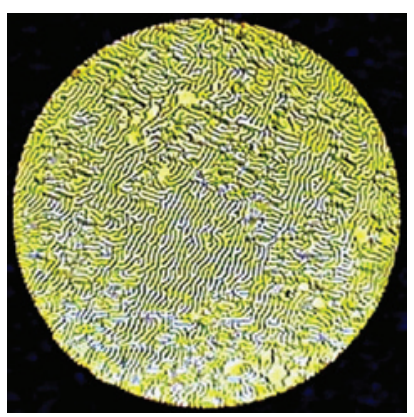

$\mathrm{b}$
Figure 87. Schlieren texture of the CB6 LC nematic phase at $60{ }^{\circ} \mathrm{C}$ (a); fingerprint texture of the mixture CB6 with $0.92 \%(+)$ HPA at $65^{\circ} \mathrm{C}(\mathrm{b}) .^{[813]}$

dependences of the CB6 system with chiral $(+) \mathrm{HPA}$ at its concentration $0.92 \%$ and $1.80 \%$ is the sudden changes in $\varepsilon$; and $\varepsilon_{\perp}$ in the mesophase (Figure $88 \mathrm{a}$ ). At the same time, no noticeable changes in the textures nature (Figure 87 ) and liquid-crystalline properties were found. It allows to suggest that a non-monotonic change in the dielectric parameters is associated with the action of a magnetic field on the helical mesophase. At temperatures and corresponding values of viscosity, at which abrupt changes in $\varepsilon, \varepsilon_{\perp}$ and $\Delta \varepsilon$ (Figure 88a) are observed, there is an obvious competition between the action of a chiral additive that tends to twist the mesophase, and a magnetic field that tends to untwist it.

Figure $88 \mathrm{~b}$ presents the temperature dependences of the kinematic viscosity of the CB6 system without a modifier and with a chiral dopant $(+)$ HPA. The introduction of a chiral dopant in amount of $0.92 \%$ is accompanied by a noticeable increase in kinematic viscosity (Figure $88 \mathrm{~b}$ ) and the jump appearance in the temperature range $50-55^{\circ} \mathrm{C}$. It should be noted that this occurs in the same temperature range as the non-monotonic changes in the permittivity components of the mixture CB6 + 0.92\% (+)HPA (Figure 88a). The reason for the appearance of anomalies in the tem-
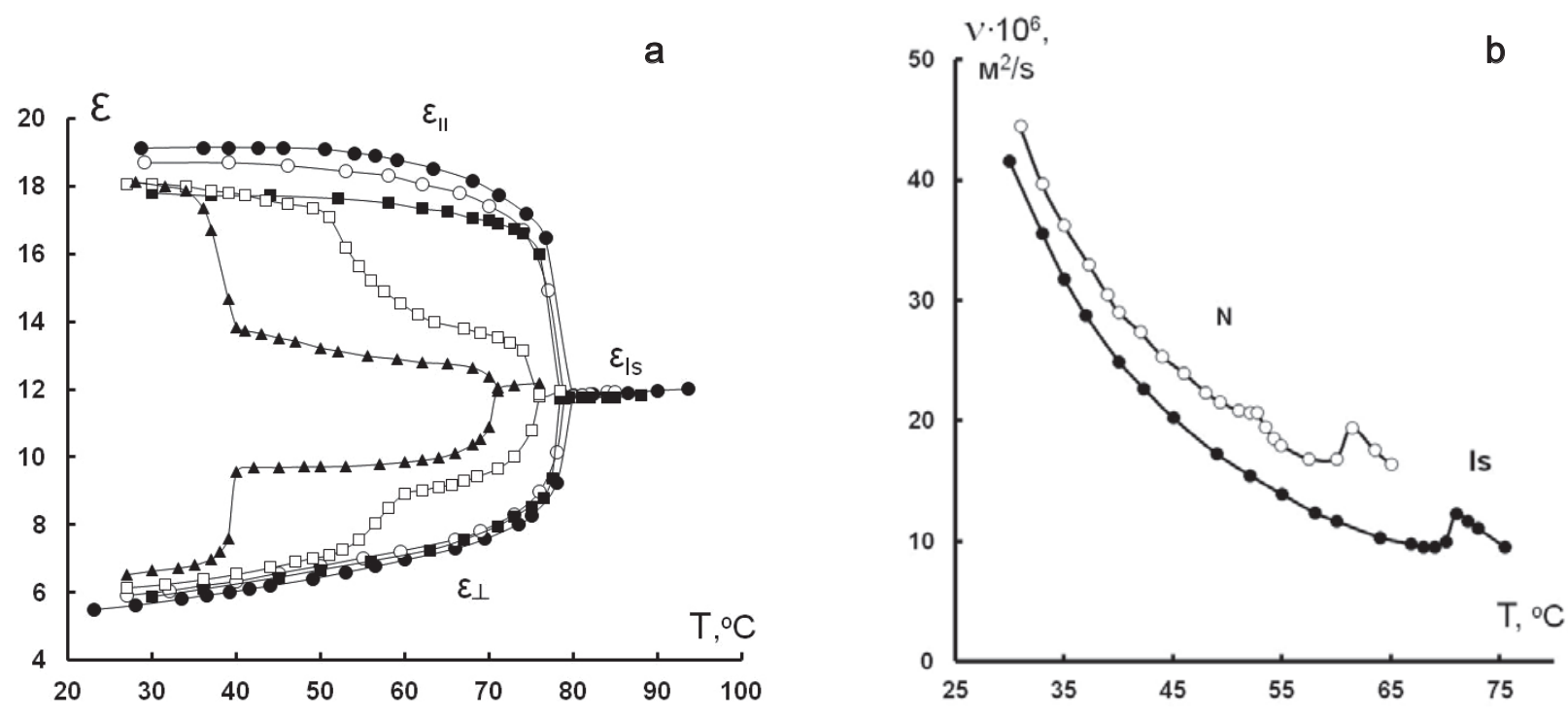

Figure 88. Temperature dependences of the permittivity components for mixtures CB6 at a concentration of $(+) \mathrm{HPA}, \%$ : - • - 0; ○-0.17; - $-0.44 ;-\square-0.92 ;-\boldsymbol{\Delta}-1,80$ (a)..$^{[813]}$ 
perature dependences of viscosity in the mesophase with the addition of a chiral macroheterocycle may be the competition of two effects - the twisting action of the chiral dopant and the orienting action of the flow tending to untwist the spiral structure of the chiral nematic. Thus, the effect of flow in a rheological experiment is similar to the influence of a magnetic field in dielectric measurements. ${ }^{\left[{ }^{[13]}\right.}$

The work $^{[814]}$ is devoted to the study of the nickel complex of 5,10,15,20-tetrakis[3',5'-di-(S)(+)(2"'-methylbutyloxy)phenyl]porphine (Ni-S(+)TPP) (see diagram) influence on the mesophase CB6 and the eutectic mixture SB2 (4-methoxybenzylidene-4'-butylaniline(MBBA) and 4-ethoxybenzylidene-4'-butylaniline (EBBA)).
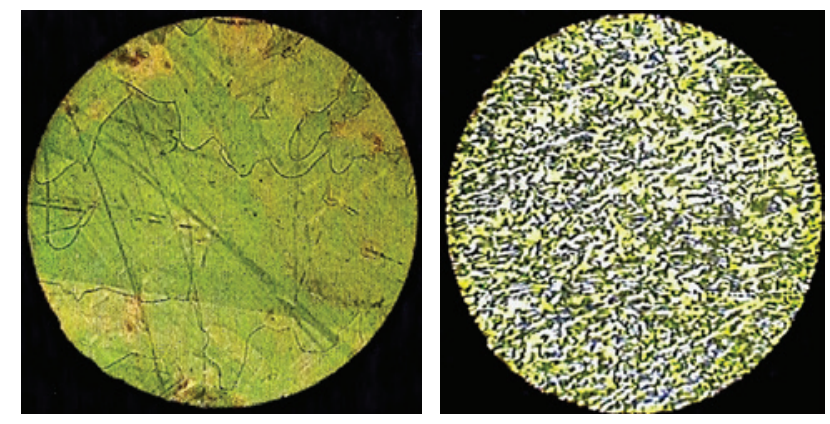

Figure 89. Textures of the initial nematic SB2 at $48{ }^{\circ} \mathrm{C}$ (a) and solution $0.55 \% \mathrm{Ni}-\mathrm{R}(+) \mathrm{TPP}+\mathrm{SB}-2$ at $48^{\circ} \mathrm{C}$.

The doping of the alkoxycyanobiphenyls mixture CB6 with the Ni-S (+) TPP complex is accompanied by a change in texture from Schlieren to the fingerprints texture, similar to that shown in Figure 87. The initial planar texture of the nematic mixture SB2, as a result of the dopant introduction, is also transformed into the texture of fingerprints (Figure 89). These data indicate the formation of chiral nematic phases in both cases. Analysis of the polarization microscopy data allowed to obtain fragments of phase diagrams related to the isotropic phase transition (Figure 90)

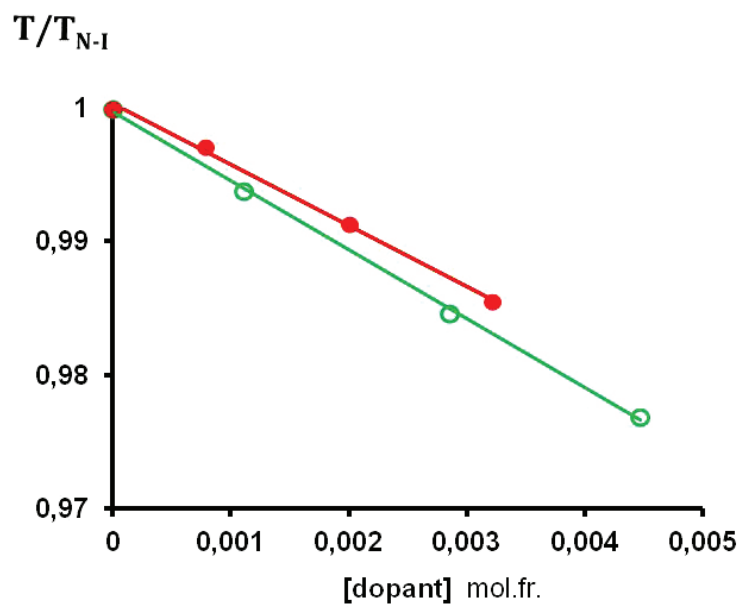

Figure 90. $\mathrm{T} / \mathrm{T}_{\mathrm{NI}}$ versus mole fraction of $\mathrm{Ni}-\mathrm{S}(+) \mathrm{TPP}$ in $\mathrm{SB} 2(-\circ-)$ and $\mathrm{CB} 6(-\bullet-) . \mathrm{T}_{\mathrm{NI}}$ is the clearing temperature of the nematic solvent. ${ }^{[814]}$ and to calculate the degree of LC destabilization as a result of the dopants introduction (Table 13), as well as to measure the values of the helix pitch (Figure 91) and to calculate the HTP (Table 13). ${ }^{[814]}$

Table 13. The slopes of the $\mathrm{T} / \mathrm{T}_{\mathrm{NI}}\left(\mathrm{f}_{2}\right)$ dependences $(\beta)$ and HTP for Ni-S(+)TPP solutions. ${ }^{\left[{ }^{[14]}\right.}$

\begin{tabular}{ccc}
\hline Solution & $\beta$, mol fraction $^{-1}$ & $\mathrm{HTP} \mu_{\mathrm{m}}^{-1}$ \\
\hline $\mathrm{CB} 6+\mathrm{Ni}-\mathrm{S}(+) \mathrm{TPP}$ & -4.43 & 126.5 \\
$\mathrm{SB} 2+\mathrm{Ni}-\mathrm{S}(+) \mathrm{TPP}$ & -5.25 & 63.5 \\
\hline
\end{tabular}

These data indicate a slightly less destabilizing effect of Ni-S (+) TPP on the CB6 mesophase as compared to SB2. It should be noted that Ni-S(+)TPP exhibits a high twisting ability with respect to LC of different chemical classes - polar cyanobiphenyls with high positive dielectric anisotropy $\Delta \varepsilon$ (CB6) and weakly polar Schiff bases with low negative $\Delta \varepsilon$ (Table 13).

Anomalous temperature dependences of the dielectric permittivity components for LC solutions $\mathrm{CB} 6+\mathrm{Ni}-\mathrm{S}(+)$ TPP and SB2 $+\mathrm{Ni}-\mathrm{S}(+)$ TPP were obtained, ${ }^{[814]}$ as well as for $(+) \mathrm{HPA}^{[813]}$ (Figure 88a). This behavior is concerned with competition between the chiral dopant, which twisted the mesophase, and the magnetic field, which tended to untwist LC helix.

On the basis of quantum chemical calculations, the formation of stable solvates of Ni-S(+)TPP with 1 and 2 alkoxycyanobiphenyl molecules due to axial coordination and inclusion was established (Figure 92a,b). Solvates involving Schiff bases (Figure 92c,d) are less stable $(\Delta E)$ owing to the impossibility of axial coordination (Table 14).

These conclusions were confirmed by analyzing the shift of the aromatic protons resonance signals of alkoxycyanobiphenyl as a result of axial ligation (Figure 93) and the responses of direct non-covalent interactions of solvate components recorded using ROESY two-dimensional correlation spectroscopy (Figure 94). Thus, the reason

$1 / \mathbf{P}_{0}, \mu m^{-}$

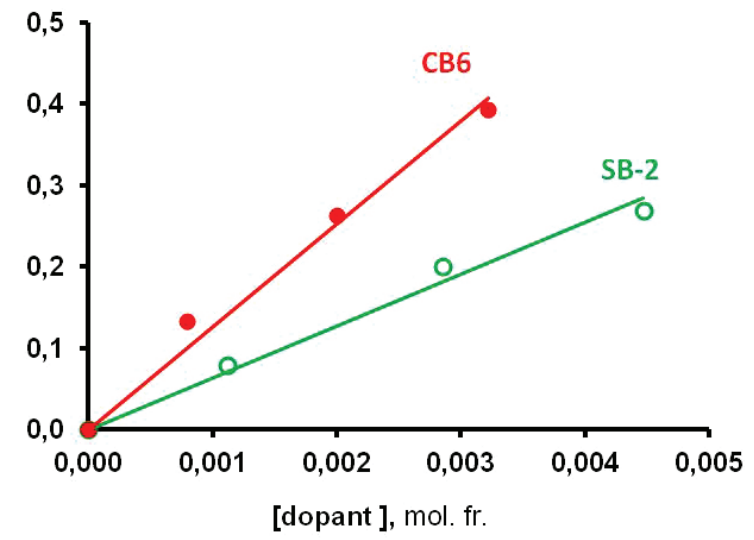

Figure 91. Reciprocal of the pitch versus molar fraction of Ni-S(+)TPP in a mixtures with CB6 and SB2 at $\mathrm{T}-\mathrm{T}_{\mathrm{N}-\mathrm{I}}=-4{ }^{\circ} \mathrm{C} \cdot{ }^{[814]}$ 
a
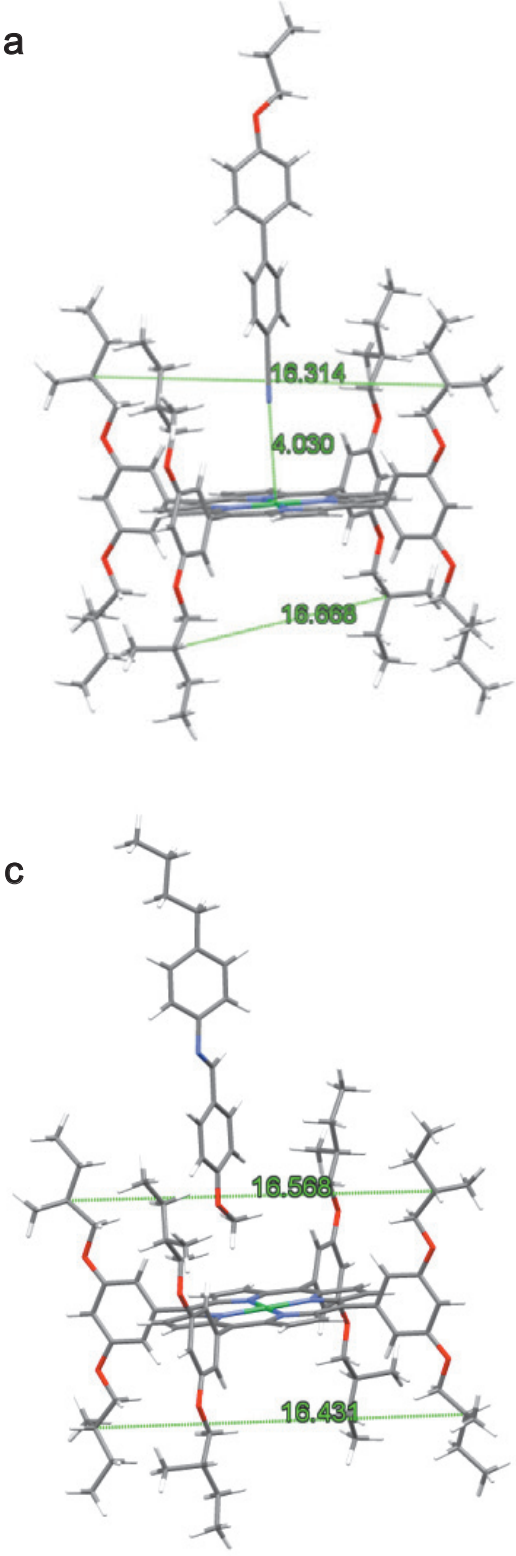

b
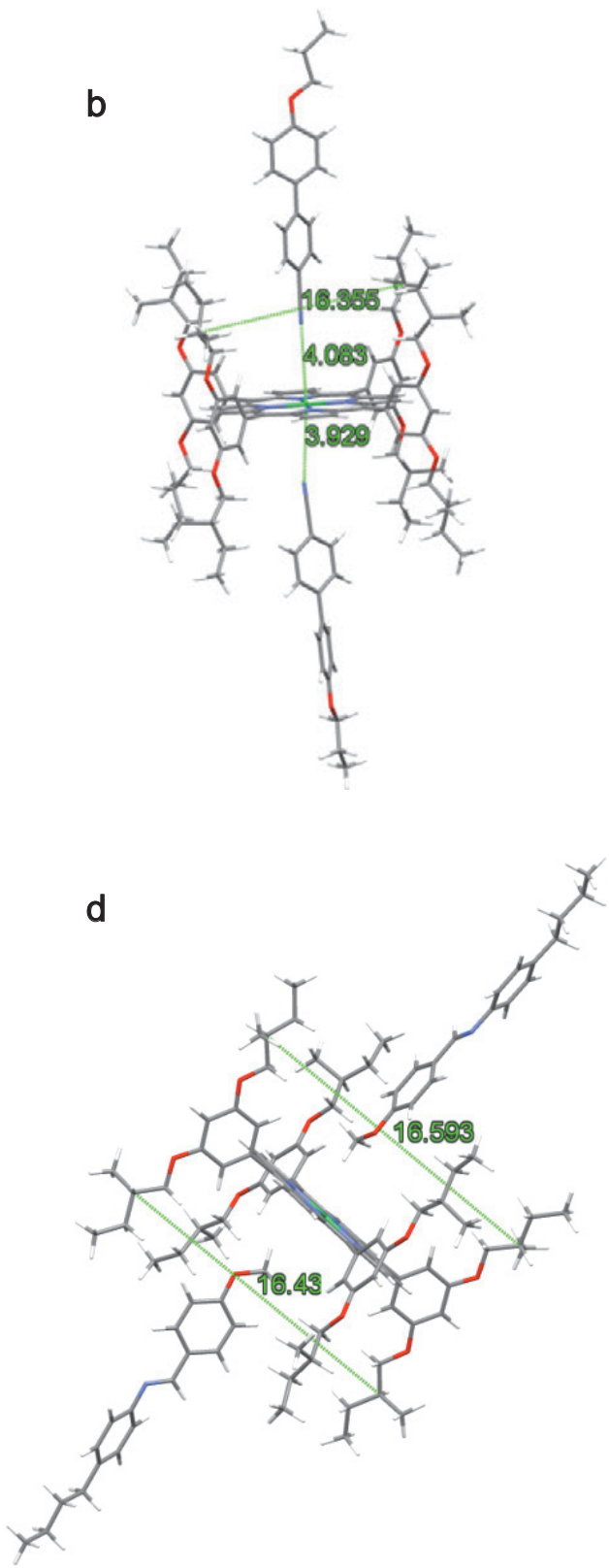

Figure 92. Structures of solvates of Ni-S(+)TPP and 3OCB with 1:1 (a) and 1:2 (b) ratio. Structures of solvates of Ni-S(+)TPP and MBBA with $1: 1(\mathrm{c})$ and $1: 2(\mathrm{~d})$ ratio. ${ }^{[814]}$

Table 14. The results of quantum chemical calculations of the chiral dopant Ni-S(+)TPP, components of LC mixtures 3OCB, MBBA and their solvates. ${ }^{[814]}$

\begin{tabular}{|c|c|c|c|c|c|c|}
\hline Structure & $E$, au & $\Delta E, \mathrm{~kJ} / \mathrm{mol}$ & $\begin{array}{c}\mathrm{C} \Xi \mathbf{N} \text { charge } \\
\text { Malliken }\end{array}$ & $\begin{array}{c}\mathrm{C} \Xi \mathrm{N} \text { charge } \\
\text { Malliken }\end{array}$ & $\begin{array}{c}\text { C-C } \Xi N \text { charge } \\
\text { Malliken }\end{array}$ & $\mathrm{r}_{\mathrm{C}^{*} \mathrm{C}^{*}}$, \\
\hline $3 \mathrm{OCB}$ & -748.2 & - & -0.314 & 0.079 & 0.090 & - \\
\hline MBBA & -828.03 & - & - & - & - & - \\
\hline $\mathrm{Ni}-\mathrm{S}(+) \mathrm{TPP}$ & -5592.5 & & - & - & - & 16.54 \\
\hline $\mathrm{Ni}-\mathrm{S}(+) \mathrm{TPP}+3 \mathrm{OCB}$ & -6340.9 & -37.9 & -0.475 & 0.261 & 0.108 & 16.31 \\
\hline $\mathrm{Ni}-\mathrm{S}(+) \mathrm{TPP}+(3 \mathrm{OCB})_{2}$ & -7089.1 & -74.6 & -0.472 & 0.262 & 0.109 & 16.36 \\
\hline $\mathrm{Ni}-\mathrm{S}(+) \mathrm{TPP}+\mathrm{MBBA}$ & -6420.6 & -7.2 & - & - & - & $\begin{array}{l}16.58 \\
16.43\end{array}$ \\
\hline $\mathrm{Ni}-\mathrm{S}(+) \mathrm{TPP}+(\mathrm{MBBA})_{2}$ & -7248.6 & -13.3 & - & - & - & $\begin{array}{l}16.59 \\
16.42\end{array}$ \\
\hline
\end{tabular}




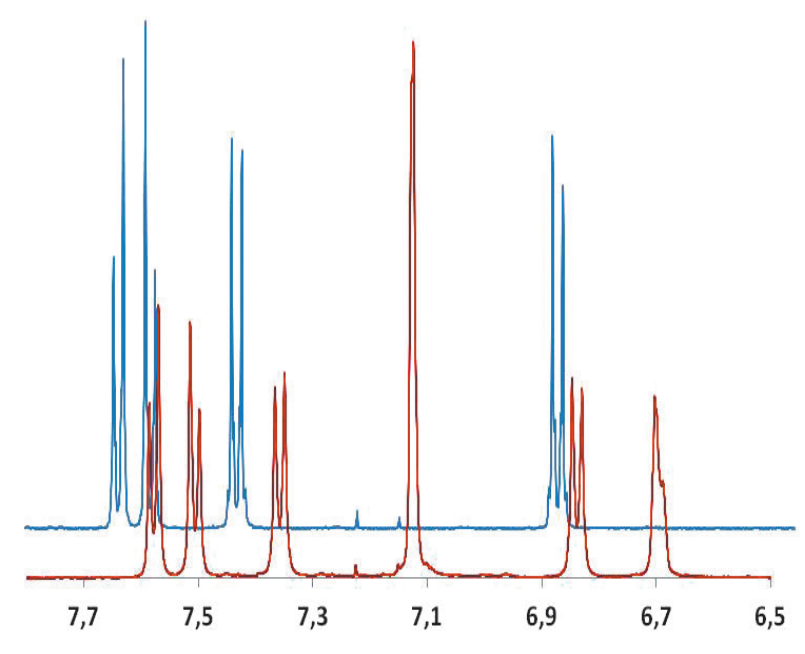

Figure 93. ${ }^{1} \mathrm{H}$ NMR spectra of $3 \mathrm{OCB}$ (blue) and $3 \mathrm{OCB}+\mathrm{Ni}-\mathrm{S}(+)$ TPP mixtures (red) with a ratio of $1: 1$ in $\mathrm{CCl}_{4}$ at $25^{\circ} \mathrm{C} \cdot{ }^{[814]}$

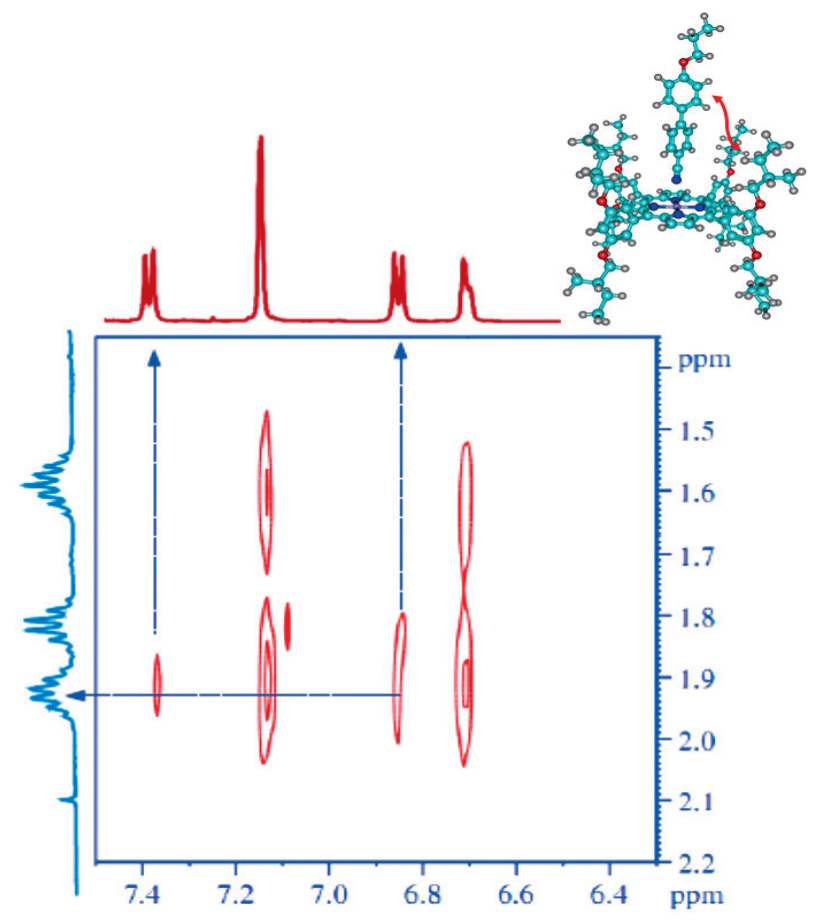

Figure 94. ${ }^{1} \mathrm{H}-{ }^{1} \mathrm{H}$ ROESY (Rotating frame Overhause Effect Spectroscopy) of mixture $3 \mathrm{OCB}+\mathrm{Ni}-\mathrm{S}(+) \mathrm{TPP}$ with molar ratio $1: 1$ in $\mathrm{CCl}_{4}$ at $25^{\circ} \mathrm{C} \cdot .^{[814]}$

for the high ability of chiral transport from optically active nickel porphyrinate to a nematic mixture of cyanobiphenyls was shown to connect with a special self-assembly mechanism combining axial coordination and inclusion of LC molecules into the chiral dopant shell. ${ }^{[814]}$

\section{Macroheterocyclic Selectors for Gas Chromatography}

One of the promising directions for diversifying the practical use of $\mathrm{MHCs}$ is the creation of stationary phases (SP) for chromatography on their basis. It should be noted that metal complexes are widely used as stationary phases in the so-called ligand exchange chromatography. [815,816] First of all, this concerns the Werner complexes. The ligand exchange chromatography is based on the formation of a labile coordination bond between the sorbate (ligand) and the metal cation with the complex formation.

Due to their high chemical selectivity, metal complexes are perspective for chromatographic separation. ${ }^{[817,818]}$ This possibility can be illustrated by the use of $\mathrm{Cu}$ (II) and $\mathrm{Ni}$ (II) bis(4-decyloxyphenyloxycarbonyl)salicylal- $N$-dodecylamine]ates ${ }^{[819]}$ for the efficient separation of dimethylpyridine isomers and heterocyclic nitrogen-containing monoand polynuclear compounds. In this regard, the use of such MHCs as porphyrins, phthalocyanines, and their metal complexes as stationary phases is of great interest.

So, in ${ }^{[820]}$, nickel tetra(4-tert-butyl-5-nitro)phthalocyaninate, $\mathrm{NiPc}(4-t-\mathrm{Bu})-4-\left(5-\mathrm{NO}_{2}\right)_{4}$, (16) was studied as a stationary phase for gas chromatography.

To estimate its structural selectivity, pairs of test sorbates were used with similar structures and boiling points: 3,4- / 3,5-lutidines and 2-, 3-, 4-picolines. Structural selectivity was determined by the Herington selectivity factor:

$$
\alpha=\tau_{3,4-} \tau_{3,5-}, \alpha=\tau_{4--} \tau_{3-},
$$

where $\tau_{3,4-}$ and $\tau_{3,5}$ are the retention times of 3,4- and 3,5-lutidines, respectively, and 4- and 3-picolines, respectively.

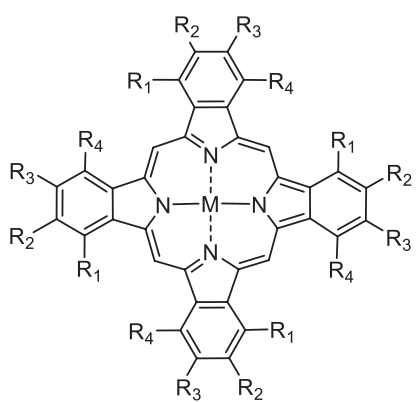

(61)

$\mathrm{R}_{1}=\mathrm{R}_{4}=\mathrm{H} ; \mathrm{R}_{2}=t-\mathrm{Bu} ; \mathrm{R}_{3}=\mathrm{NO}_{2}, \mathrm{M}=\mathrm{Cu}, \mathrm{CuPc}(4-t-\mathrm{Bu})_{4}\left(5-\mathrm{NO}_{2}\right)_{4}$

The structural selectivity of the sorbent based on $\mathrm{NiPc}(4-t-\mathrm{Bu})_{4}\left(5-\mathrm{NO}_{2}\right)_{4}$, as follows from the data in Table 15 is sufficient to recommend it as a stationary phase for gas chromatography in the quantitative analysis of organic compounds mixtures. In the opinion of the authors, ${ }^{\left[{ }^{[20]}\right.}$ this may be due to the ability of the metal phthalocyanine studied to form coordination bonds between electron donor sorbates and the metal cation in axial complexes.

Granting this, an attempt to use macrocyclic complexes of a different nature as a stationary phase was of certain interest. In this regard, the synthesis, characterization and structural selectivity study of the nickel complex of 5,10,15,20-tetrakis[3',5'-di(2''-methylbutyloxy) phenylporphine $\left(\mathrm{NiTPP}-\mathrm{R}_{8}\right.$ ) was carried out, ${ }^{[821]}$ which was the racemate of the porphyrin described in the first part of this section as an inducer of chiral nematic phases.

The test sorbates were methylpyridine (picoline) and dimethylpyridine (lutidine) isomers able to coordinate with the central Ni(II) ion. ${ }^{[822]}$ In addition, $p$ - and $m$-xylenes 
Table 15. Values of the structural selectivity coefficient $\alpha$ for $\mathrm{NiPc}(4-t-\mathrm{Bu})_{4}\left(5-\mathrm{O}_{2}\right)_{4}{ }^{[820]}$

\begin{tabular}{ccccc}
\hline \multirow{2}{*}{ Temperature, ${ }^{\circ} \mathrm{C}$} & \multicolumn{4}{c}{ Structural selectivity coefficient $\alpha$} \\
\cline { 2 - 5 } & $3,4-/ 3,5$-lutidines & 3/2-picolines & 4/3-picolines & 4/2-picolines \\
\hline 120 & 1.25 & 1.65 & 1.09 & 1.80 \\
140 & 1.23 & 1.47 & 1.08 & 1.59 \\
150 & 1.21 & 1.39 & 1.06 & 1.47 \\
160 & 1.19 & 1.32 & 1.05 & 1.40 \\
170 & 1.16 & 1.28 & 1.05 & 1.35 \\
180 & 1.26 & 1.20 & 1.05 & 1.27 \\
\hline
\end{tabular}

were chosen as standard sorbates for studying structural selectivity in gas chromatography. ${ }^{[823]}$ The retention on the porphyrin complex in the stationary phase was found to be selective with respect to the spatial structure of substituted pyridines. To estimate the structural selectivity of NiTPP- $\mathrm{R}_{8}$, Table 16 shows the values of the Herington coefficients $\alpha$ for pairs of the studied spatial isomers. The data indicate a high structural selectivity of the Ni-TPP- $\mathrm{R}_{8}$ with respect to 3,4- and 3,5-lutidines $(\alpha=1.43)$ and 4- and 3-picolines $(\alpha=1.11)$ and allow to use the NiTPP- $\mathrm{R}_{8}$ as a stationary phase for quantitative chromatographic analysis of organic compounds in their mixtures. ${ }^{[824]}$

At the same time, the selectivity of xylenes separation in the studied stationary phase is very low ( $\alpha=$ 1.005 , Table 16), apparently due to the inability to form stable complexes with the metal ion, in contrast to pyridine derivatives. In addition, the role of aliphatic hydrocarbon substituents in the chromatographic retention of sorbates was not obvious.

In this regard, quantum-chemical calculations of NiTPP (nickel complex of 5,10,15,20-tetraphenylporphine), NiTPP- $\mathrm{R}_{8}$ and their axial complexes with picolines and lutidines were performed. ${ }^{[814]}$ These calculations were limited to the complexes with 1:1 composition taking into account the infinite dilution of sorbates at chromatographic experiment.

The optimized structures shown in Figure 95 indicate a significant deviation of phenyl cores from the plane of the macrocycle. This effect is associated with steric repulsion and allows to create a hydrocarbon shell around the nickel ion. Data presented in Figure 95 and Table 17 show the calculated interaction energies and distances $\mathrm{Ni}$ - nitrogen atom of the sorbate and unambiguously indicate the formation of stable axial complexes with participation

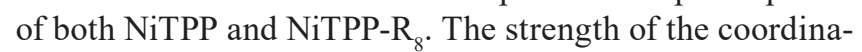
tion bond can be estimated from its length $\mathrm{r}_{\mathrm{Ni}-\mathrm{N}}$. These data (Table 17) show that the introduction of 2-methylbutoxy groups into the 3,5-positions of NiTPP leads to some weakening of the Ni-picoline coordination bond and does not affect the Ni-lutidine bond. This effect can be associated with the weak electronic influence of aliphatic substituents at the 3,5-positions of phenyl rings on the Ni orbital.

At the same time, the total stability of the axial complex is determined by the energy of its formation $(\Delta E)$. For picolin complexes, six-fold strengthening on going from

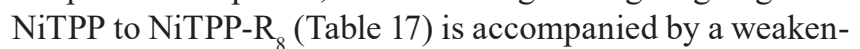
ing of the coordination bond and, thus, is associated with the interaction of the sorbate with aliphatic substituents of the shell.

This behavior confirms the importance of the spatial environment in the multicenter interaction of the substrate with the active center of the receptor. ${ }^{\left[{ }^{[25]}\right.}$ As a result of the inclusion of the sorbate into the host space, the aliphatic substituents approach each other (the distance between the opposite substituents decreases from 16.54 to $16.40 \AA$ (Table 17). This effect should be accompanied by the strengthening of the sorbate bond with the porphyrin metal complex.

Table 16. Maximum values of selectivity coefficients of Ni-TPP- $\mathrm{R}_{8}$ for pairs of spatial isomers. ${ }^{[821]}$

\begin{tabular}{|c|c|c|c|}
\hline Sorbates & $\alpha\left(\mathrm{T},{ }^{\circ} \mathrm{C}\right)$ & Sorbates & $\alpha\left(\mathrm{T},{ }^{\circ} \mathrm{C}\right)$ \\
\hline$p / m$-xylenes & $1.005(110)$ & & \\
\hline 2,3/2,4-lutidines & $1.10(130)$ & 3,4/2,6-lutidines & $4.77(110)$ \\
\hline 2,3/2,5-lutidines & $1.12(118)$ & 3,4/3,5-lutidines & $1.43(110)$ \\
\hline 2,3/2,6-lutidines & $1.99(110)$ & 3,5/2,3-lutidines & 1.69 (112) \\
\hline 2,4/2,5-lutidines & 1.07 (112) & 3,5/2,4-lutidines & 1.77 (112) \\
\hline 2,4/2,6-lutidines & $1.90(110)$ & 3,5/2,5-lutidines & 1.89 (112) \\
\hline 2,5/2,6-lutidines & $1.78(110)$ & 3,5/2,6-lutidines & 3.34 (112) \\
\hline 3,4/2,3-lutidines & $2.40(110)$ & 4/3-picolines & $1.11(118)$ \\
\hline 3,4/2,4-lutidines & $2.51(110)$ & 4/2-picolines & $2.47(90)$ \\
\hline 3,4/2,5-lutidines & $2.68(110)$ & 3/2-picolines & $2.34(90)$ \\
\hline
\end{tabular}




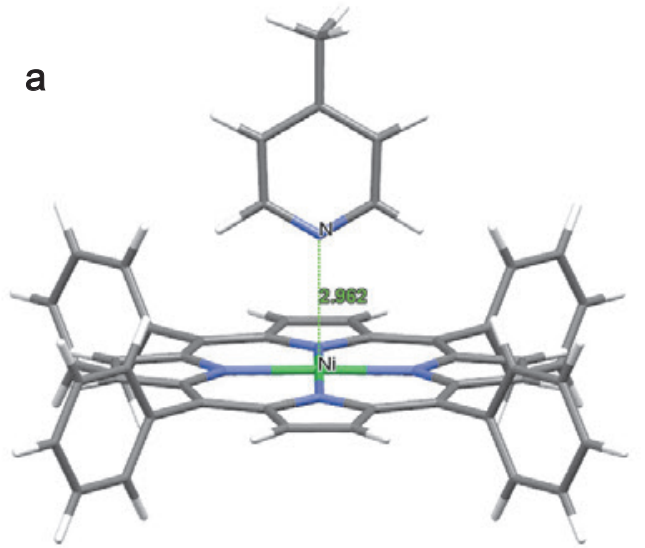

C

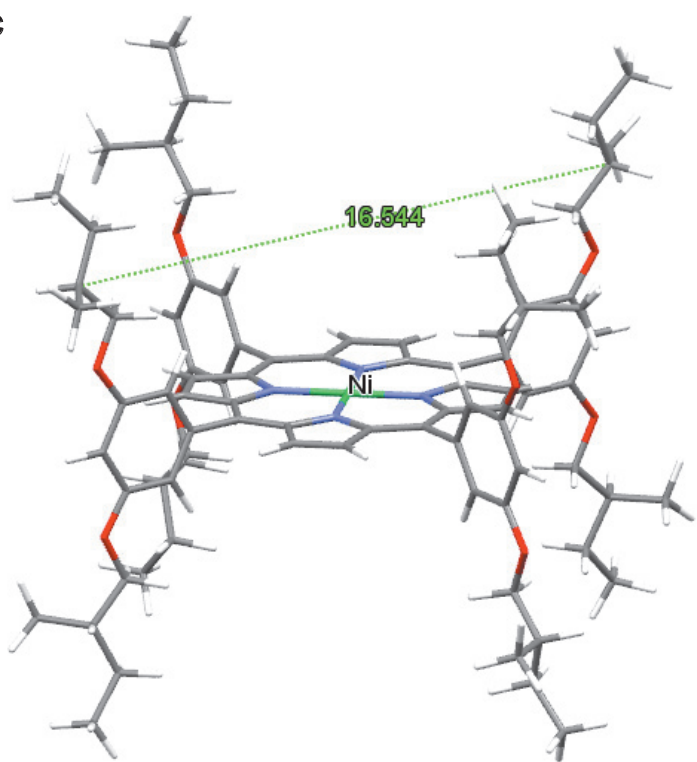

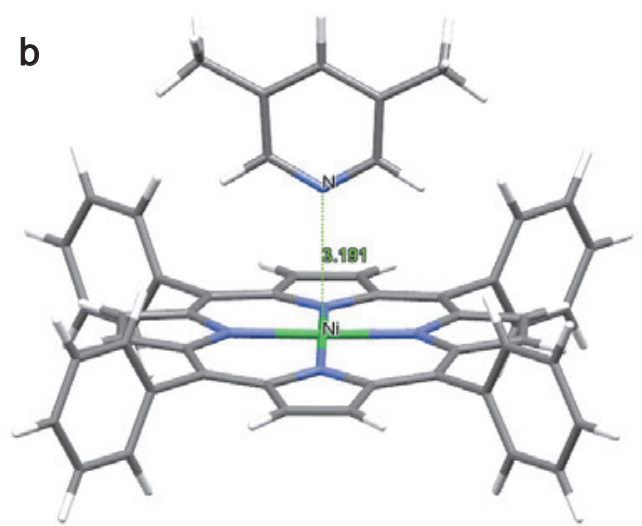

d

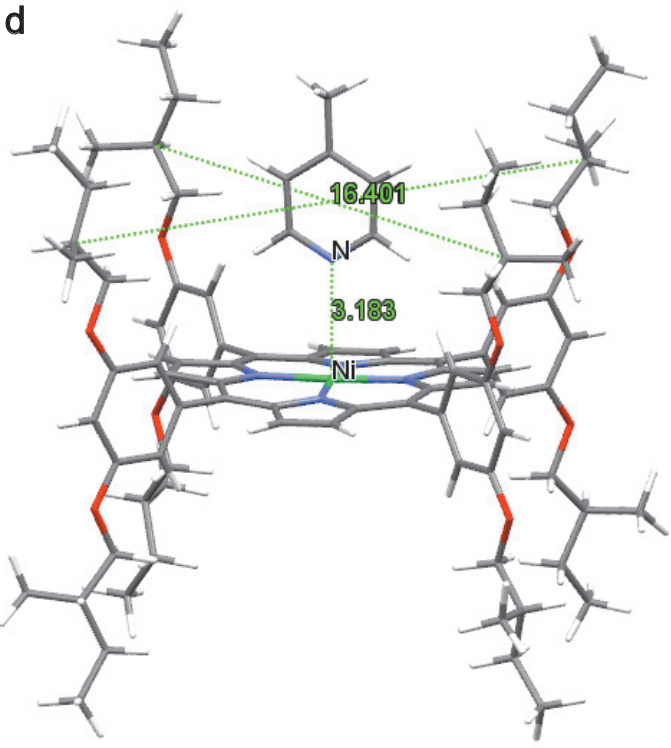

Figure 95. Molecular structure of NiTPP-R (c) and axial complexes (a) NiTPP + 4-picoline; (b) NiTPP + 3,5-lutidine;

(d) NiTPP-R + 4-picoline. . $^{[821]}$

Table 17. The energy of axial complexes formation $(\Delta E)$, the lengths of the coordination bond $\left(\mathrm{r}_{\mathrm{Ni}-\mathrm{N}}\right)$ and the distance between the tertiary carbon atoms of opposite alkyl substituents $\left(\mathrm{r}_{\mathrm{c}-\mathrm{c}}\right)^{\cdot[821]}$

\begin{tabular}{|c|c|c|c|c|c|c|}
\hline Structure & $\Delta E, \mathrm{~kJ} / \mathrm{mol}$ & $\mathrm{r}_{\mathrm{Ni}-\mathrm{N}}, \AA$ & Structure & $\mathrm{r}_{\mathrm{c}-\mathrm{c}}, \AA$ & $\Delta E, \mathrm{~kJ} / \mathrm{mol}$ & $\mathrm{r}_{\mathrm{Ni}-\mathrm{N}}, \AA$ \\
\hline NiTPP & - & - & NiTPP-R 8 & 16.54 & - & - \\
\hline NiTPP+Pyc3 & -3.2 & 2.97 & NiTPP-R + +Pyc3 & 16.40 & -18.5 & 3.03 \\
\hline NiTPP+Pyc4 & -3.2 & 2.96 & NiTPP-R $8+$ Pyc 4 & 16.40 & -18.2 & 3.18 \\
\hline NiTPP+Lu34 & -8.2 & 3.18 & NiTPP-R 8 +Lu34 & 16.42 & -6.8 & 3.18 \\
\hline NiTPP+Lu35 & -3.4 & 3.19 & NiTPP-R + +Lu35 & 16.41 & -2.0 & 3.19 \\
\hline
\end{tabular}

It is interesting to note that in the case of 3and 4-picolines, the total interaction energy is practically the same (18.5 and $18.2 \mathrm{~kJ} / \mathrm{mol}$, respectively), while for 3,4- and 3,5-lutidines, differentiation of the interaction is observed upon the introduction of substituents in the metal complex (ratio $\Delta E_{3.4} / \Delta E_{3.5}=2.4$ for NiTPP and $\Delta E_{3.4} / \Delta E_{3.5}=3.4$ for NiTPP-R , Table 17). The correspondence between $\Delta E_{3.4} / \Delta E_{3.5}\left(\Delta E_{4-} / \Delta E_{3 .}\right)$ and $\alpha$ confirms that the ratio $\Delta E_{1} / \Delta E_{2}$ for isomers can be considered as a measure of structural selectivity.

Thus, the results of the above studies ${ }^{[821,824]}$ convincingly show that the substituents on the periphery of the porphyrin cycle play a significant role in regulating the interactions of the separated sorbates and the stationary phase, and macrocyclic metal complexes can be considered as promising stationary phases in gas chromatography. 
It is important to note that self-assembly with participation of macroheterocyclic complexes including two synchronous mechanisms - axial coordination and guest-host effect ensures not only high efficiency of the chiral transfer dopantnematic LC $^{[814]}$ but are the cause of chromatographic retention and high structural selectivity of stationary phases. ${ }^{[821]}$

Representatives of another class of macroheterocyclic compounds - tetrapyrazinoporphyrazines - have been tested as stationary phases in gas chromatography. These are the camphor-substituted tetrapyrazinoporphyrazine: tetra(1',7',7'-trimethylbicyclo[2.2.1] heptano-[2',3'-b]-pyrazino)porphyrazine, Cam4PPz (62), and its copper complex, Cam4PPz-Cu (63).

A feature of these compounds is the presence of a rigid planar macrocyclic core and an aliphatic peripheral shell, which provides solubility in organic solvents sufficient for the deposition of a stationary phase on a solid support. In ${ }^{[826,827]}$, a study was carried out of the thermodynamics of dissolution and the selectivity of the separation of monoand dimethylpyridines on stationary phases Cam4PPz and $\mathrm{Cam} 4 \mathrm{PPz}-\mathrm{Cu}$, deposited on the solid carrier «Chezasorb AW-HMDS» in the presence of $5 \% \mathrm{XE}-60$ silicone.

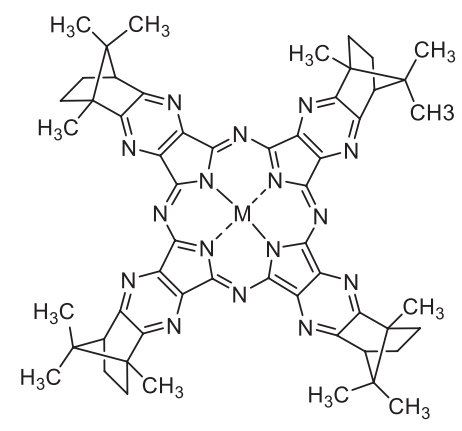

(62) $\mathrm{M}=2 \mathrm{H}\left(\mathrm{Cam}_{4} \mathrm{Pz}\right)$,

(63) $\mathrm{M}=\mathrm{Cu}\left(\mathrm{Cam}_{4} \mathrm{PPz}-\mathrm{Cu}\right)$

Table 18. Selectivity coefficients $\alpha$ for substituted pyridines on various stationary phases, deposited on "Chezasorb AWHMDS", ${ }^{[826]}$

\begin{tabular}{lccc}
\hline \multicolumn{1}{c}{ Sorbates } & XE-60 & Cam $_{4} \mathrm{PPz}$ & $\mathrm{Cam}_{4} \mathrm{PPz}-\mathrm{Cu}$ \\
\hline 3,4-lutidine/3,5-lutidine & 1.31 & 1.34 & 1.68 \\
2,5-lutidine/2,4-lutidine & 1.05 & 1.05 & 1.20 \\
3-picoline/4-picoline & 1.04 & 1.04 & 1.20 \\
3-picoline/2,6-picoline & 1.44 & 1.44 & 2.04 \\
\hline
\end{tabular}

Analysis of the data presented in Table 18 shows that the organosilicon stationary phase XE-60 itself has a certain selectivity at the separation of pyridine-based positional isomers. Its use both in an individual state and as a carrier for macrocycles is advisable, because allows to carry out not a gas-adsorption separation process, but a gas-distribution (gas-liquid) process and, thereby, increase the productivity of a chromatographic column.

The introduction of metal-free camphor-substituted pyrazinoporphyrazine $\mathrm{Cam} 4 \mathrm{Pz}$ into XE-60 placed on the surface of a solid carrier does not lead to a noticeable increase in the selectivity of SP (Table 18). This indicates that the macrocycle does not make a significant contribution to the separation process, while the role of the SP is essentially played by silicone. A completely different behavior is revealed by the Cam4PPz-Cu copper complex, the introduction of which into silicone sharply increases the separation selectivity ( $\alpha$ for 3,4 / 3,5-lutidines 1.68 and 1.20 for 3- / 4-picolines). This allows to assert that the central metal ion, able to form axial complexes with them, plays a key role in the chromatographic separation of electron-donor sorbates. Such a high structural selectivity makes it possible to recommend $\mathrm{Cam} 4 \mathrm{PPz}-\mathrm{Cu}$ for use as an selector of stationary phases in gas chromatography. ${ }^{[827]}$

In the implementation of chromatographic gas adsorption separation, a solid carrier of the stationary phase can have a significant effect on this process due to its high surface energy. In addition, in some cases, it itself can exhibit noticeable selectivity. In ${ }^{[828]}$, a study was made of the effect of the Cam4PPz-Cu deposition on the surface of the diatomite sorbent "Chromaton N-AW" on the separation selectivity of substances. Data on the selectivity of the studied sorbents are listed in Table 19.

The analysis of these results shows that the diatomite carrier "Chromaton N-AW" has a certain structural selectivity at the separation of pyridine derivatives, as well as in the analytical process of separation of the latter and aliphatic, aromatic compounds and alcohols. Judging by the value $\alpha=1$ for the $p$-xylene- $m$-xylene pair, this solid carrier does not possess regioselectivity.

The impregnation of solid carrier by $\mathrm{Cam} 4 \mathrm{PPz}-\mathrm{Cu}$ was accompanied by a noticeable increase in the selectivity coefficients of all pairs of the studied sorbates. For test pairs 3,4-lutidine-3,5-lutidine $\alpha$ is 1.61, for 3-picolin-4-picoline1.16. The high selectivity of separation of methylpyridine isomers and compounds that are able to form strong coordination bonds with the metal ion is not surprising. The coefficient $\alpha=2.95$ for a pair of 3-picoline-2,6-lutidine sorbates is very high, despite their absolutely identical boiling points $\left(144.0^{\circ} \mathrm{C}\right)$. Such a large difference in the retention time of the two electron-donor pyridine derivatives is due to significant steric restrictions in the axial coordination of 2,6-lutidine on the part of two methyl groups blocking the nitrogen atom, and the absence of such shielding in the case of 3-picoline. ${ }^{[828]}$

As shown above, the deposition of a mixture of silicon and a copper complex of camphor-substituted tetrapyrazinoporphyrazine on the surface of a solid carrier allows not only to achieve a fairly high structural selectivity, but also increasing the productivity of the chromatographic column. ${ }^{[826]} \mathrm{In}^{[829]}$, the effect of a solid carrier on the chromatographic separation of sorbates of various natures on the surface of $\mathrm{Cu}$ (II) of the tetracamphor-substituted tetrapyrazinoporphyrazine Cam4PPz-Cu complex was studied. Diatomite adsorbents "Chromaton N-AW" and "Hezasorb AWHMDS" were used as carriers, differing in that the surface of the latter is silanized and, thus, to some extent isolated from the stationary phase in Cam4PPz-Cu. This also leads to a significant increase in column performance. Thus, the number of theoretical plates of the Hezasorb AW-HMDS + Cam4PPz-Cu sorbent is 3-100 times higher than that of Chromaton N-AW + Cam4PPz-Cu. ${ }^{[829]}$ Data on the structural selectivity of the above sorbents are given in Table 20. 
Table 19. Maximum values of the selectivity coefficient. ${ }^{[828]}$

\begin{tabular}{lcc}
\hline \multicolumn{1}{c}{ Sorbates } & $\begin{array}{c}\text { Chromaton } \\
\text { N-AW }\left(140{ }^{\circ} \mathrm{C}\right)\end{array}$ & $\begin{array}{c}\text { Chromaton } \\
\text { N-AW Cam }{ }_{4} \mathrm{PPz}-\mathrm{Cu}\left(\mathrm{T},{ }^{\circ} \mathrm{C}\right)\end{array}$ \\
\hline 3,4-lutidine (179.1)-3,5-lutidine (172.2) & 1.33 & $1.61(140)$ \\
2,5-lutidine (157.0)-2,4-lutidine (158.4) & 1.19 & $1.59(140)$ \\
2,5-lutidine (157.0)-2,3-lutidine (161.2) & 1.13 & $1.68(140)$ \\
2.3- lutidine (161.2)-2,4-lutidine (158.4) & 1.13 & $1.20(140)$ \\
3- picoline (144.0)-4-picoline (145.4) & 1.13 & $1.16(140)$ \\
3- picoline (144.0)-2,6-lutidine (144.0) & 1.93 & $2.95(140)$ \\
$p$-xylene (138.4)-m-xylene (139.1) & 1.00 & $1.38(130)$ \\
$p$-xylene (138.4)-3-picoline (144.0) & 1.95 & $2.47(150)$ \\
3-picoline (144.0)-m-xylene (139.1) & 1.95 & $3.06(140)$ \\
2,6-lutidine (144.0)-p-xylene (138.4) & 1.01 & $1.20(170)$ \\
benzene (80.1)-cyclohexane (81.7) & 1.09 & $2.51(90)$ \\
propanol-2 (82.3)- cyclohexane (81.7) & 1.09 & $1.38(100)$ \\
propanol-2 (82.3)-benzene (80.1) & 1.08 & $1.65(100)$ \\
pyridine (115.6)-butanol-1 (117.9) & 1.15 & $4.82(130)$ \\
ethanol (78.5)-benzene (80.1) & 1.06 & $1.36(100)$ \\
pyridine (115.6)-epichlorohydrin (115.5) & 1.05 & $1.36(140)$ \\
DMF (153.0)-2,5-lutidine (157.0) & 1.12 & $1.56(140)$ \\
\hline
\end{tabular}

Table 20. Coefficients of sorbates structural selectivity $\alpha .^{[829]}$

\begin{tabular}{|c|c|c|}
\hline Sorbates $\left(\mathrm{T}_{\text {reflux }},{ }^{\circ} \mathrm{C}\right)$ & $\begin{array}{c}\text { Chromaton } \\
\mathrm{N}-\mathrm{AW}+\mathrm{Cam}_{4} \mathrm{PPz}-\mathrm{Cu}\end{array}$ & $\begin{array}{c}\text { Hezasorb } \\
\text { AW-HMDS }+ \text { Cam }_{4} \mathrm{PPz}-\mathrm{Cu}\end{array}$ \\
\hline 3,4-lutidine (179.1) - 3,5-lutidine (172.2) & $1.61(140)$ & $1.66(130)$ \\
\hline 2,5- lutidine (157.0) - 2,4-lutidine (158.4) & $1.59(140)$ & $1.20(160)$ \\
\hline 2,5- lutidine (157.0) - 2.3- lutidine (161.2) & $1.68(140)$ & $1.14(170)$ \\
\hline 2,3- lutidine (161.2) - 2,4-lutidine (158.4) & $1.20(140)$ & $1.11(130)$ \\
\hline 3-picoline (144.0) - 4-picoline (145.4) & $1.10(140)$ & $1.20(120)$ \\
\hline 3-picoline (144.0) - 2,6-lutidine (144.0) & $2.95(140)$ & $2.04(140)$ \\
\hline$p$-xylene (138.4) - $m$-xylene (139.1) & $1.38(130)$ & $1.02(140)$ \\
\hline$p$-xylene (138.4) - 3-picoline (144.0) & $2.47(150)$ & $1.89(130)$ \\
\hline 3-picoline (144.0) - $m$-xylene (139.1) & $3.06(140)$ & $1.91(130)$ \\
\hline 2,6-lutidine (144.0) - $p$-xylene (138.4) & $1.20(170)$ & $1.31(150)$ \\
\hline benzene (80.1) - cyclohexane (81.7) & $2.51(90)$ & $1.94(120)$ \\
\hline propanol-2 (82.3) - cyclohexane (81.7) & $1.38(100)$ & $1.33(115)$ \\
\hline propanol -2 (82.3) - benzene (80.1) & $1.65(100)$ & $1.51(115)$ \\
\hline pyridine (115.6) - butanol-1 (117.9) & $4.82(130)$ & $1.25(130)$ \\
\hline ethanol $(78.5)$ - benzene $(80.1)$ & $1.36(100)$ & $1.33(115)$ \\
\hline DMF (153.0) - 2,5-lutidine (157.0) & $1.56(140)$ & $1.40(130)$ \\
\hline ethanol (78.5) - cyclohexane (81.7) & $2.13(120)$ & $1.70(120)$ \\
\hline
\end{tabular}

Analysis of these data shows that silanation of the solid diatomite carrier is accompanied by a noticeable decrease in the selectivity of the Cam4PPz-Cu stationary phase upon separation of methyl pyridine derivatives. The only exception is the 3-picoline / 4-picoline pair, for which the $\alpha$ value increases. The general tendency towards a decrease in the coefficients of structural selectivity per- sists in the separation of the remaining sorbates (Table 20). In general, the data presented in Table 20 indicate that the nature of the surface of a solid support can significantly affect the chromatographic separation of substances, changing not only the selectivity of the macroheterocyclic stationary phase, but also the productivity of the column. 


\section{Application of Macroheterocyclic Compounds in Medicine}

\subsection{Photosensitisers Based on Chlorophyll a for PDT}

In recent years, a non-invasive method of removing malignant neoplasms, photodynamic therapy (PDT) of cancer, has taken an increasingly firm place among the methods of surgery, chemotherapy and radiation exposure traditionally used in oncology. In 2012, the Ministry of Health of the Russian Federation included PDT in the official list of methods for treating cancer. ${ }^{[830]}$ Along with minor damage to the sur- rounding healthy tissues, which is typical for surgical intervention, when using PDT, the general toxicity of the body inherent in chemotherapy is not observed, as well as serious consequences when working with radioisotopes. At the same time, certain problems should be noted that arise when removing large and pigmented tumors using PDT. In this regard, following the first porphyrin-based photosensitizers (PSs) such as Photofrin II (USA-Canada), Photosan (Germany), and Photogem (Russia), second-generation PSs based on natural and synthetic chlorins, as well as phthalocyanines were rapidly developed. The presence of an intense absorption band in the range of $660-670 \mathrm{~nm}$ in the UV-Vis spectra of these compounds made it possible to increase the depth of light exposure and the effectiveness of treatment of deeply

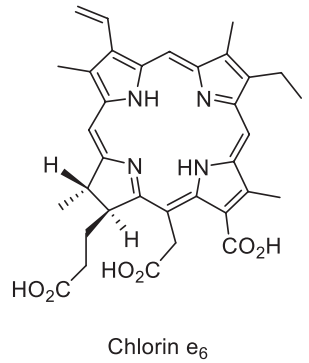

(Fotoditazine, Fotolon, Fotoran, Radachlorin)
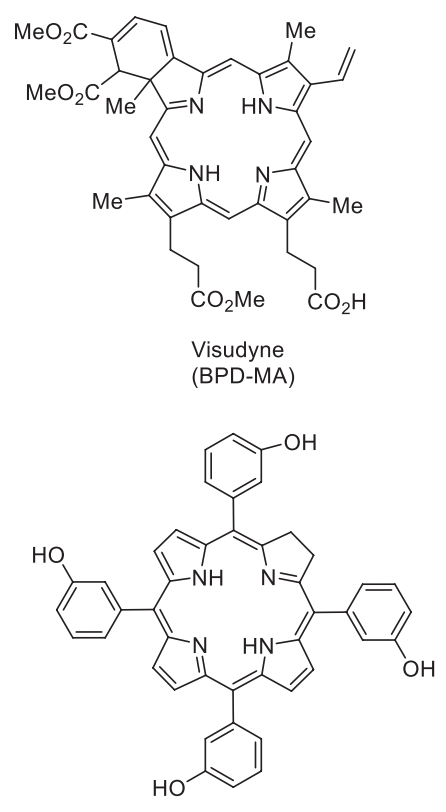

Foscan (m-THPC)

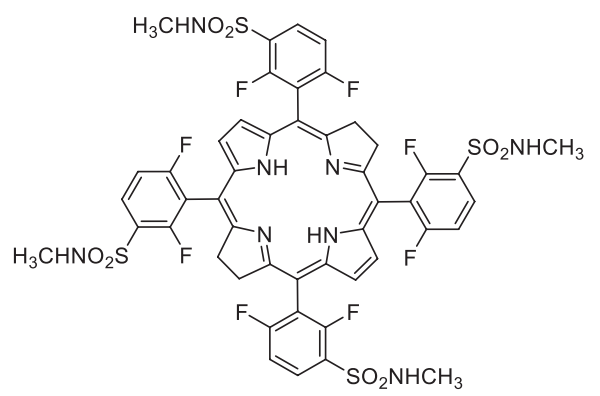

Redaporfin
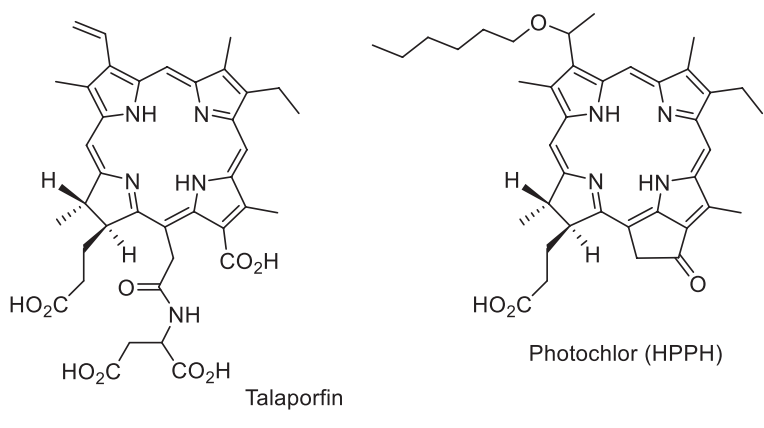

Photochlor (HPPH)

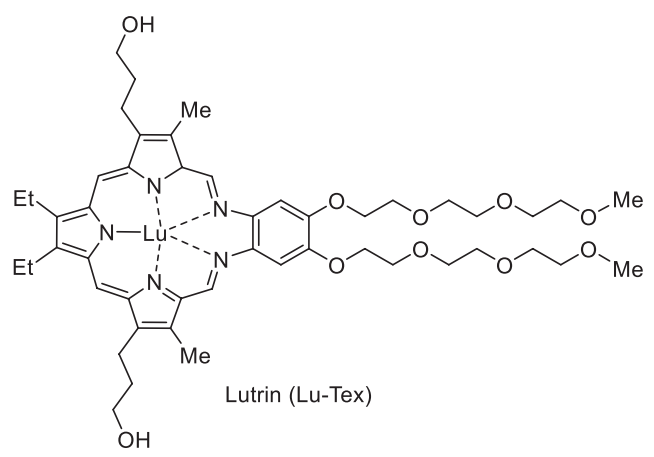

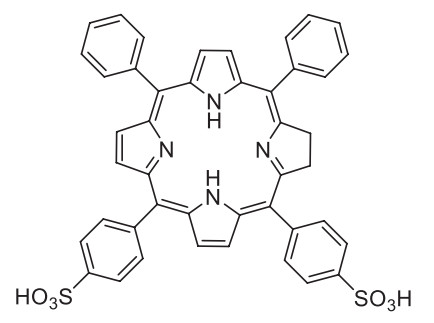

Fimaporfin

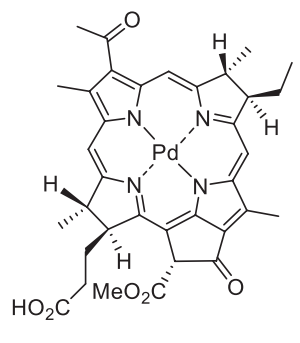

Tookad (WST09)

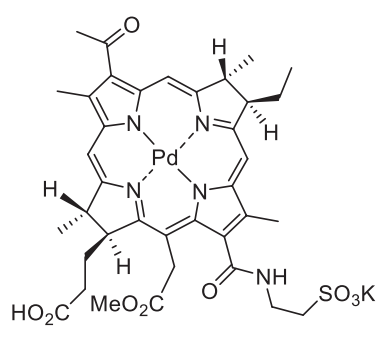

Stakel (WST11)

Figure 96. Photosensitizers for photodynamic therapy. 
located neoplasms. ${ }^{[831,832]}$ A special place among them is occupied by PSs based on natural chlorophyll $a$, which is associated with the availability of these compounds, the presence of easily transformable side substituents and structural similarity to endogenous porphyrins, which ensures low toxicity of such compounds. ${ }^{[833,834]}$ Some of the photosensitizers used, as well as those in the final stages of clinical trials, are shown in Table 21 and depicted in Figure 96.

Along with the improvement of spectral characteristics, great attention in the development of new PSs is paid to the possibility of their use for the diagnosis and visualization of tumors, since the creation of such theranostics makes it possible to reliably detect and then remove malignant neoplasms. And, finally, an extremely important role is played by the directed modification of PS and the production of certain conjugates to increase their tropism to tumors. Summarizing the basic requirements for highly effective photosensitizers, they can be formulated as follows:

- they must have intense absorption in the long-wavelength region of the spectrum;

- have a high quantum yield of singlet oxygen and possibly other forms of active oxygen;

- have spectral and other physicochemical properties for reliable diagnosis of malignant neoplasms;

- exhibit high oncospecificity and selectivity of accumulation in a certain type of tumor;

- have minimal cytotoxicity and be quickly removed from the body;

- have sufficient solubility in biological media.

The practical implementation of these requirements is fraught with certain difficulties. Thus, an increase in fluorescence, which is necessary for the diagnosis of a tumor, is usually accompanied by a decrease in the quantum yield of singlet oxygen and, as a result, a decrease in the photodynamic effect. An increase in the hydrophilicity of PS and an improvement in solubility in aqueous solutions complicate the subsequent passage of PS through the hydrophobic membrane of the cell. In this regard, when creating highly effective photosensitizers, simple modifications of substituents in the chlorin macrocycle, which can be used to improve the spectral characteristics or solubility in saline solutions, are no longer enough. It is necessary to obtain more complex conjugates by attaching certain vector molecules to the chlorin macrocycle that provide targeted delivery of these structures to the tumor, as well as external chelating fragments with various metal ions for diagnostics and visualization of tumors using fluorescence, including the infrared region of the spectrum, magnetically-resonance tomography (MRI), positron emission tomography (PET) and other physical methods.

Studies aimed at developing targeted delivery vehicles for promising PSs based on natural chlorins and increasing their selective accumulation in tumors are actively developing in two main directions. The first involves the creation of conjugates, the so-called theranostics, with certain vector molecules. The second direction is based on the use of a wide range of nanoparticles. There is a number of reviews that provide useful information on the status of work in these areas. ${ }^{[87-864]}$

Within the framework of the first direction, a fairly wide range of vector molecules is used to create theranostics with increased tropism for malignant neoplasms. These can be monoclonal antibodies and antibody fragments, ${ }^{[865,866]}$ peptides, ${ }^{[867,868]}$ carbohydrates, ${ }^{[869,870]}$ steroids, ${ }^{[871]}$ folic acid and its derivatives ${ }^{[873-875]}$ and a number of other compounds.

This section presents studies related to the development of highly effective PS based on chlorophyll $a$ derivatives with carbohydrates, peptides, tetraazamacrocycles and their metal complexes.

Much attention is paid to carbohydrates in the development of photosensitizers-theranostics, due to the fact that tumor cells of many types of cancer have an increased expression of galectins - proteins with high affinity for $\beta$-galactosides. ${ }^{[876]}$ In the synthesis of carbohydratecontaining conjugates, we used various approaches, which made it possible to attach carbohydrate fragments both to the upper part of the macrocycle at the vinyl group in pheophorbide $a$ (64a) and pyropheophorbide $a$ (64b), and on the opposite side of the macrocycle in $\mathrm{N}$-substituted purpurinimide (Scheme 19). In the first case, the starting chlorins 64a,b were treated with $40 \% \mathrm{HBr}$ in acetic acid, and the obtained $\alpha$-bromoethyl derivatives $\mathbf{6 5 a}, \mathbf{b}$ were treated with protected galactopyranose without isolation. The yield of glycochlorins 66a,b is 30-32 \%. ${ }^{[877]}$ More stable $S$-glycosylated derivative of pyropheophorbide $a \mathbf{6 7}$ was obtained by condensation of $\alpha$-bromoethyl-substituted pyropheophorbide with sodium $\beta$-D-glucopyranosyl mercaptide. The yield of the target compound was $54 \%$. ${ }^{[878]}$ The $\beta$-D-galactopyranose derivative was similarly obtained. To improve the solubility in aqueous solutions, the resulting conjugates were deacetylated.

To attach the carbohydrate fragment to the lower part of the macrocycle, the previously developed method of condensation of purpurin 18 with hydroxylamine was used. ${ }^{[879]}$ In this case, purpurin 18 (68) was condensed with $o$ - $\beta$-Dgalactopyranosyl hydroxylamine. The obtained $N$-galactopyranosylpurpurinimide (69), yield $30 \%$, is readily soluble in water, has an intense absorption band at $714 \mathrm{~nm}$ and can be used to create targeted PSs due to the addition of vector molecules to the propionic acid residue. ${ }^{[80]}$

Various derivatives of glycoconjugates were obtained using the cross-metathesis reaction. ${ }^{\left[{ }^{[81]}\right.}$ In this case, both the vinyl group already present in chlorines and the previously obtained derivatives with alkenyl substituents in the lower part of the macrocycle were used (Scheme 20). The condensation of chlorin $e_{6} \mathbf{7 2}$ with allyl- $\beta$-D-galactopyranoside tetraacetate $\mathbf{7 0}$ was carried out in the presence of a second generation Grubbs catalyst 71. The optimal yield (40\%) of glycoconjugate 73a was obtained using a fourfold excess of allyl galactazide in the presence of $25 \mathrm{~mol} \%$ catalyst. The interaction proceeds stereoselectively with the formation of the $E$-isomer. In the synthesis of conjugates with carbohydrates in the lower part of the macrocycle, mesopheophorbide $a$, obtained by hydrogenation of pheophorbide $a \mathbf{6 4 a}$, was treated with allylamine and substituted chlorin $\mathbf{7 4}$ was condensed with tetraacetate allyl- $\beta$-D-galactopyranoside $\mathbf{7 0}$ in a ratio of $1: 5$ in the presence of $5 \mathrm{~mol} \%$ Grubbs catalyst. In this case, however, the reaction is less selective than that described above for the vinyl group. As a result, two isomers 75a $E$ and $Z$ are formed in a 5:1 ratio and a total yield of $80 \%$. Individual isomers were isolated by preparative thin layer chromatography. 


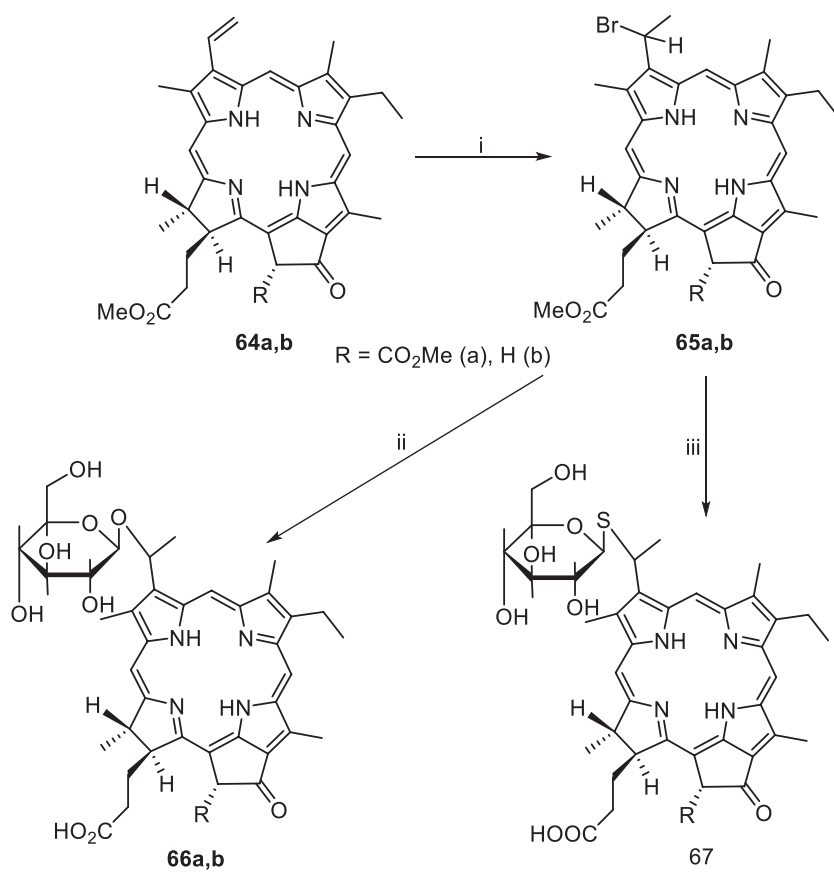

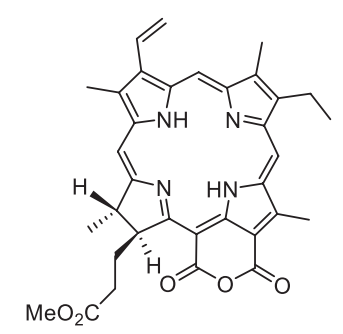

68

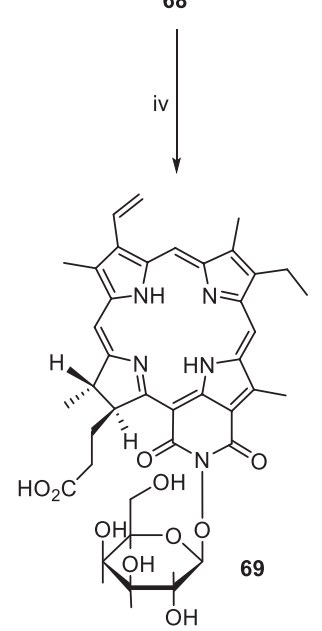

Scheme 19. Synthesis of glycoconjugates of natural chlorins.

Reagents and conditions: $\mathrm{i}-\mathrm{HBr} / \mathrm{CH}_{3} \mathrm{COOH}$; $\mathrm{ii}-1,2,3,4,6$-pentaacetyl- $\beta$-D-glucopyranose, 4-dimethylaminopyridine, $\mathrm{CHCl}_{3}, 50{ }^{\circ} \mathrm{C}, 2$ hours; iii - sodium $\beta$-D-glucopyranosyl mercaptide, DMF, $20^{\circ} \mathrm{C}, 0.5 \mathrm{~h}$; iv - $\beta$-D-galactopyranosylhydroxylamine, $\mathrm{HCl}, 20^{\circ} \mathrm{C}$.

When cross-coupling galactopyranoside $\mathbf{7 0}$ to the vinyl group of $N$-methoxypurpurinimide $\mathbf{7 6}$, we encountered certain difficulties. In contrast to chlorin $e_{6} \mathbf{7 2}$, even with prolonged heating of purpurinimide with an excess of sugar and an increase in the amount of Grubbs' catalyst to 1 equivalent, the yield of conjugate 77 did not exceed $5 \%$. This effect is apparently associated with the deactivating effect of the cycloimide fragment on the main macrocycle and vinyl group, which reduces the activity of the latter in the cross-metathesis reaction.

In this regard, for the addition of allylgalactopyranoside, mesopurpurinecycloimide $\mathbf{7 8}$ with a terminal double bond at the exocycle $\mathrm{E}$ was subsequently used. It was obtained by hydrogenation of the $\mathrm{Zn}$ complex of purpurin 18, followed by demetallation and condensation with allylamine (transformation viii, stages $1,2,3,4)$. According to NMR data, the ratio of $E / Z$ isomers of conjugate 79a was 6:1. The main isomer $E$ was isolated in $62 \%$ yield. ${ }^{[882]}$ The treatment of the obtained conjugates with sodium methoxide made it possible to obtain samples $\mathbf{7 3 b}, \mathbf{7 5 b}$ and $\mathbf{7 9 b}$, readily soluble in saline, for biological tests.

For the synthesis of chlorin $e_{6}$ glycoconjugates, we have successfully used another approach based on the $\mathrm{Cu}$ catalyzed reaction of 1,3-cycloaddition of terminal alkynes with azides according to the "click chemistry" reaction. ${ }^{[883-885]}$ Chlorin 80a, starting in the synthesis, was obtained by the reaction of pheophorbide $a \mathbf{6 4 a}$ with propargylamine (Scheme 21). Since copper is easily incorporated into the chlorin macrocycle under the conditions of the main reaction, the condensation was carried out using the $\mathrm{Zn}$ complex 80b. As a result, the conjugate 81a was obtained. Removal of zinc in a weakly acidic medium and subsequent treatment with sodium methoxide gave galactosylchlorin $\mathbf{8 1 b}$ in good yield. The more complex conjugate $\mathbf{8 2 b}$ with lactose was prepared in a similar manner.

Biological tests of the obtained glycoconjugates, performed on the Hep2 cell line, made it possible to draw two main conclusions: - galactosylchlorins obtained by the cross-metathesis reaction and containing a double bond in the spacer are significantly superior in their activity to glycoconjugates with a triazole ring; - among chlorins with a galactose residue, the leader compound is glycoconjugate $\mathbf{7 3 b}$ with a carbohydrate residue in pyrrole $\mathrm{A}\left(\mathrm{IC}_{50}=0.02 \mu \mathrm{M}\right)$, which is 8 times higher than the activity of the initial chlorin $e_{6}{ }^{[886]}$

Along with carbohydrates, amino acids and peptides are widely used in the development of theranostics for PDT. ${ }^{[867,868]}$ Thus, the well-known photosensitizer Talaporfin was obtained by condensation of chlorin $e_{6}$ with aspartic acid. Extensive research in this area is being conducted with other amino acids and peptides. Pseudopeptides as a connecting link turned out to be promising compounds in this regard. ${ }^{[889,890]}$ These ligands have increased tropism for prostate-specific membrane antigen (PSMA), a glycoprotein, the overexpression of which is characteristic of prostate cells. ${ }^{\left[{ }^{[81]}\right.}$ Using chlorin $e_{6}$, we have synthesized target PSs of a similar peptidomimetic based on urea, lysine, and glutamic acid. The effect of the position of the PSMA ligand in the macrocycle on the efficiency of the synthesized conjugates was studied.

The synthesis of conjugates containing the PSMA ligand in the lower part of the macrocycle was carried out using a $\mathrm{Cu}$-catalyzed azide-alkyne cycloaddition reaction. For this, intermediate compounds $\mathbf{8 0 b}$ and 91 were pre- 

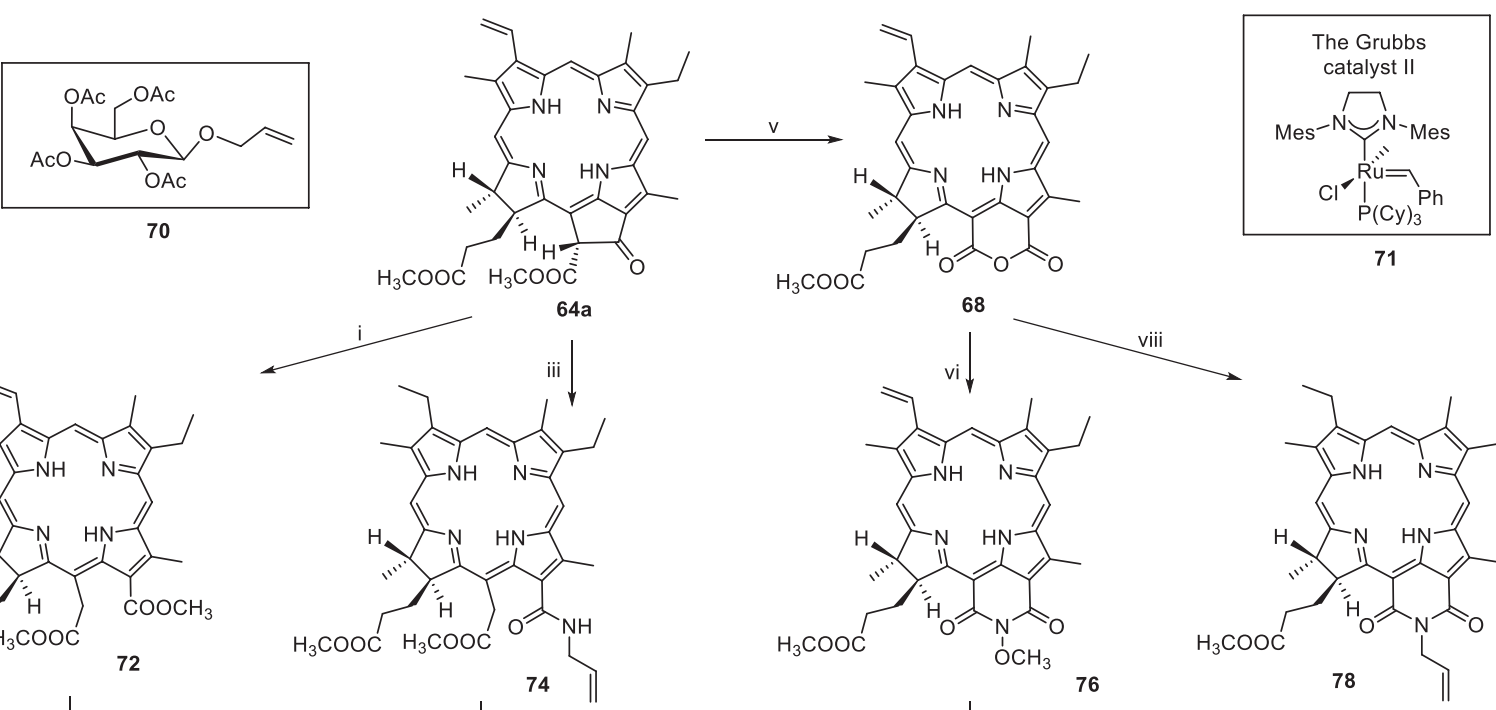

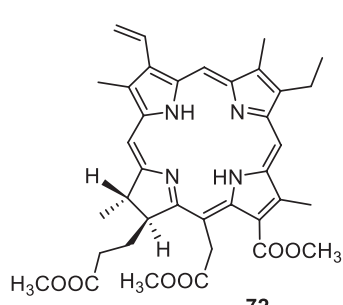

72

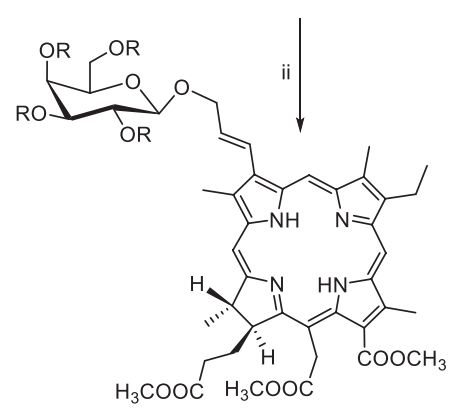

$73 a, b$
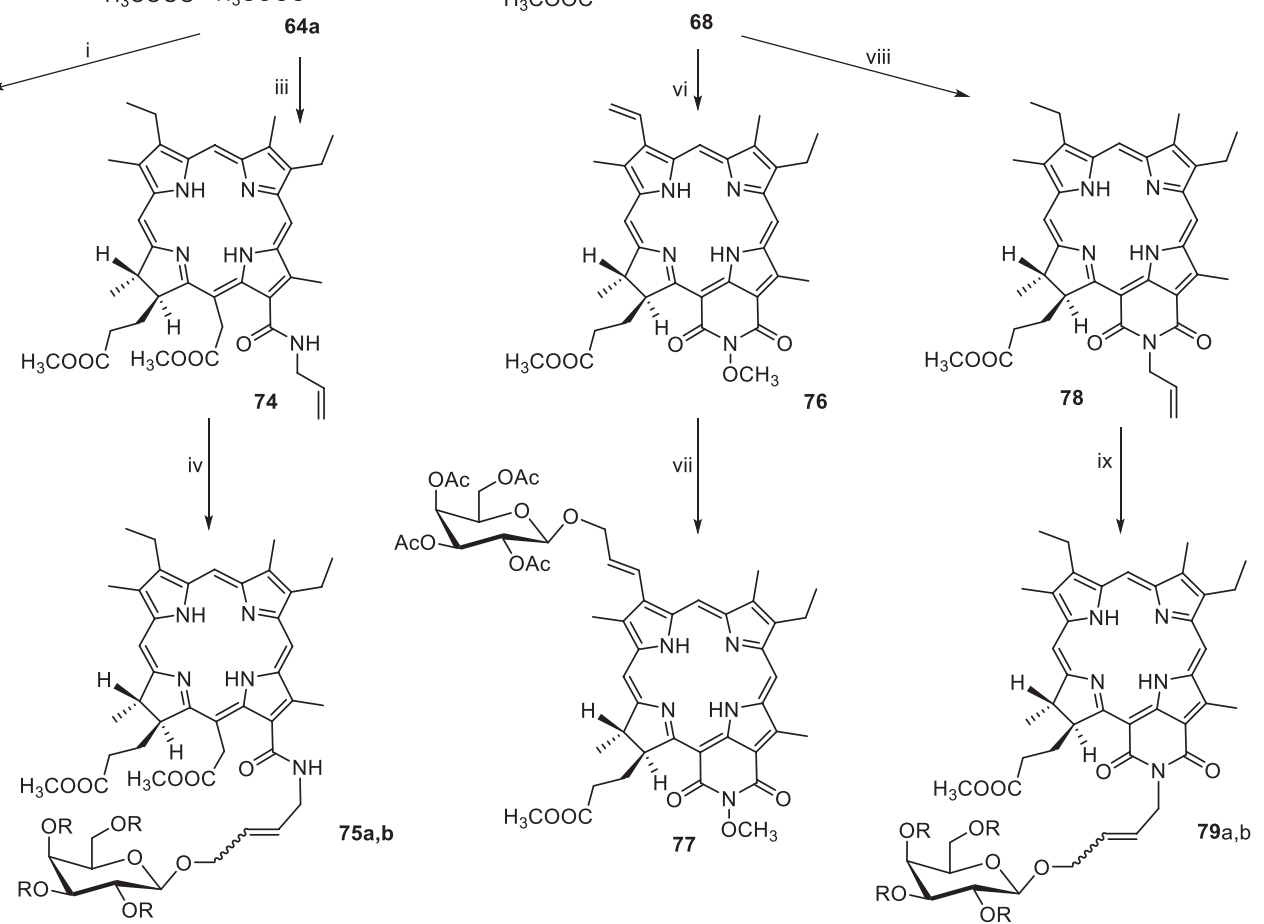

Scheme 20. Reaction of cross-metathesis of olefins in the synthesis of carbon-containing chlorins.

Reagents and reaction conditions: $\mathrm{i}-\mathrm{CH}_{3} \mathrm{ONa}, \mathrm{CH}_{3} \mathrm{OH}, \mathrm{CH}_{2} \mathrm{Cl}_{2}$; ii - 70, 71 (25 mol.\%), $\mathrm{CH}_{2} \mathrm{Cl}_{2}, \Delta, 16 \mathrm{~h}, 40$ \%; $\mathrm{iii}-\mathrm{H}_{2}, 10 \%, \mathrm{Pd} / \mathrm{C}(1)$, allylamine (2), $85 \%$; iv - 70, 71 (5 mol\%), $\mathrm{CH}_{2} \mathrm{Cl}_{2}, 4$ hours, $80 \%$; $-\mathrm{O}_{2}, \mathrm{KOH}, i-\mathrm{PrOH}(1), \mathrm{HCl}(2)$; vi - $\mathrm{NH}_{2} \mathrm{OH} \cdot \mathrm{HCl} / \mathrm{Py}_{(1)} \mathrm{CH}_{2} \mathrm{~N}_{2}(2)$; vii - 70 (excess), 71 (1 eq.), reflux, $5 \%$; viii - Zn (OAc) $(1), \mathrm{H}_{2}, 10 \% \mathrm{Pd} / \mathrm{C}$ (2), $\mathrm{HCl}(3)$, allylamine (4); ix - 7, 8, $75 \%$.

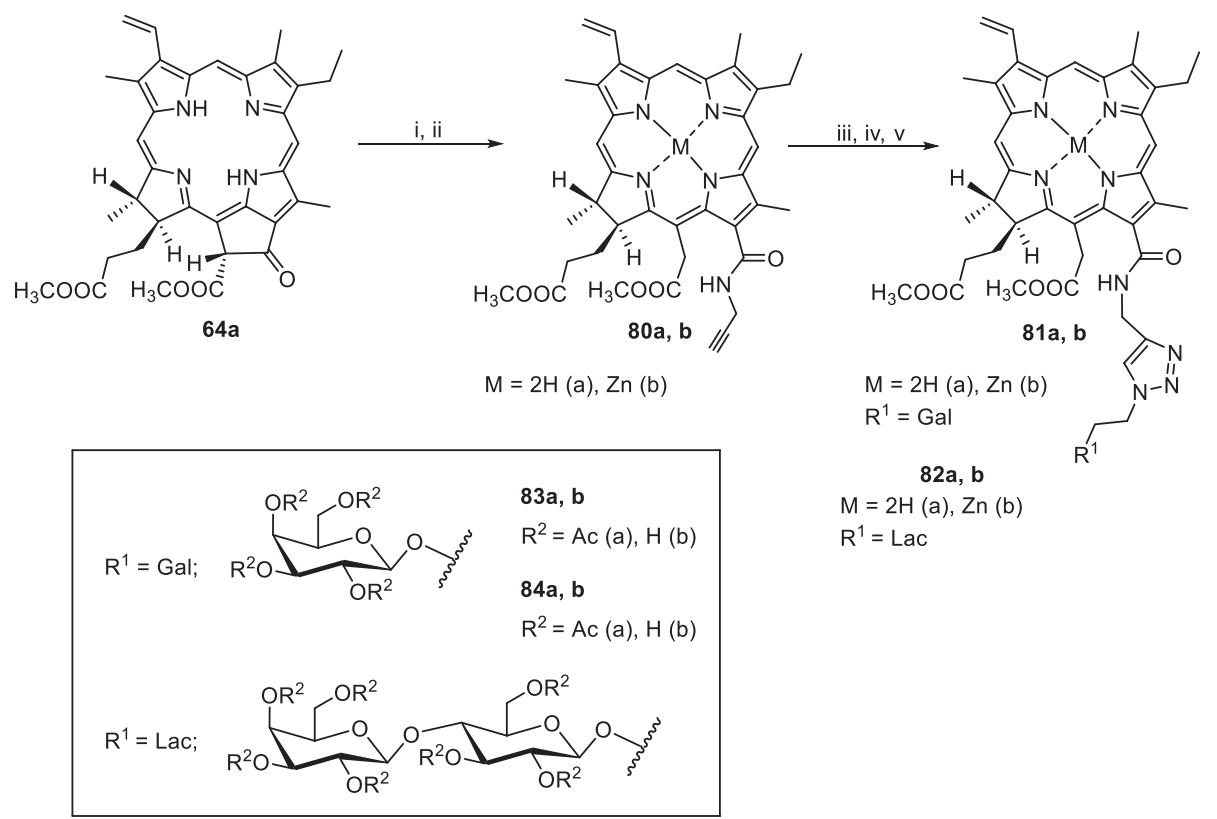

Scheme 21. Synthesis of chlorin $e_{6}$ glycoconjugates.

Reagents and reaction conditions: $\mathrm{i}$ - propargylamine, $\mathrm{CH}_{2} \mathrm{Cl}_{2}, 120 \mathrm{~h}$; ii - $\mathrm{Zn}(\mathrm{OAc})_{2} \cdot 2 \mathrm{H}_{2} \mathrm{O}, \mathrm{CH}_{2} \mathrm{Cl}_{2}, \mathrm{CH}_{3} \mathrm{ONa}$; iii - (2-azidoethyl) glycoside 83a/84a, $\mathrm{CuI}$ (10 mol\%), DIPEA, $\mathrm{CH}_{3} \mathrm{CN}$; iv $-\mathrm{CHCl}_{3}, \mathrm{HCl}$; v $-\mathrm{CH}_{2} \mathrm{Cl}_{2}, \mathrm{CH}_{3} \mathrm{ONa}, \mathrm{CH}_{3} \mathrm{OH}, 1 \mathrm{~h}$. 

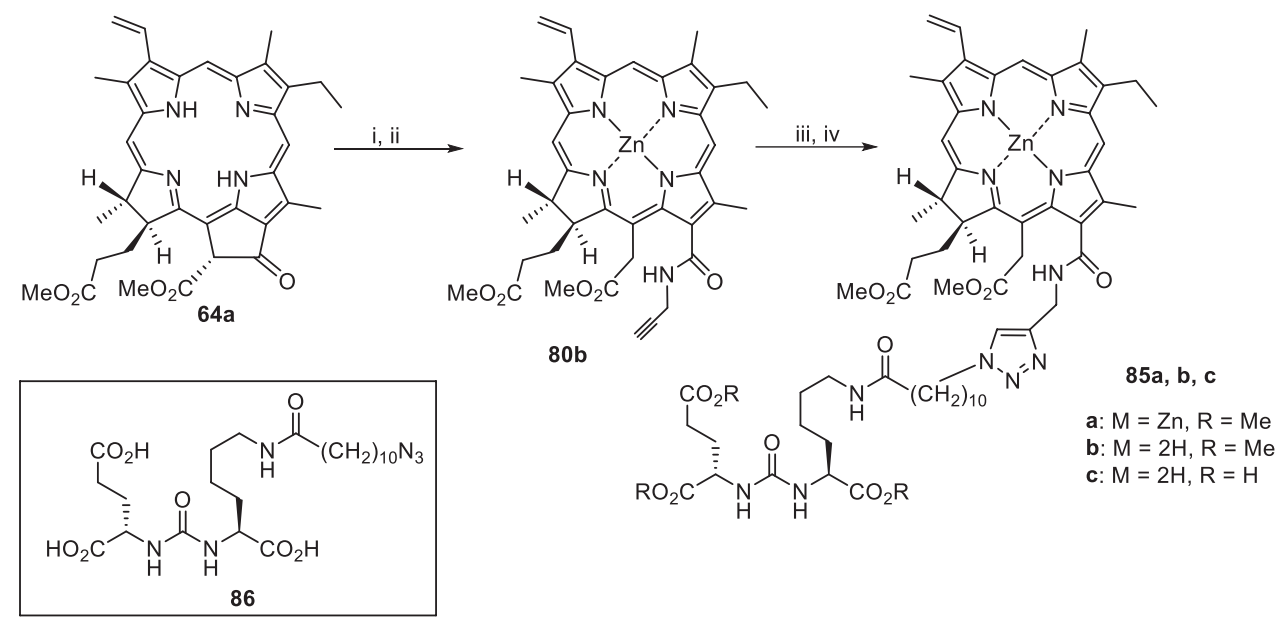

Scheme 22. Synthesis of PSMA-ligand conjugate with $13^{1}$-chlorin $e_{6}$.

Reagents and reaction conditions: $\mathrm{i}$ - propargylamine, $\mathrm{CHCl}_{3}$; $\mathrm{ii}-\mathrm{Zn}(\mathrm{OAc})_{2}, \mathrm{CHCl}_{3}$; iii - 86, CuI, DIPEA, DMF, $20^{\circ} \mathrm{C}, 16 \mathrm{~h}$; iv $-\mathrm{CF}_{3} \mathrm{COOH}, \mathrm{DMSO}, 20^{\circ} \mathrm{C}, 2 \mathrm{~h}$.

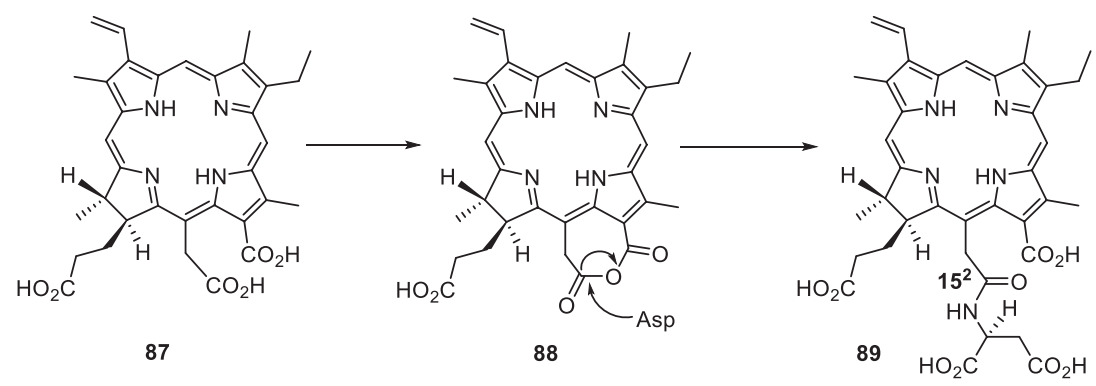

Scheme 23. The mechanism of formation of Talaporfin. ${ }^{\left[{ }^{[82]}\right.}$

liminarily obtained. In the first case, pheophorbide $a$ was treated with propargylamine, and the 13-substituted derivative was obtained in good yield (Scheme 22). Subsequent condensation of its zinc complex $\mathbf{8 0 b}$ with azide $\mathbf{8 6}$ gave the target product $\mathbf{8 5 b}$ in $62 \%$ yield calculated for the starting pheophorbide $a$ methyl ester. ${ }^{[897]}$

A more complex problem had to be solved for the regiospecific introduction of propargylamine into the 15-position of chlorin $e_{6}$. In this case, we used the development of K. Smith et al., which was used by them to prove the structure of Talaporfin. ${ }^{[892-894]}$ For a long time, it was believed that this PS represents chlorin $e_{6}$ with an aspartic acid residue at position 17, since the condensation of chlorin with an amino acid in high yield gave only one isomer, with a purity of at least $95 \%$ according to NMR data, and of the three carboxyl groups, the most reactive is the residue of propionic acid. In this regard, in the patent for obtaining of Talaporfin ${ }^{\text {[895] }}$ and numerous publications, ${ }^{[831,896]}$ its structure was depicted as 17 -substituted chlorin $e_{6}$. However, in reality, when condensation of chlorin $e_{6}$ with aspartic acid in the presence of 1 eq. of DCC a 7-membered anhydride ring initially is formed very quickly due to the condensation of substituents at the 13- and 15-positions. The subsequent nucleophilic attack of the formed anhydride by aspartic acid on the more electrophilic aliphatic carbonyl group at position $15^{2}$ leads to the formation of $15^{2}$-conjugate of chlorin $e_{6}$ with aspartic acid. A similar mechanism has been reliably proven due to the isolation of anhydride and its X-ray structural analysis. These transformations are shown in Scheme 23.

In the synthesis of isomeric theranostic 92b with the PSMA vector at position 15 , the starting chlorin $e_{6}$ was treated with 1 equiv. of 1-ethyl-3-(3-dimethylaminopropyl) carbodiimide and the resulting anhydride was condensed without isolation with propargylamine (Scheme 24). After treatment with diazomethane and introduction of a zinc ion, compound 91 was condensed with azide $\mathbf{8 6}$. Removal of zinc from the resulting conjugate 92 a allowed the target compound $92 \mathrm{~b}$ to be obtained in $43 \%$ yield based on the starting chlorin $e_{6}{ }^{[897,898]}$

In terms of studying the relationship "structure - biological activity" of chlorin $e_{6}$ conjugates with the PSMA ligand, we synthesized have theranostic with a vector in the upper part of the macrocycle at pyrrole A. ${ }^{[898]}$ The presence of a vinyl group in chlorin $e_{6}$ allowed us to introduce a vector molecule using the tetrazine-alkene addition reaction (Inverse electron demand Diels-Alder reaction, IEDDA). ${ }^{\left[{ }^{[99}\right]}$ This method is a modified Diels-Alder reaction and can be considered a "click-chemistry" method. In this case, 1,2,4,5-tetrazine acts as a "diene", and an alkene or alkyne acts as a "dienophile". [4+2]-Cycloaddition occurs with the participation of 3 and 6 carbon atoms of 1,2,4,5-tetrazine. After the addition of a dienophile and subsequent isolation of the nitrogen molecule, a 1,2-dihydropyridazine ring is formed, which is oxidized to pyridazine (Scheme 25). 


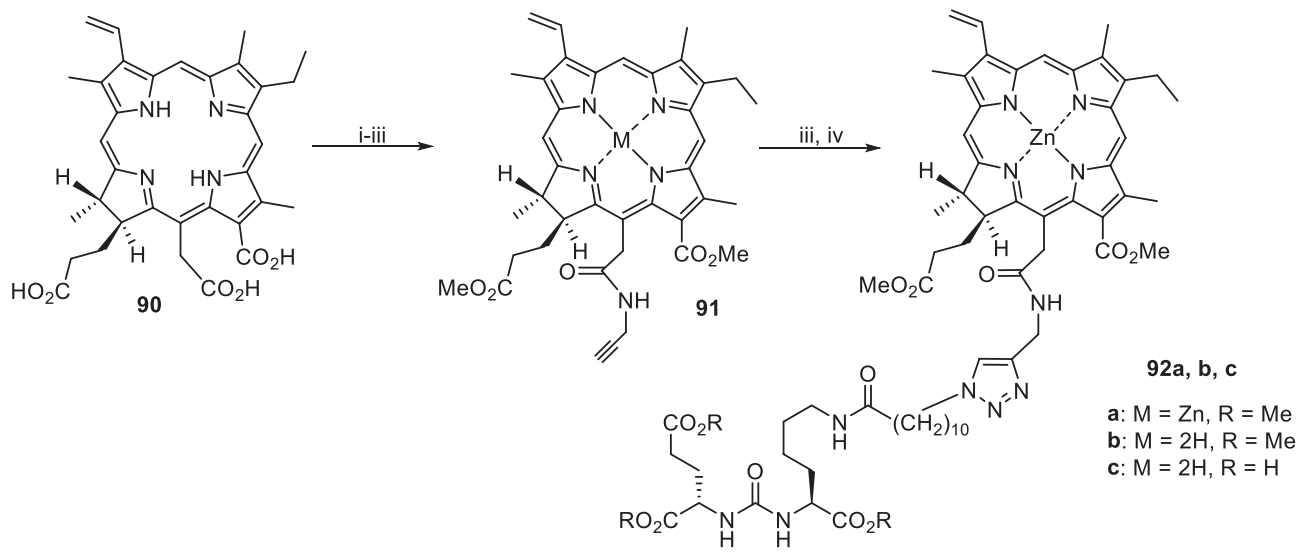

Scheme 24. Synthesis of chlorin $e_{6}$ conjugate with PSMA vector at $15^{2}$-position.

Reagents and conditions: $\mathrm{i}-\mathrm{EDC}$, DMAP, $\mathrm{HC} \equiv \mathrm{CCH}_{2} \mathrm{NH}_{2}, \mathrm{CHCl}_{3}$; ii $-\mathrm{CH}_{2} \mathrm{~N}_{2}, \mathrm{CHCl}_{3}$; $\mathrm{iii}-\mathrm{Zn}(\mathrm{OAc})_{2}$; iv - 86, CuI, DIPEA, DMF, $20^{\circ} \mathrm{C}$, 16 hours; $\mathrm{v}-\mathrm{CF}_{3} \mathrm{COOH}$.

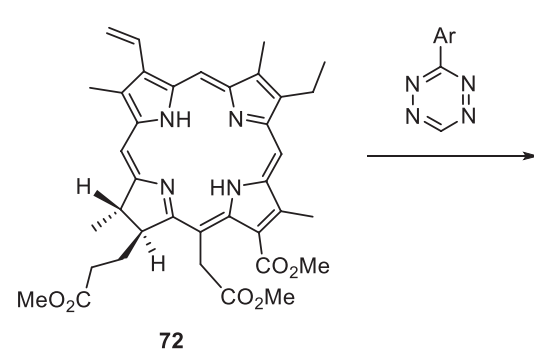

72
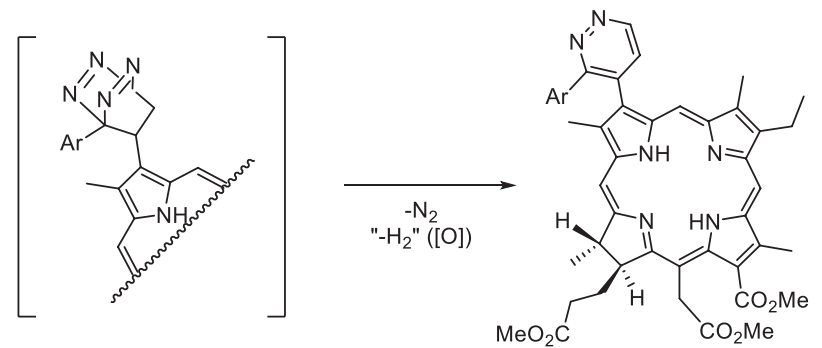

93a, b (two conformers) a: $\mathrm{Ar}=\mathrm{C}_{6} \mathrm{H}_{5}$

b: $\mathrm{Ar}=\mathrm{C}_{6} \mathrm{H}_{4} \mathrm{COOH}$

Scheme 25. Synthesis of pyridazine-substituted derivatives of chlorin $e_{6}$.

Reagents and conditions: DMF, $20^{\circ} \mathrm{C}, 8 \mathrm{~h}$.
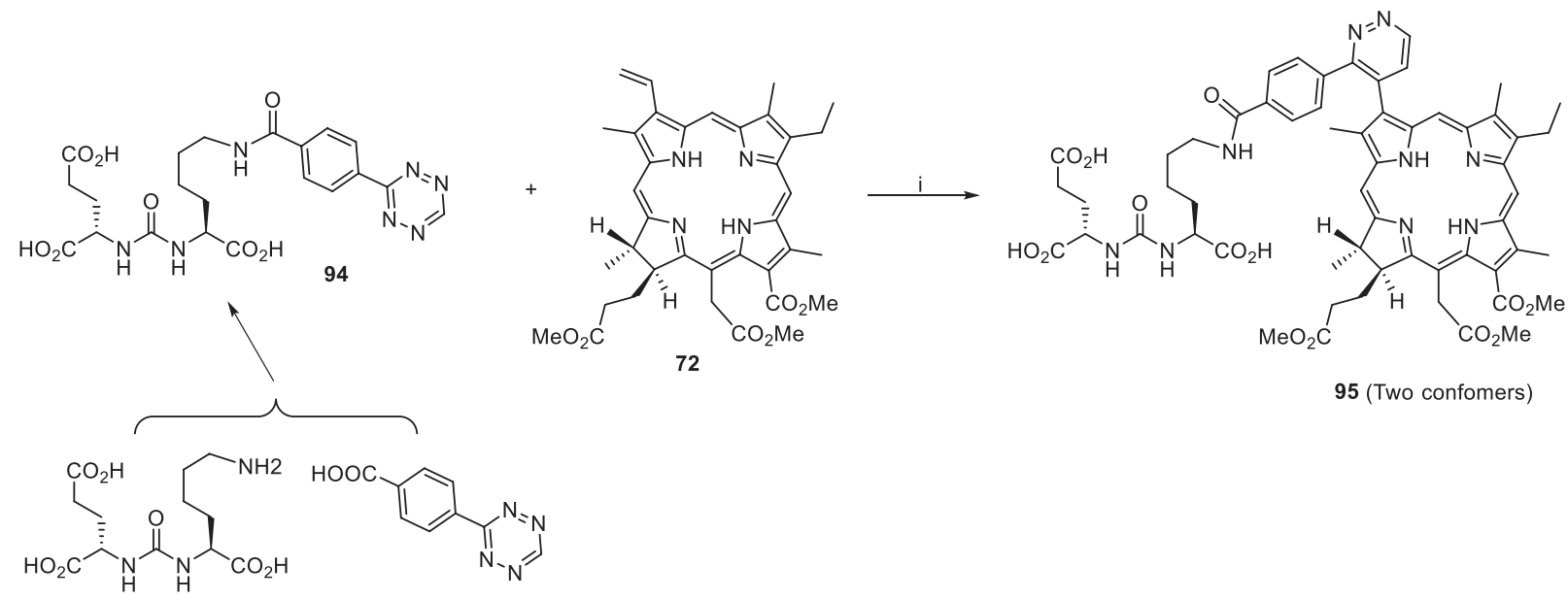

Scheme 26. Synthesis of chlorin $e_{6}$ conjugate with PSMA vector in pyrrole A.

Reagents and reaction conditions: $\mathrm{i}-\mathrm{DMF}, 20^{\circ} \mathrm{C}, 24 \mathrm{~h}$.

In order to optimize the performance of the IEDDA reaction under the conditions of our synthesis, we studied the condensation of chlorin $e_{6}$ with 3-phenyl-1,2,4,5-tetrazine in polar (methanol, DMF) and non-polar (methylene chloride, toluene) solvents. It has been found that better results can be obtained in polar solvents. Thus, when carrying out the reaction in DMF at room temperature (8 hours), the yield of the target product 93a was $76 \%$. ${ }^{1} \mathrm{H}$
NMR (COZY, NOESY, HSQS) showed the presence of two conformers in the compound in a 1:1 ratio. In a similar way, a conjugate of chlorin $e_{6}$ with carboxyphenyl-substituted tetrazine 93b was obtained, which can be used for the addition of various compounds at the carboxyl group.

A study of the photophysical properties of the obtained compounds $\mathbf{9 3 a}, \mathbf{b}$ showed that the introduction of substituted pyridazine only slightly shifts the long-wavelength band 
of chlorin $e_{6}$ from $662 \mathrm{~nm}$ to $665 \mathrm{~nm}$ (93a) and $667 \mathrm{~nm}$ (93b) and weakly affects the quantum yield of fluorescence -0.16 (93a) and 0.11 (93b), while for chlorin $e_{6}$ it is 0.16 .

Scheme 26 shows the synthesis of the chlorin $e_{6}$ conjugate, in which the PSMA vector is attached to the vinyl group of pyrrole A. For this, the ligand to PSMA was preliminarily modified with 4-(1,2,4,5-tetrazin-3-yl) benzoic acid to obtain precursor 94. The structure of conjugate 95 is confirmed by high-resolution mass spectrum and NMR spectrum. As before, in this case, two conformers are formed, differing in the location of the vector peptidomimetic. ${ }^{[000]}$

The biological activity of the obtained conjugates of chlorin $e_{6}$ with PSMA vectors $85 \mathbf{c}, 92 \mathbf{c}$, and 95 was assessed in vitro on prostate cancer cell lines, in one of which 22Rv1 cells are overexpressed PSMA receptors, while these receptors are absent in PC-3 cells. ${ }^{[901]}$ Of the two conjugates in which the PSMA vector is located in the lower part of the macrocycle, the $13^{1}$ isomer $\left(\mathrm{IC}_{50}\right.$ $1.2 \pm 0.1)$ showed the maximum phototoxicity on $22 \mathrm{Rv} 1$ cells, versus $5.3 \pm 0.1$ for the $15^{2}$ isomer. At the same time, on PC-3 cells, in the absence of PSMA, the activity of conjugate $84 \mathrm{c}$ is decreased by 7 times. However, the highest activity among the synthesized conjugates is revealed for compound 95, for which the $\mathrm{IC}_{50}$ is $0.6 \pm 0.1 \mu \mathrm{M}$. These results significantly exceed the data obtained for the currently used FS Radachlorin $\left(\mathrm{IC}_{50}=3.0 \pm 0.2 \mu \mathrm{M}\right)$. Thus, the studies performed have shown that the introduction of the PSMA vector into various positions of the macrocycle of chlorin $e_{6}$, the most widely used photosensitizer, significantly affects the phototoxicity of drugs, which should be taken into account when developing PS for the treatment of prostate cancer.

An important direction in the development of PDT for cancer is the creation of a new class of photosensitizers - theranostics, which allow both tumor therapy and their preliminary diagnosis and non-invasive imaging. For this purpose, along with the traditionally used fluorescence diagnostics, a number of new methods can be used, such as magnetic resonance imaging (MRI), single photon emission computed tomography (SPECT) and positron emission tomography (PET). Each of these methods, however, has certain advantages and limitations. Thus, SPECT and PET methods are characterized by poor resolution on a medical scanner, but the ability to visualize deep-seated tumors. MRI, on the contrary, gives a high resolution, but has a low sensitivity. In turn, fluorescence has a high resolution, but weakly penetrates into tissues. In this regard, it becomes necessary to combine various methods and create bimodal sensors for these purposes. ${ }^{[906,907]}$

Significant prospects, in this regard, are opening up when obtaining bimetallic complexes based on conjugates of natural chlorins with external chelators, which can include a wide range of metals, including lanthanides and other large metal ions. 1,4,7,10-Tetraazacyclododecanecyclene has proved to be a promising chelator for these purposes. ${ }^{[008,909]}$

We have synthesized chlorin $e_{6}$ conjugates with cyclene by condensing the latter with pheophorbide $a$ (Scheme 27). The opening of the pentane ring takes place under mild conditions, and conjugate 96 was obtained with an yield of $77 \%$. $^{[10]}$

It is known, that the introduction of metal cations into the porphyrin and chlorin macrocycles significantly changes the properties of the resulting structures and, in some cases, is accompanied by a significant decrease in the fluorescence of these compounds. ${ }^{[911,912]}$ This greatly limits the possibility of using such PSs for fluorescence diagnostics. The presence of two coordination cavities in the conjugate makes it possible to obtain homo- and heteronuclear metal complexes, which, along with PDT, will have high fluorescence and the necessary properties for other diagnostic and visualization methods.

Regioselective introduction of the palladium cation into the chlorin macrocycle of conjugate 96 was carried out under mild conditions using palladium acetate. The formation of the metal complex 97a was monitored by the shift of the absorption band from 664 to $624 \mathrm{~nm}$. Copper complex 30b was prepared in a similar manner, with the $Q$ band shifted to $640 \mathrm{~nm}$. The study of the photophysical properties of the obtained metal complexes showed that the introduction of the $\mathrm{Pd}^{2+}$ cation into the chlorin macrocycle significantly quenches the fluorescence of the starting compound. At the same time, the quantum yield of singlet oxygen generation (1,3-diphenylisobenzofuran as a chemical trap) is increased up to $98 \%$. In the case of copper complex,

Table 22. Quantum yields of fluorescence $\left(\Phi_{\mathrm{F}}\right)$ and generation of singlet oxygen $\left(\Phi_{\Delta}\right)$ of chlorin-cyclenic conjugate and its Pdcomplex.

\begin{tabular}{ccc}
\hline Compound & $\begin{array}{c}\text { Quantum yields } \\
\text { of fluorescence }\left(\Phi_{\mathrm{F}}\right)\end{array}$ & $\begin{array}{c}\text { Quantum yields } \\
\text { singlet oxygen }\left(\Phi_{\Delta}\right)\end{array}$ \\
\hline $\mathbf{9 6}$ & $0.266 \pm 0.005$ & 0.71 \\
$\mathbf{9 7 a}$ & 0.0005 & 0.98 \\
\hline
\end{tabular}

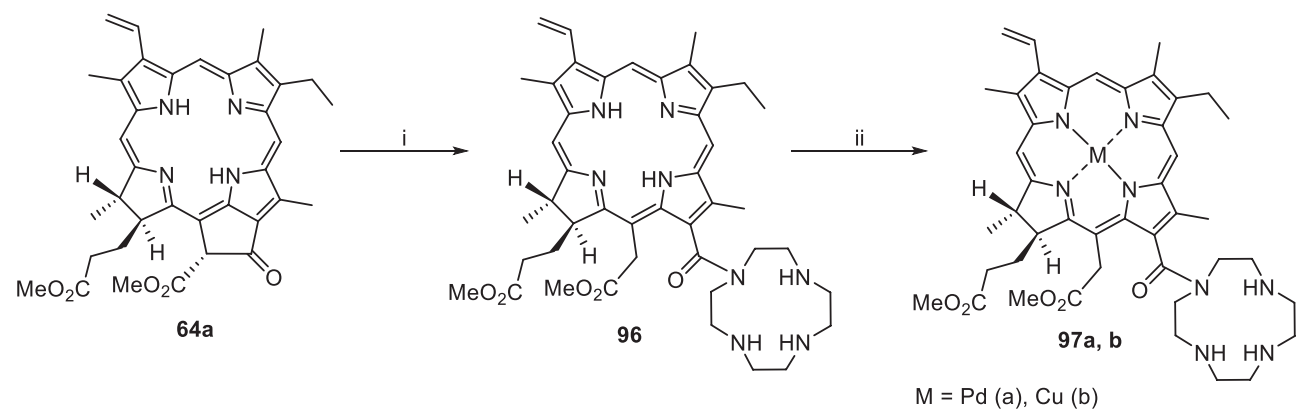

Scheme 27. Introduction of metal ions into the coordination sphere of methylpheophorbide $a$ conjugates with cyclene. Reagents and conditions: $\mathrm{i}$ - cyclene, $\mathrm{CH}_{2} \mathrm{Cl}_{2}$, DIPEA; ii - $\mathrm{Pd}(\mathrm{OAc})_{2}$ or $\mathrm{Cu}(\mathrm{OAc})_{2}, \mathrm{CH}_{2} \mathrm{Cl}_{2}$. 


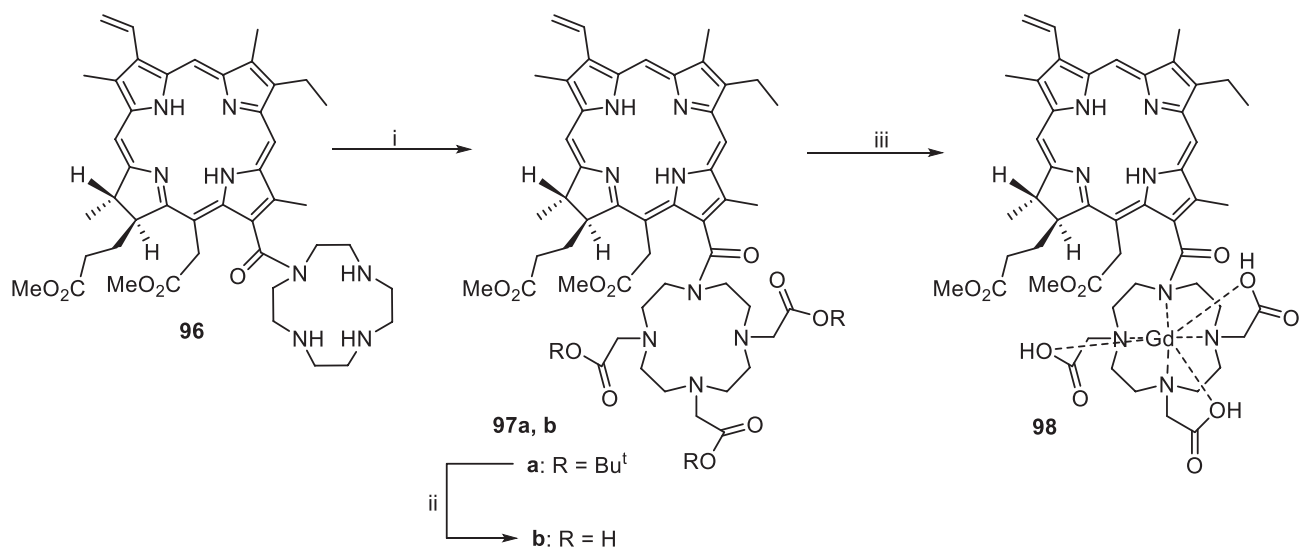

Scheme 28. Synthesis of gadolinium complex of chlorin $e_{6}$ conjugate with cyclene.

Reagents and conditions: $\mathrm{i}-\mathrm{BrCH}_{2} \mathrm{CO}_{2}{ }^{t} \mathrm{But}, \mathrm{CH}_{2} \mathrm{Cl}_{2}$, DIPEA; ii $-\mathrm{CF}_{3} \mathrm{COOH}\left(80 \%\right.$ aq.); iii $-\mathrm{GdCl}_{3} \cdot 6 \mathrm{H}_{2} \mathrm{O}, \mathrm{CH}_{2} \mathrm{Cl}{ }_{2}, \mathrm{NaOH} / \mathrm{MeOH}$.

a sharp decrease in both fluorescence and generation of singlet oxygen was observed (Table 22). ${ }^{[913]}$

Further development of studies on the creation of theranostik based on the obtained conjugate 96 was the study of the possibility of including metal cations with a large ionic radius into the cyclenic cavity. However, when trying to obtain a similar complex with gadolinium, which is known to be a part of Gadovist and Magnevist preparations used in MRI, it was found that such a complex does not have sufficient stability. To enhance the chelating properties of cyclene, we modified it by introducing residues of acetic acid at three nitrogen atoms. The method described in the literature for the direct alkylation of cyclene with bromoacetic acid turned out to be not very successful, since the resulting mono-, di- and trisubstituted derivatives have similar chromatographic mobility, which complicates the isolation of the target product. In this regard, for alkylation we used monobromoacetic acid tert-butyl ester, which was slowly added to conjugate 96 in methylene chloride in the presence of DIPEA. To unblock the carboxyl groups, the obtained triester 97a was treated with $80 \%$ TPA (Scheme 28). Conjugate 97b was condensed with $\mathrm{GdCl}_{3} \cdot 6 \mathrm{H}_{2} \mathrm{O}$ in methylene chloride with addition of a small amount of $\mathrm{NaOH}$ methanol solution while heating to $40{ }^{\circ} \mathrm{C}$. The presence of the gadolinium cation in the metal complex 98 was proved using atomic emission spectroscopy. The study of the fluorescent properties of the obtained complex showed that the presence of gadolinium cation in the immediate vicinity of the chlorin macrocycle partially quenches the fluorescence, apparently due to the release of a part of the energy of the triplet state of chlorin to the metal. Residual fluorescence, however, makes it possible to consider the obtained PS as a theranostic for complex diagnostics using PD and MRI, as well as PDT and MRI. ${ }^{[914]}$

A number of mono- and binuclear metal complexes with $\mathrm{Zn}, \mathrm{Cu}, \mathrm{Mn}, \mathrm{In}$, and $\mathrm{Pd}$ in a tetrapyrrole macrocycle and $\mathrm{Gd}$ in a cyclene have been synthesized on the basis of chlorincyclene complexes 96 and $\mathbf{9 7 b}$, the analysis of spectral and other physicochemical characteristics of which is currently being carried out in our laboratory.

Thus, the data accumulated to date make it possible to consider chlorophyll $a$ derivatives, mainly chlorin $e_{6}$, as a reliable base platform for the development of vectororiented theranostics for diagnosis and effective PDT in oncology.

\subsection{Fundamental and Applied Aspects of Bacteriochlorophyll Derivatives Chemistry}

Hydrogenated analogues of porphyrins - chlorophylls and bacteriochlorophylls - play an important role in nature, including participating in photosynthesis and related processes. The great interest in these compounds is due to the wide range of useful properties of their modified derivatives. The unique ability of bacteriochlorins for selective accumulation in tumor tissues and cause fluorescence or the photodynamic effect under the action of laser irradiation is successfully used in fluorescence diagnostics and photodynamic therapy of malignant neoplasms. ${ }^{\text {917-921] }}$

Bacteriochlorophylls are an independent group of natural chlorophylls; they are widely distributed in nature. ${ }^{\left[{ }^{[22,923]}\right.}$ To date, bacteriochlorophylls $a-g$ (99-105) are known; they differ both in the degree of the macrocycle hydrogenation and in the nature of substituents (Figure 97). The main sources of these compounds in nature are purple bacteria, green sulfur bacteria and heliobacteria. Special attention is paid to bacteriochlorophyll $a$, the $\mathrm{Q}_{\mathrm{y}}$ maximum absorption of which is not only shifted to the red region but also has the highest extinction coefficient. The source of bacteriochlorophyll is the biomass of purple bacteria such as Rh. sphaeroides, Rh. Roseapersiana and Rh.Capsulata, which produce only one type of bacteriochlorophyll. It greatly facilitates the pigment isolation and purification. ${ }^{[924-926]}$

Another group of natural bacteriochlorins are tolyporphins 106-114, which are glycosylated macrocycles isolated from lipophilic soil extracts of cyanobacteria Tolypothrixnodosa.$^{[927-929]}$ Tolyporphins A-I contain a dioxobacteriochlorin core while tolyporphin $\mathrm{K}$ is a unique monooxochlorin (Figure 98). During isolation it was found that none of these pigments contain a metal ion in the macrocycle. Moreover, it was shown that in contrast to porphyrins, chlorins and bacteriochlorins these compounds form metal complexes only with copper and silver. ${ }^{[930]}$ These compounds 


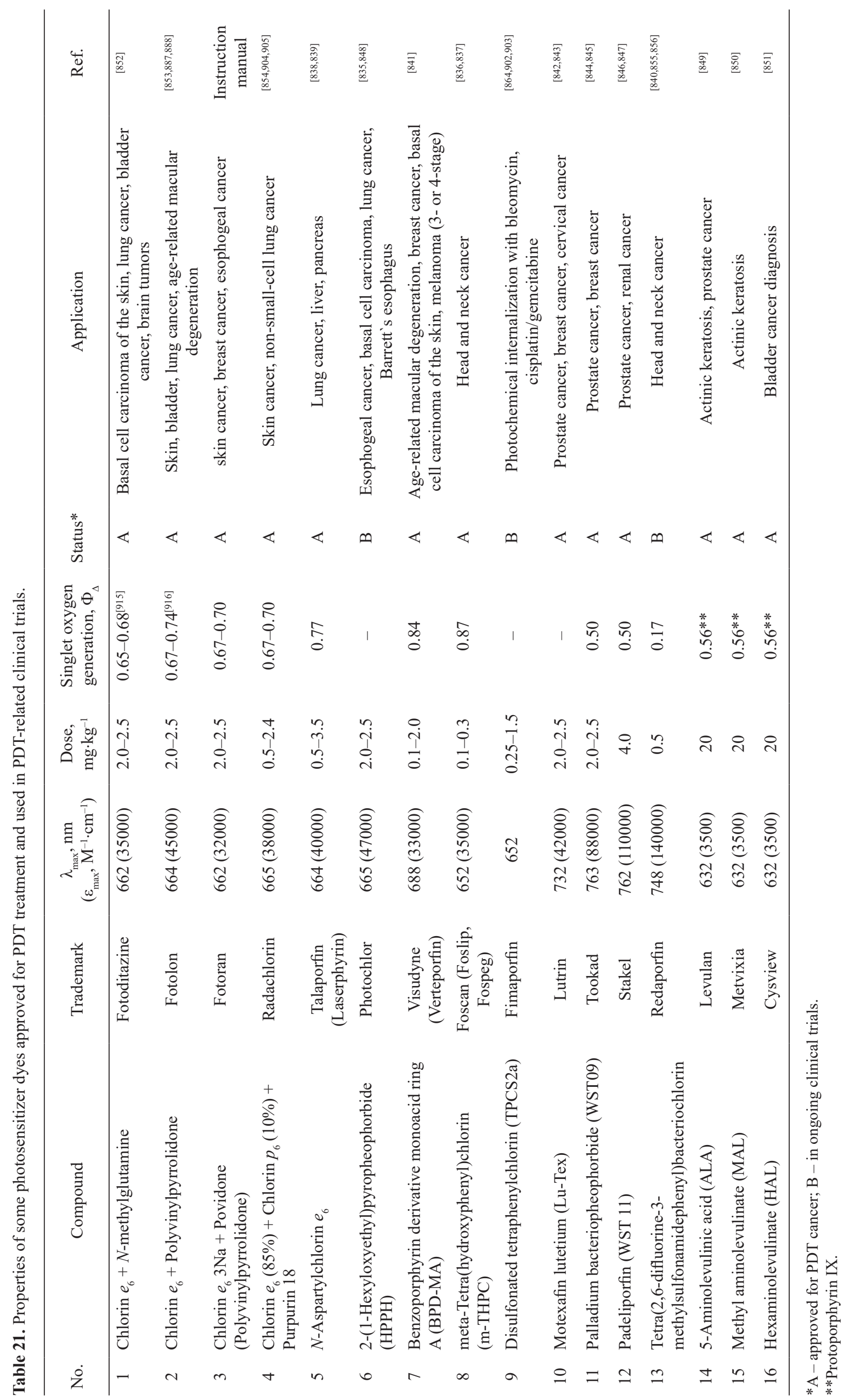




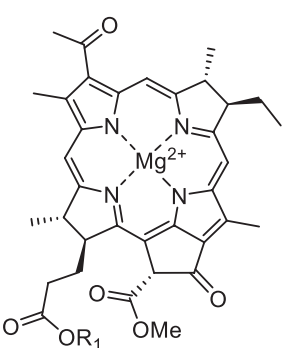

$99 \mathrm{Bcla}$

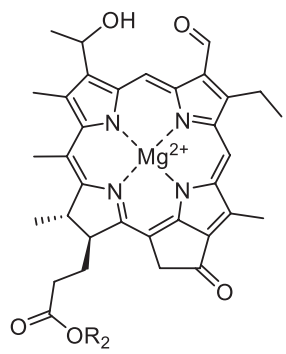

$103 \mathrm{Bcle}$

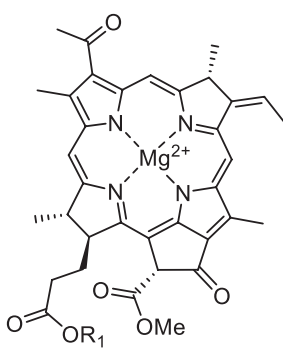

$100 \mathrm{Bcl} b$

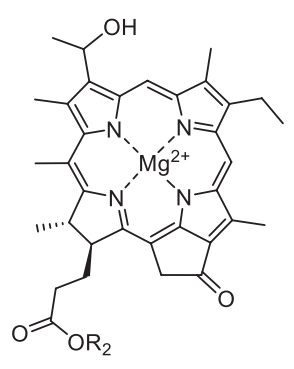

$101 \mathrm{Bcl} C$

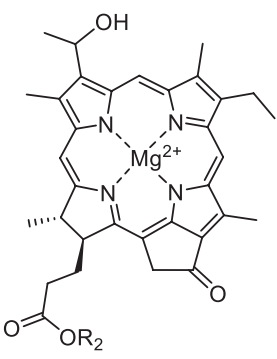

$102 \mathrm{Bcld}$<smiles>CCCC=C(C)CCCC(C)CCCC(C)CCCC(C)C</smiles>

R2:

R3:

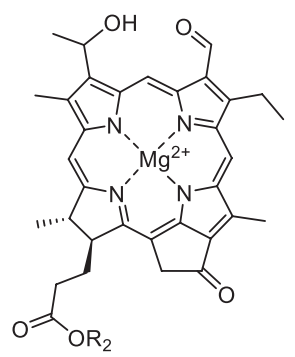

$104 \mathrm{Bcl} f$

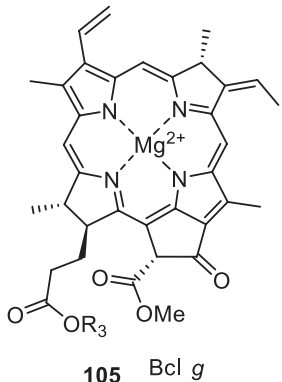

$105 \mathrm{Bcl} \mathrm{g}$

Figure 97. Bacteriochlorophylls $a-g$.

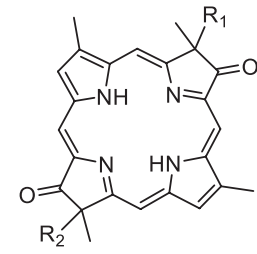

106-114

Tolyporphyrins A-
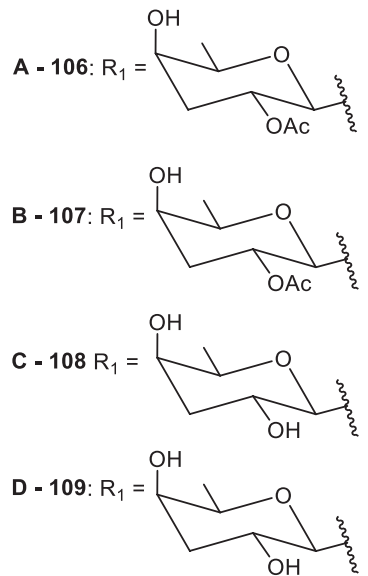
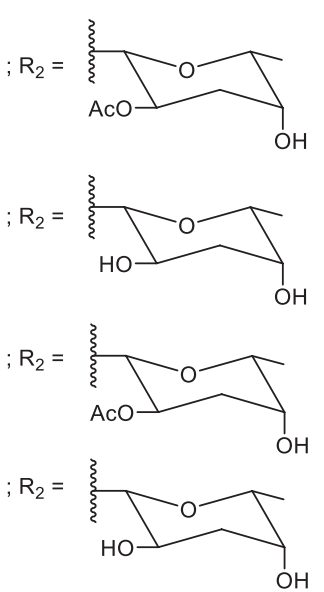

$E-110: R_{1}=O A c ; \quad R_{2}=$

F- 111: $\mathrm{R}_{1}=\mathrm{OH} ; \quad \mathrm{R}_{2}=\sum_{\mathrm{OHCO}}$

G- 112: $R_{1}=O A c ; R_{2}=O H$

$H-113: \mathrm{R}_{1}=\mathrm{OH} ; \mathrm{R}_{2}=\mathrm{OH}$

I- 114: $R_{1}=O A C ; R_{2}=O A C$

Figure 98. Tolyporphins A-I, isolated from cyanobacteria Tolypothrixnodosa.

have a maximum absorption in the region of $680 \mathrm{~nm}$, which is more typical for chlorophyll $a$ derivatives. Today, details of the structure are identified and the ways of obtaining natural tolyporphins are described. ${ }^{[931,932]}$ In addition to their ability to generate reactive oxygen species under light irradiation, it was shown that these compounds have their own antitumor activity. This makes this class of compounds very promising for biomedical applications.

In scientific papers the most widely described modifications are bacteriochlorophyll $a(\operatorname{Bchl} a, 99)$. The structure of this molecule gives opportunities for directed functionalization including the acetyl group of pyrrole $\mathrm{A}$ and propionic residue modification, the metal ion replacement and exocycle E chemical transformations (Figure 99).

It is known that natural bacteriochlorins are highly hydrophobic. ${ }^{[837]}$ To use them successfully in medicine, it is necessary to have a more balanced ratio of hydrophobic and hydrophilic substituents in the macrocycle. This is usually achieved by introducing one, two or three carboxyl groups, amino acid residues or hydroxyl-containing 


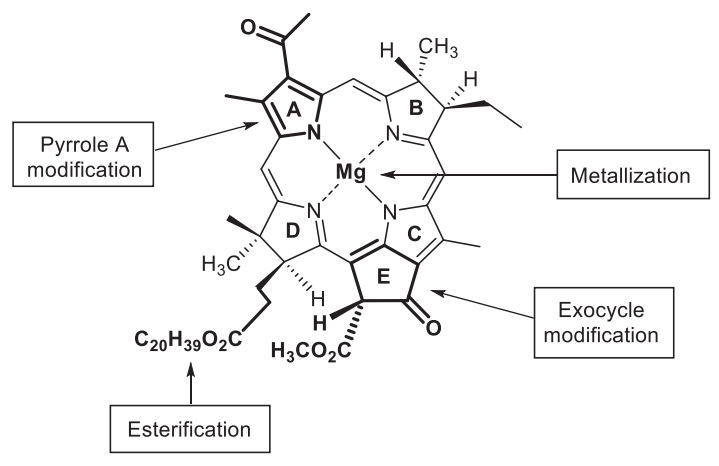

Figure 99. Possible chemical modifications of bacteriochlorophyll $a$.

substituents into the molecule. ${ }^{[933,934]}$ Thus, the introduction of serine from the propionic acid residue by enzymatic transesterification of Bchl $a$ can significantly increase its solubility in water. Such conjugates of bacteriochlorophyllide $a$ (Bchlphd $a$ ) with serine retain the photophysical properties of Bchl $a$ and generate a high ROS yield but are subjected to photo-oxidation, demetallization in a slightly acidic environment and biodegradation that limits the possibility of their use in the clinic.

Replacing the central $\mathrm{Mg}$ atom with $\mathrm{Pd}$, esterification of the propionic acid residue and transesterification of the carbomethoxyl group in the pentanone ring lead to stable derivatives of $[\mathrm{Pd}]-\mathrm{Bhlpd}$ with high photodynamic activity. ${ }^{[935]}$ The ROS quantum yield for these compounds is quite high and ranges from 1 in nonpolar solvents to 0.5 in aqueous solutions.

The introduction of sulfo groups into the bacteriochlorine macrocycle increases the hydrophilicity of the pigment molecule until it is completely dissolved in water. It has been shown that the target for such derivatives of bacteriochlorophyll $a$ is tumor vessels that leads to their necrosis because of damaging. ${ }^{\text {[917] }}$

Water-soluble negatively charged bacteriochlorins were obtained by aminolisys of pentanone exocycle in bacteriopheophorbide and its metal complexes (Figure 100). Their hydrophilic properties and ability to aggregate in aqueous solutions were studied. ${ }^{[919]}$ It was found that the opening of an exocycle in a bacteriopheophorbide significantly increases the hydrophilicity of bacteriochlorins and, as a result, increases their ability to dissolve in polar solvents, including methanol, ethanol, DMF and DMSO. In addition, compounds with an open cycle 116-127 are well soluble in aqueous solutions (PBS) up to $40 \mathrm{mg} / \mathrm{ml}$ which is significantly higher than the solubility of compounds with a pentanone ring but having polar substituents on the periphery of the macrocycle (compound $\mathbf{1 1 9}-4 \mathrm{mg} / \mathrm{ml}$ ). The distribution coefficient of $n$-octanol-water increases strongly (1:19) when the central metal ion $\mathrm{Pd}(\mathrm{II}) \mathbf{1 2 3}$ is replaced by $\mathrm{Mn}$ (III) 127. At the same time, the reduction of the carbon chain in the alkyl substituent of pigment $\mathbf{1 2 0}$ in comparison with 123 practically does not affect the distribution coefficient. All compounds with an open exocycle in aqueous solutions form aggregates (2-8 molecules), which dissociate upon

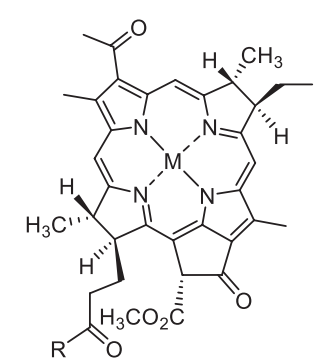

115: $\mathrm{M}=\mathrm{Mg}, \mathrm{R}=\mathrm{O}-\mathrm{CH}_{2}-\mathrm{CH}-\left(\mathrm{CH}_{2} \mathrm{OH}\right)-\mathrm{NH}_{2}$;

116: $\mathrm{M}=2 \mathrm{H}, \mathrm{R}=\mathrm{OH}$;

117: $M=P d, R=O H$ (WST09);

118: $\mathrm{M}=\mathrm{Pd}, \mathrm{R}=\mathrm{O}-\mathrm{NHS}-\mathrm{SO}_{3}^{-} \mathrm{Na}^{+}$

$119 \mathrm{M}=\mathrm{Pd}, \mathrm{R}=\mathrm{NH}-\left(\mathrm{CH}_{2}\right)_{3}-\mathrm{SO}_{3}^{-} \mathrm{Na}^{+}$

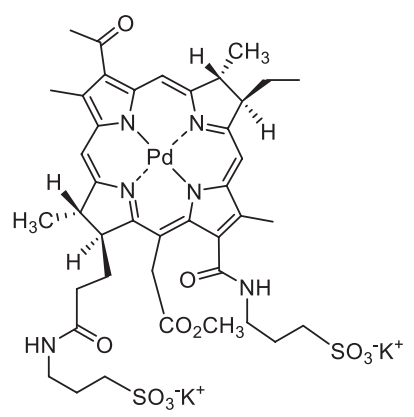

121

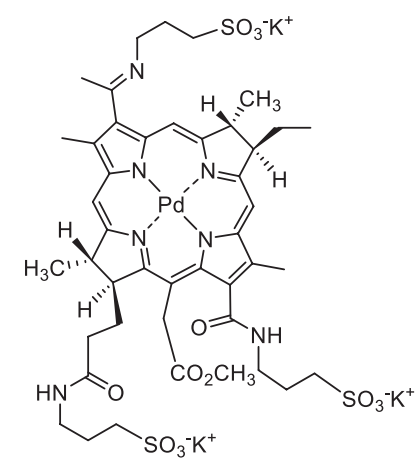

122

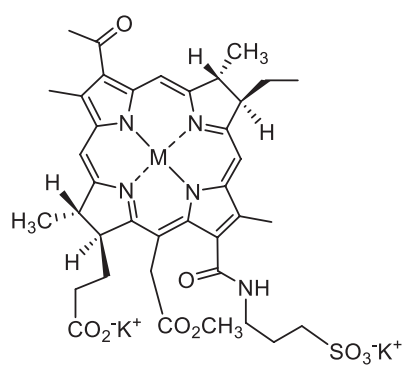

123: $M=P d(W S T 11)$;

124: $M=2 \mathrm{H}$;

125: $\mathrm{M}=\mathrm{Cu}^{\mathrm{Il}}$;

126: $M=Z n$;

127: $\mathrm{M}=\mathrm{Mn}$

Figure 100. Negatively charged PS on the basis of bacteriopheophorbide and its metal complexes. 
dilution, inclusion in micelles, and also in solutions with physiological concentrations of serum albumin.

Among the PS of the bacteriochlorin series developed at the Weissman Institute (Israel) in collaboration with French companies Steba-Biotech and Negma-Lagards, the second-generation drug Tookad, which is a Pd complex of bacteriopheophorbide a 117 , should be noted.

When acting on the Pd complex of bacteriopheophorbide with taurine (2-sulfoethylamine), the pentanone cycle opens and the dianion is formed (code name WST11) 123. ${ }^{[919]}$ The drug has good solubility in the phosphate buffer (up to $50 \mathrm{mg} / \mathrm{ml}$ ) where it is found in small aggregates. In solutions containing blood serum, it undergoes disaggregation and is present as monomers in combination with bovine serum albumin and high-density lipids.

In 2009, the article was published by Israel researchers $^{[846]}$ who showed that the cytotoxic agents in the irradiation of aqueous solutions of WST 11 are superoxide $\mathrm{O}_{2}{ }^{\bullet}$ and hydroxyl $\mathrm{OH}$ radicals while singlet oxygen was not detected in these solutions. In this case, the crucial role in the formation of a particular active form of oxygen is played by the lipophilicity of PS. Hydrophobic pigments such as Tookad are retained in the endothelial cells of the tumor vessels and damage them during irradiation by generating mainly singlet oxygen. At the same time, it was previously shown ${ }^{[937]}$ that the replacement of organic solvents with water leads to a significant increase in the concentration of superoxide and hydroxyl radicals during irradiation of Tookad solutions. The authors found that the mechanism of cytotoxic action when using water-soluble PS WST 11 belongs to type I and involves the transfer of an electron from the PS to the $\mathrm{O}_{2}$ molecule which is facilitated by the high permittivity of water. The WST11 ${ }^{+}$cation takes an electron from human serum albumin (HSA) and forms a non-covalent complex with it in blood plasma. The latter functions as a photocatalytic oxidoreductase participating in the transfer of the HSA-PS-O electron and generating superoxide and hydroxyl radicals that are main ROS in vascular PDT.

Japanese scientists have synthesized a series of bacteriopyropheophorbide $\mathbf{1 2 8}$ c derivatives with various substituents in pyrrole A (Scheme 29). ${ }^{[38]}$

The high yield of the vinylation reaction to produce compound 130 was achieved by using the outgoing mesyl group. Next, the vinyl group was oxidized to formyl 131. Since the reactivity of formyl chlorines was well studied earlier, the authors performed a series of similar transformations with the bacteriopyropheophorbide $a \mathbf{1 3 1}$ derivative resulting in compounds 132-134 (Scheme 29).

On the basis of Bchl $a$, various research groups performed studies aimed at increasing the stability of the pigment by including exocycles condensed with the main macrocycle in the molecule. The synthesis of bacteriopurpurin (BP) 138 containing an anhydride exocycle was first performed in the laboratory of Prof. A.F. Mirinov. ${ }^{[939]}$ For this purpose, bacteriochlorophyll was extracted from the biomass of purple $R h$. capsulata bacteria and then oxidized in an alkaline medium with air oxygen without isolation and additional purification. Subsequent treatment with hydrochloric acid resulted in the closure of the anhydride ring and the production of bacteriopurpurin $\mathbf{1 3 8}$.

Allomerization of bacteriochlorophyll $a$ was first observed by H. Fischer as early as 1938. ${ }^{[940]}$ Professor A. Scherz and his colleagues reported the formation of $13^{2}$-hydroxy allomers in a methanol solution of Bchl $a .^{[941]}$ The process of Bchl $a$ oxidation in BP $\mathbf{1 3 8}$ (Bchl $a$ allomerization) was studied in detail in the laboratory of Professor R. Pandey. ${ }^{[942]}$

When treating bacteriopheophytin $a \mathbf{1 3 5}$ with a solution of $5 \% \mathrm{H}_{2} \mathrm{SO}_{4}$ in methanol, along with the target methyl ester of bacteriopheophorbide $a \mathbf{1 3 6}$, a mixture of oxidation products was obtained (Scheme 30). Analysis of the latter showed that it consists of chlorin 142 and a mixture of dia-

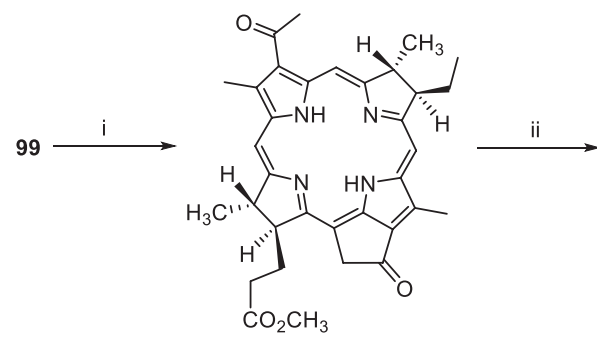

128

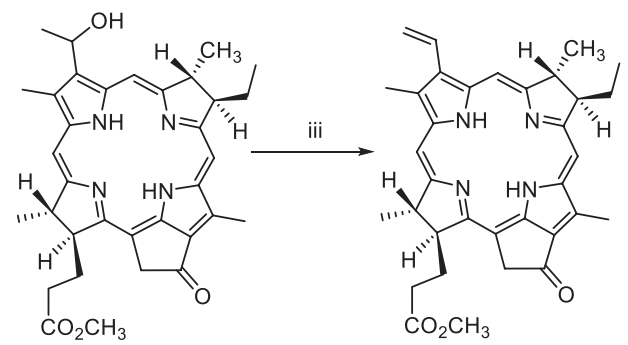

129

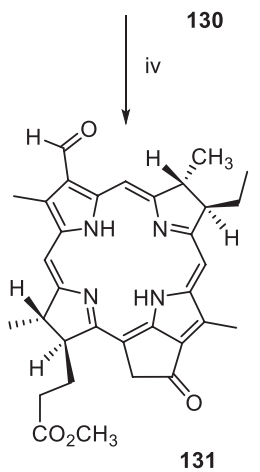

Scheme 29. Reagents and conditions: i: 1) $2 \% \mathrm{HCl}$, 2) $\mathrm{H}_{2} \mathrm{SO}_{4} / \mathrm{MeOH}$; ii: $\mathrm{NaBH}_{4}, \mathrm{MeOH} / \mathrm{CH}_{2} \mathrm{Cl}_{2}$; iii: $\mathrm{MsCl} / \mathrm{Et}_{3} \mathrm{~N}, \mathrm{CH}_{2} \mathrm{Cl}_{2}$; iv: 1) $\mathrm{OsO}_{4} / \mathrm{NaIO}_{4}, 2$ ) AcOH/THF; v: $\mathrm{NH}_{2} \mathrm{SO}_{3} \mathrm{H} / \mathrm{NaClO}_{2}$, THF, 2-methyl-2-butene; vi: ${ }^{t} \mathrm{BuNH}_{2} \cdot \mathrm{BH}_{3}, \mathrm{CH}_{2} \mathrm{Cl}_{2}$; vii: AcOH, EDC, DMAP; vii: malononitrile, $\mathrm{Et}_{3} \mathrm{~N}, \mathrm{THF}$. 

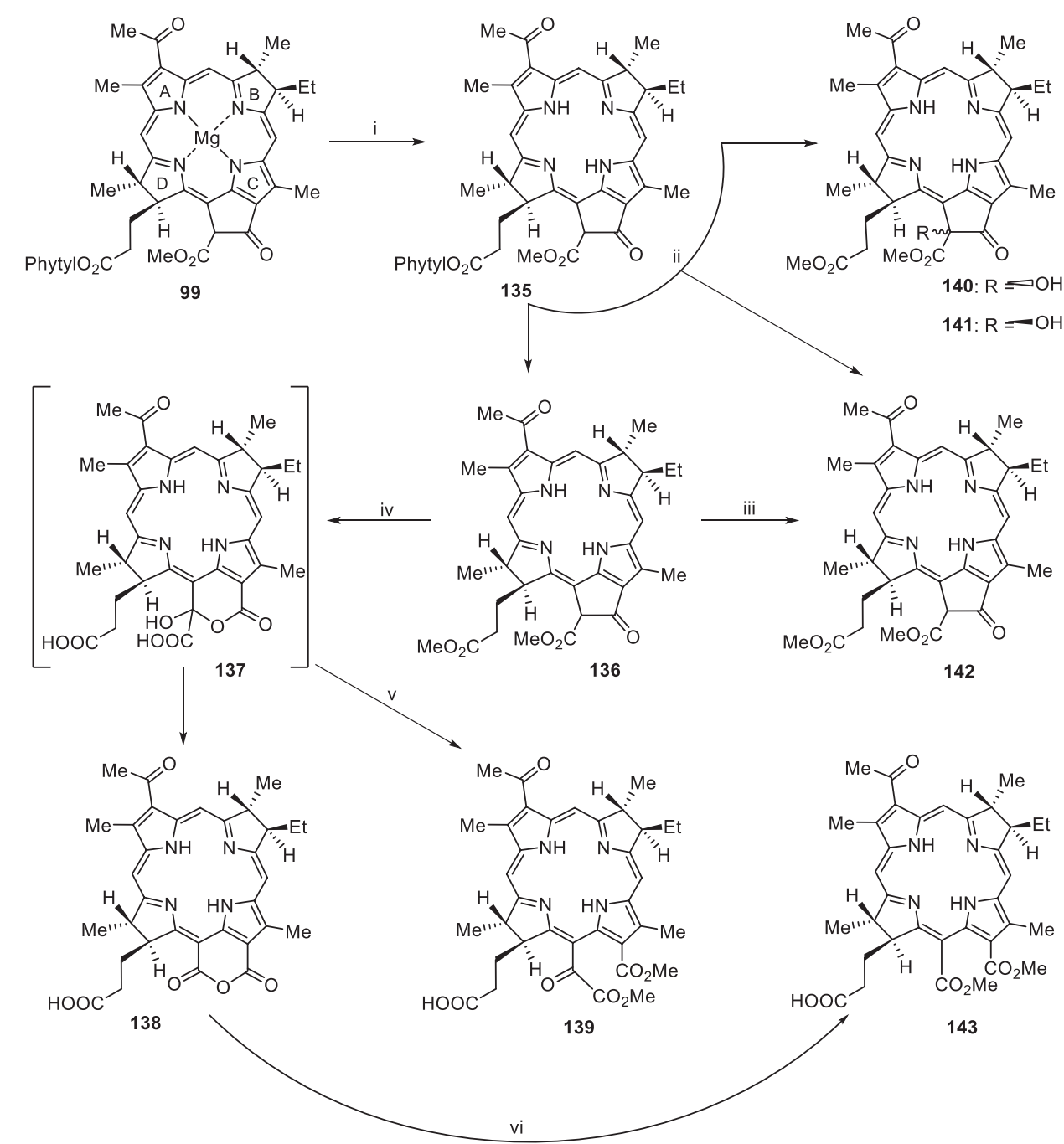

Scheme 30. Reagents and conditions: i: $0.1 \% \mathrm{HCl}$; ii: $5 \% \mathrm{H}_{2} \mathrm{SO}_{4} / \mathrm{MeOH}$; iii: $\mathrm{O}_{2}$; iv: $\mathrm{KOH} / \mathrm{C}_{3} \mathrm{H}_{7} \mathrm{OH}, \mathrm{O}_{2}$; v: $\mathrm{CH}_{2} \mathrm{~N}_{2}$; vi: $\mathrm{KOH} / \mathrm{CH}_{3} \mathrm{OH}, \mathrm{CH}_{2} \mathrm{~N}_{2}$.

stereomers $13^{2}-(R / S)$ - hydroxy derivatives 140 and 141 (epimers). The presence of a hydroxyl group in the pentanone ring was reliably proved by ${ }^{1} \mathrm{H}$ NMR spectra, with the ratio between the $\mathrm{R}$ and $\mathrm{S}$ isomers being 1:4. These detailed studies have shown that the $S$-epimer, in which the carbomethoxyl group at the $13^{2}$-position and the propionic acid residue at the 17-position of the macrocycle are directed in different directions relative to the macrocycle plane, is thermodynamically more stable under acidic conditions. ${ }^{\left[{ }^{[43}, 944\right]}$

However, working with Bchl $a$ derivatives in highly acidic conditions is difficult due to the rapid oxidation of the pigment. The authors also observed allomerization under alkaline conditions. Thus, the methyl ester of bacteriopheophorbide $a \mathbf{1 3 6}$ in propan-1-ol solution in the presence of $\mathrm{KOH}$ during air bubbling allomerizes to form "unstable bacteriochlorin" 137 which turns into BP 138 during solvent evaporation. The latter is stable only in neutral and acidic environments, and in the presence of bases there is a rapid opening of the anhydride cycle with the formation of bacteriochlorin $p_{6}$, the treatment of which with diazomethane leads to trimethyl ester $\mathbf{1 3 9}$.

Y. Saga et al. found that the treatment of bacteriopheophytin $a$ with air oxygen in the presence of catalytic amounts of titanium oxide particles also leads to allomerization.
The authors were able to isolate the phytyl ether of BP while the formation of products of oxidation of the bacteriochlorin ring, namely 3-acetylpheophytin $a$ and 3-acetylprotopheophytin $a$ was not observed. ${ }^{[945]}$

A.N. Kozyrev and co-authors synthesized a number of new bacteriochlorins using $13^{2}$-oxobacteriopyropheophorbide $\mathbf{1 4 5}$ as the starting compound which was obtained by treating the methyl ester of bacteriopheophorbide $\mathbf{1 3 6}$ with lithium hydroxide followed by oxidation with air oxygen. ${ }^{[946]}$ When it interacts with $o$-phenylenediamine and 1,10-diaminonaphthalene, bacteriochlorins 146-148 were formed with condensed rings of quinoxaline, benzimidazole and pyrimidine, respectively (Scheme 31). The absorption spectra of the obtained bacteriochlorins showed a noticeable shift of the $\mathrm{Q}_{\mathrm{y}}$ band to the red region of $816-850 \mathrm{~nm}$. At the same time, the absorption bands had high molar extinction coefficients. Treatment of the methyl ester of $13^{2}$-oxobacteriopyropheophorbide with diazomethane resulted in the formation of bacterioverdins 149-151 containing condensed six-membered methoxy substituted cyclohexenone. The reaction resulted in the formation of a mixture of verdin isomers that were separated from each other. The authors proposed a mechanism for the formation of such compounds that consisted in a nucleophilic attack 


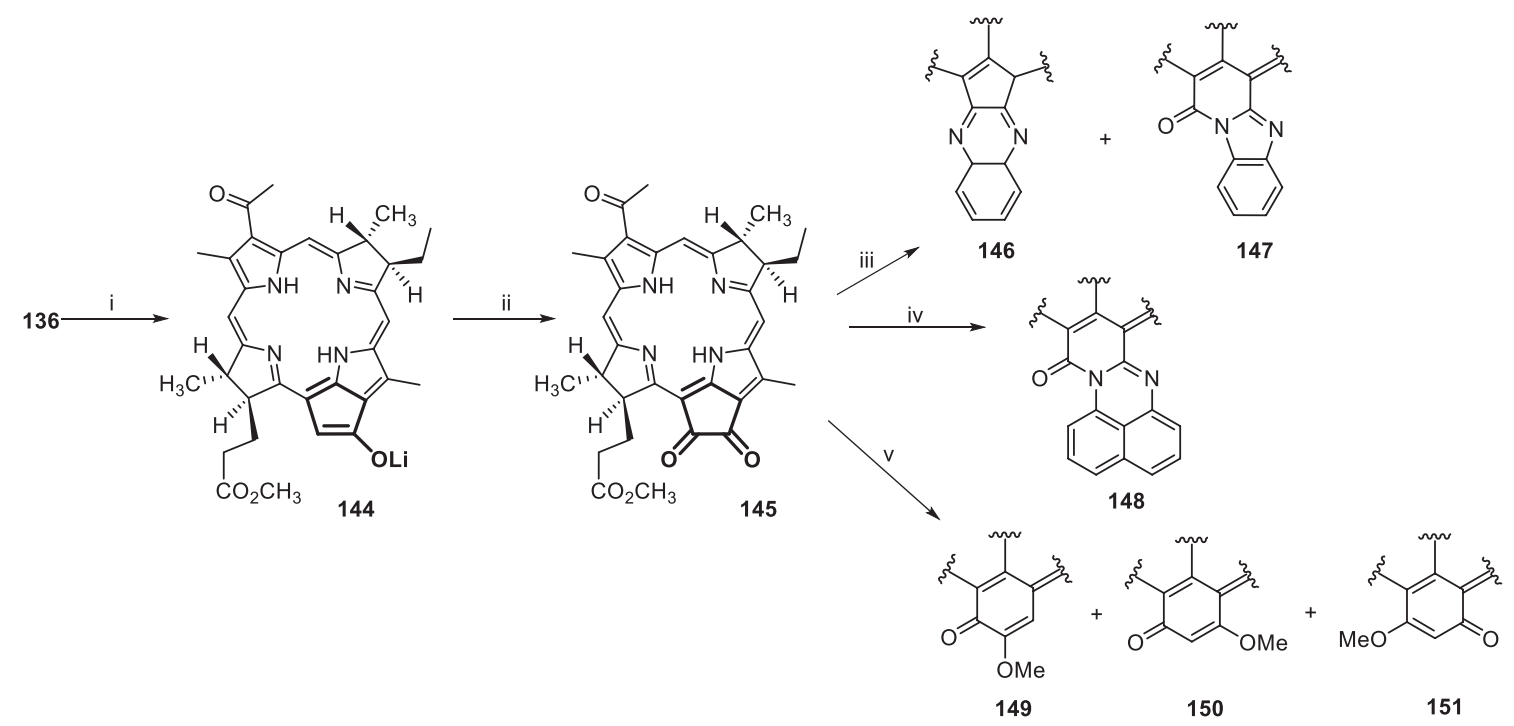

Scheme 31. Reagents and conditions: i: $\mathrm{LiOH}$; ii: $\mathrm{O}_{2}$; iii: 1,2-diphenylamine; iv: 1,9-diaminonaphthalene; v: $\mathrm{CH}_{2} \mathrm{~N}_{2}$.

of carbonyl groups of the E-ring by diazomethane followed by an increase in the size of the exocycle. Compounds 149-151 had long-wave absorption in the near-IR region $(865-890 \mathrm{~nm})$ and were highly stable at room temperature. During photophysical tests it was shown that the obtained pigments have a high reactivity with oxygen in the triplet photoexcited state that makes them potential candidates for use as photosensitizers in photodynamic therapy (PDT). However, the authors note that due to energy limitations chromophores with an absorption greater than $900 \mathrm{~nm}$ are likely to have low photodynamic activity. In addition, the unique optical properties of the resulting annulated bacteriochlorins make them promising for other applications including information storage devices and optical materials. Moreover, bacteriochlorin 145 having two keto groups is promising as a leader compound for the synthesis of a number of other chromophores.

Another direction in the synthesis of bacteriochlorophyll $a$ derivatives is the preparation of bacteriopurpurinimides (Scheme 32). A.N. Kozyrev and co-authors obtained a number of bacteriopurpurinimides, while studying in detail the mechanism of intramolecular cyclization. ${ }^{[947]}$

A mixture of amides 153a and 153b obtained by opening the anhydride cycle with bacteriopurpurin $a$ hexylamine

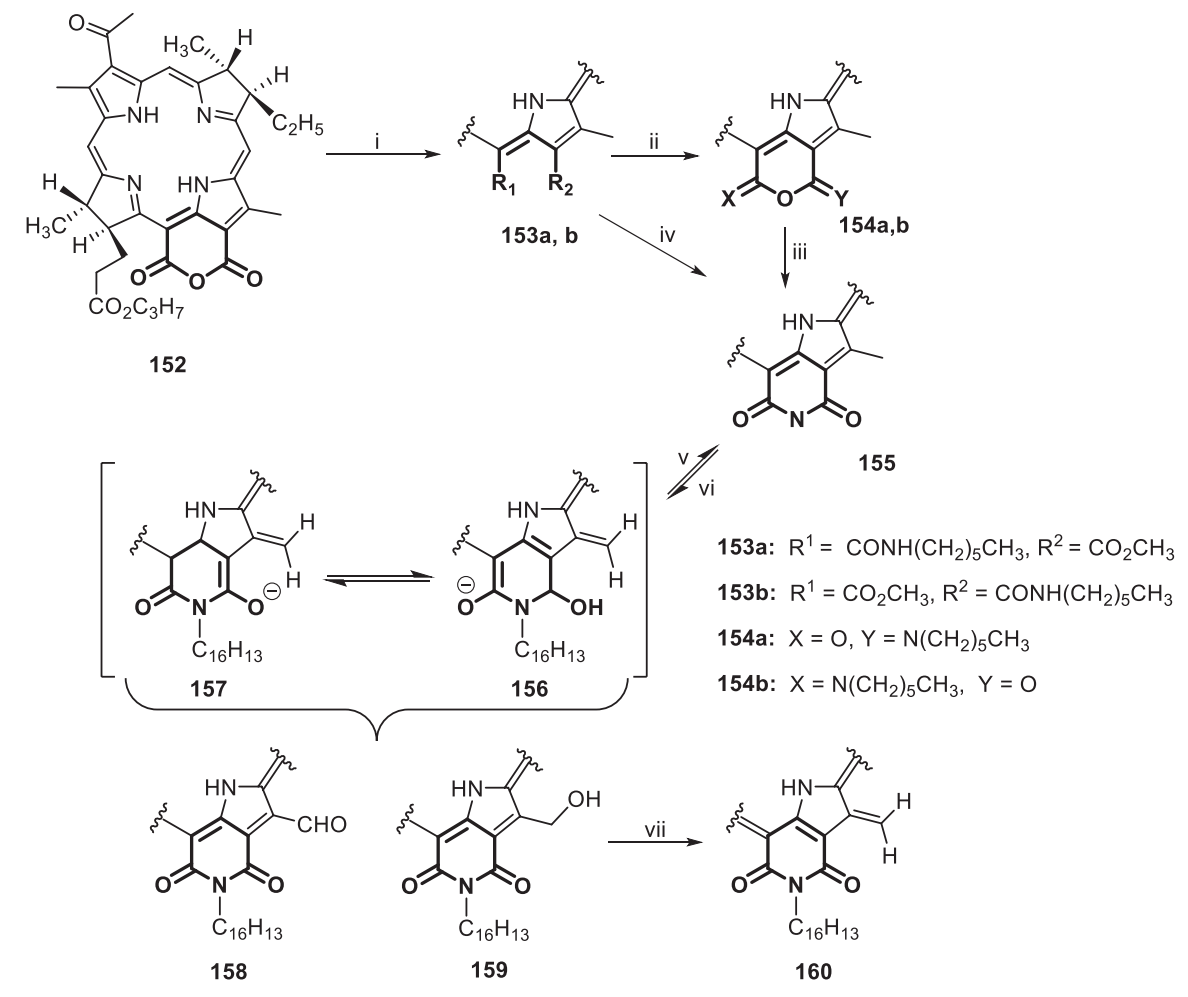

Scheme 32. Reagents and conditions: i: $\mathrm{C}_{6} \mathrm{H}_{13} \mathrm{NH}_{2}$; ii: DCC; iii: $\mathrm{DBU}$; iv: $1 . \mathrm{CH}_{2} \mathrm{~N}_{2}, 2 . \mathrm{KOH} / \mathrm{CH}_{3} \mathrm{OH}$; v: $\mathrm{KOH}$; vi: $\mathrm{HCl}$; vii: $\mathrm{H}^{+}$. 
when treated with DCC gives unstable carbodiimide derivatives that quickly turn into more stable cyclic isoimides 154a and 154b. The latter were separated chromatographically into individual isomers $\left(\lambda_{\max }=804\right.$ and $\left.796 \mathrm{~nm}\right)$ in a ratio of $6: 1$. Base-catalyzed intramolecular cyclization of isoimides leads to a $45 \%$ yield of cycloimide $155\left(\lambda_{\max }=822 \mathrm{~nm}\right)$. Similar results were obtained with amides which carboxyl groups were methylated using diazomethane before base treatment.

In the course of studying the process of intramolecular cyclization, it was found that in an alkaline medium, along with the formation of the target cycloimide 155, the 12- $\mathrm{CH}_{3}$ group is oxidized, leading to minor products: 12-formyl (4-6\%) 158 and 12-hydroxymethyl (2-4\%) 159 derivatives (Scheme 33). This oxidation, according to the authors, is a consequence of enolization, leading to tautomers $\mathbf{1 5 6}$ and 157, which are prone to oxidation in air. In turn, ketoenol tautomerism in cycloimide is possible due to the strong electron-acceptor effect of the imide exocycle conjugated to the main tetrapyrrole system. ${ }^{[948]}$

The unusual bacteriochlorin $\mathbf{1 6 0}$ which has a $Q_{y}$ band at $849 \mathrm{~nm}$ was obtained from 12-hydroxymethylcycloimide 159 under acidic conditions. 2D ROESY spectra showed the interaction of $10-\mathrm{H}$ meso-proton with the neighboring 12- $\mathrm{CH}_{2}$ group and the absence of $8-\mathrm{H}$ proton. As a possible mechanism, the elimination of the hydroxyl group under acidic conditions is proposed with the formation of a carbocation of benzyl type which is stabilized by proton separation at $\mathrm{C}-8$ of the macrocycle. ${ }^{[942]}$

Unlike natural bacteriochlorophyll $a$, isoimide and imide analogues are more stable compounds. On the basis of bacteriopurpurinimide, a series of derivatives were obtained for various applications, simultaneous modifying both the macrocycle and the acetyl group in pyrrole A being carried out. ${ }^{[882,885,949-952]}$

Fundamentally new possibilities were discovered when replacing alkylamines with bifunctional agents - hydrazine hydrate and hydroxylamine. First, the use of strong nucleophilic reagents greatly simplifies the obtaining of cycloimides. Secondly, the presence of amino- or hydroxyl groups in the synthesized cycloimides at the nitrogen atom made it possible to obtain modification series of bacteriopurpurinimides based on only one compound. ${ }^{[953,954]}$

The study of the interaction of BP with hydrazine hydrate (Scheme 33) showed that at the first stage the anhydride cycle is opened very quickly and monohydrazides 163a and 163b are formed, the acetyl group being converted into hydrazone. The assumption about the possible formation of hydrazone during the reaction and its subsequent hydrolysis in an acidic medium was confirmed by the reaction of bacteriochlorin $p_{4}$ with hydrazine. The product 162 was isolated without addition of acid. The mass spectrum of the latter confirmed the formation of hydrazone which in the presence of hydrochloric acid is easily hydrolyzed to the initial bacteriochlorin $p \mathbf{1 6 1}$.

With further reaction the absorption band at $750 \mathrm{~nm}$ shifts to $836 \mathrm{~nm}$ which indicates spontaneous cyclization of monohydrazides. This process is significantly accelerated when the reaction mass is acidified with hydrochloric acid.

$N$-Aminobacteriopurpurinimide $\mathbf{1 6 4}$ was a key compound in the synthesis of numerous functionalized derivatives of bacteriochlorophyll $a$ (Figure 101). Thus, a cationic derivative $\mathbf{1 6 5}$ was obtained which is a much more hydrophilic compound compared to the original bacteripurpurinimide $\mathbf{1 6 4}$ and therefore it is characterized by better solubility in water-alcohol solutions. This makes it convenient for biological tests and promising for medical use. Pigment $\mathbf{1 6 5}$ has been shown to have photoinduced antimicrobial and antifungicidal activity. ${ }^{[955]}$

Based on bacteriopurpurinimide 164, a new sulfurcontaining derivative of bacteriochlorophyll $a$ was obtained where the exocyclic amino group is acylated by a residue of lipoic acid; the latter is a biogenic compound that performs

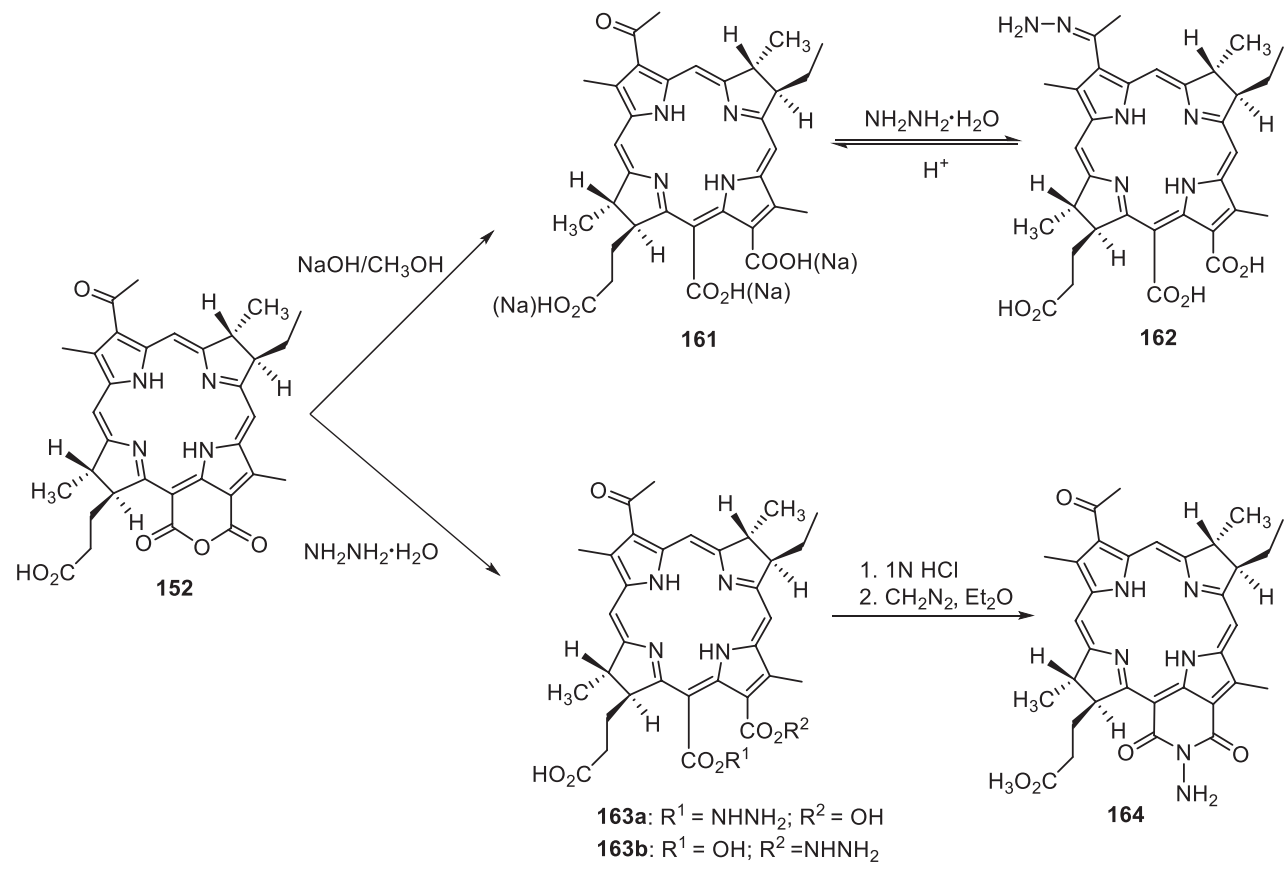

Scheme 33. The interaction of bacteriopurpurin with hydrazine hydrate. 
the function of cofactor a pyruvate dehydrogenase and alphaketoglutarate dehydrogenase complexes in the body. Due to the presence of disulfide groups in the lipoic acid molecule, the pigment acquired aurophilic properties and was immobilized on the surface of gold nanoparticles (NPS-Au) by forming S-Au bonds. The resulting particles can be used for targeted antitumor PDT. ${ }^{[956]}$

Based on pigment 164, naphthalimide and dicarboindocyanine dye, bichromophore systems 166a,b, 168 were obtained. Combining two photoactive subunits with individual spectral characteristics into a single bichromophore system can lead to a significant change in the photonics of the conjugate as a whole compared to the original chromophores due to the occurrence of energy transfer from the donor to the acceptor in the system. A significant difference in light absorption by the dye and the photosensitizer allows for separate fluorescent diagnostics and photodynamic effects making such conjugates promising theranostics.

The same bacteriopurpurinimid 164 was used as a precursor in the synthesis of boron-containing conjugates in the bacteriochlorophyll $a$ series. The addition of boron polyhedra to derivatives of natural chlorophylls provides targeted delivery of boron atoms to the tumor, which is used in the creation of drugs for borneutron capture therapy (BNCT) of cancer. ${ }^{[957,958]}$
New opportunities for the production of cycloimides in the bacteriochlorophyll $a$ series and their subsequent chemical transformations were discovered using hydroxylamine. The presence of two reaction centers (an anhydride cycle and an acetyl group) in the bacteriopurpurin molecule led to a stepwise reaction with hydroxylamine where oxime $\mathbf{1 7 0}$ is initially formed, and only when an excess of the reagent is used, the second hydroxylamine molecule reacts with the anhydride cycle, giving oxime $N$-hydroxybacteriopurpurinimide 176 (Scheme 34). The presence of a mobile hydrogen atom in the latter makes it possible to expand significantly the range of cycloimides due to acylation and alkylation reactions. The most interesting was the $O$-methyl derivative $\mathbf{1 8 2}$ formed with a high yield when diazomethane acts on cycloimide $\mathbf{1 7 6}$.

The study revealed two interesting facts. The acidity of the hydrogen atom in the oxime function is so low that the latter does not participate in the reaction with diazomethane. In addition, a thorough analysis of the $N$-methoxy derivative 182 showed that the substance is a mixture of two isomers. The isolated isomers were characterized by ${ }^{1} \mathrm{H}$ NMR spectra, including 1D NOE spectroscopy and basing on the data obtained, it was concluded that the compound exists in two stereoisomeric forms (syn- and anti-). In the course of these studies a homologous series of bac-
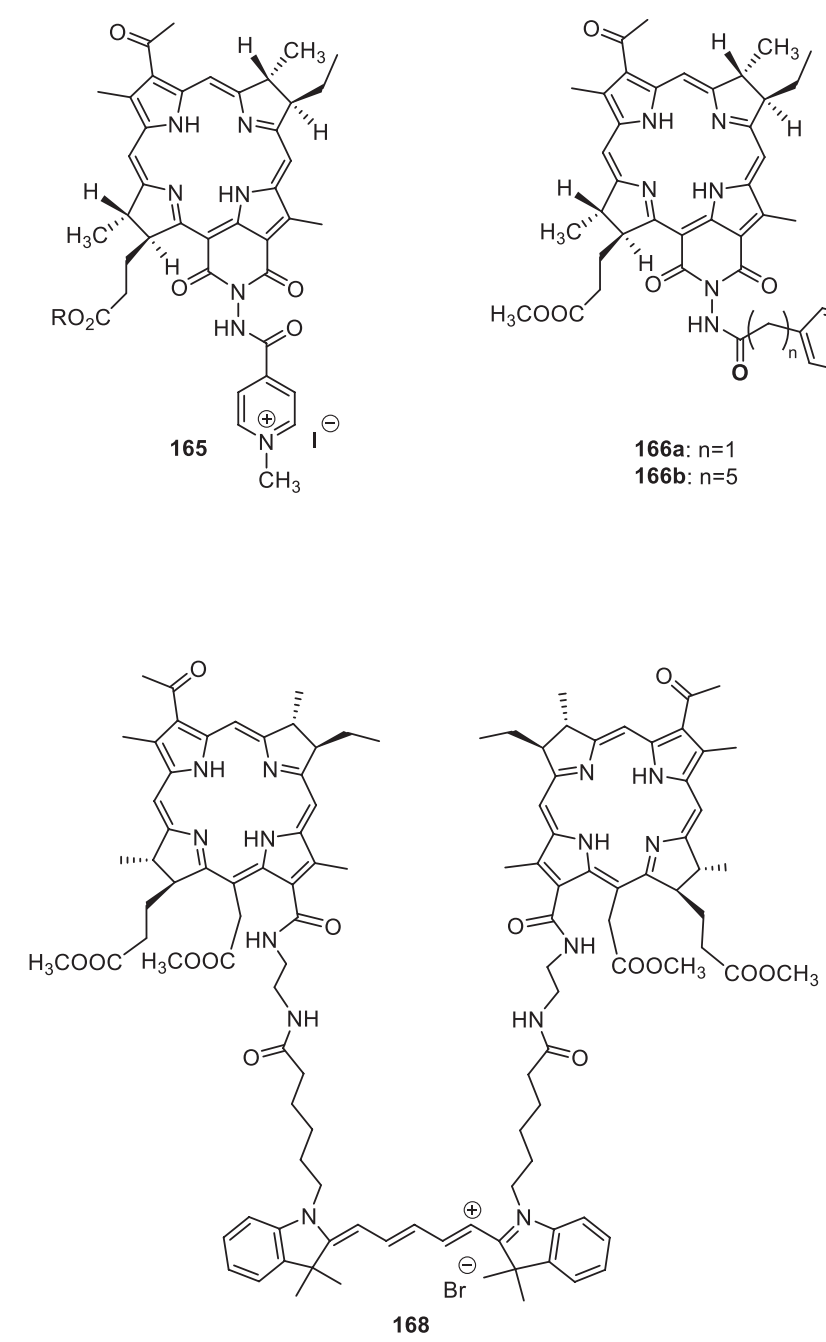
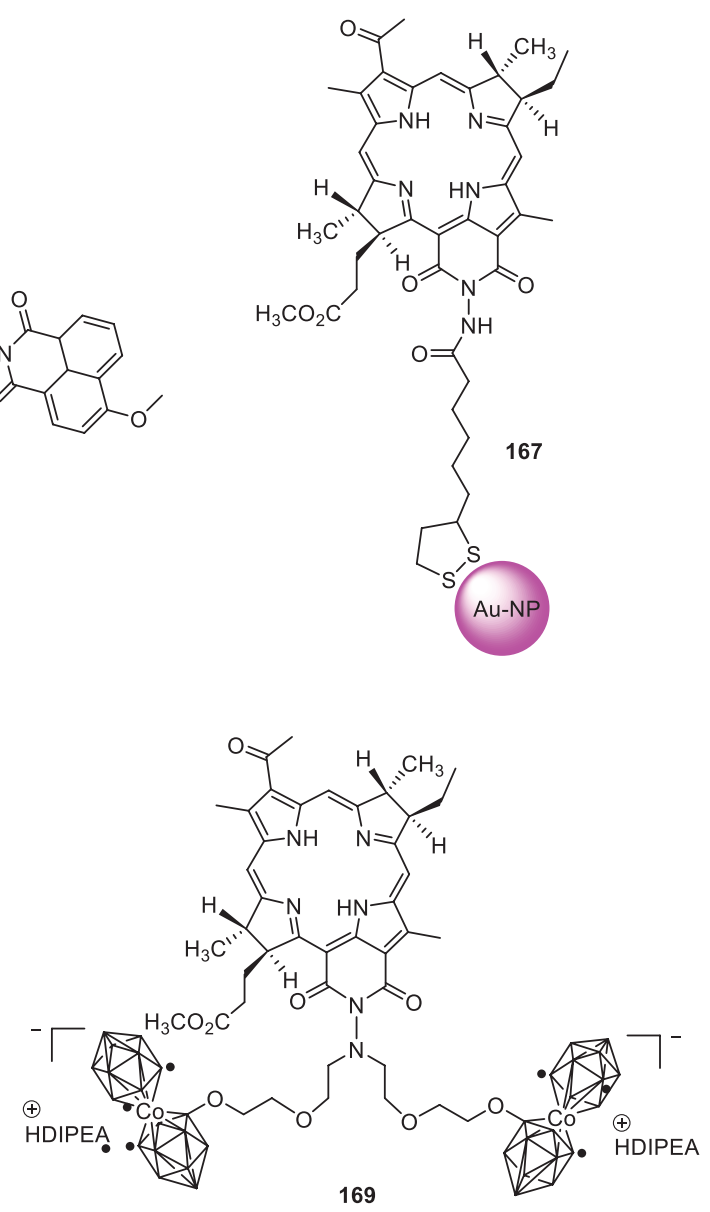

Figure 101. Functionalized derivatives of $\mathrm{N}$-aminobacteriopurpurinimide. 


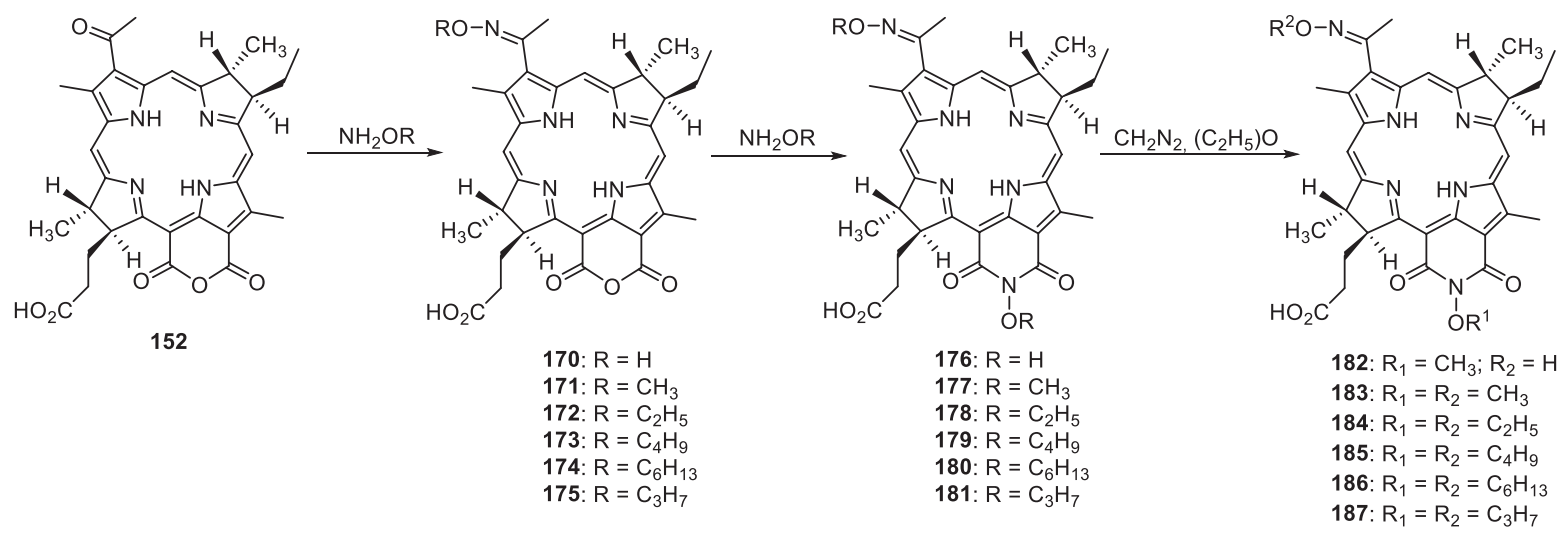

Scheme 34. The interaction of bacteriopurpurin $a$ with hydroxylamine and alkoxyamines.

teriopurpurinimides with alkyl substituents of different lengths was obtained in order to conduct screening biological tests and search for the most effective PS for PDT. ${ }^{[959]}$

In the group of Professor Grin M.A. a PS based on bacteriopurpurinimide was obtained containing a block copolymer of polyoxyethylene and polyoxypropylene (Pluronic F127) which is attached to the periphery of the bacteriochlorin macrocycle. The key pigment is the methyl ester $O$-propyloxime- $N$-propoxybacteriopurpurinimide $\quad 187$ with an intense absorption band at $800 \mathrm{~nm}$. Its dispersion in Kolliphor EL showed high photoinduced cytotoxicity on cells of various tumor lines and a significant photodynamic effect on tumors of various genesis. ${ }^{[960]}$ The disadvantages of the drug include extremely low solubility in water which leads to a decrease in its bioavailability and, consequently, the effectiveness of therapy using this compound.

Introduction to the structure of the above-mentioned PS Pluronic F127 in which the different affinity to water of the polyoxypropylene (hydrophobic) and polyoxyethylene (more hydrophilic) parts of the molecule gives it the properties of a surfactant in an aqueous solution will contribute to the self-organization of conjugate $\mathbf{1 8 8}$ molecules into nanoscale structures (Figure 102). ${ }^{[961]}$

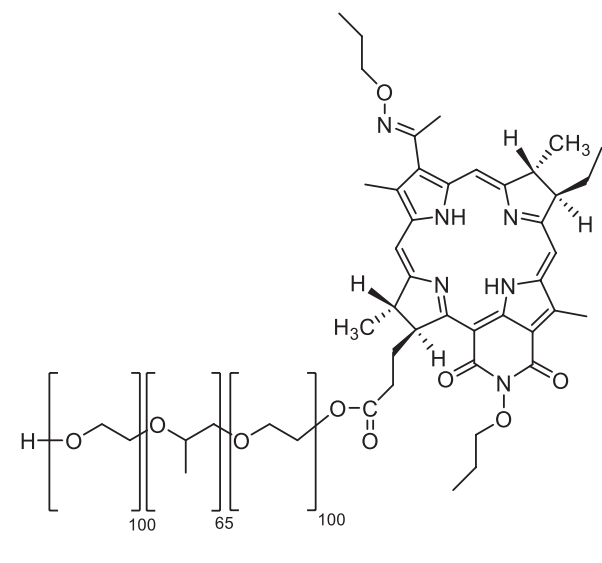

188

Figure 102. Structure of the conjugate of the methyl ester $O$-propyloxime- $N$-propoxybacteriopurpurinimide with Pluronic F 127.
Another direction in the chemistry of bacteriochlorins is the production of synthetic tetrahydroporphyrins that can be synthesized in several ways: total synthesis, modification of natural tetrapyrrole macrocycles or chemical transformations of synthetic porphyrins. The latter is the most attractive due to its simplicity and availability of raw materials. Syntheses in which meso-aryl-substituted porphyrins are reduced to bacteriochlorins by $p$-toluenesulfonylhydrazine are described. ${ }^{[34,962,963]}$ Substituents in aryl fragments allow regulating the stability, polarity and photophysical properties of pigments. One of these reduced porphyrins is compound 189 known as Redaporfin which is a substance of a clinically approved drug (Figure 103). ${ }^{[964,965]}$ One of the disadvantages of this method of synthesis is the spontaneous oxidation of the resulting bacteriochlorin back to the original products. Another disadvantage is the presence of the initial porphyrin admixture and an intermediate reduction product-chlorin, as its separation is extremely difficult.

Another approach to the bacteriochlorins synthesis is the pyrrole ring double bond oxidation in the macrocycle. The synthesis of 2,3-vic-12,13-vic-tetrahydroxybacteriochlorins meso-tetraarylporphyrins under the action of $\mathrm{OsO}_{4}$ 190 is described. ${ }^{[966,967]}$ On the basis of such compounds analogues of bacteriochlorin containing oxazoline ${ }^{[968]}$ and morpholine ${ }^{[969,970]}$ cycles were obtained. Recently, GubermanPfeffer and co-authors have reported on the synthesis of morpholinobacteriochlorins based on 5,10,15,20-tetraaryl-substituted porphyrin. ${ }^{[971]}$ The authors also synthesized their annulated derivative 194. The absorption spectra of the obtained compounds corresponded to those of typical bacteriochlorins.

Various cycloaddition reactions to porphyrins and chlorins can be used for the synthesis of bacteriochlorins. So, Nerges et al. described the synthesis of bacteriochlorins 191a-c from meso-aryl-substituted porphyrins using the Diels-Alder reaction. ${ }^{[972]}$ As a diene component isobenzofuran was taken, the method for obtaining was proposed by the same team of the authors. ${ }^{[973]}$ An interesting feature of this synthesis is that the reaction of the free base of porphyrin produces bacteriochlorin and isobacteriochlorin is obtained in the case of reaction of the Ni-complex of porphyrin. The authors note that the reactions proceed with high yields (80-99\%), regioselectively and under mild conditions, that makes this functionalization method 


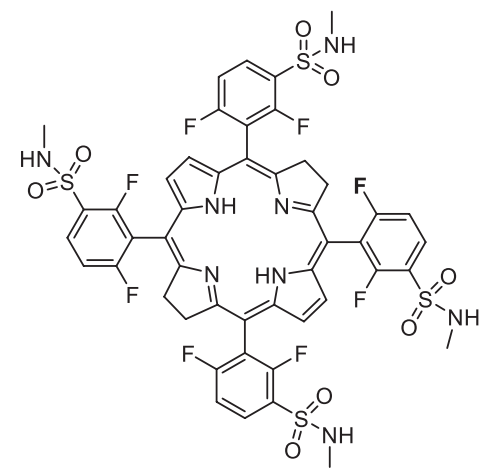

189

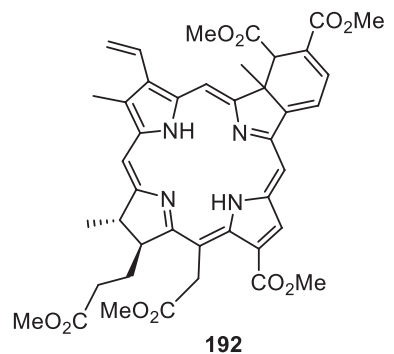

192

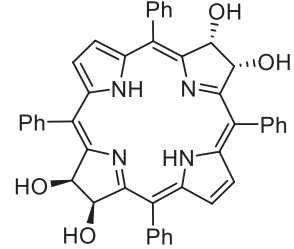

190

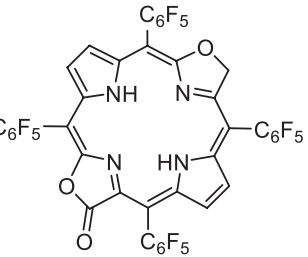

193

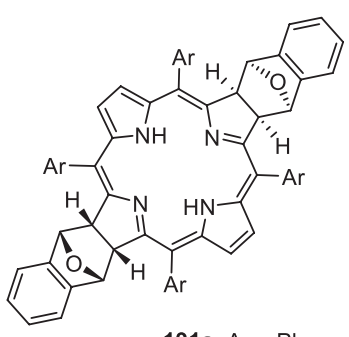

191a: $\mathrm{Ar}=\mathrm{Ph}$
191b: $\mathrm{Ar}=\mathrm{C}_{6} \mathrm{~F}_{5}$

191c: $\mathrm{Ar}=\mathrm{HO}$<smiles>CCCC(CCOC(CO)CO)Oc1c(F)c(F)c(C(F)(F)F)c(F)c1F</smiles>

Figure 103. Bacteriochlorins obtained by modification of porphyrins and chlorins.

promising for obtaining other bacteriochlorins in preparative quantitives. ${ }^{\left[{ }^{[74]}\right.}$ Bacteriochlorins can also be obtained in the course of cycloaddition reactions based on natural tetrapyrrole macrocycles. $\mathrm{Li}$ and co-authors described the preparation of benzobacteriochlorins from chlorophyll $a$ derivatives (compound 192). ${ }^{[975]}$ The most detailed cycloaddition reactions for porphyrins and chlorins are considered in the review by S.V. Dudkin and co-authors. ${ }^{[32]}$

In recent years, the Lindsey research group has developed a new method for the complete synthesis of bacterio- chlorins based on self-condensation of dihydrodipyrrinacetal containing two geminal methyl groups in the reduced pyrroline ring as well as the acetal link in the pyrroline position (Scheme 35). ${ }^{[976-978]}$ Methyl groups protect the pyrroline ring from oxidation which can lead to the formation of porphyrins and chlorins.

The synthesis of such dihydrodipyrrin-acetals can be carried out by modification of pyrrolcarbaldehyde when various functional groups can be pre-introduced allowing to vary the position of the maximum absorption of the target

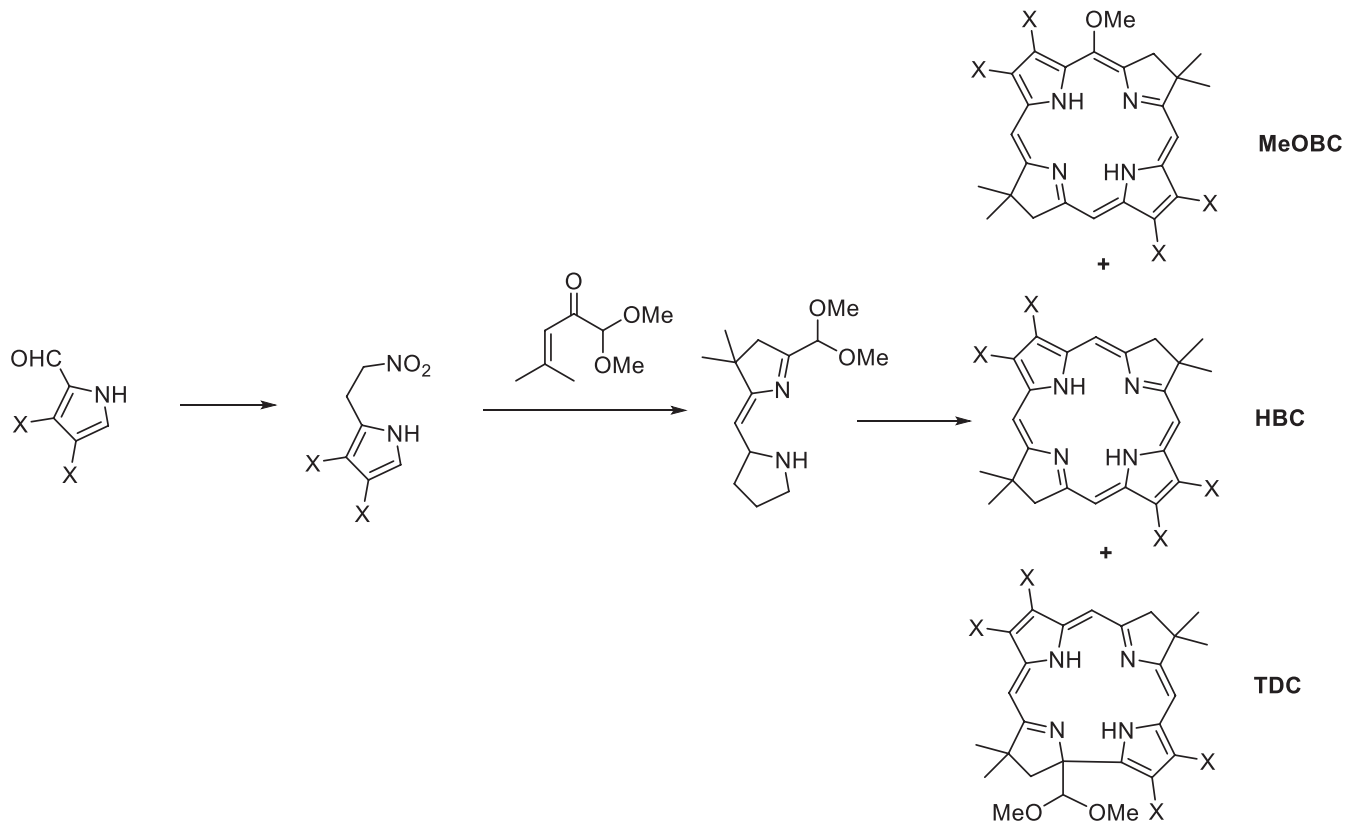

Scheme 35. General scheme for obtaining dihydrodipyrrin-acetals and their condensation. 
bacteriochlorin and its polarity. As a result of acid-catalyzed condensation of dihydrodipyrrin-acetal, three types of macrocycles can be obtained: 5-unsubstituted bacteriochlorin (HBC), 5-methoxybacteriochlorin (MeOBC) or tetradehydrocorrin (TDC). Depending on the selected catalyst as well as on the ratio of reagents, the reaction direction may be shifted towards the formation of a particular macrocycle. For example, when using $140 \mathrm{~mm} \mathrm{BF}_{3} \cdot \mathrm{OEt}_{2}$ and $18 \mathrm{~mm}$ dihydrodipyrrin-acetal, the predominant formation of HBC-type bacteriochlorin is observed. The use of TMSO-OTf and 2,6-di-tert-butylpyridine leads to the formation of a MeOBC-type macrocycle, whereas when using $\mathrm{Yb}(\mathrm{OTf})_{3}$ the TDC type pigment is obtained with a high yield $(\sim 70 \%){ }^{[962]}$

One of the factors limiting the diversity of such pigments is the instability of dihydrodipyrrin-acetals. Thus, Zhang and co-authors synthesized 2,3,12,13-tetraalkylbacteriochlorins ${ }^{[979]}$ from dialkyldihydrodipyrrin-acetals that

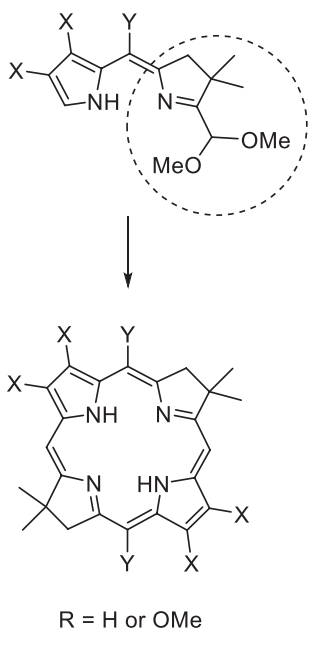

Scheme 36. "North-South" route synthesis of bacteriochlorins. were unstable under acid catalysis. As a result, to solve this problem it was necessary to obtain dialkyl derivatives of dihydrodipyrrincarbaldehyde which were subsequently introduced into the condensation reaction.

Since it is difficult to modify the meso-positions of bacteriochlorins obtained from dihydrodipyrrin-acetal, the authors proposed a different method for obtaining bacteriochlorins, called the "North-South route" (Scheme 36). ${ }^{[90]}$ This method involves the preparation of 2,2-dimethyl-substituted 1-(1,1-dimethoxymethyl) - dihydrodipyrrins. Their meso-position can be replaced by alkyl or aryl substituents. Depending on the selected conditions of acid catalysis, both di-meso substituted bacteriochlorins and tri-substituted derivatives can be obtained.

Based on the methods described above, a number of compounds with a wide range of photophysical properties were obtained. ${ }^{[981-984]}$ Currently "tunning" position of absorption maximum $\mathrm{Q}_{\mathrm{y}}$ of bacteriochlorin can be implemented in the following ways: the introduction of the pyrroles and auxochrome groups; the inclusion of the fifth exocyclic ring spanning the $13-15$ or 3-5 positions of the macrocycle, introduction of the substitutes in the meso-position (for example, methoxy group); the oxidation of the methylene link pyrroline getting oxo- and dioxobacteriochlorins (analogues of natural tolyporphin A); metallization of the macrocycle and creation of dyads.

Thus, by brominating the 15 th position of a MeOBCtype macrocycle with a subsequent Pd-catalyzed combination reaction, a number of 15-meso-aryl-substituted derivatives of $195 \mathbf{a}-\mathrm{g}$ were obtained. Their aromatic substituent included functional groups for subsequent biorthogonal reactions (Figure 104). ${ }^{[985]}$ The authors synthesized a number of functionalized cycloimides of bacteriochlorins 196a-e with an absorption range of 712-818 nm. In a number of other studies, the authors obtained $\beta, \beta$ and $\beta$, meso-annulated bacteriochlorins 197-200 (Figure 105). ${ }^{[986-988]}$

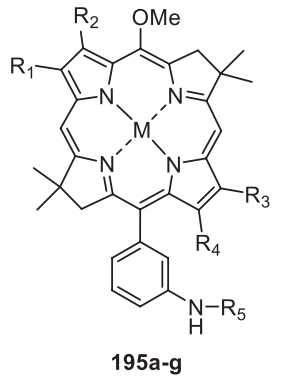

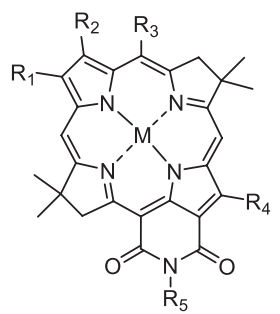

196a-e

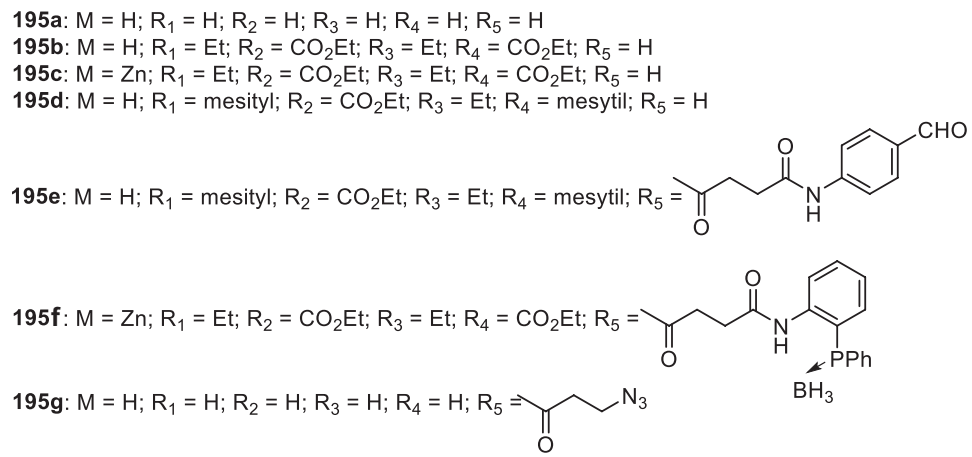

196a: $M=Z n ; R_{1}=E t ; R_{2}=C O_{2} E t ; R_{3}=O M e ; R_{4}=E t ; R_{5}=C_{2} C_{6} H_{5}$ 196b: $M=Z n ; R_{1}=E t ; R_{2}=C O_{2} E t ; R_{3}=O M e ; R_{4}=E t ; R_{5}=\left(C_{2}\right)_{12} N_{3}$ 196c: $M=\mathrm{Cu} ; \mathrm{R}_{1}=\mathrm{Et} ; \mathrm{R}_{2}=\mathrm{CO}_{2} \mathrm{Et} ; \mathrm{R}_{3}=\mathrm{OMe} ; \mathrm{R}_{4}=\mathrm{Et} ; \mathrm{R}_{5}=\left(\mathrm{CH}_{2}\right)_{12} \mathrm{~N}_{3}$ 196d: $M=\mathrm{Zn} ; \mathrm{R}_{1}=\mathrm{Et} ; \mathrm{R}_{2}=\mathrm{CO}_{2} \mathrm{Et} ; \mathrm{R}_{3}=\mathrm{OMe} ; \mathrm{R}_{4}=\mathrm{Et} ; \mathrm{R}_{5}=\left(\mathrm{CH}_{2}\right)_{12} \mathrm{NH}-\mathrm{CO}-\mathrm{CH}_{2} \mathrm{CH}_{2} \mathrm{~N}_{3}$

196e: $M=\mathrm{H} ; \mathrm{R}_{1}=\mathrm{Et} ; \mathrm{R}_{2}=\mathrm{CO}_{2} \mathrm{Et} ; \mathrm{R}_{3}=\mathrm{H} ; \mathrm{R}_{4}=\mathrm{Et} ; \mathrm{R}_{5}$

Figure 104. Functionalized bacteriochlorins for biorthogonal reactions. 


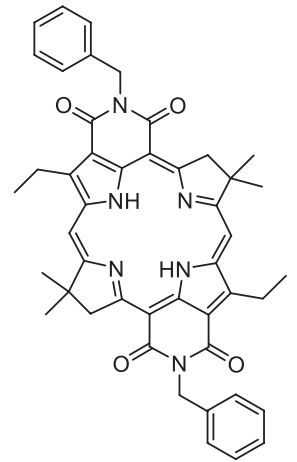

197

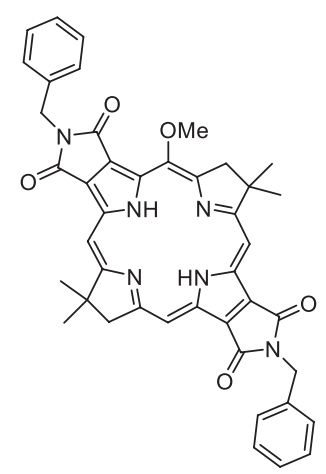

198

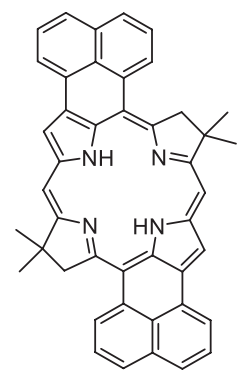

199

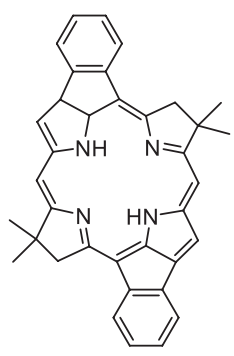

200

Figure 105. Annulated bacteriochlorins.

For the synthesis of $\beta$, meso-annulated bacteriochlorins, the Pd-catalyzed cross-combination reaction was used which allowed the introduction of a fragment of naphthalene and benzene into the macrocycle. Photophysical studies have shown that such structural motifs can significantly increase the chromatic shift of the $\mathrm{Q}_{\mathrm{y}}$ band in comparison with other auxochromic groups included in pyrroles.

The absorption of compounds 199 and 200 was 913 and $1033 \mathrm{~nm}$, respectively, which was higher than that of $\beta, \beta-$ annulated bacteriochlorins. It was established that these compounds possess extremely short lifetime of the singlet state and an extremely low quantum yield of fluorescence. In addition, it is shown that such compounds have a strong vibronic activity making them good candidates as agents for photoacoustic imaging and photothermal therapy.

Zhang and Lindsey described another method for the synthesis of $\beta$,meso-annulated bacteriochlorins containing exocyclic fragment characteristic of phorbine bacteriochlorophyll derivatives (Scheme 37). ${ }^{[989]}$ This method involves the synthesis of a number of different dihydrodipyrrines 203a-e and 204a-e (halves AD and BC) and their association by means of the Knoevenagel reaction with subsequent acid-catalyzed electrophilic aromatic substitution and Nazarov's cyclization. The proposed method, in contrast to the ones described above, allows to form exocyclic ring and the macrocycle.

The photophysical properties of the obtained 204a-e compounds were investigated. They turned out to be typical for bacteriooxophorbine-type pigments. The authors note that this method can serve as a basis for the synthesis of more complex analogues as well as for obtaining derivatives of natural bacteriopheophytin as an alternative to the method proposed by the Woodward group. ${ }^{\text {990,991] }}$

Another direction in the modification of the abovedescribed bacteriochlorins is to obtain their oxo- and dioxoderivatives under the action of various oxidants (Scheme 38). If the introduction of auxochromic groups in the composition of pyrroles $\mathrm{A}$ and $\mathrm{C}$ (the so-called Y-axis) leads to a bathochromic shift of the $\mathrm{Q}_{\mathrm{y}}$ band, then the placement of oxogroups in the pyrroline rings $\mathrm{B}$ and $\mathrm{D}$ (the $\mathrm{X}$-axis) causes a hypsochromic effect. In addition to access to the spectral absorption region of functionalized chlorins $(680-690 \mathrm{~nm})$, the pyrroline oxogroup can serve as a convenient place for further modification. The traditional method for the synthesis of such derivatives is the oxidation of pyrroles $\mathrm{B}$ and $\mathrm{D}$ in porphyrins using $\mathrm{OsO}_{4}$ to 2,3-vic-12,13-vic-tetrahydroxybacteriochlorins followed by pinacoline rearrangement. Liu et al. described the synthesis of various oxo- and dioxobacteriochlorins from bacteriochlorins obtained by the condensation of dihydrodipyrrins derivatives. ${ }^{[992]}$ When modifying compound 195a, a hypsochromic shift of the $\mathrm{Q}_{\mathrm{y}}$ band was observed from 713 to $690 \mathrm{~nm}$ for oxo- and $680 \mathrm{~nm}$ for dioxobacteriochlorins, respectively. In addition, the authors report obtaining Schiff bases based on such compounds.

Numerous studies in the field of bacteriochlorophyll $a$ and its derivatives show their significant advantages

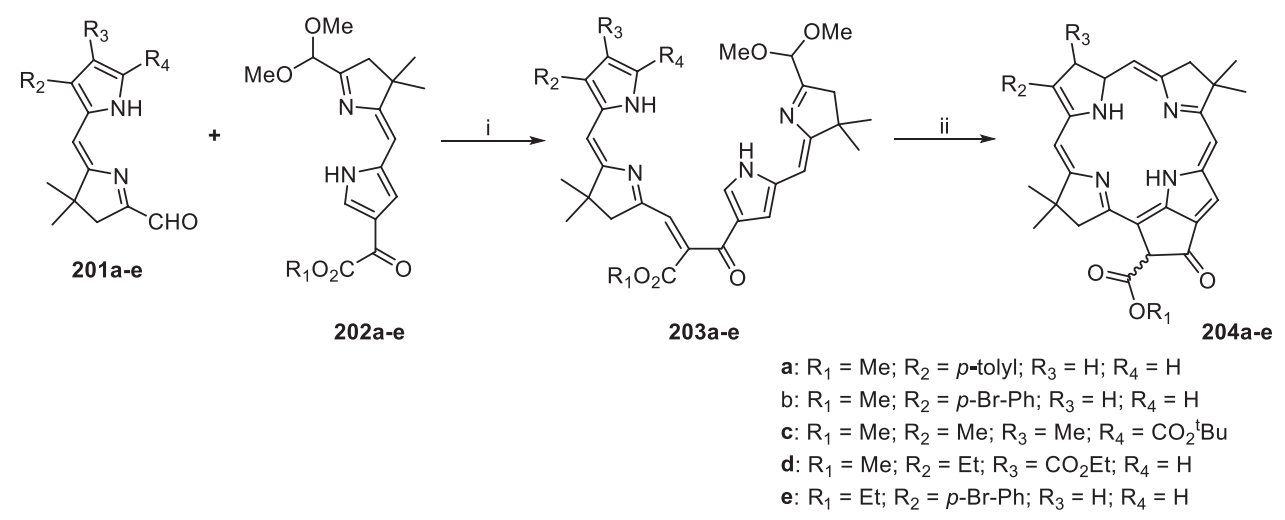

Scheme 37. Reagents and conditions: $\mathrm{i}$ - piperidine, $\mathrm{HOAc}, \mathrm{DCM}$, rt; $\mathrm{ii}-\mathrm{Yb}(\mathrm{OTf})_{3}, \mathrm{CH}_{3} \mathrm{CN}, 80^{\circ} \mathrm{C}$. 


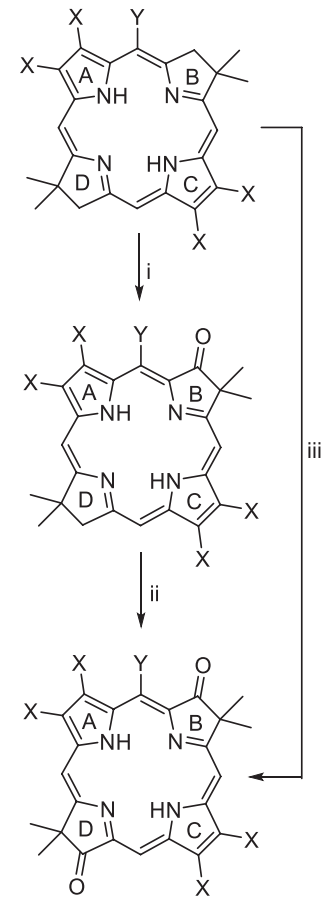

Scheme 38. General scheme of synthesis of oxoand dioxobacteriochlorins.

Reagents and conditions: $\mathrm{i}-\mathrm{MnO}_{2}$, DCM, rt. or PDC, DCM, $-30{ }^{\circ} \mathrm{C}$; ii - PDC, DCM, rt; iii - $\mathrm{CrO}_{3} \cdot \mathrm{DMP}, \mathrm{DCM}$, rt.

over porphyrins and chlorins. For example, in the field of oncology the introduction of IR-PS of the bacteriochlorin series has long been expected and is very relevant. However, the chemical and photolability of the latter, along with the limited possibilities of isolation from natural sources, make chemists search for new ways to stabilize the bacteriochlorin macrocycle and replace natural pigments with synthetic analogues. That is why this Section is structured in such a way that it begins with natural bacteriochlorophylls and sources of their production, then presents numerous ways to modify the macrocycle in order to improve photophysical characteristics, increase stability and implement the targeted nature of the bacteriochlorin derivatives action and finally concludes with a variety of approaches to the synthesis of de novo bacteriochlorins. Time will tell which bacteriochlorins will be most in demand but the research that is being conducted in the world and in our country including the Department of Chemistry and Technology of Biologically Active Compounds, Medical and Organic Chemistry named after N.A. Preobrazhensky of Russian Technological University, makes an invaluable contribution to the development of fundamental and applied chemistry of this unique class of macroheterocycles.

\subsection{The Porphyrinoid-Based Conjugates as a New Paradigm in Targeted Photodynamic Therapy of Cancer}

Photodynamic therapy (PDT) was developing more than 100 years and has become a well-established method for efficient treatment of different oncological ${ }^{[993,994]}$ and nononcological $^{[995,996]}$ diseases. PDT nowadays is successfully applying remedy for various cancers, such as breast, colon, stomach, bladder, head, brain, lung and skin cancers. ${ }^{[997]}$

The main drawbacks of the medicinal application of porphyrin-type PDT are phototoxicity and low selectivity of delivery to cancer cells. Nowadays, the so-called third generation of PS is developing. These PSs have a property of selective delivery to the cancer cells and/or multifunctional therapeutic actions. The variety of PSs using for antitumor therapy is described in plenty of comprehensive reviews. ${ }^{[831,835,998,999]}$ In this Section, we want to discuss the most promising types of PSs, in particular, conjugates of tetrapyrroles with carbohydrates, peptides, antibodies, $\mathrm{pH}$-sensitive conjugates, molecular beacons and targeted conjugates for the combined therapy.

Porphyrin conjugates bearing carbohydrate residues are promising structures for the construction of therapeutic agents demonstrating the enhanced target delivery properties, enhanced solubility in aqueous media, and simultaneously good amphiphilicity. ${ }^{[1000]}$ Carbohydrate ligands, along with peptides and antibodies, are among the most promising vectors for targeted delivery. ${ }^{[1001]}$ As vector components, carbohydrates have a number of advantages over other natural and synthetic materials: due to their enormous structural diversity, oligosaccharides and their mimetics have an amazing selectivity for protein receptors; they are biocompatible and biodegradable. ${ }^{[1002]}$

Oligo- and polysaccharides are popular scaffolds for creating nanoscale delivery systems for various therapeutic agents. ${ }^{[1003-1008]}$ The vast majority of such particles are highly biocompatible due to their natural origin. The variety of natural polysaccharides provides a wide range of particle structures (linear polymers, branched, cyclic), different chemical composition (different monosaccharides or $\alpha / \beta$ forms in a single polymer), molecular weight range and particle charge variability (positive, negative or neutral). A wide range of carbohydrate-decorated tetrapyrrole PS was developed during the last two decades ${ }^{[870,1009-1014]}$ It was shown that among different types of conjugates on the basis of synthetic neutral, cationic, dimeric, and strapped tetraphenyl-substituted chlorin photosensitizers with carbohydrates, the most efficient were neutral asymmetric monomeric porphyrins connected to mono- or disaccharide. ${ }^{[1015]}$ Importantly, that only amphiphilic chlorin derivative (compound 205) displayed a bigger efficacy than Photofrin. ${ }^{[1009,1012,1015]}$ A similar situation was observed for the conjugates based on synthetic chlorins. Asymmetric tetraphenylchlorin (TPC, compounds 206 and 207) with three glucose residues was 4-fold more active than non-substituted PS (Foscan).

Symmetric four-substituted TPC revealed low internalization, accomplished with weak photoactivity (HT29, human colon cancer cell line). At the same time, trisubstituted PS (conjugates 206 and 207) demonstrated enhanced internalization explained by active receptor-mediated endocytosis and increased phototoxicity due to the affinity to mitochondria. ${ }^{[1015-1018]}$

Both the nature of carbohydrate and its location in tetraphenylporphyrin (TPP) framework influence the therapeutic efficiency of final conjugates. Thus, in a series of modified purpurinimides bearing glucose, galactose or lactose as peripheral substituents in different cites (conjugates 208-212), the best in vitro and in vivo activity was achieved 
Macroheterocyclic Compounds - a Key Building Block in New Functional Materials and Molecular Devices

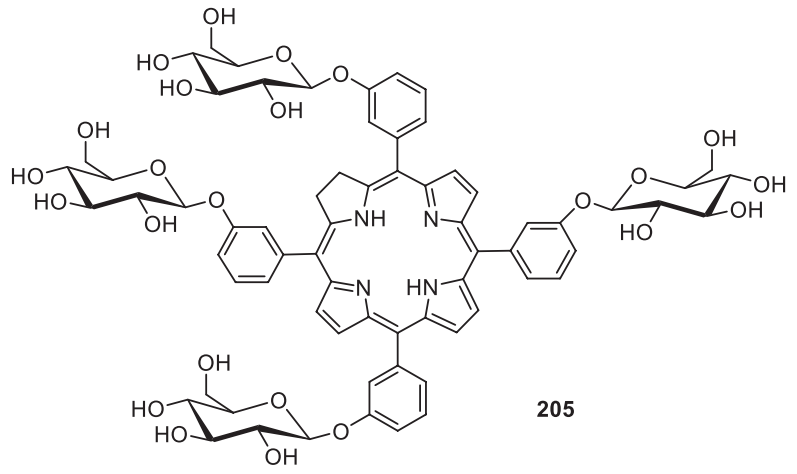

Cell survival fraction $22 \pm 4 \%$ at $2 \mathrm{mM}$ (HT29 cell line)
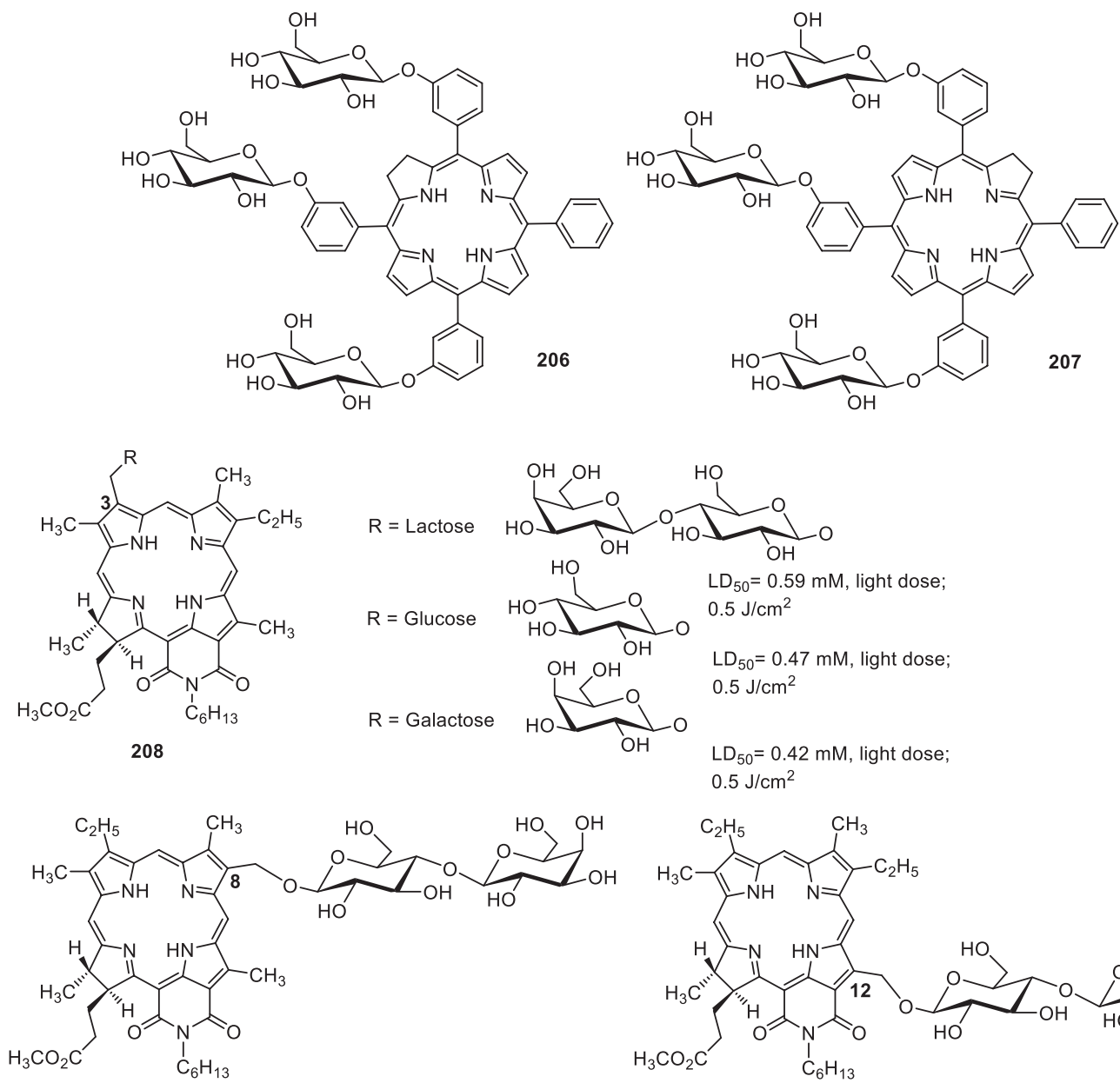

$\mathrm{LD}_{50}=0.91 \mathrm{mM}$, light dose; $1 \mathrm{~J} / \mathrm{cm}^{2}$

209

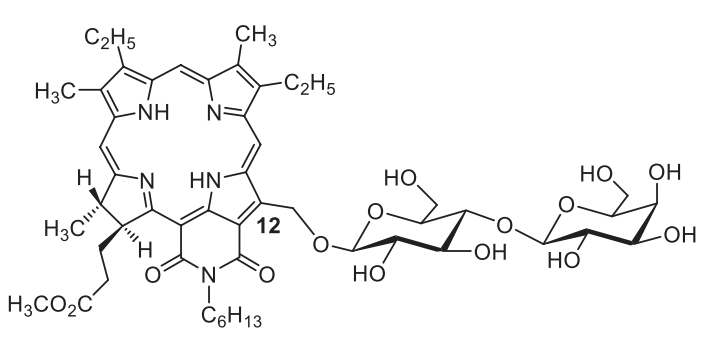

$\mathrm{LD}_{50}=0.84 \mathrm{mM}$, light dose; $1 \mathrm{~J} / \mathrm{cm}^{2}$

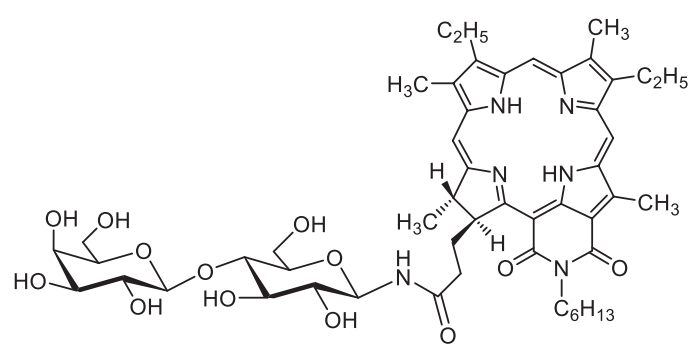

$\mathrm{LD}_{50}>1 \mathrm{mM}$, light dose; $1 \mathrm{~J} / \mathrm{cm}^{2}$

210

211

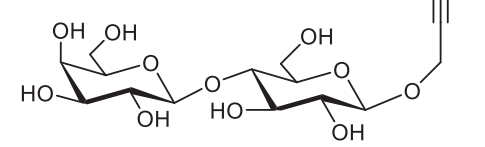

$\mathrm{LD}_{50}=0.85 \mathrm{mM}$, light dose; $1 \mathrm{~J} / \mathrm{cm}^{2}$

212 

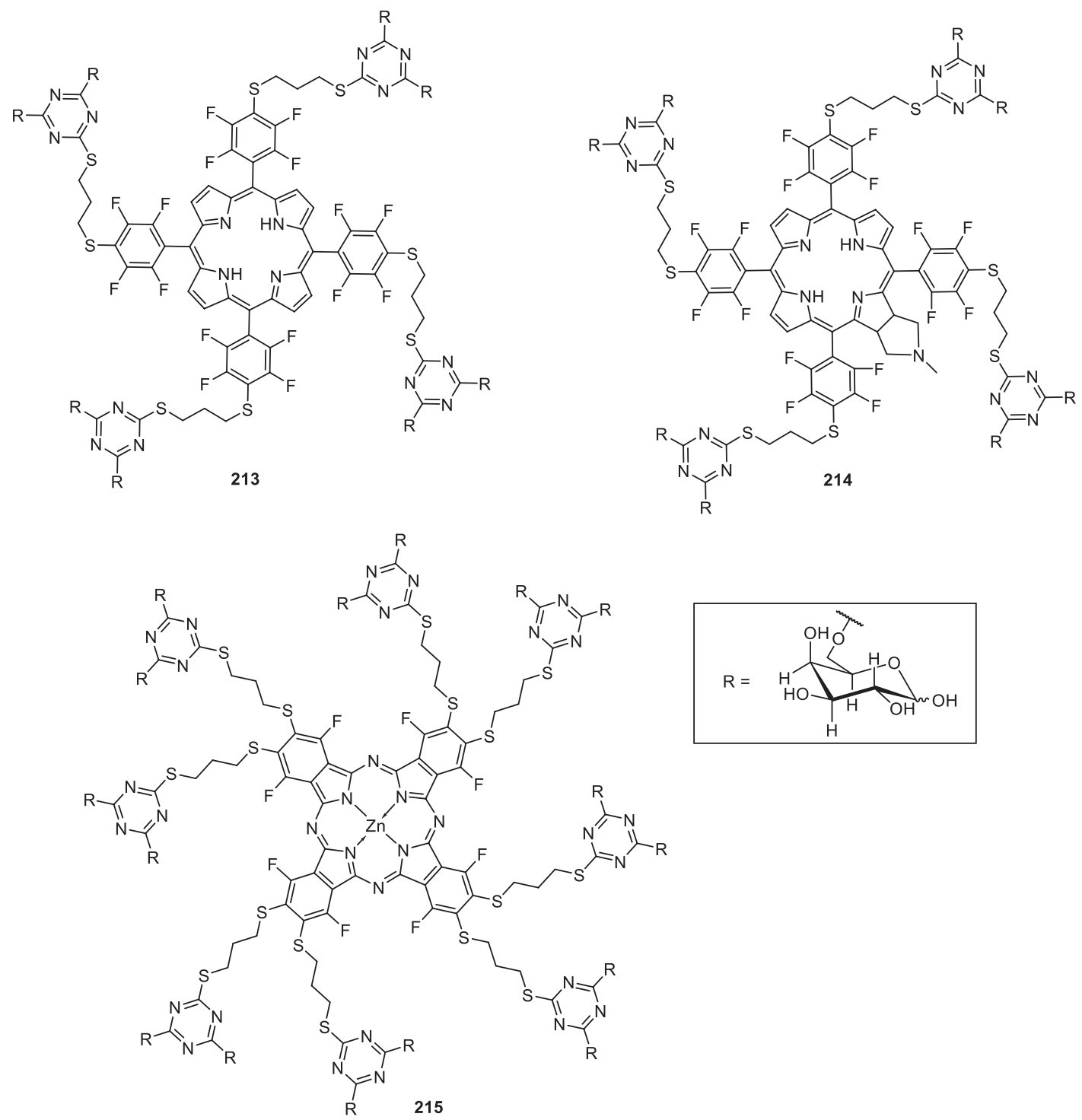

for conjugate bearing lactose in " 3 " position (conjugate 208). ${ }^{[1015]}$ Interestingly, that this conjugate is localized preferentially in lysosomes and Golgi apparatus, but not in mitochondria.

More pronounced drug delivery properties could be achieved by conjugation of PS with several vectors, containing carbohydrate moieties. Thus, decoration of porphyrins, phthalocyanines and chlorins with 1,3,5-triazines, containing two galactose fragments afforded to the formation of dendrimer-type conjugates capable of implementing multivalent interactions with lectin receptors. ${ }^{[1019,1020]}$ For example, employing of substituents with several galactose residues significantly increases the selectivity of delivery to tumor cells. This is possible due to interactions of galactose-decorated PSs with galectin-1 receptors overexpressed in many tumor cells. ${ }^{[1021,1022]}$

Conjugates 213-215 possessed good solubility in aqueous media, exhibited excellent photostability and ability to generate singlet oxygen. In comparison with porphyrinand phthalocyanine derivatives 213 and 215, chlorin conjugate 214 demonstrated better photophysical and photochemical properties. Conjugates 213-215 represent a pronounced anticancer activity both in vitro and in vivo against bladder cancer (UM-UC-3 and HT-1376 cell lines). Importantly that re-irradiation sufficiently improves the cancer treatment, even in the case of HT-1376 resistant tumors. The authors suggested that there is a predominant accumulation of conjugates in mitochondria. ${ }^{[1015]}$

The therapeutic effectiveness of conjugates 213-215 can be explained by two mechanisms. The first one deals with the interaction of conjugates with the most abundant protein of blood plasma - human serum albumin (HSA). It is known that HSA has a property to bind and to transport the antitumor agents. The increased vascular permeability in solid tumors leads to the accumulation of HSA-drug adduct in tumor interstitium resulting in a quantity of free drug circulating in the blood and its distribution, metabolism, and bioactivity. ${ }^{[1023]}$ It was shown that conjugates 213-215 form a complex with HSA in a 1:1 ratio. The second mechanism is based on specific interactions of galactose residues with galectin-1 carbohydrate-recognition domain and hydrophobic interactions of chlorin PS with the hydrophobic core of protein. ${ }^{[1020,1024]}$

Along with decorating PS with carbohydrate ligands to tumor receptors, an effective approach to the treatment of tumors is the creation of carbohydrate nanoparticles containing PS that generate singlet oxygen only at certain $\mathrm{pH}$ values. Indeed, it is known that tumors are characterized, as a rule, by acidic $\mathrm{pH}$ values. ${ }^{[1025,1026]}$ This phenomenon was used for the elaboration of polycarbohydrate delivery 
A

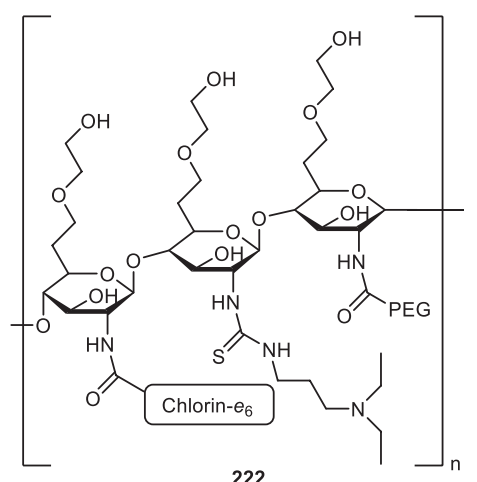

B

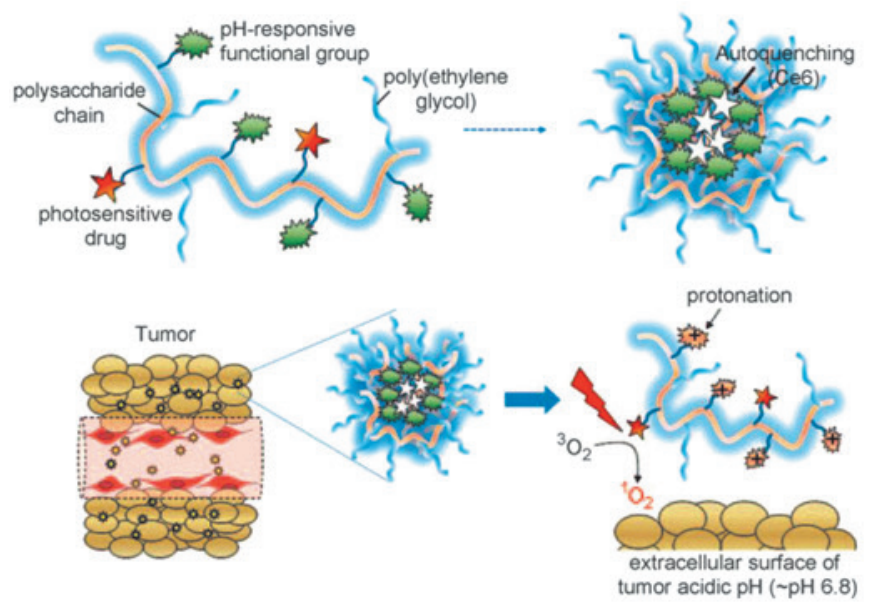

Scheme 39. (Scheme 39B, adapted with permission, from ${ }^{[869]}$ )

systems which release therapeutic agent only in acidic conditions. A PS delivery system based on chitosan shown excellent selectivity properties towards solid tumors with acidosis. $^{[869]}$ This conjugate consists of polysaccharide backbone decorated with dimethyl aminopropyl isothiocyanate substituents, polyethylene glycol, and chlorin- $e_{6}$ PS (Scheme 39A). PEG decoration gives a good solubility level for the whole conjugate in the blood (aqueous media). Amino-containing substituents play the role of $\mathrm{pH}$-sensitive switchers, which manage a macromolecule's conformation to opened chain mode. Moreover, the big size of the conjugate promotes it accumulation in tumor tissue due to enhanced permeability and retention (EPR) effect.

In solution with normal $\mathrm{pH}$, the macromolecule is self-organized in globular core with polyethylene glycol (PEG) and glycol chitosan backbone (GCS) faces outside (hydrophilic), and amino groups and PS are placed inside (hydrophobic). In this shape, PSs could not work due to autoquenching. Inside a tumor $\mathrm{pH}$ is lower $(\mathrm{p} H$ 6.5-7.0) than in other parts of body ( $\mathrm{pH}$ 7.4). ${ }^{[1027]}$ More acidic conditions of cancer tissue lead to protonation of amines, the formation of ammonium groups, and the conversion of its nature from hydrophobic to hydrophilic. This caused transformation into the uncoiled structure, liberation of PSs, and allows generating single oxygen under irradiation (Scheme 39B). It was shown on a BALB/c nu/nu mice model that conjugate mostly accumulates in the tumor in comparison with a 25-fold concentration free PS and conjugate without amino substituents. Thus, conjugation of PS with polysaccharide backbone and decoration with $\mathrm{pH}$-sensitive groups leads to the selective and self-regulating antitumor drug.

Alongside carbohydrate-based ligands, peptides, oligopeptides, and small proteins are widely used for selective delivery of therapeutic agents to the target tissues. ${ }^{[1028-1030]}$ A lot of different conjugates of photosensitizers and short peptides were elaborated. The most important are agents bearing peptides, which have an affinity to epidermal growth factor receptors, gastrin-releasing peptide receptors, integrin receptors, gonadotropine releasing hormone recep- tor, as well as peptides with affinity to cell nucleus; among protein-based conjugates, the most common are conjugates with serum proteins, serum albumins, lipoproteins, growth factor receptors and antibodies. ${ }^{[1029]}$

Van Lier et al. reported on the elaboration of PDT agent for receptor targeting and both imaging and therapeutic actions. Conjugate $\mathbf{2 1 6}$ is based on phthalocyanine and contains Bombesin, a polypeptide (Tyr-Gln-Arg-Leu-Gly-AsnGln-Trp-Ala-Val-Gly-His-Leu-Met- $\mathrm{NH}_{2}$ ), which has a strong affinity to the gastrin-releasing peptide receptors (conjugate 216). ${ }^{[1031]}$ Different types of tumor cells express receptors for Bombesin, including breast, gastric, prostate, pancreatic, colon, small cell lung cancer.

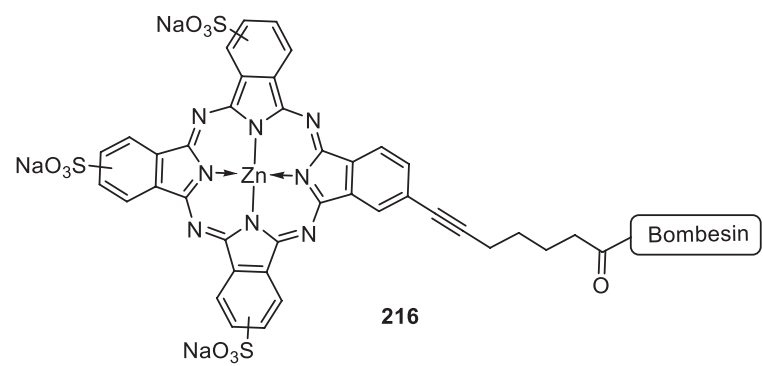

Bombesin = Tyr-GIn-Arg-Leu-Gly-Asn-GIn-Trp-Ala-Val-Gly-His-Leu-Met-NH 2

Conjugate 216 demonstrated photodynamic activity in low nano- and even picomolar concentrations towards EMT-6, A549, PC3 and MDA-MB-231 cells under irradiation with a dose of $12 \mathrm{~J} / \mathrm{cm}^{2}$.

A range of conjugates of tetrapyrrolic photosensitizers with antibodies are known. ${ }^{[863,1032]}$ These conjugates usually combine the photochemical properties of photosensitizer and the immunoreactivity of antibody without decreasing the efficacy of both parts. Recent advantages in recombinant DNA technology allow the production of different antibodies for the construction of targeted conjugates for PDT. There are more than 60 different drugs based on antibodyPS conjugates entered a clinical evaluation. ${ }^{[863]}$ 

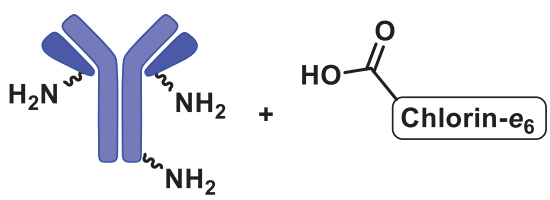

B3-H7-target antibody

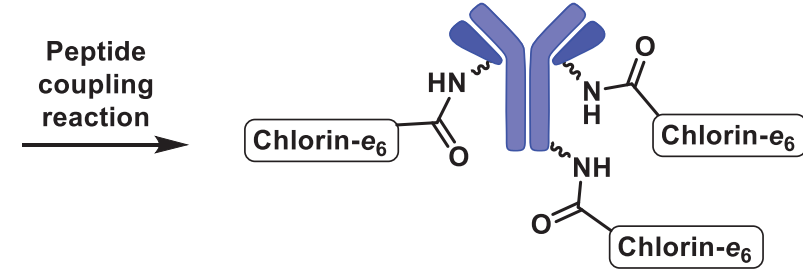

217

Antibody-PS conjugate

Scheme 40.

A recent example of chlorin $e_{6}$ conjugate with B7-H3-targeted antibody is presented in Scheme 40. ${ }^{[1033]}$ B7-H3 is an immune check point molecule, namely a cell surface receptor, highly expressed by tumor cells and tumorassociated vessels. B3-H7 was found as an important part of the regulation of immune responses for NK- and T-cells. This molecule was found to be upregulated in non-small cell lung cancer tissues, which means it could be used as an effective biomarker for antibody-driven selective drug delivery. ${ }^{[1033,1034]}$

The generation of ROS by conjugate 217 was proved at the same level as for free photosensitizer. In vivo studies with A549 tumor-bearing mice demonstrated a strong suppression of tumor growth in case of small dose of light irradiation $\left(10 \mathrm{~mW} / \mathrm{cm}^{2}, 20 \mathrm{~min}\right)$ resulted in significant decreasing of tumor weight during 45 days after PDT.

Another promising method for the development of highly selective PDT conjugates is the construction of photodynamic molecular beacons (PMB). These mol- ecules consist of photosensitizer for PDT, a cleavable linker, and a quencher. The implementation of these conjugates are based on Förster resonance energy transfer (FRET) phenomenon. ${ }^{[1035]}$

For example, Wilson and colleagues developed a conjugate of pyropheophorbide which is connected to BHQ3 (black hole quencher 3), an effective singlet oxygen quencher, through oligopeptide linker (Scheme 41, conjugate 218). ${ }^{[1036]}$ This oligopeptide is specifically cleaved by matrix metalloproteinase-7 (MMP7), an enzyme with epithelial origin, and highly expressed by breast, colon, pancreatic and nonsmall-cell lung cancer. ${ }^{[1037]}$ In healthy tissues, upon excitation of pyrotheophorbide, energy is transferred to the quencher, therefore, no single oxygen generation is observed. When conjugate 218 located in the tumor, the peptide is cut by MMP7, the quencher leaves, and the PS begins to generate reactive oxygen species (ROS) (Scheme 41).

On nude mice model bearing human nasopharyngeal epidermoid carcinoma (KB) with high MMP7 expression

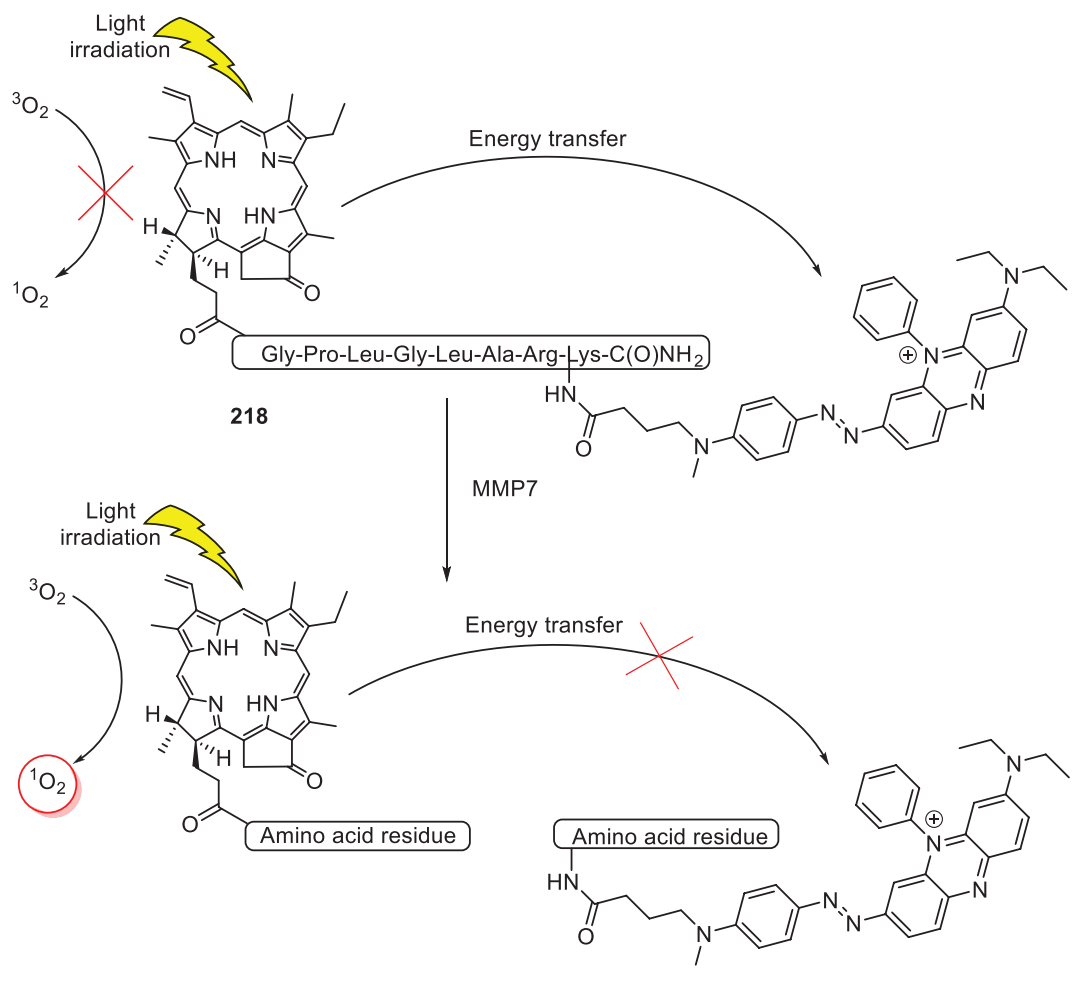

Scheme 41. 


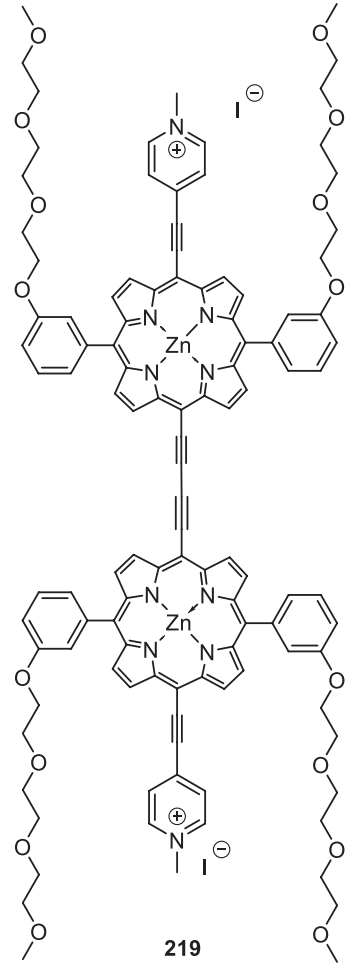

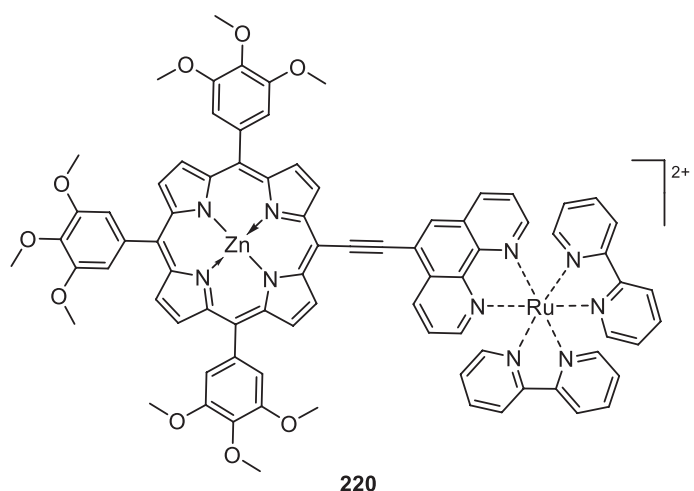

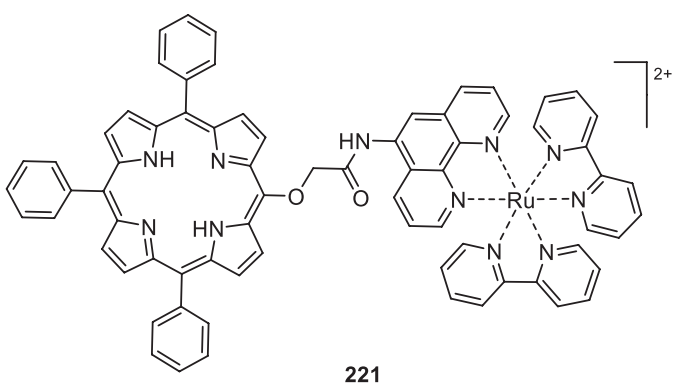

it was shown that conjugate releases free PS due to linker cleavage in 20 minutes after injection, and only in the tumor the highest concentration was reached in $3 \mathrm{~h}$. PDT treatment of tumor leads to edema immediately, reduction of tumor size - in 3 days, and complete regression - in 30 days (tumors in control experiments continued growing).

Another modern trend in porphyrin chemistry is design of two-photon sensitizers ${ }^{[1038]}$ undergoing near-infrared (NIR) excitation (700-1000 nm) allowing to perform PDT in so call "the optical therapeutic window" of biological tissues, where most biological molecules have a minimal light absorption (water, melanin, hemoglobin). ${ }^{[1038,1039]}$ Conjugates 219-221 represent examples of such two-photon tetrapyrrolic PSs. The first one has a big conjugated $\pi$-system tuned for the absorption of long-wavelength irradiation (conjugate 219). ${ }^{[1040]}$ In the second type, the porphyrin fragment is substituted with metal complex affording compound $\mathbf{2 2 0}$ with completely conjugated $\pi$-systems of porphyrin and organoruthenium frameworks and to conjugate 221, in which these two $\pi$-systems are separated with $\mathrm{sp}^{3}$-hybridized carbon atom. ${ }^{[1041,1042]}$

Excited at $920 \mathrm{~nm}$, conjugate 219 characterized by high ${ }^{1} \mathrm{O}_{2}$ quantum yield $(60 \%$ in $\mathrm{MeOH})$ is shown to be sufficiently more effective in vitro in comparison with clinically-used verteporfin. ${ }^{[1040]}$ The animal study proved that conjugate 219 is suitable for the exposure of vessels in vivo. After 7-12 minutes irradiation of an artery with $\sim 40 \mathrm{~mm}$ diameter, the blood flow was interrupted, and no photodamaging of located nearby vessels was detected. ${ }^{[1038,1040]}$

Compound 220 with completely conjugated porphyrin and organoruthenium fragments displayed a high quantum yield of singlet oxygen ( 0.93 in $\left.\mathrm{CHCl}_{3}\right)$, low dark toxicity, and high photoinduced activity. ${ }^{[1041]}$ Thus, 220 of $1 \mathrm{mM}$ concentration affords to $80 \%$ death of HK-1 cells (nasopharyngeal carcinoma) after a light dose $\left(3 \mathrm{~J} / \mathrm{cm}^{2}\right)$ irradiation at $850 \mathrm{~nm}$. Conjugate 221 shows excellent two-photoninducted PDT properties: with a concentration of $8 \mathrm{mM}$ it was needed only $0.5 \mathrm{~J} / \mathrm{cm}^{2}$ dose of light irradiation to kill $50 \%$ of cells (HK-1 line) at 800 nm. ${ }^{[1042]}$

Another important direction in the chemistry of porphyrins is an elaboration of conjugates for combined therapy. In these molecules the PS is conjugated with a second active part, usually with a chemotherapeutic drug. Numerous examples of porphyrin-, phthalocyanine-, chlorin-, bacteriochlorin-conjugates with doxorubicin, ${ }^{[1043]}$ cisplatin-type complexes, ${ }^{[1044-1047]}$ combretastatin, ${ }^{[1048-1050]}$ paclitaxe, ${ }^{[1051]}$ and other drugs ${ }^{[1052-1054]}$ are known.

The authors ${ }^{[1055]}$ proposed a promising photosensitizer based on a conjugate $\mathbf{2 2 3}$ consisting of silicon(IV) phthalocyanine, linked to the erlotinib growth factor receptor ligand and sulfonamide endoplasmic reticulum (ER) anchor. This conjugate demonstrated a synergetic effect for the acceleration of tumor cell death. Conjugate 223 localized in ER, while its analougs without ER-anchor or growth factor receptor ligand mostly accumulate in the lyzosomes and mitochondria. ${ }^{[1055]}$ Conjugate 223 displays a high photodynamic activity $\left(\mathrm{IC}_{50}\right.$ lies in a range of $4.2-25.4 \mathrm{nM}$ for A549, PC-9, and HELF cancer cell lines) under $1.5 \mathrm{~J} / \mathrm{cm}^{2}$ light irradiation at $670 \mathrm{~nm}$.

A series of natural chlorin conjugates with vandetaniban effective ligand to growth factor receptors and a tyrosine kinase inhibitor was developed in our group (conjugates 224, 225). ${ }^{[43,44,1056]}$

A cationic conjugate 224 exhibits an enhanced accumulation in A431 cells with a high level of epidermal growth factor receptor expression (EGFR) in comparison with cells with low EGFR expression (CHO and HeLa). The prepared conjugate exhibits dark and photoinduced toxicity at micromolar concentrations with $\mathrm{IC}_{50 \text { dark }} / \mathrm{IC}_{50 \text { light }}$ ration of $11-18$, which means two mechanisms of antitumor action (chemotherapy 


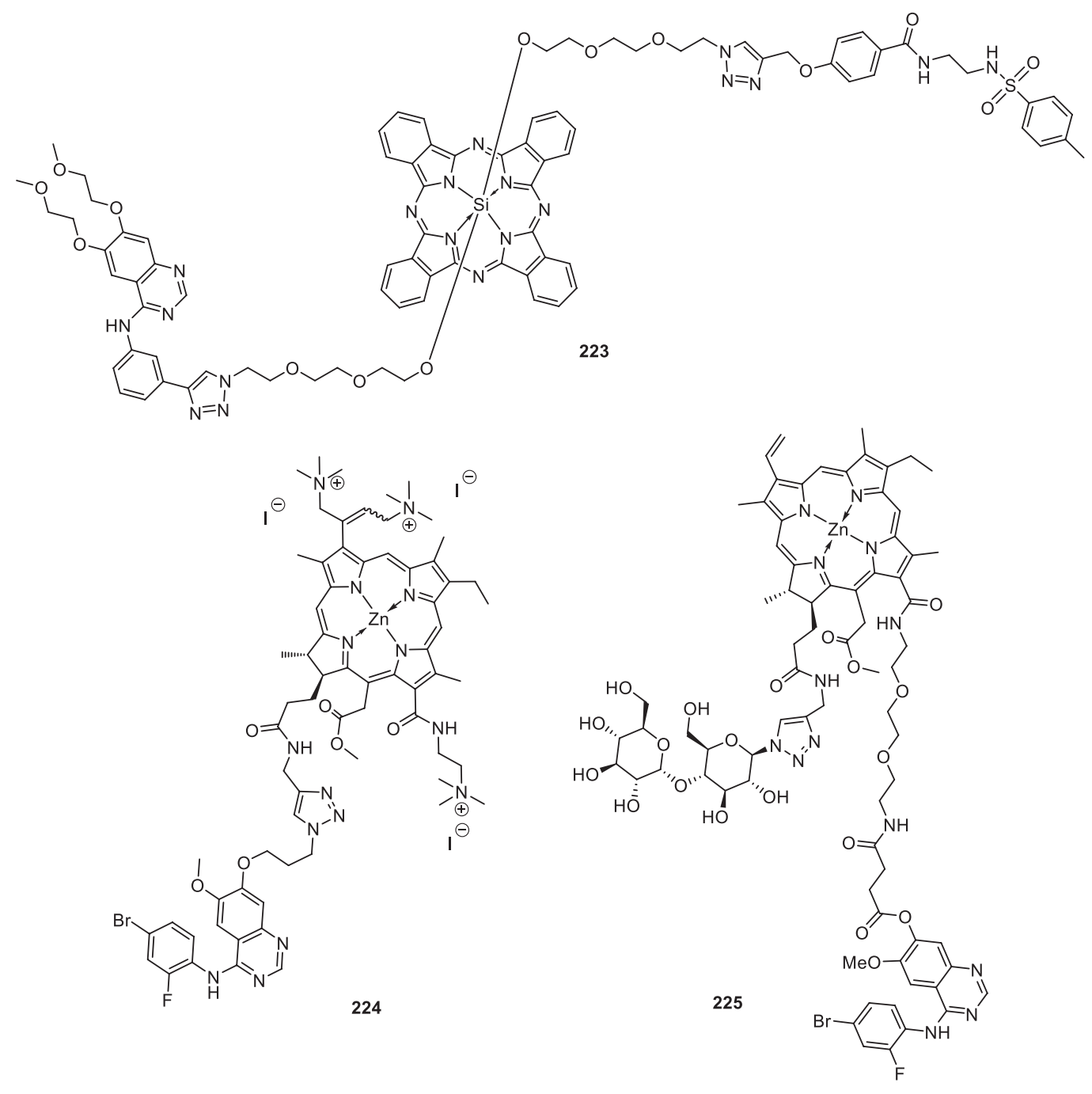

and photodynamic therapy). In tumor-bearing $\mathrm{Balb} / \mathrm{c}$ mice, the conjugate preferentially accumulates in tumor tissues with tumor-to-tissue ratio reaching 4.2 in $24 \mathrm{~h}$.

Another water-soluble conjugate 225, consisting of a chlorin $e_{6}$ photosensitizer part, a 4-arylaminoquinazoline moiety with affinity to epidermal growth factor receptors, and a hydrophilic $\beta$-D-maltose fragment, was synthesized starting from methylpheophorbide $a$ in seven steps. The prepared conjugate exhibited low levels of dark cytotoxicity and pronounced photoinduced cytotoxicity at submicromolar concentrations in vitro, with $\mathrm{IC}_{50 \text { (dark) }} /$ $\mathrm{IC}_{50 \text { (light) }}$ ratio of $\sim 368$ and a singlet oxygen quantum yield of about $20 \%$. In tumor-bearing Balb/c nude mice, conjugate 225 preferentially accumulates in the tumor tissue. Irradiation of the nude mice bearing A431 xenograft tumors after intravenous administration of the prepared conjugate with a relatively low light dose $\left(50 \mathrm{~J} / \mathrm{cm}^{2}\right)$ produced an excellent therapeutic effect with profound tumor regression and low systemic toxicity.

Thus, modern trends in the design of photosensitizers for photodynamic therapy lie in a field of the development of highly selective agents. Different approaches, such as conjugation with carbohydrates, peptides, antibodies, growth factor receptor ligands, and construction of molecular beacons, make it possible to create high-efficiency drug prototypes.

\subsection{Photodynamic Inactivation of Pathogenic Microorganisms with the Chlorin $e_{6}$-Type Sensitizers: from in vitro to in vivo Studies}

During past several years health authorities of many countries apparently have finally recognized the seriousness of the problem of antimicrobial drug resistance. ${ }^{[1057]}$ The widely accepted point of view that most, if not all, infections can be successfully treated with antibiotics ${ }^{[147,1058]}$ is left behind because microorganisms - the champions of evolution - reveal the unique ability to adapt to any antimicrobial agent to cause serious clinical challenges for humans. For example, methicillin resistant Staphylococcus aureus (MRSA) induces major clinical problems among Gram-positive pathogens ${ }^{[1057]}$ and resistance of MRSA strains towards novel antibiotics such as vancomycin is often detected. Among Gram-negative bacteria there are several pathogens such as Acinetobacter baumannii, Pseudomonas aeruginosa, etc. which are capable of resisting almost all types/classes of antibiotics. ${ }^{[1059]}$ In particular, resistance of Escherichia coli towards ciprofloxacin is detected within 10 hours in a special device containing 100 bacteria in the initial inoculation. ${ }^{[1057]}$ Thus, it is not surprising that the World Health Organization has recently published a special list of the most resistant pathogens containing 12 bacteria and bacterial families to call for developing new ways 
to fight with these dangerous microorganisms. ${ }^{[1057]}$ In principal, bacteria can be efficiently killed by various special methods such as heating, autoclaving, UV or $g$-radiation, chlorination, etc. However, none of these methods can be safely applied to humans. ${ }^{[1057]}$

Photodynamic inactivation appears to be an unique and innovative modality to kill pathogenic microorganisms both in planktonic and biofilm forms. ${ }^{[147,1058-1060]}$ This easily repeatable procedure can be efficiently used along or combined with antimicrobial therapy, efflux pumps inhibitors or membrane perturbing agents. ${ }^{[1059]}$ It involves a two-step procedure that consists of administering either locally or systemically an appropriate PS which is rapidly accumulated in bacterial cells, followed by their local illumination. The photosensitizer transfers the absorbed light energy to adjacent molecular oxygen leading to the generation of mainly singlet oxygen or additionally other high-energy reactive oxygen species (ROS). These highly reactive molecules attack bacterial cell wall components or even DNA (see Figure 106) and, hence, execute fatal oxidative damage towards microbial cells. Photodynamic inactivation has several advantages over any conventional infection treatment due to its low cost, minimal side-effects, no drug resistance, repeatability etc. ${ }^{[147,1058-1060]}$ Some disadvantages, however, do exist. These are associated with treating mainly local infections and the fact that neutral and anionic PSs clinically approved for antitumor PDT reveal weak affinity to the outer lipopolysaccharide membrane of Gram-negative bacteria. ${ }^{[1059,1061]}$ The latter leads to complications dealing with penetration into a bacterial cell and occupy a location, where ROS are able to execute fatal damage. There are several ways to increase the permeability of the Gram-negative bacteria outer membrane. ${ }^{[1061]}$ The first method includes the treatment of cells with aqueous solutions of special peptides or $\mathrm{Na}_{2} \mathrm{H}_{2}$ Edta which can bind superficially located calcium and magnesium ions that may destabilize a membrane. The second one is to use positively charged PSs or PSs conjugated with appropriate positively charged polymers. ${ }^{[147]}$ Both approaches (see below) could provide full inactivation of Gram-negative pathogens.

We consider some important results of our own extensive and continuing series of studies ${ }^{[1062-1073]}$ on the behavior of macrocyclic photosensitizers both in non-aqueous $^{[1062-1065,1073]}$ and aqueous media ${ }^{[1067,1070,1071]}$ and as well as in biological systems ${ }^{[1066,1069,1071]}$ Most of our efforts are directed to the investigation of the ability of potential PSs to inactivate archival and mainly hospital-acquired opportunistic microorganisms both in vitro and in the model of wound infection with the chlorin $e_{6}$ type PSs. These second generation PSs intensively absorb visible light at about $665 \mathrm{~nm}$ with an extinction coefficients (lge) lying within 4.5-4.6 and strongly fluorescent at longer wavelengths. Besides, chlorins studied demonstrate an appropriate solubility in water (compounds 227-231) or aqueous solutions of non-ionic surfactant Tween $80^{[1070-1072]}$ allowing therapeutic concentrations of PS to be achieved. Like most macroheterocyclic photosensitizers, chlorins are aggregated in water, however, aggregation can be easily overcomed by using micellar or polymeric surfactants, liposomes or other drug nanocarriers. . $1070,1072,1074,1075]$

The information presented below includes experimental data on singlet oxygen generation, solute distribution between aqueous and lipid-like phases in the physiological temperature range, and, what is mostly important, the results of studies on photodynamic inactivation of various pathogenic microorganisms.

All chlorin $e_{6}$-type PSs illustrated in Figure 107 were developed on the methylphephorbide $a$ platform and identified with the well-known physical chemical techniques such as ${ }^{1} \mathrm{H}$ NMR, MS, IR, UV-Vis and fluorescence emission spectra (for details, please, see original papers ${ }^{[1069,1071]}$ ). We see that most of the PSs studied contain one, two or three cationic groups. Additionally, to enhance antimicrobial activity of the chlorin-type PSs we have added to methylpheoprobide and its dicationic derivative the apolar residue of myristic acid (see compounds 230, 231 in Figure 107). This fragment

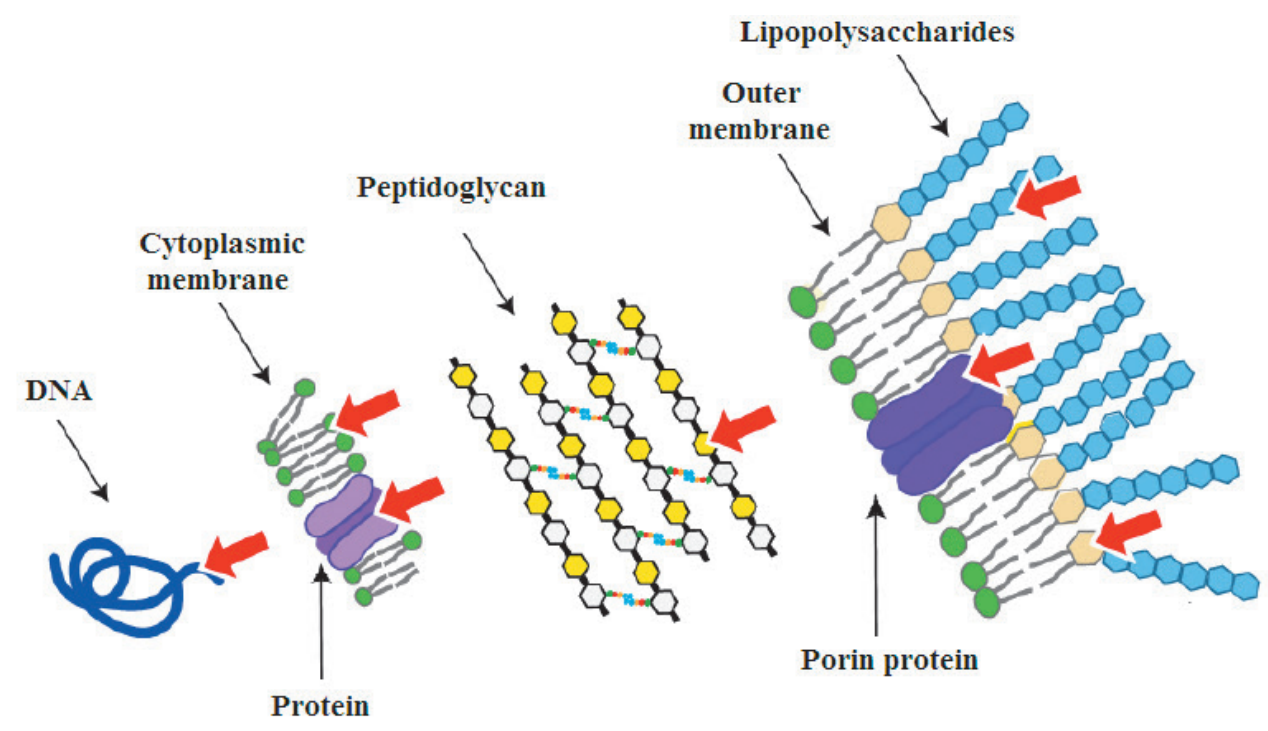

Figure 106. Most probable targets for ROS in bacterial cells (adapted from ref. ${ }^{[1059]}$ ). Note, that possible targets for Gram-positive microorganisms are restricted by the left-hand structures, i.e. they do not contain outer membrane components. 

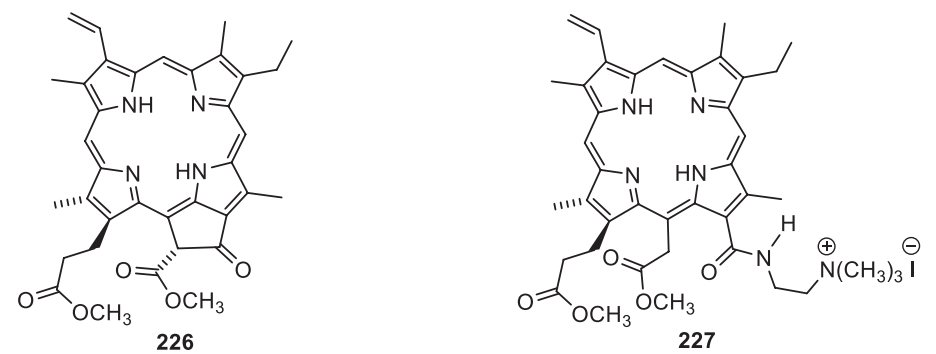

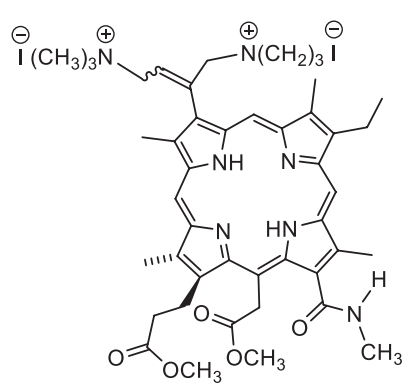

228

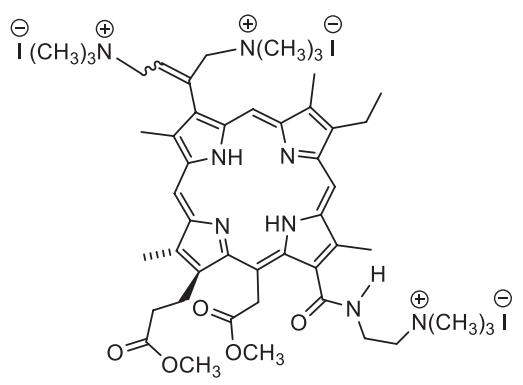

229

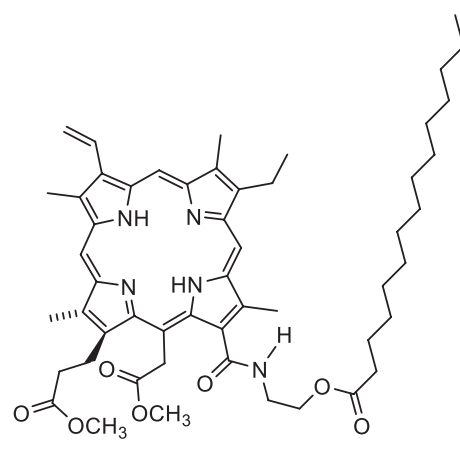

230

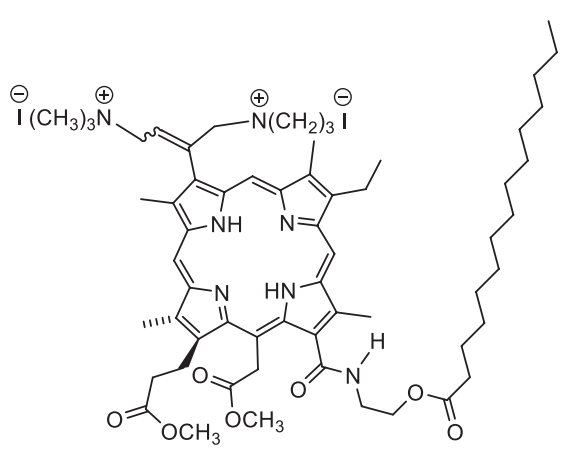

231

Figure 107. Structures of the pigments studied: 226 - pheophorbide $a$ 17(3)-methyl ester, $227-13(1)-N-\left(2-N^{\prime}, N^{\prime}, N^{\prime}-\right.$ trimethylammonioethyliodide)amide chlorin $e_{6}$ 15(2),17(3)-dimethyl ester, 228 - 3(1),3(2)-bis( $N, N, N$-trimethylaminomethyliodide) chlorin $e_{6}$ 13(1)- $N$ '-methylamide-15(2),17(3)-dimethyl ester, 229 - 3(1),3(2)-bis( $N, N, N$-trimethylaminomethyliodide) chlorin $e_{6} 13(1)-N^{\prime}-\left(2-N^{\prime \prime}, N^{\prime \prime}, N^{\prime \prime}-\right.$ trimethylammonioethyliodide)amide-15(2),17(3)-dimethyl ester, 230 - chlorin $e_{6} 13(1)-N$-(2-myristoxyethyl)amide-15(2), 17(3)-dimethyl ester, 231 - 3(1),3(2)-bis(N,N,N-trimethylaminomethyliodide) chlorin $e_{6}$ 13(1)- $N^{\prime}$-(2-myristoxyethyl)amide-15(2), 17(3)-dimethyl ester.

is known to provide a pronounced bactericidal effect towards various pathogens for the well-established antiseptic agent "Miramistin" belonging to cationic surfactants. ${ }^{[1076]}$

\section{Singlet Oxygen Quantum Yield}

We have compared the singlet oxygen quantum yields $\gamma_{4}$ estimated from direct singlet oxygen time-resolved photoluminescent measurements ${ }^{[1064,1069,1073,1077]}$ for chlorophylltype PSs containing various functional groups in benzene, 1-octanol and pyridine (Table 23). These quantities are found to be nearly independent of the sensitizer structure and vary from 0.47 to 0.67 in non-aqueous solvents. Hence, mean value $\gamma_{4}$ can be roughly estimated as $0.58 \pm 0.03$ for numerous charged or uncharged chlorophyll-ligand PS, not containing special active groups. This quantity is in a good agreement with other known literature results ${ }^{[915,1078]}$ and should be valid for compounds 230 and 231. Literature data demonstrate the aggregation of chlorin PSs in water followed by dramatic decrease of their singlet oxygen quantum yields and PDT efficiency. ${ }^{[1078]}$ That is one of the reasons for using
Table 23. Quantum yield of singlet oxygen ${ }^{1} \mathrm{O}_{2}$ for several chlorophyll pigments in non-aqueous solvents. ${ }^{[1064,1069,1073,1077]}$

\begin{tabular}{rccc}
\hline \multirow{2}{*}{ PS } & \multicolumn{3}{c}{ Solvent } \\
\cline { 2 - 4 } & Benzene & 1-Octanol & Pyridine \\
\hline $\mathbf{2 2 6}$ & 0.52 & 0.47 & 0.60 \\
$\mathbf{2 2 7}$ & - & 0.65 & 0.56 \\
$\mathbf{2 2 8}$ & - & 0.60 & 0.55 \\
$\mathbf{2 2 9}$ & - & 0.53 & 0.59 \\
$\mathbf{A}^{\mathrm{a}}$ & - & 0.52 & - \\
$\mathbf{B}^{\mathrm{b}}$ & 0.62 & 0.50 & 0.58 \\
$\mathbf{C}^{\mathrm{c}}$ & 0.61 & 0.67 & 0.66 \\
\hline
\end{tabular}

${ }^{a} 13(1)-N$-piperazinyl amide chlorin $e_{6} 15(2), 17(3)$-dimethyl ester; ${ }^{\mathrm{b}} 13(1)-N$-methylamide chlorin $e_{6} 15(2)$-diethylene glycol 17(3)-methyl ester; ${ }^{\mathrm{c}}$ chlorin $e_{6}$ trisodium salt;

Note: an experimental uncertainty is within $10 \%$. The mean value is $0.58 \pm 0.03$. 
chlorin PSs with a relatively high hydrophilicity to decrease their aggregation tendency.

\section{Affinity towards the Lipid-like Compartment}

It is known ${ }^{[1075]}$ that the human body may be viewed as a series of lipid-like membrane barriers dividing water-like filled compartments. The inner hydrophobic core of a cytoplasmic membrane is usually modeled by 1 -octanol. Similarly, aqueous phosphate buffer is used to mimic the aqueous filled compartment. ${ }^{[1075]}$ Hydrophilicity/lipophilicity of PSs may play a crucial role in their distribution between normal and malignant tissue, which is very important for treating superficially located tumors. ${ }^{[1074]}$ This point seems to be important for APDT, however, it reveals several peculiarities. Figure 106 indicates that a cytoplasmic membrane of bacteria which is modeled by OctOH is separated from an external environment by porous peptidoglycan layer (Gram-positive pathogens) and also by a negatively charged outer lipopolysaccharide membrane (Gram-negative pathogens). Since PSs are administrated usually locally in APDT, to provide better photoinactivation of Gram-negative pathogens it is reasonable to apply cationic PSs which are able to bind to the outer membrane. It seems, however, important that PS molecules contain both charged and hydrophobic parts and are not aggregated.

Table 24 compares equilibrium molalities of PSs solutions in both aqueous and a lipid-like phases as well as corresponding partition coefficients. In principal, if a solute molecule is fully ionized in a dilute aqueous solution as it is expected for compounds 227-229, the distribution coefficient should be used instead of the $P$ value. ${ }^{[1069]}$ However, taking into account the unknown degree of dissociation of PSs in a saline buffer only the $P$ values can be considered.

In fact, for methylpheophorbide $a$ (compound 226) the $P$ value is very large and strongly temperature dependent indicating its high affinity to a lipid-like phase. In contrast, the attachment of one, two or three hydrophilic trialkylammonia groups to a chlorin $e_{6}$ molecule (compounds 227-229) results in dramatic decrease of partition coefficients becom- ing nearly temperature independent. Two cationic groups of compound $\mathbf{2 2 8}$ favor the PS transfer to an aqueous phase, however, the attachment of the myristic acid residue strongly increases PS hydrophobicity and causes in very tiny solubility of compound $\mathbf{2 3 1}$ in water or aqueous phosphate buffer. Our data ${ }^{[1071]}$ clearly show that both neutral (compound 230) and cationic (compound 231, Table 24) PSs do not transfer from OctOH to PSB.

Thus, to enhance their affinity towards a water-like phase, we have exploited here the non-ionic surfactant Tween 80 (polysorbate 80 ) which is believed to be a safe and efficient stabilizing agent for aqueous formulations of many drugs. ${ }^{[1079,1080]}$ This PS formulation containing both Tween 80 and about one percent of ethanol are found to be stable for a long time. Our spectroscopic study ${ }^{[1070]}$ indicates that chlorin PSs efficiently bind to Tween 80 micelles $\left(K_{\mathrm{b}} \sim 10^{5}-10^{6}\right)$, the macrocycle being mainly located in the intermediate positions of a micelle nearby hydrophilic head groups. Apparently, it suggests that the apolar myristic residue should occupy a position into the inner core of the Tween 80 micelle, while two cationic groups are to be oriented onto a micellar surface. Hence, these charged groups are, in principal, able to bind to the negatively charged outer membrane of Gram-negative pathogens. Additionally, when a PS molecule is released from a micelle, the myristic acid residue should induce a significant disruption of a bacterial wall due to the hydrophobic interaction with its apolar units. Moreover, PS molecules bound to the surfactant micelle stay in a monomer form that provides efficient generation of singlet oxygen. ${ }^{[1078]}$

\section{Inactivation of Archival Pathogens in vitro}

The microorganisms used in our research were all classified as biosafety level two. This means that although these species are capable of causing any disease in humans, they present no health hazard to laboratory personnel when standard universal precautions are taken in handling. ${ }^{[1061]}$ The preparation of liquid bacterial cultures and PS solutions as well as photoinactivation experiments in vitro were

Table 24. Mean equilibrium molalities and partition coefficients for compounds 226-229, 231 in the 1-octanol/phosphate saline buffer biphasic system at $298-318 \mathrm{~K} \cdot .^{[1069,1071,1073]}$

\begin{tabular}{|c|c|c|c|c|c|}
\hline & \multicolumn{5}{|c|}{ PS } \\
\hline & $226^{\mathrm{a}}$ & 227 & 228 & 229 & 231 \\
\hline & \multicolumn{5}{|c|}{$298.15 \mathrm{~K}$} \\
\hline$\left(m_{\mathrm{OctOH}} / m_{\mathrm{aq}}\right) \cdot 10^{5}$ & - & $8.163 / 0.9523$ & $2.627 / 2.533$ & $1.472 / 1.525$ & $6.52 / 6.54$ \\
\hline \multirow[t]{2}{*}{$P$} & $210.1 \pm 6$ & $8.6 \pm 0.2^{b}$ & $1.04 \pm 0.02$ & $0.97 \pm 0.03$ & - \\
\hline & \multicolumn{5}{|c|}{$308.15 \mathrm{~K}$} \\
\hline$\left(m_{\mathrm{OctOH}} / m_{\mathrm{aq}}\right) \cdot 10^{5}$ & - & $8.361 / 0.738$ & $1.981 / 1.457$ & $1.412 / 1.464$ & $6.53 / 6.54$ \\
\hline \multirow[t]{2}{*}{$P$} & $151.1 \pm 1$ & $11.3 \pm 0.3$ & $1.36 \pm 0.03$ & $1.04 \pm 0.02$ & - \\
\hline & \multicolumn{5}{|c|}{$318.15 \mathrm{~K}$} \\
\hline$\left(m_{\mathrm{OctOH}} / m_{\mathrm{aq}}\right) \cdot 10^{5}$ & - & $8.064 / 0.5631$ & $1.939 / 1.212$ & $1.362 / 1.224$ & $6.53 / 6.54$ \\
\hline$P$ & $78.4 \pm 2$ & $14.3 \pm 0.3$ & $1.61 \pm 0.03$ & $1.11 \pm 0.03$ & - \\
\hline
\end{tabular}

${ }^{\mathrm{a}}$ abbreviations are identical to those in Table $23,{ }^{\mathrm{b}}$ - errors represent the twice standard deviation. 
identical to those described in our recent papers. ${ }^{[1069,1071]}$ In the first serious of experiments ${ }^{[1066,1069,1071]}$ we have studied the antimicrobial activity of our pigments towards the following opportunistic microorganisms - Gram-positive bacterium Staphylococcus aureus (6538-P ATCC=209-P FDA strain), Gram-negative bacterium Escherichia coli (M-17 strain) and yeast-like fungi Candida albicans (CCM 8261 ATCC 90028 strain). The results obtained have indicated that diluted pigment solutions provide full photodynamic inactivation of Gram-positive and fungi cells. ${ }^{[1066]}$ However, Gram-negative pathogen E. coli has revealed strong resistance both to the dark and light treatment. This result seems to be rather surprising because at least di- and tricationic PSs should demonstrate the enhanced affinity of the outer membrane of Gram-negative pathogens. Hence, either the intrinsic charge of a PS molecule is still insufficient to penetrate this barrier along or PS concentration/incubation time is too small to allow these species to cross. To provide a better penetration through the outer cell membrane we have added $\mathrm{Na}_{2} \mathrm{H}_{2}$ Edta and/or Tween 80 to each pigment solution and also two times increased the pigment concentration from $5 \cdot 10^{-5}$ to $10^{-4} \mathrm{~mol} / \mathrm{kg}$. These measures are seen from Table 25 to provide full inactivation of Gramnegative bacteria both with and without illumination. This result seems to be excellent taking into account the solute concentration and the large CFU numbers modeling wound infection. ${ }^{[1081]}$ It also indicates that dark toxicity induced by ethylenediamine tetraacetate and iodide-ions is rather high, although we see that $E$. coli survives in a $0.2 \%$ solution of $\mathrm{Na}_{2} \mathrm{H}_{2}$ Edta.

Then, to decrease dark toxicity and to observe a true photodynamic effect we have used more diluted solutions and replaced $\mathrm{Na}_{2} \mathrm{H}_{2}$ Edta by biocompartible surfactant Tween
80 (Table 25). Illumination of solutions containing pigments 227 and 228 provides almost the total killing of both Escherichia coli and Candida albicans which are well-tolerated to the treatment in the dark. Three-charged pigment 229 is seen to be the most toxic PS executing fatal damage towards Gram-negative bacteria both in the dark and under illumination. Neutral myristoil-substituted compound 230 with the concentration of $0.5 \mathrm{mmol} / \mathrm{kg}$ provides efficient killing both in the dark and under illumination. The fivefold decrease of the PS concentration leads to the appearance of photodynamic inactivation towards Staphylococcus aureus and Escherichia coli. Compound 231 containing two cationic groups is non-toxic in the dark towards Escherichia coli and Candida albicans but induces the total killing of the Gram-positive pathogen Staphylococcus aureus. ${ }^{[1071]}$

\section{Inactivation of Nosocomial Antibiotic Resistant Gram- negative Bacteria in vitro}

One of the most remarkable advantage of photodynamic inactivation is that it works equally well regardless of the antibiotic resistance status of the microbial cells. ${ }^{[1060]}$ The nosocomial antibiotic resistance microorganisms presented here contain Escherichia coli lactosonegative and Pseudomonas aeroginosa biofilm-prone strains. ${ }^{[1071]}$

Table 26 illustrates that both neutral and dicationic chlorins containing the myristic acid residue (compounds 230, 231) reveal pronounced toxicity towards Pseudomonas aeroginosa. As for Escherichia coli, compound 230 demonstrates the light dose-dependent killing, the dose of $80 \mathrm{~J} / \mathrm{cm}^{2}$ being quite enough to provide seven logs of killing (complete elimination). To observe solely photodynamic inactivation of Pseudomonas aeroginosa by compound 231, we have

Table 25. In vitro bactericidal efficacy of PSs 227-230 and additives towards archival microorganisms in the dark and under illumination. ${ }^{[1069,1071]}$

\begin{tabular}{|c|c|c|c|c|c|c|}
\hline \multirow[b]{2}{*}{ PS } & \multicolumn{6}{|c|}{ Microorganism, the initial number of CFU from here on was $10^{7}$} \\
\hline & $\begin{array}{c}\text { Staphylococcus } \\
\text { aureus }\end{array}$ & $\begin{array}{l}\text { Escherichia } \\
\text { coli }\end{array}$ & $\begin{array}{l}\text { Candida } \\
\text { albicans }\end{array}$ & $\begin{array}{c}\text { Staphylococcus } \\
\text { aureus }\end{array}$ & $\begin{array}{c}\text { Escherichia } \\
\text { coli }\end{array}$ & $\begin{array}{l}\text { Candida } \\
\text { albicans }\end{array}$ \\
\hline & \multicolumn{3}{|c|}{ Dark } & \multicolumn{3}{|c|}{$40 \mathrm{~J} / \mathrm{cm}^{2}$ light dose } \\
\hline $227^{\text {a }}$ & 0 & 0 & 0 & 0 & 0 & 0 \\
\hline $227^{\mathrm{b}}$ & 0 & $8.5 \cdot 10^{6}$ & 0 & 0 & 0 & 0 \\
\hline $228^{a}$ & 0 & 0 & 0 & 0 & 0 & 0 \\
\hline $\mathbf{2 2 8}^{\mathrm{b}}$ & 0 & $10^{7}$ & $7.5 \cdot 10^{6}$ & 0 & 0 & $5 \cdot 10^{5}$ \\
\hline $229^{a}$ & 0 & 0 & 0 & 0 & 0 & 0 \\
\hline $229^{b}$ & - & 0 & - & - & 0 & 0 \\
\hline $230^{c}$ & 0 & 0 & 0 & 0 & 0 & 0 \\
\hline $230^{\mathrm{d}}$ & $10^{7}$ & $10^{7}$ & 0 & 45 & 0 & 0 \\
\hline $1 \%$ of Tween 80 & $10^{7}$ & $10^{7}$ & $10^{7}$ & $10^{7}$ & $10^{7}$ & $10^{7}$ \\
\hline $0.5 \%$ of $\mathrm{Na}_{2} \mathrm{H}_{2} \mathrm{Edta}$ & 0 & 0 & 0 & 0 & 0 & 0 \\
\hline $0.2 \%$ of $\mathrm{Na}_{2} \mathrm{H}_{2}$ Edta & 0 & $10^{7}$ & 0 & 0 & $10^{7}$ & 0 \\
\hline
\end{tabular}

${ }^{\mathrm{a}} m=0.0001 \mathrm{~mol} / \mathrm{kg}+0.1$ mass. $\% \mathrm{Na}_{2} \mathrm{H}_{2} \mathrm{Edta} ;{ }^{\mathrm{b}} \mathrm{m}=0.0005 \mathrm{~mol} / \mathrm{kg}+1$ mass. $\%$ Tween $80 ;{ }^{\mathrm{c}} m_{\mathrm{PS}}=0.0005 \mathrm{~mol} / \mathrm{kg}+5$ mass $\%$ of EtOH $+0.5 \mathrm{mass}$ $\%$ of Tween $80 ;{ }^{\mathrm{d}} m_{\mathrm{PS}}=0.0001 \mathrm{~mol} / \mathrm{kg}+5$ mass $\%$ of EtOH +0.5 mass $\%$ of Tween 80 .

Note: the concentrations of PSs and additives are given from here on after mixing with microbial suspension. The original cell suspension (absolute control) provides $100 \%$ growth in the dark. 
Table 26. In vitro bactericidal efficacy of compounds 230, 231 compared with the anionic photosensitizer "Fotolon" [1082] towards nosocomial antibiotic resistant Gram-negative bacteria (survival CFU) in the dark and under illumination.

\begin{tabular}{|c|c|c|c|c|}
\hline \multirow[b]{2}{*}{ PS } & \multicolumn{4}{|c|}{ Microorganism } \\
\hline & $\begin{array}{c}\text { E. coli } \\
{\text { lact. } \text { negative }^{\text {a }}}^{\text {a }}\end{array}$ & $\begin{array}{l}\text { Pseudomonas } \\
\text { aeroginosa } 1^{\text {a }}\end{array}$ & $\begin{array}{l}\text { Pseudomonas } \\
\text { aeroginosa } 2 \text { a }\end{array}$ & $\begin{array}{l}\text { Pseudomonas } \\
\text { aeroginosa } 2 \mathrm{~b}\end{array}$ \\
\hline & \multicolumn{4}{|c|}{ Dark } \\
\hline 230 & $10^{7}$ & 0 & $10^{7}$ & - \\
\hline 231 & 0 & 0 & 0 & $10^{7}$ \\
\hline "Fotolon" c & $10^{5}$ & $10^{5}$ & $10^{5}$ & - \\
\hline
\end{tabular}

$40 \mathrm{~J} / \mathrm{cm}^{2}$ light dose

\begin{tabular}{lcccc}
230 & $10^{2}$ & 0 & 0 & - \\
231 & 0 & 0 & 0 & 0 \\
\hline
\end{tabular}

$80 \mathrm{~J} / \mathrm{cm}^{2}$ light dose

230

231

"Fotolon" c
0

0

$510^{2}$
0

0

$210^{3}$
0

0

$10^{4}$

${ }^{\mathrm{a}} m_{\mathrm{PS}}=0.0005 \mathrm{~mol} / \mathrm{kg}+5$ mass $\%$ of EtOH +0.5 mass $\%$ of Tween $80+0.1$ mass $\%$ of $\mathrm{Na}_{2} \mathrm{H}_{2} \mathrm{Edta} ;{ }^{\mathrm{b}} 0.0004 \mathrm{~mol} / \mathrm{kg}($ compound 231$)+5 \mathrm{mass} \%$ of EtOH +0.5 mass $\%$ of Tween $80 ;{ }^{\text {c }}$ The dosage form of "Fotolon" contained chlorin $\mathrm{e}_{6}$ with concentration of $500 \mathrm{mg} / \mathrm{l}$, which was equivalent to PS molality of $0.00075 \mathrm{~mol} / \mathrm{kg}$. The incubation time and the light dose were equal to $30 \mathrm{~min}$ and $120 \mathrm{~J} / \mathrm{cm}^{2}$, respectively. The $E$. coli strain was from the American type culture collection (ATCC), while both Pseudomonas aeroginosa strains were isolated from wound infections in a hospital. The initial CFU number was $10^{5} \cdot[1082]$

slightly decreased its concentration and totally excluded $\mathrm{Na}_{2} \mathrm{H}_{2}$ Edta from the PS formulation. These manipulations allow to avoid dark toxicity and lead to complete elimination with the fluence of $40 \mathrm{~J} / \mathrm{cm}^{2}$.

Table 26 compares photoinactivation efficacy of cationic, neutral and anionic PSs. It is clear that "Fotolon" being the molecular complex of anionic chlorin $e_{6}$ with its carrier polyvinyl pyrrolidone ${ }^{[1083]}$ is not able to eradicate Gram-negative bacteria. Although the PS concentration and the light dose are significantly larger than those for our chlorins, the killing is significantly reduced. The most probable reason is low affinity of anionic PSs to the outer membrane of Gram-negative pathogens that limits their application in APDT. Thus, both PSs containing the myristic acid residue seem to be perspective candidates to study photodynamic inactivation of pathogens in vivo using the animal model of a localized wound infection. For further research we have chosen compounds 227, 231 which reveal sufficient affinity towards a lipid-like environment and high stability in aqueous surfactant solutions due to the presence of cationic groups and, what is also important, each of them contains iodide ions which are responsible to potentiate microbial killing due to photoproduction of reactive iodine radicals. $^{[1060]}$

\section{Inactivation of Nosocomial Antibiotic Resistant Gram- negative Bacteria in the Model of Wound Infection}

The modeling of APDT of wound infections in vivo has been performing in a similar way as it was recommended by Hamblin et al. ${ }^{[1061]}$ In our study we have used the female Wistar rats at three-four months of age weighting on average 250-300 g. These animals were provided by the vivarium of Ivanovo State Medical Academy (ISMA) and all procedures with them were approved by the Ethic committee of ISMA (the approval number is ISMA EC 2017.25.10). The description of all manipulations with animals and hospital-acquired pathogens is given elsewhere. ${ }^{[1071]}$

The results of photoinactivation of two Gram-negative bacteria Escherichia coli lactosonegative and Pseudomonas aeroginosa inoculated onto a burn wound are given in Table 27. The efficacy of the neutral PS towards hospital-acquired Gram-negative pathogens is lower both in vitro and in vivo than the effect of the cationic PS. ${ }^{[1079]}$ Table 26 illustrates similar photo-induced antibacterial behavior of chlorins with one or two positively charged groups (compounds 227 and 231), where myristoil-substituted dicationic PS 231 is just slightly more effective. Thus, both compounds provide complete in vivo elimination of Pseudomonas aeruginosa (Table 27). The photodynamic effect towards Escherichia coli is rather moderate and slightly exceeds two $\log$ s of killing. This in vivo PDT effect obtained in the model of wound infection towards antibiotic-resistant Gramnegative nosocomial strains should be considered as quite impressive. The own bactericidal effect of the skin under experimental conditions chosen is also estimated in 1.0-1.5 logs of bacteria eradication.

The results presented above clearly indicate that PSs investigated are to be of particular interest for APDT. They are able to generate reactive oxygen species in a liquid phase and penetrate through bacterial membranes. Microbiological studies show that nearly all the pigments provide essential damage towards archival and hospital-acquired pathogens both in vitro and in vivo. The addition of certain agents, enhancing the outer membrane permeability, to PSs solutions provides the excellent photodynamic effect. Thus, 
Table 27. In vivo bactericidal efficacy of compounds 227 and 231 towards Escherichia coli and Pseudomonas aeruginosa after illumination with the dose of $80 \mathrm{~J} / \mathrm{cm}^{2}$.

\begin{tabular}{lccc}
\hline \multirow{2}{*}{$\begin{array}{c}\text { Microorganism, } \\
10^{7} \mathrm{CFU} / \text { burn area }\end{array}$} & \multicolumn{3}{c}{ Compound $^{\mathrm{b}}$} \\
\cline { 2 - 4 } & $\begin{array}{c}\text { Control, } \\
\text { light alone }\end{array}$ & $\mathbf{2 2 7}$ & $\mathbf{2 3 1}$ \\
\hline $\begin{array}{l}\text { Escherichia coli } \\
\text { lactosonegative }\end{array}$ & $2.6 \cdot 10^{5}$ & $3.5 \cdot 10^{3}$ & $2.2 \cdot 10^{3}$ \\
$\begin{array}{l}\text { Pseudomonas } \\
\text { aeruginosa } 2\end{array}$ & $3.7 \cdot 10^{5}$ & 0 & 0 \\
\hline
\end{tabular}

${ }^{a}$ bacteria growth in a cultural liquid was equal to $7 \cdot 10^{6}$ and $3 \cdot 10^{7}$ CFU for Escherichia coli and Pseudomonas aeruginosa, respectively; ${ }^{\mathrm{b}}$ the PS gel dosage form contained $\sim 0.25 \%$ of PS, 10 mass $\%$ of glycerol, 2 mass \% of DMSO, 1 mass \% of Tween $80,0.1$ mass $\%$ of $\mathrm{Na}_{2} \mathrm{H}_{2}$ Edta and 1.1 mass $\%$ of sodium alginate. The amount of PS added onto each burn was of $0.6-0.7 \mu \mathrm{mol}$.

the pigments studied here can be, in principal, applied as certain sensitizers for treating localized superficial infections. Ways to enhance photodynamic inactivation of antibiotic-resistant Gram-negative pathogens and their biofilms will be highlighted in future works.

\subsection{Polymeric Porphyrin-Containing Systems for Antimicrobial Photodynamic Therapy}

The progressing growth of the microbes' resistance to antibacterial drugs, first of all, antibiotics, observed in the last years, acquires an increasingly serious character. As a result, application of whole groups of such drugs becomes impossible in the treatment of infections caused by antibiotic-resistant strains of microorganisms.

A new direction of photodynamic therapy - antibacterial photodynamic therapy (APDT), or photodynamic inactivation of bacteria, viruses, and fungi may become a full alternative of antibiotic therapy in the treatment of localized infections. ${ }^{[1084,1085]}$ APDT is currently used in the treatment of acute and chronic inflammatory processes in otolaryngology, dentistry, surgery (in the treatment of purulent wounds), in burn care (in the treatment of complicated burns), gastroenterology. ${ }^{[1086,1087]}$ In should be noted that the wound and burn infections are the most serious complications by their lethality, the surgical infection being observed in $35-45 \%$ of such patients within the general structure of surgical diseases. ${ }^{[1088-1090]}$

The APDT establishment as a new direction for the application of photosensitizers (PS) in the photodynamic treatment of pathogenic bacterial cultures and the search for new PS types for those purposes is associated, first of all, with the studies of the research group led by Prof. Michael R. Hamblin (Wellman Center of Photomedicine, Boston, Massachusetts, Harvard Medical School), including those related to the photodynamic treatment of pathological cell cultures with the use of cationic and anionic photosensitizers. ${ }^{[1091-1094]}$ A number of key studies related to the creation of new types of PS systems using polymer carriers for PDT of tumors and local infections have been performed at the Roswell Park Comprehensive Cancer Center in Buffalo (Institute of Lasers, Photonics and Biophotonics, SUNY) in the group of Prof. Ravindra K. Pandey. ${ }^{[1095-1097]}$ One should also note the pioneer studies performed in the group of Dr. S. Bacci (Dermatological Dpt Casa di Cura "Santa Chiara", Florence, Italy) on the effect of the PS structure on the efficiency of the photodynamic action on pathogenic microorganisms in the treatment of chronic venous ulcers. ${ }^{[1098]}$

Both porhyrin-based PSs due to their low toxicity and high efficiency in the photogeneration of reactive oxygen species (ROS) ${ }^{[1099]}$ and non-porphyrin PSs (phenotiazines, acridines) due to their antimicrobial activity ${ }^{[100]}$ are used in APDT. It has been found in the last years that porphyrin PSs may accumulate in pathogenic microorganisms (similar to tumor cells), ${ }^{[101]}$ that initiated application of APDT for the treatment of hard-to-heal purulent wounds, trophic ulcers, fungal lesions. ${ }^{[1102,1103]}$ To date, there exists already a positive experience of the APDT use in the treatment of localized microbial infections, ${ }^{[104,1105]}$ a research on the APDT action on bacterial cultures is being conducted. ${ }^{[1106,1107]}$

An important APDT advantage is an opportunity of treating antibiotic-resistant strains of bacteria and fungi, ${ }^{[1108,1109]}$ since the multivariant character of the oxidative destruction of microbial target cells hinders the development of their resistance to the sequential cycles of the photodynamic treatment. ${ }^{[1110,1111]}$ APDT appears to be fatal even for antibiotic-resistant strains of Staphylococcus aureus, Escherichia coli and other microbes. Besides, the antimicrobial APDT action does not diminish with time during a prolonged application, in particular, when treating chronic infectious processes. The cytoplasmic membrane damage is believed to be the main mechanism for the death of pathogens in APDT, while the destruction of proteins and DNA plays only an auxiliary role. ${ }^{[112]}$

In antimicrobial PDT, a surface coating of the wound with the photosensitizer and a low-energy laser radiation with the power density of $25-30 \mathrm{~J} / \mathrm{cm}^{2}$ are usually used, since the microbial dissemination in the wound decreases and regeneration and epithelization are enhanced already at such conditions. ${ }^{[1113-1115]}$

The APDT objects are anaerobic and aerobic Grampositive and Gram-negative bacteria, as well as yeast and filamentous fungi. ${ }^{[116]}$ Irradiated triplet-excited PS molecules localized in the microbial cell membranes generate ROS effectively destroying bacterial cell walls and causing the death of the pathogenic microflora. ${ }^{[117]}$

An important aspect of antimicrobial PDT is the problem of its selectivity towards certain types of pathogenic microbes $^{[1085]}$ that may be achieved due to specific binding of PS to certain fragments of pathological cells. In particular, cationic PSs are used for selective binding to bacterial cell membranes, including those of Gram-negative bacteria, which are the most resistant to the photodynamic treatment. [1118,1119] Same purposes are also followed when using PS within macromolecular conjugates based on covalent binding between PS and fragments of biomolecules (antibodies, aminoacids, liposomes, polypeptides with the affinity towards specified target molecules), providing the photosensitizer penetration into the cell. In particular, application of chlorin $e_{6}$ conjugates with poly-L-lysine has been reported to allow decreasing the illumination dose and enhancing 
the PDT efficiency in the in vitro experiments on the PDT of the pathogenic microflora of the oral cavity. ${ }^{[1120]}$

However, the use of PS systems containing fragments of biologically active macromolecules has obvious limitations. These limitations are related not only to the difficult achieving a reproducible synthesis of such systems due to unavoidable biodiversity of the used fragments, but also to the high cost of such type of substances which restricts the opportunities for their wide application in the medicine. Problems arise also with purely scientific aspects - difficulties in studying the PS-mediated mechanisms on models, what significantly impedes a targeted search of new functionally oriented PS systems.

Therefore, the basic prospects of creation of new PS systems should evidently be linked to the search of polymerbased PS systems. ${ }^{[62]}$ The diversity of the existing types of water-soluble polymers, as well as the possibility to preset their molecular mass distributions, to create a various degree of hydrophobicity and to vary the charge density distribution open broad opportunities of tuning the properties, functionally important for such systems', - dissolution and distribution in pathological tissues of different genesis. The latter may determine the specific kinetics of the photosensitized processes initiated in vivo with the corresponding PS localization. At the same time, covalent or noncovalent (in particular, with coordinate bonds forming) PS binding to polymeric carriers may lead to reduction of the PDT side effects, ${ }^{[1121,1122]}$ in particular, due to the depression of PS binding to normal tissues, thus improving the PS bioavailability in pathological tissues and, hence, the APDT efficiency. It should be noted here, that the creation of polymercontaining PS does not exclude a possibility of using biologically active substances with their noncovalent binding to the created PS system. For example, to facilitate the PS penetration through microbial cell walls, which are usually barely passable for antibacterial agents, ${ }^{[1123]}$ the PS (deuteroporphyrin) treatment with polymyxin $\mathrm{B}$, a polycationic peptide, appeared to be efficient. ${ }^{[124]}$

Below we present the results of the studies on the development of photosensitizing APDT systems which have been conducted in the last years at the Federal Research Center of Chemical Physics of RAS. The PS systems were based on porphyrin PS (PPS) complexes with amphiphilic polymers (AP), as well as on ternary complexes of PSAP-polysaccharides (PSc), in which chitosan and sodium alginate were mainly used, due to their biocompatibility, bactericidal properties and biodegradability determining the possibility of their medicinal use. It is of note, that chitosan and chitosan-based compositions are applied in the treatment of wounds and burns, ${ }^{[1124,1125]}$ with bactericidal effect being amplified by their use in combination with traditional drugs, e.g., chlorhexidine or antibiotics. ${ }^{[1126-1128]}$

It has been demonstrated that the interaction of watersoluble porphyrins with certain AP, including Pluronics, leads to an increase in the PPS photosensitizing activity in the model photooxidation of organic substrates (tryptophan), ${ }^{[1129,1130]}$ as well as to enhanced efficiency of the photodynamic treatment of tumor cell cultures (Ehrlich carcinoma, human melanoma) and experimental tumors of laboratory animals. ${ }^{[1131,1132]}$ The size of the "polymer effect", manifesting itself as a 10-20 fold decrease of the minimal
PPS concentration to cause the $50 \%$ death of tumor cells of various types during PDT sessions, depends on the nature and molecular weight of the polymer. It is important that the amphiphilic polymers themselves did not have any significant photocytotoxic activity. At the same time, as it was shown in the experiments on laboratory animals, ${ }^{[1133,1134]}$ for PDT using PS systems based on water-soluble chlorin, photoditazine and AP, which exhibited the highest activity in the photosensitized oxidation of tryptophan (estimated by the reduced rate constant), the healing of purulent wounds, complicated burns and vascular inflammatory diseases proceeded more efficiently at all stages as compared to that during traditional PDT sessions without application of the polymer components.

The choice of the reaction of tryptophan photooxidation in water as a basic model process to test the photodynamic activity of created PS systems is determined by the fact that this reaction is used for testing the activity of PS in singlet oxygen generation in the aqueous medium. ${ }^{[1135]}$ The detected correlation of the APDT efficiency with the rate constant of tryptophan photooxidation when using one and the same (by the composition) photosensitizing system suggests, that the main agent oxidizing substrate is singlet oxygen. The crucial role of singlet oxygen in the studied photosensitized oxidation of organic substrates of different nature and the role of AP as a factor increasing the photosensitizing activity are also indicated by the results of the study on the quantum yield of singlet oxygen ${ }^{1} \mathrm{O}_{2}$ photogeneration in the $\mathrm{D}_{2} \mathrm{O}$ medium. ${ }^{[1136]}$ It should be mentioned here, that the choice of heavy water in such an experiment is determined by the longer lifetime of singlet oxygen molecules in this medium.

As it was found by ${ }^{1} \mathrm{H} \mathrm{NMR},{ }^{[1136]}$ the observed effects might be related to the formation of weakly bound complexes of water-soluble PSs with amphiphilic polymers. It was shown by ${ }^{1} \mathrm{H}$ NMR and fluorescent spectroscopy, that the increase of the PPS activity in the generation of ${ }^{1} \mathrm{O}_{2}$ and photooxidation reactions in the presence of AP in the aqueous phase was associated with the formation of complexes between AP and porphyrins resulting in the dissociation of aggregates formed by porphyrins in aqueous solutions. ${ }^{[1137,1138]}$

Established on the results of the performed experiments on the influence of PPS-AP complexes on the efficiency of the photodynamic treatment of cell cultures, a mechanism for the increase of the PPS efficiency in the AP presence has been suggested. The mechanism is based on an assumption, that PPSs may bind to cellular proteins, that in the absence of AP decrease the porphyrin's photoactivity in the ${ }^{1} \mathrm{O}_{2}$ generation. ${ }^{[139,1140]}$ When studying the activity of photoditazine (Figure 108, 232) complexes with different AP - Pluronic F-127 (a triblock copolymer of oxyethylene and oxypropylene) and polyvinylpyrrolidone (PVP) - in PDT of wounds of laboratory animals at an early stage of wound healing, it was shown, that both complexes, photoditazine- F-127 and photoditazine-PVP, had almost no hemorrhagic effect on the wound, ${ }^{[1141]}$ i.e. the use of an amphiphilic polymer might eliminate the side effects of PDT.

As it was already mentioned, polysaccharides are widely used for medical purposes due to their natural bactericidal properties. Chitosan is polycationic due to pro- 

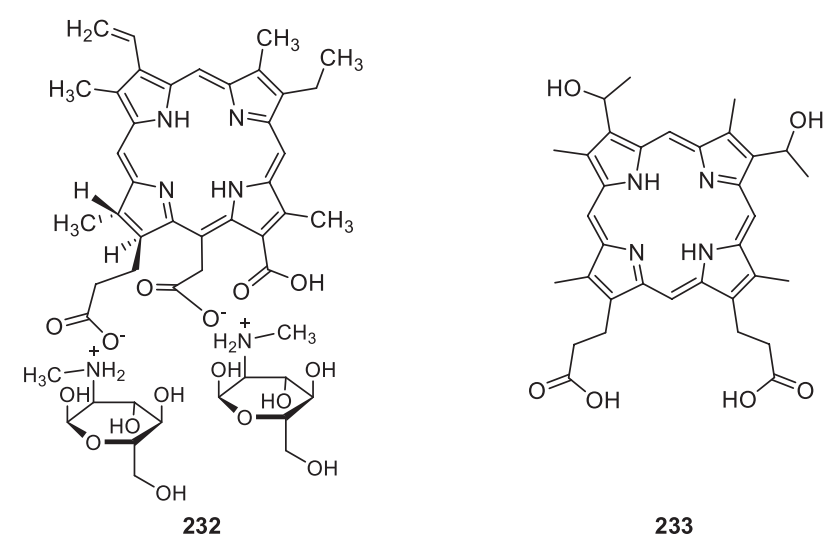

232

233

Figure 108. Structure of di- $N$-methyl-D-glucamine salt of chlorin $e_{6}$ - photoditazin (232), and hematoporphyrin (233).

tonation of the polysaccharide's aminogroups in a weakly acidic medium. It was used as a PS carrier in PDT, and it was shown, that the side effects of PDT, in particular, the hemorrhagic effect, were neutralized in the presence of chitosan during the APDT treatment of model wounds of laboratory animals. ${ }^{[142]}$ In the study, ${ }^{[1143]}$ where Photogem (a hematoporphyrin derivative, Figure 108, 233) was applied as a PS, the PS binding to chitosan reduced its toxicity.

At the same time, the interaction of chitosan as a polycation with porphyrin PSs may decrease their activity in the singlet oxygen generation. ${ }^{[144]}$ This is associated with aggregation of porphyrin molecules in the neighborhood of protonated aminogroups of chitosan. ${ }^{[145]}$ Nevertheless, the photosensitizing activity of PS-chitosan systems in the singlet ${ }^{1} \mathrm{O}_{2}$ oxygen generation may be restored and even boosted by adding amphiphilic polymers (PVP or Pluronic F-127) to the system. ${ }^{[1142,1144]}$ It was shown by ${ }^{1} \mathrm{H}$ NMR that, in the presence of AP, the photosensitizer coordinates with macromolecules of the amphiphilic polymer (through hydrophobic and hydrogen bonds) thus providing dissociation of PS aggregates and, obviously, preventing the PS from interaction with chitosan macromolecules. ${ }^{[146]}$

APDT procedures with the use of ternary PS-chitosanAP systems in the treatment of model infected wounds of laboratory animals resulted not only in the reduction of hemorrhagic manifestations (as it was proven for photoditazine-AP complexes), but also in the significant suppression of the inflammatory reaction with the simultaneous intensification of regenerative-reparative processes in the wound (growth and maturation of the granulation tissue, neoangiogenesis and collagenesis). ${ }^{[147]}$

It should be noted that application of a photodynamic treatment only does not guarantee a stable bactericidal effect and prevention of a further infection of wounds and burns. ${ }^{[117]}$ New opportunities for the enhancement of the photodynamic treatment efficiency are opened by combining APDT with biologically active substances which accelerate the wound healing, proteolytic enzymes and antioxidants being most frequently used as such substances. ${ }^{[148]}$

One of biologically active molecules with both regulatory and regenerative properties is nitric oxide, which is applied in the complex wound treatment. ${ }^{[149]}$ Nitric oxide is a short-living multifunctional signaling molecule govern-

ing intra- and intercellular processes in animal, bacterial and plant organisms and having both promoting (regulatory, protective) and inhibiting effects on the metabolism. In particular, the radical $\mathrm{NO}^{\bullet}$ form plays a vital role in the functioning of the immune, nervous, vascular systems in animals, in the growth and development of plants. ${ }^{[150,1151]}$ A small size and the absence of a charge provide high penetrability of nitric oxide (Figure 109) through the membranes of cells and cell structures.
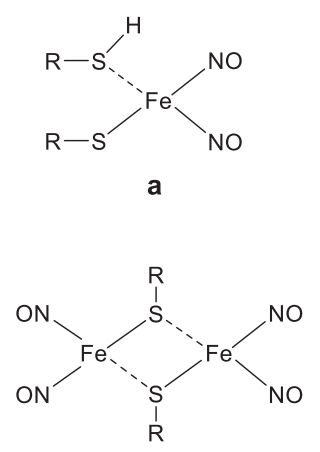

b<smiles>[R]CCC(NC(=O)CCC(N)C(=O)O)C(=O)NCC(=O)O</smiles>

Figure 109. Structure of mono- and bi-nuclear forms of DNIC with thiol-containing ligands.

The characteristic lifetime of nitric oxide in biological tissues may significantly vary. For example, in the renal tissue of rats it is $6.4 \mathrm{~s}$, in the myocardium $-0.1 \mathrm{~s}$, in the blood $(0.05-0.2) \mathrm{s}$, while in deoxygenated water NO molecules are preserved for several days. Therefore, nitric oxide appears an ideal mediator in the short-time auto- or paracrine cell signal exchange. ROS and reactive nitrogen species are found to participate in the development of pathologies associated with the oxidative stress, including atherosclerosis, ischemic heart disease, neurodegenerative diseases, cataract, cancer, diabetes. Besides, the interaction of nitric oxide and its derivatives with ROS results in the formation of reactive metabolites of nitric oxide - peroxynitrite, nitrogen dioxide, $\mathrm{NO}_{2} \mathrm{Cl}$, which are important components of the immune response in the human and animal organisms. ${ }^{[152-1154]}$ Among such processes, the interaction of ROS with dinitrosyl iron complexes (DNIC) are investigated most intensively, since such complexes are capable of being donors of $\mathrm{NO}^{-}$and $\mathrm{NO}^{+}$in biosystems and determining not only cytotoxic, but also regulatory-regenerative processes in live organisms. ${ }^{[155]}$

Since photogeneration of ROS constitutes the basis for oxidative destruction of pathological cells and tissues ${ }^{[1156]}$ (benign and malignant tumors, purulent wounds, trophic ulcers) upon the excitation of a photosensitizer administered to the lesion (PDT, APDT), there is a potential for the search of a combined effect of PDT and DNIC. In other words, one may speak about a combination of the relatively "mild" photodynamic treatment destroying pathological tissues with the simultaneous photoinitiation of the regulatoryregenerative processes resulting from the decomposition of DNIC with thiol-containing ligands and appearance of $\mathrm{NO}^{\circ}$ radicals in the reaction medium. 
In the preliminary experiments on model wounds of laboratory animals additionally inoculated with Gram-positive and Gram-negative bacterial colonies, we have established selective antimicrobial activity of PS-AP-PSc-DNIC systems, containing chitosan as PSc, towards Gram-negative bacteria. ${ }^{[1156]}$ Estimating the general contemporary trends in the APDT development aimed at the enhancement of its efficiency and suppression of side effects, one should conclude that the broad spectrum of the new possibilities related to application of polymeric PS systems, combining PDT with other therapies, as well as the undoubted achievements in the field of PDT application create a basis for a targeted scientific search of photosensitizing polymer systems for APDT.

\subsection{Attenuation of in vivo Toxicity of the Organotin Compound by the Porphyrin Modified with Peripheral 2,6-Di-tert-butylphenol Antioxidant Groups}

Design of multifunctional compounds capable of protecting the organism from heavy metal containing toxins is a challenge in medicinal chemistry. In particular, it is important to design and synthesize the agents that attenuate the toxicity of the anthropogenic tin organic compounds $\mathrm{R}_{\mathrm{n}} \mathrm{SnX}_{4-\mathrm{n}}$. These compounds are generated in vivo in the reaction of biochemical $\mathrm{C} 1$ methylation of inorganic tin containing agents. ${ }^{[1157]}$ Toxicity of organotin compounds is known to be associated with: 1) inhibition of certain enzymes and tubulin due to binding of $\mathrm{Sn}$ atom with $\mathrm{SH}$ groups; ${ }^{[1158,1159]}$ 2) formation of reactive radicals upon the homolytic cleavage of Sn-C bonds; $;^{[1160]} 3$ ) generation of reactive oxygen species (ROS) and induction of oxidative stress followed by damage of cellular structures, etc. ${ }^{[161]}$ Organotin compounds such as $\mathrm{Me}_{3} \mathrm{SnCl}$ naturally formed by $\mathrm{C} 1$ methylation, can inhibit the antioxidant enzymes and subsequently lead to disease. ${ }^{[1162]}$

The free base of the porphyrin containing 2,6-di-tertbutylphenol groups in meso-positions (meso-tetrakis(3,5di-tert-butyl-4-hydroxyphenylporphyrin; $\quad \mathrm{R}_{4}{ }_{4} \mathrm{PH}_{2} \quad$ (234)) is known to attenuate the toxicity of trimethyl tin chloride against the antioxidant enzymes catalase and superoxide dismutase; ${ }^{[1163]}$ this property attributes to a high antioxidant potency of $\mathrm{R}_{4}{ } \mathrm{PH}_{2} \cdot{ }^{[1164,1165]}$

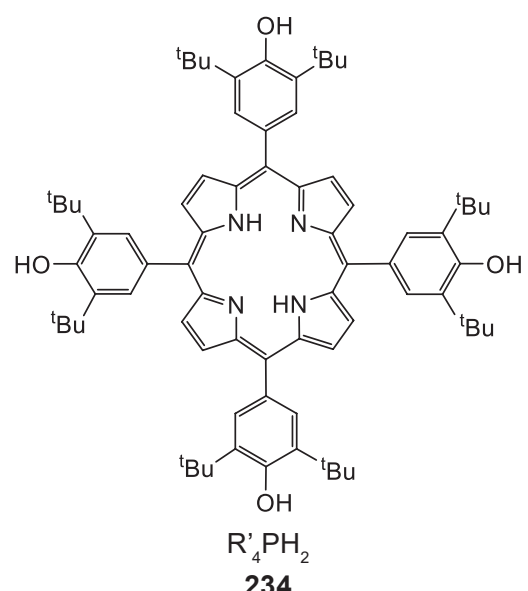

Glutathione (GSH), a component of glutathione reductase/glutathione peroxidase antioxidant system, is a major protector in the body. ${ }^{[166]}$ The reduced equivalents of NADPH in this system are formed in the pentose phosphate pathway of glucose oxidation. This pathway, an alternative to glycolysis, includes glucose-6-phosphate dehydrogenase (G6PD) as a key metabolite. ${ }^{[1167]}$ The ratio of reduced (GSH) and oxidized (GSSG) forms of intracellular glutathione is an important parameter of oxidative stress. A decrease of GSH below its physiological level indicates an altered redox status. The main function of G6PD, which contains $\mathrm{NADP}^{+}$as a coenzyme, is the reduction of $\mathrm{NADP}^{+}$to $\mathrm{NADPH}+\mathrm{H}^{+}$; the latter is needed for transition of oxidized glutathione to the reduced form. These processes make a network that regulates free radical oxidation, glutathione system and G6PD activity. ${ }^{[1168]}$

To address the protective role of $\mathrm{R}_{4}^{\prime} \mathrm{PH}_{2}$ we have synthesized this compound ${ }^{[169]}$ and evaluated its effects on glutathione and G6PD in the presence of $\mathrm{Me}_{3} \mathrm{SnCl}$. The chromatographic purification was performed on a Silica gel column followed by elution with $\mathrm{CHCl}_{3}$-hexane mixture (4:1). Trimethyl tin chloride (Fluka) was used without purification. Solutions of $\mathrm{Me}_{3} \mathrm{SnCl}$ were prepared in bidistilled water immediately before gavage. The dose of $\mathrm{Me}_{3} \mathrm{SnCl}$ in group I was $5 \mathrm{mg} / \mathrm{kg}$ of the body weight. This dose was selected based on literature data and is optimal for metabolic studies in the liver and kidneys. ${ }^{[1170]}$ The solution of porphyrin $\mathrm{R}_{4} \mathrm{PH}_{2}$ for gavage was prepared in $1 \%$ Tween-20, therefore an additional group IV was tested. The dose of $\mathrm{R}_{4} \mathrm{PH}_{2}$ was taken in 1:1 ratio with $\mathrm{Me}_{3} \mathrm{SnCl}$. The solution of $\mathrm{R}_{4}{ }_{4} \mathrm{PH}_{2}$ was injected intraperitoneally (i.p.).

One hundred female rats (Wistar, 200-220 g) were taken into the experiment. All manipulations with animals were performed in accordance with ethical principles of European Convention for the Protection of Vertebral Animals used for Experimental and Other Scientific Purposes (Strasbourg, 1986). After the completion of experiments rats were euthanized in a $\mathrm{CO}_{2}$ chamber.

Rats were divided into the groups:

$\mathrm{I}$ - single gavage of $\mathrm{Me}_{3} \mathrm{SnCl}$;

II $-\mathrm{Me}_{3} \mathrm{SnCl}$ and $\mathrm{R}_{4} \mathrm{PH}_{2}$ i.p. single administration.

III $-\mathrm{R}_{4} \mathrm{PH}_{2}$ i.p. single administration.

IV - $1 \%$ Tween-20 i.p. single administration.

$\mathrm{V}$ - control (bidistilled water gavage; the same volume as in group I).

Animals were monitored for up to 14 days. General parameters such as behavior, nutritional habits, and hair cover were evaluated daily. Rats were weighted once a week. The toxicity of trimethyl tin chloride was determined as a lower physical activity, anxiety, and aggressiveness. No lethality was registered. The rats were euthanized on 1, 7 and 14 days post treatment. The liver and kidneys were immediately removed, rinsed with ice cold saline, homogenized in $0.05 \mathrm{M}$ phosphate buffer with $\mathrm{pH} 7.4$ and $0.1 \mathrm{mM}$ EDTA using an ultrasound homogenizer. ${ }^{[1171]}$ Enzymatic activity and glutathione content were measured in supernatants after centrifugation of tissue homogenates at 2,000 g during $10 \mathrm{~min}$ in a refrigeration centrifuge. The activity of G6PD (EC 1.1.1.40) was determined spectrophotometrically based on $\mathrm{NADP}^{+}$reduction in the presence of $\mathrm{Mg}^{2+}$ at $340 \mathrm{~nm} \cdot{ }^{[1172]}$ GSH concentrations were determined in the reaction with 5,5'-dithiobis-2-nitrobenzoic acid at $412 \mathrm{~nm} .^{[1173]}$

Data were analyzed using Statistica 12.0 (StatSoft Inc., USA). The type of data distribution was determined based 
Table 28. Reduced glutathione GSH in the rat lever and kidneys ( $\mu \mathrm{mol} / \mathrm{g}$ of wet tissue) in the presence of $\mathrm{Me}_{3} \mathrm{SnCl}_{1} \mathrm{R}_{4}{ }_{4} \mathrm{PH}_{2}$ and their mixture (1:1).

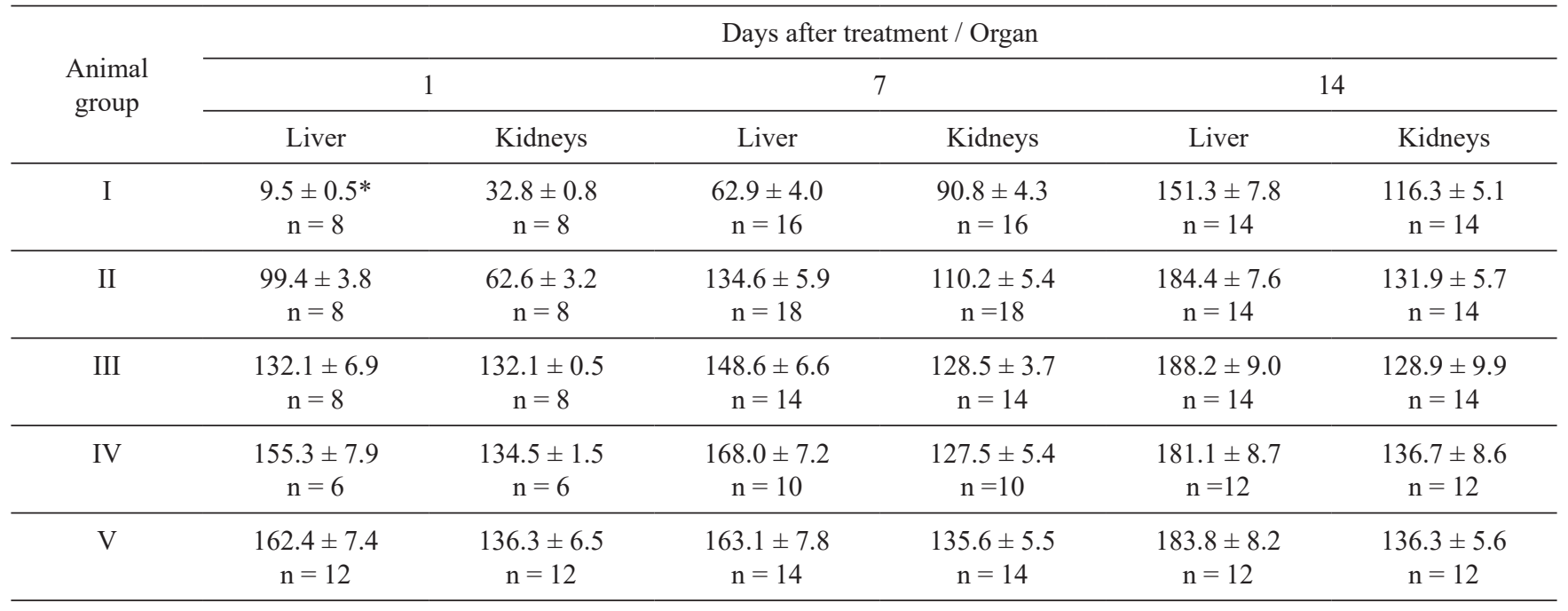

$* \mathrm{M} \pm$ SE. $\mathrm{n}$ is the number of animals per group, $\mathrm{p}$ is statistically significant difference compared to the control cohort $(\mathrm{p}<0.05)$.

on the criteria of Kolmogorov-Smirnov and Shapiro-Wilk. Differences of mean values were estimated using Student's parametrical test with $\mathrm{p}<0.05$ as a measure of statistical difference.

One day after single administration of $\mathrm{Me}_{3} \mathrm{SnCl}$ the level of reduced glutathion was dramatically decreased compared to the control animals (94.2\% and $75.9 \%$ in the liver and kidneys, respectively (Table 28).

By days 7 and 14 the GSH content in group I had risen, but never reached the initial values. In the liver GSH was decreased by $61.4 \%$ and $17.3 \%$, whereas in kidneys these parameters went down by $33.0 \%$ and $14.7 \%$ after days 7 and 14, respectively. The porphyrin $\mathrm{R}_{4} \mathrm{PH}_{2}$ (group III) caused an insignificant decrease of GSH by day 1 , but by day 14 its values were similar to the control. In group II ( $\mathrm{Me}_{3} \mathrm{SnCl}$ in combination with $\mathrm{R}_{4} \mathrm{PH}_{2}$ ) $\mathrm{GSH}$ was decreased by day 1 , although this decrease was less pronounced than after $\mathrm{Me}_{3} \mathrm{SnCl}$ alone.
The amounts of reduced glutathione in the liver or kidneys were by $38.8 \%$ or $54.1 \%$ smaller than the respective values in the control group. By day 7 these differences were even smaller, that is, $17.5 \%$ in the liver and $18.7 \%$ in kidneys. By day 14 GSH levels in the treated and control groups were similar for each organ.

No effect of Tween-20 on GSH was detectable (group IV).

Table 29 shows the results of measurements of G6PD activity. In group I the most pronounced changes were in the liver due to metabolism of $\mathrm{Me}_{3} \mathrm{SnCl}$; less toxic metabolites are excreted by kidneys.

One day after $\mathrm{Me}_{3} \mathrm{SnCl}$ administration, G6PD in the liver was decreased by $83.3 \%$; in kidneys this decrease was less pronounced (40.1\%). These changes partially reverted by day 14 although remained smaller (14.3\% in the liver and $20.1 \%$ in kidneys) than in control rats.

The effect of $\mathrm{R}_{4} \mathrm{PH}_{2}$ in group III became apparent by day 7; after 14 days some increase of G6PD activity

Table 29. Activity of glucose-6-phosphate dehydrogenase G6PD in the rat lever and kidneys ( $\mu \mathrm{mol} / \mathrm{g}$ of wet tissue) in the presence of $\mathrm{Me}_{3} \mathrm{SnCl}, \mathrm{R}_{4}{ }_{4} \mathrm{PH}_{2}$ and their mixture (1:1).

\begin{tabular}{|c|c|c|c|c|c|c|}
\hline \multirow{3}{*}{$\begin{array}{l}\text { Animal } \\
\text { group }\end{array}$} & \multicolumn{6}{|c|}{ Days after treatment / Organ } \\
\hline & \multicolumn{2}{|c|}{1} & \multicolumn{2}{|c|}{7} & \multicolumn{2}{|c|}{14} \\
\hline & Liver & Kidneys & Liver & Kidneys & Liver & Kidneys \\
\hline I & $\begin{array}{c}0.1 \pm 0.01 * \\
\mathrm{n}=8\end{array}$ & $\begin{array}{c}0.3 \pm 0.02 \\
\mathrm{n}=8\end{array}$ & $\begin{aligned} 0.3 & \pm 0.02 \\
\mathrm{n} & =16\end{aligned}$ & $\begin{aligned} 0.3 & \pm 0.04 \\
\mathrm{n} & =16\end{aligned}$ & $\begin{aligned} 0.6 & \pm 0.02 \\
\mathrm{n} & =14\end{aligned}$ & $\begin{aligned} 0.4 & \pm 0.02 \\
\mathrm{n} & =14\end{aligned}$ \\
\hline II & $\begin{array}{c}0.3 \pm 0.02 \\
\mathrm{n}=8\end{array}$ & $\begin{array}{c}0.4 \pm 0.04 \\
\mathrm{n}=8\end{array}$ & $\begin{aligned} 0.5 & \pm 0.01 \\
\mathrm{n} & =18\end{aligned}$ & $\begin{array}{c}0.5 \pm 0.2 \\
\mathrm{n}=18\end{array}$ & $\begin{aligned} 0.8 & \pm 0.03 \\
\mathrm{n} & =14\end{aligned}$ & $\begin{aligned} 0.5 & \pm 0.02 \\
\mathrm{n} & =14\end{aligned}$ \\
\hline III & $\begin{array}{c}0.6 \pm 0.02 \\
\mathrm{n}=8\end{array}$ & $\begin{array}{c}0.5 \pm 0.03 \\
\mathrm{n}=8\end{array}$ & $\begin{aligned} 0.7 & \pm 0.02 \\
\mathrm{n} & =14\end{aligned}$ & $\begin{aligned} 0.4 & \pm 0.02 \\
\mathrm{n} & =14\end{aligned}$ & $\begin{aligned} 0.6 & \pm 0.02 \\
\mathrm{n} & =14\end{aligned}$ & $\begin{aligned} 0.5 & \pm 0.02 \\
\mathrm{n} & =14\end{aligned}$ \\
\hline IV & $\begin{array}{c}0.6 \pm 0.07 \\
\mathrm{n}=6\end{array}$ & $\begin{array}{c}0.5 \pm 0.02 \\
\mathrm{n}=6\end{array}$ & $\begin{aligned} 0.7 & \pm 0.01 \\
\mathrm{n} & =10\end{aligned}$ & $\begin{array}{c}0.5 \pm 0.03 \\
\mathrm{n}=10\end{array}$ & $\begin{array}{c}0.7 \pm 0.3 \\
\mathrm{n}=12\end{array}$ & $\begin{aligned} 0.5 & \pm 0.02 \\
\mathrm{n} & =12\end{aligned}$ \\
\hline $\mathrm{V}$ & $\begin{aligned} 0.6 & \pm 0.05 \\
\mathrm{n} & =12\end{aligned}$ & $\begin{array}{c}0.5 \pm 0.02 \\
\mathrm{n}=12\end{array}$ & $\begin{aligned} 0.6 & \pm 0.03 \\
n & =14\end{aligned}$ & $\begin{aligned} 0.5 & \pm 0.02 \\
\mathrm{n} & =14\end{aligned}$ & $\begin{aligned} 0.7 & \pm 0.03 \\
\mathrm{n} & =12\end{aligned}$ & $\begin{array}{c}0.5 \pm 0.02 \\
\mathrm{n}=12\end{array}$ \\
\hline
\end{tabular}

$* \mathrm{M} \pm$ SE. $\mathrm{n}$ is the number of animals per group, $\mathrm{p}$ is statistically significant difference compared to the control cohort ( $\mathrm{p}<0.05$ ). 
in the liver $(16.7 \%)$ and a decrease $(20.1 \%)$ in kidneys were detectable. The increased G6PD activity suggests a stimulation of the pentose phosphate shunt concomitant with accumulation of pentose and reduced $\mathrm{NADPH}+\mathrm{H}^{+}$, that are needed for detoxication, antioxidant defence and repair processes. We therefore tend to interpret this effect as beneficial.

In group II ( $\mathrm{Me}_{3} \mathrm{SnCl}$ plus $\left.\mathrm{R}_{4} \mathrm{PH}_{2}\right)$ G6PD went down by day 1 , but to a lesser extent than after $\mathrm{Me}_{3} \mathrm{SnCl}$ alone: $50.0 \%$ in the liver and $20.1 \%$ in kidneys. Combination with $\mathrm{R}_{4}{ }_{4} \mathrm{PH}_{2}$ attenuated the enzyme inhibitory effect of $\mathrm{Me}_{3} \mathrm{SnCl}$ by $16.7 \%$ in the liver by day 14 , whereas this parameter in kidneys was the same as in the control group.

In group IV (Tween-20) there was no effect on G6PD.

Tin containing compounds are capable of altering the redox balance via different mechanisms including ROS generation and oxidative stress. ${ }^{[1161]}$ This process can be attenuated with cytoprotective antioxidants. On the other hand, tin compounds are known to inhibit the enzymes. ${ }^{[174]}$ $\mathrm{Me}_{3} \mathrm{SnCl}$ interacts with $\mathrm{SH}$-groups in the proteins. ${ }^{[175]}$ Inorganic salts of the tin formed during metabolic dealkylation of $\mathrm{Me}_{3} \mathrm{SnCl}$ in the liver can also bind $\mathrm{SH}$ groups. Thus, the protective effect of $\mathrm{R}_{4}{ }^{2} \mathrm{PH}_{2}$ that has antioxidant phenol groups and the tetrapyrrolic macrocycle, can be due to inhibition of radical processes as well as to metal ion chelation.

Figures 110 and 111 present data on glutathione levels and G6PD activity in the presence of $\mathrm{Me}_{3} \mathrm{SnCl}$ with or without $\mathrm{R}_{4} \mathrm{PH}_{2} . \mathrm{Me}_{3} \mathrm{SnCl}$ alone potently decreased $\mathrm{GSH}$ content whereas $\mathrm{R}_{4} \mathrm{PH}_{2}$ demonstrated a protective effect concomitant with GSH restoration up to the level in intact rats.

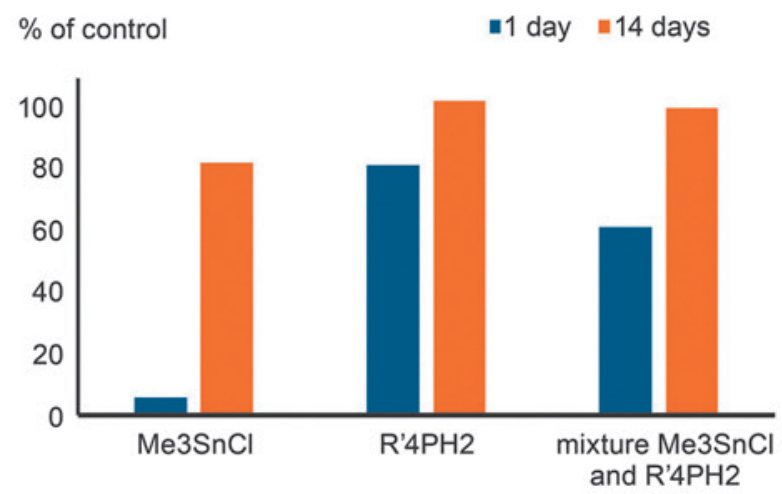

Figure 110. Time course of reduced glutathione (GSH) in the rat liver in the presence of $\mathrm{Me}_{3} \mathrm{SnCl}, \mathrm{R}_{4}{ }_{4} \mathrm{PH}_{2}$ and their mixture (1:1).

The activity of G6PD dropped dramatically after single administration of $\mathrm{Me}_{3} \mathrm{SnCl}$ (5 mg/kg; Table 29, Figure 111). In a separate experiment we showed that $\mathrm{R}_{4}^{\prime} \mathrm{PH}_{2}$ alone had virtually no effect on G6PD activity. Combination of $\mathrm{Me}_{3} \mathrm{SnCl}$ and $\mathrm{R}_{4}{ }_{4} \mathrm{PH}_{2}$ (1:1) G6PD was inhibited to a lesser extent compared to $\mathrm{Me}_{3} \mathrm{SnCl}$ alone. By day 14 G6PD was restored by this combination.

The glutathione system and particularly glutathione regulate the enzymes such as pyrophosphatase, phosphoglyceraldehyde dehydrogenase, and hemoglobin reductase. The decrease of GSH is critical for the activity of GSH

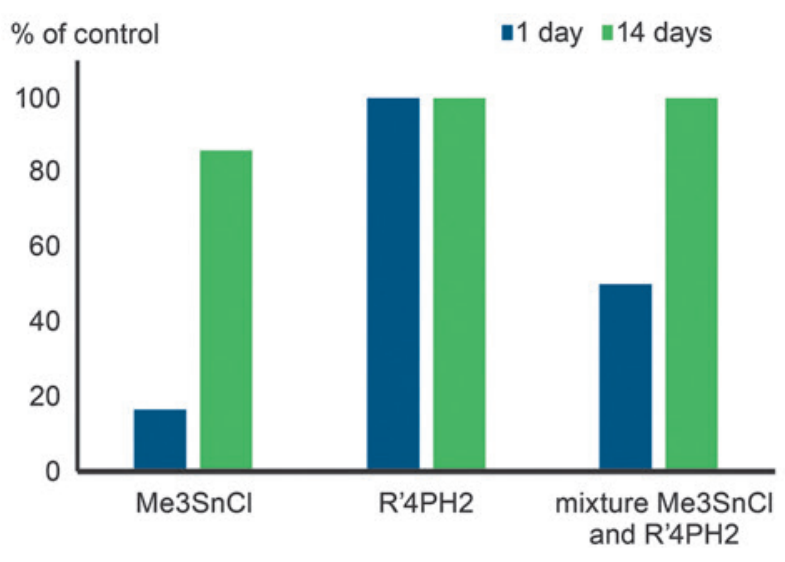

Figure 111. Time course of glucose-6-phosphate dehydrogenase (G6PD) activity in the rat liver in the presence of $\mathrm{Me}_{3} \mathrm{SnCl}$, $\mathrm{R}^{\prime}{ }_{4} \mathrm{PH}_{2}$ and their mixture (1:1).

dependent enzymes. Likewise, GSH content is regulated by G6PD. We found that both parameters changed concomitantly. By day 1 after $\mathrm{Me}_{3} \mathrm{SnCl}$ injection each parameter was decreased. In group of $\mathrm{R}_{4} \mathrm{PH}_{2}$ in combination with trimethyl tin chloride both GSH and G6PD also went down in parallel although not as dramatically as in the case of $\mathrm{Me}_{3} \mathrm{SnCl}$ alone. GSH levels dropped down to a bigger extent than G6PD activity.

The results presented herein demonstrated that the porphyrin $\mathrm{R}_{4} \mathrm{PH}_{2}$ attenuated the toxic effect of trimethyltin chloride on GSH content and G6PD activity in the rat liver and kidneys. Early (day 1) drop of each parameter was less pronounced in animals treated with the combination; by day 14 the combination restored GSH and G6PD to normal values. One may hypothesize that $\mathrm{R}_{4}{ }_{4} \mathrm{PH}_{2}$ being antioxidant, is oxidized in vivo and decreases ROS. In turn, this causes a decrease of synthesis and regeneration of GSH. In kidneys GSH concentrations remain unaltered because the intact $\mathrm{R}_{4} \mathrm{PH}_{2}$ does not enter this organ due to fast metabolic conversion, both in situ and in the liver.

Overall, we demonstrated that the porphyrin $\mathrm{R}_{4} \mathrm{PH}_{2}$ attenuated the toxic effects of the tin compound on the antioxidant defense system. We tend to attribute the protective efficacy of $\mathrm{R}_{4}{ }_{4} \mathrm{PH}_{2}$ to its 2,6-di-tert-butylphenol fragments as antioxidant moieties, as well as to the free base of the porphyrin as a chelator.

\subsection{Interaction of Macroheterocycles with Globular Proteins}

The new COVID-19 viral disease causing a severe acute respiratory syndrome was first reported in China, in December 2019. The disease has rapidly spread and turned into a pandemic since then. In fact, the study of the virus and how to fight with it is now vital, which makes this work relevant and timely and explains why the scientific community is so interested in this problem. It is known so far that the SARS-CoV-2 composition includes RNA, some nonstructural and four structural proteins: S-protein, envelope, membrane, and nucleocapsid proteins. ${ }^{[1176]}$ Among these proteins, S-protein plays a significant role in the virus 
attachment, integration, and entry into the cells and, consequently, it acts as a target for synthesizing antibodies, entry inhibitors, and various vaccines. SARS-CoV-2, as well as its predecessor - the coronavirus causing a severe acute respiratory syndrome (SARS-CoV), uses the ACE2 human receptor (a membrane protein, an angiotensin-converting enzyme) for the virus entry into the host cells. The SARSCoV-2 genome has been sequenced ${ }^{[1177]}$ and a genetic comparison has been made between SARS-CoV-2 and SARS$\mathrm{CoV} \cdot{ }^{[1178]}$ Computer modelling has shown that the S-proteins of SARS-CoV-2 and SARS-CoV have almost identical $3 \mathrm{D}$ structures in the receptor-binding domain (RBD). ${ }^{[179]}$ The authors ${ }^{[179]}$ have found that the amino acid sequences of the SARSCoV-2 and SARS-CoV S-proteins are $76.5 \%$ identical and have a high homology degree. ${ }^{[1179]}$ The transmembrane S-glycoprotein of SARS-CoV-2 forms a homotrimer protruding from the virus surface and including two functional elements; an S1-subunit responsible for binding with the host cell receptor and an S2-subunit responsible for cell membrane fusion. ${ }^{[180]}$ The S1-subunit contains RBD, the most variable coronavirus genome part in the S-protein. Six RBD amino acids are critical to the binding with the ACE2 receptors. ${ }^{[181]}$ The interaction is complementary, for example, the glutamine residue (394) in the SARS-CoV-2 RBD corresponds to the lysine residue (31) on the human ACE2 receptor. ${ }^{[182,1183]}$

Another important part of the SARS-CoV-2 S-glycoprotein - the polybasic cleavage site (RRAR, PRRA (proline-arginine-arginine-alanine) amino acid sequence is located at the $\mathrm{S} 1 / \mathrm{S} 2$ subunit border. It is effectively broken down by furin and other proteases of the host cell, such as TMPRSS2, plays a role in increasing the virulence, and widens the range of possible host organisms. ${ }^{[1178,1184,1185]}$ Such cleavage is thought to be a precondition for membrane fusion.

An analysis of the available literature data allows us to conclude that most of the studies have focused on two targets of the virus inactivation: its S-protein and host protein structures (ACE2 and TMPRSS2). Inhibiting the functions of the serine protease ${ }^{[1178,1186,1187]}$ or angiotensin-converting enzyme ${ }^{[188-1192]}$ may lead to even more negative effects in a patient and make the situation worse in case of multiorgan failure. Therefore, studies focusing on the virus S-protein as a target for virus inactivation can have much better prospects. Research is now being done in various directions, first of all, in the field of using monoclonal antibodies. ${ }^{[1193-1197]}$ The main idea of such works is evident: the amino acid sequences of the S-protein and its receptorbinding domain are known, which allows researchers to synthesize antibodies (of protein nature) with high binding affinity to the S1 subunit or its part. ${ }^{[193,1194]}$ However, the use of antibodies in Covid 19 treatment raises a lot of questions. The main one is the unavoidable immune response to the antibody administration, which may even lead to a cytokine storm as the immune system of such patients is already unbalanced. Cytokine storm is the main cause of death in Covid 19 patients. ${ }^{[1195,1196]}$ That is why most clinical treatment protocols include immunosuppressive drugs. ${ }^{[1197,1198]}$

The second research direction is the use of small molecules capable of binding with the key S1-subunit fragments. A number of works have focused on computer modelling of the S-protein interaction with drugs that are already used in medicine. ${ }^{[1199,1200]}$ As the results of the virtual screening of low molecular weight compounds showed, some preparations, such as some antihypertensive drugs (rescinnamine, iloprost, and prazosin), antifungal drugs (posaconazole and itraconazole), antibacterial drugs (sulfasalazine, azlocillin, penicillin, and cefsulodine), and dabigatran etexilate, an anticoagulant drug, demonstrated high binding affinity to the S-protein. Some natural flavonoids also demonstrated high binding affinity to the S-protein. However, most of the afore-mentioned compounds are not localized in the S-protein binding region with ACE2. The only compound that could target the binding site between the S-protein and ACE2 is hesperidin. Hesperidin binds to the S-protein RBD region through hydrophobic interactions and formation of a hydrogen bond between the Tyr440 RBD amino acid residue and hesperidin. ${ }^{[199]}$ A similar computer-based strategy of finding a compound binding the S-protein in the potential contact area with ACE2 was proposed in work ${ }^{[1200]}$. The authors selected four compounds - pemirolast, isoniazid pyruvate, nitrofurantoin, and eriodictyol - with high affinity binding and required localization.

As it has been said above, RBD in the S-protein is the most variable part of the coronavirus genome. It means

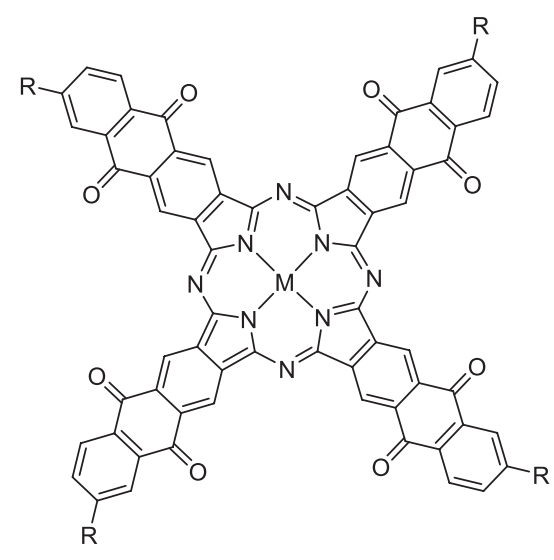

235

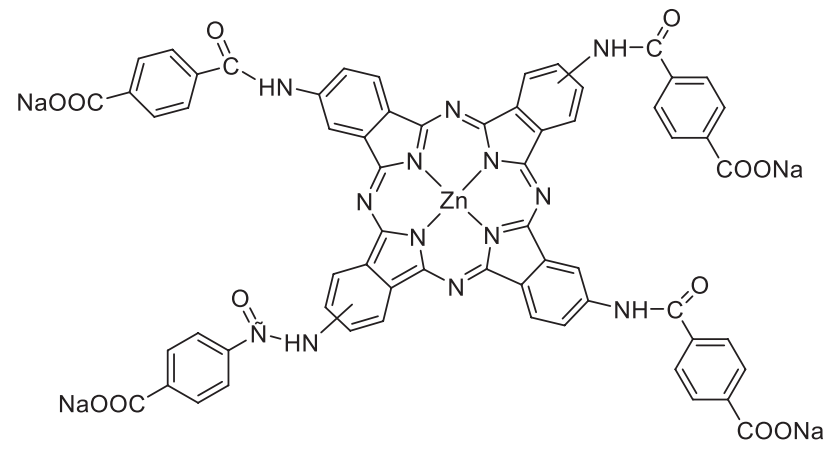

236

Figure 112. Molecules of tetraantraquinoporphyrazine $\left(\mathrm{M}=\mathrm{AlOH}, \mathrm{Co} ; \mathrm{R}=\mathrm{COONa}, \mathrm{SO}_{3} \mathrm{Na}\right)$, and $\mathrm{ZnPc}\left(4-\mathrm{NH}-\mathrm{CO}-\mathrm{C}_{6} \mathrm{H}_{4}-\mathrm{COONa}\right)_{4}$. 
that modelling of a drug compound inhibiting this protein part may not lead to the desired results. We propose another strategy. It is based on forming a complex of macroheterocyclic compounds (MHC) with protein structures, in which the $\mathrm{MHC}$ is responsible for cross-linking of the protein polypeptide chains. We have earlier ${ }^{[1201,1202]}$ synthesized MHCs (Figure 112) that enable the cross-linking of the albumin polypeptide chains belonging to different protein domains.

We were able to synthesize a number of MHCs that built into the protein domains providing the cross-linking of the polypeptide chains belonging to different subdomains. The obtained complexes possessed very high thermal stability (Table 30). Thermal destruction of individual albumin is known to start from the stage of breakage of the disulfide bonds stabilizing the native protein conformation. This stage covers the temperature interval from 46 to $60{ }^{\circ} \mathrm{C}$, and the protein spreads (melts) losing its native conformation. The built-in MHC compounds effectively prevent the protein melting, increasing the thermal stability by $1.5-2$ times (Table 30).

Table 30. Thermal stability of BSA and its complexes with MHCs.

\begin{tabular}{lcc}
\hline \multicolumn{1}{c}{ System } & $\mathrm{T}_{\text {melt }} \pm 5,{ }^{\circ} \mathrm{C}$ & Ref \\
\hline BSA & 57 & ${ }^{[1203]}$ \\
BSA- $\mathrm{ZnPc}\left(4-\mathrm{NH}-\mathrm{CO}-\mathrm{C}_{6} \mathrm{H}_{4}-\mathrm{COONa}\right)_{4}$ & 56 & ${ }^{[1203]}$ \\
BSA- $\left(\mathrm{ZnPc}\left(4-\mathrm{NH}-\mathrm{CO}-\mathrm{C}_{6} \mathrm{H}_{4}-\mathrm{COONa}\right)_{4}\right)_{2}$ & 90 & ${ }^{[1202]}$ \\
BSA-NaCl-HOAlPc$\left(\mathrm{SO}_{3} \mathrm{Na}_{4}\right.$ & 68 & ${ }^{[1201]}$ \\
BSA-NaCl-HOAlTAP $(\mathrm{COONa})_{4}$ & 120 & {$[1201]$} \\
BSA-NaCl-HOAlTAP $\left(\mathrm{PhSO}_{3} \mathrm{Na}\right)_{4}$ & 128 & {$\left[{ }^{[1201]}\right.$} \\
BSA-NaCl-HOAlTAP $\left(\mathrm{SO}_{3} \mathrm{Na}\right)_{4}$ & 129 & {$[1201]$} \\
\hline
\end{tabular}

We believe that a similar approach would allow us to prepare an MHC that could effectively bind with various polypeptide chains of the virus S-protein and thus prevent its binding with host cells.

Since the virus $\mathrm{S}$-protein is globular, ligands that can be bound inside it must be predominantly hydrophobic. Besides, they must be soluble in physiological media (as a potential drug compound). And the main condition: they must have long peripheral substituents enabling the binding of various polypeptide chains of the virus S-protein.

Porphyrin is a hydrophobic molecule, and it is the substituents, such as sulpho- or $N$-methylpyridyl groups, that make it soluble in aqueous media, including all physiological media at $\mathrm{pH}$ from 6 to 7.5 .

The screening allowed us to select 2 structures containing molecules of the residue of the amino acid - tyrosine - at their periphery: a) cationic porphyrin, in which the tyrosyl residue is bound to the porphyrin molecule via the amino acid hydroxyl group and alkyl spacer (PorAm); b) anionic porphyrin, in which the tyrosyl residue is bound to the porphyrin molecule via the amino acid amino group (TyrSP) (Figure 113).

After that we modelled the interaction of the obtained structures with the coronavirus S-protein by the molecular docking method. Spike protein structure of SARS-CoV-2 $(6 \mathrm{VYB})^{[1204]}$ as downloaded from the Protein Data Bank. The porphyrin structures were calculated by method DFT/B3LYP/6-311G** in Gaussian 09 Rev. D.01. ${ }^{[1205]}$ Molecular docking of the proteins with porphyrins was performed using AutoDock Vina ${ }^{[1206]}$ and visualized with MGLTools 1.5.6. Firstly, the ligand and protein coordinate files were prepared respectively to include the information needed by AutoDock and the PDBQT files were created. Then the three-dimension of the grid box was set in AutoDockTools to create the grid parameter file. Afterwards, AutoGrid was used to generate the grid maps and AutoDock Vina was run for receptor-ligand docking. After the docking was completed, the results were shown in Python Molecule Viewer 1.5.6 from MGLTools 1.5.6, and then the binding energy and receptor-ligand interactions were evaluated.

The highest binding energy was found in TyrSP and equalled $11.5 \mathrm{kcal} / \mathrm{mol}$. In case of PorAm, the energy of binding with the coronavirus S-protein was $9.1 \mathrm{kcal} / \mathrm{mol}$ (Figure 114). The PorAm porphyrin builds into the protein globule between the S1 and S3 chains and stays very close to the S1 ARG765 chain and S3 LEU303 chain of the S-protein. TyrSP is located near the S1 ILE569 subunit, S2 LYS304 chain, and S3 SER746 chain. Both porphyrins are located rather far from the SARS-CoV-2 RBD, which means that the porphyrins are probably unable to inhibit the S-protein binding with ACE2. However, their localization and binding energy will, evidently, allow these porphyrins to inhibit the functioning of the PRRA polybasic cleavage site, i.e. inhibit the most important cycle of the virus - its internalization into the host cell.

Thus, TyrSP can stabilize the structure of the S-protein by forming specific bonds between the tyrosyl substituents of the porphyrin with the serine, leucine, and isoleucine amino acid residues of various S-protein chains. PorAm has lower energy of binding with the protein than TyrSp, which is probably caused by the interaction complementarity. The higher binding energy in TyrSP is evidently explained by the fact that it binds to all three chains of the S-protein, while PorAm binds only to two chains, i.e. it is a multicentre interaction.

The proposed strategy of synthesizing the selected porphyrins is presented in Figure 113.

To confirm our hypothesis about the stabilization of the S-protein through its binding with the tyrosyl residues of the porphyrin, it is suggested to conduct in vitro studies of the synthesized porphyrins with the coronavirus S-protein.

Thus, we have modelled two macroheterocyclic compounds that are soluble in water and are potentially suitable for cross-linking of the coronavirus S-protein polypeptide subunits.

The interaction of these MHCs with the coronavirus S-protein belongs to the multicentre interaction type and in case of TyrSP, it is complementary.

The obtained results indicate effective porphyrin binding inside the S-protein globule and cross-linking of the S-protein subunits, which is expected to prevent the fusion of the virus particles with the host membrane cell. 
a)

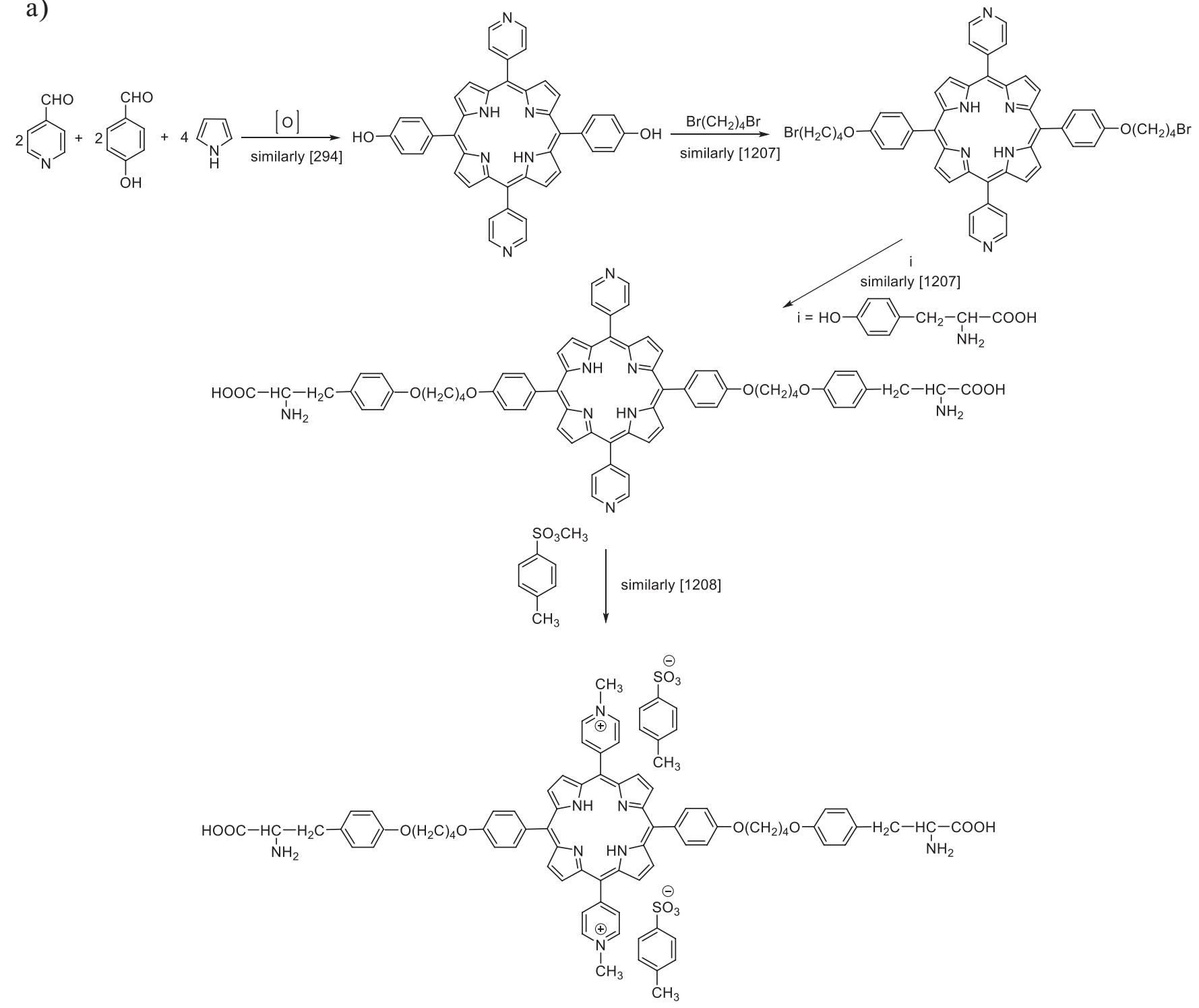

b)

similarly [294]

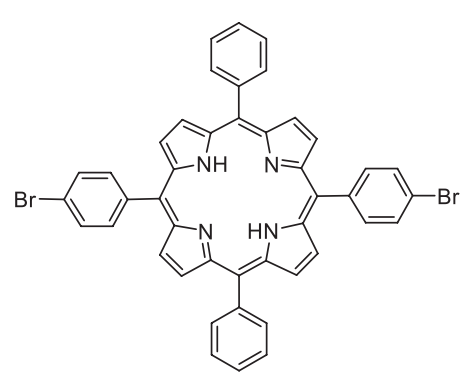

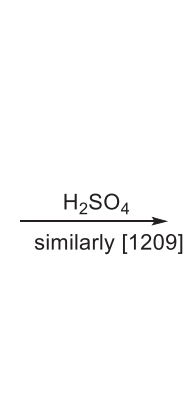<smiles>O=C(O)c1ccccc1</smiles>

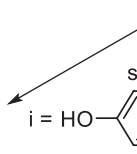

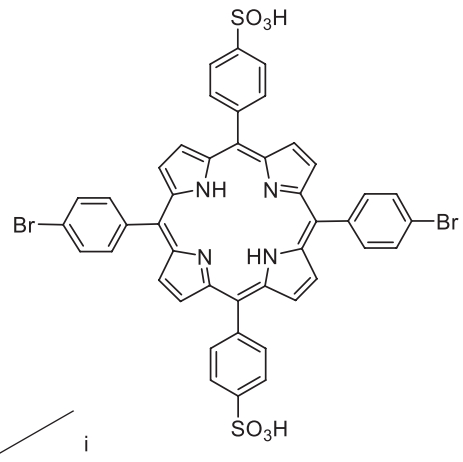

$\mathrm{OH}$

similarly [1210]

$\stackrel{1}{\mathrm{~N}} \mathrm{H}_{2}$

Figure 113. The proposed synthesis strategy: (a) PorAm, (b) TyrSP. 
a)

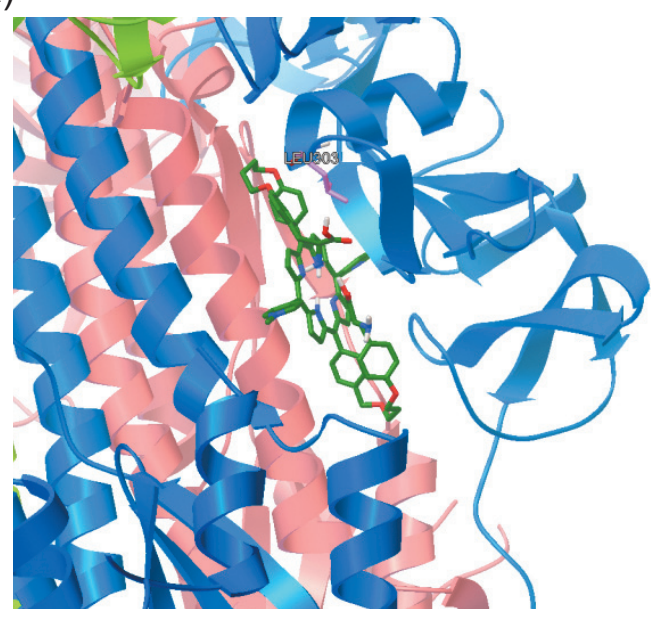

b)

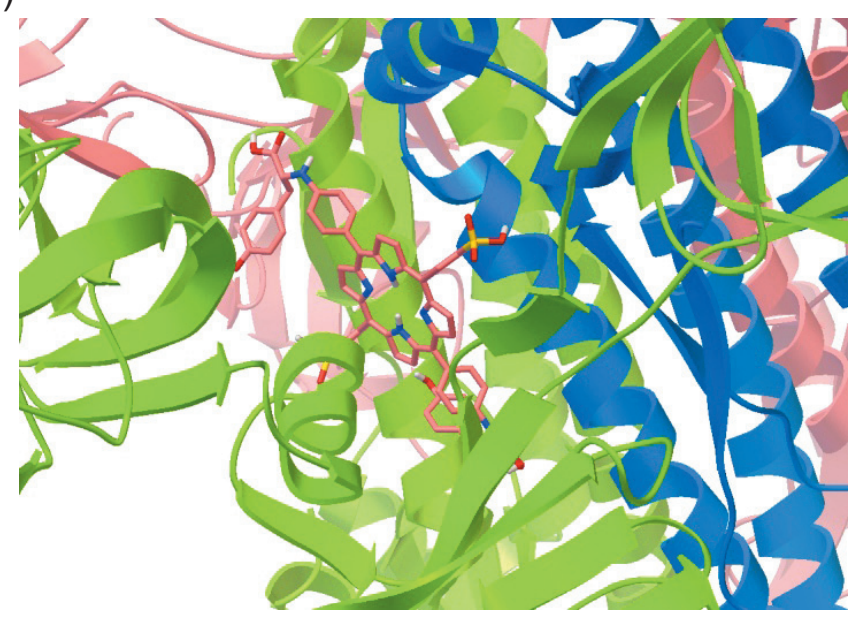

Figure 114. Molecular docking results of Spike protein with a) PorAm b) TyrSP.

\subsection{Thermal Stabilization of Protein by Macroheterocycles}

Physiological properties of proteins are greatly influenced by temperature effects. The vast majority of proteins denature at $40-60{ }^{\circ} \mathrm{C}$. The temperature at which $50 \%$ of the native protein undergoes heat denaturation is called the melting temperature. One can hardly overestimate the practical significance of the thermal effects studies on the protein systems of plants, animals and humans, and with each year an increasing number of practical applications appear. For example, in the study of thermal effects on cells it was found that high temperature primarily suppresses various repair systems, ${ }^{[1211,1212]}$ inhibiting some protein factors that take part in the repairing systems.

Oncocells have a defective thermoprotection mechanism, which causes their increased death during the thermal exposure. For example, the heat stress $\left(43^{\circ} \mathrm{C}\right.$ for 180 minutes $)$ of the cell cultures of lung tissue (normal and malignant) results in a decrease in the number of healthy cells by $8 \%$, and cancer cells by $78 \%{ }^{[1213]}$ Even more encouraging data are demonstrated by the thermoexposure combined with chemotherapy. ${ }^{[1214]}$ As a result, in recent years the hyperthermic approach to the treatment of oncologic diseases has been intensively developed. According to the report published in the ClinicalTrials.gov system (National Institutes of Health, USA) in 2000-2014. 109 clinical trials of tumor therapy using some form of hyperthermia have been registered. ${ }^{[1215]}$ Moreover, more than a third were undertaken in the last year of the report, and this fact indicates a growing interest in the clinical use of hyperthermia.

In the field of chemical technology an increase in the thermal stability of proteins is promising for their application as catalysts. The use of bio-catalysts (enzymes) provides numerous advantages compared with the traditional catalysts, in particular, high selectivity, efficiency, environmental friendliness, etc. The large-scale use of enzymes in technological processes is hindered by their low thermal stability, which does not allow the specialists to include proteins in the technological process. That is why so much attention has been paid to the study of the thermal stability of enzymes: over the period 1998-2008 more than 200 thousand papers were published in which the heat resistance of enzymes was explored, and over the last decade (2009-2018) the number of publications has doubled.

Existing approaches to increasing the thermal stability of enzymes, for example, using osmolytes or encapsulation, do not allow the biocatalyst to be incorporated into existing technological schemes. This is explained by the fact that osmolytes provide thermal stability of proteins only in huge concentrations, measured in units and tens of mol/1. This causes an increase in the viscosity of the reactants, accelerated corrosion of the metal parts of the reactors, regeneration costs, and so on. Encapsulation of the protein changes the chemistry of the catalysis process, the latter becomes heterogeneous, and the stage of mass transfer of the substrate and reaction products through the capsule wall is added. Some spatial constraints appear that cannot be solved in the case of bulk substrates (for example, lignin), and it makes this method of stabilizing proteins also unacceptable for industrial use.

The next promising direction, not related to the changes in the properties of the medium, but which allows one to increase the thermal stability of proteins, is their chemical modification. This approach is based on the use of mutagenesis. Its implementation requires information on the structure of the protein to identify aminoacid residues in the adjacent polypeptide chains or the domains that can be replaced with cysteine, and the replacement must be carried out in such a way that additional disulfide bridges are formed. After that, the induced mutagenesis is performed, and then the mutant protein is synthesized. It is clear that the method is very laborious; it requires detailed X-ray data, which for the vast majority of enzymes are lacking. One of the first works in this direction was published in 1989:[1216] using oligonucleotide-directed mutagenesis, six variants of lysozyme of phage T4 were created with new intrachain disulfide bonds. For this, two, four or six specific aminoacid residues in the polynucleotide chain were replaced by cysteine residues. A as a result, one, two and three disulfide bonds were formed. Amino acid residues replaced by cysteine residues were located in the enzyme close to each other, 
so that when new disulfide bonds were formed, the overall protein conformation did not change significantly. After mutagenesis, mutant genes were identified and expressed in E. coli, and the recombinant proteins were purified, their enzymatic activity and thermal stability were determined. The results of these experiments showed that the thermal stability of the enzyme increases when new disulfide bonds are formed, however, sufficient enzyme activity remains only in two cases out of three (one additional bond - $100 \%$ activity, thermal stability is $6^{\circ} \mathrm{C}$ higher; two bonds $-95 \%$ activity, heat stability is $15^{\circ} \mathrm{C}$ higher).

Another positive result ${ }^{[1217,1218]}$ was achieved when obtaining the thermostable mutant xylanase Bacillus circulans - an enzyme that can be used in paper production at the pulp bleaching stage but the production technology necessitates the functioning of the enzyme at relatively high temperatures $\left(60-80{ }^{\circ} \mathrm{C}^{[1213]}\right)$. In the xylanase polypeptide chains, a part of the amino acid residues has been replaced by the cysteine in the positions providing the formation of S-S bonds. Thus, eight xylanase derivatives were obtained. All of them possessed a higher thermal stability than the natural enzyme. At the same time, three of them were as active at $60{ }^{\circ} \mathrm{C}$ as the native protein, and one containing the disulfide bond between the $\mathrm{N}$ - and $\mathrm{C}$-termini was more active and retained over $85 \%$ of its activity after two hours exposure at $60^{\circ} \mathrm{C}$.

It should be noted that the modification of the primary structure of the protein does not always ensure the desired result. If it is possible to achieve thermal stability of a protein, then, as a rule, its catalytic activity decreases.

The opposite approach is based on increasing the thermal stability of proteins by replacing amino acid residues with low thermal stability. It is known that as the temperature increases, one of the first processes to be proceeded is the one of aspargin and glutamine residues deamidation with the formation of ammonia. The authors, ${ }^{[1219]}$ using oligonucleotide-directed mutagenesis, replaced two asparagine residues in the triosephosphate isomerase molecule. Replacement of one of them with a threonine or isoleucine residue resulted in an increase of the enzyme thermal stability, but the resulting protein had low enzymatic activity. It is obvious that the binding of a ligand (substrate) is so specific that any change in the aminoacid residues always results in a decrease of the catalytic activity or a complete loss of this function. ${ }^{[1220,1221]}$

Knowledge on the mechanisms and methods for increasing/decreasing the proteins thermal stability is essential when solving problems of pharmacology, microbiology, food industry or for increasing the storage period of the protein preparations, etc.

\section{Effect of Low Molecular Weight Substances on the Thermostability of Serum Albumin}

Low molecular weight thermoprotectors are known to be the external protein stabilizers, and they should not have a negative impact on its main functions. ${ }^{[1222]}$ The variety of thermoprotective substances is great, but several main classes of chemical compounds can be distinguished: carbohydrates and their derivatives, amino acids and their derivatives, polyols, betaines and ectoins.
Historically, the search for thermoprotectors began with the study of low molecular weight substances produced by mesophylls and extremophiles cells under the heat stress conditions. The melting point of the protein extremophiles is $80-110^{\circ} \mathrm{C} \cdot .^{[1223-1225]}$ It is noteworthy that the proteins of these living beings are built from the same twenty aminoacids as the ones of the living organisms under normal climatic conditions. It is evident that in the extremophiles the structure of the protein and the polypeptide sequence provide, on the one hand, the molecular stability of the protein, and on the other, structural flexibility. Numerous studies of the polypeptide sequences of mesophylls and extremophiles allowed the researchers to formulate a number of general regularities that distinguish heat-resistant proteins. ${ }^{[1223]}$ They are characterized by: a decrease in the proportion of the uncharged polar aminoacid residues and an increase in the proportion of the charged amino acid residues, ${ }^{[1226,1227]}$ as well as the predominance of $\beta$-folding over $\alpha$-helices. These studies underlie the principles of the design of the thermostable proteins. In addition, it was found that at high temperatures various substances are accumulated in the cells of extremophiles and mesophylls. Under thermal stress, neutral or zwitterionic compounds are accumulated in the mesophyll cells, and anionic compounds are accumulated in the extremophiles. Mannosylglycerate, trehalose, glycine, ectoin are the most common thermoprotectors among thermophilic prokaryotes. [1228-1231] In [1232], when comparing the thermoprotective properties of endogenous and exogenous organic compounds ((2S)-2-(1-O- $\alpha$-D-mannopyranosyl)propionate, 2-(1-O- $\alpha-\mathrm{D}-$ mannopyranosyl)acetate, (2R)-2-(1-O- $\alpha$-D-glucopyranosyl) glycerate and 1-O-(2-glyceryl)- $\alpha$-D-mannopyranoside, trehalose, glycerin, ectoin, dimyoinositol-1,3'-phosphate and diglycerol phosphate) and inorganic salts with respect to enzymes, it has been convincingly proved ${ }^{[1232]}$ that the charged organic compounds are more preferable for thermal protein stabilization, but the effectiveness of each of them for different proteins will differ. At the same time, the thermostability of the protein increases at best by $15^{\circ} \mathrm{C}$ with the used concentrations of thermoprotectors from 0.5 to $3.5 \mathrm{M}$.

What are the mechanisms underlying the stabilization of proteins by means of low molecular weight substances? An unequivocal answer to this question is absent yet, and the opinions of the researchers are often diametrically opposed. Some believe that the effect of thermal stabilization is based on any change in the properties of a solvent, on a change in the amount of "free" water capable of hydrating protein. Others attribute the influence of thermoprotectors directly to the interaction of low-molecular substances with protein, for example, considering that protein stabilization with sucrose, glycerol and related compounds is accomplished by forming a protective coating (shell) that prevents the unfolding of proteins. ${ }^{[1233]}$.

The first point of view was further developed in the study of the thermodynamics of the processes of protein melting in the presence of low molecular weight compounds. ${ }^{[1234-1238]}$ As a result, the following was postulated:

1) the processes of the protein denaturation, structure stabilization and precipitation (salting out) require a high concentration of the added agent (from 1 to $10 \mathrm{M}$ );

2) the interaction of the protein with low molecular weight thermoprotectors is a low-energy one; 
3) all low molecular weight substances stabilizing the protein structure do not interact with the protein specifically, and in their presence the protein hydration changes; ${ }^{[1239]}$

4) denaturations are specifically bound by the proteins.

However these postulates need to be revised. To demonstrate this thesis, we will consider the literature data and the data obtained by the authors on the thermoprotectors of bovine serum albumin (BSA).

According to numerous literature data, the process of thermal denaturation of albumin is irreversible regardless of the external factors, ${ }^{[1240,1241]}$ and the destruction of the disulfide bridges, which stabilize the albumin structure, underlies the triggering mechanism for the denaturation of the albumin. Two peaks are recorded on the DSC of BSA curves: endo- (up to $65^{\circ} \mathrm{C}$ ) and the exothermic one following it (above $65^{\circ} \mathrm{C}$ ). The first one, as it was unequivocally proved in ${ }^{[1242]}$, is due to the change in the native conformation of the protein. For albumin heated to $70{ }^{\circ} \mathrm{C}$, IR spectral changes owing to the transition of the $\alpha$-helix to $\beta$-folds are recorded, as well as a significant decrease in the absorption intensity in the region of 476 and $525 \mathrm{~cm}^{-1}$ (SS stretching vibrations). All this suggests the change in albumin structure and the loss of its native conformation. ${ }^{[1243]}$

The melting temperature of the protein significantly depends on the presence of electrolytes, ${ }^{[1244]}$ heating rate, phase state, etc. Thermal denaturation of the fat-free BSA, according to DSC data, occurs at $56^{\circ} \mathrm{C}$, and in the presence of the background electrolyte it takes place at $69^{\circ} \mathrm{C}$. A similar effect of electrolytes was reported in the papers written by Ito ${ }^{[245]}$ who studied the heat denaturation of BSA $(20 \%$ solution in $0.1 \mathrm{M}$ phosphate buffer at $\mathrm{pH} 7$ ). The strong stabilizing effect on the native structure of BSA with the addition of $\mathrm{NaCl}$ in the range of $0-1.0 \mathrm{M}$ was reported in. ${ }^{[1246-1248]}$ In this research the melting point of the protein varied from 56 to $72{ }^{\circ} \mathrm{C}$, and the magnitude of the thermal effect of denaturation was from 567 to $1100 \mathrm{~kJ} / \mathrm{mol}$.

The temperature of the thermal resistance of BSA depends on whether the protein is defatted. Binding of the oleic acid by a protein results in a decrease in the thermal resistance of albumin by 10 degrees. ${ }^{[1249]}$ Fatty acids have an "wedging effect" on the domains, thereby contributing to the weakening of seventeen disulfide bonds.

The use of water-alcohol solutions with a volume fraction of propanol at least $4 \%{ }^{[1250]}$ can increase the thermal stability of albumin up to $4{ }^{\circ} \mathrm{C}$, and the ethanol introduced into the solution in the same amount does not exhibit thermoprotective properties. ${ }^{[1251]}$ The authors attribute this phenomenon to a stronger interaction of the hydrophobic part of the alcohol molecule with the protein, and this interaction results in an increase in the proportion of alphastructuring.

A similar effect is demonstrated by the presence of trehalose in the $0.5 \mathrm{M}$ BSA solution. The melting point of the protein increases by $3.7^{\circ} \mathrm{C}$, with a decrease in the size of the globule and an increase in alpha helicity from $45.85 \%$ to $48.81 \%$. The affinity of the trehalose-stabilized protein was significantly reduced in relation to thioflavin, curcumin and resveratrol. ${ }^{[1252]}$ Polyethyleneglycols, with a relative small molecular weight, introduced into the albumin solutions, contrary to the expectations of the authors, ${ }^{[1253]}$ reduce the melting temperature by $10^{\circ} \mathrm{C}$.
Judging by the literature data, sucrose exhibits the greatest thermostabilizing effect: with an increase in the sucrose concentration from 0 to $40 \mathrm{wt} . \%$, the melting temperature increases from 72.9 to $79.2^{\circ} \mathrm{C}$. Quite unexpected is the interpretation of the results proposed by the authors. They believe that the role of the thermoprotector is reduced to a specific surface interaction of the sucrose with a protein globule, and this suggestion is highly doubtful, given the concentrations of the sucrose used. ${ }^{[1254]}$

The attempts to establish the molecular mechanism of action of arginine, glycine, and guanidine hydrochloride on the thermostability of albumin were undertaken in ${ }^{[1255]}$. With the addition of $0.5 \mathrm{M}$ glycine, the thermal stability of the protein increases by $7-9{ }^{\circ} \mathrm{C}$, while arginine and guanidine hydrochloride, added with same concentrations, result in a decrease in the thermal melting of the protein by 5 and $10{ }^{\circ} \mathrm{C}$, respectively. The authors suggest that glycine may affect the thermal stability of a protein due to the competitive interaction with water and due to the energy increase required for the hydration of the protein unfolding. The thermostabilizing effect of sucrose at the concentration of $1 \mathrm{M}$ on the albumin was reported in ${ }^{[1256]}$, a distinctive feature of this paper being a detailed analysis of the secondary and tertiary structure of the protein against the background of sucrose solution.

In methodological terms, it is impossible not to note the work ${ }^{[1257]}$ in which it was shown, that the errors in the interpretation of the data on thermal action may be due to thermally induced aggregation of the bovine serum albumin.

One can cite an enormous number of the works presented in the literature on this topic, the abundance of which on the one hand indicates the significance of this phenomenon, ${ }^{[1222]}$ and on the other, demonstrates the similarity of the results obtained due to the established dogmas and approaches.

In a series of our previous works, ${ }^{[1201,1258-1261]}$ when analyzing the structure of serum albumin in blood, it was suggested, that macroheterocyclic compounds belonging to the classes of porphyrins and phthalocyanines can act as thermoprotectors. At the same time, they should not occupy traditional protein binding sites in the subdomains IB, IIIA, IIA, but should be located among the domains, providing their additional contraction. The choice of macroheterocyclic compounds is due to the wide possibilities of chemical modification of both peripheral substituents and the macroring itself. Thus, the task was reduced to the search for such structures of the macroheterocyclic compounds that would not intercalate into the protein, leaving the traditional binding sites not involved, but located among the domains and providing H-binding or hydrophobic stabilization of the protein globule. Thermoprotective properties and complexing ability with respect to bovine serum albumin of blood was evaluated for three related classes of the macroheterocyclic compounds: porphyrins, phthalocyanines, and tetraanthraquinone porphyrazines. These macroheterocyclic compounds differ in the length of the aromatic system, the contours of conjugation, hydrophobic/hydrophilic character. Porphyrin compounds were mainly intercalates and, at best, the stability of the protein increased by $10{ }^{\circ} \mathrm{C}$. During the transition from porphyrin 
to phthalocyanines and tetraanthraquinone porphyriazines, the desired effect was achieved. In particular, the traditional binding sites of bovine serum albumin remained vacant, and thermal stability increased, depending on the nature of the thermoprotector, by $14{ }^{\circ} \mathrm{C}$ for phthalocyanines, by $33{ }^{\circ} \mathrm{C}$ for $\mathrm{J}$-dimer of the substituted phthalocyanine and by $73{ }^{\circ} \mathrm{C}$ in the case of tetraanthraquinone porphyrazines. The concentration of macroheterocyclic compound did not exceed $10^{-5} \mathrm{~mol} / \mathrm{l}$. Thus, it can be concluded that the action of the thermoprotector is energetically strong and directional, it indicates a new trend in the stabilization of proteins.

This review considers some aspects of the application of macroheterocyclic compounds for practical purposes. The authors would like to express the hope that the presented material will encourage new researchers to study the inexhaustible useful properties of this class of compounds.

Acknowledgements. The review was financially supported by a number of Foundations, Programs and grants: Section 1 (Koiman O.I. et al.) - by the Russian Foundation for Basic Research (RFBR) (project No. 18-03-00986); Section 2 (Beletskaya I.P. et al.) - by the RFBR grant No.18-0300709; Section 3 (Tomilova L.G. et al.) - by the ERA.Net RUS Plus Plasmon Electrolight project (No. 18-53-76006 ERA); Section 4 (Martynov A.G. et al.) - by the Russian Science Foundation (RSF) (project No. 18-73-10174); Section 5 (Konarev D.V. et al.) - by the RSF (project No. 17-1301215); Section 6 (Lomova T.N. et al.) - partially by the RFBR (project No. 18-43-370022-r-a) and by the Program of the State Academies of Sciences (subject No.00922014-0002, 0092-2014-0003); Section 7 (Zenkevich E.I. et al.) - by the program BSPSR "Convergence-2020 3.03", Grant of President of Republic of Belarus in Science (EZ, 2020), Volkswagen project No. I/79 435 (Germany) as well as in part by the European Union's Horizon 2020 program within a Training Period in the Research and Innovation Action Bio4Comp "Parallel Network-Based Biocomputation" (Grant agreement 732482, 2017-2021); Section 8 (Zhdanova K.A. et al.) - by the RFBR (project No. 20-3370218); Section 9 (Stuzhin P.A. et al.) - by RSF (project No 17-13-01522); Section 10 (Rusakova N.V. et al.) - by the National Academy of Sciences of Ukraine; Section 11 (Parfenyuk V.I. et al.) - by the RFBR (project No. 17-0300678); Section 12 (Vashurin A.S., Koifman O.I.) - by the Grant of President of Russian Federation (MK-204.2019.3); Section 13 (Makarov S.V., Koifman O.I. et al.) - within the framework of the state assignment for the implementation of research work (No.FZZW-2020-0008); Section 14 (Mamardashvili N.Zh., Koifman O.I.) - by the RFBR (project No. 19-03-00078 A); Section 15 (Kurtikyan T.S. et al.) - by the State Science Committee of the Republic of Armenia (project No.18T-1D242); Section 16 (Burmistrov V.A., Koifman O.I. et al.) - by the Ministry of Science and Higher Education of Russia (grant agreement No.07515-2020-782) in part of selectors for gas chromatography, the investigation of inductors for liquid crystal phases was carried out within the framework of the state assignment for the implementation of research work (No. FZZW-20200008); Section 17.1. (Mironov A.F. et al.) was supported by the RFBR (project No.18-03-00961); Section 17.2. (Grin M.A. et al.) - by the RFBR (project No. 19-03-00302) and by the Ministry of Science and Higher Education of the Russian Federation (project No.0706-2020-0019); Section 17.3. (Fedorov A.Yu. et al.) - by the RFBR NNIO_a (project No. 18-503-12087); Section 17.4. (Kustov A.V. et al.) - by the RFBR (projects No. 20-03-00153 and 20-5300038); Section 17.5. (Solovieva A.N. et al.) - by the RFBR (project No. 18-29-06019MK); Section 17.6. (Milaeva E.R. et al.) - by the RSF (project No. 19-13-00084) and the RFBR (projects No. 18-03-00203 and 20-03-00471); Section 17.7. (Syrbu S.A., Lebedeva N.Sh. et al.) - by the RFBR (project No. 20-04-60067); Section 17.8. (Lebedeva N.Sh. et al.) - by the RFBR (project No. 19-03-00468).

\section{References}

1. Milgrom L.R. The Colours of Life: An Introduction to the Chemistry of Porphyrins and Related Compounds. Oxford: University Press, 1997. 256 p.

2. Battersby A.R. Nat. Prod. Rep. 2000, 17, 507-526.

3. Tetrapyrroles: Birth, Life and Death (Warren M.J., Smith A.G., Eds.). N.Y.: Springer Science \& Business Media, 2009. $406 \mathrm{p}$.

4. Serebrennikova O.V. Geochemistry of Porphyrins: Conditions of Accumulation and Direction of Metalloporphyrin Transformation in Sendimentary Rocks. In: Advances in Porphyrin Chemistry. Vol. 3 (Golubchikov O.A., Ed.). SPb.: St-PbGU, 2001. p. 326-349 (in Russ.).

5. Keely B.J. Geochemistry of Chlorophylls. In: Chlorophylls and Bacteriochlorophylls. Advances in Photosynthesis and Respiration. Vol. 25 (Grimm B., Porra R.J., Rüdiger W., Scheer H., Eds.). Netherlands: Springer, 2006. p. 535-561.

6. Bandaranayake W.M. Nat. Prod. Rep. 2006, 23, 223-255.

7. Kepp K.P. Coord. Chem. Rev. 2017, 344, 363-374.

8. Collman J.P., Boulatov R., Sunderland C.J., Fu L. Chem. Rev. 2004, 104, 561-588.

9. Anderson J.L.R., Chapman S.K. Dalton Trans. 2005, 1, 13-24.

10. Bertini I., Cavallaro G., Rosato A. Chem. Rev. 2006, 106, 90-115.

11. Dereven'kov I.A., Salnikov D.S., Silaghi-Dumitrescu R., Makarov S.V., Koifman O.I. Coord. Chem. Rev. 2016, 309, 68-83.

12. Saga Y., Yamashita M., Imanishi M., Kimura Y., Masaoka Y., Hidaka T., Nagasawa Y. ACS Omega 2020, 5, 6817-6825.

13. Lindsey J.S. Chem. Rev. 2015, 115, 6534-6620.

14. Malyasova A.S., Khelevina O.G., Koifman O.I. Ross. Khim. Zh. 2017, 61, 3-10 (in Russ.).

15. Krasnovsky Jr. A.A. Ross. Khim. Zh. 2017, 61, 17-41 (in Russ.).

16. Scheer H. An Overview of Chlorophylls and Bacteriochlorophylls: Biochemistry, Biophysics, Functions and Applications. In: Chlorophylls and Bacteriochlorophylls. Advances in Photosynthesis and Respiration. Vol. 25 (Grimm B., Porra R.J., Rüdiger W., Scheer H., Eds.). Netherlands: Springer, 2006. p. 1-26.

17. Gunderson V.L., Wasielewski M.R. Supramolecular Chlorophyll Assemblies for Artificial Photosynthesis. In: Handbook of Porphyrin Science. Vol. 20 (Kadish K.M., Smith K.M., Guilard R., Eds.). World Scientific Publishing Co. Pte. Ltd.: Singapore, 2012. p. 45-105.

18. Borbas K.E. Chlorins. In: Handbook of Porphyrin Science. Vol. 36 (Kadish K.M., Smith K.M., Guilard R., Eds.) 
World Scientific Publishing Co. Pte. Ltd.: Singapore, 2016. p. 1-149.

19. Storz J.F. Physiology 2016, 31, 223-232.

20. Yonetani T., Kanaori K. Biochim. Biophys. Acta 2013, 1834, 1873-1884.

21. Wilks A. Antioxidants \& Redox Signaling 2002, 4, 603-614.

22. Rovira C., Kunc K., Hutter J., Parrinello M. Inorg. Chem. 2001, 40, 11-17.

23. Jones A.R. Photochem. Photobiol. Sci. 2017, 16, 820-834.

24. Shell T.A., Lawrence D.S. Acc. Chem. Res. 2015, 48, 2866-2874.

25. Proinsias K., Giedyk M., Gryko D. Chem. Soc. Rev. 2013, 42, 6605-6619.

26. Ishihara S., Labuta J., Van Rossom W., Ishikawa D., Minami K., Hill J.P., Ariga K. Phys. Chem. Chem. Phys. 2014, 16, 9713-9746.

27. Mirkovic T., Ostroumov E.E., Anna J.M., van Grondelle R., Govindjee, Scholes G.D. Chem. Rev. 2017, 117, 249-293.

28. Aratani N., Kim D., Osuka A. Acc. Chem. Res. 2009, 42, 1922-1934.

29. Otsuki J. J. Mater. Chem. A 2018, 6, 6710-6753.

30. Liu Y., Zhang S., Lindsey J.S. Nat. Prod. Rep. 2018, 35, 879-901.

31. Taniguchi M., Lindsey J.S. Chem. Rev. 2017, 117, 344-535.

32. Dudkin S.V., Makarova E.A., Lukyanets E.A. Russ. Chem. Rev. 2016, 85, 700-730.

33. Chen C.-Y., Sun E., Fan D., Taniguchi M., McDowell B.E., Yang E., Diers J.R., Bocian D.F., Holten D., Lindsey J.S. Inorg. Chem. 2012, 51, 9443-9464.

34. Brückne C., Samankumara L., Ogikubo J., Syntheses of Bacteriochlorins and Isobacteriochlorins. In: Handbook of Porphyrin Science. Vol. 17 (Kadish K.M., Smith K.M., Guilard R., Eds.). Singapore: World Scientific Publishing Co. Pte. Ltd., 2012. p. 1-112.

35. Szyszko B., Latos-Grażyński L. Chem. Soc. Rev. 2015, 44, 3588-3616.

36. Berezin D.B., Karimov D.R., Kustov A.V. Corroles and Their Derivatives: Synthesis, Properties and Prospects for Practical Application (Koifman O.I., Ed.). Moscow: LENAND, 2018. 304 p. (in Russ.).

37. Paolesse R. Synthesis and Modification of Porphyrinoids. In: Topics in Heterocyclic Chemistry. Vol. 33. Springer-Verlag: Berlin, 2014. p. 1-34.

38. Barata J.F.B., Neves M.G.P.M.S., Faustino M.A.F., Tomé A.C., Cavaleiro J.A.S. Chem. Rev. 2017, 117, 3192-3253.

39. Porphyrins: Structure, Properties, Synthesis (Enikolopyan N.S., Ed.). Moscow: Nauka, 1985. 333 p. (in Russ.)

40. Askarov K.A., Ageeva T.A., Rashidova S.T. Wastes of Sericulture. Ways of Their Processing, Application Prospects (Koifman O.I., Ed.). Moscow: Khimia, 2008. 219 p. (in Russ.).

41. Mironov A.F. Ross. Khim. Zh. 2017, 61, 42-68 (in Russ.).

42. Grin M.A., Mironov A.F. Rus. Chem. Bull., Int. Ed. 2016, 65, 333-349.

43. Nyuchev A.V., Otvagin V.F., Gavryushin A.E., Romanenko Yu.I., Koifman O.I., Belykh D.V., Schmalz H.-G., Fedorov A.Yu. Synthesis 2015, 47, 3717-3726.

44. Otvagin V.F., Nyuchev A.V., Kuzmina N.S., Grishin I.D., Gavryushin A.E., Romanenko Yu.V., Koifman O.I., Belykh D.V., Peskova N.N., Shilyagina N.Yu., Balalaeva I.V., Fedorov A.Yu. Eur. J. Med. Chem. 2018, 144, 740-750.

45. Koifman O.I., Ponomarev G.V. A Method of Obtaining Methylpheophorbide, 2013, Patent RF No. 2490273.

46. Ponomarev G.V., Koifman O.I. Photosensitizer and Method of its Preparation, 2014, Patent RF No. 2523380.

47. Chissov V.I., Skobelkin O.K., Mironov A.F. et.al. Pirogov Russian Journal of Surgery 1994, 12, 3-6.
48. Hayashi T., Hisaeda Y. Acc. Chem. Res. 2002, 35, 35-43.

49. Lindsey J.S. Acc. Chem. Res. 2010, 43, 300-311.

50. Beletskaya I.P., Tyurin V.S., Uglov A., Stern C., Guilard R. Survey of Synthetic Routes for Synthesis and Substitution in Porphyrins. In: Handbook of Porphyrin Science. Vol. 23 (Kadish K.M., Smith K.M., Guilard R., Eds.). World Scientific Publishing, 2012. p. 81-278.

51. Smith K.M. New J. Chem. 2016, 40, 5644-5649.

52. Brückner C. Acc. Chem. Res. 2016, 49, 1080-1092.

53. Smith R.M. Strategies of the Synthesis of Octaalkylporphyrin Systems. In: The Porphyrin Handbook. Vol. 1 (Kadish K.M., Smith K.M., Guilard R., Eds.). Academic Press: San Diego, 2000. p. 1-43.

54. Lindsey J.S. Synthesis of meso-Substituted Porphyrins. In: The Porphyrin Handbook. Vol. 1 (Kadish K.M., Smith K.M., Guilard R., Eds.). Academic Press: San Diego, 2000. p. $45-118$.

55. Semeykin A.S., Syrbu S.A., Koifman O.I. Izv. Vyssh. Uchebn. Zaved., Khim. Khim Tekhnol. 2004, 47, 46-55.

56. Lash T.D. Synthesis of Novel Porphyrinoid Chromophores. In: The Porphyrin Handbook. Vol. 2 (Kadish K.M., Smith K.M., Guilard R., Eds.). Academic Press: San Diego, 2000. p. $125-196$.

57. Cheprakov A.V. The Synthesis of $\pi$-Extended Porphyrins. In: Handbook of Porphyrin Science. Vol. 13 (Kadish K.M., Smith K.M., Guilard R., Eds.). World Scientific Publishing Co. Pte. Ltd.: Singapore, 2011. p. 1-149.

58. Ishkov Yu.V., Zhilina Z.I., Vodzinskii S.V. In: Advances in Porphyrin Chemistry. Vol. 4 (Golubchikov O.A., Ed.). SPb.: St-PbGU, 2003. p. 31-44 (in Russ.).

59. Smith B.E., Lash T.D. Tetrahedron 2010, 66, 4413-442.

60. Hiroto S., Miyake Y., Shinokubo H. Chem. Rev. 2016, 117, 2910-3043.

61. Tanaka T., Osuka A. Chem. Soc. Rev. 2015, 44, 943-969.

62. Koifman O.I., Ageeva T.A. Porphyrin Polymers: Synthesis, Properties, Applications. Moscow: LENAND, 2018. 304 p.

63. Matano Y. Chem. Rev. 2017, 117, 3138-3191.

64. Claessens C.G., Hahn U., Torres T. Chem. Rec. 2008, 8, 75-97.

65. Kobayashi N. Synthesis and Characterization of Chiral Phthalocyanines. In: Handbook of Porphyrin Science. Vol. 23 (Kadish K.M., Smith K.M., Guilard R., Eds.). World Scientific, 2012. p. 373-440.

66. Lomova T.N., Tyulyaeva E.Y., New Trends in the Direct Synthesis of Phthalocyanine/Porphyrin Complexes. In: Direct Synthesis of Metal Complexes (Kharisov B., Ed.). Elsevier, 2018. p. 239-278.

67. Koifman O.I., Ageeva T.A. Russ. Chem. Bull. 2015, 64, 2001-2011.

68. Ageeva T.A., Golubev D.V., Gorshkova A.S., Ionov A.M., Koifman O.I., Mozhchil R.N., Rumyantseva V.D., Sigov A.S., Fomichev V.V. Macroheterocycles 2018, 11, 155-161.

69. Ageeva T.A., Golubev D.V., Gorshkova A.S., Ionov A.M., Kohylova E.V., Koifmah O.I., Mozhchil R.N., Rozhkova E.P., Rumyantseva V.D., Sigov A.S., Fomichev V.V. Macroheterocycles 2019, 12, 148-153.

70. McConnell I., Li G., Brudvi G.W. Chem. Biol. 2010, 17, 434-447.

71. Bottari G., Trukhina O., Ince M., Torres T. Coord. Chem. Rev. 2012, 256, 2453-2477.

72. Drain C.M., Varotto A., Radivojevic I. Chem. Rev. 2009, 109, 1630-1658.

73. Morisue M., Kobuke Y. Supramolecular Organization of Porphyrins and Phthalocyanines by Use of Biomimetic Coordination Methodology. In: Handbook of Porphyrin Science. Vol. 32 (Kadish K.M., Smith K.M., Guilard R., Eds.). World Scientific Publishing, 2014. p. 1-126. 
74. Mamardashvili G.M., Mamardashvili N.Zh., Koifman O.I. Russ. Chem. Rev. 2008, 77, 59-75.

75. Koifman O.I., Mamardashvili N.Zh. Nanotechnologies in Russia 2009, 4, 253-261.

76. Beletskaya I., Tyurin V.S., Tsivadze A.Y., Guilard R., Stern C. Chem. Rev. 2009, 109, 1659-1713.

77. Koifman O.I., Ageeva T.A. Polymer Sci. Ser. C 2014, 56, 84-103.

78. Khelevina O.G., Malyasova A.S., Koifman O.I. Russ. J. Gen. Chem. 2020, 90, 1646-1659.

79. Ageeva T.A., Bush A.A., Golubev D.V., Gorshkova A.S., Kamentsev K.E., Koifman O.I., Rumyantseva V.D., Sigov A.S., Fomichev V.V. J. Organomet. Chem. 2020, 922, 121355.

80. Percástegui E.G., Jancik V. Coord. Chem. Rev. 2020, 407, 213165.

81. Functional Materials Based on Tetrapyrrole Macroheterocyclic Compounds (Koifman O.I., Ed.). Moscow: LENAND, 2019. $848 \mathrm{p}$.

82. Chan K.S., Zhou X., Luo B.S., Mak T.C.W. J. Chem. Soc. Chem. Commun. 1994, 271.

83. Chan K.S., Zhou X.A., Au M.T., Tam C.Y. Tetrahedron 1995, 51, 3129.

84. Hyslop A.G., Kellett M.A., Iovine P.V., Therien M.J. J. Am. Chem. Soc. 1998, 120, 12676.

85. Ali H., van Lier J.E. Tetrahedron 1994, 50, 11933.

86. Shanmugathasan S., Johnson C.K., Edwards C., Matthews E.K., Dolphin D., Boyle R.W. J. Porphyrins Phthalocyanines 2000, 4, 228.

87. Chang J.C., Ma C.J., Lee G.H., Peng S.M., Yeh C.Y. Dalton Trans. 2005, 1504.

88. Gauler R., Risch N. Eur. J. Org. Chem. 1998, 1193.

89. Sergeeva N.N., Scala A., Bakar M.A., O’Riordan G., O’Brien J., Grassi G., Senge M.O. J. Org. Chem. 2009, 74, 7140.

90. Santos F.D., Cunha A.C., de Souza M.C.B.V., Tome A.C., Neves M.G.P.M.S., Ferreira V.F., Cavaleiro J.A.S. Tetrahedron Lett. 2008, 49, 7286.

91. Chen Y., Zhang X.P. J. Org. Chem. 2003, 68, 4432.

92. Takanami T., Hayashi M., Hino F., Suda K. Tetrahedron Lett. 2003, 44, 7353.

93. Khan M.M., Ali H., van Lier J.E. Tetrahedron Lett. 2001, 42, 1615.

94. Gao G.-Y., Chen Y., Zhang X.P. J. Org. Chem. 2003, 68, 6215.

95. Wang K., Osuka A., Song J. ACS Central Science, 2020, 6, 2159-2178.

96. Gao G.-Y., Chen Y., Zhang X.P. Org. Lett. 2004, 6, 1837.

97. Gao G.-Y., Ruppel J.V., Allen B., Chen Y., Zhang X.P. J. Org. Chem. 2007, 72, 9060.

98. Artamkina G.A., Sazonov P.K., Shtern M.M., Grishina G.V., Veselov I.S., Semeikin A.S., Syrbu S.A., Koifman O.I., Beletskaya I.P. Synlett 2008, 45-48.

99. Artamkina G.A., Sazonov P.K., Shtern M.M., Grishina G.V., Veselov I.S., Semeikin A.S., Syrbu S.A., Koifman O.I., Beletskaya I.P. Russ. J. Org. Chem. 2008, 44, 421.

100. Mikhalitsyna E.A., Tyurin V.S., Beletskaya I.P. J. Porphyrins Phthalocyanines 2015, 19, 874.

101. Tyurin V.S., Mikhalitsyna E.A., Semeikin A.S., Beletskaya I.P. Macroheterocycles 2015, 8, 358.

102. Polevaya Y.P., Tyurin V.S., Beletskaya I.P. J. Porphyrins Phthalocyanines 2014, 18, 20.

103. Yaschuk Y.P., Tyurin V.S., Beletskaya I.P. Macroheterocycles 2012, 5, 302.

104. Locos O.B., Arnold D.P. Org. Biomol. Chem. 2006, 4, 902.

105. Song J., Jang S.Y., Yamaguchi S., Sankar J., Hiroto S., Aratani N., Shin J.Y., Easwaramoorthi S., Kim K.S., Kim D., Shinokubo H., Osuka T. Angew. Chem. Int. Ed. 2008, 47, 6004.
106. Mikhalitsyna E.A., Tyurin V.S., Khrustalev V.N., Lonin I.S., Beletskaya I.P. Dalton Trans. 2014, 43, 3563.

107. Mikhalitsyna E.A., Tyurin V.S., Zamilatskov I.A., Khrustalev V.N., Beletskaya I.P. Dalton Trans. 2012, 41, 7624.

108. Ranyuk E.R., Filatov M.A., Averin A.D., Cheprakov A.V., Beletskaya I.P. Synthesis 2012, 44, 393.

109. Yakushev A.A., Averin A.D., Maloshitskaya O.A., Syrbu S.A., Koifman O.I., Beletskaya I.P. Macroheterocycles 2016, 9, 65.

110. Yakushev A.A., Averin A.D., Maloshitskaya O.A., Syrbu S.A., Koifman O.I., Beletskaya I.P. Mendeleev Commun. 2016, 26, 199.

111. Averin A.D., Yakushev A.A., Maloshitskaya O.A., Syrbu S.A., Koifman O.I., Beletskaya I.P. Russ. Chem. Bull. 2017, 66, 1456.

112. Yakushev A.A., Averin A.D., Maloshitskaya O.A., Koifman O.I., Syrbu S.A., Beletskaya I.P. Macroheterocycles 2018, $11,135$.

113. Yakushev A.A., Chernichenko N.M., Anokhin M.V., Averin A.D., Buryak A.K., Denat F., Beletskaya I.P. Molecules 2014, 19, 940.

114. Yakushev A.A., Averin A.D., Sakovich M.V., Vatsouro I.M., Kovalev V.V., Syrbu S.A., Koifman O.I., Beletskaya I.P. J. Porphyrins Phthalocyanines 2019, 23, 1551.

115. Braun A., Tcherniac J. Berichte der Deutschen Chemischen Gesellschaft 1907, 40, 2709-2714.

116. Linstead R. P. J. Chem. Soc. (Resumed) 1934, 1016-1017.

117. Berezin B.D. Coordination Compounds of Porphyrins and Phthalocyanine. New York - Toronto: J. Wiley, 1981. 278 p.

118. Leznoff C.C., Lever A.B.P. In: Phthalocyanines. Properties and Applications (Leznoff C.C., Lever A.B.P., Eds.). New York: VCH, 1989, Vol. 1. 448 p.; 1993, Vol. 2. 305 p.; 1993, Vol. 3. 303 p.; 1996, Vol. 4. 536 p.

119. Allen C.M., Sharman W.M., Van Lier J.E. J. Porphyrins Phthalocyanines 2001, 5, 161-169.

120. Walter M.G., Rudine A.B., Wamser C.C. J. Porphyrins Phthalocyanines 2010, 14, 759-792.

121. Wang H., Qi D., Xie Z., Cao W., Wang K., Shang H., Jiang J. Chem. Commun. 2013, 49, 889-891.

122. Fukuda T., Biyajima T., Kobayashi N. J. Am. Chem. Soc. 2010, 132, 6278-6279.

123. Horii Y., Horie Y., Katoh K., Breedlove B.K., Yamashita M. Inorg. Chem., 2018, 57, 565-574.

124. Wang H., Kobayashi N., Jiang J. Chem. Eur. J. 2012, 18, 1047-1049.

125. Korostei Y.S., Tarasova V.G., Pushkarev V.E., Borisova N.E., Vorobiev A.K., Tomilova L.G. Dyes Pigm. 2018, 159, $573-575$

126. Korostei Y.S., Pushkarev V.E., Tolbin A.Y., Dzuban A.V., Chernyak A.V., Konev D.V., Medvedeva T.O., Talantsev A.D., Sanina N.A., Tomilova L.G. Dyes Pigm. 2019, 170, 107648.

127. Chen Y., Liu C., Ma F., Qi D., Liu Q., Sun H.-L., Jiang J. Chem. Eur. J. 2018, 24, 8066-8070.

128. Pushkarev V.E., Tolbin A.Y., Zhurkin F.E., Borisova N.E., Trashin S.A., Tomilova L.G., Zefirov N.S. Chem. Eur. J. 2012, 18, 9046-9055.

129. Korostei Y.S., Tolbin A.Y., Dzuban A.V., Pushkarev V.E., Sedova M.V., Maklakov S.S., Tomilova L.G. Dyes Pigm. 2018, 149, 201-211.

130. Wang K., Qi D., Wang H., Cao W., Li W., Jiang J. Chem. Eur. J. 2012, 18, 15948-15952.

131. Balashova I.O., Pushkarev V.E., Shestov V.I., Tomilova L.G., Koifman O.I., Ponomarev G.V. Macroheterocycles 2015, 8, 233-238.

132. Tomilova L.G., Pushkarev V.E., Balashova I.O., Shestov V.I., Ponomarev G.V., Koifman O.I., Platonova Y.B., Volov A.N. 2020, Patent $R F$ No 2722309. 
133. Quartarolo A.D., Pérusse D., Dumoulin F., Russo N., Sicilia E. J. Porphyrins Phthalocyanines 2013, 17, 980-988.

134. Yang K., Wang J., Zhao Z., Zhao F., Wang K., Zhang X., Zhang F. Org. Electron. 2020, 83, 105739.

135. Li W., Liu W., Zhang X., Yan D., Liu F., Zhan C. Macromol. Rapid Commun. 2019, 40, 1900353.

136. Kobayashi N., Furuyama T., Satoh K. J. Am. Chem. Soc. 2011, 133, 19642-19645.

137. Lukyanets E.A., Nemykin V.N. J. Porphyrins Phthalocyanines 2010, 14, 1-40.

138. Kobayashi N., Nakajima S.-I., Ogata H., Fukuda T. Chem. Eur. J. 2004, 10, 6294-6312.

139. Muranaka A., Yonehara M., Uchiyama M. J. Am. Chem. Soc. 2010, 132, 7844-7845.

140. Ince M., Hausmann A., Martínez-Díaz M.V., Guldi D.M., Torres T. Chem. Commun. 2012, 48, 4058-4060.

141. Dubinina T.V., Tomilova L.G., Zefirov N.S. Russ. Chem. Rev. 2013, 82, 865-895.

142. Dubinina T.V., Trashin S.A., Borisova N.E., Boginskaya I.A., Tomilova L.G., Zefirov N.S. Dyes Pigm. 2012, 93, 1471-1480.

143. Makarov S.G., Suvorova O.N., Litwinski C., Ermilov E.A., Röder B., Tsaryova O., Dülcks T., Wöhrle D. Eur. J. Inorg. Chem. 2007, 2007, 546-552.

144. Dubinina T.V., Ivanov A.V., Borisova N.E., Trashin S.A., Gurskiy S.I., Tomilova L.G., Zefirov N.S. Inorg. Chim. Acta 2010, 363, 1869-1878.

145. Dubinina T.V., Paramonova K.V., Trashin S.A., Borisova N.E., Tomilova L.G., Zefirov N.S. Dalton Trans. 2014, 43, 2799-2809.

146. Dubinina T.V., Pushkarev V.E., Trashin S., Paramonova K.V., Tomilova L.G. Macroheterocycles 2012, 5, 366-370.

147. Berendonk T.U., Manaia C.M., Merlin C., Fatta-Kassinos D., Cytryn E., Walsh F., Bürgmann H., Sørum H., Norström M., Pons M.-N., Kreuzinger N., Huovinen P., Stefani S., Schwartz T., Kisand V., Baquero F., Martinez J.L. Nat. Rev. Microbiology 2015, 13, 310-317.

148. Cizmas L., Sharma V.K., Gray C.M., McDonald T.J. Environ. Chem. Lett. 2015, 13, 381-394.

149. Larsson D.G.J. Upsala J. Med. Sci. 2014, 119, 108-112.

150. EU Action on Antimicrobial Resistance, https://ec.europa. eu/health/antimicrobial-resistanceleu-action-on-antimicrobial-resistance en (date of access 20.11.2020).

151. WHO multi-country survey reveals widespread public misunderstanding about antibiotic resistance, https://www.who. int/en/news-room/detail/16-11-2015-who-multi-countrysurvey-reveals-widespread-public-misunderstandingabout-antibiotic-resistance (date of access 20.11.2020).

152. Unceta N., Sampedro M.C., Bakar N.K.A., Gómez-Caballero A., Goicolea M.A., Barrio R.J. J. Chromatogr. A 2010, 1217, 3392-3399.

153. Berna M.J., Ackermann B.L., Murphy A.T. Anal. Chim. Acta 2004, 509, 1-9.

154. Ferrer I., Zweigenbaum J.A., Thurman E.M. J. Chromatogr. A 2010, 1217, 5674-5686.

155. Feier B., Gui A., Cristea C., Săndulescu R. Anal. Chim. Acta 2017, 976, 25-34.

156. Cristea C., Florea A., Tertiş M., Săndulescu R. Immunosensors. In: Biosensors. Micro and Nanoscale Applications (Rinken T., Ed.). IntechOpen, 2015.

157. Trashin S., Rahemi V., Ramji K., Neven L., Gorun S.M., De Wael K. Nat. Commun. 2017, 8, 16108.

158. Khan S.U., Trashin S.A., Korostei Y.S., Dubinina T.V., Tomilova L.G., Verbruggen S.W., De Wael K., ChemPhotoChem 2020, 4, 300-306.

159. Merkel P.B., Kearns D.R. J. Am. Chem. Soc. 1972, 94, 7244-7253.
160. Davies M.J. Methods 2016, 109, 21-30.

161. Valduga G., Nonell S., Reddi E., Jori G., Braslavsky S.E. Photochem. Photobiol. 1988, 48, 1-5.

162. Tanielian C., Wolff C., Esch M. J. Phys. Chem. 1996, 100, 6555-6560.

163. del Rey B., Keller U., Torres T., Rojo G., Agullo-Lopez F., Nonell S., Marti C., Brasselet S., Ledoux I., Zyss J. J. Am. Chem. Soc. 1998, 120, 12808-12817.

164. Dubinina T.V., Osipova M.M., Zasedatelev A.V., Krasovskii V.I., Borisova N.E., Trashin S.A., Tomilova L.G., Zefirov N.S. Dyes Pigm. 2016, 128, 141-148.

165. Wilkinson F., Helman W.P., Ross A.B. J. Phys. Chem. Ref. Data 1993, 22, 113-262.

166. Potz R., Goeldner M., Hueckstaedt H., Cornelissen U., Tutass A., Homborg H. ChemInform 2000, 31(16).

167. Nyokong T. Coord. Chem. Rev. 2007, 251, 1707-1722.

168. Burtsev I.D., Dubinina T.V., Platonova Y.B., Kosov A.D., Pankratov D.A., Tomilova L.G. Mendeleev Commun. 2017, 27, 466-469.

169. Platonova Y.B., Morozov A.S., Burtsev I.D., Korostei Y.S., Ionidi V.Y., Romanovsky B.V., Tomilova L.G. Mendeleev Commun. 2018, 28, 198-199.

170. Burtsev I.D., Platonova Y.B., Volov A.N., Tomilova L.G. Polyhedron 2020, 188, 114697.

171. Moiseeva E.O., Platonova Y.B., Konev D.V., Trashin S.A., Tomilova L.G. Mendeleev Commun. 2019, 29, 212-214.

172. Novikov R.A., Levina A.A., Borisov D.D., Volodin A.D., Korlyukov A.A., Tkachev Y.V., Platonova Y.B., Tomilova L.G., Tomilov Y.V. Organometallics 2020, 39, 2580-2593.

173. Burtsev I.D., Platonova Y.B., Volov A.N., Tomilova L.G. Macroheterocycles 2020, 13, 126-129.

174. Platonova Y.B., Volov A.N., Tomilova L.G. J. Catalysis 2019, 373, 222-227.

175. Platonova Y.B., Volov A.N., Tomilova L.G. Bioorg. Med. Chem. Lett. 2020, 30, 127351.

176. Platonova Y.B., Volov A.N., Tomilova L.G. J. Catalysis 2020, 391, 224-228.

177. Leyzerovich N.N., Shvedene N.V., Blikova Y.N., Tomilova L.G., Pletnev I.V. Electroanalysis 2001, 13, 246-252.

178. Shvedene N.V., Otkidach K.N., Gumerov M.R., Tarakanov P.A., Tomilova L.G. J. Anal. Chem. 2015, 70, 72-80.

179. Blikova Y.N., Ivanov A.V., Tomilova L.G., Shvedene N.V. Russ. Chem. Bull. 2003, 52, 150-153.

180. Shvedene N.V., Abashev M.N., Arakelyan S.A., Otkidach K.N., Tomilova L.G., Pletnev I.V. J. Solid State Electrochem. 2019, 23, 543-552.

181. Tolbin A.Y., Tomilova L.G. Russ. Chem. Rev. 2011, 80, 531-551.

182. Shvedene N.V., Otkidach K.N., Ondar E.E., Osipova M.M., Dubinina T.V., Tomilova L.G., Pletnev I.V. J. Anal. Chem. 2017, 72, 95-104.

183. Sheik-Bahae M., Said A.A., Wei T., Hagan D.J., Stryland E.W.V. IEEE J. Quantum Electronics 1990, 26, 760-769.

184. Oluwole D.O., Ngxeke S.M., Britton J., Nyokong T. J. Photochem. Photobiol., A: Chemistry 2017, 347, 146-159.

185. Sekhosana K.E., Amuhaya E., Nyokong T. Polyhedron 2016, 105, 159-169.

186. Mgidlana S., Şen P., Nyokong T. J. Mol. Struct. 2020, 1220, 128729.

187. Bankole O.M., Osifeko O., Nyokong T. J. Photochem. Photobiol., A: Chemistry 2016, 329, 155-166.

188. Kuzmina E.A., Dubinina T.V., Borisova N.E., Tarasevich B.N., Krasovskii V.I., Feofanov I., Dzuban A.V., Tomilova L.G. Dyes Pigm. 2020, 174, 108075.

189. Kuzmina E.A., Dubinina T.V., Zasedatelev A.V., Baranikov A.V., Makedonskaya M.I., Egorova T.B., Tomilova L.G. Polyhedron 2017, 135, 41-48. 
190. Kuzmina E.A., Dubinina T.V., Dzuban A.V., Krasovskii V.I., Maloshitskaya O.A., Tomilova L.G. Polyhedron 2018, 156, 14-18.

191. Pan F., Gao S., Chen C., Song C., Zeng F. Mater. Sci. Eng.: $R$ : Reports 2014, 83, 1-59.

192. Chen Y., Song H., Jiang H., Li Z., Zhang Z., Sun X., Li D., Miao G. Appl. Phys. Lett. 2014, 105, 193502.

193. Tseng R.J., Huang J., Ouyang J., Kaner R.B., Yang Nano Lett. 2005, 5, 1077-1080.

194. Kotova M.S., Drozdov K.A., Dubinina T.V., Kuzmina E.A., Tomilova L.G., Vasiliev R.B., Dudnik A.O., Ryabova L.I., Khokhlov D.R. Scientific Reports 2018, 8, 9080.

195. Gonzalez-Anton R., Osipova M.M., Garcia-Hernandez C., Dubinina T.V., Tomilova L.G., Garcia-Cabezon C., RodriguezMendez M.L. Electrochimica Acta 2017, 255, 239-247.

196. Martynov A.G., Safonova E.A., Tsivadze A.Y., Gorbunova Y.G. Coord. Chem. Rev. 2019, 387, 325-347.

197. Yamashita M. Bull. Chem. Soc. Jpn. 2020, bcsj.20200257.

198. Sessoli R., Gatteschi D., Caneschi A., Novak M.A. Nature 1993, 365, 141-143.

199. Ishikawa N., Sugita M., Ishikawa T., Koshihara S.-Y., Kaizu Y. J. Am. Chem. Soc. 2003, 125, 8694-8695.

200. Ishikawa N., Sugita M., Ishikawa T., Koshihara S., Kaizu Y. J. Phys. Chem. B 2004, 108, 11265-11271.

201. Wang H., Wang B.W., Bian Y., Gao S., Jiang J. Coord. Chem. Rev. 2016, 306, 195-216.

202. Ishikawa N., Iino T., Kaizu Y. J. Am. Chem. Soc. 2002, 124, 11440-11447.

203. Ishikawa N., Otsuka S., Kaizu Y. Angew. Chem. Int. Ed. 2005, 44, 731-3.

204. Holmberg R.J., Polovkova M.A., Martynov A.G., Gorbunova Y.G., Murugesu M. Dalton Trans. 2016, 45, 9320-9327.

205. Lan Y., Klyatskaya S., Ruben M., Fuhr O., Wernsdorfer W., Candini A., Corradini V., Lodi Rizzini A., del Pennino U., Troiani F., Joly L., Klar D., Wende H., Affronte M. J. Mater. Chem., C 2015, 3, 9794-9801.

206. Polovkova M.A., Martynov A.G., Birin K.P., Nefedov S.E., Gorbunova Y.G., Tsivadze A.Y. Inorg. Chem. 2016, 55, 9258-9269.

207. Sakaue S., Fuyuhiro A., Fukuda T., Ishikawa N. Chem. Commun. 2012, 48, 5337.

208. Katoh K., Breedlove B.K., Yamashita M. Chem. Sci. 2016, 7, 4329-4340.

209. Sugimoto H., Higashi T., Maeda A., Mori M., Masuda H., Taga T. J. Chem. Soc., Chem. Commun. 1983, 1234-1235.

210. Maeda A., Sugimoto H. J. Chem. Soc. Faraday Trans. 2 1986, 82, 2019.

211. Ge J., Qiu Y., Wang H., Su J., Wang P., Chen Z. Chem. - An Asian J. 2020, 15, 3013-3019.

212. Ge J.-Y., Wang H.-Y., Li J., Xie J.-Z., Song Y., Zuo J.-L. Dalton Trans. 2017, 46, 3353-3362.

213. Fukuda T., Kuroda W., Ishikawa N. Chem. Commun. 2011, 47, 11686.

214. Katoh K., Horii Y., Yasuda N., Wernsdorfer W., Toriumi K., Breedlove B.K., Yamashita M. Dalton Trans. 2012, 41, 13582-13600.

215. Horii Y., Katoh K., Sugimoto K., Nakanishi R., Breedlove B.K., Yamashita M. Chem. Eur. J. 2019, 3098-3104.

216. Morita T., Damjanović M., Katoh K., Kitagawa Y., Yasuda N., Lan Y., Wernsdorfer W., Breedlove B.K., Enders M., Yamashita M. J. Am. Chem. Soc. 2018, 140, 2995-3007.

217. Tolbin A.Y., Pushkarev V.E., Shulishov E.V., Tomilova L.G. J. Porphyrins Phthalocyanines 2012, 16, 341-350.

218. Horii Y., Katoh K., Breedlove B.K., Yamashita M. Chem. Commun. 2017, 53, 8561-8564.

219. Horii Y., Kishiue S., Damjanović M., Katoh K., Breedlove B.K., Enders M., Yamashita M. Chem. Eur. J. 2018, 24, $4320-4327$.
220. Martynov A.G., Polovkova M.A., Berezhnoy G.S., Sinelshchikova A.A., Dolgushin F.M., Birin K.P., Kirakosyan G.A., Gorbunova Y.G., Tsivadze A.Y. Inorg. Chem. 2020, 59, 9424-9433.

221. Martynov A.G., Gorbunova Y.G. Polyhedron 2010, 29, 391-399.

222. Martynov A.G., Gorbunova Y.G., Tsivadze A.Y. Dalton Trans. 2011, 40, 7165-7171.

223. Birin K.P., Gorbunova Y.G., Tsivadze A.Y. Magn. Reson. Chem. 2010, 48, 505-515.

224. Gorbunova Y.G., Martynov A.G., Birin K.P., Tsivadze A.Y. Russ. J. Inorg. Chem. 2021, in press.

225. Wöhrle D., Schnurpfeil G., Makarov S.G., Kazarin A., Suvorova O.N. Macroheterocycles 2012, 5, 191-202.

226. The Porphyrin Handbook. Vols. 1-14 (Kadish K.M., Smith K.M., Guilard R., Eds.). San Diego: Academic Press, 2010.

227. Inabe T., Tajima H. Chem. Rev. 2004, 104, 5503-5534.

228. Yu D.E.C., Matsuda M., Tajima H., Kikuchi A., Taketsugu T., Hanasaki N., Naito T., Inabe T. J. Mater. Chem. 2009, 19, $718-723$.

229. Konarev D.V., Kuzmin A.V., Simonov S.V., Khasanov S.S., Otsuka A., Yamochi H., Saito G., Lyubovskaya R.N. Dalton Trans. 2012, 41, 13841-13847.

230. Konarev D.V., Khasanov S.S., Ishikawa M., Otsuka A., Yamochi H., Saito G., Lyubovskaya R.N. Inorg. Chem. 2013, 52, 3851-3859.

231. Konarev D.V., Zorina L.V., Khasanov S.S., Hakimova E.U., Lyubovskaya R.N. New J. Chem. 2012, 36, 48-51.

232. Konarev D.V., Kuzmin A.V., Faraonov M.A., Ishikawa M., Nakano Y., Khasanov S.S., Otsuka A., Yamochi H., Saito G., Lyubovskaya R.N. Chem. Eur. J. 2015, 21, 1014-1028.

233. Konarev D.V., Faraonov M.A., Kuzmin A.V., Khasanov S.S., Nakano Y., Batov M.S., Norko S.I., Otsuka A., Yamochi H., Saito G., Lyubovskaya R.N. New J. Chem. 2017, 41, $6866-6874$.

234. Konarev D.V., Khasanov S.S., Kuzmin A.V., Nakano Y., Ishikawa M., Otsuka A., Yamochi H., Saito G., Lyubovskaya R.N. Cryst. Growth Des. 2017, 17, 753-762.

235. Konarev D.V., Kuzmin A.V., Khasanov S.S., Batov M.S., Otsuka A., Yamochi H., Kitagawa H., Lyubovskaya R.N. CrystEngComm 2018, 20, 385-401.

236. Konarev D.V., Kuzmin A.V., Batov M.S., Khasanov S.S., Otsuka A., Yamochi H., Kitagawa H., Lyubovskaya R.N. ACS Omega 2018, 3, 14875-14888.

237. Konarev D.V., Kuzmin A.V., Nakano Y., Faraonov M.A., Khasanov S.S., Otsuka A., Yamochi H., Saito G., Lyubovskaya R.N. Inorg. Chem. 2016, 55, 1390-1402.

238. Konarev D.V., Kuzmin A.V., Fatalov A.M., Khasanov S.S., Yudanova E.I., Lyubovskaya R.N. Chem. Eur. J. 2018, 24, $8415-8423$

239. Konarev D.V., Zorina L.V., Khasanov S.S., Shestakov A.F., Fatalov A.M., Otsuka A., Yamochi H., Kitagawa H., Lyubovskaya R.N. Inorg. Chem. 2018, 57, 583-589.

240. Konarev D.V., Kuzmin A.V., Shestakov A.F., Khasanov S.S., Lyubovskaya R.N. Dalton Trans. 2019, 48, 4961-4972.

241. Konarev D.V., Kuzmin A.V., Khasanov S.S., Otsuka A., Yamochi H., Saito G., Lyubovskaya R.N. Eur. J. Inorg. Chem. 2016, 4099-4103.

242. Konarev D.V., Kuzmin A.V., Nakano Y., Khasanov S.S., Otsuka A., Yamochi H., Kitagawa H., Lyubovskaya R.N. Dalton Trans. 2018, 47, 4661-4671.

243. Konarev D.V., Kuzmin A.V., Khasanov S.S., Otsuka A., Yamochi H., Kitagawa H., Lyubovskaya R.N. Inorg. Chim. Acta 2020, 510, 119732.

244. Konarev D.V., Troyanov S.I., Kuzmin A.V., Nakano Y., Khasanov S.S., Otsuka A., Yamochi H., Saito G., Lyubovskaya R.N. Organometallics 2015, 34, 879-889. 
245. Kubiak R., Janczak J. J. Alloys Compd. 1992, 189, 107111.

246. Janczak J., Kubiak R. Acta Crystallogr., Sect. C: Cryst. Struct. Commun. 2003, 59, m237-m240.

247. Janczak J., Kubiak R., Śledź M., Borrmann H., Grin Y. Polyhedron 2003, 22, 2689-2697.

248. Janczak J., Kubiak R. Inorg. Chim. Acta 2003, 342, 64-76.

249. Konarev D.V., Khasanov S.S., Ishikawa M., Otsuka A., Yamochi H., Saito G., Lyubovskaya R.N. Chem. Asian J. 2017, 12, 910-919.

250. Zhou W., Thompson J.R., Leznoff C.C., Lez D.B. Chem. Eur. J. 2017, 23, 2323-2331.

251. Hiroto S., Furukawa K., Shinokubo H., Osuka A. J. Am. Chem. Soc. 2006, 128, 12380-12381.

252. Wong E.W.Y., Leznoff D.B. J. Porphyrins Phthalocyanines 2012, 16, 154-162.

253. Konarev D.V., Zorina L.V., Khasanov S.S., Lyubovskaya R.N. Dalton Trans. 2012, 41, 9170-9175.

254. Konarev D.V., Karimov D.R., Khasanov S.S., Shestakov A.F., Otsuka A., Yamochi H., Kitagawa H., Lyubovskaya R.N. Dalton. Trans. 2017, 46, 13994-14001.

255. Konarev D.V., Khasanov S.S., Kumin A.V., Nakano Y., Ishikawa M., Otsuka A., Yamochi H., Saito G., Lyubovskaya R.N. Inorg. Chem. 2017, 56, 1804-1813.

256. Kitagawa T., Lee Y., Takeuchi K. Chem. Commun. 1999, 1529-1530.

257. Ishikawa N. Phthalocyanine-Based Magnets. In: Functional Phthalocyanine Molecular Materials. Vol. 135 (Jiang J., Ed.). Berlin: Struct. Bonding, 2010. p. 211-228.

258. Barraclough C.G., Martin R.L., Mitra S., Sherwood R.C. J. Chem. Phys. 1970, 53, 1638-1642.

259. Barraclough C.G., Gregson A.K., Mitra S. J. Chem. Phys. 1974, 60, 962-968.

260. Mitra S., Gregson A.K., Hatfield W., Weller R. Inorg. Chem. 1983, 22, 1729-1732.

261. Evangelisti M., Bartolome J., de Jongh L.J., Filoti G. Phys. Rev. B 2002, 66, 144410.

262. Korepanov V.I., Sedlovets D.M. Macroheterocycles 2019 , $12,232-243$.

263. Giménez-Agulló N., de Pipaón C.S., Adriaenssens L., Filibian M., Martínez-Belmonte M., Escudero-Adán E.C., Carretta P., Ballester P., Galán-Mascarós J.R. Chem. Eur. J. 2014, 20, 12817-12825.

264. Zhang P., Guo Y.-N., Tang J. Coord. Chem. Rev. 2013, 257, $1728-1763$.

265. Magnani N. Int. J. Quantum Chemistry 2014, 114, 755-759.

266. Kan J., Wang H., Sun W., Cao W., Tao J., Jiang J. Inorg. Chem. 2013, 52, 8505-8510.

267. Wang K., Zeng S., Wang H., Dou J., Jiang J. Inorg. Chem. Front. 2014, 1, 167-171.

268. Lomova T.N. Axially Coordinated Metalloporphyrins in Science and Application. Moscow: URSS, 2018. 700 p. (in Russ.).

269. Beltran-Lopez J.F., Sazatornil M., Palacios E., Burriel R. J. Therm. Anal. Calorim. 2016, 125, 579-583.

270. Liu J.-L., Chen Y.-C., Guo F.-S., Tong M.-L. Coord. Chem. Rev. 2014, 281, 26-49.

271. Ishikawa N., Sugita M., Wernsdorfer W. Angew. Chem. Int. Ed. 2005, 44, 2931-2935.

272. Sharples J.W., Collison D. Polyhedron SI 2013, 66, 15-27.

273. Korolev V.V., Korolev D.V., Lomova T.N., Mozhzhukhina E.G., Zakharov A.G. Russ. J. Phys. Chem. A 2012, 86, 504-508.

274. Korolev V.V., Romanov A.S., Arefyev I.M. Russ. J. Phys. Chem. A 2006, 80, 464-466.

275. Korolev V.V., Korolev D.V., Ramazanova A.G. J. Therm. Anal. Calorim. 2019, 136, 937-941.
276. Korolev V.V., Klyueva M.E., Arefyev I.M., Ramazanova A.G., Lomova T.N., Zakharov A.G. Macroheterocycles 2008, 1, 68-71.

277. Klyueva M.E., Korolev V.V., Arefyev I.M., Lomova T.N. J. Porphyrins Phthalocyanines 2008, 12, 584.

278. Korolev V.V., Aref'ev I.M., Lomova T.N., Klyueva M.E., Zakharov A.G., Korolev D.V. Russ. J. Phys. Chem. A 2010, 84, 1631-1635.

279. Korolev V.V., Aref'ev I.M., Lomova T.N., Ovchenkova E.N., Klyueva M.E., Zakharov A.G., Korolev D.V. Russ. J. Phys. Chem. A 2012, 86, 1165-1170.

280. Lomova T.N., Korolev V.V., Ramazanova A.G., Ovchenkova E.N. J. Porphyrins Phthalocyanines 2015, 19, 1262-1269.

281. Korolev V.V., Lomova T.N., Ramazanova A.G., Mozhzhukhina E.G. Synth. Met. 2016, 220, 502-507.

282. Korolev V.V., Lomova T.N., Ramazanova A.G., Mozhzhukhina E.G. Mendeleev Commun. 2016, 26, 301-303.

283. Korolev V.V., Lomova T.N., Ramazanova A.G., Korolev D.V., Mozhzhukhina E.G. J. Organomet. Chem. 2016, 819, 209-215.

284. Lomova T.N., Korolev V.V., Bichan N.G., Ovchenkova E.N., Ramazanova A.G., Balmasova O.V., Gruzdev M.S. Synth. Met. 2019, 253, 116-121.

285. Korolev V.V., Lomova T.N., Ramazanova A.G. Radioelektr. Nanosistem. Inform. Tekhnol. [Radio Electronics. Nanosystems. Information Technology] 2019, 11, 199-216 (in Russ.).

286. Korolev V.V., Lomova T.N., Maslennikova A.N., Korolev D.V., Shpakovsky D.B., Zhang J., Milaeva E.R. J. Magn. Magn. Mater. 2016, 401, 86-90.

287. Miller J.S., Calabrese J.C., McLean R.S., Epstein A.J. $A d v$. Mater. 1992, 4, 498-501.

288. Miller J.S., Vazquez C., Calabrese J.C., McLean R.S., Epstein A.J. Adv. Mater. 1994, 6, 217-221.

289. Böhm A., Vazquez C., McLean R.S., Calabrese J.C., Kalm S.E., Manson J.L., Epstein A.J., Miller J.S. Inorg. Chem. 1996, 35, 3083-3088.

290. Brandon E.J., Arif A.M., Burkhart B.M., Miller J.S. Inorg. Chem. 1998, 37, 2792-2798.

291. Zhang Y.J. Alloys and Compounds 2019, 787, 1173-1186.

292. Ovchenkova E.N., Bichan N.G., Tsaturyan A.A., Kudryakova N.O., Gruzdev M.S., Gostev F.E., Shelaev I.V., Nadtochenko V.A., Lomova T.N. J. Phys. Chem. C 2020, 124, 4010-4023.

293. Andreenko A.S., Belov K.P., Nikitin S.A. Uspekhi Fiz. Nauk [Advances in Physical Sciences] 1989, 158, 553-579 (in Russ.).

294. Milaeva E.R., Gerasimova O.A., Zhang J., Shpakovsky D.B., Syrbu S.A., Semeykin A.S., Koifman O.I., Kireeva E.G., Shevtsova E.F., Bachurin S.O., Zefirov N.S. J. Inorg. Biochem. 2008, 102, 1348-1358.

295. Stuzhin P.A., Hamdush M., Ziener U. Inorg. Chim. Acta 1995, 236, 131-139.

296. Edwards W.D., Weiner B., Zerner M.C. J. Phys. Chem. 1988, 92, 6188-6197.

297. Korolev D.V. Abstract of the Candidate's Dissertation in Chemistry. Ivanovo: G.A. Krestov Institute of Solution Chemistry of Russian Academy of Sciences, 2012. 16 p.

298. Lomova T.N., Korolev V.V., Zakharov A.G. Mater. Sci. Eng. B 2014, 186, 54-63.

299. Anikin M.S., Tarasov E.N., Kudrevatykh N.V., Osadchenko V.H., Zinin A.V. Acta Phys. Pol., A 2015, 127, 635-637.

300. Piskorsky P., Korolev D.V., Valeev R.A., Morgunov R.B., Kunitsyna E.I. Physics and Engineering of Permanent Magnets (Kablov E.N., Ed.). Moscow: VIAM, 2018. 392 p. (in Russ.).

301. Bichan N.G., Ovchenkova E.N., Gruzdev M.S., Lomova T.N. Zh. Strukt. Khimii [Russ. J. Struct. Chem.] 2018, 59, 734-741 (in Russ.). 
302. Lomova T.N., Korolev V.V., Ramazanova A.G., Balmasova O.V., Mozhzhukhina E.G. In: XIII Int. Conference "Synthesis and Application of Porphyrins and Their Analogs" (ICPC-13). Ivanovo: Ivanovo State University of Chemistry and Technology, 2019. p. 125.

303. Lomova T.N., Andrianova L.G., Berezin B.D. Zh. Fiz. Khim. 1987, 61, 2921-2928 (in Russ,).

304. Korolev V.V., Lomova T.N., Ramazanova A.G., Balmasova O.V., Mozhzhukhina E.G. J. Porphyrins Phthalocyanines 2019, 23, 1110-1117.

305. Gurek A.G., Basova T., Luneau D., Lebrun C., Kol'tsov E., Hassan A.K., Ahsen V. Inorg. Chem. 2006, 45, 1667-1676.

306. Tishin A.M., Spichcin Y.I. The Magnetocaloric Effect and its Applications. Institute of Physics Publishing, Bristol and Philadelphia, 2003. $476 \mathrm{p}$.

307. Korolev D.V., Korolev V.V., Lomova T.N., Mozhzhukhina E.G., Zakharov A.G. In: IV Regional Conference of Young Scientists Theoretical and Experimental Chemistry of Liquid-phase Systems (Krestovsky Reading). Ivanovo: G.A. Krestov Institute of Solution Chemistry of the Russian Academy of Sciences, 2010. p. 28 (in Russ.).

308. Kudrevatykh N.V., Volegov A.S. Magnetism of Rare Earth Metals and Their Intermetallic Compounds. Yekaterinburg, 2015, 198 p. (in Russ.) .

309. Shultz D.A., Sandberg K.A. J. Phys. Org. Chem. 1999, 12, $10-18$.

310. Xiang L.C., Liu Q., Guo C.-C., Tan Z. J. Porphyrins Phthalocyanines 2010, 14, 825-831.

311. Mironov A.F. Macroheterocycles 2011, 4, 186-208.

312. Yoon I., Demberelnyamba D., Li J.Z., Shim Y.K. In: Handbook of Porphyrin Science. Vol. 33 (Kadish K.M., Smith K.M., Guilard R., Eds.). Singapore: World Scientific Publishing Co Pte Ltd, 2014. p. 167.

313. Oluwole D., Yagodin A.V., Britton J., Martynov A.G., Gorbunova Y.G., Tsivadze A.Yu., Nyokong T. Dalton Trans. 2017, 46, 16190-16198.

314. Lan Y., Klyatskaya S., Ruben M. In: Lanthanides and Actinides in Molecular Magnetism (Layfeld R.A., Murugesu M., Eds.). Wiley-VCH Verlag GmbH \& Co. KGaA, 2015. p. 223.

315. Damjanovic' M., Morita T., Katoh K. Yamashita M., Enders M. Chem. Eur. J. 2015, 21, 14421-14432.

316. Bryant D.A., Hunter C.N., Warren M.J. J. Biol. Chem. 2020 , 295, 6888-6925.

317. Mathew S., Yella A., Gao P., Humphry-Baker R., Curchod B.F., Ashari-Astani N., Tavernelli I., Rothlisberger U., Nazeeruddin M.K., Grätzel M. Nat. Chem. 2014, 6, 242-247.

318. La Rosa M., Payne E.H., Credi A. ChemistryOpen 2020, 9 , 200-213.

319. Lemon C.M., Karnas E., Han X., Bruns O.T., Kempa T.J., Fukumura D., Bawendi M.G., Jain R.K., Duda D.G., Nocera J. Am. Chem. Soc. 2015, 137, 9832-9842.

320. Zenkevich E.I., Gaponenko S.V., Sagun E.I., von Borczyskowski C. Reviews in Nanoscience and Nanotechnology (Chen W., Zhao Y., Juzenas P., Eds.). USA: American Scientific Publishers, 2013, 2(3). p. 184-207.

321. Jeong Y.-H., Son M., Yoon H., Kim P., Lee D.-H., Kim D., Jang W.-D. Angew. Chem. Int. Ed. 2014, 53, 6925-6928.

322. Shao S., Rajendiran V., Jonathan F., Lovell J.F. Coord. Chem. Rev. 2019, 379, 99-120.

323. Zenkevich E.I., von Borczyskowski C. Formation Principles and Excited States Relaxation in Self-Assembled Complexes: Multiporphyrin Arrays and "Semiconductor CdSe/ZnS Quantum Dot-Porphyrin" Nanocomposites. In: Handbook of Porphyrin Science with Application to Chemistry, Physics, Materials Science, Engineering, Biology and Medicine. Vol. 22 - Biophysical and Physicochemical Studies of Tetrapyr- roles (Kadish K., Smith K.M., Guilard R., Eds.). Singapore: World Scientific Publishing Co. Pte. Ltd., 2012. p. 67168.

324. Fukuzumi S., Lee Y.-M., Nam W. ChemPhotoChem 2018, 2, 121-135.

325. Hood D., Sahin T., Parkes-Loach P.S., Jiao J., Harris Michelle A., Dilbeck P., Niedzwiedzki D.M., Kirmaier C., Loach P.A., Bocian D.F., Lindsey J.S., Holten D. ChemPhotoChem 2018, 2, 300-313.

326. Lee H., Hong K.I., Jang W.D. Coord. Chem. Rev. 2018, 354, 46-73.

327. Khadria A., Fleischhauer J., Boczarow I., Wilkinson J.D., Kohl M.M., Anderson H.L. Science 2018, 4, 153-16.

328. Huang W.B., Gu W., Huang H.X., Wang J.B., Shen W.X., Lv Y.Y., Shen J. Dyes Pigm. 2017, 143, 427-435.

329. Gaponenko S.V., Demir H.V. Applied Nanophotonics. Cambridge: Cambridge University Press, 2018.

330. Pal K. Hybrid Nanocomposites: Fundamentals, Synthesis, and Applications. USA: Jenny Stanford Publishing, 1st Edit., 2019. $412 \mathrm{p}$.

331. Pleus R.C., Murashov V. Physico-Chemical Properties of Nanomaterials. USA: Pan Stanford, 2018. 338 p.

332. Petersen N.O. Foundations for Nanoscience and Nanotechnology. London: CRC Press, 2017.

333. Self-Assembled Organic-Inorganic Nanostructures: Optics and Dynamics (Zenkevich E., von Borczyskowski C., Eds.) Singapore: Pan Stanford, 2016. 408 p.

334. Liu M., Voznyy O., Sabatini R., García de Arquer F.P., Munir R., Balawi A.H., Lan X., Fan F., Walters G., Kirmani A.R., Hoogland S., Laquai F., Amassian A., Sargent E.H. Nat. Mater. 2017, 16, 258-263.

335. Safi M., Domitrovic T., Kapur A., Zhan N., Aldeek F., Johnson J.E., Mattoussi H. Bioconjugate Chem. 2017, 28, 64-74.

336. Kundu S., Patra A. Chem. Rev. 2017, 117, 712-757.

337. Sayevich V., Guhrenz C., Dzhagan V.M., Sin M., Werheid M., Cai B., Borchardt L., Widmer J., Zahn D.R.T., Brunner E., Lesnyak V., Gaponik N., Eychmueller A. ACS Nano 2017, $11,1559-157$

338. Kovalenko M.V., Manna L., Cabot, Hens Z., Talapin D.V., Kagan C.R., Klimov V.I., Rogach A.L., Reiss P., Milliron D.J., Guyot-Sionnnest P., Konstantatos G., Parak W.J., Hyeon T., Korgel B.A., Murray C.B., Heiss W. ACS Nano 2015, 9, 1012-1057.

339. Orlova A.O., Gromova Yu.A., Maslov V.G., Prudnikau A.V., Artemyev M.V., Fedorov A.V., Baranov A.V. J. Applied Phys. 2013, 113, 214305.

340. Wolfbeis O.S. Chem. Soc. Rev. 2015, 44, 4743-4768.

341. McBride J.R, Pennycook T.J., Pennycook S.J., Rosenthal S.J. ACS Nano 2013, 7, 8358-8365.

342. Stupak A., Blaudeck T., Zenkevich E., Krause S., von Borczyskowski C. Phys. Chem. Chem. Phys. 2018, 20, 18579-18600.

343. Plehn T., Ziemann D., May V. Phys. Chem. Chem. Phys. 2018, 20, 26870-26884.

344. Boles M.A., Ling D., Hyeon T., Talapin D.V. Nat. Mater. 2016, 15, 141-153.

345. Duim H., Fang H.-H., Adjokatse S., ten Brink G.H., Marques M.A.L., Kooi B.J., Blake G.R., Botti S., Loil M.A. Appl. Phys. Rev. 2019, 6, 031401.

346. Zeng B., Palui G., Zhang C., Zhan N., Wang W., Ji X., Chen B., Mattoussi H. Chem. Mater. 2018, 30, 225-238.

347. Brown P.R., Kim D., Lunt R.R., Zhao N., Bawendi M.G., Grossman J.C., Bulovi V. ACS Nano 2014, 8, 5863-5872.

348. Kilin D.S., Tsemekham K., Zenkevich E.I., Prezho O.V. von Borczyskowski C. J. Photochem. Photobiol. A 2007, 190, 342-351.

349. Voznyy O. J. Phys. Chem. C 2011, 115, 15927-15932. 
350. Kilina S.V., Tamukong P.K., Kilin D.S. Acc. Chem. Res. 2016, 49, 2127-2135.

351. Zenkevich E., Shulga A., Cichos F., Petrov E.P., Blaudeck T., von Borczyskowski C. J. Phys. Chem. B 2005, 109, 8679-8692.

352. Blaudeck T., Zenkevich E.I., Cichos F., von Borczyskowski C. J. Phys. Chem. C 2008, 112, 20251-20257.

353. Blaudeck T., Zenkevich E.I., Abdel-Mottaleb M., Szwaykowska K., Kowerko D., Cichos F., von Borczyskowski C. ChemPhysChem 2012, 13, 959-972.

354. Zenkevich E.I., Blaudeck T., Kowerko D., Stupak A.P., Cichos F., von Borczyskowski C. Macroheterocycles 2012, $5,98-114$.

355. Zhong C., Sangwan V.K., Kang J., Luxa J., Sofer Z., Hersam M.C., Weiss E.A. J. Phys. Chem. Lett. 2019, 10, 493-499.

356. Raevskaya A., Rozovik O., Novikova A., Selyshchev O., Stroyuk O., Dzhagan V., Goryacheva I., Gaponik N., Zahn D.R.T., Eychmüller A. RSC Adv. 2018, 8, 7550-7557.

357. Pong B.-K., Trout B.L., Lee J.-Y. Langmuir 2008, 24, 5270-5276.

358. Schapotschnikow P., Hommersom B., Vlugt T.J.H. J. Phys. Chem. C 2009, 113, 12690-12698.

359. Kowerko D., Krause S., Amecke N., Abdel-Mottaleb M., Schuster J. von Borczyskowski C. Int. J. Mol. Sci. 2009, 10, 5239-5256.

360. Kowerko D., Schuster J., Amecke N., Abdel-Mottaleb M., Dobrawa R., Würthner F., von Borczyskowski C. Phys. Chem. Chem. Phys. 2010, 12, 4112-4123.

361. Chernook A.V., Shulga A.M., Zenkevich E.I., Rempel U., von Borczyskowski C. J. Phys. Chem. 1996, 100, 1918-1926.

362. Chernook A.V., Rempel U., von Borczyskowski C., Zenkevich E.I., Shulga A.M. Chem. Phys. Lett. 1996, 254, 229-241.

363. Lecture Notes in Nanoscale Science and Technology. Corel Shell Quantum Dots. Synthesis, Properties and Devices Vol. 18. (Tong X., Wang Z.M., Eds.). Switzerland: Springer Nature AG. 2020.

364. Zhang C., Jin Z., Zeng B., Wang W., Palui G., Mattoussi H. J. Phys. Chem. B 2020, 124, 4631-4650.

365. Stroyuk O., Raievska O., Zahn D.R.T. Unique Luminescent Properties of Composition-/Size-Selected Aqueous Ag-In-S and Core/Shell Ag-In-S/ZnS Quantum Dots. In: Lecture Notes in Nanoscale Science and Technology. Core/Shell Quantum Dots. Synthesis, Properties and Devices. Vol. 18. (Tong X., Wang Z.M., Eds.) Switzerland: Springer Nature AG, 2020. p. 67-122.

366. Agranovich V.M., Galanin M.D. Electronic Excitation Energy Transfer in Condensed Matter. Amsterdam, New York: North-Holland Pub. Co., 1982. 371 p.

367. Zenkevich E.I., Sagun E.I., Shulga A.M., Knyukshto V.N., Yarovoi A.A., Stupak A.P., von Borczyskowski C. Opt. Spectrosc. 2007, 103, 998-1009.

368. Klimov V. Nanocrystall Quantum Dots. Washington: CRS Press LLC, 2010.

369. Dabbousi B.O., Redriguez-Vejo J., Mikulec F.V., Heine J.R., Mattousi H., Ober R., Jensen K.F., Bawendi M.G. J. Phys. Chem. B 1997, 101, 9463-9475.

370. Zenkevich E.I., Blaudeck Th., Heidernätsch M., Cichos F., von Borczyskowski C. Theor. Exper. Chem. 2009, 45, 23-34.

371. Zenkevich E.I., Stupak A.P., Kowerko D., von Borczyskowski C. Chem. Phys. 2012, 406, 21-29.

372. Zenkevich E., Stupak A., Göhler C., Krasselt C., von Borczyskowski C. ACS Nano 2015, 9, 2886-2903.

373. Inerbaev T.M., Masunov A.E., Khondaker S.I., Dobrinescu A., Plamadă A.-V., Kawazoe Y. J. Chem. Phys. 2009, 131, 044106.

374. Kilina S., Kilin D., Tretiak S. Chem. Rev. 2015, 115, 5929-5978.

375. Dayal S., Burda C. J. Am. Chem. Soc. 2007, 129, 7977-7981.
376. Liptay T.J., Ram R.J. Appl. Phys. Lett. 2006, 89, 223132

377. De Mello Donegá. Nanoparticles. Berlin, Heidelberg: Springer-Verlag, 2014

378. Fischer T., Heinrich K., Spudat C., Martin J., Otto T., Gessner T., Kroll L. Microelectron. Eng. 2015, 146, 57-61.

379. Möbius M., Ma X.-Y., Martin J., Doty M.F., Otto T., Gessner T. Proc. SPIE 2015, 9370, 93701X.

380. Möbius M., Martin J., Hartwig M., Baumann R.R., Otto T., Gessner T. AIP Adv. 2016, 6, 085309.

381. Korten T., Nitzsche B., Gell C., Ruhnow F., Leduc C., Diez S. Fluorescence Imaging of Single Kinesin Motors on Immobilized Microtubules. In: Methods in Molecular Biology (Single Molecule Analysis) Vol. 783. (Peterman E., Wuite G., Eds.) Springer Protocols: Humana Press, 2011. p. 121-137.

382. Korten S., Albet-Torres N., Paderi F., ten Siethoff L., Diez S., Korten T., te Kronnie G., Månsson A. Lab Chip 2013, 13, 866-876.

383. Selyshchev O., Dzhagan V., Zenkevich E., Stroyuk A., Raevskaya A., Sheinin V., Kulikova O., Koifman O., Zahn D.R.T. Electronic Interaction Between Ag-In-S, Ag-In-S/ ZnS Quantum Dots and Quaternary Amine Aromatic Molecules - A Photoluminescence Quenching Study. In: Book of Abstracts of the 14th International Symposium on Functional $\pi$-Electron Systems (June 2-7) 2019, Berlin. p. \#106.

384. Sheinin V., Kulikova O., Zenkevich E., Selyshchev O., Dzhagan V., Stroyuk A., Raevskaya A., Koifman O., Zahn D.R.T. Tetra(N-methyl-4-pyridyl)porphyrin Sonde Report on the Surface of AIS/ZnS/GSH Quantum Dots in Water. In: Book of Abstr. of the $1^{\text {st }}$ International Conference on Noncovalent Interactions (2-6 September) 2019, Lisbon. p. P82.

385. Zenkevich E., Sheinin V., Kulikova O., Selyshchev O., Dzhagan V., Stroyuk O., Raievska O., Koifman O., von Borczyskowski C., Zahn D.R.T. Self-Assembled Nanocomposites Based on Semiconductor Quantum Dots and Porphyrin Molecules: Interface Chemistry, Optical Properties and Energy Relaxation Processes. In: Book of Abstracts of Webinar on Materials Science and Nanotechnology. Coalesce Research Group, 33 Market Point Dr., Greenwille SC 29607, USA (July 29-30) 2020. p. 11.

386. Carella A., Borbone F., Centore R. Front. Chem. 2018, 6, 481.

387. Arooj Q., Wilson G.J., Wang F. Materials 2019, 12, 4024.

388. Iftikhar H., Sonai G.G., Hashmi S.G., Nogueira A.F., Lund P.D. Materials 2019, 12, 1998.

389. Widhiyanuriyawan D., Trihutomo P., Soeparman S., Yuliati L. Scientific World Journal 2020, 2020, 7910702.

390. Koyyada G., Chitumalla R.K., Thogiti S., Kim J.H., Jang J., Chandrasekharam M., Jung J.H. Molecules 2019, 24, 3554.

391. Luceño-Sánchez J.A., Díez-Pascual A.M., Capilla R.P. Int. J. Mol. Sci. 2019, 20, 976

392. Ezhov A.V., Vyalba F.Yu., Zhdanova K.A., Mironov A.F., Zhizhin K.Yu., Bragina N.A. Fine Chem. Technol. 2018, 13(2), 21-30 (in Russ.).

393. Longo C., De Paoli M.-A. J. Braz. Chem. Soc. 2003, 14, 889-901.

394. Ezhov A.V., Zhdanova K.A., Bragina N.A., Mironov A.F. Macroheterocycles 2016, 9, 337-352.

395. Vittal R., Ho K.-C. Renewable and Sustainable Energy Reviews 2017, 70, 920-935.

396. Eguchi K., Koga H., Sekizawa K., Sasaki K. J. Ceramic Soc. Japan 2000, 108, 1067-1071.

397. Septiawan T.Y., Sumardiasih S., Obina W.M., Supriyanto A., Khairuddin, Cari C. AIP Conference Proceedings 2017, $1868,060010$.

398. Banik A., Ansari M.S., Qureshi M. ACS Omega 2018, 3, 14482-14493. 
399. Saeidi M., Abrari M., Ahmadi M. Appl. Phys. A 2019, 125, 409.

400. Valerio T.L., Maia G.A.R., Gonçalves L.F., Viomar A., Banczek E. do P., Rodrigues P.R.P. Mater. Res. 2019, 22(suppl. 1), e20180864.

401. Chen L.H., Xue B.F., Luo Y.H. Chin. Phys. Lett. 2007, 24, 555-558.

402. Chen D., Zhang Q., Wang G. Electrochem. Commun. 2007, 9, 2755-2759.

403. Jung Y.S., Yoo B., Lim M.K., Kim K.J. Electrochim. Acta 2009, 54, 6286-6291.

404. Gu P., Yang D., Zhu X., Sun H., Wangyang P., Li J., Tian H. AIP Adv. 2017, 7, 105219.

405. Lu J.F., Bai J., Xu X.B., Li Z.H., Cao K., Cui J., Wang M.K. Chin. Sci. Bull. 2012, 57(32), 4131-4142.

406. Wang M., Grätzel C., Zakeeruddin S.M., Grätzel M. Energy Environ. Sci. 2012, 5, 9394-9405.

407. Stergiopoulos T., Rozi E., Karagianni C.-S., Falaras P. Nanoscale Res. Lett. 2011, 6, 307.

408. Cao Y., Zhang J., Bai Y., Li R., Zakeeruddin S.M., Grätzel M., Wang P. J. Phys. Chem., C 2008, 112, 13775-13781.

409. Önen T., Karakuş M.Ö., Coşkun R., Çetin H. J. Photochem. Photobiol., A: Chemistry 2019, 385, 112082.

410. Shen S.-Y., Dong R.-X., Shih P.-T., Ramamurthy V., Lin J.-J., Ho K.-C. ACS Appl. Mater. Interfaces 2014, 6(21), 18489-18496.

411. Pujiarti H., Arsyad W.S., Shobih, Muliani L., Hidayat R. IOP Conf. Series: J. Physics: Conf. Series 2018, 1011, 012020.

412. Duan Y., Tang Q., Chen Y., Zhao Z., Lv Y., Hou M., Yang P., He B., Yu L. J. Mater. Chem., A 2015, 3, 5368-5374.

413. Nam S.-H., Lee K.H., Yu J.-H., Boo J.-H. Appl. Sci. Converg. Technol. 2019, 28, 194-206.

414. Shalini S., Balasundaraprabhu R., Satish Kumar T., Prabavathy N., Senthilarasu S., Prasanna S. Int. J. Energy Res. 2016, 40, 1303-1320.

415. Sharma K., Sharma V., Sharma S.S. Nanoscale Res. Lett. 2018, 13, 381.

416. Qin Y., Peng Q. Int. J. Photoenergy 2012, 2012, 291579.

417. Abate A., Planells M., Hollman D.J., Stranks S.D., Petrozza A., Kandada A.R.S., Vaynzof Y., Pathak S.K., Robertson N., Snaith H.J. Adv. Energy Mater. 2014, 4, 1400166.

418. Hu Y., Ivaturi A., Planells M., Boldrini C.L., Biroli A.O., Robertson N. J. Mater. Chem., A 2016, 4, 2509.

419. Hwang S., Lee J.H., Park C., Lee H., Kim C., Park C., Lee M.H., Lee W., Park J., Kim K., Park N.G., Kim C. Chem. Commun. 2007, 46, 4887.

420. Mane S.B., Cheng C.F., Sutanto A.A., Datta A., Dutta A., Hung C.H. Tetrahedron 2015, 71, 7977.

421. Al-horaibi S.A., Gaikwad S.T., Rajbhoj A.S. Adv. Mat. Lett. 2018, 9, 353-362.

422. Chen G., Sasabe H., Igarashi T., Hong Z., Kido J. J. Mater. Chem., A 2015, 3, 14517.

423. Khopkar S., Shankarling G. Dyes Pigm. 2019, 170, 107645.

424. Qin C., Wong W.-Y., Han L. Chem. Asian. J. 2013, 8, 1706-1719.

425. Jamalullail N., Mohamad I.S., Norizan M.N., Baharum N.A. In: IEEE 15th Student Conference on Research and Development (SCOReD) 2017, p. 344-349.

426. Pamain A., Pogrebnaya T., King'ondu C.K. Res. J. Eng. Appl. Sci. 2014, 3, 332-336.

427. Liu Q., Gao N., Liu D., Liu J., Li Y. Appl. Sci. 2018, 8, 1697.

428. Selopal G.S., Wu H.-P., Lu J., Chang Y.-C., Wang M., Vomiero A., Concina I., Diau E.W.-G. Scientific Reports 2016, 6, 18756.

429. Tsao H.N., T. Moehl C.Yi, Yum J.H., Zakeeruddin S.M., Nazeeruddin M.K., Grätzel M., ChemSusChem 2011, 4, 591-594.
430. Angaridis P., Lazarides T., Coutsolelos A.C. Polyhedron 2014, 82, 19-32.

431. Ladomenou K., Kitsopoulos T.N., Sharma G.D., Coutsolelos A.G. $R S C A d v$. 2014, 4, 21379-21404.

432. Obraztsov I., Kutner W., D'Souza F. Sol. RRL 2017, 1, 1600002.

433. Zhang L., Cole J.M. ACS Appl. Mater. Interfaces 2015, 7, 3427-3455.

434. Daphnomili D., Landrou G., Singh S.P., Thomas A., Yesudas K., Bhanuprakash K., Sharma G.D., Coutsolelos A.G. RSC Adv. 2012, 2, 12899-12908.

435. Lu J., Xu X., Li Z., Cao K., Cui J., Zhang Y., Shen Y., Li Y., Zhu J., Dai S., Chen W., Cheng Y., Wang M. Chem. Asian. J. 2013, 8, 956-962.

436. Mai C.-L., Moeh T., Hsieh C.-H., Decoppet J.-D., Zakeeruddin S.M., Grätzel M., Yeh C.-Y. ACS Appl. Mater. Interfaces 2015, 7, 14975-14982.

437. Brennan B.J., Portolés M.J.L., Liddell P.A., Moore T.A., Moore A.L., Gust D. Phys. Chem. Chem. Phys. 2013, 15, 16605-16614.

438. Odobel F., Blart E., Lagrée M., Villieras M., Boujtita H., El Murr N., Caramoric S., Bignozzi C.A. J. Mater. Chem. 2003, $13,502-510$.

439. López-Duarte I., Wang M., Humphry-Baker R., Ince M., Martínez-Díaz M.V., Nazeeruddin M.K., Torres T., Grätzel M. Angew. Chem. Int. Ed. 2012, 51, 1895-1898.

440. He H., Gurunga A., Si L. Chem. Commun. 2012, 48 , 5910-5912.

441. Ma T., Inoue K., Noma H., Yao K., Abe E. J. Photochem. Photobiol., A: Chemistry 2002, 152, 207-212.

442. Gou F., Jiang X., Li B., Jing H.-W., Zhu Z. ACS Appl. Mater. Interfaces 2013, 5, 12631-12637.

443. Gou F., Jiang X., Fang R., Jing H., Zhu Z. ACS Appl. Mater Interfaces 2014, 6, 6697-6703.

444. Zhang N., Zhang B., Yan J., Xue X., Peng X., Li Y., Yang Y., Ju C., Fan C., Feng Y. Renewable Energy 2015, 77, 579-585.

445. Keawin T., Tarsang R., Sirithip K., Prachumrak N., Sudyoadsuk T., Namuangruk S., Roncali J., Kungwan N., Promarak V., Jungsuttiwong S. Dyes Pigm. 2017, 136, 697-706.

446. Ishida M., Park S.W., Hwang D., Koo Y.B., Sessler J.L., Kim D.Y., Kim D. J. Phys. Chem., C 2011, 115, 19343-19354.

447. Matsuzaki H., Murakami T.N., Masaki N., Furube A., Kimura M., Mori S. J. Phys. Chem., C 2014, 118, 1720517212

448. Si L., He H. J. Phys. Chem., A 2014, 118, 3410-3418.

449. Imahori H., Hayashi S., Hayashi H., Oguro A., Eu S., Umeyama T., Matano Y. J. Phys. Chem., C 2009, 113, 18406-18413.

450. Kroeze J.E., Hirata N., Koops S., Nazeeruddin Md.K., Schmidt-Mende L., Grätzel M., Durrant J.R. J. Am. Chem. Soc. 2006, 128, 16376-16383.

451. Zhang L., Cole J.M. J. Mater. Chem., A 2017, 5, 19541-19559.

452. Xue X., Zhang W., Zhang N., Ju C., Peng X., Yang Y., Liang Y., Feng Y., Zhang B. RSC Adv. 2014, 4, 8894-8900.

453. Chen J., Ko S., Liu L., Sheng Y., Han H., Li X. New J. Chem. 2015, 39, 3736-3746.

454. Lu F., Zhang J., Zhou Y., Zhao Y., Zhang B., Feng Y. Dyes Pigm. 2016, 125, 116-123.

455. Magnano G., Marinotto D., Cipolla M.P., Trifiletti V., Listorti A., Mussini P.R., Di Carlo G., Tessore F., Manca M., Orbelli Biroli A., Pizzotti M. Phys. Chem. Chem. Phys. 2016, 18, 9577-9585.

456. Higashino T., Kawamoto K., Sugiura K., Fujimori Y., Tsuji Y., Kurotobi K., Ito S., Imahori H. ACS Appl. Mater. Interfaces 2016, 8, 15379-15390.

457. Ananthakumar S., Balaji D., Kumar J.R., Babu S.M. SN Appl. Sci. 2019, 1, 186. 
458. Bessho T., Zakeeruddin S.M., Yeh C.-Y., Diau E.W.-G., Grätzel M. Angew. Chem. Int. Ed. 2010, 49, 66466649.

459. Michaels H., Rinderle M., Freitag R., Benesperi I., Edvinsson T., Socher R., Gagliardi A., Freitag M. Chem. Sci. 2020, 11, 2895-2906.

460. Truta L.A.A.N.A., Moreira F.T.C., Sales M.G.F. Biosens. Bioelectron. 2018, 107, 94-102.

461. Guo W., Xue X., Wang S., Lin C., Wang Z.L. Nano Lett. 2012, 12, 2520-2523.

462. Pu X., Song W., Liu M., Sun C., Du C., Jiang C., Huang X., Zou D., Hu W., Wang Z.L. Adv. Energy Mater. 2016, 6, 1601048

463. Yun S., Qin Y., Uhl A.R., Vlachopoulos N., Yin M., Li D., Han X., Hagfeldt A. Energy Environ. Sci. 2018, 11, 476-526.

464. Honda K, Fujishima A. Nature 1972, 238, 37-38.

465. Watanabe M. Sci. Technol. Adv. Mater. 2017, 18(1), 705-723.

466. Youngblood W.J., Lee S.-H.A., Maeda K., Mallouk T.E. Acc. Chem. Res. 2009, 42, 1966-1973.

467. Gan J., Lu X., Tong Y. Nanoscale 2014, 6, 7142-7164.

468. Abe R. J. Photochem. Photobiol., C: Photochem. Rev. 2010, 11, 179-209.

469. Yun S., Vlachopoulos N., Qurashi A., Ahmad S., Hagfeldt A. Chem. Soc. Rev. 2019, 48, 3705-3722.

470. Swierk J.R., Mallouk T.E. Chem. Soc. Rev. 2013, 42, 2357-2387.

471. Swierk J.R., Méndez-Hernádez D.D., McCool N.S., Liddell P., Terazono Y., Pahk I., Tomlin J.J., Oster N.V., Moore T.A., Moore A.L., Gust D., Mallouk T.E. Proc. Nat. Acad. Sci. 2015, 112, 1681-1686.

472. Sherman B.D., Bergkamp J.J., Brown C.L., Moore A.L., Gust D., Moore T.A. Energy Environ Sci. 2016, 9, 1812-1817.

473. Kang S.H., Jeong M.J., Eom Y.K., Choi I.T., Kwon S.M., Yoo Y., Kim J., Kwon J., Park J.H., Kim H.K. Adv. Energy Mater. 2017, 7, 1602117.

474. Melville O.A., Lessard B.H., Bender T.P. ACS Appl. Mater. Interfaces 2015, 7, 13105-13118.

475. de la Torre G., Bottari G., Torres T. Adv. Energy Mater. 2017, 7, 1601700.

476. Brinkmann H., Kelting C., Makarov S., Tsaryova O., Schnurpfeil G., Wöhrle D., Schlettwein D. Phys. Status Solidi (A) Appl. Mater. Sci. 2008, 205, 409-420.

477. Stuzhin P.A. Fluorinated Phthalocyanines and Their Analogues. In: Fluorine in Heterocyclic Chemistry. Vol. 1. 5-Membered Heterocycles and Macrocycles (Nenajdenko V.G., Ed.). Heidelberg: Springer, 2014. p. 621-681.

478. Stuzhin P.A., Ercolani C. Porphyrazines with Annulated Heterocycles. In: Porphyrin Handbook. Vol. 15 (Kadish K.M., Smith K.M., Guilard R., Eds.). Amsterdam: Elsevier Science, 2003. p. 263-364.

479. Donzello M.P., Ercolani C., Novakova V., Zimcik P., Stuzhin P.A. Coord. Chem. Rev. 2016, 309, 107-179.

480. Novakova V., Donzello M.P., Ercolani C., Zimcik P., Stuzhin P.A. Coord. Chem. Rev. 2018, 361, 1-73.

481. Lonchakov A.V., Rakitin O.A., Gritsan N.P., Zibarev A.V. Molecules 2013, 18, 9850-9900.

482. Rakitin O.A. Tetrahedron Lett. 2020, 61, 152230.

483. Konstantinova L.S., Knyazeva E.A., Rakitin O.A. Rev. Org. Preparations and Procedures Int. 2014, 46, 475-544.

484. Stuzhin P.A., Bauer E.M., Ercolani C. Inorg. Chem. 1998, 37, 1533-1539.

485. Bauer E.M., Ercolani C., Galli P., Popkova I.A., Stuzhin P.A. J. Porphyrins Phthalocyanines 1999, 3, 371-379.

486. Donzello M.P., Ercolani C., Stuzhin P.A. Coord. Chem. Rev. 2006, 250, 1530-1561.

487. Cozzolino A., Yang Q., Vargas-Baca I. Cryst. Growth Des. 2010, 10, 4959-4964.
488. Stuzhin P.A., Mikhailov M.S., Yurina E.S., Bazanov M.I., Koifman O.I., Pakhomov G.L., Travkin V.V., Sinelshchikova A.A. Chem. Comm. 2012, 48, 10135-10137.

489. Otlyotov A.A., Ryzhov I.V., Kuzmin I.A., Zhabanov Y.A., Mikhailov M.S., Stuzhin P.A. Int. J. Molec. Sci. 2020, 21, 2923.

490. Stuzhin P.A., Ivanova S.S., Hamdoush M., Kirakosyan G.A., Kiselev A., Popov A., Sliznev V., Ercolani C. Dalton Trans. 2019, 48, 14049-14061.

491. Bauer E.M., Cardarilli D., Ercolani C., Stuzhin P.A., Russo U. Inorg. Chem. 1999, 38, 6114-6120.

492. Donzello M.P., Viola E., Giustini M., Ercolani C., Monacelli F. Dalton Trans. 2012, 41, 6112-6121.

493. Angeloni S., Bauer E.M., Ercolani C., Popkova I.A., Stuzhin P.A. J. Porphyrins Phthalocyanines 2001, 5, 881888.

494. Tarakanova E.N., Hamdoush M., Eroshin A.V., Ryzhov I.V., Zhabanov Y.A., Stuzhin P.A. Polyhedron 2021, 193, 114877.

495. Donzello M.P., Agostinetto R., Ivanova S.S., Fujimori M., Suzuki Y., Yoshikawa H., Shen J., Awaga K., Ercolani C., Kadish K.M., Stuzhin P.A. Inorg. Chem. 2005, 44, 8539-8551.

496. Miyoshi Y., Takahashi K., Fujimoto T., Yoshikawa H., Matsushita M.M., Ouchi Y., Kepenekian M., Robert V., Donzello M.P., Ercolani C., Awaga K. Inorg. Chem. 2012, $51,456-462$.

497. Onay H., Yerli Y., Öztürk R. Transition Met. Chem. 2009, $34,163-166$

498. Hamdoush M., Ivanova S.S., Pakhomov G.L., Stuzhin P.A. Macroheterocycles 2016, 9, 230-233.

499. Hamdoush M., Nikitin K., Skvortsov I., Somov N., Zhabanov Yu., Stuzhin P.A. Dyes Pigm. 2019, 170, 107584.

500. Kudrik E.V., Bauer E.M., Ercolani C., Chiesi-Villa A., Rizzoli C., Gaberkorn A., Stuzhin P.A. Mendeleev Commun. 2001, 11(2), 45-47.

501. Donzello M.P., Ercolani C., Gaberkorn A.A., Kudrik E.V., Meneghetti M., Marcolongo G., Rizzoli C., Stuzhin P.A. Chem. Eur. J. 2003, 9, 4009-4024.

502. Gaberkorn A.A., Donzello M.-P., Stuzhin P.A. Russ. J. Org Chem. 2006, 42, 929-935.

503. Stuzhin P.A., Pimkov I.V., Ul-Haq A., Ivanova S.S., Popkova I.A., Volkovich D.I., Kuzmitskii V.A., Donzello M.-P. Russ. J. Org. Chem. 2007, 43, 1854-1863.

504. Svec J., Zimcik P., Novakova L., Rakitin O.A., Amelichev S., Stuzhin P.A., Novakova V. Eur. J. Org. Chem. 2015, 3, 596-604.

505. Stuzhin P.A. Synthesis, Structure and Physico-chemical properties of Azaporphyrins and Porphyrazines. Diss. doc. chem sci. Ivanovo: ISUCT, 2004.

506. Volkovich D.I., Kuzmitsky V.A., Stuzhin P.A. J. Appl. Spectrosc. 2008, 75, 621-636.

507. Cai X., Zhang Y., Zhang X., Jiang J. J. Molec. Struc.: THEOCHEM 2007, 812, 63-70.

508. Donzello M.-P., Ercolani C., Kadish K.M., Ricciardi G., Rosa A., Stuzhin P.A. Inorg. Chem. 2007, 46, 4145-4157.

509. Tverdova N.V., Giricheva N.I., Savelyev D.S., Mikhailov M.S., Vogt N., Koifman O.I., Stuzhin P.A., Girichev G.V. Macroheterocycles 2017, 10, 27-30.

510. Zhabanov Y.A., Sliznev V.V., Ryzhov I.V., Stuzhin P.A. J. Porphyrins Phthalocyanines 2020, 24, 1146-1154.

511. Zhabanov Yu.A., Tverdova N.V., Giricheva N.I., Girichev G.V., Stuzhin P.A. J. Porphyrins Phthalocyanines 2017, 21, 439-452.

512. Fujimori M., Suzuki Y., Yoshikawa H., Awaga K. Angew. Chem. Int. Ed. 2003, 42, 5863-5865.

513. Suzuki Y., Fujimori M., Yoshikawa H., Awaga K. Chem. Eur. J. 2004, 10, 5158-5164. 
514. Donzello M.P., Fujimori M., Miyoshi Y., Yoshikawa H., Viola E., Awaga K., Ercolani C. J. Porphyrins Phthalocyanines 2010, 14, 343-348.

515. Miyoshi Y., Kubo M., Fujinawa T., Suzuki Y., Yoshikawa H., Awaga K. Angew. Chem. Int. Ed. 2007, 46, 5532-5536.

516. Eguchi K., Heutz S., Awaga K. J. Porphyrins Phthalocyanines 2017, 21, 322-326.

517. Eguchi K., Nanjo C., Awaga K., Tseng H.-H., Robaschik P., Heutz S. Phys. Chem. Chem. Phys. 2016, 18, 17360-17365.

518. Stuzhin P.A., Mikhailov M.S., Travkin V.V., Gudkov E.Y., Pakhomov G.L. Macroheterocycles 2012, 5, 162-165.

519. Stuzhin P.A., Mikhailov M.S., Travkin V.V., Pakhomov G.L. Zinc(II) Tetra(1,2,5-thiadiazolo)porphyrazine Complex in Thin Film Photovoltaic Structures. In: Recent Developments in Coordination, Bioinorganic, and Applied Inorganic Chemistry. Vol. 11 (Melnik M., Segl'a P., Tatarko M., Eds.). Bratislava: Press of Slovak University of Technology, 2013. p. 318-323. ISBN 978-80-227-3918-4.

520. Hou J., Wang Y., Eguchi K., Nanjo C., Takaoka T., Sainoo Y., Awaga K., Komeda T. Appl. Surface Sci. 2018, 440, 16-19.

521. Wang Y., Hou J., Eguchi K., Nanjo C., Takaoka T., Sainoo Y., Awaga K., Komeda T. ACS Omega 2020, 5, 6676-6683.

522. Miyoshi Y., Fujimoto T., Yoshikawa H., Matsushita M.M., Awaga K., Yamada T., Ito H. Org. Electron. 2011, 12, 239-243.

523. Fujimoto T., Miyoshi Y., Matsushita M.M., Awaga K. Chem. Commun. 2011, 47, 5837-5839.

524. Nanjo C., Fujimoto T., Matsushita M.M., Awaga K. J. Phys. Chem., C 2014, 118, 14142-14149.

525. Koptyaev A.I., Khamdoush M., Fedoseev A.N., Travkin V.V., Pakhomov G.L. Macroheterocycles 2018, 11, 412-417.

526. Pakhomov G.L., Travkin V.V., Hamdoush M., Zhabanov Yu.A., Stuzhin P.A. Macroheterocycles 2017, 10, 548-551.

527. Park J.M., Lee J.H., Jang W.-D. Coord. Chem. Rev. 2020, 407, 213157-213185.

528. Konovalova N.V., Evstigneeva R.P., Luzgina V.N. Russ. Chem. Rev. 2001, 70, 939-970.

529. Borovkov V.V., Mamardashvili N.Zh., Inoue Y. Russ. Chem. Rev. 2006, 75, 737-748.

530. Kruk N.N. J. Appl. Spectrosc. 2008, 75, 461-482.

531. Flamigni L., Gryko D.T. Chem. Soc. Rev. 2009, 38, 16351646.

532. De Souza F., Chitta R., Ohkubo K. et al. J. Am. Chem. Soc. 2008, 130, 14263-14272.

533. Adeyemi O.O., Malinovskii V.L., Biner S.M. et al. Chem. Commun. 2012, 48, 9589-9591.

534. Andrianova L.G., Lomova T.N., Berezin B.D. Zh. Neorg. Khim. 1984, 29, 1697-1701 (in Russ.).

535. Miyazaki Y., Satake A., Kobuke Y. J. Mol. Cat. A: Chem. 2008, 283, 129-139.

536. Ni Y., Marchal G., Yu G. et al. Acad. Radiol. 1995, 2, 687-699.

537. He H., Zhu X., Hou A. Dalton Trans. 2004, 23, 4064-4073.

538. He H., Wong W.-K., Li K.-F., Cheah K.-W. Synth. Met. 2004, $143,81-87$.

539. He H., Sykes A.G., May P.S., He G. Dalton Trans. 2009, 36, 7454-7461.

540. Foley T., Harrison B., Knefely A. et al. Inorg. Chem. 2003, 42, 5023-5032.

541. Xin Z., Wei L., Ming J., Fa L. Synth. React. Inorg. Met. Org. Nano-Met. Chem. 2000, 30, 1747-1758.

542. Li D.-M., Zhao Z.-X., Liu S.-Q. et al. Synth. Commun. 2000, 30, 4017-4026.

543. Li D.-M., Zhao Z.-X., Sun H.-R. et al. Synth. React. Inorg. Met. Org. Nano-Met. Chem. 2000, 30, 1899-1915.

544. Zhao Z., Liu G. Synth. React. Inorg. Met. Org. Nano-Met. Chem. 2002, 32, 465-473.
545. Wong W.-K., Hou A., Guo J. et al. J. Chem. Soc., Dalton Trans. 2001, 20, 3092-3098.

546. Jiang F.-L., Wong W.-K., Zhu X.-J. et al. Eur. J. Inorg. Chem. 2007, 21, 3365-3374.

547. Zhu X.-J., Zhang T., Zhao S. et al. Eur. J. Inorg. Chem. 2011, $22,3314-3320$.

548. Hindre F., Plouzennec M., Certaines J. et al. J. Magn. Reson. Imaging 1993, 3, 59-65.

549. Li G., Slansky A., Dobhal M.P., Goswami L.N. Bioconjugate Chem. 2005, 16, 32-42.

550. Nah M., Oh J.B., Kim H.K. et al. J. Phys. Chem. A. 2007, 111, 6157-6164.

551. Beeby A., Dickins R., Fitzgerald S. et al. Chem. Commun. 2000, 13, 1183-1184.

552. Kang T.S., Harrison B.S., Foley T.J. et al. Adv. Mat. 2003, 15 , 1093-1097.

553. Guo L., Yan B. Inorg. Chem. Commun. 2011, 14, 1833-1837.

554. Zhu X., Wong W.-K., Wong W.-Y., Yang X. Eur. J. Inorg. Chem. 2011, 4651-4674.

555. Mironov A.F. Russ. Chem. Rev. 2013, 82, 333-351.

556. Shuhui B., Hu J., Wang Q., Liu X., Zhen Z. Photochem. Photobiol. Sci. 2008, 7, 474-479.

557. Tolbin A., Pushkarev V., Tomilova L. Mendeleev Commun. 2008, 18, 94-95.

558. Kasuga K., Tsutsui M. Coord. Chem. Rev. 1980, 32, 67-95.

559. Smola S.S., Snurnikova O.V., Fadeyev E.N. et al. Macroheterocycles 2012, 5, 343-349.

560. Semenishyn N.N., Smola S.S., Rusakova N.V. et al. Macroheterocycles 2017, 10, 268-272.

561. Semenishyn N.N., Smola S.S., Rusakova N.V. et al. Macroheterocycles 2018, 11, 262-268.

562. Gross Z., Galili N., Saltsman I. Angew. Chem. Int. Ed. 1999, $38,1427-1429$.

563. Semenishyn N., Gross Z. Dalton Trans. 2013, 42, 3775-3778.

564. Buckley H.L., Anstey M.R., Gryko D.T., Arnold J. Chem. Commun. 2013, 49, 3104-3106.

565. Semenishyn N.N., Rusakova N.V. Macroheterocycles 2016, 9, 163-168.

566. Day N.U., Wamser C.C., Walter M.G. Polym. Int. 2015, 64, 833-857.

567. Asif Mahmood et al. J. Mater. Chem. A. 2018, 6, 1676916797.

568. Shirakawa H. et al. J. Chem. Soc., Chem. Commun. 1977, 16, 578-580.

569. Mac Diarmid A.G. Synthetic Metals 2001, 125, 11-22.

570. Heeger A.J. Rev. Modern Phys. 2001, 73, 681-700.

571. Liu L., Yang D., Tian H., Ji Y. Optics Commun. 2012, 285, 171-177.

572. Light Scattering in Solids I. Introductory Concepts (Cardona M., Ed.). Springer Berlin Heidelberg 1983. XV 366 p.

573. Troger F. et al. Springer Berlin Heidelberg 2012, 161-251.

574. Bower D.I. Infrared Dichroism, Polarized Fluorescence and Raman Spectroscopy. In: Structure and Properties of Oriented Polymers (Ward I.M., Ed.). Springer Netherlands, 1997. p. 181-233.

575. Sosorev A.Yu., Trukhanov V.A., Maslennikov D.R. et al. ACS Appl. Mater. Interfaces 2020, 12, 9507-9519.

576. Kuzmin S.M., Chulovskaya S.A., Parfenyuk V.I., Koifman O.I. Mendeleev Commun. 2019, 29, 309-311.

577. Kuzmin S.M., Chulovskaya S.A., Parfenyuk V.I. Adv. Colloid Interface Sci. 2018, 253, 23-34.

578. Kuzmin S.M., Chulovskaya S.A., Parfenyuk V.I. Electrochim. Acta 2018, 292, 256-267.

579. Chulovskaya S.A., Kuzmin S.M., Parfenyuk V.I. Macroheterocycles 2015, 8, 259-265.

580. Kuzmin S.M., Chulovskaya S.A., Parfenyuk V.I. J. Electroanal. Chem. 2016, 772, 80-88. 
581. Kuzmin S.M., Chulovskaya S.A., Tesakova M.V., Semeikin A.S., Parfenyuk V.I. Macroheterocycles 2014, 7, 218-224.

582. Kuzmin S.M., Chulovskaya S. A., Parfenyuk V.I. Macroheterocycles 2013, 6, 334-339.

583. Tesakova M.V., Semeikin A.S., Parfenyuk V.I. J. Porphyrins Phthalocyanines 2016, 20, 793-803.

584. Kuz'min S.M., Chulovskaya S.A., Parfenyuk V.I. Russ. J. Electrochem. 2014, 50, 429-437.

585. Lunt R.R., Benziger J.B., Forrest S.R. Adv. Mater. 2010, 22, 1233-1236.

586. Mikhnenko O.V., Lin J., Shu Y., Anthony J.R., Blom P.W.M., Nguyen T.-Q. et al. Phys. Chem. Chem. Phys. 2012, 14, 14196-14201.

587. Menke S.M., Holmes R.J. Energy Environ Sci. 2014, 7, 499-512.

588. Walter M.G., Wamser C.C. J. Phys. Chem. C. 2010, 114, $7563-7574$.

589. Kuzmin S.M., Chulovskaya S.A., Parfenyuk V.I. Electrochim. Acta 2020, 342, 136064.

590. Kuzmin S.M., Chulovskaya S.A., Parfenyuk V.I., Koifman O.I. Mendeleev Commun. 2020. DOI 10.1016/j.mencom.2020.11.030.

591. Fateeva A., Devautour-Vinot S., Heymans N., Devic T., Grenèche J.-M., Wuttke S., Miller S., Lago A., Serre C., Weireld G.D., Maurin G., Vimont A., Férey G. Chem. Mater. 2011, 23, 4641-4651.

592. Fateeva A. Chater P.A., Ireland C.P., Tahir A.A., Khimyak Y.Z., Wiper P.V., Darwent J.R., Rosseinsky M.J. Angew. Chem. Int. 2012, 51, 7440-7444.

593. Yang X.L., Xie M.H., Zou C., He Y., Chen B., O'Keeffe M. et al. J. Am. Chem. Soc. 2012, 13, 10638-10645.

594. Kuzmin S.M., Chulovskaya S.A., Parfenyuk V.I. J. Porphyrins Phthalocyanines 2015, 19, 1053-1062.

595. Parfenyuk V.I., Tesakova M.V., Chulovskaya S.A., Kuzmin S.M. Macroheterocycles 2019, 12, 154-164.

596. Tesakova M.V., Koifman O.I., Parfenyuk V.I. J. Porphyrins Phthalocyanines 2018, 22, 632-639.

597. Kuzmin S.M., Chulovskaya S.A., Parfenyuk V.I. Mater. Chem. Phys. 2020, 241, 122394.

598. Popov I.A., Kuzmin S.M., Chulovskaya S.A., Semeikin A.S., Parfenyuk V.I. Macroheterocycles 2012, 5, 131-135.

599. Kuzmin S.M., Chulovskaya S.A., Koifman O.I., Parfenyuk V.I. Electrochem. Commun. 2017, 83, 28-32.

600. Tesakova M.V., Lutovac M., Parfenyuk V.I. J. Porphyrins Phthalocyanines 2018, 22, 1047-1053.

601. Filimonova Yu.A., Chulovskaya S.A., Kuzmin S.M., Parfenyuk V.I. Electroplating and Surface Treatment 2020, 28, 21-28 (in Russ.).

602. Masa J., Ozoemena K., Schuhmann W., Zagal J.H. J. Porphyrins Phthalocyanines 2012, 16, 762-784.

603. Tryk D.A., Cabrera C.R., Fujishima A., Spataru N. In: The Electrochemical Society Proceedings (Prakash J., Chu D., Scherson D., Enayetullah M., Tae Bae I., Eds.). Pennington, New Jersey, 2005. p. 45.

604. Taylor R.J., Humffray A.A. J. Electroanal. Chem. Interfacial Electrochem. 1975, 64, 63-84.

605. Qin H., Xu L., Zhong D. J. Phys. Chem. C 2020, 124, 5167-5173.

606. Hu Q., Rezaee E., Li M., Chen Q., Cao Y., Mayukh M., McGrath D.V., Xu Z.X. ACS Appl. Mater. Interfaces 2019, $11,36535-36543$.

607. Song C., Li Y., Gao C., Zhang H., Chuai Y., Song D. Mater. Lett. 2020, 270, 127666.

608. Boileau N.T., Melville O.A., Mirka B., Cranston R., Lessard B.H. RSC Adv. 2019, 9, 2133-2142.

609. Lo P.C., Rodríguez-Morgade M.S., Pandey R.K., Ng D., Torres T., Dumoulin F. Chem. Soc. Rev. 2020, 49, 1041-1056.
610. Valli F., García Vior M.C., Roguin L.P., Marino J. Free Radic. Biol. Med. 2020, 152, 743-754.

611. Su H.C., Tran T.T., Bosze W., Myung N.V. Sens. Actuators Rep. 2020, 2, 100011.

612. Wang L., Wang L., Yang G., Xie Q., Zhong S., Su X., Hou Y., Zhang B. Langmuir 2020, 36, 4532-4539.

613. Morlanés N., Almaksoud W., Rai R.K., Ould-Chikh S., Ali M.M., Vidjayacoumar B., Al-Sabban B.E., Albahily K., Basset J.M. Catal. Sci. Technol. 2020, 10, 844-852.

614. Neamtu M., Nadejde C., Brinza L., Dragos O., Gherghel D., Paul A. Sci. Rep. 2020, 10, 1-12.

615. Thomas A.L. Phthalocyanine Research and Applications. CRC Press, 1990. 304 p.

616. Terao R., Nakazono T., Parent A.R., Sakai K. Chempluschem 2016, 81, 1064-1067.

617. Filippova A., Vashurin A., Znoyko S., Kuzmin I., Razumov M., Chernova A., Shaposhnikov G., Koifman O. J. Mol. Struct. 2017, 1149, 17-26.

618. Vashurin A., Filippova A., Znoyko S., Voronina A., Lefedova O., Kuzmin I., Maizlish V., Koifman O. J. Porphyrins Phthalocyanines 2015, 19, 983-996.

619. Vashurin A., Kuzmin I., Razumov M., Golubchikov O., Koifman O. Macroheterocycles 2018, 11, 11-20.

620. Filippova A.A., Kerner A.A., Znoiko S.A., Tikhomirova T.V., Vashurin A.S. Russ. J. Inorg. Chem. 2020, 65, 247-254.

621. Voronina A.A., Filippova A.A., Znoiko S.A., Vashurin A.S. Maizlish V.E. Russ. J. Inorg. Chem. 2015, 60, 1407-1414.

622. Reid N., Barat R. Chem. Eng. Commun. 2016, 203, 714-723.

623. Saka E.T., Çağlar Y. Catal. Lett. 2017, 147, 1471-1477.

624. Dai D., Yang Z., Yao Y., Chen L., Jia G., Luo L. Catal. Sci. Technol. 2017, 7, 934-942.

625. Sun X., Wang L., Tan Z. Catal. Lett. 2015, 145, 1094-1102.

626. Baturhan O.E., Sağlam M.B., Özkaya A.R. Synth. Met. 2020, $263,116351$.

627. Chen B., Bu Y., Yang J., Nian W., Hao S. Chem. Eng. J. 2020, 399, 125675.

628. Li L., Wu H., Chen H., Zhang J., Xu X., Wang S., Wang S., Sun H. Chemosphere 2020, 256, 127160.

629. Wang C., Shao N., Xu J., Zhang Z., Cai Z. J. Hazard. Mater. 2020, 388, 121751.

630. Gong J., Li D., Huang J., Ding L., Tong Y., Li K., Zhang C. Catal. Lett. 2014, 144, 487-497.

631. Vashurin A., Kuzmin I., Mayzlish V., Razumov M., Golubchikov O., Koifman O. J. Serbian Chem. Soc. 2016, 81, 1025-1036.

632. Vashurin A., Maizlish V., Pukhovskaya S., Voronina A., Kuzmin I., Futerman N., Golubchikov O., Koifman O. J. Porphyrins Phthalocyanines 2015, 19, 573-581.

633. Tugba S.E., Tekintas K. J. Mol. Struct. 2020, 1215, 128189.

634. Vashurin A., Erzunov D., Kazaryan K., Tonkova S., Tikhomirova T., Filippova A., Koifman O. Dyes Pigm. 2020, 174, 108018.

635. Makarov S.G., Ketkov S.Y., Wöhrle D. Chem. Commun. 2020, 56, 5653-5656.

636. Vashurin A., Marfin Y., Tarasyuk I., Kuzmin I., Znoyko S., Goncharenko A., Rumyantsev E. Appl. Organomet. Chem. 2018, 32, e4482.

637. Vashurin A.S. Russ. Chem. Bull. 2016, 65, 2220-2228.

638. Tripathi D., Negi H., Singh R.K., Singh U.P., Srivastava V.C. J. Coord. Chem. 2019, 72, 2982-2996.

639. Yang Y., Li M., Ren Y., Li Y., Xia C. Int. J. Hydrogen Energy 2018, 43, 3797-3802.

640. Thiruppathiraja T., Arokiyanathan A., Aazaad B., Silviya R., Lakshmipathi S. Int. J. Hydrogen Energy 2020, 45, $8540-8548$.

641. Guo S., Li D., Gao B., Li Y., Zhang H., Li Y., Duan Q. J. Coord. Chem. 2019, 72, 1146-1155. 
642. Huai M., Yin Z., Wei F., Wang G., Lu J., Zhuang L. Chem. Phys. Lett. 2020, 754, 137655.

643. Ma D.D., Han S.G., Cao C., Li X., Wu X.T., Zhu Q.L. Appl. Catal. B Environ. 2020, 264, 118530.

644. Li K., Zhu J., Liu Q., Li Z., Zhao J. J. Electrochem. Soc. 2020, 167, 040506.

645. Silva N., Castro-Castillo C., Oyarzún M., Ramírez S., Gutierrez-Ceron C., Marco J., Silva J., Zagal J. Electrochim. Acta 2019, 308, 295-306.

646. Yan X., Xu X., Liu Q., Guo J., Kang L., Yao J. J. Power Sources 2018, 389, 260-266.

647. Mahmiani Y., Sevim A., Gül A. J. Photochem. Photobiol. A Chem. 2016, 321, 24-32.

648. Koç Keşir M., Dilber G., Sökmen M., Durmuş M. J. Sol-Gel Sci. Technol. 2020, 93, 687-694.

649. Zhou Z., Chen A., Kong A., Fan X., Zhang X., Shan Y. J. Electrochem. Soc. 2018, 165, H658-H666.

650. Qian M., Ma J., Materials M. Trans. Tech. Publ. 2017, 748, 433-437.

651. Wang R., Liu Y., Zuo P., Zhang Z., Lei ., Liu Y. Environ. Sci. Pollut. Res. 2020, 27, 18831-18842.

652. Huang Y., Yang Z., Yang S., Xu Y. J. Adv. Nanomater. 2017, 2, 146-153.

653. Chauhan P., Yan N. RSC Adv. 2015, 5, 37517-37520.

654. Ziyadova T.M., Burmistrov V.A., Maizlish V.E., Koifman O.I. Russ. J. Phys. Chem A 2017, 91, 460-463.

655. Ziyadova T.M., Burmistrov V.A., Maizlish V.E., Koifman O.I. Kinet. Catal. 2016, 57, 313-318.

656. Mapukata S., Kobayashi N., Kimura M., Nyokong T. J. Photochem. Photobiol. A Chem. 2019, 379, 112-122.

657. Zhu Z., Chen Y., Gu Y., Wu F., Lu W., Xu T., Chen W. Water Res. 2016, 93, 296-305.

658. Bridwell-Rabb J., Drennan C.L. Curr. Opin. Chem. Biol. 2017, 37, 63-70.

659. Giedyk M., Goliszewska K., Gryko D. Chem. Soc. Rev. 2015, 44, 3391-3404.

660. Zelder F. Chem. Commun. 2015, 51, 14004-14017.

661. Lexa D., Saveant J.-M. Acc. Chem. Res. 1983, 16, 235-243.

662. Dereven'kov I.A., Hannibal L., Dürr M., Salnikov D.S., Bui Thi T.T., Makarov S.V., Koifman O.I., Ivanović-Burmazović I. J. Organomet. Chem. 2017, 839, 53-59.

663. Dereven'kov I.A., Bui Thi T.T., Salnikov D.S., Makarov S.V. Russ. J. Phys. Chem. A 2016, 90, 596-600.

664. Lexa D., Saveant J.M., Zickler J. J. Am. Chem. Soc. 1980, 102, 2654-2663.

665. Birke R.L., Huang Q., Spataru T., Gosser D.K. Jr. J. Am. Chem. Soc. 2006, 128, 1922-1936.

666. Sajan A., Birke R.L. Electroanalysis 2016, 28, 2743-2753.

667. Dereven'kov I.A., Makarov S.V., Bui Thi T.T., Makarova A.S., Koifman O.I. Eur. J. Inorg. Chem. 2018, 2987-2992.

668. Pallares I.G., Moore T.C., Escalante-Semerena J.C., Brunold T.C. J. Am. Chem. Soc. 2016, 138, 3694-3704.

669. Dürichen H., Diekert G., Studenik S. Protein Sci. 2019, 28, 1902-1908.

670. Johnston R.C., Zhou J., Smith J.C., Parks J.M. J. Phys. Chem. A 2016, 120, 7307-7318.

671. Kumar M., Hirao H., Kozlowski P.M. J. Biol. Inorg. Chem. 2012, 17, 1107-1121.

672. Kumar M., Kozlowski P.M. Angew. Chem. Int. Ed. 2011, 50, 8702-8705.

673. Bhat S.A., Rashid N., Rather M.A., Pandit S.A., Ingole P.P., Bhat M.A. Electrochim. Acta 2020, 337, article number 135730 (doi: 10.1016/j.electacta.2020.135730).

674. Robertson W.D., Bovell A.M., Warncke K. J. Biol. Inorg. Chem. 2013, 18, 701-713.

675. Lexa D., Savéant J.-M. J. Chem. Soc., Chem. Commun. 1975, 872-874.
676. Shimakoshi H., Hisaeda Y. ChemPlusChem 2014, 79, $1250-1253$.

677. Tian H., Shimakoshi H., Imamura K., Shiota Y., Yoshizawa K., Hisaeda Y. Chem. Commun. 2017, 53, 9478-9481.

678. Shimakoshi H., Luo Z., Tomita K., Hisaeda Y. J. Organomet. Chem. 2017, 839, 71-77.

679. Ogawa A., Oohora K., Hayashi T. Inorg. Chem. 2018, 57, 14644-14652.

680. Grodkowski J., Neta P. J. Phys. Chem. A 2000, 104, 18481853.

681. Wang Y., Chen Z. Talanta 2010, 82, 534-539.

682. Ji J., Chung Y., Kwon Y. J. Mat. Chem. C 2020, 8, 2749-2755.

683. Shahadat H.M., Younus H.A., Ahmad N., Shiguo Z., Zhuiykov S., Verpoort F. Chem. Commun. 2020, 56, 1968-1971.

684. Shimakoshi H., Hisaeda Y. Curr. Opin. Electrochem. 2018, 8, 24-30.

685. Tahara K., Pan L., Ono T., Hisaeda Y. Beilstein J. Org. Chem. 2018, 14, 2553-2567.

686. Thordarson P. Chem. Soc. Rev. 2011, 40, 1305-1323.

687. Zaitseva S.V., Zdanovich S.A., Koifman O.I. Macroheterocycles 2012, 5, 81-86.

688. Iwamoto H., Nishi S., Haino T. Chem. Commun. 2011, 47, 12670-12672.

689. Kundrat O., Kas M., Tkadlecova M., Lang K., Cvacka J., Stibor I., Lhotak P. Tetrahedron Lett. 2007, 48, 6620-6623.

690. Sallas F., Darcy R. Eur. J. Org. Chem. 2008, 6, 957-969.

691. Korendovych I.V., Roesner R.A., Rybak-Akimova E.V. $A d v$. Inorg. Chem. 2007, 59, 109-173.

692. Puglisi A., Purrello R., Rizzarelli E., Sortino S., Vecchio G. New J. Chem. 2007, 31, 1499-1506.

693. Hosokawa K., Miura Y., Kiba T., Kakuchi T., Sato S. Chem. Lett. 2008, 37, 60-61.

694. Fathalla M., Li S., Diebold U., Alb A., Jayawickramarajah J. Chem. Commun. 2009, 4209-4211.

695. Kralova J., Kejik Z., Briza T., Pouckova P., Kral A., Martasek P., Kral V. J. Med. Chem. 2010, 53, 128-138.

696. Guo Y., Zhang P., Chao J., Shuang S., Dong C. Spectrochim. Acta A 2008, 71A, 946-950.

697. Fathalla M., Neuberger A., Li S.-C., Schmehl R., Diebold U., Jayawickramarajah J. J. Am. Chem. Soc. 2010, 132, 9966-9967.

698. Guo Y.-J., Chao J.-B., Pan J.-H. Spectrochim. Acta A 2007, 68A, 231-236.

699. Kiba T., Suzuki H., Hosokawa K., Kobayashi H., Baba S., Kakuchi T., Sato S. J. Phys. Chem. B 2009, 113, 11560-11563.

700. Ermilov E.A., Menting R., Lau J.T.F., Leng X., Roeder B., Ng D.K.P. Phys. Chem. Chem. Phys. 2011, 13, 17633-17641.

701. Samaroo D., Vinodu M., Chen X., Drain C.M. J. Comb. Chem. 2007, 9, 998-1011.

702. Tsuda A. Bull. Chem. Soc. Jpn. 2009, 82, 11-28.

703. Goldberg I. CrystEngComm 2008, 10, 637-645.

704. Boyd P.D.W., Reed C.A. Acc. Chem. Res. 2005, 38, 235-242.

705. Jurow M., Schuckman A.E., Batteas J.D., Drain C.M. Coord. Chem. Rev. 2010, 254, 2297-2310.

706. Chen Y., Zhang Y., Liu Y. Isr. J. Chem. 2011, 51, 515-524.

707. Endo T. Chem. Rec. 2011, 11, 146-157.

708. Kraus T. Curr. Org. Chem. 2011, 15, 802-814.

709. De Rossi R.H., Silva O.F., Vico R.V., Gonzalez C.J. Pure Appl. Chem. 2009, 81, 755-765.

710. Ballester P., Claudel M., Durot S., Kocher L., Schoepff L., Heitz V. Chem. Eur. J. 2015, 21, 15339-15348.

711. Maufroy A., Favereau L., Anne F.B., Pellegrin Y., Blart E., Hissler M., Jacquemin D., Odobel F. J. Mater. Chem. A 2015, 3, 3908-3917.

712. Koifman O.I., Mamardashvili N.Z., Antipin I.S. Synthetic Receptors on the Basis of Porphyrins and Their Conjugates 
with Calix[4]arenes (Konovalov A.I., Ed.). Moscow, 2006. $248 \mathrm{p}$.

713. Mamardashvili G.M., Zvezdina S.V., Mamardashvili N.Z. Russ. J. Gen. Chem. 2011, 81, 594-601.

714. Kulikova O.M., Mamardashvili N.Z. Russ. J. Org. Chem. 2010, 46(8), 1244-1248.

715. Rossom W.V., Kundrat O., Ngo T.H., Lhotak P., Dehaen W., Maes W. Tetrahedron Lett. 2010, 51, 2423-2426.

716. Nakazawa J., Mizuki M., Shimazaki Y., Tani F., Naruta Y. Org. Lett. 2006, 8, 4275-4278.

717. Durot S., Taesch J., Heitz V. Chem. Rev. 2014, 114, 8542 8578.

718. Qiu W.-G., Li Z.-F., Bai G.-M., Meng S.-N., Dai H.-X., He H. Spectrochim. Acta A 2007, 66A, 1189-1193.

719. Guo Y.-J., Guo L., Pan J.-H. Phys. Chem. Liq. 2007, 45, 261-269.

720. Tsuchiya Y., Yamano A., Shiraki T., Sada K., Shinkai S. Chem. Lett. 2011, 40, 99-101.

721. Callari F.L., Mazzaglia A., Scolaro L.M., Valli L., Sortino S. J. Mater. Chem. 2008, 18, 802-805.

722. Yu M., Chen Y., Zhang N., Liu Y. Org. Biomol. Chem. 2010, $8,4148-4154$.

723. Favereau L., Warnan J., Anne F.B., Pellegrin Y., Blart E., Jacquemin D., Odobel F. J. Mater. Chem. A 2013, 1, 7572-7575.

724. Bichan N.G., Tyulyaeva E.Yu., Khodov I.A., Lomova T.N. J. Mol. Struct. 2014, 1061, 82-89.

725. Nguyen N.T., Mamardashvili G.M., Kulikova O.M., Scheblykin I.G., Mamardashvili N.Z., Dehaen W. RSC Adv. 2014, 4, 19703-19709.

726. Albrecht K., Kasai Y., Kimoto A., Yamamoto K. Macromolecules 2008, 41, 3793-3800.

727. Albrecht K., Kasai Y., Kuramoto Y., Yamamoto K. Chem. Comun. 2013, 46, 6861-6863.

728. Wang L., Li H., Deng J., Cao D. Curr. Org. Chem. 2013, 17, 3078-3091.

729. Su S., Ding Y., Li Y., Wu Y., Nie G. Biomaterials 2016, 80, 169-178.

730. Tsolekile N., Nelana S., Oluwafemi O.S. Molecules 2019, 249140, 2669.

731. Mamardashvili N., Maltseva O., Ivanova Y., Mamardashvili G. Tetrahedron Lett. 2008, 49, 3752-3756.

732. Paolesse R., Monti D., Nardis S., Di Natale C., PorphyrinBased Chemical Sensors In: Handbook of Porphyrin Science (Kadish K.M., Smith K.M., Guilard R., Eds.). World Scientific Publishing Co., 2013. p. 121-215.

733. Cioates C., Van Staden S.-R., Van Staden F. J. Solid State Sci. Techn. 2020, 9, 051005.

734. Gottfried M. J. Surf. Sci. Rep. 2015, 70, 259-379.

735. Fagadar-Cosma G., Birdeanu M., Fagadar-Cosma E. J. Res. Updates in Polym. Sci. 2016, 5, 39-51.

736. Rodriguez-Mendez M., Antoniode de Saja J. Molecular Materials for Gas Sensor and Sensor Arrays. In: $A d v$. Nanomat. Inexpens. Gas Microsen. (Elsevier B.V., Liobet E., Eds.) 2020. p. 37-54.

737. Paolesse R., Nardis S., Monti D., Stefanelli M., Di Natale C. Chem. Rev. 2017, 117, 2517-2583.

738. Gouterman M. Optical Spectra and Electronic Structure of Porphyrins and Related Rings. In: The Porphyrins (Dolphin D., Ed.). New York: Academic, 1978. p. 1-128.

739. Di Amico A., Di Natale C., Paolesse R., Macagnano A., Mantini A. Sens. Actuators 2000, B65, 209-215.

740. Rivera M., Rivera J.M., Amelines-Sarria O., Wang Y.A. $A d v$. Mater. Phys. Chem. 2018, 8, 441-457.

741. Celiesiute R., Ramanaviciene A., Gicevicius M., Ramanavicius A. Critical Rev. Anal. Chem. 2018, 49, 195-208.

742. Supriya S., Shetti V.S., Hegde G. New J. Chem. 2018, 42, 12328-12348.
743. Itagaki Y., Deki K., Nakashima S.-I., Sadaoka Y. Sens. Actuat. B Chem. 2005, 108, 393-397.

744. Ma X., Sun J., Wang M., Hu M., Chen G.H., Huang J. Sens. Actuat. B 2006, 114, 1035-1042.

745. Korposh S.O., Takahara N., Ramsden J.J., Lee S.-W., Kunitake T. J. Biol. Phys. Chem. 2006, 6, 125-132.

746. Muthukumar P., John S.A. Sens. Actuat., B 2011, 159, 238-244.

747. Kalimuthu P., Sivanesan A., John S.A. J. Chem. Sci. 2012, $124,1315-1325$.

748. Cano M., Castillero P., Roale J., Pedrosa J.M., Brittle S., Richardson T., González-Elipe A.R., Barranco A. Sensor. Actuat. B Chem. 2010, 150, 764-769.

749. Lv Y., Wu J., Xu Z.K. Sensor. Actuat. B-Chem. 2010, 148 233-239.

750. Lin F.-W., Xu X.-L., Wan L.-S., Wu J., Xu Z.K. $R S C A d v$ 2015, 5, 30472-30477.

751. Hu M., Kang W., Cheng B., Li Z., Zhao Y., Li L. Microchem. Acta 2016, 183(5), 1713-1720.

752. Hu M., Kang W., Zhao Y., Shia J., Cheng B. RSC Adv. 2017, 7, 26849-26856.

753. Hu M., Kang W., Zhong Z., Cheng B., Xing W. Ind. Eng. Chem. Res. 2018, 57(34), 11668-11674.

754. Wang B., Chen Z., Zuo X., Wu Y., He C., Wang X., Li Z. Sensor. Actuat., B-Chem. 2011, 160, 1-6.

755. Muthukumar P., John S.A. Sensor. Actuat. B-Chem. 2012, $174,74-80$

756. Castillero P., Roales J., Lopes-Costa T., Sanchez-Valencia J.R., Barranco A., Gonzalez-Elipe A.R., Pedrosa J.M. Sensors 2017, 17, 24-38.

757. Kim J., Lim S.-H., Yoon Y., Thangadurai T.D., Yoon S. Tetrahedron Lett. 2011, 52, 2645-2648.

758. Korposh S., James S.W., Lee S.-W., Topliss S.M., Cheung S.C., Batty W.J., Tatam R.P. Opt. Express 2010, 18, 13227 13238.

759. Jarzebinska R., Korposh S., James S., Batty W., Tatam R., Lee S.-W. Anal. Lett. 2012, 45(10), 1297-1309.

760. Wang T., Korposh S., James S.W., Tatam R. P., Lee S-W. Sens. Actuators, B 2016, 228, 573-580.

761. Korposh S., Kodaira S., Selyanchyn R., Ledezma F.H., James S.W., Lee S.-W. Opt. Laser Tech. 2018, 101, 1-10.

762. Sawada K., Tanaka T., Yokoyama T., Yamachi R., Oka Y., Masai H., Terao J., Uchida K. Jpn. J. Appl. Phys. 2020, 59, $1-6$.

763. Richardson T.H., Brook R.A., Davis F., Hunter C.A. Coll. Sur. A: Physicochem. Eng. Aspects 2006, 284-285, 320-325.

764. Roales J., Pedrosa J.M., Castillero P., Cano M., Richardson T.H. Thin Solid Films 2011, 519, 2025-2030.

765. Roales J., Pedrosa J.M., Guillen M.G., Lopes-Costa T., Castillero P., Barranco A., González-Elipe A.R. Sensors 2015, 15, 11118-11132.

766. Mc Donagh C., Burke C.S., Mc Craith B.D. Chem. Rev. 2008, 108, 400-422.

767. Peter C., Schmitt K., Apitz M., Woellenstein J. Microsyst. Technol. 2012, 18, 925-930.

768. Abudukeremu H., Kari N., Zhang Y., Wang J., Nizamidin P., Abliz S., Yimit A. J. Mater. Sci. 2018, 53, 10822-10834.

769. Kutilike B., Kari N., Zhang Y., Nizamidin P., Yimit A. Meas. Sci. Technol. 2020, 31, 055105.

770. Diab N., Schuhmann W. Electrochim. Acta 2001, 47, 265-273.

771. Miki H., Matsubara F., Nakashima S., Ochi S., Nakagawa K., Matsuguchi M., Sadaoka Y. Sens. Actuat. 2016, 231, 458-468.

772. Shiba S., Yamada K., Matsuguchi M. Sensors 2020, 20, 1295.

773. Khan A.H., Rao M.V., Li Q. Sensors 2019, 19(905), 1-39. 
774. Strianese M., Lamberti M., Pellecchi C. Dalton Trans. 2017, 46, 1872-1879.

775. Vikrant K., Kumar V., Ok Y., Kim K., Deep A. Trends Anal. Chem. 2018, 105, 263-281.

776. Chen J., Zhu Y., Kaskel S. Angew. Chem. Int. Ed. 2020. DOI:10.1002/anie.201909880.

777. Ma Y., Su H., Kuang X., Li X., Zhang T., Tang, B. Anal. Chem. 2014, 86, 11459-11463.

778. Tuerdi G., Kari N., Yan Y., Nizamidin P., Yimit A. Sensors 2017, 17(12), 2717.

779. Mamtmin G., Kari N., Abdurahman R., Nizamidin P., Yimit A. Opt. Las. Tech. 2020, 128, 106260.

780. Maimaiti A., Abdurahman R., Kari N., Ma Q., Wumaier K., Nizamidin P., Yimit A. J. Modern Opt. 2020, 67, 507514.

781. Amao Y., Okura I. J. Porphyrins Phthalocyanines 2009, 13, 1111-1122.

782. Hutter L.H., Muller B.J., Koren K., Borisov S.M., Klimant I. J. Mater. Chem. C 2014, 2, 7589-7598.

783. Kimura T., Watanabe S., Sawada S., Shibasaki Y., Oishi Y. J. Polym. Sci., A: Pol. Chem. 2017, 55, 1086-1094.

784. Arunkumar C., Kooriyaden F. R., Zhang X., Sujatha S., Zhao J. New J. Chem. 2017, 41, 4908-4917.

785. Biring S., Sadhu A.S., Deb M. Sensors 2019, 19, 5124.

786. Mao Y., Gao Y., Wu S., Wu S., Shi J., Zhou B., Tian Y. Sens. Actuat. B: Chem. 2017, 251, 495-502.

787. Mao Y., Mei Z., Wen J., Li G., Tian Y., Zhou B., Tian Y. Sens. Actuat. 2018, B257, 944-953.

788. Mao Y., Akram M., Shi J., Wen J., Yang C., Jiang J., Zhou B., Tian Y. Sens. Actuat. B: Chem. 2019, 282, 885-895.

789. Spencer J.A., Ferraro F., Roussakis E., Klein A., Wu J., Runnels J.M., Zaher W., Mortensen L.J., Alt C., Turcotte R., Yusuf R., Cote D., Vinogradov S.A., Scadden D.T., Lin C.P. Nature 2014, 508, 269-273.

790. The Porphyrin Handbook. Biochemistry and Binding: Activation of Small Molecules. Vol. 4 (Kadish K.M., Smith K.M., Guilard R., Eds.). New York: Academ. Press, 2000. $341 \mathrm{p}$.

791. Porphyrins: Spectroscopy, Electrochemistry, Applications (Enikolopyan N.S., Ed.). Moscow: Nauka, 1987. $384 \mathrm{p}$.

792. Mamardashvili G.M., Mamardashvili N.Zh., Koifman O.I. Russ. Chem. Rev. 2005, 74, 765-780.

793. Zaitsev S.Yu. Supramolecular Nanoscale Systems at the Interface of the Phases. Concepts and Perspectives for Bionanotechnologies. Moscow: LENAND, 2010. $212 \mathrm{p}$.

794. Kitzerow H-S, Bahr C. Chirality in Liquid Crystals. New York (NY): Springer-Verlag Inc., 2001.

795. Kelly S.M., O’Neill M. Liquid Crystals for Electro-Optic Applications. In: Handbook of Advanced Electronic and Photonic Materials and Devices Vol. 7: Liquid Crystals, Display and Laser Materials (Nalwa H.S., Ed.). N.Y. etc.: Academic Press, 2000. p. 1-66.

796. Onuchak L.A., Arutunov J.I., Kuraeva J.G., et.al. Method for Analysis of Structural and Optical Isomers, 2014. Patent RF No. RU 2528126.

797. Matt B., Pondman K.M., Asshoff S.J., et al. Angew. Chem. Int. Ed. 2014, 53, 12446.

798. Eelkema R. Liq. Cryst. 2011, 38, 1641-1652.

799. Cachelin P., Green J.P., Peijs T., Heeney M., Bastiaansen W.M. Adv. Opt. Mater. 2016, 4, 592-596.

800. Iwan A., Boharewicz B., Tazbir I., Hamplová V., Bubnov A. Solid-State Electronics 2015, 104, 53-60.

801. Ishida Y., Kai Y., Kato S., et al. Angew. Chem. Int. Ed. 2008, 47, 8241-8245.

802. Van Delden R.A., Koumura N., Harada N., Feringa B.L. PNAS 2002, 99, 4945-4949.
803. Gottarelli G., Spada G.P. Mol. Cryst. Liq. Cryst. 1985, 123, 377-388.

804. Celebre G., de Luca G., Maiorino M., et al. J. Am. Chem. Soc. 2005, 127, 11736-11744.

805. Watanabe G., Yoshida J. J. Phys. Chem. B 2016, 120, 6858-6864.

806. Yoshida J., Watanabe G., Kakizawa K., et al. Inorg. Chem. 2013, 52, 11042-11050.

807. Engelmann M., Braun M., Kuball H-G. Liq. Cryst. 2007, 34, $73-77$.

808. Braun M., Hahn A., Engelmann M., et al. Chem. Eur. J. 2005, 11, 3405-3412.

809. Handbook of Porphyrin Science with Applications to Chemistry, Physics, Materials Science, Engineering, Biology and Medicine. Vol. 7. Physicochemical Characterization (Kadish K.M., Smith K.M., Guilard R., Eds.). World Scientific Publishing: Co. Pte. Ltd., 2010.

810. Kobayashi N. Optically Active Porphyrin Systems Analyzed by Circular Dichroism. In: Handbook of Porphyrin Science. Vol. 7, Ch. 33 (Kadish K.M., Smith K.M., Guilard R., Eds.). Singapore: World Scientific, 2010. p.147-240.

811. Lu H., Kobayashi N. Chem Rev. 2016, 116, 6184-6261.

812. Lehmann M., Dechant M., Gerbig L., et al. Liq. Cryst. 2019, 46, 1985-1994.

813. Burmistrov V.A., Novikov I.V., Aleksandriiskii V.V., et al. J. Mol. Liq. 2019, 287, 110961.

814. Burmistrov V.A., Novikov I.V., Alexandriiskii V.V., Semeikin A.S., Koifman O.I. Liq. Cryst. 2020. DOI:10.108 0/02678292.2020.1817583.

815. Davankov V.A. Ligand Exchange Chromatography. Encyclopedia of Separation Science. Vol. 5 (Wilson I.D., Adlard E.R., Cooke M., Pool C.F., Eds.). Amsterdam: Acad.Press, 2000. p. 2369-2380.

816. Davankov V., Navratil J., Walton H. Ligand Exchange Chromatography. USA: SRC-Press, 1988. 209 p.

817. Wenzel T.J., Yarmaloff L.W., St. Cyr L.Y., O'Meara L.J., Donatelli M., Bauer R.W. J. Chromatogr. 1987, 396, 51-64.

818. Burmistrov V.A., Rodicheva J.A., Trifonova I.P., Koifman O.I. Modern Tendencies in Materials Functionalization by Macroheterocycles. In: Functional Materials Based on Tetrapyrrole Macrocyclic Compounds. Ch. 1 (Koifman O.I., Ed.). URSS, 2019. p. 17-62.

819. Krestov A.G., Blokhina S.V., Galyametdinov Yu.G., Ol'khovich M.V., Lokhanov V.V. Russ. J. Phys. Chem. 1993, 67(1), 151-154.

820. Kuvshinov G.V., Maizlish V.E., Kuvshinova S.A., Burmistrov V.A., Koifman O.I. Macroheterocycles 2016, 9, 244-249.

821. Burmistrov V.A., Semeikin A.S., Kuvshinov G.V., Aleksandriiskii V.V., Lubimova T.V., Kuvshinova S.A., Koifman O.I. J. Porphyrins Phthalocyanines 2017, 21, 103-109.

822. Sanders J.K.M., Bampos N., Clude-Watson Z., Darling S.L., Hawley J.C., Kim H.-J., Mak C.C., Webb S.J. Axial Coordination Chemistry of Metalloporphyrins. In: The Porphyrin Handbook. Vol. 3, Ch. 15 (Kadish K.M., Smit K.M., Guilard R., Eds.). New York: Academ. Press, 2000. p. 1-47.

823. Grajek H., Witkiewicz Z., Purchała M., Drzewin'ski W. Chromatographia 2016, 79, 1217-1245.

824. Burmistrov V.A., Semeikin A.S., Lubimova T.V., Novikov I.V., Litov K.M., Aleksandriiskii V.V., Kuvshinova S.A., Koifman O.I. Nickel Complex of 5,10,15,20-tetrakis [3',5'di-(2'-methylbutyloxy)phenyl]-porphin Exhibiting the Stationary Phase Property for Gas Chromatography, 2015, Patent RF No. 2557655.

825. Allenmark S.G. Chromatographic Enantioseparation: Methods Applications. Chichester: Horwood, 1988. 224 p.

826. Kuvshinov G.V., Koifman O.I. Russ. J. Phys. Chem. A 2018, 92, 2025-2031. 
827. Kuvshinov G.V., Kuvshinova S.A., Burmistrov V.A., Koifman O.I. Sorbent for Gas Chromatography, 2017, Patent RF No. 2621337.

828. Kuvshinov G.V., Kuvshinova S.A., Koifman O.I. J. Anal. Chem. 2017, 72, 1172-1177.

829. Kuvshinov G.V., Koifman O.I. Russ. J. Gen. Chem. 2019, 89, 1279-1285.

830. Executive Orders No.1629n dated 29.12.2012 and No.915n dated 15.11.2012 of the Ministry of Health of the Russian Federation.

831. Abrahamse H., Hamblin M. Biochem. J. 2016, 473, 347-364.

832. Kwiatkowski S., Knap B., Przystupski D. et al. Biomedicine \& Pharmacotherapy 2018, 106, 1098-1107.

833. Zhang X.H., Zhang L.J., Sun J.J. et al. Biomedicine \& Pharmacotherapy 2016, 81, 265-272.

834. Mironov A.F. Russ. J. Gen. Chem. 2019, 89, 1952-1983.

835. Ethirajan Y., Chen Y., Joshi P., Pandey R.K. Chem. Soc. Rev. 2011, 40, 340-362.

836. Senge M.O., Brandt J.C. Photochem. Photobiol. 2011, 87, 1240-1296.

837. Bonnett R. Chemical Aspects of Photodynamic Therapy. Amsterdam: Gordon and Breach Science Publishers, 2000.

838. Muragaki Y., Akimoto J., Manuyama T., et al. J. Neurosung. 2013, 119, 845-852.

839. Kobayashi W., Liu Q., Nakagawa H., et al. Oral. Oncol. 2006, 42, 45-49.

840. Saavedra R., Rocha L.B., Dabrowski J.M., et al. Chem. Med. Chem. 2014, 9, 390-398.

841. Shan W.M., Lim T.H., Pece A., et al. Graefes. Arch. Clin. Exp. Ophthalmol. 2010, 248, 613-626.

842. Sessler J.L., Miller R.A. Biochem. Pharmacol. 2000, 59, 733-739.

843. Josefsen L.B., Boyle R.W. Metal-Based Drugs 2008, 200, $1-24$.

844. Scherz A., Salomon Y. The Story of Tookad, From Bench to Bedside. In: Handbook of Photomedicine (Hamblin M.R., Huang Y.Y., Eds.). CRC Press, Boca Raton, FL, 2014.

845. Chevalier S., Anidjar M., Scarlata E., et al. J. Urol. 2011, 196, 302-309.

846. Ashur I., Goldschmidt R., Pinkas I., et al. J. Phys. Chem. A 2009, 113, 8027-8037.

847. Scherz A., Salomon Y., Coleman. J. Photodiagn. Photodyn. Ther. 2017, 17, 22-31.

848. Lobel J., MacDonald I.J., Ciesielski M.Y., et al. Laser Surg. Med. 2001, 29, 397-405.

849. Peng Q., Berg K., Moan J., et al. Photochem. Photobiol. 1997, 65, 235-251.

850. Krammer B., Plaetzer K. Photochem. Photobiol. Sci. 2008, 7, 283-289.

851. Furre I.E., Shahzidi S., Luksiene Z., et al. Cancer Res. 2005, 65, 11051-11060.

852. Romanko Y.S., Tsyb A.F., Kaplan M.A., et al. Bull. Exp. Biol. Med. 2004, 138, 584-589.

853. Petrov P., Trukacheva T., Kaplan M., 2006, European Patent Application No EP 1610821.

854. Biswas R., Moon J.H., Ahn J.C. Photochem. Photobiol. 2014, 90, 1108-1118.

855. Pucelic B.L., Arnaut G., Stochel G., et al. ACS Appl. Mater. Interfaces 2016, 8, 22039-22055.

856. Santos L.L., Oliveira J., Monteiro E., et al. Case Rep. Oncol. 2018, 11, 769-776.

857. Ormond A.B., Freeman H.S. Materials 2013, 6, 817-840.

858. Pereira P.M.R., Korsak B., Sarmento B., et al. Org. Biomol. Chem. 2015, 13, 2518-2529.

859. Moret F., Reddi E. J. Porphyrins Phthalocyanines 2017, 21, 1-18.

860. Pucelik B., Sułek A., Dąbrowski J.M. Coord. Chem. Rev. 2020, 416, 213340.
861. Mironov A.F., Zhdanova K.A., Bragina N.A. Russ. Chem. Rev. 2018, 87, 859-881.

862. Sandland J., Malatesti N., Boyle R. Photodiagnosis Photodyn. Ther. 2018, 23, 281-294.

863. Boyle R.W., Sandland J. Bioconjugate Chem. 2019, 30, 975-993.

864. Hamblin M.R. Photochem. Photobiol. 2020, 96, 506-516.

865. Sato K., Nagaya T., Choyke P.L., et al. Theranostics 2015, 5 , 698-709.

866. Parslow A.C., Parakh S., Lee F.T. Biomedicines 2016, 4, 14-31.

867. You H., Yoon H.E., Jeong P.M., et al. Bioorg. Med. Chem. 2015, 23, 1453-1462.

868. Yang N.J., Hinner M.J. Meth. Mol. Biol. 2015, 126, 29-53.

869. Park S.Y., Baik H.J., Oh Y.T., et al. Angew. Chem. Int. Ed. Engl. 2011, 50, 1644-1647.

870. Singh S., Aggarwal A., Dinesh N.V.S., et al. Chem. Rev. 2015, 115, 10261-10306.

871. El-Akra N., Noirot A., Faye J.C., et al. Photochem. Photobiol. Sci. 2006, 5, 996-999.

872. Stefflova K., Li H., Chen J., et al. Bioconjugate Chem. 2007, 18, 379-388.

873. Gravier J., Schneider R., Frochot C., et al. J. Med. Chem. 2008, 51, 3867-3877.

874. Suvorov N.V., Mironov A.F., Grin M.A. Russ. Chem. Bull. 2017, 1982-2008.

875. Barondes S.H., Castronovo V., Cooper D.N.W., et al. Cell 1994, 76, 597-598

876. Zheng X., Pandey R.K. Anti-Cancer Agents in Med. Chem. 2008, 8, 241-268.

877. Aksenova A.A., Sebyakin Yu.L., Mironov A.F. Russ. J. Bioorg. Chem. 2000, 26, 111-124.

878. Aksenova A.A., Sebyakin Yu.L., Mironov A.F. Russ. J. Bioorg. Chem. 2001, 27, 124-129.

879. Mironov A.F., Lebedeva V.S. Tetrahedron Lett. 1998, 39, 905-908.

880. Lebedeva V.S., Ruziev R.D., Popov A.V., et al. Mendeleev Commun. 2007, 17, 212-213.

881. Lonin I.S. Ph.D. Thesis in Chemical Science "Synthesis and Examination of Properties of Natural Chlorins and Bacteriochlorins". Moscow, 2009 (in Russ.).

882. Lonin I.S., Lakhina A.A., Grin M.A., et al. Mendeleev Commun. 2012, 22, 157-158.

883. Grin M.A., Lonin I.S., Makarov A.I., et al. Mendeleev Commun. 2008, 18, 135-137.

884. Lonin I.S., Makarov A.I., Lakhina A.A., et al. J. Porphyrins Phthalocyanines 2008, 12, 619.

885. Grin M.A., Lonin I.S., Lakhina A.A., Ol'shanskaya E.S., Makarov A.I., Sebyakin Y.L., Guryeva L.Yu., Toukach P.V., Kononikhin A.S., Kuzmin V.A., Mironov A.F. J. Porphyrins Phthalocyanines 2009, 13, 336-345.

886. Grin M.A., Plotnikova E.A., Plyutinskaya A.D., et al. Russ. Biother. J. 2012, 11, 14

887. Petrov P., Trukhacheva T., Isakov G., et al. Acta Bioopt. Inform. Med. 2004, 10, 6-7.

888. Trukhacheva T.V., Shlyahtin S.V., Isakov G.A., et al. Fotolon - A Novel Solution for PDT. Review of the Results of Pharmaceutical, Pharmacological and Clinical Trials. Minsk: RUP "Belmedpreparaty", 2009. p. 64. ISBN 978-5-89552-367-4.

889. Kularatne S.A., Venkatesh C., Santhapuram H.K.R., et al. J. Med. Chem. 2010, 53, 7767-7777.

890. Hillier S.M., Maresca K.P., Lu G., et al. J. Nucl. Med. 2013, 54, 1369-1376.

891. Stoermer D., Liu Q., Hall M.R., et al. Bioorg. Med. Chem. Lett. 2003, 13, 2097-2100.

892. Hargus J.A., Fronczek F.R., Vicente M.G.H., Smith K.M. Photochem. Photobiol. 2007, 83, 1006-1015. 
893. Jinadasa R.G.W., Hu X., Vicente M.G.H., Smith K.M. J. Med. Chem. 2011, 54, 7464-7476.

894. Jinadasa R.G.W., Zhou Z., Vicente M.G.H., Smith K.M. Org. Biomol. Chem. 2016, 14, 1049-1064.

895. Bommer J.C., Ogden B.F. Tetrapyrrole Therapeutic Agents, 1987, U.S. Patent 4,693.885.

896. Pandey R.K., Zheng G. Porphyrins as Photosensitizers in Photodynamic Therapy. In: The Porphyrin Handbook. Vol. 6 (Kadish K. M., Smith K. M., Guilard R., Eds.). Boston: Academic Press, 2000. p. 157-230.

897. Suvorov N.V. Ph.D. Thesis in Chemical Science "Modified Natural Chlorins of Targeted Action Against Tumor Cells of Various Genesis". Moscow, 2019.

898. Grin M.A., Suvorov N.V., Machulkin A.E., et al., 2018, Patent RU 2670087 C1.

899. Oliveira B.L., Guo Z., Bernardes G.J.L. Chem. Soc. Rev. 2017, 46, 4895-4950.

900. Suvorov N.V., Cheskov D.A., Mironov A.F., et al. Mendeleev Commun. 2019, 29, 206-207.

901. Suvorov N.V., Machulkin A.E., Ivanova A.V., et al. J. Porphyrins Phthalocyanines 2018, 22, 1030-1038.

902. Selbo P.K., Bostad M., Olsen C.E., et al. Photochem. Photobiol. Sci. 2015, 14, 1433-1450.

903. Sultan A.A., Jerjes W., Berg K., et al. Lancet Oncol. 2016, 17, 1217-1229.

904. Kochneva E.V., Filonenko E.V., Vakulovskaya E.G., et al. Photodiagnosis Photodyn. Ther. 2010, 7, 258-267.

905. Shiryaev A.A., Musaev G.K., Levkin V.V., et al. Photodiagnosis Photodyn. Ther. 2019, 26, 218-223.

906. Baker M. Nature 2010, 463, 977-980.

907. Thorp-Greewood F.L., Coogan M.P. Dalton Trans. 2011, 40, 6129-6143.

908. Mewis R.E., Archibald S.J. Coord. Chem. Rev. 2010, 254, 1682-1712.

909. Rashid H.U., Khan K., Yaseen M., et al. Rev. Roum. Chim. 2014, 59, 27-33.

910. Grin M.A., Brusov S.S., Shchepelina E.Y., et al. Mendeleev Commun. 2017, 27, 338-340.

911. Mironov A.F. Transition Metal Complexes of Porphyrins and Porphyrinoids. In: Handbook of Porphyrin Science. Vol 18. Applications and Materials (Kadish K.M., Smith K.M., Guillard R., Eds). World Scientific, 2012. p. 304-413.

912. Rumyantseva V.D., Shchelkunova A.E., Gorshkova A.S., Alekseev Yu.V., Shumilova N.M., Shilov I.P., Ivanov A.V., Mironov A.F. Fine Chem. Technol. 2017, 12, 72-80.

913. Smirnov A.S., Grin M.A., Mironov A.F. Fine Chem. Technol. 2019, 14, 95-103.

914. Brusov S.S. Ph.D. Thesis in Chemical Science "Natural Chlorins with Photoinduced Antibacterial, Antitumor Activity and Diagnostic Potential". Moscow, 2018.

915. Zenkevich E., Sagun E., Knyukshto V., Shulga A., Mironov A., Efremova O., Bonnett R., Songca S.P., Kassem M.J. Photochem. Photobiol. B: Biol. 1996, 33, 171-180.

916. Parkhats M.V., Galievsky V.A., Stashevsky A.S., Trukhacheva T.V., Dzhagarov B.M. Opt. Spectrosc. 2009, 107, 974-980.

917. Henderson B.W., Sumlin A.B., Owcharczak B.L., Dougherty T.J. Photochem.Photobiol. B 1991, 10, 303-313.

918. Koudinova N.V., Pinthus J.H., Brandis A., Brenner O., Bendel P., Ramon J., Eshhar Z., Scherz A., Salomon Y. Int. J. Cancer 2003, 104, 782-789.

919. Brandis A., Mazor O., Neumark E., Rozenbach V.-Belkin, Salomon Y., Scherz A. Photochem. Photobiol. 2005, 81, 983-993.

920. Grin M.A., Mironov A.F., Shtil A.A. Anti-Cancer Agents in Med. Chem. 2008, 8, 683-697.

921. Grin M.A., Mironov A.F. In: Chemical Processes with Participation of Biological and Related Compounds
(Lomova T.N., Zaikov G.T., Eds.). Boston, Brill: Leiden, 2008. p. 5-43.

922. Eisner U. J. Chem. Soc. 1957, 3461-3469.

923. Scheer H. Chlorophylls. Boston, London: CRC Press, Boca Raton Ann Arbor, 1991. p. 115-143.

924. Mironov A.F., Efremov A.V. 1996, Patent RF No 2144085.

925. Tsygankov A.A., Laurinavichene T.V., Gogotov I.N. Biotechnol. Tech. 1994, 8, 575-578.

926. Tsygankov A.A., Laurinavichene T.V., Bukatin V.E., Gogotov I.N., Hall D.O. Biochem. Microbiol. 1997, 33, 485-490.

927. Prinsep M.R., Caplan F.R., Moore R.E., Patterson G.M.L., Smith C.D. J. Am. Chem. Soc. 1992, 114, 385-387.

928. Prinsep M.R., Patterson G.M.L., Larsen L.K., Smith C. D. Tetrahedron 1995, 51, 10523-10530.

929. Prinsep M.R., Patterson G.M.L., Larsen L.K., Smith C. D. J. Nat. Prod. 1998, 61, 1133-1136.

930. Prinsep M.R., Appleton T.G., Hanson G.R., Lane I., Smith C.D., Puddick J., Fairlie, D.P. Inorg. Chem. 2017, 56, 5577-5585.

931. Minehan T.G., Kishi Y. Angew. Chem. Int. Ed. 1999, 38, 923-925.

932. Minehan T.G., Cook-Blumberg L., Kishi Y., Prinsep M.R., Moore R.E. Angew. Chem. Int. Ed. 1999, 38, 926-928.

933. Hoebeke M., Schuitmaker H.J., Jannink L.E., et al. Photochem. Photobiol. 1997, 66, 502-508.

934. Fiedor L., Rosenbach-Belkin V., Sai M., Scherz A. Plan. Physiol. Biochem. 1996, 34, 393-398.

935. Scherz A., Salomon Y., Brandis A., Scheer H. 2000, PCT Patent WO00/33833.

936. Azzouzi A.R., Barret E., Bennet J., Moore C., Taneja S., Muir G., Villers A., Coleman J., Allen C., Scherz A., Emberton M. World J. Urology 2015, 33, 945-953.

937. Vakrat-Haglili Y., Weiner L., Brumfeld V., Brandis A., Salomon Y., McIlroy B., Wilson B.C., Pawlak A., Rozanowska M., Sarna T., Scherz A. J. Am. Chem. Soc. 2005, 127, 6487-6497.

938. Sasaki S., Tamiaki H. J. Org. Chem. 2006, 71, 2648-2654.

939. Mironov A.F., Kozyrev A.N., Brandis A.S. Proc. SPIE 1992, 1922, 204-208.

940. Fischer H., Lambrecht R., Mittenzwei H.Z. Physiol. Chem. 1939, 1, 253-259.

941. Hartwich G., Fiedor L., Simonin I., Cmiel E., Schafer W., Noy D., Scherz A., Scheer H. J. Am. Chem. Soc. 1998, 120, 3675-3683.

942. Kozyrev A.N., Chen Y., Goswami L.N., Tabaczynski W.A., Pandey R.K. J. Org.Chem. 2006, 71, 1949-1960.

943. Waielewski M.R., Svec W.A. J. Org. Chem. 1980, 45, 1969-1974.

944. Chen Y., Potter W.R., Missert J.R., Morgan J., Pandey R.K. Bioconjugate Chem. 2007, 18, 1460-1473.

945. Saga Y., Ishitani A., Takahashi N., Kawamura K. Bioorg. Med. Chem. Lett. 2015, 25, 639-641.

946. Kozyrev A., Ethirajan, M., Chen P., Ohkubo K., Robinson B.C., Barkigia K.M., Pandey R.K. J. Org. Chem. 2012, 77, 10260-10271.

947. Kozyrev A.N., Zheng G., Zhu C.F. Tetrahedron Lett. 1996, 37, 6431-6434.

948. Pandey R.K., Sumlin A.B., Constantine S., Aoudia M., Potter W.R., Bellnier D.A., Henderson B.W., Rodgers M.A., Smith K.M., Dougherty T.J. Photochem. Photobiol. 1996, 64, 194-204.

949. Grin M.A., Lonin I.S., Likhosherstov L.M., Novikova O.S., Plyutinskaya A.D., Plotnikova E.A., Kachala V.V., Yakubovskaya R.I., Mironov A.F. J. Porphyrins Phthalocyanines 2012, 16, 1094-1109.

950. Gorshkova A.S., Rumyantseva V.D., Mironov A.F. Fine Chem. Technol. 2018, 13(2), 5-20. 
951. Patel N., Pera P., Joshi P., Dukh M., Tabaczynski W.A., Siters K.E., Pandey R.K. J. Med. Chem. 2016, 59, 9774-9787.

952. Plotnikova E.A., Stramova V.O., Morozova N.B., Plyutinskaya A.D., Ostroverkhov P.V., Grin M.A., Mironov A.F., Yakubovskaya R.I., Kaprin A.D. Biomed. Photon. 2019, 8, $18-23$.

953. Mironov A.F., Grin M.A., Tsiprovskiy A.G., Kachala V.V., Karmakova T.A., Plyutinskaya A.D., Yakubovskaya R.I. J. Porphyrins Phthalocyanines 2003, 7, 725-730.

954. Mironov A.F., Grin M.A., Tsiprovskiy A.G., Dzardanov D.V., Golovin K.V., Feofanov A.V. 2004, Patent RF No. 2223274.

955. Brusov S.S., Grin M.A., Meerovich G.A., Mironov A.F., Romanova Yu. M., Tiganova I.G. 2017, Patent RF No. 2610566.

956. Pantyushenko I.V., Rudakovskaya P.G., Starovoitova A.V., Mikhailovskaya A.A., Abakumov M.A., Kaplan M.A., Tsigankov A.A., Majouga A.G., Grin M.A., Mironov A.F. Biochemistry 2015, 80, 752-762.

957. Mironov A.F., Grin M.A. J. Porphyrins Phthalocyanines 2008, 12, 1163-1172.

958. Grin M.A., Brittal D.I., Tsiprovskiy A.G., Bregadze V.I., Mironov A.F. Macroheterocycles 2010, 3, 222-227.

959. Meerovich I.G., Tsyprovskiy A.G., Meerovich G.A., Barkanova S.V., Borisova L.M., Oborotova N.A., Baryshnikov A.Yu., Mironov A.F. Proc. SPIE 2007, 6427, 64270W1-W9.

960. Chissov V.I., Yakubovskaya R.I., Mironov A.F., Grin M.A., Plotnikova E.A., Morozova N.B., Tsigankov A.A. 2012, Patent RF No. 2521327.

961. Grin M.A., Filonenko E.V., Mironov A.F., Suvorov N.B., Pankratov A.A., Grigor'evukh N.I. 2020, Patent RF No. 2720806.

962. Aravindu K., Krayer M., Kim H.-J., Lindsey J.S. New J. Chem. 2011, 35, 1376-1384.

963. Senge M.O., Wiehe A., Ryppa C. Adv. Photosynth. Respir. 2006, 25, 27-37.

964. Lange C., Bednarski P.J. Curr. Pharmac. Design 2016, 22, 6956-6974.

965. Karwicka M., Pucelik B., Gonet M., Elas M., Dąbrowski J.M. Scientific Reports 2019, 9, 12655.

966. Bruhn T., Brückner C. J. Org. Chem. 2015, 80, 4861-4868.

967. Samankumara L.P., Zeller M., Krause J.A., Brückner C. Org. Biomol. Chem. 2010, 8, 1951-1965.

968. Ke X.S., Yang B.Y., Cheng X., Chan S.L.F., Zhang J.L. Chem. Eur. J. 2014, 20, 4324-4333.

969. MacGowan S.A., Senge M.O. Chem. Commun. 2011, 47, $11621-11623$.

970. MacGowan S.A., Senge M.O. Biochim. Biophys. Acta 2016, 1857, 427-442.

971. Guberman-Pfeffer M.J., Greco J.A., Samankumara L.P., Zeller M., Birge R.R., Gascon J.A., Brückner C. J. Am. Chem. Soc. 2017, 139, 548-560.

972. Herges R., Peters M.K. 2020, Patent EP 3653226.

973. Peters M.K., Herges R. Beilstein J. Org. Chem. 2017, 13, 2659-2662.

974. Peters M.K., Röhricht F., Näther C., Herges R. Org. Lett. 2018, 20, 7879-7883.

975. Li G., Graham A., Chen Y., Dobhal M.P., Morgan J., Zheng G., Kozyrev A., Oseroff A., Dougherty T.J., Pandey R.K. J. Med. Chem. 2003, 46, 5349-5359.

976. Kim H.-J., Lindsey J.S. J. Org. Chem. 2005, 70, 5475-5486.

977. Krayer M., Ptaszek M., Kim H.-J., Meneely K.R., Fan D., Secor K., Lindsey J.S. J. Org. Chem. 2010, 75, 1016-1039.

978. Reddy K.R., Lubian E., Pavan M.P., Kim H.-J., Yang E., Holten D., Lindsey J.S. New J. Chem. 2013, 37, 1157-1173.

979. Zhang S., Kim H.-J., Tang Q., Yang E., Bocian D.F., Holten D., Lindsey J.S. New J. Chem. 2016, 40, 5942-5956.

980. Liu Y., Lindsey J.S. J. Org. Chem. 2016, 81, 11882-11897.
981. Esemoto N.N., Yu Z., Wiratan L., Satraitis A., Ptaszek M. Org. Lett. 2016, 18, 4590-4593.

982. Bennion M.C., Burch M.A., Dennis D.G., Lech M.E., Neuhaus K., Fendler N.L., Parris M.R., Cuadra J.E., Dixon C.F., Mukosera G.T., Blauch D.N., Hartmann L., Snyder N.L., Ruppel, J.V. Eur. J. Org. Chem. 2019, 2019, 6496-6503.

983. Ogata F., Nagaya T., Maruoka Y., Akhigbe J., Meares A., Lucero M.Y., Satraitis A., Fujimura D., Okada R., Inagaki F., Choyke P.L., Ptaszek M., Kobayashi H. Bioconjugate Chem. 2019, 30, 169-183.

984. Ballatore M.B., Milanesio M.E., Fujita H., Lindsey J.S., Durantini E.N. J. Biophotonics 2020, 13, e201960061.

985. Jiang J., Taniguchi M., Lindsey J.S. New J. Chem. 2015, 39, $4534-4550$

986. Vairaprakash P., Yang E., Sahin T., Taniguchi M., Krayer M., Diers J.R., Wang A., Niedzwiedzki D.M., Kirmaier C., Lindsey J.S., Bocian D.F., Holten D. J. Phys. Chem. B 2015, 119, 4382-4395.

987. Krayer M., Yang E., Diers J.R., Bocian D.F., Holten D., Lindsey J.S. New J. Chem. 2011, 35, 587-601.

988. Fujita H., Jing H., Krayer M., Allu S., Veeraraghavaiah G., Wu Z., Jiang J., Diers J.R., Magdaong N.C.M., Mandal A.K., Roy A., Niedzwiedzki D.M., Kirmaier C., Bocian D.F., Holten D., Lindsey J.S. New J. Chem. 2019, 43, 7209 7232.

989. Zhang S., Lindsey J.S. J. Org. Chem. 2020, 82, 2489-2504.

990. Woodward R.B., Ayer W.A., Beaton J.M., Bickelhaupt F., Bonnett R., Buchschacher P., Closs G.L., Dutler H., Hannah J., Hauck F.P., Itô S., Langemann A., Le Goff E., Leimgruber W., Lwowski W., Sauer J., Valenta Z., Volz H. J. Am. Chem. Soc. 1960, 82, 3800-3802.

991. Woodward R.B. Pure Appl. Chem. 1961, 2, 383-404

992. Liu M., Chen C.-Y., Hood D., Taniguchi M., Diers J.R., Bocian D.F., Holten D., Lindsey J.S. New J. Chem. 2017, 41, 3732-3744.

993. van Straten D., Mashayekhi V., de Bruijn H.S., Oliveira S., Robinson D. J. Cancers (Basel) 2017, 9, 1-54.

994. Agostinis P., Berg K., Cengel K.A., Foster T.H., Girotti A.W., Gollnick S.O., Hahn S.M., Hamblin M.R., Juzeniene A., Kessel D., Korbelik M., Moan J., Mroz P., Nowis D., Piette J., Wilson B.C., Golab J. Cancer J. Clin. 2017, 61, 250-281.

995. Babilas P., Schreml S., Landthaler M., Szeimies R.-M. Photodermatol. Photoimmunol. Photomed. 2010, 26, 118-132.

996. Kharkwal G.B., Sharma S.K., Huang Y.Y., Dai T., Hamblin M.R. Lasers Surg. Med. 2011, 43, 755-767.

997. Allison R.R., Sibata C.H. Photodiagnosis Photodyn. Ther. 2010, 7, 61-75.

998. Knap B., Przystupski D., Saczko J., Ewa K., Knapczop K., Kotli J., Michel O., Kotowski K. Kulbacka J. 2018, 106, 1098-1107.

999. Zhang J., Jiang C., Paulo J., Longo F., Bentes R., Zhang H., Alexandre L. Acta Pharm. Sin. B 2018, 8, 137-146.

1000. Cai L., Gu Z., Zhong J., Wen D., Chen G., He L., Wu J., Gu Z. Drug Discov. Today 2018, 23, 1126-1138.

1001. Yoo J., Park C., Yi G., Lee D., Koo H. Cancers (Basel) 2019, 11,640 .

1002. Chen F., Huang G., Huang H. Future Med. Chem. 2020, 12, 161-171.

1003. Kang B., Opatz T., Landfester K., Wurm F.R. Chem. Soc Rev. 2015, 44, 8301-8325.

1004.Zhao K., Li D., Shi C., Ma X., Rong G., Kang H., Wang X., Sun B. Curr. Drug Deliv. 2016, 13, 494-499.

1005. Lee E., Lee J., Lee I.-H., Yu M., Kim H., Chae S.Y., Jon S. J. Med. Chem. 2008, 51, 6442-6449.

1006. Khatun Z., Nurunnabi M., Reeck G.R., Cho K.J., Lee Y. J. Control. Release 2013, 170, 74-82. 
1007. Hyung Park J., Kwon S., Lee M., Chung H., Kim J.-H., Kim Y.-S., Park R.-W., Kim I.-S., Bong Seo S., Kwon I. C., Young Jeong S. Biomaterials 2006, 27, 119-126.

1008. Park J.H., Cho Y.W., Son Y.J., Kim K., Chung H., Jeong S.Y., Choi K., Park C.R., Park R.-W., Kim I.-S., Kwon I.C. Colloid Polym. Sci. 2006, 284, 763-770.

1009. Voszka I., Galántai R., Maillard P., Csík G. J. Photochem. Photobiol. B Biol. 1999, 52, 92-98.

1010. Kaldapa C., Blais J.C., Carré V., Granet R., Sol V., Guilloton M., Spiro M., Krausz P. Tetrahedron Lett. 2000, 41, 331-335.

1011. Davoust E., Granet R., Krausz P., Carré V., Guilloton M. Tetrahedron Lett. 1999, 40, 2513-2516.

1012. Oulmi D., Maillard P., Guerquin-Kern J.L., Huel C., Momenteau M. J. Org. Chem. 1995, 60, 1554-1564.

1013. Sol V., Blais J.C., Carré V., Granet R., Guilloton M., Spiro M., Krausz P. J. Org. Chem. 1999, 64, 4431-4444.

1014. Kuzmina N.S., Otvagin V.F., Krylova L.V., Nyuchev A.V., Romanenko Y.V., Koifman O.I., Balalaeva I.V., Fedorov A.Y. Mendeleev Commun. 2020, 30, 159-161.

1015. Pandey S.K., Zheng X., Morgan J., Missert J.R., Liu T.H., Shibata M., Bellnier D.A., Oseroff A.R., Henderson B.W., Dougherty T.J., Pandey R.K. Mol. Pharm. 2007, 4, 448-464.

1016. Laville I., Figueiredo T., Loock B., Pigaglio S., Maillard P., Grierson D.S., Carrez D., Croisy A., Blais J. Bioorg. Med. Chem. 2003, 11, 1643-1652.

1017. Laville I., Pigaglio S., Blais J.C., Loock B., Maillard P., Grierson D.S., Blais J. Bioorg. Med. Chem. 2004, 12, 3673-3682.

1018. Bautista-Sanchez A., Kasselouri A., Desroches M.C., Blais J., Maillard P., de Oliveira D.M., Tedesco A.C., Prognon P., Delaire J. J. Photochem. Photobiol. B Biol. 2005, 81, 154-162.

1019. Silva S., Pereira P.M.R., Silva P., Almeida Paz F.A., Faustino M.A.F., Cavaleiro J.A.S., Tomé J.P.C. Chem. Commun. 2012, 48, 3608-3610.

1020. Pereira P.M.R., Silva S., Bispo M., Zuzarte M., Gomes C., Girão H., Cavaleiro J.A.S., Ribeiro C.A.F., Tomé J.P.C., Fernandes R. Bioconjugate Chem. 2016, 27, 2762-2769.

1021. Liu F., Rabinovich G.A. Nat. Rev. Cancer 2005, 5, $29-41$.

1022. Thijssen V.L., Heusschen R., Caers J., Griffioen A.W. Biochim. Biophys. Acta 2015, 1855, 235-247.

1023. Yang F., Zhang Y., Liang H. Int. J. Mol. Sci. 2014, 15, 3580-3595.

1024. D'Auria S., Petrova L., John C., Russev G., Varriale A., Bogoeva V. Mol. Biosyst. 2009, 5, 1331-1336.

1025. Tannock I.F., Rotin D. Cancer Res. 1989, 49, 4373-4384.

1026. Huber V., Camisaschi C., Berzi A., Ferro S., Lugini L., Triulzi T., Tuccitto A., Tagliabue E., Castelli C., Rivoltini L. Semin. Cancer Biol. 2017, 43, 74-89.

1027. Lee E.S., Kim D., Youn Y.S., Oh K.T., Bae Y.H. Angew. Chem. Int. Ed. 2008, 47, 2418-2421.

1028. Biscaglia F., Gobbo M. Pept. Sci. 2018, 110, e24038.

1029. Almeida-Marrero V., Van De Winckel E., Anaya-Plaza E., Torres T., De La Escosura A. Chem. Soc. Rev. 2018, 47, 7369-7400.

1030. Lin Y., Zhou T., Bai R., Xie Y. J. Enzyme Inhib. Med. Chem. 2020, 35, 1080-1099.

1031. Ranyuk E., Cauchon N., Klarskov K., Guérin B., Van Lier J.E. J. Med. Chem. 2013, 56, 1520-1534.

1032. Bullous A.J., Alonso C.M.A., Boyle R.W. Photochem. Photobiol. Sci. 2011, 10, 721-750.

1033. Zhu L., Liu J., Zhou G., Ng H.M., Ang I.L., Ma G., Liu Y., Yang S., Zhang F., Miao K., Poon T.C.W., Zhang X., Yuan Z., Deng C.X., Zhao Q. Chem. Commun. 2019, 55, 14255-14258.

1034. Picarda E., Ohaegbulam K.C., Zang X. Clin. Cancer Res. 2016, 22, 3425-3431.

1035. Teunissen A.J.P., Pérez-Medina C., Meijerink A., Mulder W.J.M. Chem. Soc. Rev. 2018, 47, 7027-7044.
1036. Zheng G., Chen J., Stefflova K., Jarvi M., Li H., Wilson B.C. Proc. Natl. Acad. Sci. U.S.A. 2007, 104, 8989-8994.

1037. Overall C.M., Kleifeld O. Nat. Rev. Cancer 2006, 6, 227239.

1038. Bolze F., Jenni S., Sour A., Heitz V. Chem. Commun. 2017, $53,12857-12877$.

1039. Kobayashi H., Ogawa M., Alford R., Choyke P. L., Urano Y. Chem. Rev. 2010, 110, 2620-2640.

1040. Collins H.A., Khurana M., Moriyama E.H., Mariampillai A., Dahlstedt E., Balaz M., Kuimova M.K., Drobizhev M., Yang V.X.D., Phillips D., Rebane A., Wilson B.C., Anderson H.L. Nat. Photonics 2008, 2, 420-424.

1041. Ke H., Wang H., Wong W.K., Mak N.K., Kwong D.W.J., Wong K.L., Tam H.L. Chem. Commun. 2010, 46, 66786680.

1042. Poon C.T., Chan P.S., Man C., Jiang F.L., Wong R.N.S., Mak N.K., Kwong D.W.J., Tsao S.W., Wong W.K. J. Inorg. Biochem. 2010, 104, 62-70.

1043. Ke M., Chen S., Peng X., Zheng Q., Zheng B., Yeh C., Huang J. Eur. J. Med. Chem. 2017, 127, 200-209.

1044. Abu-Surrah A., Kettunen M. Curr. Med. Chem. 2006, 13, 1337-1357.

1045. Brunner H., Schellerer K.M. Monatsh. Chem. 2002, 133, 679-705.

1046. Lottner C., Bart K. C., Bernhardt G., Brunner H. J. Med. Chem. 2002, 45, 2064-2078.

1047. Lottner C., Knuechel R., Bernhardt G., Brunner H. Cancer Lett. 2004, 203, 171-180.

1048. Bio M., Rajaputra P., Nkepang G., Awuah S.G., Hossion A.M.L., You Y. J. Med. Chem. 2013, 56, 3936-3942.

1049. Rajaputra P., Bio M., Nkepang G., Thapa P., Woo S., You Y. Bioorg. Med. Chem. 2016, 24, 1540-1549.

1050. Bio M., Rajaputra P., Nkepang G., You Y. J. Med. Chem. 2014, 57, 3401-3409.

1051. Thapa P., Li M., Bio M., Rajaputra P., Nkepang G., Sun Y., Woo S., You Y. J. Med. Chem. 2016, 59, 3204-3214.

1052. Zhou X.Q., Meng L.B., Huang Q., Li J., Zheng K., Zhang F.L., Liu J.Y., Xue J.P. ChemMedChem 2015, 10, 304-311.

1053. Tomanová P., Rimpelová S., Jurášek M., Buděšínský M., Vejvodová L., Ruml T., Kmoníčková E., Drašar P. B. Steroids 2015, 97, 8-12.

1054. Tuncel S., Trivella A., Atilla D., Bennis K., Savoie H., Albrieux F., Delort L., Billard H., Dubois V., Ahsen V., Caldefie-Chézet F., Richard C., Boyle R.W., Ducki S., Dumoulin F. Mol. Pharm. 2013, 10, 3706-3716.

1055. Zhao X., Ma H., Chen J., Zhang F., Jia X., Xue J. Eur. J. Med. Chem. 2019, 182, 111625.

1056. Otvagin V.F., Kuzmina N.S., Krylova L.V., Volovetsky A.B., Nyuchev A.V., Gavryushin A.E., Meshkov I.N., Gorbunova Y.G., Romanenko Y.V., Koifman O.I., Balalaeva I.V., Fedorov A.Y. J. Med. Chem. 2019, 62, 11182-11193.

1057. Maisch T., Eichner A., Späth A., Gollmer A., König B., Regensburger J., Bäumler W. PLOS ONE 2014, 1-18.

1058. Wainwright M., Maisch T., Nonell S., Plaetzer K., Almeida A., Tegos G.P., Hamblin M.R. Lancet Infect Dis. 2017, 17(2), e49-e55.

1059. Yao L., Rong Q., Zaat S.A.J., Breukink E., Heger M. J. Clin. Transl. Res. 2015, 1(3), 140-167.

1060. Hamblin M.R. Curr. Opin. Microbiol. 2016, 33, 67-73.

1061. Huang L., Dai T., Hamblin M.R. Antimicrobial Photodynamic Inactivation and Photodynamic Therapy for Infections. In: Photodynamic Therapy. Methods and Protocols (Gomer C.J., Ed.). New York: Springer, 2010. p. 155-174.

1062. Kustov A.V., Smirnova N.L., Berezin M.B. Thermochim. Acta 2011, 521, 224-226.

1063. Kustov A.V., Smirnova N.L., Berezin D.B., Berezin M.B. J. Chem. Thermodyn. 2015, 89, 123-126. 
1064. Berezin D.B., Karimov D.R., Venediktov E.A., Kustov A.V., Makarov V.V., Romanenko Y.V. Macroheterocycles 2015, 8 , 384-388.

1065. Kustov A.V., Smirnova N.L., Berezin D.B., Berezin M.B. J. Chem. Thermodyn. 2015, 83, 104-109.

1066. Kustov A.V., Garas'ko E.V., Belykh D.V., Khudyaeva I.S., Startseva O.M., Makarov V.V., Strel'nikov A.I., Berezin D.B. Usp. Sovrem. Estestvozn. 2016, 12, 263-268 (in Russ.).

1067. Kustov A.V., Belykh D.V., Smirnova N.L., Khudyaeva I.S., Berezin D.B. J. Chem. Thermodyn. 2017, 115, 302-306.

1068. Kustov A.V., Antonova O.A., Smirnova N.L., Khudyaeva I.S., Belykh D.V., Berezin D.B. Thermochim. Acta 2018, 669 , 169-172.

1069. Kustov A.V., Belykh D.V., Smirnova N.L., Venediktov E.A., Kudayarova T.V., Kruchin S.O., Berezin D.B. Dyes Pigm. 2018, 149, 553-559.

1070. Berezin D.B., Kustov A.V., Krestyaninov M.A., Shukhto O.V., Batov D.V., Kukushkina N.V. J. Mol. Liq. 2019, 283, 532-536.

1071. Kustov A.V., Kustova T.V., Belykh D.V., Khudyaeva I.S., Berezin D.B. Dyes Pigm. 2020, 173, 107948.

1072. Berezin D.B., Makarov V.V., Znoyko S.A., Mayzlish V.E., Kustov A.V. Mendeleev Commun. 2020, 30, 621-623.

1073. Kustov A.V., Belykh D.V., Startseva O.M., Kruchin S.O., Venediktov E.A., Berezin D.B. Pharm. Anal. Acta 2016, $7(5), 480-484$.

1074. Yakavets I., Millard M., Zorin V., Lassalle H.-P., Bezdetnaya L. J. Contr. Release 2019, 304, 268-287.

1075. Smith D.A., Van de Waterbeemd H., Walker D.K., Mannhold R., Kubinyi H., Timmerman H. Pharmacokinetics and Metabolism on Drug Design. In: Methods and Principles in Medicinal Chemistry (Mannhold R., Kubinyi H., Timmerman H., Eds.). Weinheim: Wiley-VCH Verlag, 2001. 141 p.

1076. Fromm-Dornieden C., Rembe J.D., Schäfer N., Böhm J., Stuermer E.K. J. Med. Microbiol. 2015, 64, 407-414.

1077. Venediktov E.A., Tulikova E.Yu., Rozhkova E.P., Belykh D.V., Khudyaeva I.S., Berezin D.B. Macroheterocycles 2017, 10, 295-300.

1078. Dabrowski J.M. Adv. Inorg. Chem. 2017, 70, 343-394.

1079. Schwartzberg L.S., Navari R.M. Adv. Ther. 2018, 35, 754-767.

1080. Mahmood M.E., Al-Koofee D.A.F. Global J. Sc. Front. Res. Chem. 2013, 13(4), 1-7.

1081. Kustov A.V., Berezin D.B., Koifman O.I. Antimicrobial and Antiviral Photodynamic Therapy: Mechanisms, Targets and Prospects for Clinical Applications. In: Functional Materials Based on Tetrapyrrole Macrocyclic Compounds (Koifman O.I., Ed.). Moscow: Lenand, 2019. p. 532-581 (in Russ.).

1082. Drulis-Kawa Z., Bednarkiewicz A., Bugla G., Stręk W., Doroszkiewicz W. Adv. Clin. Exp. Med. 2006, 15, 279-283.

1083. Isakau H.A., Parkhats M.V., Knyukshto V.N., Dzhagarov B.M., Petrov E.P., Petrov P.T. J. Photochem. Photobiol. B: Biol. 2008, 92, 165-174.

1084. Hamblin M.R., Hasan T. Photochem. Photobiol. Sci. 2004, 3(5), 436-450

1085. Bertoloni G., Rossi F., Valduga G., Jori G., Ali H., van Lier J.E. Microbios 1992, 71(286), 33-46.

1086. Lambrechts S.A.G., Demidova T.N., Aalders M.C.G. Photochem. Photobiol. Sci. 2005, 4, 503.

1087. Awad M.M., Tovmasyan A., Craik J.D., Batinic-Haberle I., Benov L.T. Appl. Microbiol. Biotechnol. 2016, 100, 7679-7688.

1088. Geynits A.V., Sorokaty A.E., et al. Laser Medicine 2007, 1(3), 45.

1089. Gostishchev V.K. General Surgery. Moscow: GEOTARMEDIA, 2016. 736 p.
1090. Briskin B.S., Proshin A.V., Lebedev V.V., Yakobishvili Ya.I. Infections in Surgery 2003, 1(4), 11.

1091. Hamblin M.R., Abrahamse H. Antibiotics (Basel) 2020, 9(2), E53.

1092. Hamblin M.R., Dai T. Photodiagnosis Photodyn Ther. 2010 , 7(2), 134-136.

1093. Sharma S.K., Dai T., Kharkwal G.B., Huang Y.Y., Huang L., De Arce V.J., Tegos G.P., Hamblin M.R. Curr. Pharm. Des. 2011, 17, 1303-1319.

1094. St Denis T.G., Dai T., Izikson A., Astrakas C., Anderson R.R., Hamblin M.R., Tegos G.P. Virulence 2011, 2, 509520.

1095. Cacaccio J., Durrani F., Cheruku R.R., Borah B., Ethirajan M., Tabaczynski W., Pera P., Missert J.R., Pandey R.K. Photochem. Photobiol. 2020, 96, 625-635.

1096. Jenkins S.V., Srivatsan A., Reynolds K.Y., Gao F., Zhang Y., Heyes C.D., Pandey R.K., Chen J. J. Colloid Interface Sci. 2016, 461, 225-231.

1097. Srivatsan A., Pera P., Joshi P., Wang Y., Missert J.R., Tracy E.C., Tabaczynski W.A., Yao R., Sajjad M., Baumann H., Pandey R.K. Bioorg. Med. Chem. 2015, 23, 3603-17.

1098. Grandi V., Bacci S., Corsi A., Sessa M., Puliti E., Murciano N., Scavone F., Cappugi P., Pimpinelli N. Photodiagn. Photodyn. Therapy 2018, 21, 252-256.

1099. Akhlyustina E.V. J. Physics: Conf. Series 2019, 1189.

1100. Kurochkina A.Yu., Plavsky V.Yu., Yudina N.A. Med. J. 2010, 2, 131-133.

1101. Casas A., Fukuda H., Di Venosa G., Batlle A. Br. J. Cancer 2001, 85, 279-284.

1102. Zeina B., Greenman J., Corry D., Purcell W.M. Br. J. Dermatol. 2002, 146, 568-573.

1103. Stranadko E.F., Kuleshov I.Yu., Karakhan G.I. Laser Medicine 2010, 14(2), 52-56.

1104. Shin F.E., Tolstykh P.I., Stranadko E.F., Solovieva A.B., Ivanov A.V., Eliseenko V.I., Mamantov P.G., Shin E.F., Kuleshov I.Yu. Laser Medicine 2009, 3-4, 55-60.

1105. Malik Z., Ladan H., Nitzan Y., Smetana Z. Antimicrobal and Antiviral Activity of Porphyrin Photosensitation. In: Photodynamic Therapy of Cancer (Jori G., Moan J., Star W., Eds.). Proc. SPIE 2078, 1994. p. 305-312.

1106. Paolo L.R., Segalla A., Bertoloni G., et al. J. Photochem. Photobiol. 2000, 59(1-3), 152-158.

1107. Kato I.T., Prates R.A., Sabino C.P., Fuchs B.B., Tegos G.P., Mylonakis E., Hamblin M.R., Ribeiro M.S., Antimicrob. Agents Chemother. 2013, 57(1), 445-451.

1108. Jori G., Brown S.B. Photochem. Photobiol. Sci. 2004, 3, 403-405.

1109. Dai T., Fuchs B.B., Coleman J.J., Prates R.A., Astrakas C., St. Denis T., Ribeiro M.S., Mylonakis E., Hamblin M.R., Tegos G.P. Front. Microbiol. 2012, 120.

1110. Jori J. J. Environ. Path. Toxcol. Oncol. 2006, 25, 505-519.

1111. Vera D.M., Haynes M.H., Ball A.R., Dai D.T., Astrakas C., Kelso M.J., Hamblin M.R., Tegos G.P. Photochem. Photobiol. 2012, 88, 499-511.

1112. Wilson B.C. In: Handbook of Photonics for Biomedical Science (Tuchin V.V., Ed.). London: CRC Press, Taylor \& Francis Group, 2010. p. 649-686.

1113. Harris F., Pierpoint L. Med. Res. Rev. 2012, 32, 1292-327.

1114. Li X., Guo H., Tian Q., Zheng G., Hu Y., Fu Y., Tan H. J. Surg. Res. 2013, 184(2), 1013-1021.

1115. Wozniak A., Grinholc M. Front Microbiol. 2018, 9, 930.

1116. Grinholc M., Nakonieczna J., Fila G., Taraszkiewicz A., Kawiak A., Szewczyk G., Sarna T., Lilge L., Bielawsk K.P. Appl. Microbiol. Biotechnol. 2015, 99, 4031-4043.

1117. Hamblin M.R. Photochem. Photobiol. 2012, 88, 496-498.

1118. Thota S., Wang M., Jeon S., Maragani S., Hamblin M.R., Chiang L.Y. Molecules 2012, 17, 5225-5243. 
1119. Malik Z., Hanania J., Nitzan Y. J. Photochem. Photobiol. B 1990, 5, 281-293.

1120. Stranadko E.F., Koraboev U.M., Tolstykh M.P. Surgery 2000, 9, 67-70.

1121. Lu Z.R., Ye F., Vaidya A. J. Control. Release 2007, 122, 269-277.

1122. Knop K., Mingotaud A.-F., El-Akra N., Violleau F., Souchard J.-P. Photochem. Photobiol. Sci. 2009, 8, 396-404.

1123. Nitzan Y., Gutterman M., Malik Z., Ehrenberg B. Photochem. Photobiol. 1992, 55, 89-96.

1124. Strakhovskaya M.G., Belenikina N.S., Nikitina V.V., Kovalenko S.Yu., Kovalenko I.B., Averyanov A.V., Rubin A.B., Galochkina T.V. Clinical Practice 2013, 4, 25-30.

1125. Loke W.K., Lau S.K., Yong L.L., Khor E. Sum C.K. J. Biomed. Mater. Res. 2000, 53, 8.

1126. Muzzarelli R.A.A., Morganti P., Morganti G., Palombo P., Palombo M., Biagini G., Belmonte M.M., Giantomassi F., Orlandi F., Muzzarelli C. Carbohydrate Polymers 2007, 70, 27434.

1127. Aoyagi S., Onishi H., Machida Y. Int. J. Pharm. 2007, 330, $138-45$.

1128. Solak E.K., Kaya S. J. Gazi University Health Sciences 2020, 2, 11-17.

1129. Zhientaev T.M., Melik-Nubarov N.S., Litmanovich E.A., Aksenova N.A., Glagolev N.N., Solovieva A.B. Polymer Science, Ser. A 2009, 5, 757-767.

1130. Solovieva A.B., Melik-Nubarov N.S., Zhiyentayev T.M., Tolstih P.I., Kuleshov I.I., Aksenova N.A., Litmanovich E.A., Glagolev N.N., Timofeeva V.A., Ivanov A.V. Laser Physics 2009, 19(4), 1-8.

1131. Solovieva A.B., Glagolev N.N., Ivanov A.V., Konoplyannikov A.G., Melik-Nubarov N.S., Rogovina S.Z., Zhientaev T.M. An Agent for the Treatment of Malignant tumors by the Method of Photodynamic Therapy, 2008, RF patent No. 2314806.

1132. Gorokh Yu.A., Aksenova N.A., Solovieva A.B., Olshevskaya V.A., Zaitsev A.V., Lagutina M.A., Luzgina V.N., Mironov A.F., Kalinin V.N. Russ. J. Phys. Chem., A 2011, $85,871-875$.

1133. Solovieva A.B., Tolstykh P.I., Ivanov A.V., Glagolev N.N., Shinn F.E., Kuleshov I.Yu. A Method of Treating Extensive Purulent Wounds of Soft Tissues, 2010, RF patent No. 2396994.

1134. Huang L., Xuan Y., Koide Y., Zhiyentayev T., Tanaka M., Hamblin M.R. Lasers Surg. Med. 2012, 44, 490-499.

1135. Wagner J.R., Ali H., Langlois R., Brasseur N., van Lier J.E. Photochem. Photobiol. 1987, 45, 587-594.

1136. Aksenova N.A., Oles T., Sarna T., Glagolev N.N., Chernjak A.V., Volkov V.I., Kotova S.L., Melik-Nubarov N.S., Solovieva A.B. Laser Physics 2012, 22, 1642-1649.

1137. Solovieva A.B., Tolstih P.I., Melik-Nubarov N.S., Zhientaev T.M., Kuleshov I.G., Glagolev N.N., Ivanov A.V., Karahanov G.I., Tolstih M.P., Timashev P.S. Laser Phyics 2010, 5, 1068-1074.

1138. Aksenova N.A., Zhientaev T.M., Brilkina A.A., Dubasova L.V., Ivanov A.V., Timashev P.S., Melik-Nubarov N.S., Solovieva A.B. Photonics \& Lasers in Medicine 2013, 2, 189-198.

1139. Zhiyentayev T.M., Boltaev U.T., Solov'eva A.B., Aksenova N.A., Glagolev N.N., Chernjak A.V., Melik-Nubarov N.S. Photochem. Photobiol. 2014, 90, 171-182.

1140. Tsvetkov V.B., Solov'eva A.B., Melik-Nubarov N.S. Phys. Chem. Chem. Phys. 2014, 16, 10903-10913.

1141. Rudenko T.G., Shekhter A.B., Guller A.E., Aksenova N.A., Glagolev N.N., Ivanov A.V., Aboyants R.K., Kotova S.L., Solovieva A.B. Photochem. Photobiol. 2014, 90, 14131422 .
1142. Solovieva A.B., Kardumian V.V., Aksenova N.A., Belovolova L.V., Glushkov M.V., Bezrukov E.A., Sukhanov R.B., Kotova S.L., Timashev P.S. Sci. Rep. 2018, 8, 8042.

1143. Fontana C.R., dos Santos D.S. Jr., Bosco J.M., Spolidorio D.M., Marcantonio R.A. Drug Deliv. 2008, 15, 417-422.

1144. Aksenova N.A., Timofeeva V.A., Rogovina S.Z., Timashev P.S., Glagolev N.N., Solovieva A.B. Polymer Science, Ser. B 2010, 52, 122-128.

1145. Glagolev N.N., Rogovina S.Z., Solov'eva A.B., Aksenova N.A., Kotova S.L. Russ. J. Phys. Chem. 2006, 80, Suppl. 1., S72-S76.

1146. Kardumyan V.V., Aksenova N.A., Chernyak A.A., Glagolev N.N., Volkov V.I., Solovieva A.B. Laser Phys. 2015, 25, 6002.

1147. Solovieva A.B., Spokoiny A.L., Rudenko T.G., Shekhter A.B., Glagolev N.N., Aksenova N.A. Clinical Practice 2016, $2,45-49$.

1148. Tolstykh P.I., Shin F.E., Tamrazova O.B., Derbenev V.A., Kuleshov I.Yu., Solovieva A.B., Vasyagin S.N. Military Medical J. 2010, 8, 41.

1149. Rushai A.K., Makarenko A.V., Bodachenko K.A., Kolosova T.A. Clinical Medicine 2013, 14, 101-104.

1150. Drexler H. Cardiovasc Res. 1999, 43, 572-579.

1151. Shumaev K.B., Gubkin A.A., Gubkina S.A., Gudkov L.L., Sviryaeva I.V., Timoshin A.A., Topunov A.F., Vanin A.F., Ruuge E.T.O. Biophysics 2006, 51, 472-477.

1152. Santos C.X.C., Anilkumar N., Zhang M., Brewer A.C., Shah A.M. Free Radical Biology \& Medicine 2011, 50, 777793.

1153. Murray J., Taylor S.W., Zhang B., Ghosh S.S., Capaldi R.A. J. Biol. Chem. 2003, 278, 37223-37230.

1154. Vanin A.F. Biochemistry 1998, 63, 924-938.

1155. Mironov A.F. Photodynamic Therapy for Cancer. In: Advances in Porphyrin Chemistry. Vol. 1 (Golubchikov O.A., Ed.). SPb.: St-PbGU, 1997. p. 357-374.

1156. Solovieva A.B., Vanin A.F., Shekhter A.B., Glagolev N.N., Aksenova N.A., Mikoyan V.D., Kotova S.L., Rudenko T.G., Fayzullin A.L., Timashev P.S. Nitric Oxide 2019, 83, 24-32.

1157. Davies A.G. Tin Organometallics. In: Comprehensive Organometallic Chemistry. Vol. 3. London: Elsevier, 2007. p. 809-883.

1158. Desai A., Mitchison T.J. Ann. Rev. Cell. Dev. Biol. 1997, 13, $83-117$.

1159. Milaeva E.R., Shpakovsky D.B., Gracheva Y.A., Antonenko T.A., Osolodkin D.I., Palyulin V.A., Shevtsov P.N., Neganova M.E., Vinogradova D.V., Shevtsova E.F. J. Organomet. Chem. 2015, 782, 96-102.

1160. Milaeva E., Petrosyan V., Berberova N., Pimenov Y., Pellerito L. Bioinorg. Chem. Appl. 2004, 18, 69-91.

1161. Benedetti M, Giuliani M.E., Regoli F. Ann. NY Acad. Sci. 2015, 1340, 8-19.

1162. Aschner M., Aschner J.L. Neurosc. Biobehav. Rev. 1992, 16, 427-435.

1163. Milaeva E.R., Tyurin V.Yu., Gracheva Yu.A., Dodochova M.A., Pustovalova L.M., Chernyshev V.N. Bioinorg. Chem. Appl. 2006, Article ID 64927, 1-5.

1164. Tyurin V.Yu., Zhang J., Glukhova A.P., Milaeva E.R. Macroheterocycles 2011, 4, 211-212.

1165. Milaeva E.R., Tyurin V.Yu., Shpakovsky D.B., Gerasimova O.A., Zhang J., Gracheva Yu.A. Heteroatom Chem. 2006, $17,475-480$

1166. Forman H.J., Zhang H., Rinna A. Mol. Aspects Med. 2009 , $30,1-12$.

1167. Cappellini M.D., Fiorelli G. Lancet 2008, 371, 9606, 64-74.

1168. Levy H.R., Christoff M. Biochem J. 1983, 214, 959-965.

1169. Milgrom L.R., Jones C.C., Harriman A. J. Chem. Soc. Perkin Trans. 1988, 2, 71-79. 
1170. Gerbec E.N., Messing R.D., Starber S.B. Brain Res. Bull. 1988, 346-351.

1171. Steckelbroeck S., Stoffel-Wagner B., Reichelt R., Schramm J., Bidlingmaier F., Siekmann L., Klingmuller D. J. Neuroendocrinol. 1999, 11, 457-464.

1172. Pashkov A.N., Popov S.S., Semenikhina A.V., Rakhmanova T.I. Bull. Experim. Biol. Med. 2005, 139, 520-524.

1173. Sedlak J., Lindsey R.H. Anal. Biochem. 1968, 2, 192-205.

1174. Geloso M.C., Corvino V., Cavallo V., Toesca A., Guadagni E., Passalacqua R., Michetti F. Neurosci. Lett. 2004, 357, 103-107.

1175. Kook S.C., Wong K., Ng Meng, Kumar Das V.G. Appl. Organomet. Chem. 1991, 5, 409-415.

1176. Bosch B.J., van der Zee R., de Haan C.A.M., Rottier P.J.M. J. Virol. 2003, 77, 8801-8811.

1177. Lu R., Zhao X., Li J., et al. The Lancet 2020, 395, 565-574.

1178. Hoffmann M., Kleine-Weber H., Schroeder S., et al. Cell 2020, 2, 271-280.

1179. Xu X., Chen P., Wang J., Feng J., et al. Science China Life Sciences 2020, 63, 457-460.

1180. Wan Y., Shang J., Graham R., et al. J. Virol. 2020, 94, e00127-20.

1181. Zhang H., Penninger J.M., Li Y., Zhong N., Slutsky A.S. Intensive Care Medicine 2020, 46, 586-590.

1182. Yan R., Zhang Y., Li Y., Xia L., Guo Y., Zhou Q. Science 2020, 361, 1444-1448.

1183. Zhao Y., Zhao Z., Wang Y., Zhou Y., Ma Y., Zuo W. Am. J. Respir. Critical Care Med. 2020, 202, 756-759.

1184. Coutard B., Valle C., de Lamballerie X., Canard B., Seidah N.G., Decroly E. Antiviral Res. 2020, 176(104742), 1-5.

1185. Matsuyama S., Nao N, Shirato K., et al. Proc. Nat. Acad. Sci. USA 2020, 117, 7001-7003.

1186. Kawase M., Shirato K., van der Hoek L., Taguchi F., Matsuyama S. J. Virol. 2012, 86, 6537-6545.

1187. Zhou Y., Vedantham P., Lu K., et al. Antiviral Res. 2015, 116, $76-84$.

1188. Kuba K., Imai Y., Rao S., et al. Nat. Med. 2005, 11, 875-879.

1189. Monteil V., Kwon H., Prado P., et al. Cell 2020, 181, 905-913.

1190. Gheblawi M., Wang K., Viveiros A., et al. Circ. Res. 2020, $126,1456-1474$.

1191. Jiang S., Hillyer C., Du L. Trends in Immunology 2020, 5, 355-359.

1192. Wang C., Li W., Drabek D., et al. Nat. Commun. 2020, 11, 2251.

1193. Widjaja I., Wang C., Haperen R., et al. Emerg. Microbes Infect. 2019, 8, 516-530.

1194. Hwang W.C., Lin Y., Santelli E., et al. J. Biol. Chem. 2006, 281, 34610-34616.

1195. Pedersen S.F., Ho Y.-C. J. Clin. Invest. 2020, 130, 2202-2205.

1196. Woo Y.L., Kamarulzaman A., Augustin Y., Staines H., Altice F. J. Petrol. 2020. DOI: 10.31219/osf.io/mxsvw.

1197. Bussalino E., Maria A. De, Russo R., et al. Am. J. Transplant. 2020, 7, 1922-1924.

1198. Sarzi-Puttini P., Giorgi V., Sirotti S., Marotto D., et al. Clinical and Experimental Rheumatology 2020, 38, 337-342.

1199. Wu C., Liu Y., Yang Y., et al. Acta Pharm. Sin. B 2020, 10, 766-788.

1200. Smith M., Smith J. ChemRxiv 2020. DOI: 10.26434/chemrxiv.11871402.

1201. Lebedeva N., Popova T., Kozbial M., et al. J. Porphyrins Phthalocyanines 2011, 15, 223-229.

1202. Lebedeva N.S., Gubarev Y.A., Koifman O.I. Mendeleev Commun. 2015, 25, 307-309.

1203. Lebedeva N., Malkova E., Vyugin A. Koifman O., Gubarev Y. Biochip J. 2016, 10, 1-8.

1204. Walls A.C., Park Y.J., Tortorici M.A., et al. Cell 2020, 181, 281-292.
1205. Frisch M.J., Trucks G.W., Schlegel H.B., et al. Gaussian Inc., Wallingford 2013, 121, 150-166.

1206. Trott O., Olson A.J. J. Comput. Chem. 2010, 31, 455-461.

1207. Syrbu S.A., Semeikin A.S., Berezin B.D., Koifman O.I. Khim. Geterotsikl. Soed. [Chem. Heterocycl. Compds.] 1989, 10, 1373-1377.

1208. Koifman O.I., Ponomarev G.V., Syrbu S.A., Zharov E.V., Sergeeva T.V., Lukovkin A.V., 2014, Patent RF No. 2535097.

1209. Kruper W.J., Chamberlin Jr.T.A., Kochanny M. J. Org. Chem. 1989, 11, 2753-2756.

1210. Sharma K.K., Mandloi M., Rai N., Jain R. RSC Adv. 2016, 6 , 96762-96767.

1211. van den Tempel N., Horsman M.R., Kanaar R. Int. J. Hyperthermia 2016, 32, 446-454.

1212. Mallory M., Gogineni E., Jones G.C., Greer L., Simone II C.B. Crit. Rev. Oncol. Hematol. 2016, 97, 56-64.

1213. Kumar V., Marin-Navarro J., Shukla P. World J. Microbiol. Biotechnol. 2016, 32, 34.

1214. Issels R.D., Lindner L.H., Verweij J., Wessalowski R., Reichardt P., Wust P., Ghadjar P., Hohenberger P., Angele M., Salat C. JAMA Oncology 2018, 4, 483-492.

1215. Cihoric N., Tsikkinis A., van Rhoon G., Crezee H., Aebersold D.M., Bodis S., Beck M., Nadobny J., Budach V., Wust P. International Journal of Hyperthermia 2015, 31, 609-614.

1216. Matsumura M., Signor G., Matthews B.W. Nature 1989, 342, 291.

1217. Zhang S., Zhang K., Chen X., Chu X., Sun F., Dong Z. Biochem. Biophys. Res. Commun. 2010, 395, 200-206.

1218. Joo J.C., Pack S.P., Kim Y.H., Yoo Y.J. J. Biotechnol. 2011, $151,56-65$.

1219. Ahern T.J., Casal J.I., Petsko G.A., Klibanov A.M. Proc. Nat. Acad. Sci. 1987, 84, 675-679.

1220. Buß O., Rudat J., Ochsenreither K. Comput. Struct. Biotech. J. 2018, 16, 25-33.

1221. Glick B.R., Pasternak J.J., Patten C.L. Molecular Biotechnology: Principles and Applications of Recombinant DNA, $4^{\text {th }}$ ed, Washington, DS:ASM PRESS, 2010. $1000 \mathrm{p}$.

1222. Wlodarczyk S.R., Custódio D., Pessoa Jr.A., Monteiro G. Eur. J. Pharm. Biopharm. 2018, 131, 92-98.

1223. Fields P.A. Comp. Biochem. Physiol. Part A: Molecular \& Integrative Physiology 2001, 129, 417-431.

1224. Vieille C., Zeikus G.J. Microbiol. Mol. Biol. Rev. 2001, 65, $1-43$.

1225. Bonch-Osmolovskaya E., Miroshnichenko M., Kostrikina N., Chernych N., Zavarzin G. Arch Microbiol. 1990, 154, 556-559.

1226. Haney P.J., Badger J.H., Buldak G.L., Reich C.I., Woese C.R., Olsen G.J. Proc. Nat. Acad. Sci. 1999, 96, 3578-3583.

1227. Chakravarty S., Varadarajan R. FEBS Lett. 2000, 470, 65-69.

1228. Empadinhas N., da Costa M.S. Int. Microbiol. 2006, 9, 199-206.

1229. Kaur P., Ghai N., Sangha M.K. African J. Biotech. 2009, 8, 619-625.

1230. Bursy J., Kuhlmann A.U., Pittelkow M., Hartmann H., Jebbar M., Pierik A.J., Bremer E. Appl. Environ. Microbiol. 2008, 74, 7286-7296.

1231. Fischer D., Geyer A., Loos E. The FEBS J. 2006, 273, 137-149.

1232. Faria T.Q., Mingote A., Siopa F., Ventura R., Maycock C., Santos H. Carbohydr. Res. 2008, 343, 3025-3033.

1233. Grigoryan K., Markarian S., Aznauryan M. Problems of Cryobiology and Cryomedicine 2009, 19, 3-10.

1234. Timasheff S.N. Annu. Rev. Biophys. Biomol. Struct. 1993, 22, 67-97.

1235. Timasheff S.N. Biochemistry 2002, 41, 13473-13482.

1236. Liu Y., Bolen D. Biochemistry 1995, 34, 12884-12891. 
1237. Michels J.J., Fiammengo R., Timmerman P., Huskens J., Reinhoudt D.N. J. Inclusion Phenom. Macrocycl. Chem. 2001, 41, 163-172.

1238. Raffaini G., Ganazzoli F. J. Inclusion Phenom. Macrocycl. Chem. 2013, 76, 213-221.

1239. Arakawa T., Bhat R., Timasheff S.N. Biochemistry 1990, 29, 1914-1923.

1240. Barone G., Capasso S., Del Vecchio P., De Sena C., Fessas D., Giancola C., Graziano G., Tramonti P. J. Therm. Anal. Calorim. 1995, 45, 1255-1264.

1241. Barone G., Giancola C., Verdoliva A. Thermochim. Acta 1992, 199, 197-205.

1242. Farruggia B., Rodriguez F., Rigatuso R. J. Protein Chem. 2001, 20, 81-89.

1243. Lebedeva N., Malkova E., Gubarev Y., V’yugin A., Borisov A. Int. J. Org. Chem. 2013, 3, 225-228.

1244. Wang S.-L., Lin S.-Y., Li M.-J., Wei Y.-S., Hsieh T.-F. Biophys Chem. 2005, 114, 205-212.

1245. Itoh T., Wada Y., Nakanishi T. Agric. Biol. Chem. 1976, 40, 1083-1086.

1246. Baier S.K., McClements D.J. J. Agric. Food Chem. 2003, 51, 8107-8112.

1247. Yamasaki M., Yano H., Aoki K. Int. J. Biol. Macromol. 1991, 13, 322-328.

1248. Baier S.K., McClements D.J. Food Res Int. 2003, 36, 1081-1087.
1249. Michnik A. J. Therm. Anal. Calorim. 2003, 71, 509519.

1250. Tankovskaia S.A., Abrosimova K.V., Paston S.V. J. Mol. Struct. 2018, 1171, 243-252.

1251. Michnik A., Drzazga Z. J. Therm. Anal. Calorim. 2007, 88, 449-454.

1252. Das A., Basak P., Pattanayak R., Kar T., Majumder R., Pal D., Bhattacharya A., Bhattacharyya M., Banik S. P. Int. J. Biol. Macromol. 2017, 105, 645-655.

1253. Samanta N., Mahanta D.D., Hazra S., Kumar G.S., Mitra R.K. Biochimie 2014, 104, 81-89.

1254. Baier S., McClements D.J. J. Agric. Food Chem. 2001, 49, 2600-2608.

1255. Platts L., Falconer R.J. Int. J. Pharm. 2015, 486, 131-135.

1256. Shil S., Das N., Sengupta B. Sen P. ACS Omega 2018, 3, 16633-16642.

1257. Dasgupta M., Kishore N. PloS one 2017, 12, e0172208.

1258. Lebedeva N.S., Gubarev Y.A., Lyubimtsev A.V., Yurina E.S., Koifman O.I. Macroheterocycles 2017, 10, 37-42.

1259. Lebedeva N.S., Gubarev Y.A., Yurina E.S., Syrbu S.A. J. Mol. Liq. 2018, 265, 664-667.

1260. Lebedeva N.S., Malkova E., Popova T., Kutyrev A., Syrbu S., Parfenyuk E., Vyugin A. Spectrochim. Acta Part A: Mol. Biomol. Spectrosc. 2014, 118, 395-398.

1261. Lebedeva N.S., Yurina E.S., Guseinov S.S., Gubarev Y.A, Syrbu S.A. Dyes Pigm. 2019, 162, 266-271.

\section{Cite as:}

Koifman O.I., Ageeva T.A., Beletskaya I.P., Averin A.D., Yakushev A.A., Tomilova L.G., Dubinina T.V., Tsivadze A.Yu., Gorbunova Yu.G., Martynov A.G., Konarev D.V., Khasanov S.S., Lyubovskaya R.N., Lomova T.N., Korolev V.V., Zenkevich E.I., Blaudeck T. , Ch. von Borczyskowski, Zahn D.R.T., Mironov A.F., Bragina N.A., Ezhov A.V., Zhdanova K.A., Stuzhin P.A., Pakhomov G.L., Rusakova N.V., Semenishyn N.N., Smola S.S., Parfenyuk V.I., Vashurin A.S., Makarov S.V., Dereven’kov I.A., Mamardashvili N.Zh., Kurtikyan T.S., Martirosyan G.G., Burmistrov V.A., Aleksandriiskii V.V., Novikov I.V., Pritmov D.A., Grin M.A., Suvorov N.V., Tsigankov A.A., Fedorov A.Yu., Kuzmina N.S., Nyuchev A.V., Otvagin V.F., Kustov A.V., Belykh D.V., Berezin D.B., Solovieva A.B., Timashev P.S., Milaeva E.R., Gracheva Yu.A., Dodokhova M.A., Safronenko A.V., Shpakovsky D.B., Syrbu S.A., Gubarev Yu.A., Kiselev A.N., Koifman M.O., Lebedeva N.Sh., Yurina E.S. Macroheterocyclic Compounds - a Key Building Block in New Functional Materials and Molecular Devices Macroheterocycles 2020, 13, 311-467, DOI: $10.6060 / \mathrm{mhc} 200814 \mathrm{k}$ 